\title{
OAK RIDGE RESERVATION ENVIRONMENTAL REPORT FOR 1989
}

\section{VOLUME 2: DATA PRESENTATION}

Project director

F. C. Kornegay

Project coordinator

D. C. West

Technical coordinators
S. T. Goodpasture
C. W. Kimbrough
M. F. Tardiff

Coordinating editors
V. A. Jacobs
A. R. Wilson

Date Published: October 1990

\section{Prepared by \\ Office of Environmental Compliance Documentation MARTIN MARIETTA ENERGY SYSTEMS, INC. \\ and \\ Environmental Management Staff}

Oak Ridge Y-12 Plant, Oak Ridge National Laboratory, and Oak Ridge Gaseous Diffusion Plant MARTIN MARIETTA ENERGY SYSTEMS, INC.

P.O. Box 2008

Oak Ridge, Tennessee 37831 for the

U.S. DEPARTMENT OF ENERGY Under Contract No. DE-AC05-840R21400

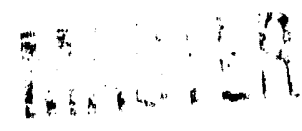




\section{CONTENTS}

1. INTRODUCTION ................................... I

2. ENVIRONMENTAL MONITORING SUMMARY $\ldots \ldots \ldots \ldots \ldots \ldots \ldots \ldots \ldots$

2.1 AIRBORNE DISCHARGES, AMBIENT AIR MONITORING, AND METEOROLOGICAL MONITORING ...................... 9

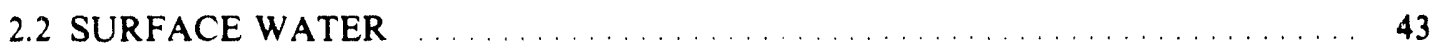

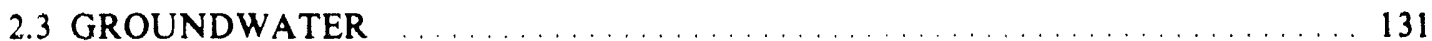

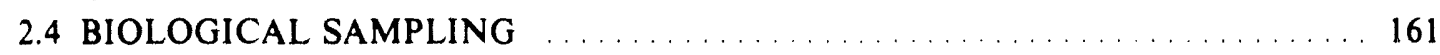

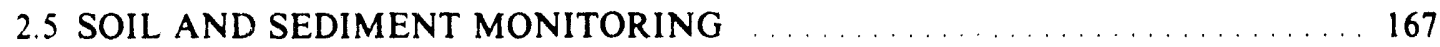

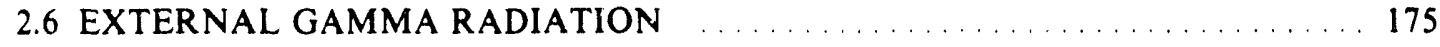

3. POTENTIAL RADIATION AND CHEMICAL DOSE TO THE PUBLIC $\ldots \ldots \ldots 179$

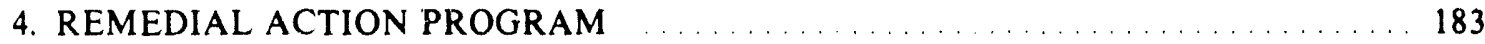

5. SOLID WASTE MANAGEMENT PROGRAM $\ldots \ldots \ldots \ldots \ldots \ldots \ldots \ldots \ldots \ldots \ldots \ldots$

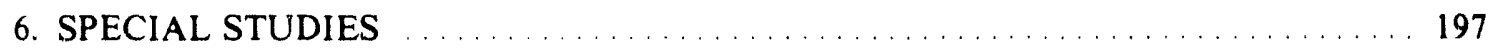

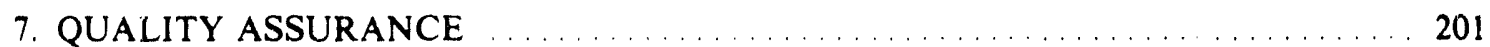




\section{INTRODUCTION}




\section{INTRODUCTION AND GENERAL INFORMATION}

The first two volumes of this report are devoted to a presentation of environmental data and supporting narratives for the U.S. Department of Energy's (DOE's) Oak Ridge Reservation (ORR) and surrounding environs during 1989. Volume 1 includes all narrative descriptions, summaries, and conclusions and is intended to be a "stand-alone" report for the ORR for the reader who does not want to review in detail all of the
1989 data. Volume 2 includes the detailed data summarized in a format to ensure that all environmental data are represented in the tables. Narratives are not included in Vol. 2. The tables in Vol. 2 are addressed in Vol. 1. For this reason, Vol. 2 cannot be considered a stand-alone report but is intended to be used in conjunction with Vol. 1. 


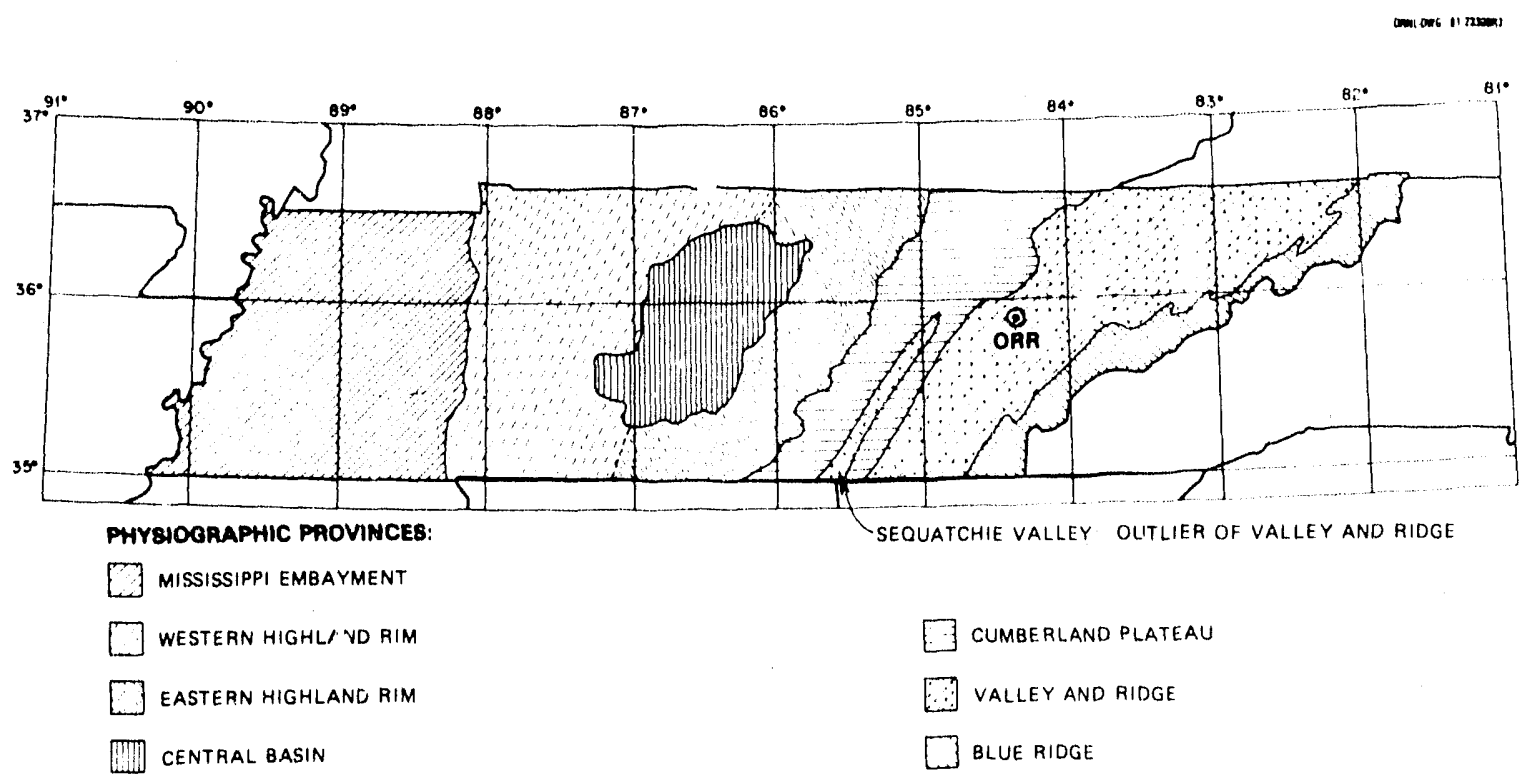

Fig. 1.3.1. Physiographic map of Tennessee.

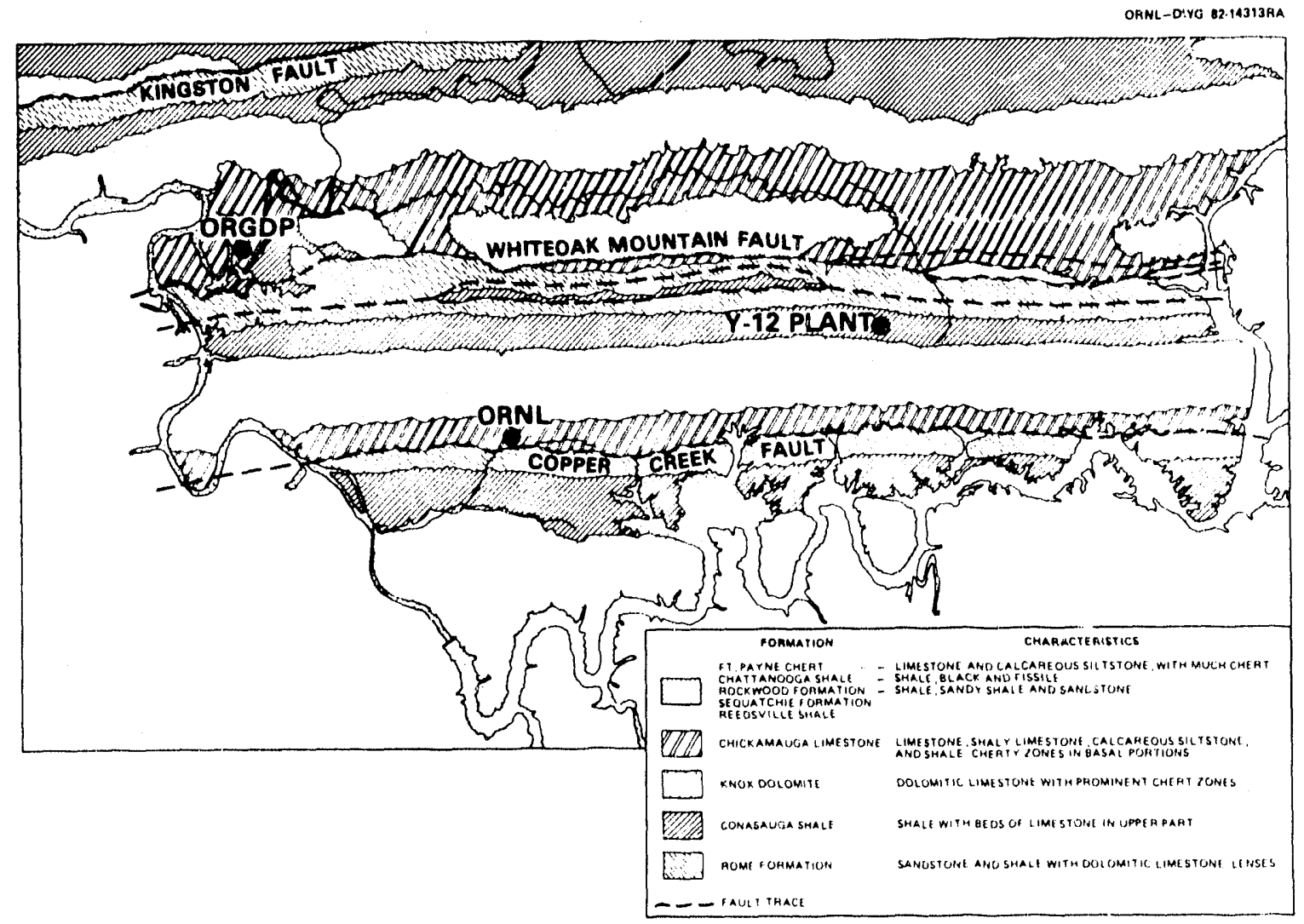

Fig. 1.3.2. Geologic map of the Department of Energy's Oak Ridge Reservation. 
Table 1.1.1. Administrative ${ }^{a}$ units on the ORR in 1989

\begin{tabular}{|c|c|c|}
\hline \multirow{2}{*}{ Description } & \multicolumn{2}{|c|}{ Area } \\
\hline & Hectares & Acres \\
\hline Oak Ridge Reservation ${ }^{b}$ & 12,684 & 31,343 \\
\hline Oak Ridge Y-12 Plant & 328 & 811 \\
\hline Oak Ridge National Laboratory & 445 & 1,100 \\
\hline Oak Ridge Gaseous Diffusion Plant & 688 & 1,700 \\
\hline Oak Ridge Associated Universities ${ }^{c}$ & 121 & 298 \\
\hline Total & 14,266 & 35,252 \\
\hline
\end{tabular}

${ }^{a}$ Administrative units are those units that are managed by a major installation or by central Energy Systems.

${ }^{6}$ The Oak Ridge Reservation actually encompasses all of the contiguous land owned by DOE in the Oak Ridge Area; however, as an "administrative unit," it is all of the land area not controlled by the other units. Each unit includes some land outside the designated fenced area. The total combined fenced area of the three major facilities is 810 ha (2000 acres).

'Oak Ridge Associated Universities manages the Scarboro Facility, as well as, other facilities within the Oak Ridge area. 
Table 1.2.1. Populations of central East

Tennessee towns"

Town/city Population

Anderson County

\begin{tabular}{lr} 
Clinton & 5,245 \\
Lake City & 2,335 \\
Norris & 1,374 \\
Oak Ridge r r r & 27,662 \\
Oliver Springs & 3,600 \\
\multicolumn{2}{c}{ Blount County } \\
Friendsville & 694 \\
Alcoa & 6,870 \\
Maryville & 17,478 \\
\multicolumn{2}{c}{ Knox County }
\end{tabular}

Knoxville $\quad 183,139$

Loudon County

Greenback $\quad 546$

Lenoir City $\quad 5,446$

Loudon $\quad 3,940$

Morgan County

Wartburg

Roane County

$\begin{array}{ll}\text { Harriman } & 8,303 \\ \text { Kingston } & 4,441\end{array}$

Rockwood $\quad 5,767$

Sevier County

Sevierville $\quad 4,566$

Union County

Luttrell 962

Maynardville $\quad 924$

Campbell County

$\begin{array}{ll}\text { Caryville } & 2,039 \\ \text { Jellico } & 2,769 \\ \text { Jacksboro } & 1,620 \\ \text { LaFollette } & 8,176\end{array}$

"Source: 1980 Census of Population, U.S. Depariment of Commerce, Bureau of the Census. 


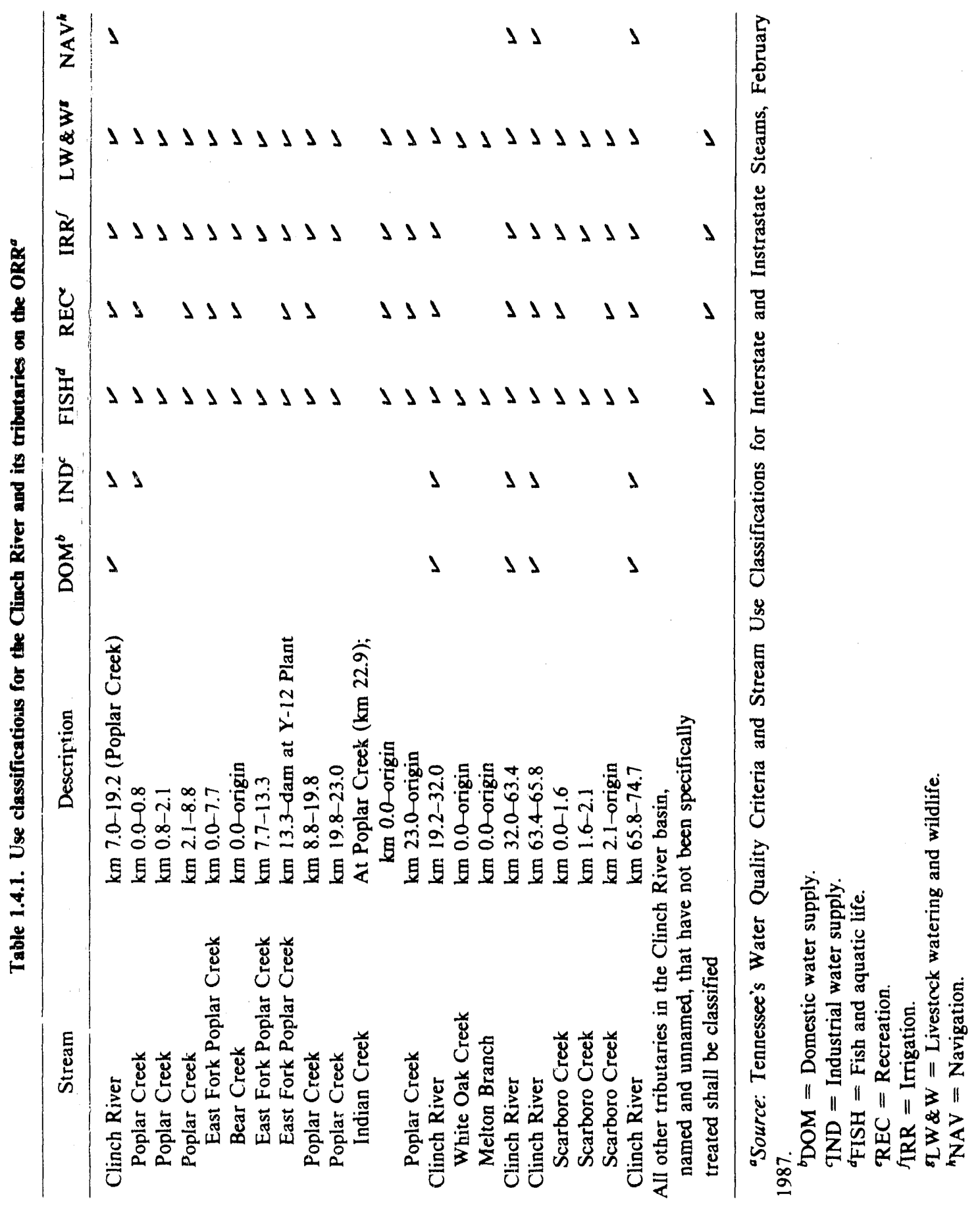


2. ENVIRONMENTAL MONITORING SUMMARY

\author{
2.1 AIRBORNE DISCHARGES, \\ AMBIENT AIR MONITORING, \\ AND METEOROLOGICAL MONITORING
}




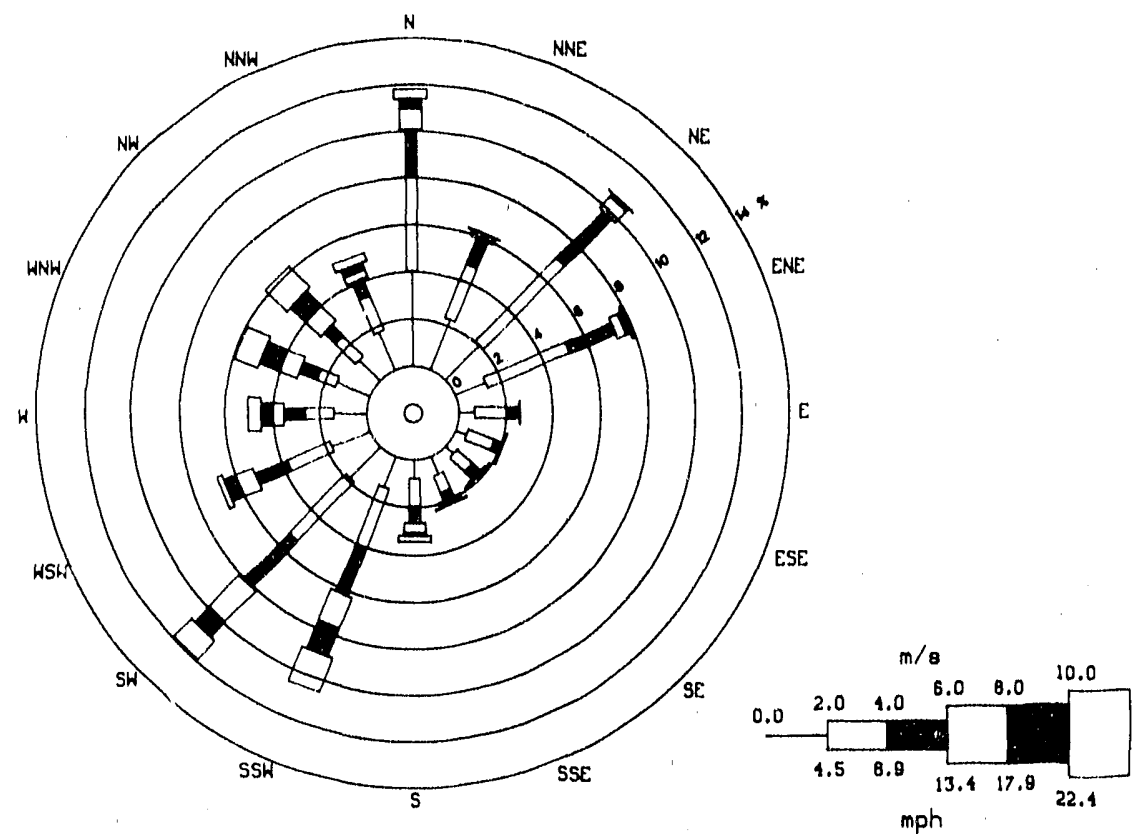

Fig. 2.1.1. 1989 Wind rose for ORGDP tower MT1 (10-m level), with 85.5\% of possible data.

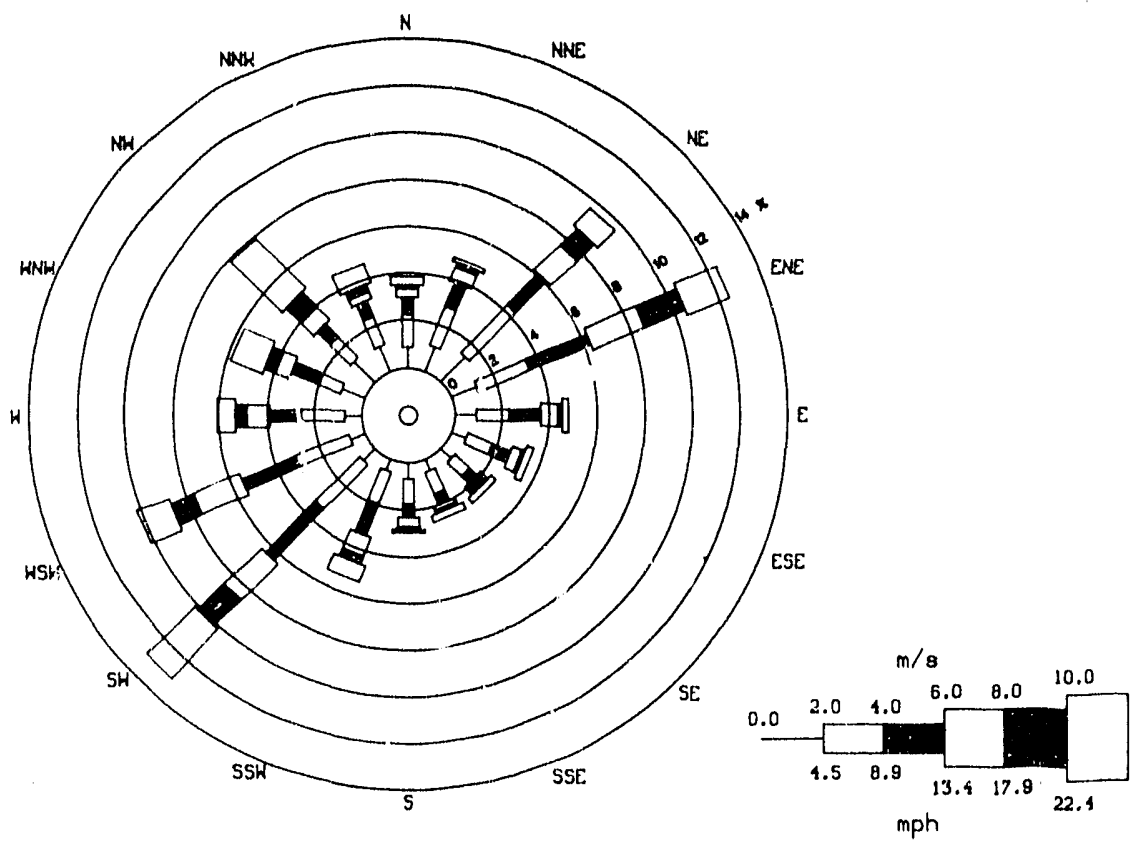

Fig. 2.1.2. 1989 Wind rose for ORGDP tower MT1 (60-m level), with $88.3 \%$ of possible data. 
ORNII-DWU 90-5277

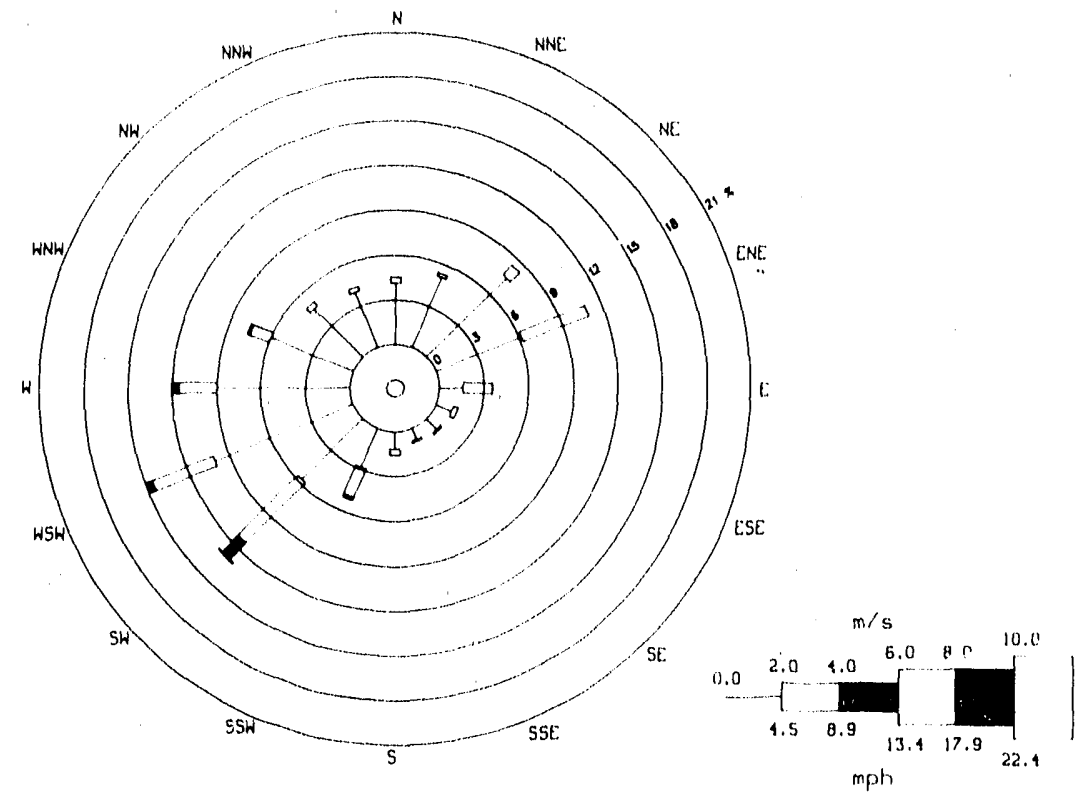

Fig. 2.1.3. 1989 Wind rose for ORNL tower MT2 $(10-\mathrm{m}$ level), with $71.5 \%$ of possible data.

ORNLIDWG 90-5278

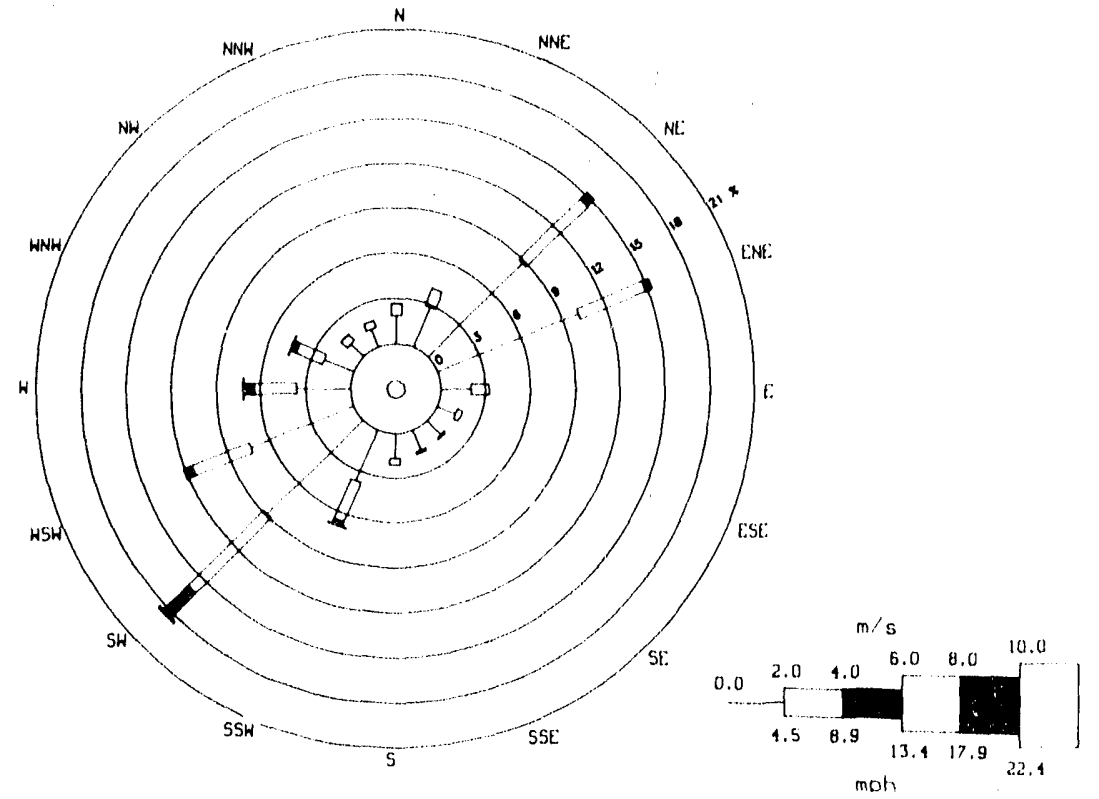

Fig. 2.1.4. 19, Wind rose for ORNL tower MT2 (30-m level), with $71.2 \%$ of possible data. 


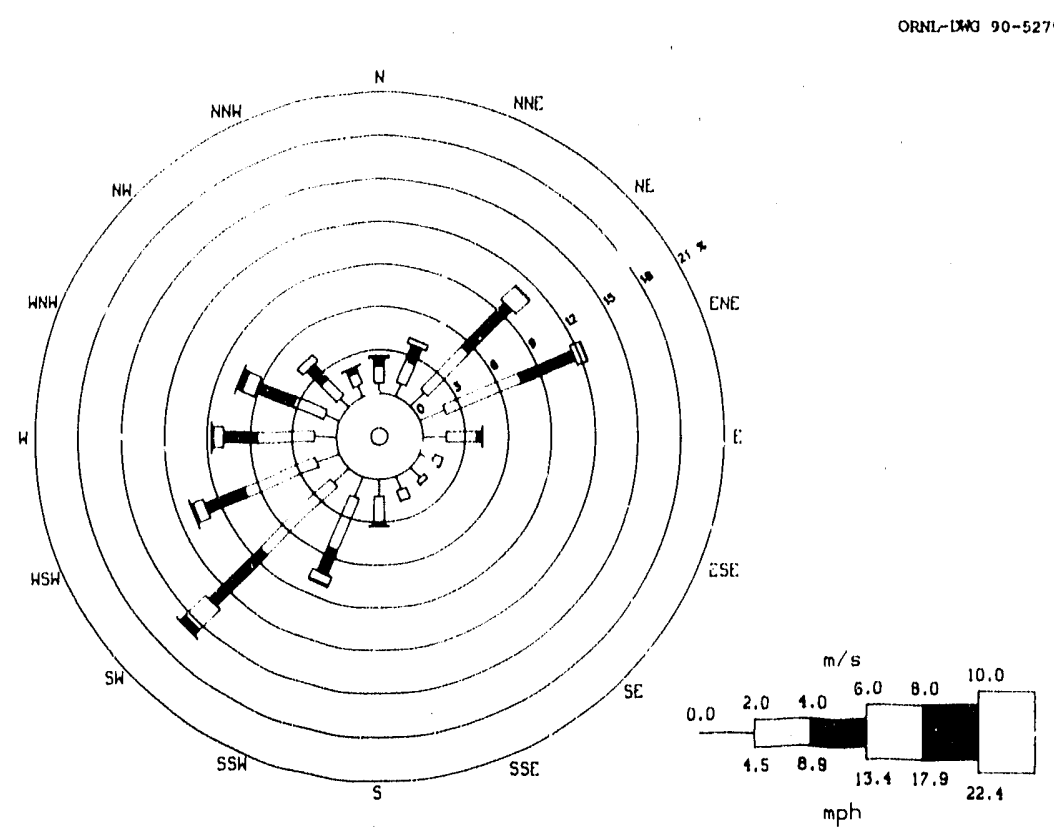

Fig. 2.1.5. 1989 Wind rose for ORNL tower MT2 (100-m-level), with $75.0 \%$ of possible data.

ORNII-DWG 90-5275

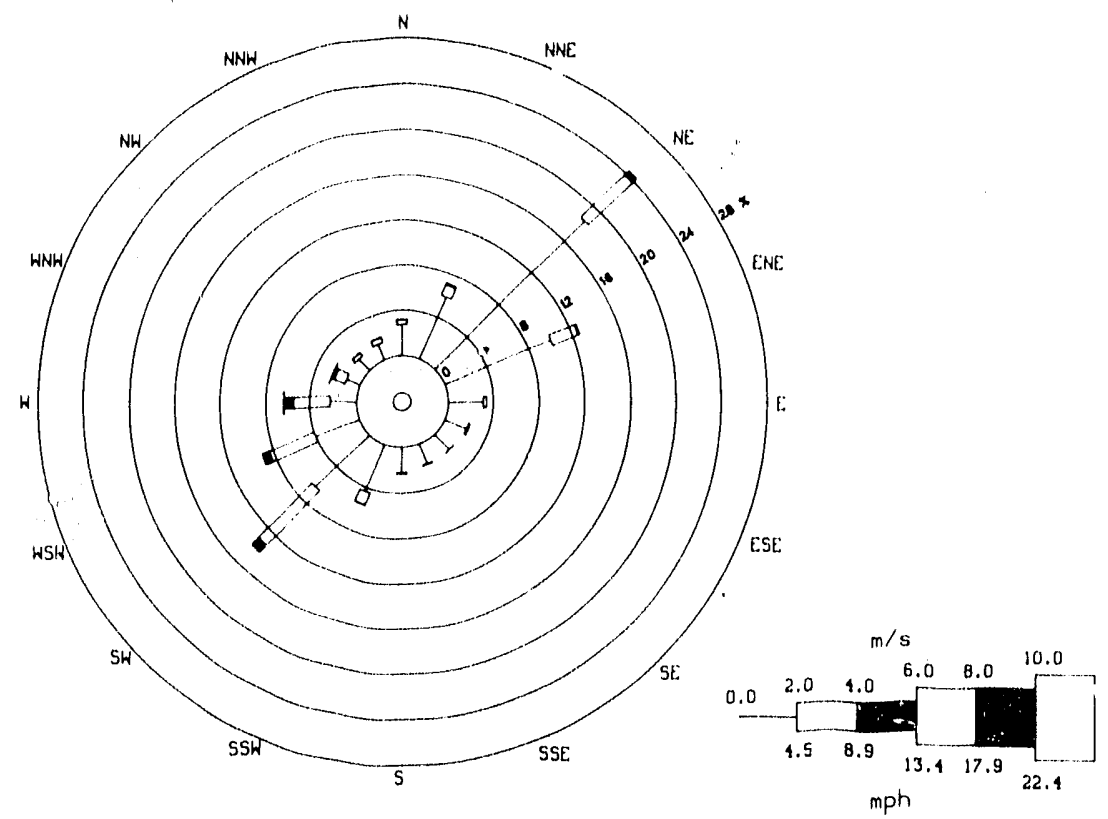

Fig. 2.1.6. 1989 Wind rose for ORNL tower MT3 $(10-\mathrm{m}$ level), with $82.7 \%$ of possible data. 


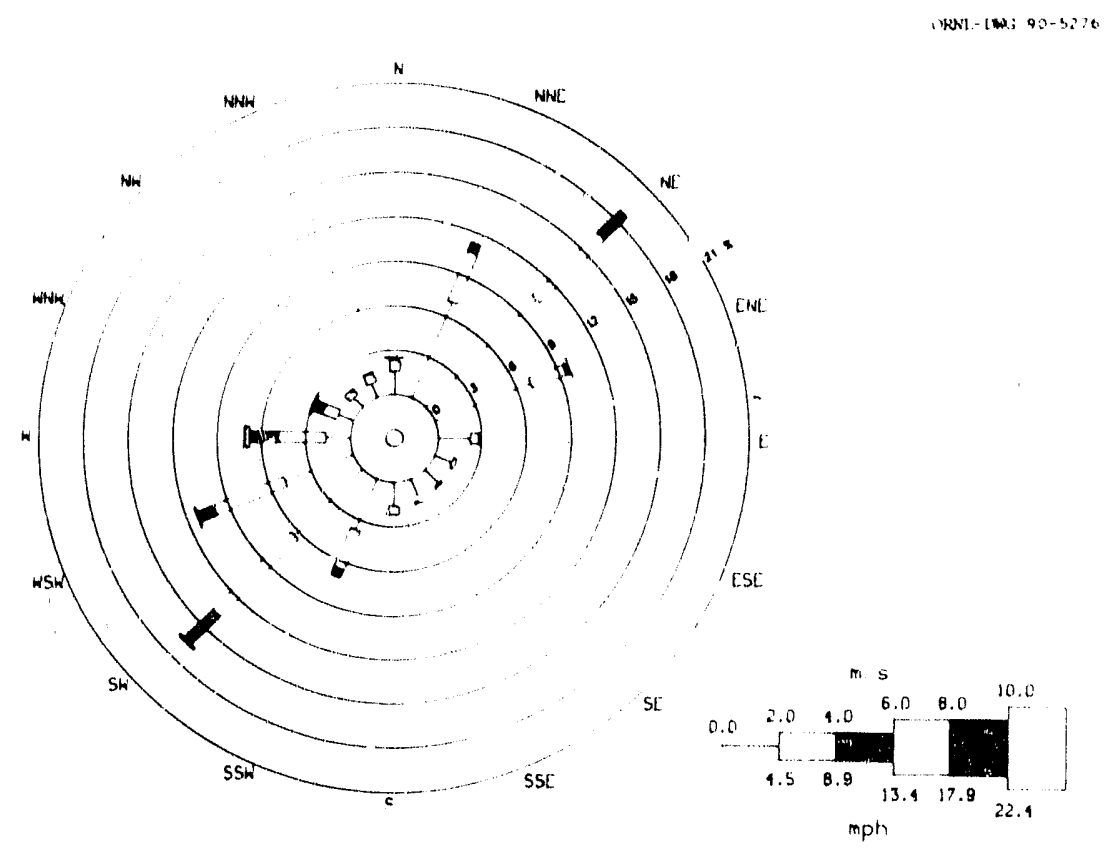

Fig. 2.1.7. 1989 Wind rose for ORNL tower MT3 (30-m level, with 83.0\% of possible data.

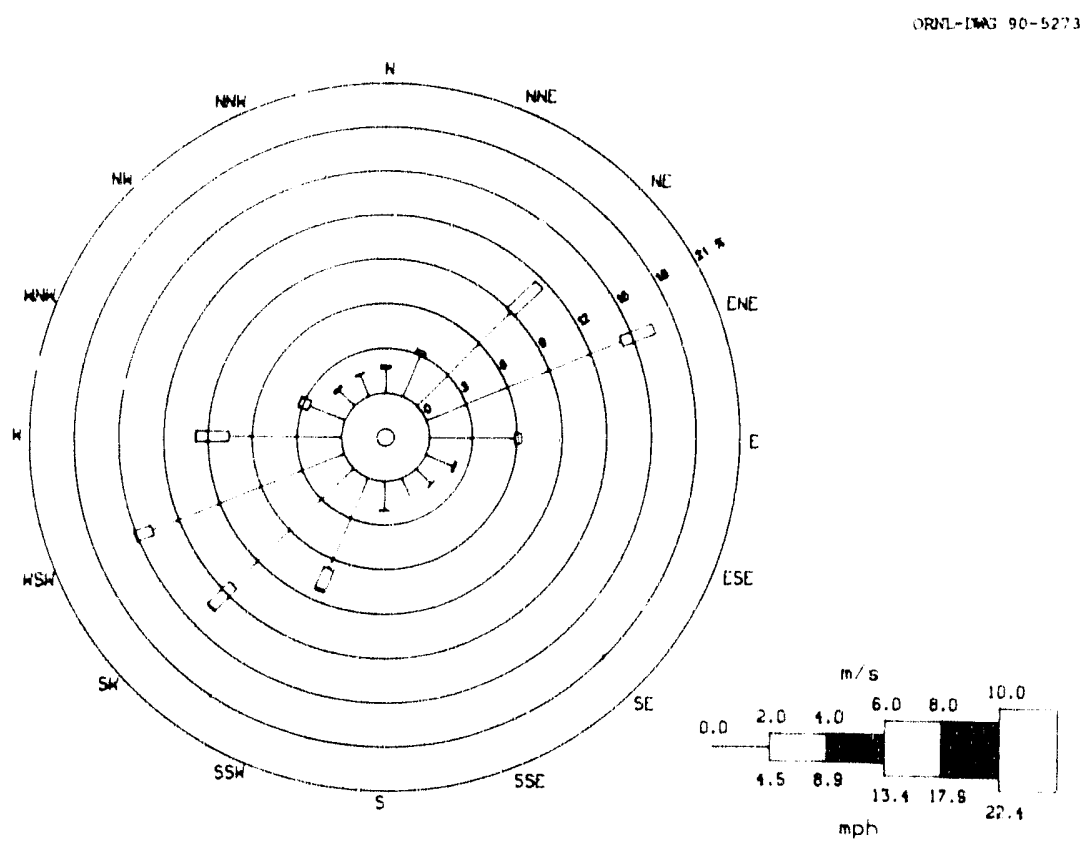

Fig. 2.1.8. 1989 Wind rose for ORNL tower MT4 (10-m level), with $78.1 \%$ of possible data. 


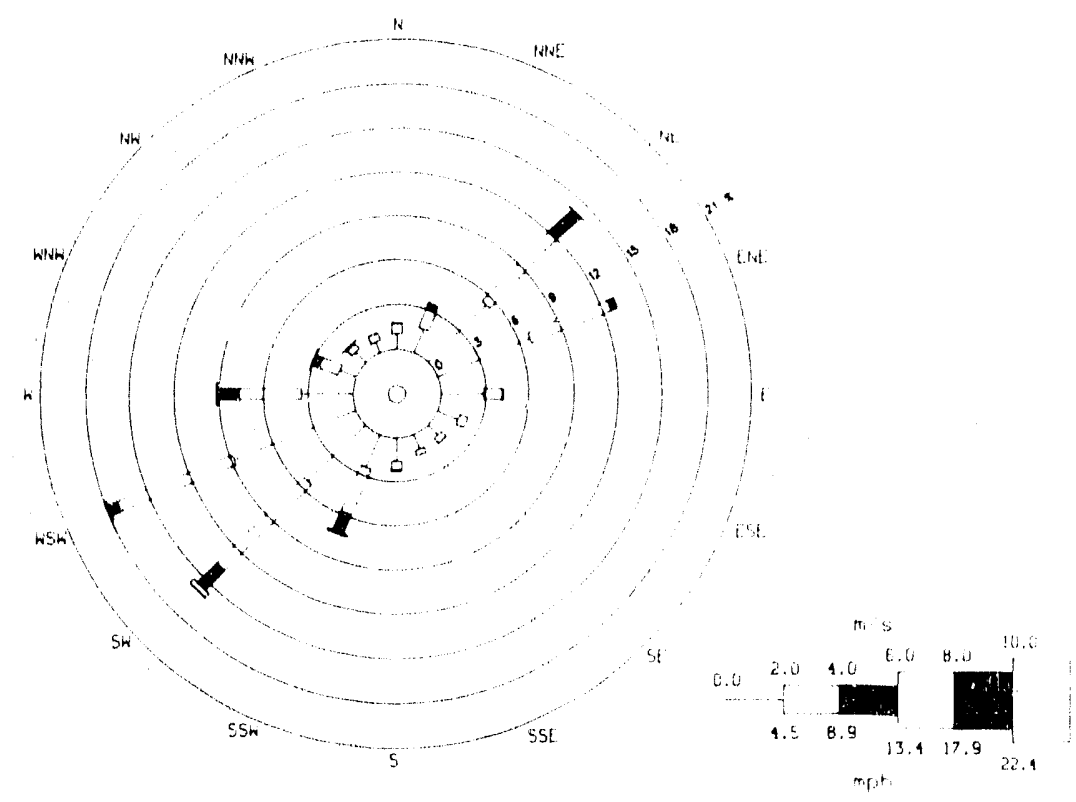

Fig. 2.1.9. 1989 Wind rose for ORNL tower MT4 $(30 \mathrm{~m}$ level), with $84.4 \%$ of possible data.

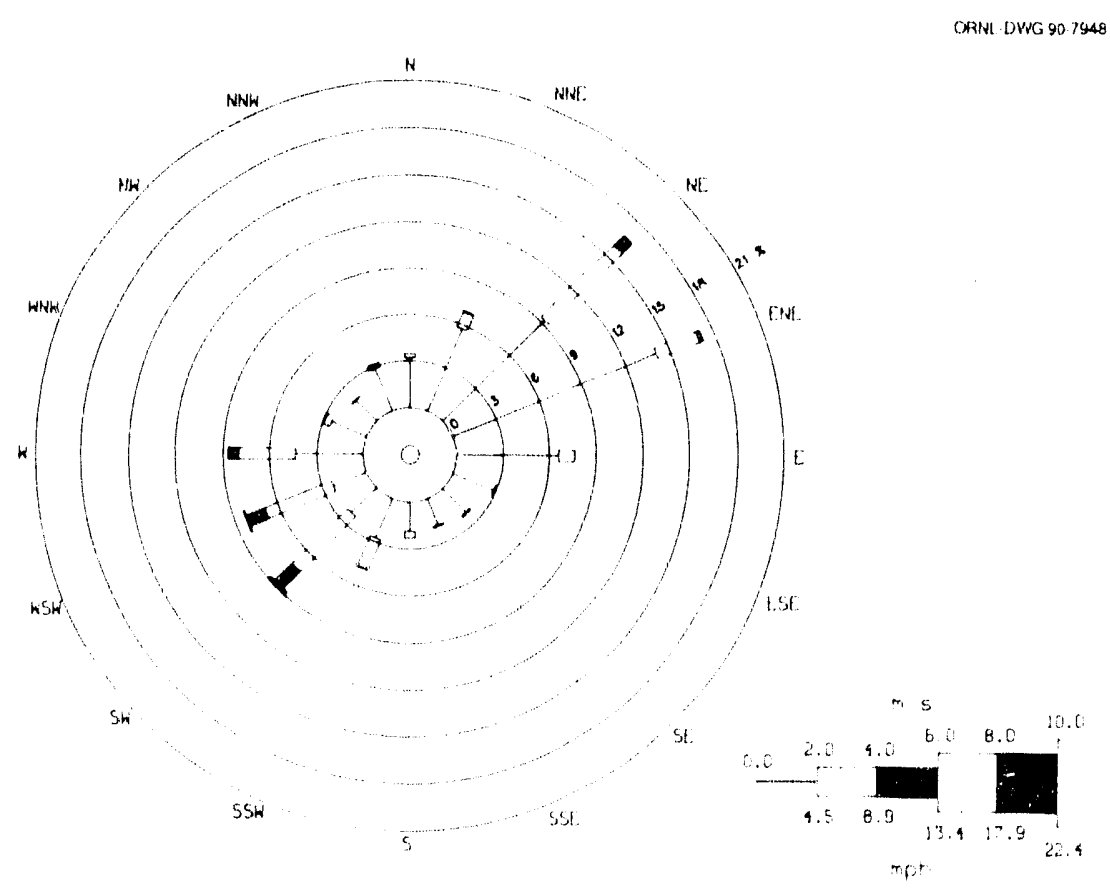

Fig. 2.1.10. 1989 Wind rose for Y-12 tower MTE (east) $(10-\mathrm{m}$ level), with $97.9 \%$ of possible data. 


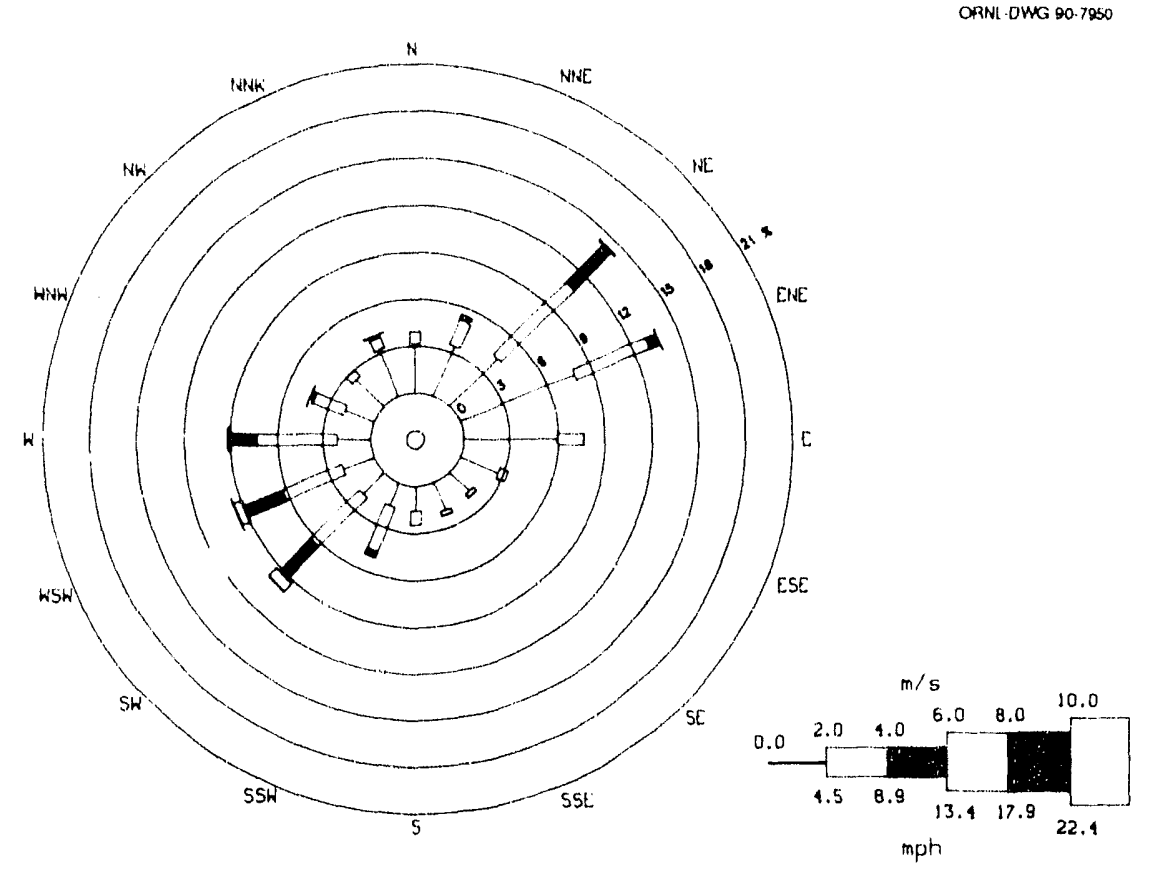

Fig. 2.1.11. 1989 Wind rose for Y-12 tower MTE (east) (30-m level), with 97.6\% of possible data.

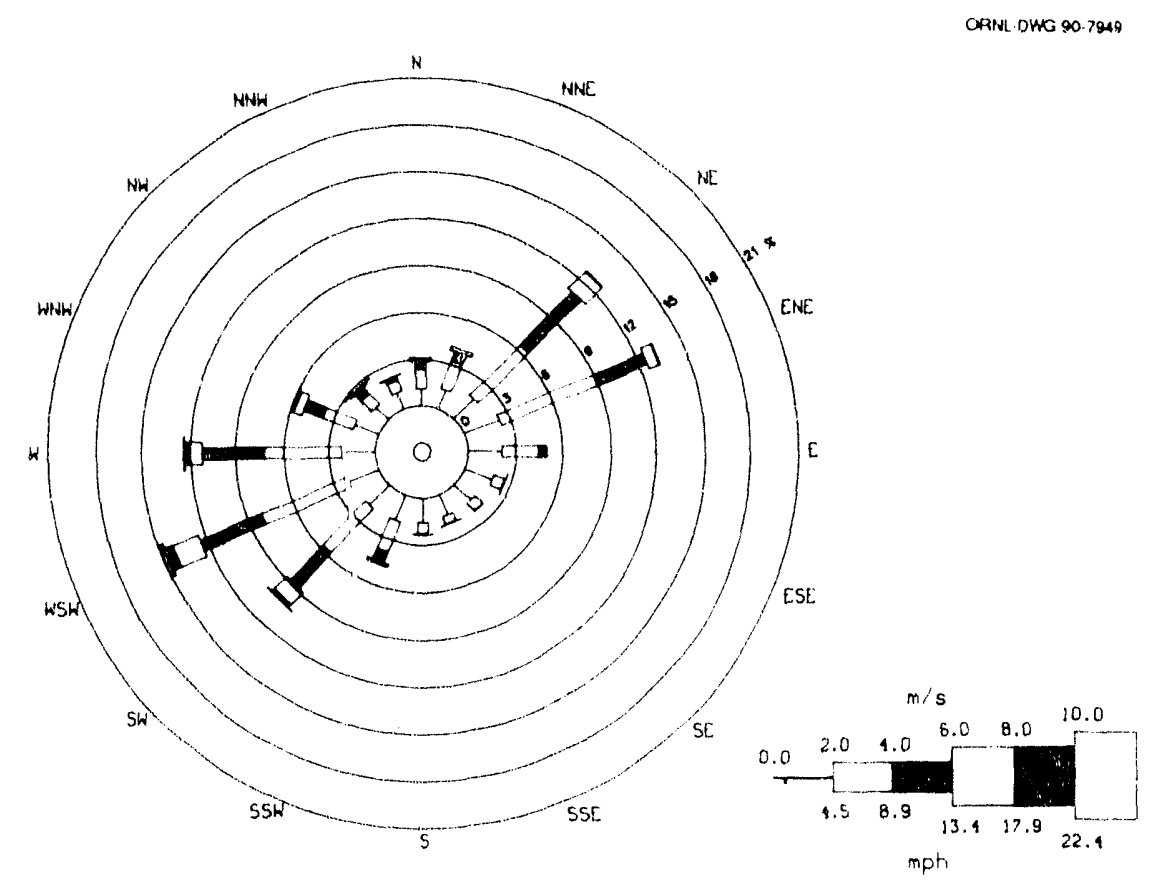

Fig. 2.1.12. 1989 Wind rose for $\mathrm{Y}-12$ tower MTE (east) $(100-\mathrm{m}$ level), with $97.5 \%$ of possible data. 


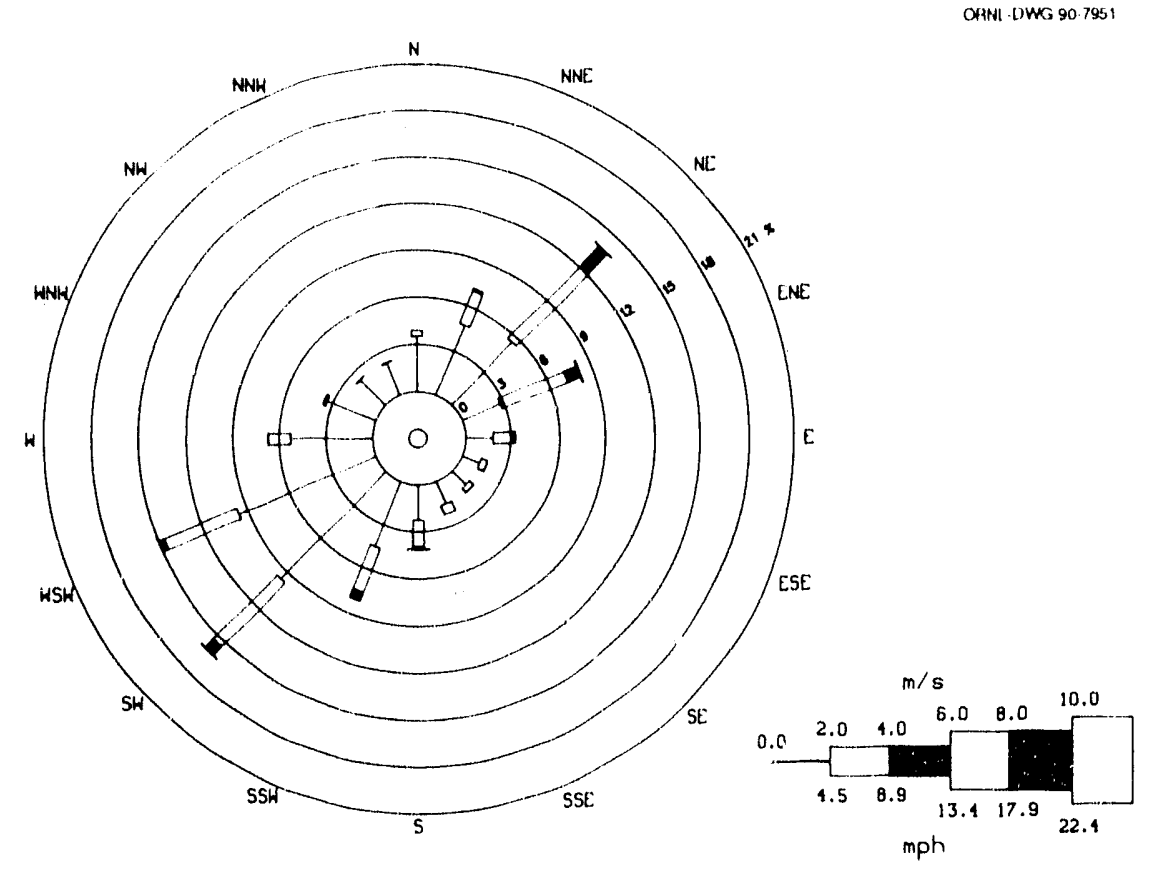

Fig. 2.1.13. 1989 Wind rose for $\mathrm{Y}-12$ tower MTW (west) (10-m level), with $97.2 \%$ of possible data.

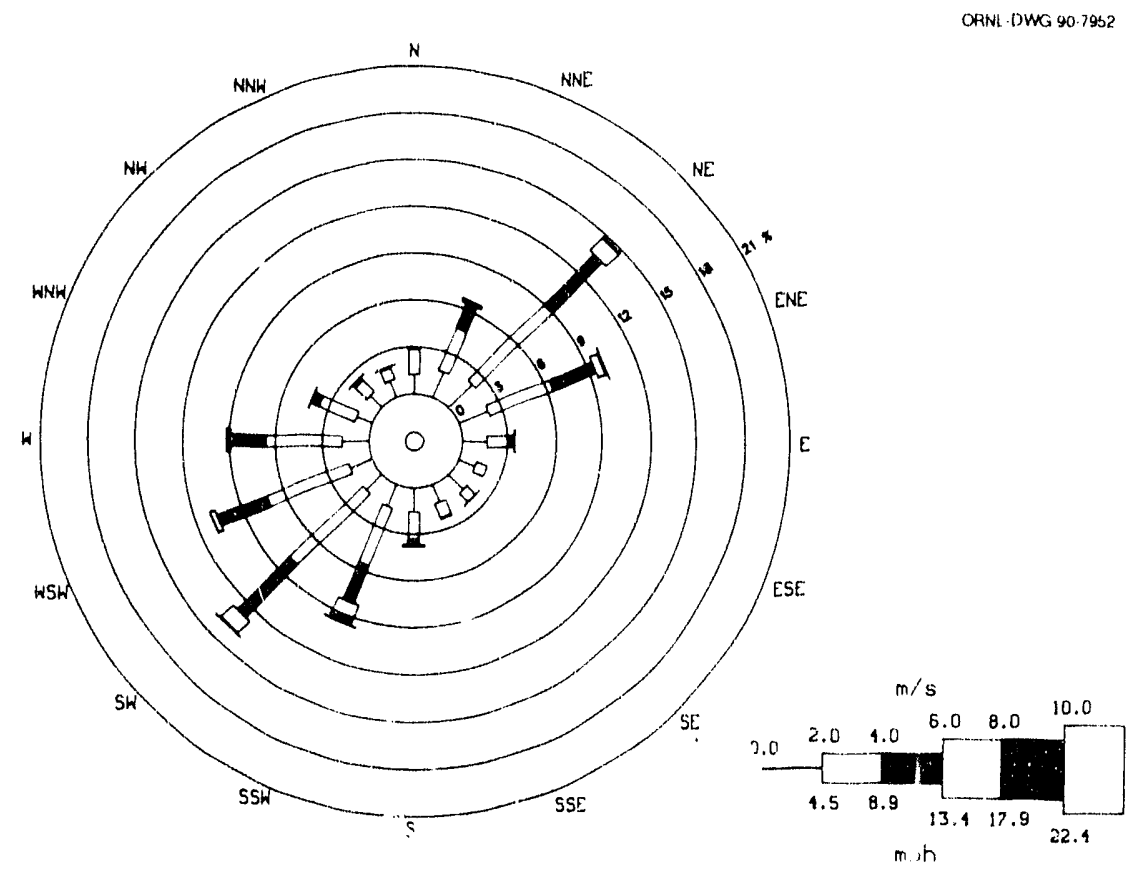

Fig. 2.1.14. 1989 Wind rose for Y-12 tower MTW (west) $(60-\mathrm{m}$ level), with $92.5 \%$ of possible data. 


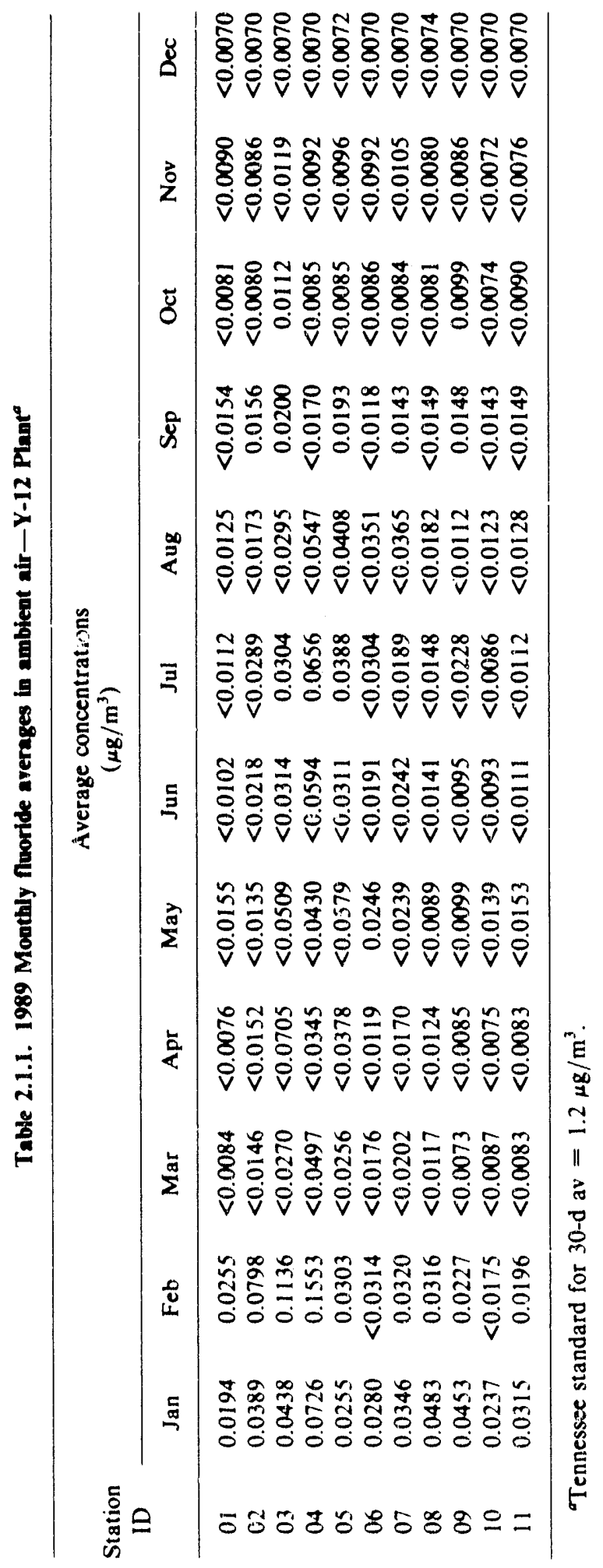


Table 2.1.2. $1989{ }^{234} \mathrm{U},{ }^{235} \mathrm{U},{ }^{236} \mathrm{U}$, and ${ }^{234} \mathrm{U}$ in air at the Y-12 Plant"

\begin{tabular}{|c|c|c|c|c|}
\hline \multirow{2}{*}{$\begin{array}{l}\text { Station } \\
\text { number }\end{array}$} & \multicolumn{4}{|c|}{$\begin{array}{l}\text { Concentration } \\
\left(10^{-15} \mu \mathrm{Ci} / \mathrm{cm}^{3}\right)\end{array}$} \\
\hline & lst Quarter & 2nd Quarter & 3rd Quarter & 4th Quarter \\
\hline \multicolumn{5}{|c|}{${ }^{234} U$} \\
\hline 1 & $1.74 \pm 0.300$ & $3.75 \pm 0.321$ & $0.401 \pm 0.0788$ & $0.367 \pm 0.0587$ \\
\hline 2 & $1.79 \pm 0.293$ & $1.61 \pm 0.152$ & $1.15 \pm 0.149$ & $0.551 \pm 0.0868$ \\
\hline 3 & $2.30 \pm 0.379$ & $0.959 \pm 0.152$ & $2.69 \pm 0.279$ & $1.11 \pm 0.0133$ \\
\hline 4 & $2.50 \pm 0.391$ & $3.25 \pm 0.289$ & $2.36 \pm 0.268$ & $1.49 \pm 0.181$ \\
\hline 5 & $3.94 \pm 0.0826$ & $4.95 \pm 0.381$ & $3.09 \pm 0.314$ & $0.985 \pm 0.137$ \\
\hline 6 & $2.28 \pm 0.365$ & $4.04 \pm 0.513$ & $2.36 \pm n .245$ & $0.817 \pm 0.120$ \\
\hline 7 & $2.27 \pm 0.327$ & $2.11 \pm 0.178$ & $1.40 \pm 0.162$ & $0.831 \pm 0.0975$ \\
\hline 8 & $1.95 \pm 0.342$ & $1.43 \pm 0.136$ & $2.46 \pm 0.278$ & $0.864 \pm 0.112$ \\
\hline 9 & $1.33 \pm 0.221$ & $1.09 \pm 0.114$ & $0.487 \pm 0.0600$ & $0.494 \pm 0.0740$ \\
\hline 10 & $1.23 \pm 0.198$ & $0.875 \pm 0.103$ & $0.815 \pm 0.116$ & $0.340 \pm 0.0531$ \\
\hline 11 & $1.16 \pm 0.189$ & $1.83 \pm 0.165$ & $1.54 \pm 0.173$ & $0.361 \pm 0.0602$ \\
\hline 12 & $2.54 \pm 0.453$ & $1.83 \pm 0.165$ & $0.561 \pm 0.912$ & $0.360 \pm 0.0561$ \\
\hline \multicolumn{5}{|c|}{${ }^{235} U$} \\
\hline 1 & $b$ & $0.137 \pm 0.0369$ & $0.0129 \pm 0.0130$ & $0.0145 \pm 0.0145$ \\
\hline 2 & $0.106 \pm 0.0337$ & $0.0881 \pm 0.0295$ & $0.012 \pm 0.0194$ & $0.0718 \pm 0.0281$ \\
\hline 3 & $0.103 \pm 0.0338$ & $0.0183 \pm 0.0183$ & $0.179 \pm 0.0433$ & $0.109 \pm 0.0308$ \\
\hline 4 & $0.0124 \pm 0.0361$ & $0.146 \pm 0.0392$ & $0.0777 \pm 0.0303$ & $0.039 \pm 0.0242$ \\
\hline 5 & $0.444 \pm 0.122$ & $0.193 \pm 0.0395$ & $0.180 \pm 0.0434$ & $0.00969 \pm 0.00168$ \\
\hline 6 & $0.0874 \pm 0.0295$ & $0.221 \pm 0.0972$ & $0.129 \pm 0.0373$ & $0.0214 \pm 0.0153$ \\
\hline 7 & $0.0954 \pm 0.0278$ & $0.0808 \pm 0.024$ & $0.460 \pm 0.0210$ & $0.0539 \pm 0.0196$ \\
\hline 8 & $0.268 \pm 0.0677$ & $0.0841 \pm 0.026$ & $0.120 \pm 0.0382$ & $0.0772 \pm 0.0294$ \\
\hline 9 & $0.055 \pm 0.0224$ & $0.0398 \pm 0.018$ & $0.0441 \pm 0.0159$ & $0.151 \pm 0.0151$ \\
\hline 10 & $0.05 i 6 \pm 0.0209$ & $0.0453 \pm 0.0242$ & $0.0406 \pm 0.0251$ & $0.01+1 \pm 0.0101$ \\
\hline 11 & $0.044 \pm 0.0191$ & $0.068 \pm 0.0232$ & $0.0974 \pm 0.0306$ & $0.0324 \pm 0.0165$ \\
\hline 12 & $0.244 \pm 0.0649$ & $0.068 \pm 0.0232$ & $0.0228 \pm 0.0163$ & $0.0355 \pm 0.0162$ \\
\hline \multicolumn{5}{|c|}{${ }^{236} \mathrm{U}$} \\
\hline 1 & $b$ & 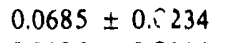 & $b$ & $0.0189 \pm 0.0111$ \\
\hline 2 & $0.0405 \pm 0.0177$ & $0.0196 \pm 0.0114$ & $b$ & $0.0168 \pm 0.0120$ \\
\hline 3 & $0.0282 \pm 0.0148$ & $0.0155 \pm 0.0156$ & $0.0515 \pm 0.02$ & $0.0450 \pm 0.0175$ \\
\hline 4 & $0.0318 \pm 0.0150$ & $0.0644 \pm 0.0233$ & $b$ & $0.0245 \pm 0.0184$ \\
\hline 5 & $0.0748 \pm 0.0341$ & $0.114 \pm 0.0272$ & $0.00728 \pm 0.00731$ & $0.00174 \pm 0.0124$ \\
\hline 6 & $0.0463 \pm 0.0189$ & $0.274 \pm 0.0908$ & $b$ & $0.0181 \pm 0.0130$ \\
\hline 7 & $0.0314 \pm 0.0135$ & $0.0334 \pm 0.0138$ & $0.00748 \pm 0.00752$ & $0.0110 \pm 0.0784$ \\
\hline 8 & $0.201 \pm 0.0526$ & $0.0187 \pm 0.0109$ & $0.0269 \pm 0.0157$ & $0.0211 \pm 0.0159$ \\
\hline 9 & $0.0397 \pm 0.0173$ & $0.0198 \pm 0.0115$ & $0.0182 \pm 0.00921$ & $0.0347 \pm 0.0158$ \\
\hline 10 & $0.0245 \pm 0.0127$ & $0.0226 \pm 0.0131$ & $0.0344 \pm 0.0246$ & $0.00585 \pm 0.00587$ \\
\hline 11 & $0.0245 \pm 0.0 ! 27$ & $0.0313 \pm 0.0142$ & $0.0144 \pm 0.0103$ & $0.0275 \pm 0.0140$ \\
\hline 12 & $0.154 \pm 0.0446$ & $0.0313 \pm 0.0142$ & $b$ & $0.0177 \pm 0.0104$ \\
\hline \multicolumn{5}{|c|}{${ }^{238} U$} \\
\hline 1 & $0.127 \pm 0.376$ & $0.125 \pm 0.0316$ & $0.156 \pm 0.0443$ & $0.0876 \pm 0.0241$ \\
\hline 2 & $0.144 \pm 0.0367$ & $0.140 \pm 0.0256$ & $0.144 \pm 0.0368$ & $0.0120 \pm 0.0325$ \\
\hline 3 & $0.171 \pm 0.0423$ & $0.568 \pm 0.0405$ & $0.159 \pm 0.0349$ & $0.161 \pm 0.0338$ \\
\hline 4 & $0.205 \pm 0.0459$ & $0.246 \pm 0.046$ & $0.313 \pm 0.0603$ & $0.311 \pm 0.0573$ \\
\hline 5 & $3.94 \pm 0.826$ & $0.165 \pm 0.0322$ & $0.171 \pm 0.0374$ & $0.178 \pm 0.0423$ \\
\hline 6 & $0.142 \pm 0.0362$ & $0.344 \pm 0.0978$ & $0.153 \pm 0.0335$ & $0.160 \pm 0.0403$ \\
\hline 7 & $0.218 \pm 0.0439$ & $0.161 \pm 0.0321$ & $0.137 \pm 0.0331$ & $0.109 \pm 0.0252$ \\
\hline 8 & $0.380 \pm 0.0820$ & $0.234 \pm 0.040$ & $0.453 \pm 0.0760$ & $0.225 \pm 0.0437$ \\
\hline 9 & $0.182 \pm 0.0440$ & $0.158 \pm 0.0341$ & $0.168 \pm 0.0301$ & $0.155 \pm 0.0348$ \\
\hline 10 & $0.162 \pm 0.0384$ & $0.110 \pm 0.286$ & $0.174 \pm 0.0408$ & $0.125 \pm 0.0283$ \\
\hline 11 & $0.209 \pm 0.046$ & $0.1486 \pm 0.0328$ & $0.398 \pm 0.0626$ & $0.944 \pm 0.0259$ \\
\hline 12 & $0.166 \pm 0.0445$ & $0.1486 \pm 0.0328$ & $0.0932 \pm 0.0926$ & $0.0527 \pm 0.0173$ \\
\hline
\end{tabular}

aSee Fig. 2.1.17 in Vol. I for station locations.

'No data available. 
Table 2.1.3. 1989 Sulfur dioxide in air at the Y-12 Planta,

\begin{tabular}{|c|c|c|c|c|}
\hline \multicolumn{2}{|c|}{$\begin{array}{l}\text { Month/ } \\
\text { station ID }\end{array}$} & $\begin{array}{c}\text { Monthly av } \mathrm{SO}_{2} \\
(\text { ppm) }\end{array}$ & $\begin{array}{c}\mathrm{Max} \\
(\mathrm{ppm})\end{array}$ & $\begin{array}{c}\mathrm{Max} \\
(\mathrm{ppm})\end{array}$ \\
\hline \multicolumn{5}{|l|}{ January } \\
\hline & East & 0.010 & 0.033 & 0.105 \\
\hline & West & 0.009 & 0.034 & 0.097 \\
\hline \multirow[t]{3}{*}{ February } & $\cdots$ & $\ldots$ & & \\
\hline & East & 0.009 & 0.025 & 0.065 \\
\hline & West & 0.010 & 0.017 & 0.026 \\
\hline \multicolumn{5}{|l|}{ March } \\
\hline & East & 0.011 & 0.033 & 0.079 \\
\hline & West & 0.011 & 0.026 & 0.055 \\
\hline \multicolumn{5}{|l|}{ April } \\
\hline & East & 0.007 & 0.016 & 0.038 \\
\hline & West & 0.005 & 0.010 & 0.023 \\
\hline \multicolumn{5}{|l|}{ May } \\
\hline & East & 0.009 & 0.017 & 0.041 \\
\hline & West & 0.004 & 0.010 & 0.023 \\
\hline \multicolumn{5}{|l|}{ June } \\
\hline & East & 0.009 & 0.024 & 0.106 \\
\hline & West & 0.005 & 0.010 & 0.023 \\
\hline \multicolumn{5}{|l|}{ July } \\
\hline & West & 0.007 & 0.014 & 0.016 \\
\hline \multicolumn{5}{|l|}{ August } \\
\hline & East & 0.019 & 0.034 & 0.090 \\
\hline \multirow{2}{*}{\multicolumn{5}{|c|}{ September }} \\
\hline & & & & \\
\hline & East & 0.012 & 0.041 & 0.123 \\
\hline & West & 0.003 & 0.008 & 0.015 \\
\hline \multicolumn{5}{|l|}{ October } \\
\hline & East & 0.011 & 0.019 & 0.050 \\
\hline \multirow{2}{*}{\multicolumn{5}{|c|}{ November }} \\
\hline & & & & \\
\hline & East & 0.015 & 0.030 & 0.058 \\
\hline & West & 0.006 & 0.011 & 0.018 \\
\hline \multicolumn{5}{|l|}{ December } \\
\hline & East & 0.024 & 0.063 & 0.113 \\
\hline & West & 0.010 & 0.042 & 0.083 \\
\hline
\end{tabular}

"See Vol. 1, Fig. 2.1.17 for station locations.

'Tennessee $24-\mathrm{h}$ average standard is $0.14 \mathrm{ppm}$; Tennessee 3 -h average standard is $0.5 \mathrm{ppm}$. 
Table 2.1.4. 1989 total suspended particulates in air at the Y-12 Plante

\begin{tabular}{|c|c|c|c|c|c|}
\hline \multirow{2}{*}{$\begin{array}{c}\text { Date sample } \\
\text { collected }\end{array}$} & \multicolumn{2}{|c|}{$\begin{array}{l}\text { Concentration }{ }^{b, c} \\
\left(\mu \mathrm{g} / \mathrm{m}^{3}\right)\end{array}$} & \multirow{2}{*}{$\begin{array}{l}\text { Date sample } \\
\text { collected }\end{array}$} & \multicolumn{2}{|c|}{$\begin{array}{l}\text { Concentration } \\
\left(\mu \mathrm{g} / \mathrm{m}^{3}\right)\end{array}$} \\
\hline & East & West & & East & West \\
\hline $1 / 3$ & 67.0 & 100.7 & $7 / 3$ & $62.8^{e}$ & $289.6^{b, e}$ \\
\hline $1 / 8$ & 18.4 & 6.0 & $7 / 9$ & $20.2^{e}$ & $d$ \\
\hline $1 / 15$ & 8.5 & 3.2 & $7 / 15$ & $73.6^{e}$ & $122.7^{e}$ \\
\hline $1 / 24$ & 51.1 & 31.4 & $7 / 21$ & $40.5^{e}$ & 28.6 \\
\hline $2 / 5$ & 53.6 & 56.7 & $7 / 27$ & $80.6^{e}$ & $136.3^{e}$ \\
\hline $2 / 13$ & 3.7 & $d$ & $8 / 2$ & $58.7^{e}$ & $42.9^{e}$ \\
\hline $2 / 20$ & $d$ & $d$ & $8 / 3$ & $69.9^{e}$ & $57.7^{e}$ \\
\hline $2 / 27$ & 9.1 & 6.0 & $8 / 14$ & $97.1^{e}$ & $87.4^{c}$ \\
\hline $3 / 4$ & 30.1 & 31.4 & $8 / 20$ & $93.9^{e}$ & $92.0^{e}$ \\
\hline $3 / 10$ & 72.3 & 56.7 & $8 / 26$ & $40.5^{e}$ & $d$ \\
\hline $3 / 16$ & 129.2 & 145.4 & $9 / 1$ & $d$ & $d$ \\
\hline $3 / 22$ & 110.0 & 86.0 & $9 / 7$ & $d$ & $d$ \\
\hline $3 / 28$ & 86.0 & $d$ & $9 / 13$ & $d$ & $d$ \\
\hline $4 / 5$ & 6.9 & $d$ & $9 / 19$ & $d$ & $d$ \\
\hline $4 / 10$ & $d$ & $d$ & $9 / 25$ & $d$ & $d$ \\
\hline $4 / 16$ & 49.1 & $d$ & $10 / 1$ & $8.4^{e}$ & $9.9^{e}$ \\
\hline $4 / 22$ & 32.7 & $d$ & $10 / 7$ & $d$ & $d$ \\
\hline $4 / 28$ & 53.4 & $d$ & $10 / 13$ & $d$ & $d$ \\
\hline $5 / 4$ & 16.3 & $d$ & $10 / 19$ & $d$ & $d$ \\
\hline $5 / 10$ & 29.3 & $d$ & $10 / 25$ & 67.1 & 54.5 \\
\hline $5 / 16$ & $d$ & $d$ & $10 / 31$ & 30.0 & 13.8 \\
\hline $5 / 22$ & $d$ & $d$ & $11 / 6$ & 18.0 & 27.0 \\
\hline $5 / 28$ & $d$ & $d$ & $11 / 12$ & 19.6 & 39.5 \\
\hline $6 / 3$ & $35.6^{e}$ & $d$ & $11 / 18$ & 22.5 & 20.0 \\
\hline $6 / 9$ & $40.6^{e}$ & $d$ & $11 / 24$ & 22.0 & 17.2 \\
\hline $6 / 15$ & 14.4 & $68.2^{e}$ & $11 / 30$ & 25.8 & 22.9 \\
\hline $6 / 21$ & 44.0 & $385.6^{b, c}$ & $12 / 6$ & 13.2 & 101.0 \\
\hline \multirow[t]{3}{*}{$6 / 27$} & $124.7^{e}$ & $59.8^{e}$ & $12 / 18$ & 38.8 & $275.9^{e}$ \\
\hline & & & $12 / 24$ & 25.7 & 25.4 \\
\hline & & & $12 / 30$ & 9.8 & 45.6 \\
\hline
\end{tabular}

${ }^{a}$ See Fig. 2.1.17 in Vol. 1 for station locations.

${ }^{b}$ Tennessee primary air quality standard $=260 \mu \mathrm{g} /\left(\mathrm{m}^{3} \cdot 24 \mathrm{~h}\right)$.

Tennessee secondary air quality standard $=150 \mu \mathrm{g} /\left(\mathrm{m}^{3} \cdot 24 \mathrm{~h}\right)$.

${ }^{d}$ Invalid sample or no sample (downtime).

'Low air flow: $<35 \mathrm{cfm}$. 
Table 2.1.5. 1989 gross alpha and beta in air at the Y-12 Plant*

\begin{tabular}{|c|c|c|c|c|}
\hline \multirow{2}{*}{$\begin{array}{l}\text { Station } \\
\text { number }\end{array}$} & \multicolumn{4}{|c|}{$\begin{array}{l}\text { Concentration } \\
\left(10^{-15} \mu \mathrm{Ci} / \mathrm{cm}^{3}\right)\end{array}$} \\
\hline & 1st Quarter & 2nd Quarter & 3rd Quarter & 4th Quarter \\
\hline \multicolumn{5}{|c|}{ Gross alpha } \\
\hline 1 & $4.49 \pm 0.717$ & $1.74 \pm 0.608$ & $2.11 \pm 0.540$ & $1.49 \pm 0.435$ \\
\hline 2 & $3.43 \pm 0.661$ & $2.41 \pm 0.646$ & $2.38 \pm 0.557$ & $2.57 \pm 0.533$ \\
\hline 3 & $5.94 \pm 0.790$ & $5.62 \pm 0.809$ & $3.69 \pm 0.636$ & $3.33 \pm 0.560$ \\
\hline 4 & $3.96 \pm 0.690$ & $4.08 \pm 0.734$ & $2.84 \pm 0.585$ & $3.78 \pm 0.623$ \\
\hline 5 & $5.54 \pm 0.770$ & $5.75 \pm 0.816$ & $3.43 \pm 0.620$ & $3.48 \pm 0.574$ \\
\hline 6 & $4.42 \pm 0.714$ & $3.92 \pm 1.01$ & $6.00 \pm 0.759$ & $2.90 \pm 0.551$ \\
\hline 7 & $6.20 \pm 0.802$ & $2.74 \pm 0.664$ & $3.23 \pm 0.609$ & $2.84 \pm 0.562$ \\
\hline 8 & $5.17 \pm 0.780$ & $3.41 \pm 0.70$ & $4.75 \pm 0.694$ & $3.22 \pm 0.560$ \\
\hline 9 & $3.63 \pm 0.672$ & $2.21 \pm 0.635$ & $3.10 \pm 0.601$ & $1.60 \pm 0.481$ \\
\hline 10 & $4.22 \pm 0.703$ & $2.48 \pm 0.649$ & $3.30 \pm 0.613$ & $1.99 \pm 0.505$ \\
\hline 11 & $3.17 \pm 0.647$ & $3.01 \pm 0.678$ & $2.24 \pm 0.548$ & $2.88 \pm 0.571$ \\
\hline 12 & $3.83 \pm 0.683$ & $2.44 \pm 0.646$ & $3.30 \pm 0.613$ & $2.84 \pm 0.554$ \\
\hline \multicolumn{5}{|c|}{ Gross beta } \\
\hline 1 & $16.0 \pm 1.39$ & $15.8 \pm 1.36$ & $19.7 \pm 1.54$ & $15.9 \pm 1.22$ \\
\hline 2 & $16.6 \pm 1.42$ & $18.7 \pm 1.52$ & $21.2 \pm 1.62$ & $19.5 \pm 1.45$ \\
\hline 3 & $20.9 \pm 1.65$ & $19.8 \pm 1.57$ & $20.5 \pm 1.58$ & $24.6 \pm 1.73$ \\
\hline 4 & $16.3 \pm 1.40$ & $20.4 \pm 1.61$ & $21.8 \pm 1.65$ & $24.5 \pm 1.76$ \\
\hline 5 & $18.9 \pm 1.54$ & $18.2 \pm 1.49$ & $21.5 \pm 1.63$ & $22.8 \pm 1.63$ \\
\hline 6 & $20.7 \pm 1.63$ & $17.8 \pm 1.75$ & $20.1 \pm 1.56$ & $21.8 \pm 1.58$ \\
\hline 7 & $18.0 \pm 1.49$ & $20.5 \pm 1.62$ & $23.5 \pm 1.75$ & $19.2 \pm 1.44$ \\
\hline 8 & $18.0 \pm 1.52$ & $21.6 \pm 1.68$ & $25.0 \pm 1.83$ & $21.9 \pm 1.58$ \\
\hline 9 & $19.1 \pm 1.55$ & $18.2 \pm 1.49$ & $23.3 \pm 1.74$ & $22.1 \pm 1.60$ \\
\hline 10 & $17.9 \pm 1.48$ & $16.3 \pm 1.39$ & $20.9 \pm 1.60$ & $19.0 \pm 1.42$ \\
\hline 11 & $15.3 \pm 1.35$ & $17.7 \pm 1.46$ & $24.0 \pm 1.77$ & $20.0 \pm 1.50$ \\
\hline 12 & $19.5 \pm 1.57$ & $19.8 \pm 1.58$ & $21.0 \pm 1.61$ & $23.0 \pm 1.65$ \\
\hline
\end{tabular}

${ }^{\circ}$ See Fig. 2.1.17 in Vol. 1 for station locations. 
Table 2.1.6. Long-lived gross alpha activity in air-ORR, 1989

\begin{tabular}{|c|c|c|c|c|c|}
\hline \multirow{2}{*}{ Location } & \multirow{2}{*}{$\begin{array}{l}\text { No. of } \\
\text { samples }\end{array}$} & \multicolumn{4}{|c|}{$\begin{array}{l}\text { Concentration } \\
\left(10^{-19} \mu \mathrm{Ci} / \mathrm{mL}\right)\end{array}$} \\
\hline & & $\operatorname{Max}$ & Min & Av & $\begin{array}{l}\text { Std. } \\
\text { error }\end{array}$ \\
\hline \multicolumn{6}{|c|}{ ORNL PAM stations ${ }^{a}$} \\
\hline 3 & 26 & 1.7 & -0.73 & 0.73 & 0.10 \\
\hline 4 & 11 & 1.2 & -1.1 & 0.17 & 0.25 \\
\hline 7 & 26 & 1.5 & -1.5 & 0.52 & 0.17 \\
\hline 9 & 17 & 1.7 & -1.6 & 0.42 & 0.25 \\
\hline 20 & 20 & 2.1 & -1.4 & 0.73 & 0.21 \\
\hline 21 & 26 & 2.1 & -1.4 & 0.73 & 0.18 \\
\hline 22 & 27 & 2.1 & -1.6 & 0.61 & 0.19 \\
\hline $\begin{array}{l}\text { Network } \\
\text { summary }\end{array}$ & 153 & 2.1 & -1.6 & 0.60 & 0.070 \\
\hline \multicolumn{6}{|c|}{ Reservation PAM stations ${ }^{a}$} \\
\hline 8 & 6 & 2.5 & -2.0 & -0.56 & 0.64 \\
\hline 23 & 23 & 2.3 & -1.7 & 0.71 & 0.19 \\
\hline 31 & 11 & 2.1 & -1.4 & 0.0059 & 0.37 \\
\hline 33 & 26 & 2.0 & -1.1 & 0.60 & 0.15 \\
\hline 34 & 26 & 1.6 & -1.5 & 0.57 & 0.17 \\
\hline 36 & 11 & 1.1 & -1.7 & -0.21 & 0.34 \\
\hline 40 & 23 & 1.7 & -1.5 & 0.69 & 0.18 \\
\hline 41 & 24 & 1.8 & -1.1 & 0.58 & 0.18 \\
\hline 42 & 26 & 1.4 & -1.6 & 0.44 & 0.19 \\
\hline 43 & 25 & 1.7 & -1.5 & 0.54 & 0.17 \\
\hline 44 & 28 & 1.8 & -1.3 & 0.53 & 0.16 \\
\hline 45 & 24 & 2.2 & -0.98 & 0.71 & 0.16 \\
\hline 46 & 19 & 2.6 & -1.1 & 0.82 & 0.24 \\
\hline $\begin{array}{l}\text { Network } \\
\text { summary }\end{array}$ & 272 & 2.6 & -2.0 & 0.53 & 0.057 \\
\hline \multicolumn{6}{|c|}{ RAM stations ${ }^{b}$} \\
\hline 51 & 15 & 1.2 & -0.74 & 0.088 & 0.16 \\
\hline 52 & 22 & 4.6 & -1.6 & 1.3 & 0.31 \\
\hline 53 & 14 & 2.8 & -1.4 & 0.74 & 0.29 \\
\hline 55 & 8 & 1.3 & -1.5 & -0.059 & 0.31 \\
\hline 56 & 13 & 2.2 & -1.6 & -0.19 & 0.35 \\
\hline 57 & 15 & 2.8 & -0.90 & 0.89 & 0.26 \\
\hline 58 & 21 & 2.5 & -3.5 & 1.2 & 0.37 \\
\hline $\begin{array}{l}\text { Network } \\
\text { summary }\end{array}$ & 108 & 4.6 & -3.5 & 0.68 & 0.13 \\
\hline $\begin{array}{l}\text { Overall } \\
\text { summary }\end{array}$ & 533 & 4.6 & -3.5 & 0.58 & 0.044 \\
\hline
\end{tabular}

${ }^{a}$ See Vol. 1, Fig. 2.1.18.

${ }^{b}$ See Vol. 1, Fig. 2.1.19. 
Table 2.1.7. Long-lived gross beta activity in air-ORR, 1989

\begin{tabular}{|c|c|c|c|c|c|}
\hline \multirow{2}{*}{ Location } & \multirow{2}{*}{$\begin{array}{l}\text { No. of } \\
\text { samples }\end{array}$} & \multicolumn{4}{|c|}{$\begin{array}{c}\text { Concentration } \\
\left(10^{-15} \mu \mathrm{Ci} / \mathrm{mL}\right)\end{array}$} \\
\hline & & $\operatorname{Max}$ & $\operatorname{Min}$ & $A v$ & $\begin{array}{l}\text { Std. } \\
\text { error }\end{array}$ \\
\hline \multicolumn{6}{|c|}{ ORNL PAM stations ${ }^{a}$} \\
\hline 3 & 26 & 38 & 17 & 25 & $\cdot 2$ \\
\hline 4 & 11 & 36 & 15 & 24 & 2.0 \\
\hline 7 & 26 & 35 & 13 & 22 & 1.1 \\
\hline 9 & 17 & 37 & 13 & 21 & 1.6 \\
\hline 20 & 20 & 45 & 18 & 27 & 1.6 \\
\hline 21 & 26 & 44 & 17 & 26 & 1.3 \\
\hline 22 & 27 & 42 & 18 & 25 & 1.2 \\
\hline $\begin{array}{l}\text { Network } \\
\text { summary }\end{array}$ & 153 & 45 & 13 & 24 & 0.52 \\
\hline \multicolumn{6}{|c|}{ Reservation PAM stations } \\
\hline 8 & 6 & 42 & 22 & 35 & 3.1 \\
\hline 23 & 23 & 38 & 17 & 23 & 1.1 \\
\hline 31 & 11 & 32 & 18 & 24 & 1.6 \\
\hline 33 & 26 & 40 & 12 & 21 & 1.5 \\
\hline 34 & 26 & 40 & 9.3 & 21 & 1.5 \\
\hline 36 & 11. & 38 & 9.7 & 22 & 2.6 \\
\hline 40 & 23 & 29 & 9.1 & 20 & 1.2 \\
\hline 41 & 21 & 31 & 11 & 21 & 1.1 \\
\hline 42 & 26 & 30 & 11 & 20 & 1.1 \\
\hline 43 & 25 & 40 & 10 & 21 & 1.5 \\
\hline 44 & 28 & 36 & 5.0 & 20 & 1.3 \\
\hline 45 & 24 & 38 & 9.0 & 23 & 1.5 \\
\hline 46 & 19 & 39 & 11 & 24 & 1.5 \\
\hline $\begin{array}{l}\text { Network } \\
\text { summary }\end{array}$ & 272 & 42 & 5.0 & 22 & 0.42 \\
\hline \multicolumn{6}{|c|}{$R A M$ stations $^{b}$} \\
\hline 51 & 15 & 31 & 9.4 & 20 & 1.7 \\
\hline 52 & 22 & 56 & 4.1 & 28 & 2.1 \\
\hline 53 & 14 & 43 & 9.9 & 26 & 3.0 \\
\hline 55 & 8 & 18 & 1.5 & 10 & 2.0 \\
\hline 56 & 13 & 34 & 2.7 & 23 & 2.3 \\
\hline 57 & 15 & 44 & 19 & 31 & 2.4 \\
\hline 58 & 21 & 50 & 23 & 32 & 1.9 \\
\hline $\begin{array}{l}\text { Network } \\
\text { summary }\end{array}$ & 108 & 56 & 1.5 & 26 & 1.0 \\
\hline $\begin{array}{l}\text { Overall } \\
\text { summary }\end{array}$ & 533 & 56 & 1.5 & 23 & 0.34 \\
\hline
\end{tabular}

${ }^{a}$ See Vol, 1, Fig. 2.1.18.

${ }^{b}$ See Vol. 1, Fig. 2,1.19. 
Table 2.1.8. ${ }^{131}$ I concentrat/ ns in air-ORR, 1989

\begin{tabular}{|c|c|c|c|c|c|c|}
\hline \multirow{2}{*}{ Location } & \multirow{2}{*}{$\begin{array}{l}\text { No. if } \\
\text { samples }\end{array}$} & \multicolumn{4}{|c|}{$\begin{array}{l}\text { Concentration } \\
\left(10^{-15} \mu \mathrm{Ci} / \mathrm{mL}\right)\end{array}$} & \multirow{2}{*}{$\begin{array}{l}\mathrm{DCG}^{a} \\
(\%)\end{array}$} \\
\hline & & $\operatorname{Max}$ & Min & Av & $\begin{array}{l}\text { Std. } \\
\text { error }\end{array}$ & \\
\hline \multicolumn{7}{|c|}{ ORNL PAM stations } \\
\hline 3 & 26 & 13 & -19 & -0.63 & 1.1 & $<0.01$ \\
\hline 4 & 11 & 3.0 & -0.59 & 0.51 & 0.32 & $<0.01$ \\
\hline 7 & 26 & 2.3 & -3.7 & 0.018 & 0.24 & $<0.01$ \\
\hline 9 & 17 & 8.7 & -2.4 & 1.8 & 0.81 & $<0.01$ \\
\hline 20 & 20 & 6.1 & -6.0 & 0.11 & 0.59 & $<0.01$ \\
\hline 21 & 26 & 8.9 & -2.6 & 0.94 & 0.49 & $<0.01$ \\
\hline 22 & 27 & 8.8 & -8.3 & 0.32 & 0.54 & $<0.01$ \\
\hline $\begin{array}{l}\text { Network } \\
\text { summary }\end{array}$ & 153 & 13 & -19 & 0.36 & 0.26 & $<0.01$ \\
\hline
\end{tabular}

Reservation PAM stations

\begin{tabular}{ccccccc}
8 & 6 & 2.3 & 0.65 & 1.4 & 0.26 & $<0.01$ \\
23 & 23 & 4.5 & -2.3 & 0.51 & 0.37 & $<0.01$ \\
31 & 11 & 1.4 & -1.3 & 0.24 & 0.24 & $<0.01$ \\
33 & 11 & 2.7 & -1.1 & 0.46 & 0.32 & $<0.01$ \\
34 & 26 & 3.6 & -7.8 & -0.20 & 0.48 & $<0.01$ \\
36 & 11 & 22 & -0.53 & 2.5 & 1.9 & $<0.01$ \\
40 & 23 & 2.3 & -2.0 & 0.59 & 0.18 & $<0.01$ \\
41 & 24 & 3.7 & -1.6 & 0.27 & 0.29 & $<0.01$ \\
42 & 11 & 2.1 & -1.1 & 0.34 & 0.31 & $<0.01$ \\
43 & 11 & 2.1 & -1.4 & 0.21 & 0.33 & $<0.01$ \\
44 & 28 & 9.4 & -3.8 & 0.20 & 0.41 & $<0.01$ \\
45 & 24 & 4.4 & -4.3 & 0.14 & 0.30 & $<0.01$ \\
46 & 19 & 5.7 & -2.2 & 0.81 & 0.46 & $<0.01$ \\
Network & 228 & 22 & -7.8 & 0.44 & 0.14 & $<0.01$ \\
summary & & & & & & \\
Overall & 381 & 22 & -19 & 0.41 & 0.13 & $<0.01$ \\
summary & & & & & & \\
\hline
\end{tabular}

${ }^{a}$ Percentage derived concentration guide (DCG) = maximum/DCG $\times 100$. The DCG for ${ }^{131} \mathrm{I}$ is $400,000 \times 10^{-15}$ ${ }_{\mu} \mathrm{Ci} / \mathrm{mL}$.

${ }^{b}$ See Vol. 1, Fig. 2.1.18.

Table 2.1.9. Estimated average total tritium activity in air-ORNL, 1989

\begin{tabular}{lcc}
\hline & \multicolumn{3}{c}{$\begin{array}{c}\text { Concentration } \\
\left(10^{-6} \mathrm{pCi} / \mathrm{mL}\right)\end{array}$} \\
\cline { 2 - 3 } Location $^{a}$ & Total & $\begin{array}{c}\mathrm{DCG}^{6} \\
(\%)\end{array}$ \\
\hline 3 & 35 & 0.035 \\
8 & 25 & 0.025 \\
\hline
\end{tabular}

${ }^{a}$ See Vol. 1, Fig. 2.1.18.

${ }^{b}$ Percentage derived concentration guide $(\mathrm{DCG})=$ maximum $/ \mathrm{DCG}$ $\times 100$. The DCG for tritium is $0.1 \mathrm{pCi} / \mathrm{mL}$. This assumes that $50 \%$ of the tritium is absorbed through the skin. 
Table 2.1.10. 1989 contisuous air monitoring data

\begin{tabular}{|c|c|c|c|c|c|c|}
\hline \multirow[b]{2}{*}{ Analysis } & \multicolumn{5}{|c|}{ Concentration $\left(10^{-15} \mu \mathrm{Ci} / \mathrm{m}^{\mathrm{L}}\right)$} & \multirow[b]{2}{*}{$\begin{array}{c}\text { Percentage } \\
\text { DCG }\end{array}$} \\
\hline & $\begin{array}{c}\text { Station } \\
\\
34\end{array}$ & $\begin{array}{c}\text { Percentage }^{b} \\
\text { DCG }\end{array}$ & $\begin{array}{c}\text { Station } \\
40\end{array}$ & $\begin{array}{c}\text { Percentage } \\
\text { DCG }\end{array}$ & $\begin{array}{c}\text { Station } \\
41\end{array}$ & \\
\hline${ }^{60} \mathrm{Co}$ & 0 & $<0.010$ & 0.14 & $<0.010$ & -0.21 & $<0.010$ \\
\hline${ }^{137} \mathrm{Cs}$ & 0.058 & $<0.010$ & -0.058 & $<0.010$ & -0.037 & $<0.010$ \\
\hline${ }^{238} \mathrm{Pu}$ & 0.00016 & $<0.010$ & 0.00036 & $<0.01 .0$ & -0.00099 & $<0.010$ \\
\hline${ }^{239} \mathrm{P}_{\mathrm{L}}$ & -0.00054 & $<0.010$ & -0.0017 & $<0.010$ & -0.0031 & $<0.010$ \\
\hline${ }^{228} \mathrm{Th}$ & 0.033 & 0.084 & 0.038 & 0.094 & 0.033 & 0.083 \\
\hline${ }^{230} \mathrm{Th}$ & 0.0061 & 0.015 & 0.0097 & 0.024 & 0.010 & 0.026 \\
\hline${ }^{232} \mathrm{Th}$ & 0.0058 & 0.083 & 0.0091 & 0.13 & 0.0073 & 0.10 \\
\hline Total Sr & 0.016 & $<0.010$ & -0.012 & $<0.010$ & -0.0063 & $<0.010$ \\
\hline${ }^{234} U$ & 0.033 & 0.036 & 0.49 & 0.54 & 0.094 & 0.10 \\
\hline${ }^{235} \mathrm{U}$ & 0.0019 & $<0.010$ & 0.027 & 0.027 & 0.010 & 0.010 \\
\hline \multirow[t]{2}{*}{${ }^{238} \mathrm{U}$} & 0.014 & 0.014 & 0.061 & 0.061 & 0.021 & 0.021 \\
\hline & $\begin{array}{c}\text { Station } \\
45 \\
\end{array}$ & $\begin{array}{c}\text { Percentage } \\
\text { DCG }\end{array}$ & $\begin{array}{c}\text { Station } \\
46 \\
\end{array}$ & $\begin{array}{c}\text { Percentage } \\
\text { DCG }\end{array}$ & LAMs & $\begin{array}{c}\text { Percentage } \\
\text { DCG }\end{array}$ \\
\hline${ }^{60} \mathrm{Co}$ & -0.13 & $<0.010$ & 0.26 & $<0.010$ & 0.0041 & $<0.010$ \\
\hline${ }^{137} \mathrm{Cs}$ & -0.075 & $<0.010$ & -0.0057 & $<0.010$ & 0.038 & $<0.010$ \\
\hline${ }^{238} \mathrm{Pu}$ & -0.00099 & $<0.010$ & -0.0024 & $<0.010$ & 0.0013 & $<0.010$ \\
\hline${ }^{239} \mathrm{Pu}$ & -0.0023 & $<0.010$ & -0.002 & $<0.010$ & 0.00042 & $<0.010$ \\
\hline${ }^{228} \mathrm{Th}$ & 0.031 & 0.077 & 0.036 & 0.091 & 0.0096 & 0.024 \\
\hline${ }^{230} \mathrm{Th}$ & 0.0084 & 0.021 & 0.0065 & 0.016 & 0.0056 & 0.014 \\
\hline${ }^{232} \mathrm{Th}$ & 0.0065 & 0.093 & 0.0061 & 0.088 & (..0059 & 0.084 \\
\hline Total Sr & 0.056 & $<0.010$ & 0.10 & $<0.010$ & 0.059 & $<0.010$ \\
\hline${ }^{234} \mathrm{U}$ & 0.33 & 0.36 & 0.36 & 0.40 & 0.062 & 0.069 \\
\hline${ }^{235} \mathrm{U}$ & 0.022 & 0.022 & 0.015 & 0.015 & 0.0039 & $<0.010$ \\
\hline \multirow[t]{2}{*}{${ }^{238} \mathrm{U}$} & 0.077 & 0.077 & 0.052 & 0.052 & 0.015 & 0.015 \\
\hline & PAMs & $\begin{array}{c}\text { Percentage } \\
\text { DCG }\end{array}$ & RAMs & $\begin{array}{c}\text { Percentage } \\
\text { DCG }\end{array}$ & & \\
\hline${ }^{60} \mathrm{Co}$ & 0.020 & $<0.010$ & -0.019 & $<0.010$ & & \\
\hline${ }^{137} \mathrm{Cs}$ & -0.0081 & $<0.010$ & -0.0090 & $<0.010$ & & \\
\hline${ }^{238} \mathrm{Pu}$ & -0.00011 & $<0.010$ & 0.00051 & $<0.010$ & & \\
\hline${ }^{239} \mathrm{Pu}_{\mathrm{u}}$ & -0.00015 & $<0.010$ & -0.0015 & $<0.010$ & & \\
\hline${ }^{228} \mathrm{Th}$ & 0.011 & 0.028 & 0.019 & 0.047 & & \\
\hline${ }^{230} \mathrm{Th}$ & 0.0054 & 0.014 & 0.0090 & 0.022 & & \\
\hline${ }^{232} \mathrm{Th}$ & 0.0053 & 0.075 & 0.0078 & 0.11 & & \\
\hline Total Sr & 0.0087 & $<0.010$ & 0.015 & $<0.010$ & & \\
\hline${ }^{234} \mathrm{U}$ & 0.086 & 0.096 & 0.015 & 0.017 & & \\
\hline${ }^{235} \mathrm{U}$ & 0.0051 & $<0.010$ & 0.0016 & $<0.010$ & & \\
\hline${ }^{238} \mathrm{U}$ & 0.020 & 0.020 & 0.016 & 0.016 & & \\
\hline
\end{tabular}

aSee Figs. 2.1.18 and 2.1.19 in Vol. 1.

${ }^{6}$ Percentage of $\mathrm{DCG}=$ average/derived concentration guide $(\mathrm{DCG}) \times 100$. The DCG for ${ }^{60} \mathrm{Co}$ is $8 \times 10^{-11} \mu \mathrm{Ci} / \mathrm{ml}$; ${ }^{137} \mathrm{Cs}$ is $4 \times 10^{-10} \mu \mathrm{Ci} / \mathrm{ml} ;{ }^{238} \mathrm{Pu}$ is $3 \times 10^{-14} \mu \mathrm{Ci} / \mathrm{ml} ;{ }^{239} \mathrm{Pu}$ is $2 \times 10^{-14} \mu \mathrm{Ci} / \mathrm{ml} ;{ }^{228} \mathrm{Th}$ is $4 \times 10^{-14} \mu \mathrm{Ci} / \mathrm{ml} ;{ }^{230} \mathrm{Th}$ is $4 \times 10^{-14} \mu \mathrm{Ci} / \mathrm{ml} ;{ }^{232} \mathrm{Th}$ is $7 \times$ $10^{-15} \mu \mathrm{Ci} / \mathrm{ml}$; Total $\mathrm{Sr}$ is $9 \times 10^{-12} \mu \mathrm{Ci} / \mathrm{ml} ;{ }^{234} \mathrm{U}$ is $9 \times 10^{-14} \mu \mathrm{Ci} / \mathrm{ml} ;{ }^{235} \mathrm{U}$ is $1 \times 10^{-13}$ $\mu \mathrm{Ci} / \mathrm{ml} ;{ }^{238} \mathrm{U}$ is $1 \times 10^{-13}$. Source for DCG is DOE Order 5400.5 , "Radiation Protection of the Public and the Environment," Chapter III. 
Table 2.1.11. Air permits at the Y-12 Plant

\begin{tabular}{|c|c|c|c|}
\hline $\begin{array}{l}\text { Y-12 Plant } \\
\text { source number }\end{array}$ & $\begin{array}{l}\text { Emission source } \\
\text { reference number }\end{array}$ & $\begin{array}{l}\text { Permit } \\
\text { number }\end{array}$ & $\begin{array}{l}\text { Permit } \\
\text { type }^{a}\end{array}$ \\
\hline Two gasoline tanks & $01-0020-62$ & $015114 \mathrm{P}$ & $\mathrm{O}$ \\
\hline$Y-1501-2-A(01)$ & $01-0020-70$ & $023506 \mathrm{P}$ & $\mathrm{O}$ \\
\hline$Y-1501-2-A(02)$ & $01-0020-70$ & $023506 \mathrm{P}$ & $\mathrm{O}$ \\
\hline$Y-9201-1-A(01)$ & $01-0020-15$ & 025974P & $\mathrm{O}$ \\
\hline $\mathrm{Y}-9201-1-\mathrm{A}(02)$ & $01-0020.15$ & $025974 \mathrm{P}$ & 0 \\
\hline Y-9201-1-A(03) & $01-0020-15$ & $025974 \mathrm{P}$ & $\mathrm{O}$ \\
\hline Y.9201-1-A(04) & $01 \sim 0020-15$ & $025974 \mathrm{P}$ & 0 \\
\hline$Y-9201-1-A(05)$ & $01-0020-15$ & $025974 \mathrm{P}$ & 0 \\
\hline$Y-9201-1-A(15)$ & $01-0020-15$ & $025974 \mathrm{P}$ & 0 \\
\hline$Y-9201-1-A(19)$ & $01-0020-15$ & $\mathrm{n} 25974 \mathrm{P}$ & 0 \\
\hline$Y-9201-1-B(16)$ & $01-0020-59$ & $025898 \mathrm{P}$ & 0 \\
\hline$Y-9201-1-B(18)$ & $01-0020-59$ & 025898P & $\mathrm{O}$ \\
\hline$Y-9201-1-C(3)$ & $01-0020-17$ & $012665 \mathrm{P}$ & $\mathrm{O}$ \\
\hline$Y-9201-1-C(4)$ & $01-0020-17$ & $012665 \mathrm{P}$ & 0 \\
\hline$Y-9201-1-D(09)$ & $01-0020-61$ & $025958 \mathrm{P}$ & $\mathrm{O}$ \\
\hline$Y-9201-1-D(10)$ & $01-0020-61$ & $025958 \mathrm{P}$ & 0 \\
\hline$Y-9201-1-D(11)$ & $01-0020-61$ & $025958 \mathrm{P}$ & 0 \\
\hline$Y-9201-1-D(13)$ & $01-0020-61$ & $025958 \mathrm{P}$ & 0 \\
\hline$Y-9201-2-C(01)$ & $01-0020-67$ & $015146 \mathrm{P}$ & 0 \\
\hline$Y-9201-2-C(02)$ & $01-0020-67$ & $015146 \mathrm{P}$ & 0 \\
\hline$Y-9201-2-C(03)$ & $01-0020-67$ & $015146 \mathrm{P}$ & $\mathrm{O}$ \\
\hline$Y-9201-3-A(01)$ & $01-0020-55$ & $013002 \mathrm{P}$ & 0 \\
\hline$Y-9201-5-A(C 1)$ & $01-0020-29$ & $025964 \mathrm{P}$ & 0 \\
\hline$Y-9201-5-A(02)$ & $01-0020-29$ & $02.5964 \mathrm{P}$ & 0 \\
\hline$Y-9201-5-A(03)$ & $01-0020-29$ & $025964 \mathrm{P}$ & 0 \\
\hline Y-9201-5-A(04) & $01-0020-29$ & $025964 \mathrm{P}$ & 0 \\
\hline$Y-9201-5-A(05)$ & $01-0020-29$ & $025964 \mathrm{P}$ & 0 \\
\hline$Y-9201-5-A_{i}(06)$ & $01-0020-29$ & $025964 \mathrm{P}$ & 0 \\
\hline Y-9201-5-A(07) & $01-0020-29$ & $025964 \mathrm{P}$ & 0 \\
\hline $\mathrm{Y}-9201-5-\mathrm{A}(08)$ & $01-0020-29$ & 025964P & 0 \\
\hline Y-9201-5-A(09) & $01-0020-29$ & $025964 \mathrm{P}$ & O \\
\hline$Y-9201-5-A(10)$ & $01-0020-29$ & 025964P & 0 \\
\hline$Y-9201-5-B(01)$ & $01-0020-21$ & $025956 \mathrm{P}$ & 0 \\
\hline$Y-9201-5-B(02)$ & $01-0020-21$ & $025956 \mathrm{P}$ & 0 \\
\hline$Y-9201-5-B(03)$ & $01-0020-21$ & $025956 \mathrm{P}$ & 0 \\
\hline$Y-9201-5-B(04)$ & $01-0020-21$ & 025956P & 0 \\
\hline$Y-9201-5-B(05)$ & $01-0020-21$ & $025956 \mathrm{P}$ & 0 \\
\hline$Y-9201-5-B(06)$ & $01-0020-21$ & $025956 \mathrm{P}$ & $\mathbf{O}$ \\
\hline$Y-9201-5-B(07)$ & $01-0020-21$ & $025956 \mathrm{P}$ & 0 \\
\hline Y-9201-5-C(01) & $01-1020-43$ & 025949p & 0 \\
\hline$Y-9201-5-D(01)$ & $01-1020-44$ & $025902 \mathrm{P}$ & 0 \\
\hline$Y-9201-5-D(02)$ & $01-1020-44$ & $025902 \mathrm{P}$ & 0 \\
\hline$Y-9201-5-E(01)$ & $01-1020-70$ & 025983P & 0 \\
\hline$Y-9201-5-E(02)$ & $01-1020-70$ & 025983P & $\mathrm{O}$ \\
\hline$Y-9201-5-E(08)$ & $01-1020-70$ & $025983 \mathrm{P}$ & $\mathrm{O}$ \\
\hline$Y-9201-5-F(01)$ & $01-0020-36$ & $025973 \mathrm{P}$ & $\mathrm{O}$ \\
\hline$Y-9201-5-F(02)$ & $01-0020-36$ & $025973 \mathrm{P}$ & 0 \\
\hline$Y-9201-5-F(03)$ & $01-0020-36$ & $025973 \mathrm{P}$ & $\mathrm{O}$ \\
\hline
\end{tabular}


Table 2.1.11 (continued)

\begin{tabular}{|c|c|c|c|}
\hline $\begin{array}{l}\text { Y-12 Plant } \\
\text { source number }\end{array}$ & $\begin{array}{l}\text { Emission source } \\
\text { reference number }\end{array}$ & $\begin{array}{l}\text { Permit } \\
\text { number }\end{array}$ & $\begin{array}{l}\text { Permit } \\
\text { type }\end{array}$ \\
\hline$Y-9201-5 . F(04)$ & $01.0020-36$ & $025973 \mathrm{P}$ & $\mathrm{O}$ \\
\hline$Y^{\prime}-9201-5-F(05)$ & $01-0020-36$ & $025973 \mathrm{P}$ & $\mathrm{O}$ \\
\hline$Y-9201-5-G(01)$ & $01-0020.44$ & $025896 \mathrm{P}$ & $\mathrm{O}$ \\
\hline$Y-9201-5-G(02)$ & $01-0020-44$ & $025896 \mathrm{P}$ & $\mathrm{O}$ \\
\hline$Y-9201-5-G(03)$ & $01-0020.44$ & $025896 \mathrm{P}$ & $\mathrm{O}$ \\
\hline$Y-9201-5-G(04)$ & $01-0020.44$ & $025896 \mathrm{P}$ & 0 \\
\hline$Y-9201-5-G(05)$ & 01.0020 .44 & $025896 \mathrm{P}$ & o \\
\hline$Y-9201-5-G(06)$ & 01.0020 .44 & $025896 \mathrm{P}$ & 0 \\
\hline$Y-9201-5-G(07)$ & $01-0020-44$ & $025896 \mathrm{P}$ & $\mathrm{O}$ \\
\hline$Y-9201-5-H(01)$ & $01-0020-16$ & $026019 \mathrm{P}$ & $\mathrm{O}$ \\
\hline $\mathrm{Y} .9201-5-\mathrm{H}(02)$ & 01.0020 .16 & $026019 \mathrm{P}$ & $\mathrm{O}$ \\
\hline$Y^{\prime}-9201-5-H(03)$ & $01-0020-16$ & $026019 P$ & $\mathrm{O}$ \\
\hline$Y-9201-5-H(04)$ & $01-0020-16$ & $026019 P$ & $\mathrm{O}$ \\
\hline$Y-9201-5 \cdot H(05)$ & $01-0020-16$ & $026019 \mathrm{P}$ & $\mathrm{O}$ \\
\hline$Y-9201-5-H(06)$ & $01-0020-16$ & $026019 \mathrm{P}$ & $\mathrm{O}$ \\
\hline Y-9201-5-H(07) & $01.0020-16$ & $026019 \mathrm{P}$ & 0 \\
\hline $\mathrm{Y} \cdot 9201-5-\mathrm{H}(08)$ & $01-0020-16$ & $026019 \mathrm{P}$ & $\mathrm{O}$ \\
\hline Y-9201-5IN-A(01) & $01-0020-18$ & $025950 \mathrm{P}$ & 0 \\
\hline$Y-9201-5 N-B(03)$ & $01-0020-30$ & $025962 \mathrm{P}$ & $\mathrm{O}$ \\
\hline$Y-9201-5 N-B(04)$ & $01-0020-30$ & $025962 \mathrm{P}$ & $\mathrm{O}$ \\
\hline$Y^{\prime}-9201-5 N-B(05)$ & $01-0020-30$ & $025962 \mathrm{P}$ & $\mathrm{O}$ \\
\hline$Y-9201-5 N-B(06)$ & $01-0020-30$ & $025962 \mathrm{P}$ & $\mathrm{O}$ \\
\hline$Y-920 !-5 N-B(07)$ & $01-0020-30$ & $025962 \mathrm{P}$ & $\mathrm{O}$ \\
\hline Y-9201-5N-B(08) & $01-0020-30$ & $025962 \mathrm{P}$ & $\mathrm{O}$ \\
\hline Y $-9201-5 \mathrm{~N}-\mathrm{B}(09)$ & 01.0020 .30 & $025962 \mathrm{P}$ & $\mathrm{O}$ \\
\hline Y'-9201-5N-B(10) & $01-0020-30$ & $025962 \mathrm{P}$ & 0 \\
\hline$Y-9201-5 \mathrm{~N}-\mathrm{B}(11)$ & 01.0020 .30 & $025962 \mathrm{P}$ & $\mathrm{O}$ \\
\hline$Y \cdot 9201 \cdot 5 N \cdot B(12)$ & $01-0020-30$ & $325962 \mathrm{p}$ & $\mathrm{O}$ \\
\hline$Y \cdot 9202 \cdot A \cdot(01)$ & $01-0020-21$ & $021086 \mathrm{P}$ & $\mathrm{O}$ \\
\hline$Y \cdot 9202 \cdot A \cdot(n 2)$ & $01-0020-21$ & $021086 \mathrm{P}$ & $\mathrm{O}$ \\
\hline Y-9202-A-(03) & $01.0020-21$ & $021086 \mathrm{P}$ & $\mathrm{O}$ \\
\hline Y-9202-A-(04) & $01-0020.21$ & $021086 \mathrm{P}$ & $\mathrm{O}$ \\
\hline$Y \cdot 9203-C(1,2)$ & $01-1020-84$ & $0997819 \mathrm{P}$ & $\mathrm{C}$ \\
\hline$Y-9204-2-A(01)$ & 01.0020 .46 & $026107 \mathrm{P}$ & $\mathrm{O}$ \\
\hline$Y-9204-2-A(02)$ & $01-0020.46$ & $026107 \mathrm{P}$ & 0 \\
\hline$Y-9204-2-A(03)$ & $01.0020-46$ & $026107 P$ & 0 \\
\hline $\mathrm{Y}-9204-2-\mathrm{A}(04)$ & $01-0020-46$ & $02610 \% \mathrm{P}$ & $\mathrm{O}$ \\
\hline$Y .9204-2-. A(05)$ & 01.0020 .46 & $026107 \mathrm{P}$ & $\mathrm{O}$ \\
\hline$Y \cdot 9204-2-A(06)$ & $01-0020.46$ & $026107 \mathrm{P}$ & 0 \\
\hline$Y-9204-2-A(07)$ & 01.0020 .46 & $026107 \mathrm{P}$ & 0 \\
\hline$Y-9204-2-A(08)$ & 01.0020 .46 & $026107 \mathrm{P}$ & $\mathrm{O}$ \\
\hline$Y-9204 \cdot 2 \cdot A(09)$ & 01.0020 .46 & $026107 \mathrm{P}$ & $\mathrm{O}$ \\
\hline$Y \cdot 9204-2 \cdot A(10)$ & $01-0020-46$ & $026107 \mathrm{P}$ & $\mathrm{O}$ \\
\hline$Y-9204-2 \cdot A(11)$ & 01.0020 .46 & $026107 P$ & $\mathrm{O}$ \\
\hline$Y \cdot 9204-2 \cdot A(12)$ & 01.0020 .46 & $026107 \mathrm{P}$ & $\mathrm{O}$ \\
\hline$Y-9204-2-A(13)$ & 01.0020 .46 & $026107 \mathrm{P}$ & $\mathrm{O}$ \\
\hline$Y-9204-2-B(14)$ & 01.0020 .71 & $025954 \mathrm{P}$ & 0 \\
\hline Y.9204-2-B(15) & $01-0020.71$ & $025954 \mathrm{P}$ & $\mathrm{O}$ \\
\hline$Y-9204 \cdot 2 \cdot B(16)$ & $01.0020-71$ & $025954 \mathrm{P}$ & $\mathrm{O}$ \\
\hline$Y \cdot 9204 \cdot 2 \cdot B(17)$ & 01.0020 .71 & $025954 \mathrm{P}$ & 0 \\
\hline Y-9204-2-B(18) & $01.0020-71$ & $025954 \mathrm{P}$ & $\mathrm{O}$ \\
\hline $\mathrm{Y}^{\prime} \cdot 9204-2-\mathrm{B}(19)$ & $01-0020-71$ & $025954 \mathrm{P}$ & $\mathrm{O}$ \\
\hline$Y-9204-2-B(20)$ & $01-0020.71$ & $025954 \mathrm{P}$ & 0 \\
\hline$Y-9204-2-B(2 i)$ & 01.0020 .71 & $025954 \mathrm{P}$ & $\mathrm{O}$ \\
\hline
\end{tabular}


Table 2.1.11 (continued)

\begin{tabular}{|c|c|c|c|}
\hline $\begin{array}{l}\text { Y-12 Plant } \\
\text { source number }\end{array}$ & $\begin{array}{l}\text { Emission source } \\
\text { reference number }\end{array}$ & $\begin{array}{l}\text { Permit } \\
\text { number }\end{array}$ & $\begin{array}{l}\text { Permit } \\
\text { type }^{a}\end{array}$ \\
\hline Y.9204-2-B(22) & $01-0020-71$ & $025954 \mathrm{P}$ & 0 \\
\hline$Y-9204-2-B(23)$ & $01-0020-71$ & $025954 \mathrm{P}$ & 0 \\
\hline Y.9204-2-B(24) & $01-0020-71$ & $025954 \mathrm{P}$ & 0 \\
\hline$Y-9204-2-B(25)$ & $01-0020.71$ & $025954 \mathrm{P}$ & 0 \\
\hline Y-9204-2-B(26) & $01-0020-71$ & $025954 \mathrm{P}$ & 0 \\
\hline$Y-9204-2-B(27)$ & $01-0020.71$ & 025954P & 0 \\
\hline Y-9204-2-B(28) & $01-0020-71$ & $025954 \mathrm{P}$ & 0 \\
\hline$Y-9204-2-C(29)$ & $01 \cdot 1020-19$ & $025900 \mathrm{P}$ & 0 \\
\hline$Y-9204-2 \cdot C(30)$ & $01-1020-19$ & 025900P & 0 \\
\hline$Y-9204-2-C(31)$ & $01-1020-19$ & 025900P & $\therefore$ \\
\hline$Y-9204-2-C(32)$ & $01-1020-19$ & $025900 \mathrm{P}$ & 0 \\
\hline$Y-9204-2-C(33)$ & $01-1020-19$ & 025900P & 0 \\
\hline$Y-9204-2 \cdot C(34)$ & $01-1020-19$ & 025900P & 0 \\
\hline$Y-9204-2-C(35)$ & $01-1020-19$ & 025900P & 0 \\
\hline$Y-9204-2-C(36)$ & $01-1020-19$ & 025900P & 0 \\
\hline$Y-9204-<-C(37)$ & $01-1020-19$ & 025900P & 0 \\
\hline$Y-9204-2 \cdot C(38)$ & $01-1020-19$ & 025900P & 0 \\
\hline Y-9204-2-C(39) & $01-1020-19$ & 025900P & 0 \\
\hline$Y-9204-2-C(40)$ & $01-1020-19$ & 025900P & 0 \\
\hline$Y-9204-2-C(41)$ & $01-1020-19$ & 025900P & 0 \\
\hline Y.9204-2-C(42) & $01-1020-19$ & 025900P & 0 \\
\hline$Y-9204-2-C(43)$ & $01-1020-19$ & 025900P & 0 \\
\hline Y-9204-2-C: (44) & $01-1020-19$ & 025900P & 0 \\
\hline$Y-9204-2-C(45)$ & $01-1020-19$ & $025900 \mathrm{P}$ & 0 \\
\hline$Y-9204-2-C(46)$ & $01-1020-19$ & 025900P & 0 \\
\hline$Y-9204-2-C(47)$ & $01-1020-19$ & 025900P & $?$ \\
\hline Y-9204-2-C(48) & $01-1020-19$ & 025900P & 0 \\
\hline Y-9204-2-C(49) & $01-1020-19$ & 025900P & 0 \\
\hline$Y-9204-2-C(50)$ & $01-1020-19$ & 025900P & 0 \\
\hline$Y-9204-2-C(51)$ & $01-1020-19$ & 025900P & 0 \\
\hline$Y-9204-2-D(52)$ & $01-1020-57$ & $025967 \mathrm{P}$ & 0 \\
\hline$Y-9204-2-D(53)$ & $01-1020-57$ & $025967 \mathrm{P}$ & 0 \\
\hline$Y-9204-2-D(54)$ & $01-1020-57$ & $025967 \mathrm{P}$ & 0 \\
\hline$Y-9204-2-D(55)$ & $01 \cdots 1020-57$ & 025967P & $\mathrm{O}$ \\
\hline$Y-9204-2-D(56)$ & $01-1020-57$ & $025967 \mathrm{P}$ & 0 \\
\hline$Y-9201-2-D(57)$ & $01-1020-57$ & 025967P & 0 \\
\hline$Y-9204-2 \cdot D(58)$ & $01-1020-57$ & $025967 \mathrm{P}$ & $\mathrm{O}$ \\
\hline Y-9204-2-D(59) & $01-1020-57$ & 025967P & 0 \\
\hline$Y-9204-2 \cdot D(60)$ & $01-1020-57$ & $025967 \mathrm{P}$ & $\mathrm{O}$ \\
\hline Y.9204-2-D(61) & $01-1020-57$ & $025967 \mathrm{P}$ & 0 \\
\hline$Y-9204-2-D(62)$ & $01-1020.57$ & $025967 \mathrm{P}$ & 0 \\
\hline$Y-9204-2-D(63)$ & $01-1020-57$ & $025967 \mathrm{P}$ & 0 \\
\hline$Y-9204-2-D(64)$ & $01-1020-57$ & $025967 \mathrm{P}$ & $\mathrm{O}$ \\
\hline$Y-9204-2-D(65)$ & $01-1020-57$ & $025967 \mathrm{P}$ & 0 \\
\hline$Y-9204-2-D(66)$ & $01-1020-57$ & $025967 \mathrm{P}$ & 0 \\
\hline$Y-9204-2-D(67)$ & $01-1020-57$ & $025967 \mathrm{P}$ & 0 \\
\hline$Y-9204-2-E(068)$ & $01-1020-55$ & 025959P & 0 \\
\hline$Y-9204-2-E(069)$ & $01-1020.55$ & 025959P & 0 \\
\hline$Y-9204-2-E(070)$ & $01-1020-55$ & 025959P & 0 \\
\hline$Y-9204-2-E(071)$ & $01-1020-55$ & $025959 \mathrm{P}$ & 0 \\
\hline$Y-9204-2-E(072)$ & $01-1020-55$ & $025959 \mathrm{P}$ & 0 \\
\hline$Y-9204-2-E(073)$ & $01-1020-55$ & $025959 \mathrm{P}$ & 0 \\
\hline$Y-9204-2-E(074)$ & $01-1020-55$ & 025959P & $\mathrm{O}$ \\
\hline Y-9204-2-E(075) & $01-1020-55$ & $025959 \mathrm{P}$ & 0 \\
\hline$Y-9204-2-E(076)$ & $01-1020-55$ & 025959P & 0 \\
\hline
\end{tabular}


Table 2.1.11 (continued)

\begin{tabular}{|c|c|c|c|}
\hline $\begin{array}{l}\text { Y-12 Plant } \\
\text { source number }\end{array}$ & $\begin{array}{l}\text { Emission source } \\
\text { reference number }\end{array}$ & $\begin{array}{l}\text { Permit } \\
\text { number }\end{array}$ & $\begin{array}{c}\text { Permit } \\
\text { type }\end{array}$ \\
\hline$Y-9204-2-E(077)$ & $01-1020-55$ & 025959P & $\mathrm{O}$ \\
\hline$Y-9204-2-E(078)$ & $01-1020-35$ & 025959P & $\mathrm{O}$ \\
\hline Y-9204-2-E(079) & $01-1020-55$ & $025959 \mathrm{P}$ & 0 \\
\hline$Y-9204-2 \cdot E(080)$ & $01-1020-55$ & 025959P & $\mathrm{O}$ \\
\hline$Y-9204-2-E(081)$ & $01-1020-55$ & $025959 \mathrm{P}$ & 0 \\
\hline$Y-9204-2 \cdot F(082)$ & 01.0020 .51 & $025897 \mathrm{P}$ & 0 \\
\hline$Y-9204-2-F(083)$ & $01.0020-51$ & $025897 \mathrm{P}$ & $\mathrm{O}$ \\
\hline$Y-9.204-2 \cdot F(084)$ & $01-0020-51$ & $025897 \mathrm{P}$ & 0 \\
\hline$Y-9204-2-F(085)$ & $01-0020.51$ & $025897 \mathrm{P}$ & $\mathrm{O}$ \\
\hline$Y-9204-2-F(086)$ & $01-0020.51$ & $025897 \mathrm{P}$ & $\mathrm{O}$ \\
\hline$Y-9204-2-F(087)$ & $01-0020-51$ & $025897 \mathrm{P}$ & $\mathrm{O}$ \\
\hline$Y-9204-2-G(08.8)$ & $01-1020-72$ & $028350 \mathrm{P}$ & 0 \\
\hline$Y .9204-2 \cdot G(089)$ & $01-1020-72$ & $028350 \mathrm{P}$ & $\mathrm{O}$ \\
\hline$Y-9204-2-G(090)$ & $01-1020-72$ & $028350 \mathrm{P}$ & 0 \\
\hline$Y-9204-2 . G(091)$ & $01-1020-72$ & $028350 \mathrm{P}$ & $\mathrm{O}$ \\
\hline$Y-9204-2-G(092)$ & 01.1020 .72 & $028350 \mathrm{P}$ & $\mathrm{O}$ \\
\hline$Y-9204-2-G(093)$ & $01-1020-72$ & $028350 \mathrm{P}$ & 0 \\
\hline$Y .9204-2-H(01)$ & $01-1020-42$ & $025952 \mathrm{P}$ & 0 \\
\hline$Y-9204-2-H(02)$ & $01-1020-42$ & $025952 \mathrm{P}$ & 0 \\
\hline$Y-9204-2 \cdot H(03)$ & $01-1020-42$ & $025952 \mathrm{P}$ & 0 \\
\hline$Y-9204-2-H(04)$ & $01-1020-42$ & $025952 \mathrm{P}$ & $\mathrm{O}$ \\
\hline Y-9204-2-H(05) & $01 \cdot 1020-42$ & $025952 \mathrm{P}$ & 0 \\
\hline$Y-9204-2 \cdot H(06)$ & $01-1020-42$ & $025952 \mathrm{P}$ & $\mathrm{O}$ \\
\hline$Y-9204-2-I(94)$ & $01-102.0-71$ & $026067 \mathrm{P}$ & $\mathrm{O}$ \\
\hline$Y .9204-2-I(95)$ & $01-1020-71$ & $026067 \mathrm{P}$ & $\mathrm{O}$ \\
\hline Y.9204-2E-A(01) & $01-0020-68$ & $024598 \mathrm{P}$ & 0 \\
\hline Y-9204-2E-A(02) & $01-0020-68$ & $024598 \mathrm{P}$ & $\mathrm{O}$ \\
\hline Y-9204-2E-A(03) & 01.0020 .68 & $024598 \mathrm{P}$ & 0 \\
\hline$Y-9204-2 E_{\cdots} A(04)$ & $01-0020-68$ & $024598 \mathrm{P}$ & 0 \\
\hline$Y-9204-2 E-A(05)$ & $01-0020-68$ & $024598 \mathrm{P}$ & $\mathrm{O}$ \\
\hline Y.9204-2E-A(06) & $01-0020.68$ & $024598 \mathrm{P}$ & 0 \\
\hline$Y-9204-2 E-A(07)$ & $01-0020-68$ & $024598 \mathrm{P}$ & 0 \\
\hline Y.9204-2E-A(08) & $01-0020-68$ & $024598 \mathrm{P}$ & $\mathrm{O}$ \\
\hline Y.9204-2E-A(09) & $01-0020-68$ & $024598 \mathrm{P}$ & 0 \\
\hline Y-9204-2E-Á(10) & $01-0020-68$ & $024598 \mathrm{P}$ & $\mathrm{O}$ \\
\hline$Y-9204-2 E-A(11)$ & $01.0020-68$ & 024598P & 0 \\
\hline Y-9204-2E-A(16) & $01-0020-68$ & 024598P & $\mathrm{O}$ \\
\hline$Y-9204-2 E-A(17)$ & $01-0020-68$ & $024598 \mathrm{P}$ & 0 \\
\hline Y-9204-2E-A(18) & $01-0020-68$ & $024598 \mathrm{P}$ & 0 \\
\hline Y-9204-2E-A(19) & $01-0020-68$ & $024598 \mathrm{P}$ & 0 \\
\hline$Y-9204-2 E-B(12)$ & $01-1020-41$ & $025953 \mathrm{P}$ & 0 \\
\hline$Y-9204-2 E-B(14)$ & $01-1020.41$ & $025953 \mathrm{P}$ & $\mathrm{O}$ \\
\hline$Y .9204-2 E-B(15)$ & $01-1020-41$ & $025953 \mathrm{P}$ & $\mathrm{O}$ \\
\hline$Y-9204-2 E-C(12)$ & $01-1020-68$ & $022890 \mathrm{P}$ & $\mathrm{O}$ \\
\hline Y.9204-2E-C(13) & $01 \cdot 1020-68$ & $022890 \mathrm{P}$ & $\mathrm{O}$ \\
\hline
\end{tabular}


Table 2.1.11 (continued)

\begin{tabular}{|c|c|c|c|}
\hline $\begin{array}{l}\text { Y-12 Plant } \\
\text { source number }\end{array}$ & $\begin{array}{l}\text { Emission source } \\
\text { reference number }\end{array}$ & $\begin{array}{l}\text { Permit } \\
\text { number }\end{array}$ & $\begin{array}{l}\text { ermit } \\
\text { type }\end{array}$ \\
\hline Y-904-4-AJ-106 & 01-0020-89 & $018208 \mathrm{P}$ & 0 \\
\hline$Y-9204-4-A(01)$ & $01-1020-56$ & 996205P & $\mathrm{O}$ \\
\hline Y-9204-4-A(02) & $01-1020-56$ & $996205 \mathrm{P}$ & 0 \\
\hline Y-9204-4-A(03) & $01-1020-56$ & 996205P & 0 \\
\hline$Y-9204-4 \cdot A(04)$ & $01-1020-56$ & 996205P & $\mathrm{O}$ \\
\hline $\mathrm{Y}-9204-4-\mathrm{A}(05)$ & $01-1020-56$ & 996205P & $\mathrm{O}$ \\
\hline$Y-9204-4-A(06)$ & $01-1020-56$ & $996205 \mathrm{P}$ & 0 \\
\hline Y-9204-4-A(07) & $01-1020-56$ & 996205P & $\mathrm{O}$ \\
\hline Y-9204-4-A(08) & $01-1020-56$ & 996205P & 0 \\
\hline Y-9204-4-A(09) & $01-1020-56$ & 996205P & $\mathrm{O}$ \\
\hline Y-92,04-4-A(10) & $01-1020-56$ & $996205 \mathrm{P}$ & $\mathrm{O}$ \\
\hline Y-9204-4-A(11) & $01-1020-56$ & $996205 \mathrm{P}$ & 0 \\
\hline$Y-9204-4-A(12)$ & $01-1020-56$ & 996205P & 0 \\
\hline Y-9204-4-A(13) & $01-1020-56$ & $996205 \mathrm{P}$ & 0 \\
\hline Y-9204-4-A(14) & $01-1020-56$ & 996205P & $\mathrm{O}$ \\
\hline Y-9204-4-A(15) & $01-1020-56$ & 996205P & 0 \\
\hline$Y-9204-4-A(17)$ & $01-1020-56$ & 996205P & 0 \\
\hline$Y-9204-4-A(18)$ & $01-1020-56$ & 996205P & 0 \\
\hline Y-9204-4-B(01) & $01-0020-72$ & $025961 \mathrm{P}$ & 0 \\
\hline Y-9204-4-B(02) & $01-0020-72$ & $025961 \mathrm{P}$ & 0 \\
\hline$Y-9204-4-B(03)$ & $01-0020-72$ & 025961P & $\mathrm{O}$ \\
\hline$Y-9204-4-B(04)$ & $01-0020-72$ & $025961 \mathrm{P}$ & $\mathrm{O}$ \\
\hline Y-9204-4-B(05) & $01-0020-72$ & $025961 \mathrm{P}$ & 0 \\
\hline Y-9204-4-B(06) & $01-0020-72$ & $025961 \mathrm{P}$ & $\mathrm{O}$ \\
\hline$Y-9204-4-B(07)$ & $01-0020-72$ & $025961 \mathrm{P}$ & 0 \\
\hline Y-9204-4-B(08) & $01-0020-72$ & $025961 \mathrm{P}$ & $\mathrm{O}$ \\
\hline Y-9204-4-B(09) & $01-0020-72$ & $025961 \mathrm{P}$ & 0 \\
\hline$Y-9204-4-B(10)$ & $01-0020-72$ & $025961 \mathrm{P}$ & $\mathrm{O}$ \\
\hline Y-9204-4-B(11) & $01-0020-72$ & $025961 \mathrm{P}$ & 0 \\
\hline Y-9204-4-C(01) & $01-1020-36$ & 025968P & 0 \\
\hline Y-9204-4-D(01) & $01-1020-35$ & 025963P & 0 \\
\hline Y-9204-4-E(01) & $01-0020-33$ & $025002 \mathrm{P}$ & 0 \\
\hline Y-9204-4-E(02) & $01-0020-33$ & $025002 \mathrm{P}$ & $\mathrm{O}$ \\
\hline$Y-9204-4-E(03)$ & $01-0020-33$ & $025002 \mathrm{P}$ & 0 \\
\hline$Y-9204-4-E(04)$ & $01-0020-33$ & $025002 \mathrm{P}$ & 0 \\
\hline Y-9204-4-E(05) & $01-0020-33$ & $025002 \mathrm{P}$ & 0 \\
\hline $\mathrm{Y}-9206-\mathrm{A}(01)$ & $01-0020-48$ & $012892 \mathrm{P}$ & $\mathrm{O}$ \\
\hline$Y-9206-A(02)$ & $01-0020-48$ & $012892 \mathrm{P}$ & 0 \\
\hline Y-9206-A(03) & $01-0020-48$ & $012892 \mathrm{P}$ & 0 \\
\hline$Y-9206-B(01)$ & $01-0020-03$ & 026765P & 0 \\
\hline$Y-9206-B(02)$ & $01-0020-03$ & $026765 \mathrm{P}$ & $\mathrm{O}$ \\
\hline Y-9206-B(03) & $01-0020-03$ & $026765 \mathrm{P}$ & $\mathrm{O}$ \\
\hline Y-9206-B(04) & $01-0020-03$ & $026765 \mathrm{P}$ & 0 \\
\hline Y-9206-B(05) & $01-0020-03$ & $026765 \mathrm{P}$ & $\mathrm{O}$ \\
\hline$Y-9206-B(06)$ & $01-0020-03$ & 026765P & $\mathrm{O}$ \\
\hline Y-9206-B(07) & $01-0020-03$ & 026765P & 0 \\
\hline Y-9206-B(08) & $01.0020-03$ & $026765 \mathrm{P}$ & $\mathrm{O}$ \\
\hline Y-9206-B(09) & $01-0020-03$ & $026765 \mathrm{P}$ & o \\
\hline Y-9206-B(10) & $01-0020-03$ & 026765P & 0 \\
\hline Y-9206-B(11) & $01-0020-03$ & $026765 \mathrm{P}$ & $\mathrm{O}$ \\
\hline$Y-9206-B(12)$ & $01-0020-03$ & $026765 \mathrm{P}$ & $\mathrm{O}$ \\
\hline Y-9206-B(13) & $01-0020-03$ & $026765 \mathrm{P}$ & 0 \\
\hline$Y-9206-C(01)$ & $01-1020-24$ & 026766P & 0 \\
\hline Y-9206-C(02) & $01-1020-24$ & $026766 \mathrm{P}$ & 0 \\
\hline
\end{tabular}


Table 2.1.11 (continued)

\begin{tabular}{|c|c|c|c|}
\hline $\begin{array}{l}\text { Y-12 Plant } \\
\text { source number }\end{array}$ & $\begin{array}{l}\text { Emission source } \\
\text { reference number }\end{array}$ & $\begin{array}{l}\text { Permit } \\
\text { number }\end{array}$ & $\begin{array}{l}\text { Permit } \\
\text { type }^{a}\end{array}$ \\
\hline Y-9206-D(13) & $01-1020-38$ & J25901P & 0 \\
\hline Y-9212-A(01) & $01-1020-72$ & 0997756P & $\mathrm{C}$ \\
\hline $\mathrm{Y}-9212-\mathrm{A}(02)$ & $01-1020-72$ & 0997756P & C \\
\hline$Y-9212-A(03)$ & $01-1020-72$ & 0997756P & $\mathrm{C}$ \\
\hline$Y-9212-A(04)$ & $01-1020-72$ & 0997756P & $\mathrm{C}$ \\
\hline$Y-9212-A(05)$ & $01-1020-72$ & 0997756P & C \\
\hline$Y-9212-A(06)$ & $01-1020-72$ & 0997756P & $\mathrm{C}$ \\
\hline $\mathrm{Y}-9212-\mathrm{A}(07)$ & $01-1020-72$ & 0997756P & $\mathrm{C}$ \\
\hline Y-9212-A(08) & $01-1020-72$ & 0997756P & C \\
\hline Y.9212-A(10) & 01.1020 .72 & 0997756P & C \\
\hline $\mathrm{Y}-9212-\mathrm{A}(11)$ & $01-1020-72$ & 0997756P & $\mathrm{C}$ \\
\hline $\mathrm{Y}-9212-\mathrm{A}(12)$ & $01.1020-72$ & $0997756 \mathrm{P}$ & $\mathrm{C}$ \\
\hline Y-9212-A(13) & $01-1020-72$ & $0997756 \mathrm{P}$ & C \\
\hline Y-9212-A(14) & $01 \cdot 1020-72$ & 0997756P & $\mathrm{C}$ \\
\hline Y-9212-A(15) & $01-1020-72$ & 0997756P & $\mathrm{C}$ \\
\hline$Y-9212-A(16)$ & $01-1020-72$ & 0997756P & $\mathrm{C}$ \\
\hline Y-9212-A(17) & $01-1020-72$ & 0997756P & $\mathrm{C}$ \\
\hline$Y-9212-A(18)$ & $01-1020-72$ & 0997756P & $\mathrm{C}$ \\
\hline Y-9212-A(19) & $01-1020-72$ & 0997756P & $\mathrm{C}$ \\
\hline Y-9212-A(20) & $01-1020-72$ & 0997756P & $\mathrm{C}$ \\
\hline$Y-9212-A(21)$ & $01-1020-72$ & 0997756P & $\mathrm{C}$ \\
\hline$Y-9212-A(22)$ & $01.1020-72$ & $0997756 \mathrm{P}$ & $\mathrm{C}$ \\
\hline Y-9212-A(23) & $01-1020-72$ & 0997756P & $\mathrm{C}$ \\
\hline Y-9212-A(24) & $01-1020-72$ & $0997756 \mathrm{P}$ & $\mathrm{C}$ \\
\hline $\mathrm{Y}-9212-\mathrm{A}(25)$ & $01 \cdot 1020-72$ & 0997756P & $\mathrm{C}$ \\
\hline $\mathrm{Y}-9212-\mathrm{A}(26)$ & $01-1020-72$ & 0997756P & $\mathrm{C}$ \\
\hline Y-9212-A(27) & $01-1020-72$ & $0997756 \mathrm{P}$ & $\mathrm{C}$ \\
\hline Y-9212-A(28) & $01-1020-72$ & 0997756P & $\mathrm{C}$ \\
\hline$Y-9212-B(01)$ & $01-0020-02$ & $025955 \mathrm{P}$ & 0 \\
\hline$Y-9212-B(02)$ & $01-0020-02$ & 025955P & $\mathrm{O}$ \\
\hline$Y-9212-B(03)$ & $01-0020-02$ & 025955P & 0 \\
\hline Y-9212-B(04) & $01-0020-02$ & $025955 \mathrm{P}$ & O \\
\hline$Y-9212-C(01)$ & $01-0020-05$ & $025984 \mathrm{P}$ & $\mathrm{O}$ \\
\hline Y-9212-C(02) & $01-0020-05$ & 025984P & $\mathrm{O}$ \\
\hline Y-9212-C(03) & $01-0020-05$ & $025984 \mathrm{P}$ & $\mathrm{o}$ \\
\hline$Y-9212-C(04)$ & $01-0020-05$ & $025984 \mathrm{P}$ & 0 \\
\hline Y-9212-C(05) & $01.0020-05$ & 025984P & $\mathrm{O}$ \\
\hline Y-9212-C(06) & $01-0020-05$ & $025984 \mathrm{P}$ & o \\
\hline Y-9212-C(07) & $01-0020-05$ & $025984 \mathrm{P}$ & $\mathrm{o}$ \\
\hline Y-9212-C(08) & $01-0020-05$ & $025984 \mathrm{P}$ & 0 \\
\hline Y-9212.C(09) & $01-0020-05$ & $025984 \mathrm{P}$ & $\mathrm{O}$ \\
\hline$Y-9212-D(01)$ & $01-1020-46$ & $025904 \mathrm{P}$ & $\mathrm{o}$ \\
\hline$Y-9212-E(01)$ & $01-1020-48$ & $025969 \mathrm{P}$ & 0 \\
\hline Y-9212-E(02) & $01-1020-48$ & 025969P & 0 \\
\hline$Y-9212-F(01)$ & 01.1020 .49 & $025960 \mathrm{P}$ & 0 \\
\hline$Y-9212-F(03)$ & $01-1020-49$ & 025960P & $\mathrm{O}$ \\
\hline
\end{tabular}


Table 2.1.11 (continued)

\begin{tabular}{|c|c|c|c|}
\hline $\begin{array}{c}\text { Y-12 Plant } \\
\text { source number }\end{array}$ & $\begin{array}{l}\text { Emission source } \\
\text { reference number }\end{array}$ & $\begin{array}{l}\text { Permit } \\
\text { number }\end{array}$ & $\begin{array}{c}\text { Permit } \\
\text { type }\end{array}$ \\
\hline$Y-9212-F(04)$ & $01-1020-49$ & $025960 \mathrm{P}$ & 0 \\
\hline$Y-9212-F(05)$ & $01-1020-49$ & $025960 \mathrm{P}$ & $\mathrm{O}$ \\
\hline$Y-9212-G(01)$ & $01-1020-47$ & 022942P & 0 \\
\hline Y-9215-A(01) & $01-0020-37$ & $022130 \mathrm{P}$ & 0 \\
\hline$Y-9215-B(02)$ & $01-0020-38$ & $012880 \mathrm{P}$ & $\mathrm{O}$ \\
\hline$Y-9215-B(05)$ & $01-1020-51$ & $022882 \mathrm{P}$ & 0 \\
\hline$Y-9215-B(10)$ & $01-1020-51$ & 028299P & $\mathrm{O}$ \\
\hline$Y-9215-B(18)$ & $01-1020-51$ & 028299P & 0 \\
\hline$Y-9215-B(20)$ & $01-1020-51$ & 028299P & 0 \\
\hline$Y-9215-C(02)$ & $01-1020-52$ & 025948P & $\mathrm{O}$ \\
\hline$Y-9215-C(03)$ & $01-1020-52$ & 025948P & 0 \\
\hline$Y-9215 \cdot C(10)$ & $01-1020-52$ & $025948 \mathrm{P}$ & 0 \\
\hline$Y-9215-C(11)$ & $01-1020-52$ & $025948 \mathrm{P}$ & $\mathrm{O}$ \\
\hline$Y-9215-C(17)$ & $01-1020-52$ & $025948 \mathrm{P}$ & $\mathrm{O}$ \\
\hline Y-9215-C(19) & $01-1020-52$ & 025948P & $\mathrm{O}$ \\
\hline$Y \cdot 9215-D(12)$ & $01-1020-53$ & $025966 \mathrm{P}$ & 0 \\
\hline$Y-9215-D(13)$ & $01-1020-53$ & 025966P & $\mathrm{O}$ \\
\hline$Y-9215-D(14)$ & $01-1020-53$ & 025966P & $\mathrm{O}$ \\
\hline$Y-9215-D(15)$ & $01-1020-53$ & $025966 \mathrm{P}$ & $\mathrm{O}$ \\
\hline$Y-9215-E(06)$ & $01-1020-54$ & $025972 \mathrm{P}$ & $\mathrm{O}$ \\
\hline$Y-9215-E(07)$ & $01-1020-54$ & $025972 \mathrm{P}$ & $\mathrm{O}$ \\
\hline Y-9215-E(08) & $01-1020-54$ & $025972 \mathrm{P}$ & $\mathrm{O}$ \\
\hline$Y-9401-2-B(02)$ & $01-0020-06$ & $012461 P$ & 0 \\
\hline$Y-9401-2-C(03)$ & $01-0020-07$ & $012462 P$ & $\mathrm{O}$ \\
\hline$Y-9401-2-D(04)$ & $01-0020-08$ & $012463 \mathrm{P}$ & $\mathrm{O}$ \\
\hline$Y-9401-2-E(05)$ & $01-0020-09$ & $021446 \mathrm{P}$ & $\mathrm{O}$ \\
\hline$Y-9401-2-F(06)$ & $01-0020-10$ & 021446P & $\mathrm{O}$ \\
\hline$Y-9401-2-G(07)$ & $01-0020-88$ & $021446 \mathrm{P}$ & $\mathrm{O}$ \\
\hline$Y-9401-2-H(08)$ & $01-0020-88$ & $021446 \mathrm{P}$ & $\mathrm{O}$ \\
\hline$Y-9401-2-J(10)$ & $01-0020-88$ & $021446 \mathrm{P}$ & $\mathrm{O}$ \\
\hline$Y-9401-2-K(11)$ & $01-0020-88$ & 021446P & 0 \\
\hline$Y-9401-2-L(12)$ & $01-0020-88$ & $021446 \mathrm{P}$ & $\mathrm{O}$ \\
\hline$Y-9401-2-M(13)$ & $01-0020-88$ & $021446 \mathrm{P}$ & $\mathrm{O}$ \\
\hline$Y-9401-2-N(14)$ & $01-0020-88$ & 021446P & $\mathrm{O}$ \\
\hline$Y-9401-2 \cdot P(15)$ & $01-0020-88$ & 021446P & $\mathrm{O}$ \\
\hline$Y-9401-2-Q(16)$ & $01-0020-88$ & 021446P & $\mathrm{O}$ \\
\hline$Y-9401-2-R(17)$ & $01-0020-88$ & $021446 \mathrm{P}$ & $\mathrm{O}$ \\
\hline$Y-9401-2-S(18)$ & $01-0020-88$ & 021446P & $\mathrm{O}$ \\
\hline$Y-9401-3-A$ & $01-1020-31$ & $027419 \mathrm{~F}$ & 0 \\
\hline$Y-9401-3-B(1)$ & $01 \cdot 1020-32$ & $027419 \mathrm{~F}$ & $\mathrm{O}$ \\
\hline$Y-9401-3-C$ & $01-1020-33$ & $027419 \mathrm{~F}$ & $\mathrm{O}$ \\
\hline$Y-9401-3-D(2)$ & $01-1020-34$ & $02741.9 \mathrm{~F}$ & $\mathrm{O}$ \\
\hline$Y-9401-3-E$ & $01-0030-39$ & $012881 \mathrm{P}$ & $\mathrm{O}$ \\
\hline$Y-9401-3-F(04)$ & $01-1020-27$ & $023498 \mathrm{P}$ & 0 \\
\hline$Y-9401-3-G(01)$ & $01-1020-61$ & $026472 \mathrm{P}$ & $\mathrm{O}$ \\
\hline$Y-9401-3-G(02)$ & $01-1020-61$ & $926472 P$ & $\mathrm{O}$ \\
\hline
\end{tabular}


Table 2.1.11 (continued)

\begin{tabular}{|c|c|c|c|}
\hline $\begin{array}{l}\text { Y-12 Plant } \\
\text { source number }\end{array}$ & $\begin{array}{l}\text { Emission source } \\
\text { reference number }\end{array}$ & $\begin{array}{l}\text { Permit } \\
\text { number }\end{array}$ & $\begin{array}{l}\text { Permit } \\
\text { type }^{a}\end{array}$ \\
\hline$Y-9401-3-H(01)$ & $01-1020-61$ & $495278 \mathrm{P}$ & $\mathrm{C}$ \\
\hline$Y-9401-3-I(1)$ & $01-1020-66$ & $023852 \mathrm{P}$ & $\mathrm{O}$ \\
\hline Y-9401-3-J-1 & $01-1020-82$ & $997626 \mathrm{P}$ & $\mathrm{C}$ \\
\hline$Y-9401-4-A(01)$ & $01-0020-65$ & 022240P & $\mathrm{O}$ \\
\hline$Y-9401-5-A(01)$ & $01-0020-92$ & $026108 \mathrm{P}$ & $\mathrm{O}$ \\
\hline$Y-9404-11-A(1)$ & $01-1020-81$ & $028426 \mathrm{P}$ & $\mathrm{O}$ \\
\hline$Y-9404-11-A(2)$ & $01-1020-81$ & $028426 \mathrm{P}$ & $\mathrm{O}$ \\
\hline$Y \cdot 9404-11-A(3)$ & $01-1020-81$ & $028426 \mathrm{P}$ & 0 \\
\hline$Y-9404-11-A(4)$ & $01-1020-81$ & $028426 \mathrm{P}$ & $\mathrm{O}$ \\
\hline$Y-9404 \cdot 5-B(02)$ & $01-0020-25$ & $012866 \mathrm{P}$ & $\mathrm{O}$ \\
\hline$Y-9404-5-B(03)$ & $01-0020-25$ & $012866 \mathrm{P}$ & $\mathrm{O}$ \\
\hline$Y-9404-9-C(03)$ & $01-0020-40$ & $012882 \mathrm{P}$ & 0 \\
\hline Y.9404-9-D(04) & $01-0020.40$ & $012882 \mathrm{P}$ & 0 \\
\hline$Y-9.404-9-E(05)$ & $01-0020-40$ & $012882 \mathrm{P}$ & 0 \\
\hline$Y-9616-7-A(01)$ & $01-1020-74$ & $026502 \mathrm{P}$ & $\mathrm{O}$ \\
\hline$Y-9616-7-A(02)$ & $01-1020-74$ & $026502 \mathrm{P}$ & $\mathrm{O}$ \\
\hline $\mathrm{Y}-9616-7-\mathrm{A}(03)$ & $01-1020-74$ & $026502 \mathrm{P}$ & $\mathrm{O}$ \\
\hline$Y-9616-7-A(04)$ & $01-1020-74$ & $026502 \mathrm{P}$ & $\mathrm{O}$ \\
\hline$Y-9616-7-A(05)$ & $01-1020-74$ & $026502 \mathrm{P}$ & 0 \\
\hline$Y-9616-7-A(06)$ & $01-1020 \cdot 74$ & $026502 \mathrm{P}$ & $\mathrm{O}$ \\
\hline$Y-9616-7-A(07)$ & $01-1020-74$ & $026502 \mathrm{P}$ & $\mathrm{O}$ \\
\hline$Y-9616-7-A(08)$ & $01-1020-74$ & $026502 \mathrm{P}$ & $\mathrm{O}$ \\
\hline$Y-9616-7-A(09)$ & $01-1020-74$ & $026502 \mathrm{P}$ & $\mathrm{O}$ \\
\hline$Y-9616-7-A(10)$ & $01-1020-74$ & $026502 \mathrm{P}$ & $\mathrm{O}$ \\
\hline$Y-9616-7-A(11)$ & $01-1020-74$ & $026502 \mathrm{P}$ & $\mathrm{O}$ \\
\hline$Y-9616-7-A(12)$ & $01-1020-74$ & $026502 \mathrm{P}$ & $\mathrm{O}$ \\
\hline$Y-9616-7-B(1)$ & $01-1020-80$ & $0997583 \mathrm{P}$ & $\mathrm{C}$ \\
\hline$Y-9620-2 A$ & $01-0020-5 n$ & $012894 \mathrm{P}$ & $\mathrm{O}$ \\
\hline Y.9623-A(01) & $01-1020-25$ & 025970P & $\mathrm{O}$ \\
\hline Y-9623-A(02) & $01-1020-25$ & $025970 \mathrm{P}$ & 0 \\
\hline Y.9623-A(03) & $01-1020-25$ & 025970P & $\mathrm{O}$ \\
\hline Y-9623-A(04) & $01-1020-25$ & $025970 \mathrm{P}$ & $\mathrm{O}$ \\
\hline$Y-9623-A(05)$ & $01-1020-25$ & $025970 \mathrm{P}$ & 0 \\
\hline$Y-9623-A(06)$ & $01-1020-25$ & 025970P & $\mathrm{O}$ \\
\hline$Y-9712-A(01)$ & $01-1020-65$ & $023851 \mathrm{P}$ & $\mathrm{O}$ \\
\hline$Y-9720-19-A(01)$ & $01-0020-41$ & $012885 \mathrm{P}$ & $\mathrm{O}$ \\
\hline Y.9720-19-C(01) & $01-0020-23$ & $012864 \mathrm{P}$ & 0 \\
\hline Y-9720-19-D(03) & $01-0020-27$ & $012869 \mathrm{P}$ & $\mathrm{O}$ \\
\hline$Y-9720-20-A(01)$ & $01-1020-39$ & $025971 \mathrm{P}$ & 0 \\
\hline$Y-9720-41-A(01)$ & $01-1020-63$ & $028384 \mathrm{P}$ & $\mathrm{O}$ \\
\hline$Y-9720-41-A(02)$ & $01-1020-63$ & $028384 \mathrm{P}$ & $\mathrm{O}$ \\
\hline$Y-9720-41-A(03)$ & $01-1020-63$ & $028384 \mathrm{P}$ & $\mathrm{O}$ \\
\hline
\end{tabular}


Table 2.1.11 (continued)

\begin{tabular}{|c|c|c|c|}
\hline $\begin{array}{c}\text { Y-12 Plant } \\
\text { source n'.mber }\end{array}$ & $\begin{array}{l}\text { Emission source } \\
\text { reference number }\end{array}$ & $\begin{array}{l}\text { Permit } \\
\text { number }\end{array}$ & $\begin{array}{c}\text { Permit } \\
\text { type }^{a}\end{array}$ \\
\hline$Y-9720-41-A(04)$ & $01-1020-63$ & $028384 \mathrm{P}$ & $\mathrm{O}$ \\
\hline$Y-9720-41-A(05)$ & $01-1020-63$ & $028384 \mathrm{P}$ & $\mathrm{O}$ \\
\hline$Y-9720-5-A$ & $01-1020-75$ & 027379P & $\mathrm{O}$ \\
\hline$Y-9720-6-B(01)$ & $01-0020-75$ & $015154 \mathrm{P}$ & 0 \\
\hline$Y-9720-6-C(01)$ & $01-0020-76$ & $015155 \mathrm{P}$ & $\mathrm{O}$ \\
\hline$Y-9720-6-C(02)$ & $01-0020-76$ & $015155 \mathrm{P}$ & 0 \\
\hline$Y-9720-6-E(01)$ & $01-0020-83$ & $016548 \mathrm{P}$ & $\mathrm{O}$ \\
\hline$Y-9737-A(01)$ & $01-0020-22$ & $012863 P$ & $\mathrm{O}$ \\
\hline Y-9737-B(02) & $01-0020-78$ & $015157 \mathrm{P}$ & 0 \\
\hline$Y-9737-C(02)$ & $01-0020-78$ & $015157 \mathrm{P}$ & 0 \\
\hline$Y-9737-D(02)$ & $01-0020-78$ & $015157 \mathrm{P}$ & 0 \\
\hline$Y-9737-E(02)$ & $01-0020-78$ & $015157 \mathrm{P}$ & 0 \\
\hline$Y-9737-F(02)$ & $01-0020-78$ & $015157 \mathrm{P}$ & 0 \\
\hline$Y-9737-G(03)$ & $01-0020-79$ & $015160 \mathrm{P}$ & 0 \\
\hline$Y-9737-H(04)$ & $01-0020-79$ & $015160 \mathrm{P}$ & 0 \\
\hline$Y-9737-I(05)$ & $01-0020-79$ & $015160 \mathrm{P}$ & 0 \\
\hline$Y-9738-A(01)$ & $01-0020-14$ & 025975P & 0 \\
\hline$Y-9738-A(02)$ & $01-0020-14$ & $025975 \mathrm{P}$ & 0 \\
\hline Y-9738-A(03) & $01-0020-14$ & $025975 \mathrm{P}$ & $\mathrm{O}$ \\
\hline Y-9738-A(04) & $01-0020-14$ & $025975 \mathrm{P}$ & 0 \\
\hline Y.9738-A(05) & $01-0020-14$ & $025975 \mathrm{P}$ & 0 \\
\hline$Y-9739-A(01)$ & $01-1020-78$ & 028105P & 0 \\
\hline$Y-9739-B(02)$ & $01-1020-78$ & $028105 P$ & 0 \\
\hline$Y-9754-2-A(01)$ & $01-0020-52$ & $012897 \mathrm{P}$ & 0 \\
\hline$Y-9767-4-A(01)$ & $01-0020-35$ & $012877 \mathrm{P}$ & 0 \\
\hline$Y-9808-A(01)$ & $01-1020-22$ & $026109 \mathrm{P}$ & 0 \\
\hline$Y-9809-A(01)$ & $01-0020-93$ & 025899P & 0 \\
\hline$Y-9811-A(01)$ & $01-0020-42$ & $012886 \mathrm{P}$ & 0 \\
\hline$Y-9811-B(02)$ & $01-1020-45$ & 025903P & $\mathrm{O}$ \\
\hline Y-9812-A(01) & $01-1020-29$ & $022474 \mathrm{P}$ & 0 \\
\hline$Y-9812-A(02)$ & $01-1020-29$ & $022474 \mathrm{P}$ & $\mathrm{O}$ \\
\hline$Y-9812-A(03)$ & $01-1020-29$ & $022474 \mathrm{P}$ & 0 \\
\hline$Y-9815-A(03)$ & $01-0020-11$ & $025895 \mathrm{P}$ & $\mathrm{O}$ \\
\hline$Y-9815-A(04)$ & $01-0020-11$ & $025895 \mathrm{P}$ & 0 \\
\hline$Y-9815-A(05)$ & $01-0020-11$ & $025895 \mathrm{P}$ & 0 \\
\hline$Y-9815-A(06)$ & $01-0020-11$ & $025895 \mathrm{P}$ & $\mathrm{O}$ \\
\hline Y-9815-A(07) & $01-0020-11$ & $025895 \mathrm{P}$ & 0 \\
\hline Y-9815-A(08) & $01-0020-11$ & $025895 \mathrm{P}$ & $\mathrm{O}$ \\
\hline$Y-9818-A(01)$ & $01-0020-12$ & $025965 \mathrm{P}$ & $\mathrm{O}$ \\
\hline$Y-9818-A(02)$ & $01-0020-12$ & $025965 \mathrm{P}$ & 0 \\
\hline$Y-9818-A(03)$ & $01-0020-12$ & $025965 \mathrm{P}$ & 0 \\
\hline$Y-9818-A(04)$ & $01-0020-12$ & $025965 \mathrm{P}$ & 0 \\
\hline $\mathrm{Y}-9818-\mathrm{A}(05)$ & $01.0020-12$ & $025965 \mathrm{P}$ & 0 \\
\hline$Y-9818-A(06)$ & $01-0020-12$ & $025965 \mathrm{P}$ & 0 \\
\hline
\end{tabular}


Table 2.1.11 (continued)

\begin{tabular}{cccc}
\hline $\begin{array}{c}\text { Y-12 Plant } \\
\text { source number }\end{array}$ & $\begin{array}{c}\text { Emission source } \\
\text { reference number }\end{array}$ & $\begin{array}{c}\text { Permit } \\
\text { number }\end{array}$ & $\begin{array}{c}\text { Permit } \\
\text { type }^{a}\end{array}$ \\
\hline Y-9818-A(07) & $01-0020-12$ & $025965 \mathrm{P}$ & $\mathrm{O}$ \\
Y-9818-A(08) & $01-0020-12$ & $025965 \mathrm{P}$ & $\mathrm{O}$ \\
Y-9818-A(09) & $01-0020-12$ & $025965 \mathrm{P}$ & $\mathrm{O}$ \\
Y-9818-A(10) & $01-0020-12$ & $025965 \mathrm{P}$ & $\mathrm{O}$ \\
Y-9818-A(11) & $01-0020-12$ & $025965 \mathrm{P}$ & $\mathrm{O}$ \\
Y-9818-A(12) & $01-0020-12$ & $025965 \mathrm{P}$ & $\mathrm{O}$ \\
Y-9929-F(01) & $01-0020-39$ & $012881 \mathrm{P}$ & $\mathrm{O}$ \\
Y-9995-A(01) & $01-0020-21$ & $021086 \mathrm{P}$ & $\mathrm{O}$ \\
Y-9998-A(01) & $01-0020-13$ & $025957 \mathrm{P}$ & $\mathrm{O}$ \\
Y-9998-A(02) & $01-0020-13$ & $025957 \mathrm{P}$ & $\mathrm{O}$ \\
Y-9998-A(03) & $01-0020-13$ & $025957 \mathrm{P}$ & $\mathrm{O}$ \\
Y-9998-A(04) & $01-0020-13$ & $025957 \mathrm{P}$ & $\mathrm{O}$ \\
Y-9998-A(05) & $01-0020-13$ & C25957P & $\mathrm{O}$ \\
Y-9998-A(06) & $01-0020-13$ & $025957 \mathrm{P}$ & $\mathrm{O}$ \\
Y-9998-B(01) & $01-1020-40$ & $026110 \mathrm{P}$ & $\mathrm{O}$ \\
Y-9998-C(01) & $01-1020-84$ & $0997769 \mathrm{P}$ & $\mathrm{C}$ \\
Y-Townsite & $01-0020-53$ & $012889 \mathrm{~F}$ & $\mathrm{O}$ \\
\hline
\end{tabular}

${ }^{a} \mathrm{O}=$ operating; $\mathrm{C}=$ construction. 
Table 2.1.12. Air permits at ORNL

\begin{tabular}{|c|c|c|c|c|}
\hline $\begin{array}{c}\text { ORNL } \\
\text { source } \\
\text { number }\end{array}$ & $\begin{array}{l}\text { Emission } \\
\text { source } \\
\text { reference } \\
\text { number }\end{array}$ & $\begin{array}{l}\text { Permit } \\
\text { number }\end{array}$ & Source & type $^{a}$ \\
\hline$X-1506$ & $73-0112-02$ & $012452 \mathrm{P}$ & Hood & 0 \\
\hline $\mathrm{X}-2000-02$ & $73-0112-75$ & $024473 \mathrm{P}$ & Furnace, ovens, hoods, pumps & 0 \\
\hline X-2000-09 & $73-0112-32$ & $024135 \mathrm{P}$ & Laser with wet scrubber & 0 \\
\hline$X-2013-01$ & $73-0112-65$ & 026944P & Parts washer (degreaser) & $\mathbf{o}$ \\
\hline$X-2013-04$ & $73-0112-52$ & $024913 \mathrm{P}$ & Vapor blaster & 0 \\
\hline X-2018-02 & $73-0112-13$ & 024250P & Parts washer (degreaser) & 0 \\
\hline $\mathrm{X}-2018-03$ & $73-0112-44$ & $024117 \mathrm{P}$ & Oven & 0 \\
\hline$X-2026-06$ & $73-0112-77$ & 024759P & Rad laboratory & 0 \\
\hline $\mathrm{X}-2510-\mathrm{T} 1$ & $73-0112-63$ & $024402 \mathrm{P}$ & Tank, propane & 0 \\
\hline$X-2519-01$ & $73-0112-65$ & 026944P & Parts washer (degreaser) & 0 \\
\hline$X-2519-1 / 5$ & $73-0112-03$ & $028027 \mathrm{P}$ & 5 boilers at steam plant and ash & 0 \\
\hline $\mathrm{X}-2519-\mathrm{T} 1$ & $73-0112-57$ & $024915 \mathrm{P}$ & Tank, sulfuric acid & 0 \\
\hline $\mathrm{X}-2522$-TIA & $73-0112-10$ & $024114 \mathrm{P}$ & Tank, fuel oil & 0 \\
\hline X 2525-01 & $73-0112-14$ & $013013 \mathrm{P}$ & Degreaser & 0 \\
\hline$X-2525-02$ & $73-0112-65$ & 026944P & Parts washer (degreaser) & 0 \\
\hline$X-2525-03$ & $73-0112-65$ & 026944P & Parts washer (degreaser) & 0 \\
\hline$X-2525-04$ & $73-0112-38$ & 023809P & Machine shop & 0 \\
\hline$X-2525-06$ & $73-0112-95$ & 027257P & Machine shop & 0 \\
\hline$X-2525-08$ & $73-0112-62$ & $024949 \mathrm{P}$ & Spray booth and oven & 0 \\
\hline$X-2525-11$ & $73-0112-49$ & $024151 \mathrm{P}$ & Electroplating shop & 0 \\
\hline$X-2525-13$ & $73-0112-54$ & 027392P & Vapor blaster and buffers & 0 \\
\hline $\mathrm{X}-2525-\mathrm{T} 1$ & $73.0112-72$ & $024475 \mathrm{P}$ & Tank, waste oil & 0 \\
\hline $\mathrm{X}-2525-\mathrm{T} 2$ & $73-0112-72$ & 024475P & Tank, waste oil & 0 \\
\hline$X-2547-01$ & $73-0112-27$ & 028439P & Spray booth & 0 \\
\hline$X-2547-02$ & $73-0112.65$ & 026944P & Parts washer (degreaser) & 0 \\
\hline$X-3003-06$ & $73-0112-29$ & $023760 \mathrm{P}$ & Tank, sulfur hexafluoride & 0 \\
\hline$X-3003-08$ & $73.0112-29$ & $023760 \mathrm{P}$ & Tank, sulfur hexafluoride & 0 \\
\hline $\mathrm{X}-3004-\mathrm{T} 1$ & $73-0112-46$ & $024136 \mathrm{P}$ & Tank, nitric acid & 0 \\
\hline$X-3004-T 2$ & $73-0112-46$ & $024136 \mathrm{P}$ & Tank, nitric acid & 0 \\
\hline $\mathrm{X}-3004-\mathrm{T} 3$ & $73-\mathrm{C} 112-46$ & $024136 \mathrm{P}$ & Tank, nitric acid & 0 \\
\hline$X-3005-01$ & $73-0112-65$ & 026944P & Parts washer (degreaser) & 0 \\
\hline$X-3005-02$ & $73-0112-18$ & $027214 \mathrm{P}$ & Parts washer (degreaser) & 0 \\
\hline$X-3012.01$ & $73-0112-74$ & 024449P & Furnace & 0 \\
\hline$X-30: 2-02$ & $73-0112-50$ & $024252 \mathrm{P}$ & Degreaser & 0 \\
\hline$X-3025-01$ & $73-0112-65$ & $026944 \mathrm{P}$ & Parts washer (degreaser) & 0 \\
\hline$X-3039-01$ & $73-0112-93$ & $026525 \mathrm{P}$ & Off gas and hot cell ventilat: & o \\
\hline$X-3074-01$ & $73-0112-65$ & $026944 \mathrm{P}$ & Parts washer (degreaser) & 0 \\
\hline $\mathrm{X}-3103-\mathrm{Tl}$ & $73.0112-42$ & $024113 \mathrm{P}$ & Tank, sulfuric acid & 0 \\
\hline$X-3104-03$ & $73-0112-81$ & $024511 \mathrm{P}$ & Carpenter shop & 0 \\
\hline $\mathrm{X}-3117-\mathrm{T} 1$ & $73-0112-42$ & $024115 \mathrm{P}$ & Tank, sulfuric acid & 0 \\
\hline$X-3500-02$ & $73-0112-65$ & $026944 \mathrm{P}$ & Parts washer (degreaser) & 0 \\
\hline$X-3500-12$ & $73-0112-73$ & 024450P & Furnace & 0 \\
\hline$X-3502-01$ & $73-0112-05$ & $013022 \mathrm{P}$ & Spray booth & 0 \\
\hline$X-3502-02$ & $73-0112-06$ & $013023 \mathrm{P}$ & Spray booth & 0 \\
\hline$X-3502-03$ & $73-0112-07$ & $013024 \mathrm{P}$ & Spray booth & 0 \\
\hline$X-3502-09$ & $73-0112-94$ & 027194P & Hood, gluing & 0 \\
\hline $\mathrm{X}-3502-\mathrm{SV} 1$ & $73-0112-39$ & $023808 \mathrm{P}$ & Oven, curing & 0 \\
\hline $\mathrm{X}-3502-\mathrm{SV} 2$ & $73-0112-40$ & $023807 \mathrm{P}$ & Oven, tempering & 0 \\
\hline$X-3502-S V 4$ & $73-0112-30$ & 024309P & Cyclone and carpentry shop & 0 \\
\hline$X-3504-01$ & $73-0112-80$ & $024451 \mathrm{P}$ & Oven & 0 \\
\hline$X-3544-01$ & $73-0112-70$ & $025552 \mathrm{P}$ & PWTP & $\mathrm{OM}$ \\
\hline$X-3587-1$ & $73-0112-56$ & 99755IP & Electroplating shop & $\mathrm{CM}$ \\
\hline$X-3608-01$ & $73-0112-37$ & $995888 \mathrm{P}$ & NRWTP air stripper and tanks & 0 \\
\hline $\mathrm{X}-4508-08$ & $73-0112-61$ & $025121 \mathrm{P}$ & Acid etching process & 0 \\
\hline
\end{tabular}


Table 2.1.12 (continued)

\begin{tabular}{|c|c|c|c|c|}
\hline $\begin{array}{l}\text { ORNL } \\
\text { source } \\
\text { number }\end{array}$ & $\begin{array}{l}\text { Emission } \\
\text { source } \\
\text { reference } \\
\text { number }\end{array}$ & $\begin{array}{l}\text { Permit } \\
\text { number }\end{array}$ & Source & $\begin{array}{l}\text { Permit } \\
\text { type }\end{array}$ \\
\hline$X-4508-09$ & $73-0112-55$ & $024306 \mathrm{P}$ & Sand blaster & $\mathrm{O}$ \\
\hline$X .4508-16$ & $73-0112-51$ & 024909P & Spray booth & $\mathrm{O}$ \\
\hline $\mathrm{X}-4508-\mathrm{T} 1$ & $73-0112-64$ & 024403P & Tank, freon & $\mathrm{O}$ \\
\hline$X-4515-00$ & $73-0112-68$ & $025239 \mathrm{P}$ & HTML & $\mathrm{O}$ \\
\hline$X-5500-00$ & $73-0112-29$ & $023760 \mathrm{P}$ & Tank & $\mathrm{O}$ \\
\hline$X-6000-01$ & $73-0112-65$ & $026944 \mathrm{P}$ & Parts washer (degreaser) & $\mathrm{O}$ \\
\hline$X-6000-02$ & $73-0112-59$ & $024308 \mathrm{P}$ & Vapor blaster & $\mathrm{O}$ \\
\hline$X-6005-00$ & 73-0112-29 & $023760 \mathrm{P}$ & Tanh, sulfur hexafluoride & $\mathrm{O}$ \\
\hline$X-6010-00$ & $73-0112-85$ & $025282 \mathrm{P}$ & ORELA & $\mathrm{O}$ \\
\hline$X-6010-01$ & $73-0112-65$ & $026944 \mathrm{P}$ & Parts washer (degreaser) & $\mathrm{O}$ \\
\hline$X-7002-01$ & $73-0112-19$ & $024251 \mathrm{P}$ & Parts washer (degreaser) & $\mathrm{O}$ \\
\hline$X-7002-03$ & 73.0112 .08 & $013025 \mathrm{P}$ & Spray booth & $\mathrm{O}$ \\
\hline$X-7002-03$ & $73-0112-65$ & $026944 \mathrm{P}$ & Parts washer (degreaser) & $\mathrm{O}$ \\
\hline$X-7002-04$ & $73-0112-65$ & $026944 \mathrm{P}$ & Parts washer (degreaser) & $\mathrm{O}$ \\
\hline $\mathrm{X}-7002-\mathrm{T} 1$ & $73-0112-88$ & 025659P & Tank, waste oil & $\mathrm{O}$ \\
\hline$X-7003-01$ & $73-0112-79$ & $024452 \mathrm{P}$ & Furnace & 0 \\
\hline$X-7003-01$ & $73-0112-65$ & $026944 \mathrm{P}$ & Parts washer (degreaser) & $\mathrm{O}$ \\
\hline$X-7005-00$ & $73-0112-45$ & $024118 \mathrm{P}$ & Machining tools & $\mathrm{O}$ \\
\hline$X-7005-3-7$ & $73-0112-26$ & $028438 \mathrm{P}$ & Lead shop & $\mathrm{O}$ \\
\hline$X-7007-1 / 2$ & $73-0112.09$ & $024134 \mathrm{P}$ & Spray booth & $\mathrm{O}$ \\
\hline$X-7012-01$ & $73-0112-65$ & 026944P & Parts washer (degreaser) & $\mathrm{O}$ \\
\hline $\mathrm{X}-7021-00$ & $73-0112.58$ & $024307 \mathrm{P}$ & Grinding shop & $\mathrm{O}$ \\
\hline $\mathrm{X}-7021-\mathrm{T} 1$ & $73-0112-58$ & $025660 \mathrm{P}$ & Tank, waste oil & $\mathrm{O}$ \\
\hline$X-7025-00$ & $73-0112-92$ & $026070 \mathrm{P}$ & TTFF & $\mathrm{O}$ \\
\hline$X-7057-01$ & $73-0112-76$ & $024760 \mathrm{P}$ & Sand blaster & $\mathrm{O}$ \\
\hline$X-7069-C / D$ & $73-0112-11$ & $013030 \mathrm{P}$ & Tank, gasoline & $\mathrm{O}$ \\
\hline $\mathrm{X}-7069-\mathrm{T}$ & $73-0112-60$ & $026726 \mathrm{P}$ & Two gasoline tanks & 0 \\
\hline $\mathrm{X}-7075-\mathrm{T} 1$ & $73-0112-90$ & $025661 \mathrm{P}$ & Tank, waste oil & $\mathrm{O}$ \\
\hline $\mathrm{X}-7075-\mathrm{T} 2$ & $73-0112-90$ & $025661 \mathrm{P}$ & Tank, photographic waste & $\mathrm{O}$ \\
\hline $\mathrm{X}-7075-\mathrm{T} 3$ & $73-0112-90$ & $025661 \mathrm{P}$ & Tank, photographic waste & $\mathrm{O}$ \\
\hline$X-7503-00$ & $73-0112-83$ & $025254 \mathrm{P}$ & iviolten salt reactor & $\mathrm{O}$ \\
\hline$X-7600-01$ & $73-0112-20$ & $017930 \mathrm{P}$ & Nuclear fuel reprocessing & $\mathrm{O}$ \\
\hline $\mathrm{X}-7601-\mathrm{T} 1$ & $73-0112-47$ & $024137 \mathrm{P}$ & Tank, nitric acid & 0 \\
\hline$X-7602-01$ & $73-0112.24$ & 027090P & Boiler, hot water & $\mathrm{O}$ \\
\hline$X-7603-01$ & $73-0112-25$ & $022743 \mathrm{~F}$ & Boiler, steam & $\mathrm{O}$ \\
\hline$X-7606-01$ & $73.0112-65$ & 026944P & Parts washer (degreaser) & $\mathrm{O}$ \\
\hline$X-7667-0$ & $\mathrm{~N} / \mathrm{A}$ & & Chemical detonation site & $\mathrm{OB}$ \\
\hline$X-7822-00$ & $73-0112-86$ & 025340P & Solid waste shredder & $\mathrm{O}$ \\
\hline$X-7830-01$ & $73-0112-71$ & $027132 \mathrm{P}$ & EASC & $\mathrm{O}$ \\
\hline$X-7831-00$ & $73-0112.84$ & $025281 \mathrm{P}$ & Baler and box compactor & $\mathrm{O}$ \\
\hline $\mathrm{X}-7900-\mathrm{T} 1$ & $73-0112-43$ & $024116 \mathrm{P}$ & Tank, nitric acid & $\mathrm{O}$ \\
\hline $\mathrm{X}-7900-\mathrm{T} 2$ & $73-0112-43$ & $024116 \mathrm{P}$ & Tank, nitric acid & 0 \\
\hline $\mathrm{X}-7900-\mathrm{T} 3$ & $73-0112-66$ & $025162 \mathrm{P}$ & Tank, nitric acid & $\mathrm{O}$ \\
\hline $\mathrm{X}-7900-\mathrm{T} 4$ & $73-0112-66$ & $025162 \mathrm{P}$ & Tank, nitric acid & 0 \\
\hline $\mathrm{X}-7903-\mathrm{Tl}$ & $73-0112-48$ & $024138 \mathrm{P}$ & Tank, sulfuric acid & $\mathrm{O}$ \\
\hline$X-7910-01$ & $73-0112-65$ & $026944 \mathrm{P}$ & Parts washer (degreaser) & $\mathrm{O}$ \\
\hline$X-7911-00$ & $73-0112-82$ & $025249 \mathrm{P}$ & HFIR, TRU, and TURF & $\mathrm{O}$ \\
\hline$X-7934-02$ & $73-0112-53$ & $\mathrm{C} 24912 \mathrm{P}$ & Silver recovery system & $\mathrm{O}$ \\
\hline$X-7935-01$ & $73-0112-78$ & $027393 \mathrm{P}$ & Equipment cleaning facility & $\mathrm{O}$ \\
\hline $\mathrm{X}-4500 \mathrm{~N} I-93$ & $73-0112-65$ & $026944 \mathrm{P}$ & Parts washer (degreaser) & $\mathrm{O}$ \\
\hline $\mathrm{X}-4500 \mathrm{~S} 1-01$ & $73-0112-87$ & $026021 \mathrm{P}$ & Parts washer (degreaser) & 0 \\
\hline$X-4500 S 3-50$ & $73-0112-31$ & $024 C 88 \mathrm{P}$ & Mercury purification system & 0 \\
\hline
\end{tabular}

${ }^{a} \mathrm{O}=$ operating; $\mathrm{M}=$ under modification; $\mathrm{C}=$ construction; $\mathrm{OB}=$ open burning. 
Table 2.1.13. Air Permits at ORGDP

\begin{tabular}{|c|c|c|c|c|}
\hline $\begin{array}{c}\text { ORGDP } \\
\text { source number }\end{array}$ & $\begin{array}{c}\text { Emission } \\
\text { source } \\
\text { reference } \\
\text { number } \\
\text { (73-XXXX-XX) }\end{array}$ & $\begin{array}{l}\text { Permit } \\
\text { number }\end{array}$ & Source & $\begin{array}{l}\text { Permit } \\
\text { type }^{a}\end{array}$ \\
\hline K-402 8-16-990-cool-P-162539 & $0106-28$ & 024500P & Misc. chemical storage (18 tanks) & 0 \\
\hline K-402 9 PC & $0101-42$ & $012660 \mathrm{P}$ & Gas diffusion purge cascade & $\mathbf{0}$ \\
\hline K-402 9-16-989-cool-P-162554 & $0106-28$ & 024500P & Misc. chemical storage (18 tanks) & 0 \\
\hline K-502 2-327298 Freon R-114 & $0106-28$ & 024500P & Misc. chemical storage (18 tanks) & 0 \\
\hline K-502 2-327300 Freon R-114 & $0106-28$ & $024500 \mathrm{P}$ & Misc. chemical storage (18 tanks) & 0 \\
\hline K-602 WAP & $0106-93$ & 024297P & Evacuation of cascade cells & 0 \\
\hline K-602 2-325172 Freon R-114 & $0106-28$ & $024500 \mathrm{P}$ & Misc. chemical storage (18 tanks) & 0 \\
\hline K-602 4-325285 Freon R-114 & $0106-28$ & $024500 \mathrm{P}$ & Misc. chemical storage (18 tanks) & $\mathbf{O}$ \\
\hline K-832 chromate $T$ & $1106-10$ & $024947 \mathrm{P}$ & Storage tank & 0 \\
\hline K-892 chromate T & $1106-10$ & $024947 \mathrm{P}$ & Storage tank & 0 \\
\hline K .892 lime silo & $1106-10$ & $025120 \mathrm{P}$ & Lime storage silo & $\mathbf{o}$ \\
\hline K.892 sulfuric acid tank, N & $0106-28$ & $024500 \mathrm{P}$ & Misc. chemical storage (18 tanks) & 0 \\
\hline K-892 sulfuric acid tank, S & $0106-28$ & $024500 \mathrm{P}$ & Misc. chemical storage (18 tarks) & $\mathbf{o}$ \\
\hline K-894 sulfuric acid tank & $0106-28$ & $024500 \mathrm{P}$ & Misc. chemical storage (18 tanks) & 0 \\
\hline K-896 soda silo & $1106-24$ & $024758 \mathrm{P}$ & Soda ash silo & 0 \\
\hline K-902 WAP \& jet & $0106-93$ & $024298 \mathrm{P}$ & Evacuation of cascade cells & $\mathbf{o}$ \\
\hline K-902 3-324383 Freon R-114 & $0106-28$ & $024500 \mathrm{P}$ & Misc. chemical storage (18 tanks) & 0 \\
\hline K-902 3-324389 & & & Storage tank & \\
\hline K-902 3-324469 Freon R-114 & $0106-28$ & $024500 \mathrm{P}$ & Misc. chemical storage (18 tanks) & $\mathbf{0}$ \\
\hline K-902 3-324470 Freon R-114 & $0106-28$ & 024500P & Misc. chemical storage (18 tanks) & 0 \\
\hline K-902 5 PCB $(1-4)$ & $1106-10$ & 024947P & PCB storage tanks (4) & $\mathbf{o}$ \\
\hline K-902 6 PCB (1-3) & $1106-10$ & 024947P & PCB storage tanks (4) & 0 \\
\hline K-902 6 PCB & $1106-10$ & 024947P & PCB storage tank & 0 \\
\hline K-1001 C Blueprint 1 and 2 & $1106-11$ & 024943P & Blueprint machines (2) & 0 \\
\hline K-1001 Opti-copy & $1106-07$ & $024395 P$ & Photographic reproduction & 0 \\
\hline K-1004 L oven & 0106-95 & $024299 \mathrm{P}$ & Sintering operation & 0 \\
\hline $\mathrm{K}-1004 \mathrm{~T}$ center $\mathrm{b}$ hood & $1106-04$ & $024756 \mathrm{P}$ & Resin and hardener mixer with hoods & 0 \\
\hline $\mathrm{K}-1004 \mathrm{~T}$ hood & $1106-04$ & 024498P & Resin and hardener mixer with nood & $\mathbf{0}$ \\
\hline K-1004 T hood-methchlor & $1106-15$ & $025493 \mathrm{P}$ & Ultrasonic epoxy parts cleaner & $\mathbf{0}$ \\
\hline $\mathrm{K}-1004 \mathrm{~T}$ south oven & $1106-01$ & $024304 \mathrm{P}$ & Matrix composites cure & 0 \\
\hline $\mathrm{K}-1004 \mathrm{~T}$ west $\mathrm{n}$ oven & $0106-96$ & 024301P & Matrix composites cure & $\mathbf{0}$ \\
\hline $\mathrm{K}-1004 \mathrm{~T}$ wind 1 & $1106-27$ & $025843 \mathrm{P}$ & Winding and coating operations & 0 \\
\hline $\mathrm{K}-1004 \mathrm{~T}$ wind 2 & $1106-27$ & $025843 \mathrm{P}$ & Winding and coating operativas & 0 \\
\hline $\mathrm{K}-1004 \mathrm{~T}$ wind 3 & $1106-27$ & $025843 \mathrm{P}$ & Wirding and coating operations & $\mathbf{0}$ \\
\hline $\mathrm{K}-1004 \mathrm{~T}$ wind 4 & $1106-27$ & 025843P & Winding and coating operations & 0 \\
\hline K-1010 bond hood & $1106-06$ & $024502 \mathrm{P}$ & Parts clean and adhesives mix & 0 \\
\hline $\mathrm{K}-1010$ bond oven & $0106-90$ & $024270 \mathrm{P}$ & Matrix composites cure & 0 \\
\hline $\mathrm{K}-1023 \mathrm{R}$ oven 323.586 & $0106-91$ & $024271 \mathrm{P}$ & Matrix composites cure & $\mathbf{O}$ \\
\hline K-1024 FT-1 & $0106-18$ & $025655 \mathrm{P}$ & Filter-testing facility & o \\
\hline K-1035 plate 1 & $0106-99$ & 024305P & Potting and developing ops & 0 \\
\hline K-1035 plate 2 & $1106-05$ & $024503 P$ & Instrument-cleaning operation & $\mathbf{o}$ \\
\hline K-1035 plate 3 & $0106-97$ & $024302 \mathrm{P}$ & Acid cleaning and decontamination & $\mathrm{O}$ \\
\hline K-1035 plate 4 & & & Degreaser and cleaner & \\
\hline K-1035 plate 5 & $0106-98$ & 024303P & Printed circuit board Mfg. & $\mathbf{0}$ \\
\hline K-1037 AVLIS furnace & $0106-81$ & $023119 \mathrm{P}$ & AVLIS furnace & $\mathbf{O}$ \\
\hline K-1037 AVLIS grieve oven & $0106-80$ & $023118 \mathrm{P}$ & AVLIS grieve oven (TB-500) & $\mathbf{o}$ \\
\hline K-1037 AVLIS quincy oven & $0106-79$ & $023120 \mathrm{P}$ & AVLIS quincy oven (73-6 OOM) & $\mathbf{0}$ \\
\hline
\end{tabular}


Table 2.1.13 (continued)

\begin{tabular}{|c|c|c|c|c|}
\hline $\begin{array}{c}\text { ORGDP } \\
\text { source number }\end{array}$ & $\begin{array}{c}\text { Emission } \\
\text { source } \\
\text { reference } \\
\text { number } \\
(73-X X X X-X X)\end{array}$ & $\begin{array}{l}\text { Permit } \\
\text { number }\end{array}$ & Source & $\begin{array}{l}\text { Permit } \\
\text { type }^{a}\end{array}$ \\
\hline K-1037 dry spray booth & $0106-76$ & $994500 \mathrm{P}$ & DeVilbiss spray booth ELD \#432 & PTC \\
\hline K-1037 grit blast facility & 0106-77 & $022111 \mathrm{P}$ & Grit blast facility with baghouse & o \\
\hline K-1037 MLBH mechanical lab & $0106-84$ & $023662 \mathrm{P}$ & Mechanical lab, cut and shape parts & 0 \\
\hline K. 1200 center bay & $0106-87$ & $026548 \mathrm{P}$ & Center bay & 0 \\
\hline K-1200 center bay hood & $0106-87$ & $026548 \mathrm{P}$ & Center bay hood & 0 \\
\hline K-1200 center bay oven & $0106-87$ & $026548 \mathrm{P}$ & Center bay oven & 0 \\
\hline $\mathrm{K}-1200 \mathrm{FAE}$ & 0106-86 & 9954-92P & Isotope separation facility & PTC \\
\hline K-1200 north bay oven & $0106-922$ & $024272 \mathrm{P}$ & Matrix composites cure & 0 \\
\hline K-1202 ST-1 & $1106-20$ & $024911 \mathrm{P}$ & Solvents storage tank & 0 \\
\hline K-1232 acetic acid tank, N & $1106-23$ & $024614 \mathrm{P}$ & Acetic acid tank, $\mathbf{N}$ & 0 \\
\hline K-1232 acetic acid tank, S & $1106-23$ & $02414 \mathrm{P}$ & Acetic acid tank, $\mathbf{S}$ & 0 \\
\hline $\mathrm{K}-1232$ lime storage silo & & & Lime storage silo & \\
\hline $\mathrm{K}-1300 \mathrm{~B}$ & $0106-37$ & $012505 \mathrm{P}$ & Hazardous ops emergency vent & o \\
\hline K-1302 stack & $0106-42$ & & Process effluent emissions point & $\mathrm{O}$ \\
\hline K-1401 BOP assembly hood & & & BOP assembly hood & 0 \\
\hline K-1401 composite machining & $0106-88$ & $025514 \mathrm{P}$ & Composite machining process & 0 \\
\hline K-1401 foam pack & $1106-12$ & $025490 \mathrm{P}$ & Foam-packing operation & 0 \\
\hline K-1401 H-304 w acid tank & $1106-30$ & $025656 \mathrm{P}$ & Acid cleaning of steel parts & $\mathbf{O}$ \\
\hline K-1401 H-306 acid tank & $1106-30$ & $025656 \mathrm{P}$ & Acid cleaning of steel parts & 0 \\
\hline $\mathrm{K}-1401 \mathrm{HCL}$ tank E & $0106-228$ & $024500 \mathrm{P}$ & $\mathrm{HCl}$ storage tank & 0 \\
\hline K-1401 LH glove box 1,2 & $1106-03$ & 026679P & LH-glove box 1,2 & $\mathbf{O}$ \\
\hline K-1401 LH glove box 3 & $1106-03$ & $026679 \mathrm{P}$ & LH-glove box 3 & 0 \\
\hline K-1401 machine shop & $1106-09$ & 025585P & Grinding \& machining stations & 0 \\
\hline K-1401 seal shop acid cleaning & $1106-14$ & 025492P & Seal shop acid-machining stations & $\mathrm{O}$ \\
\hline K-1401 seal shop cleaning & $1106-17$ & 025495P & Cleaning process & 0 \\
\hline K-1401 seal shop 1 & $1106-13$ & $025491 \mathrm{P}$ & Seal shop process & 0 \\
\hline K-1401 slope testing stand & $1106-29$ & 9963838 & Uranium hexafluoride converters & PTC \\
\hline $\mathrm{K}-1401 \mathrm{~T} \cdot 104$ & $1106-32$ & $025658 \mathrm{P}$ & Acid cleaning of steel parts & 0 \\
\hline $\mathrm{K}-1401$ tricho e $\operatorname{tank}$ & $1106-10$ & $024947 \mathrm{P}$ & Trichloroethane storage tank & 0 \\
\hline K-1401 000 oven, NE & $0106-89$ & 995772P & Electric bake oven & PTC \\
\hline $\mathrm{K}-\mathrm{i} 401000$ oven, NE-2 & $0106-89$ & $997364 \mathrm{P}$ & Oven for curing & PTC \\
\hline K-1401 121659 & 0106-09 & $016306 \mathrm{P}$ & Trichloroethane degreaser & 0 \\
\hline K-1407 A lime-silo & $1106-25$ & $0224455 \mathrm{P}$ & Lime storage silo & 0 \\
\hline $\mathrm{K}-1407 \mathrm{H}-\mathrm{F}-210$, lime bin CNF & $1106-18$ & 025443P & Hydrated lime storage silo & 0 \\
\hline K-1413 propane LBD121422 & $0106-28$ & 024500P & Propane storage $\tan k$ & 0 \\
\hline K-1414 diesel & $1106-02$ & $024335 \mathrm{P}$ & Underground storage tank & 0 \\
\hline K-1414 UG methanol, unleaded gas & $0106-28$ & 024500P & Methanol/gasoline storage tank & $\mathrm{o}$ \\
\hline K-1419 F-200CBP & $1106-19$ & 025243P & Sulfuric acid storage tank & $\mathrm{O}$ \\
\hline K-1419 F-4860-CBP & $1106-19$ & $0252243 \mathrm{P}$ & Sulfuric acid storage tank & $\mathrm{o}$ \\
\hline K-141920 & $0106-83$ & 025250P & Scrubber \& cleaning facility & $\mathrm{O}$ \\
\hline K-1420 CP-02 & $0106-46$ & $026164 \mathrm{~F}$ & Concrete batch plant & $\mathrm{O}$ \\
\hline $\mathrm{K}-1420 \mathrm{Al}$ & $0106-82$ & 024396P & Fiämmable waste storage tank & 0 \\
\hline $\mathrm{K}-1420 \mathrm{I}-1$ incinerator & 0106-08 & $015691 \mathrm{P}$ & Waste incinerator & 0 \\
\hline $\mathrm{K}-1420$ nitric acid tank, NE & $1106-22$ & $024453 \mathrm{P}$ & Nitric acid tank, NE & 0 \\
\hline $\mathrm{K}-1420$ nitric acid tank, NW & $1106-22$ & 024453P & Nitric acid tank, NW & $\mathrm{o}$ \\
\hline K-1420 nitric acid tank, SE & $1106-22$ & 024453P & Nitric acid tank, SE & 0 \\
\hline $\mathrm{K}-1420$ nitric acid tank, SW & $1106-22$ & $024453 \mathrm{P}$ & Nitric acid tank, SW & $\mathrm{o}$ \\
\hline $\mathrm{K}-1420$ & $0106-70$ & 023798P & Phillips vapor degreaser & 0 \\
\hline
\end{tabular}


Table 2.1.13 (continued)

\begin{tabular}{|c|c|c|c|c|}
\hline $\begin{array}{c}\text { ORGDP } \\
\text { source number }\end{array}$ & $\begin{array}{c}\text { Emission } \\
\text { source } \\
\text { reference } \\
\text { number } \\
\text { (73-XXXX-XX) }\end{array}$ & $\begin{array}{l}\text { Permit } \\
\text { number }\end{array}$ & Source & $\begin{array}{l}\text { Permit } \\
\text { type }^{a}\end{array}$ \\
\hline K-1420 237306 vapor degreaser & $0106-49$ & 023797P & Detrex vapor degreaser & 0 \\
\hline$K-1423$ process & 0106-37 & $023001 \mathrm{P}$ & Toll enrichment facility & 0 \\
\hline K-1435 TSCA incinerator & $0106-78$ & 9962541 & TSCA insinerator & PTC \\
\hline K.1435 C tank farm & $0106-75$ & $024105 \mathrm{P}$ & Hazardous liquid wastes & 0 \\
\hline $\mathrm{K}-1501 \mathrm{~A} 1, \mathrm{~A} 2, \mathrm{~A} 3$ & $\begin{array}{l}0106-01 \\
02,03,04 \\
05,06,17\end{array}$ & $027049 \mathrm{~F}$ & Steam plant & $\mathbf{O}$ \\
\hline K-1501 sulfuric acid tank & 0106.28 & $024500 \mathrm{P}$ & Sulfuric acid storage tank & 0 \\
\hline $\mathrm{K}-1505 \mathrm{E}$ & $0106-39$ & 023796P & Coal sizing \& conveying system & 0 \\
\hline K-1515 north alum tank & $0106-28$ & 024500P & Alum sulfate storage tank & $\mathbf{0}$ \\
\hline K-1515 south alum tank & $0106-28$ & 024500P & Alum sulfate storage tank & $\mathbf{0}$ \\
\hline K.1580 blueprint & $1106-16$ & $025494 \mathrm{P}$ & Blueprint machine & 0 \\
\hline Y.12 SDDP & $1106-33$ & $996949 \mathrm{P}$ & Sludge detoxification demo project & PTC \\
\hline
\end{tabular}

${ }^{\circ} \mathrm{O}=$ operating; PTC $=$ permit to construct. 


\subsection{SURFACE WATER}


Table 2.2.1. Radionuclide concentrations in surface water around ORNL in 1989

\begin{tabular}{|c|c|c|c|c|c|c|}
\hline \multirow{2}{*}{ Radionuclide } & \multirow{2}{*}{$\begin{array}{l}\text { No. of } \\
\text { samples }\end{array}$} & \multicolumn{4}{|c|}{$\begin{array}{l}\text { Concentration } \\
(\mathrm{pCi} / \mathrm{L})\end{array}$} & \multirow{2}{*}{$\begin{array}{l}\text { Percentage } \\
\text { of } \mathrm{DCG}^{c}\end{array}$} \\
\hline & & Max & Min & Av & $\begin{array}{l}\text { Std. } \\
\text { error }\end{array}$ & \\
\hline \multicolumn{7}{|c|}{ Melton Hill Dam } \\
\hline${ }^{241} \mathrm{Am}$ & 4 & 1.5 & -0.032 & 0.50 & 0.35 & 1.7 \\
\hline${ }^{244} \mathrm{Cm}$ & 2 & 1.4 & 0.24 & 0.84 & 0.59 & 1.4 \\
\hline${ }^{60} \mathrm{Co}$ & 12 & 22 & -16 & 3.1 & 3.5 & 0.062 \\
\hline${ }^{137} \mathrm{Cs}$ & 12 & 14 & -24 & -5.3 & 2.8 & $<0.0010$ \\
\hline Gross alpha & 12 & 19 & -3.8 & 5.8 & 1.9 & $d$ \\
\hline Gross beta & 12 & 43 & -49 & 9.9 & 8.2 & $d$ \\
\hline${ }^{238} \mathrm{Pu}$ & 4 & 0.027 & -0.063 & 0.0027 & 0.023 & 0.0068 \\
\hline${ }^{239} \mathrm{Pu}$ & 4 & 0.054 & -0.13 & -0.016 & 0.040 & $<0.001$ \\
\hline Total $\mathrm{Sr}^{e}$ & 5 & 4.1 & -0.95 & 1.7 & 0.94 & 0.17 \\
\hline${ }^{3} \mathrm{H}$ & 4 & 1200 & -81 & 470 & 280 & 0.024 \\
\hline \multicolumn{7}{|c|}{ White Oak Creek headwaters } \\
\hline${ }^{241} \mathrm{Am}$ & 4 & 0.38 & -0.081 & 0.13 & 0.098 & 0.43 \\
\hline${ }^{244} \mathrm{Cm}$ & 1 & 0.35 & 0.35 & 0.35 & $d$ & 0.59 \\
\hline${ }^{60} \mathrm{Co}$ & 12 & 49 & --51 & 0.86 & 7.4 & 0.017 \\
\hline${ }^{137} \mathrm{Cs}$ & 12 & 24 & -24 & 0.27 & 4.1 & 0.0090 \\
\hline Gross alpha & 12 & 46 & -5.4 & 9.4 & 4.1 & $d$ \\
\hline Gross beta & 12 & 46 & 2.7 & 17 & 3.6 & $d$ \\
\hline${ }^{238} \mathrm{Pu}$ & 4 & 0.059 & -0.022 & 0.016 & 0.020 & 0.039 \\
\hline${ }^{239} \mathrm{Pu}$ & 4 & 0.027 & -0.089 & -0.015 & 0.026 & $<0.001$ \\
\hline Total $\mathrm{Sr}^{e}$ & 4 & 1.6 & -1.6 & 0.47 & 0.72 & 0.047 \\
\hline${ }^{3} \mathrm{H}$ & 4 & 620 & -110 & 310 & 150 & 0.016 \\
\hline \multicolumn{7}{|c|}{7500 bridge } \\
\hline${ }^{60} \mathrm{Co}$ & 12 & 54 & -24 & 13 & 6.1 & 0.25 \\
\hline${ }^{137} \mathrm{Cs}$ & 12 & 180 & -5.4 & 70 & 14 & 2.3 \\
\hline Total $\mathrm{Sr}^{e}$ & 12 & 240 & 49 & 88 & 16 & 8.8 \\
\hline${ }^{3} \mathrm{H}$ & 12 & 24000 & 2400 & 7700 & 1700 & 0.38 \\
\hline \multicolumn{7}{|c|}{ First Creek } \\
\hline${ }^{60} \mathrm{Co}$ & 12 & 57 & -7.6 & 8.4 & 5.3 & 0.17 \\
\hline${ }^{137} \mathrm{Cs}$ & 12 & 51 & -22 & 5.6 & 5.3 & 0.19 \\
\hline Total $\mathrm{Sr}^{e}$ & 12 & 510 & 190 & 310 & 31 & 31 \\
\hline \multicolumn{7}{|c|}{ Fifth Creek } \\
\hline${ }^{60} \mathrm{Co}$ & 12 & 51 & -14 & 10 & 4.8 & 0.20 \\
\hline${ }^{137} \mathrm{Cs}$ & 12 & 14 & -27 & -6.4 & 3.5 & $<0.001$ \\
\hline Total Sre & 12 & 54 & 1.4 & 34 & 3.5 & 3.4 \\
\hline \multicolumn{7}{|c|}{ Melton Branch 2} \\
\hline${ }^{60} \mathrm{Co}$ & 12 & 54 & -11 & 17 & $=-$ & 0.34 \\
\hline${ }^{137} \mathrm{Cs}$ & 12 & 41 & -2.7 & 9.4 & . & 0.31 \\
\hline Total $\mathrm{Sr}^{\mathrm{e}}$ & 12 & 13 & -2.4 & 2.6 & ' & 0.26 \\
\hline${ }^{3} \mathrm{H}$ & 12 & $46 C 500$ & 890 & 41000 & 38 & 2.0 \\
\hline
\end{tabular}


Table 2.2.1 (continued)

\begin{tabular}{|c|c|c|c|c|c|c|}
\hline \multirow{2}{*}{ Radionuclide } & \multirow{2}{*}{$\begin{array}{c}\text { Number of } \\
\text { samples }\end{array}$} & \multicolumn{4}{|c|}{$\begin{array}{l}\text { Concentration } \\
(\mathrm{pCi} / \mathrm{L})\end{array}$} & \multirow{2}{*}{$\begin{array}{l}\text { Percentage } \\
\text { of } \mathrm{DCG}^{c}\end{array}$} \\
\hline & & $\operatorname{Max}$ & Min & Av & $\begin{array}{l}\text { Std. } \\
\text { error }^{b}\end{array}$ & \\
\hline \multicolumn{7}{|c|}{ Northwest tributary } \\
\hline${ }^{60} \mathrm{Co}$ & 12 & 41 & -35 & -0.52 & 6.1 & $<0.001$ \\
\hline${ }^{137} \mathrm{Cs}$ & 12 & 24 & -27 & 4.3 & $3: 8$ & 0.14 \\
\hline Total $\mathrm{Sr}^{e}$ & 12 & 78 & 23 & 54 & 4.4 & 5.4 \\
\hline \multicolumn{7}{|c|}{ Raccoon Creek } \\
\hline${ }^{60} \mathrm{Co}$ & 12 & 24 & -16 & 0.78 & 3.8 & 0.016 \\
\hline${ }^{137} \mathrm{Cs}$ & 12 & 30 & -19 & 3.7 & 3.8 & 0.12 \\
\hline Total Sre & 12 & 81 & 11 & 24 & 5.6 & 2.4 \\
\hline
\end{tabular}

${ }^{a}$ Locations are shown in Fig. 2.2.4 in Vol. 1.

bStandard error of the mean.

'Average concentration as a percentage of the derived concentration guide (DCG).

${ }^{d}$ Not applicable.

Total radioactive $\mathrm{Sr}\left({ }^{89} \mathrm{Sr}+{ }^{90} \mathrm{Sr}\right)$. 
Table 2.2.2. Radionuclide concentrations in water around ORGDP in 1989

\begin{tabular}{|c|c|c|c|c|c|}
\hline \multirow{2}{*}{ Parameter } & \multirow{2}{*}{$\begin{array}{c}\text { Number of } \\
\text { samples }\end{array}$} & \multicolumn{3}{|c|}{$\begin{array}{l}\text { Concentration } \\
(\mathrm{pCi} / \mathrm{L})\end{array}$} & \multirow{2}{*}{$\begin{array}{l}\text { Percentage } \\
\text { of } \mathrm{DCG}^{a}\end{array}$} \\
\hline & & $\operatorname{Max}$ & Min & Av & \\
\hline \multicolumn{6}{|c|}{ West Fork Poplar Creek } \\
\hline${ }^{237} \mathrm{~Np}$ & 4 & 0.08 & -0.73 & -0.14 & $<0.01$ \\
\hline $239 / 240 \mathrm{Pu}$ & 4 & 1.2 & -0.08 & 0.44 & 1.5 \\
\hline${ }^{99} \mathrm{Tc}$ & 4 & 43 & -1529 & -402 & $<0.01$ \\
\hline${ }^{137} \mathrm{Cs}$ & 4 & 0 & 0 & 0 & $<0.01$ \\
\hline $\mathrm{U}^{b}$ & 4 & $<0.7$ & $<0.7$ & $<0.7$ & NA \\
\hline $\mathrm{U}(\mathrm{mg} / \mathrm{L})$ & 4 & $<0.001$ & $<0.001$ & $<0.001$ & NA \\
\hline \multicolumn{6}{|c|}{$K-1710$} \\
\hline${ }^{237} \mathrm{~Np}$ & 12 & 0.28 & -0.66 & -0.09 & $<0.01$ \\
\hline${ }^{239 / 240} \mathrm{Pu}$ & 12 & 0.79 & -0.99 & 0.06 & 0.2 \\
\hline${ }^{99} \mathrm{Tc}$ & 12 & 265 & -1529 & -247 & $<0.01$ \\
\hline${ }^{137} \mathrm{Cs}$ & 12 & 0 & 0 & 0 & $<0.01$ \\
\hline$U^{b}$ & 12 & 1.30 & $<0.7$ & $<1.10$ & $<0.2$ \\
\hline$U(\mathrm{mg} / \mathrm{L})$ & 12 & 0.0020 & $<0.0010$ & $<0.0017$ & NA \\
\hline \multicolumn{6}{|c|}{$K-716$} \\
\hline${ }^{237} \mathrm{~Np}$ & 11 & 0.91 & -0.7 & 0.095 & $<0.01$ \\
\hline${ }^{239 / 240} \mathrm{Pu}$ & 11 & 1.0 & -0.72 & 0.22 & 0.7 \\
\hline${ }^{99} \mathrm{Tc}$ & 11 & 321 & -1529 & -258 & $<0.01$ \\
\hline${ }^{137} \mathrm{Cs}$ & 11 & 0 & 0 & 0 & $<0.01$ \\
\hline $\mathrm{U}^{b}$ & 11 & 1.3 & $<0.7$ & $<0.9$ & $<0.2$ \\
\hline$U(\mathrm{mg} / \mathrm{L})$ & 11 & 0.0020 & $<0.0010$ & $<0.0013$ & NA \\
\hline \multicolumn{6}{|c|}{$K-1513$} \\
\hline${ }^{237} \mathrm{~Np}$ & 12 & 0.26 & -0.65 & -0.11 & $<0.01$ \\
\hline${ }^{239 / 240} \mathrm{Pu}$ & 12 & 9 & -0.39 & 0.87 & 2.9 \\
\hline${ }^{99} \mathrm{Tc}$ & 12 & 298 & -1529 & -233 & $<0.0 \mathrm{i}$ \\
\hline${ }^{137} \mathrm{Cs}$ & 12 & 0 & 0 & 0 & $<0.01$ \\
\hline $\mathrm{U}^{b}$ & 12 & $<0.7$ & $<0.7$ & $<0.7$ & NA \\
\hline $\mathrm{U}(\mathrm{mg} / \mathrm{L})$ & 12 & $<0.001$ & $<0.001$ & $<0.001$ & NA \\
\hline
\end{tabular}


Table 2.2.2. (continued)

\begin{tabular}{|c|c|c|c|c|c|}
\hline \multirow{2}{*}{ Parameter } & \multirow{2}{*}{$\begin{array}{l}\text { Number of } \\
\text { samples }\end{array}$} & \multicolumn{3}{|c|}{$\begin{array}{l}\text { Concentration } \\
(\mathrm{pCi} / \mathrm{L})\end{array}$} & \multirow{2}{*}{$\begin{array}{l}\text { Percentage } \\
\text { of } \mathrm{DCG}^{a}\end{array}$} \\
\hline & & $\operatorname{Max}$ & Min & Av & \\
\hline \multicolumn{6}{|c|}{$K .901$ at 892} \\
\hline${ }^{237} \mathrm{~Np}$ & 1 & 0 & 0 & 0 & $<0.01$ \\
\hline $239 / 240 \mathrm{Pu}$ & 1 & 0.06 & 0.06 & 0.06 & 0.2 \\
\hline${ }^{99} \mathrm{Tc}$ & 1 & -29 & -29 & -29 & $<0.01$ \\
\hline${ }^{137} \mathrm{Cs}$ & 1 & 0 & 0 & 0 & $<0.01$ \\
\hline $\mathrm{U}^{b}$ & $i$ & $<0.7$ & $<0.7$ & $<0.7$ & NA \\
\hline $\mathrm{U}(\mathrm{mg} / \mathrm{L})$ & 1 & $<0.001$ & $<0.001$ & $<0.001$ & NA \\
\hline \multicolumn{6}{|c|}{$K-1770$} \\
\hline${ }^{237} \mathrm{~Np}$ & 12 & 0.25 & -0.65 & -0.11 & $<0.01$ \\
\hline${ }^{239 / 240} \mathrm{Pu}$ & 12 & 0.72 & -0.77 & 0.11 & 0.4 \\
\hline${ }^{99} \mathrm{Tc}$ & 12 & 198 & -1529 & -224 & $<0.01$ \\
\hline${ }^{137} \mathrm{Cs}$ & 12 & 0 & 0 & 0 & $<0.01$ \\
\hline $\mathrm{U}^{b}$ & 12 & 0.7 & $<0.7$ & $<0.7$ & $<0.1$ \\
\hline $\mathrm{U}(\mathrm{mg} / \mathrm{L})$ & 12 & 0.001 & $<0.001$ & $<0.001$ & NA \\
\hline \multicolumn{6}{|c|}{ Clinch River } \\
\hline${ }^{237} \mathrm{~Np}$ & 4 & 0.16 & -0.66 & -0.17 & $<0.01$ \\
\hline${ }^{239 / 240} \mathrm{Pu}$ & 4 & 0.79 & -0.08 & 0.16 & 0.5 \\
\hline${ }^{99} \mathrm{Tc}$ & 4 & 148 & -1529 & -359 & $<0.01$ \\
\hline${ }^{137} \mathrm{Cs}$ & 4 & 0 & 0 & 0 & $<0.01$ \\
\hline$U^{b}$ & 4 & $<0.7$ & $<0.7$ & $<0.7$ & NA \\
\hline $\mathrm{U}(\mathrm{mg} / \mathrm{L})$ & 4 & $<0.001$ & $<0.001$ & $<0.001$ & NA \\
\hline \multicolumn{6}{|c|}{ Mitchell Branch } \\
\hline Gross Alpha & 3 & 1 & -1 & 0.03 & NA \\
\hline Gross Beta & 3 & 13 & 1 & 5 & NA \\
\hline Gross Gamma & 3 & 0 & 0 & 0 & NA \\
\hline
\end{tabular}

${ }^{a}$ Average concentration as a percentage of the derived concentration guide (DCG) from DOE Order 5400.5 .

${ }^{6}$ The specific activity for natural uranium of $1.49 \times 1^{6} \mathrm{~g} / \mathrm{Ci}$; was used to determine $\mathrm{pCi} / \mathrm{L}$. 
Table 2.2.3. 1989 ORGDP concentrations at West Fork Poplar Creek

\begin{tabular}{|c|c|c|c|}
\hline \multirow[t]{2}{*}{ Parameter } & \multicolumn{3}{|c|}{ Concentration } \\
\hline & Max & Min & Av \\
\hline 1,1,1-Trichloroethane, $\mu \mathrm{g} / \mathrm{L}$ & $<5$ & $<5$ & $<5$ \\
\hline 1,1,2,2-Tetrachloroethane, $\mu \mathrm{g} / \mathrm{L}$ & $<5$ & $<5$ & $<5$ \\
\hline 1,1,2-Trichloroethane, $\mu \mathrm{g} / \mathrm{L}$ & $<5$ & $<5$ & $<5$ \\
\hline 1,1-Dichloroethane, $\mu \mathrm{g} / \mathrm{L}$ & $<5$ & $<5$ & $<5$ \\
\hline 1,1-Dichloroethene, $\mu \mathrm{g} / \mathrm{L}$ & $<5$ & $<5$ & $<5$ \\
\hline 1,2,4-Trichlorobenzene, $\mu \mathrm{g} / \mathrm{L}$ & $<10$ & $<10$ & $<10$ \\
\hline 1,2-Dichlorobenzene, $\mu \mathrm{g} / \mathrm{L}$ & $<10$ & $<10$ & $<10$ \\
\hline 1,2-Dichloroethane, $\mu \mathrm{g} / \mathrm{L}$ & $<5$ & $<5$ & $<5$ \\
\hline 1,2-Dichloropropane, $\mu \mathrm{g} / \mathrm{L}$ & $<5$ & $<5$ & $<5$ \\
\hline 1,3-Dichlorobenzene, $\mu \mathrm{g} / \mathrm{L}$ & $<10$ & $<10$ & $<10$ \\
\hline 1,4-Dichlorobenzene, $\mu \mathrm{g} / \mathrm{L}$ & $<10$ & $<10$ & $<10$ \\
\hline 2,4,6-Trichlorophenol, $\mu \mathrm{g} / \mathrm{L}$ & $<10$ & $<10$ & $<10$ \\
\hline 2,4-Dichlorophenol, $\mu \mathrm{g} / \mathrm{L}$ & $<10$ & $<10$ & $<10$ \\
\hline 2,4-Dimethylphenol, $\mu \mathrm{g} / \mathrm{L}$ & $<10$ & $<10$ & $<10$ \\
\hline 2,4-Dinitrophenol, $\mu \mathrm{g} / \mathrm{L}$ & $<10$ & $<10$ & $<10$ \\
\hline 2,4-Dinitrotoluene, $\mu \mathrm{g} / \mathrm{L}$ & $<10$ & $<10$ & $<10$ \\
\hline 2,6-Dinitrotoluene, $\mu \mathrm{g} / \mathrm{L}$ & $<10$ & $<10$ & $<10$ \\
\hline 2-Chloroethylvinyl ether, $\mu \mathrm{g} / \mathrm{L}$ & $<10$ & $<10$ & $<10$ \\
\hline 2-Chloronaphthalene, $\mu \mathrm{g} / \mathrm{L}$ & $<10$ & $<10$ & $<10$ \\
\hline 2-Chlorophenol, $\mu \mathrm{g} / \mathrm{L}$ & $<10$ & $<10$ & $<10$ \\
\hline 2-Nitrophenol, $\mu \mathrm{g} / \mathrm{L}$ & $<10$ & $<10$ & $<10$ \\
\hline 3,3'-Dichlorobenzidine, $\mu \mathrm{g} / \mathrm{L}$ & $<20$ & $<20$ & $<20$ \\
\hline 4,6-Dinitro-2-methylphenol, $\mu \mathrm{g} / \mathrm{L}$ & $<50$ & $<50$ & $<50$ \\
\hline 4-Bromophenyl-phenylether, $\mu \mathrm{g} / \mathrm{L}$ & $<10$ & $<10$ & $<10$ \\
\hline 4-Chloro-3-methylphenol, $\mu \mathrm{g} / \mathrm{L}$ & $<10$ & $<10$ & $<10$ \\
\hline 4-Chlorophenyl-phenylether, $\mu \mathrm{g} / \mathrm{L}$ & $<10$ & $<10$ & $<10$ \\
\hline 4-Nitrophenol, $\mu \mathrm{g} / \mathrm{L}$ & $<50$ & $<50$ & $<50$ \\
\hline Acenaphthene, $\mu \mathrm{g} / \mathrm{L}$ & $<10$ & $<10$ & $<10$ \\
\hline Acenaphthylene, $\mu \mathrm{g} / \mathrm{L}$ & $<10$ & $<10$ & $<10$ \\
\hline Ammonia nitrogen, $\mathrm{mg} / \mathrm{L}$ & $<0.2$ & $<0.2$ & $<0.2$ \\
\hline Anthracene, $\mu \mathrm{g} / \mathrm{L}$ & $<10$ & $<10$ & $<10$ \\
\hline Arsenic, $\mathrm{mg} / \mathrm{L}$ & $<0.005$ & $<0.005$ & $<0.005$ \\
\hline Benzene, $\mu \mathrm{g} / \mathrm{L}$ & $<5$ & $<5$ & $<5$ \\
\hline Benzidine, $\mu \mathrm{g} / \mathrm{L}$ & $<10$ & $<10$ & $<10$ \\
\hline Benzo(a)anthracene, $\mu \mathrm{g} / \mathrm{L}$ & $<10$ & $<10$ & $<10$ \\
\hline Benzo(a)pyrene, $\mu \mathrm{g} / \mathrm{L}$ & $<10$ & $<10$ & $<10$ \\
\hline Benzo(b)fluoranthene, $\mu \mathrm{g} / \mathrm{L}$ & $<10$ & $<10$ & $<10$ \\
\hline $\operatorname{Benzo}(\mathrm{g}, \mathrm{h}, \mathrm{i})$ perylene, $\mu \mathrm{g} / \mathrm{L}$ & $<10$ & $<10$ & $<10$ \\
\hline Benzo(k)fluoranthene, $\mu \mathrm{g} / \mathrm{L}$ & $<10$ & $<10$ & $<10$ \\
\hline Bromodichloromethane, $\mu \mathrm{g} / \mathrm{L}$ & $<5$ & $<5$ & $<5$ \\
\hline Bromoform, $\mu \mathrm{g} / \mathrm{L}$ & $<5$ & $<5$ & $<5$ \\
\hline Bromomethane, $\mu \mathrm{g} / \mathrm{L}$ & $<10$ & $<10$ & $<10$ \\
\hline Butylbenzylphthalate, $\mu \mathrm{g} / \mathrm{L}$ & $<10$ & $<10$ & $<10$ \\
\hline Cadmium, mg/L & $<0.002$ & $<0.002$ & $<0.002$ \\
\hline Carbon tetrachloride, $\mu \mathrm{g} / \mathrm{L}$ & $<5$ & $<5$ & $<5$ \\
\hline Chemical oxygen demand (COD), $\mathrm{mg} / \mathrm{L}$ & $<5$ & $<5$ & $<5$ \\
\hline Chlorobenzene, $\mu \mathrm{g} / \mathrm{L}$ & $<5$ & $<5$ & $<5$ \\
\hline Chloroethane, $\mu \mathrm{g} / \mathrm{L}$ & $<10$ & $<10$ & $<10$ \\
\hline Chloroform, $\mu \mathrm{g} / \mathrm{L}$ & $<5$ & $<5$ & $<5$ \\
\hline Chloromethane, $\mu \mathrm{g} / \mathrm{L}$ & $<10$ & $<10$ & $<10$ \\
\hline
\end{tabular}


Table 2.2.3 (Continued)

\begin{tabular}{|c|c|c|c|}
\hline \multirow[t]{2}{*}{ Parameter } & \multicolumn{3}{|c|}{ Concentration } \\
\hline & $\operatorname{Max}$ & Min & Av \\
\hline Chromium, mg/L & $<0.010$ & $<0.010$ & $<0.010$ \\
\hline Chrysene, $\mu \mathrm{g} / \mathrm{L}$ & $<10$ & $<10$ & $<10$ \\
\hline Copper, mg/L & $<0.0040$ & $<0.0040$ & $<0.0040$ \\
\hline Cyaride, $\mathrm{mg} / \mathrm{L}$ & $<0.1$ & $<0.1$ & $<0.1$ \\
\hline Di-n-butylphthalate, $\mu \mathrm{g} / \mathrm{L}$ & $<10$ & $<10$ & $<10$ \\
\hline Dibenz(a,h)anthracene, $\mu \mathrm{g} / \mathrm{L}$ & $<10$ & $<10$ & $<10$ \\
\hline Dibromochloromethane, $\mu \mathrm{g} / \mathrm{L}$ & $<5$ & $<5$ & $<5$ \\
\hline Diethylphthalate, $\mu \mathrm{g} / \mathrm{L}$ & $<10$ & $<10$ & $<10$ \\
\hline Dimethylphthalate, $\mu \mathrm{g} / \mathrm{L}$ & $<10$ & $<10$ & $<10$ \\
\hline Dissolved solids, mg/L & 160 & 98 & 136 \\
\hline Ethylbenzene, $\mu \mathrm{g} / \mathrm{L}$ & $<5$ & $<5$ & $<5$ \\
\hline Fluoranthene, $\mu \mathrm{g} / \mathrm{L}$ & $<10$ & $<10$ & $<10$ \\
\hline Fluorene, $\mu \mathrm{g} / \mathrm{L}$ & $<10$ & $<10$ & $<10$ \\
\hline Fluoride, mg/L & $<0.1$ & $<0.1$ & $<0.1$ \\
\hline Hexachlorobenzene, $\mu \mathrm{g} / \mathrm{L}$ & $<10$ & $<10$ & $<10$ \\
\hline Hexachlorobutadiene, $\mu \mathrm{g}_{\prime}^{\prime} \mathrm{L}$ & $<10$ & $<10$ & $<10$ \\
\hline Hexachlorocyclopentadiene, $\mu \mathrm{g} / \mathrm{L}$ & $<10$ & $<10$ & $<10$ \\
\hline Hexachloroethane, $\mu \mathrm{g} / \mathrm{L}$ & $<10$ & $<10$ & $<10$ \\
\hline Indeno(1,2,3-cd)pyrene, $\mu \mathrm{g} / \mathrm{L}$ & $<10$ & $<10$ & $<10$ \\
\hline Isophorone, $\mu \mathrm{g} / \mathrm{L}$ & $<10$ & $<10$ & $<10$ \\
\hline Lead, $\mathrm{mg} / \mathrm{L}$ & 0.0047 & $<0.0040$ & $<0.0042$ \\
\hline Manganese, mg/L & 0.19 & 0.11 & 0.16 \\
\hline Mercury, mg/L & $<0.0002$ & $<0.0002$ & $<0.0002$ \\
\hline Methylene chloride, $\mu \mathrm{g} / \mathrm{L}$ & $<10$ & $<10$ & $<10$ \\
\hline N-nitroso-di-n-propylamine, $\mu \mathrm{g} / \mathrm{L}$ & $<10$ & $<10$ & $<10$ \\
\hline $\mathrm{N}$-ritrosodimethylamine, $\mu \mathrm{g} / \mathrm{L}$ & $<10$ & $<10$ & $<10$ \\
\hline $\mathrm{N}$-nitrosodiphenylamine, $\mu \mathrm{g} / \mathrm{L}$ & $<10$ & $<10$ & $<10$ \\
\hline Naphthalene, $\mu \mathrm{g} / \mathrm{L}$ & $<10$ & $<10$ & $<10$ \\
\hline Nickel, mg/L & $<0.05$ & $<0.05$ & $<0.05$ \\
\hline Vitrate nitrogen, $\mathrm{mg} / \mathrm{L}$ & 0.36 & 0.20 & 0.24 \\
\hline Nitrobenzene, $\mu \mathrm{g} / \mathrm{L}$ & $<10$ & $<10$ & $<10$ \\
\hline Pentachlorophenol, $\mu \mathrm{g} / \mathrm{L}$ & $<50$ & $<50$ & $<50$ \\
\hline Phenanthrene, $\mu \mathrm{g} / \mathrm{L}$ & $<10$ & $<10$ & $<10$ \\
\hline Phenol, $\mu \mathrm{g} / \mathrm{L}$ & $<10$ & $<10$ & $<10$ \\
\hline Pyrene, $\mu \mathrm{g} / \mathrm{L}$ & $<10$ & $<10$ & $<10$ \\
\hline Sodium, mg/L & 4.1 & 2.8 & 3.4 \\
\hline Sulfate, $\mathrm{mg} / \mathrm{L}$ & 43.0 & 36.0 & 38.5 \\
\hline Suspended solids, mg/L & 21.0 & 7.0 & 14.5 \\
\hline Tetrachloroethene, $\mu \mathrm{g} / \mathrm{L}$ & $<5$ & $<5$ & $<5$ \\
\hline Toluene, $\mu \mathrm{g} / \mathrm{L}$ & $<5$ & $<5$ & $<5$ \\
\hline Trichloroethene, $\mu \mathrm{g} / \mathrm{L}$ & $<5$ & $<5$ & $<5$ \\
\hline Vinyl chloride, $\mu \mathrm{g} / \mathrm{L}$ & $<10$ & $<10$ & $<10$ \\
\hline Zinc, mg/L & 0.033 & $<0.020$ & $<0.022$ \\
\hline bis(2-Chloroethoxy)methane, $\mu \mathrm{g} / \mathrm{L}$ & $<10$ & $<10$ & $<10$ \\
\hline bis(2-Chloroethyl)ether, $\mu \mathrm{g} / \mathrm{L}$ & $<10$ & $<10$ & $<10$ \\
\hline bis(2-Chloroisopropyl)ether, $\mu \mathrm{g} / \mathrm{L}$ & $<10$ & $<10$ & $<10$ \\
\hline bis(2-Ethylhexyl)phthalate, $\mu \mathrm{g} / \mathrm{L}$ & $<10$ & $<10$ & $<10$ \\
\hline cis-1,3-Dichloropropene, $\mu \mathrm{g} / \mathrm{L}$ & $<5$ & $<5$ & $<5$ \\
\hline di-n-Octylphthalate, $\mu \mathrm{g} / \mathrm{L}$ & $<10$ & $<10$ & $<10$ \\
\hline $\mathrm{pH}$ & 8.6 & 7.6 & \\
\hline trans-1,2-Dichloroethene, $\mu \mathrm{g} / \mathrm{L}$ & $<5$ & $<.5$ & $<5$ \\
\hline trans-1,3-Dichloropropene, $\mu \mathrm{g} / \mathrm{L}$ & $<5$ & $<5$ & $<5$ \\
\hline
\end{tabular}


Table 2.2.4. 1989 ORGDP concentrations at Clinch River

\begin{tabular}{|c|c|c|c|}
\hline \multirow[t]{2}{*}{ Parameter } & \multicolumn{3}{|c|}{ Concentration } \\
\hline & $\operatorname{Max}$ & Min & Av \\
\hline 1,1,1-Trichloroethane, $\mu \mathrm{g} / \mathrm{L}$ & $<5$ & $<5$ & $<5$ \\
\hline 1,1,2,2-Tetrachloroethane, $\mu \mathrm{g} / \mathrm{L}$ & $<5$ & $<5$ & $<5$ \\
\hline 1,1,2-Trichloroethane, $\mu \mathrm{g} / \mathrm{L}$ & $<5$ & $<5$ & $<5$ \\
\hline 1,1-Dichloroethane, $\mu \mathrm{g} / \mathrm{L}$ & $<5$ & $<5$ & $<5$ \\
\hline 1,1-Dichloroethene, $\mu \mathrm{g} / \mathrm{L}$ & $<5$ & $<5$ & $<5$ \\
\hline 1,2,4-Trichlorobenzene, $\mu \mathrm{g} / \mathrm{L}$ & $<10$ & $<10$ & $<10$ \\
\hline 1,2-Dichlorobenzene, $\mu \mathrm{g} / \mathrm{L}$ & $<10$ & $<10$ & $<10$ \\
\hline 1,2-Dichloroethane, $\mu \mathrm{g} / \mathrm{L}$ & $<5$ & $<5$ & $<5$ \\
\hline 1,2-Dichloropropane, $\mu \mathrm{g} / \mathrm{L}$ & $<5$ & $<5$ & $<5$ \\
\hline 1,3-Dichlorobenzene, $\mu \mathrm{g} / \mathrm{L}$ & $<10$ & $<10$ & $<10$ \\
\hline 1,4-Dichlorobenzene, $\mu \mathrm{g} / \mathrm{L}$ & $<10$ & $<10$ & $<10$ \\
\hline 2,4,6-Trichlorophenol, $\mu \mathrm{g} / \mathrm{L}$ & $<10$ & $<10$ & $<10$ \\
\hline 2,4-Dichlorophenol, $\mu \mathrm{g} / \mathrm{L}$ & $<10$ & $<10$ & $<10$ \\
\hline 2,4-Dimethylphenol, $\mu \mathrm{g} / \mathrm{L}$ & $<10$ & $<10$ & $<10$ \\
\hline 2,4-Dinitrophenol, $\mu \mathrm{g} / \mathrm{L}$ & $<10$ & $<10$ & $<10$ \\
\hline 2,4-Dinitrotoluene, $\mu \mathrm{g} / \mathrm{L}$ & $<10$ & $<10$ & $<10$ \\
\hline 2,6-Dinitrotoluene, $\mu \mathrm{g} / \mathrm{L}$ & $<10$ & $<10$ & $<10$ \\
\hline 2-Chloroethylvinyl ether, $\mu \mathrm{g} / \mathrm{L}$ & $<10$ & $<10$ & $<10$ \\
\hline 2-Chloronaphthalene, $\mu \mathrm{g} / \mathrm{L}$ & $<10$ & $<10$ & $<10$ \\
\hline 2-Chlorophenol, $\mu \mathrm{g} / \mathrm{L}$ & $<10$ & $<10$ & $<10$ \\
\hline 2-Nitrophenol, $\mu \mathrm{g} / \mathrm{L}$ & $<10$ & $<10$ & $<10$ \\
\hline 3,3'-Dichlorobenzidine, $\mu \mathrm{g} / \mathrm{L}$ & $<20$ & $<20$ & $<20$ \\
\hline 4,6-Dinitro-2-methylphenol, $\mu \mathrm{g} / \mathrm{L}$ & $<50$ & $<50$ & $<50$ \\
\hline 4-Bromophenyl-phenylether, $\mu \mathrm{g} / \mathrm{L}$ & $<10$ & $<10$ & $<10$ \\
\hline 4-Chloro-3-methylphenol, $\mu \mathrm{g} / \mathrm{L}$ & $<10$ & $<10$ & $<10$ \\
\hline 4-Chlorophenyl-phenylether, $\mu \mathrm{g} / \mathrm{L}$ & $<10$ & $<10$ & $<10$ \\
\hline 4-Nitrophenol, $\mu \mathrm{g} / \mathrm{L}$ & $<50$ & $<50$ & $<50$ \\
\hline Acenaphthene, $\mu \mathrm{g} / \mathrm{L}$ & $<10$ & $<10$ & $<10$ \\
\hline Acenaphthylene, $\mu \mathrm{g} / \mathrm{L}$ & $<10$ & $<10$ & $<10$ \\
\hline Ammonia nitrogen, $\mathrm{mg} / \mathrm{L}$ & $<0.2$ & $<0.2$ & $<0.2$ \\
\hline Anthracene, $\mu \mathrm{g} / \mathrm{L}$ & $<10$ & $<10$ & $<10$ \\
\hline Arsenic, mg/L & $<0.005$ & $<0.005$ & $<0.005$ \\
\hline Benzene, $\mu \mathrm{g} / \mathrm{L}$ & $<5$ & $<5$ & $<5$ \\
\hline Benzidine, $\mu \mathrm{g} / \mathrm{L}$ & $<10$ & $<10$ & $<10$ \\
\hline Benzo(a)anthracene, $\mu \mathrm{g} / \mathrm{L}$ & $<10$ & $<10$ & $<10$ \\
\hline Benzo(a)pyrene, $\mu \mathrm{g} / \mathrm{L}$ & $<10$ & $<10$ & $<10$ \\
\hline Benzo(b)fluoranthene, $\mu \mathrm{g} / \mathrm{L}$ & $<10$ & $<10$ & $<10$ \\
\hline $\operatorname{Benzo}(g, h, i)$ perylene, $\mu \mathrm{g} / \mathrm{L}$ & $<10$ & $<10$ & $<10$ \\
\hline Benzo(k)fluoranthene, $\mu \mathrm{g} / \mathrm{L}$ & $<10$ & $<10$ & $<10$ \\
\hline Bromodichloromethane, $\mu \mathrm{g} / \mathrm{L}$ & $<5$ & $<5$ & $<5$ \\
\hline Bromoform, $\mu \mathrm{g} / \mathrm{L}$ & $<5$ & $<5$ & $<5$ \\
\hline Bromomethane, $\mu \mathrm{g} / \mathrm{L}$ & $<10$ & $<10$ & $<10$ \\
\hline Butylbenzylphthalate, $\mu \mathrm{g} / \mathrm{L}$ & $<10$ & $<10$ & $<10$ \\
\hline Cadmium, mg/L & $<0.002$ & $<0.002$ & $<0.002$ \\
\hline Carbon tetrachloride, $\mu \mathrm{g} / \mathrm{L}$ & $<5$ & $<5$ & $<5$ \\
\hline Chemical oxygen demand (COD), $\mathrm{mg} / \mathrm{L}$ & $<8$ & $<5$ & $<6$ \\
\hline Chlorobenzene, $\mu \mathrm{g} / \mathrm{L}$ & $<5$ & $<5$ & $<5$ \\
\hline Chloroethane, $\mu \mathrm{g} / \mathrm{L}$ & $<10$ & $<10$ & $<10$ \\
\hline Chloroform, $\mu \mathrm{g} / \mathrm{L}$ & $<5$ & $<5$ & $<5$ \\
\hline Chloromethane, $\mu \mathrm{g} / \mathrm{L}$ & $<10$ & $<10$ & $<10$ \\
\hline
\end{tabular}


Table 2.2.4 (Continued)

\begin{tabular}{|c|c|c|c|}
\hline & \multicolumn{3}{|c|}{ Concentration } \\
\hline & $\operatorname{Max}$ & Min & Av \\
\hline Chromium, mg/L & $<0.010$ & $<0.010$ & $<0.010$ \\
\hline Chrysene, $\mu \mathrm{g} / \mathrm{L}$ & $<10$ & $<10$ & $<10$ \\
\hline Copper, mg/L & 0.0044 & $<0.0040$ & $<0.0041$ \\
\hline Cyanide, $\mathrm{mg} / \mathrm{L}$ & $<0.1$ & $<0.1$ & $<0.1$ \\
\hline Di-n-butylphthalate, $\mu \mathrm{g} / \mathrm{L}$ & $<10$ & $<10$ & $<10$ \\
\hline Dibenz(a,h)anthracene, $\mu \mathrm{g} / \mathrm{L}$ & $<10$ & $<10$ & $<10$ \\
\hline Dibromochloromethane, $\mu \mathrm{g} / \mathrm{L}$ & $<5$ & $<5$ & $<5$ \\
\hline Diethylphthalate, $\mu \mathrm{g} / \mathrm{L}$ & $<10$ & $<10$ & $<10$ \\
\hline Dimethylphthalate, $\mu \mathrm{g} / \mathrm{L}$ & $<10$ & $<10$ & $<10$ \\
\hline Dissolved solids, $\mathrm{mg} / \mathrm{L}$ & 176 & 132 & 152 \\
\hline Ethylbenzene, $\mu \mathrm{g} / \mathrm{L}$ & $<5$ & $<5$ & $<5$ \\
\hline Fluoranthene, $\mu \mathrm{g} / \mathrm{L}$ & $<10$ & $<10$ & $<10$ \\
\hline Fluorene, $\mu \mathrm{g} / \mathrm{L}$ & $<10$ & $<10$ & $<10$ \\
\hline Fluoride, $\mathrm{mg} / \mathrm{L}$ & $<0.1$ & $<0.1$ & $<0.1$ \\
\hline Hexachlorobenzene, $\mu \mathrm{g} / \mathrm{L}$ & $<10$ & $<10$ & $<10$ \\
\hline Hexachlorobutadiene, $\mu \mathrm{g} / \mathrm{L}$ & $<10$ & $<10$ & $<10$ \\
\hline Hexachlorocyclopentadiene, $\mu \mathrm{g} / \mathrm{L}$ & $<10$ & $<10$ & $<10$ \\
\hline Hexachloroethane, $\mu \mathrm{g} / \mathrm{L}$ & $<10$ & $<10$ & $<10$ \\
\hline Indeno( 1,2,3-cd)pyrene, $\mu \mathrm{g} / \mathrm{L}$ & $<10$ & $<10$ & $<10$ \\
\hline Isophorone, $\mu \mathrm{g} / \mathrm{L}$ & $<10$ & $<10$ & $<10$ \\
\hline Lead, $\mathrm{mg} / \mathrm{L}$ & $<0.0040$ & $<0.0040$ & $<0.0040$ \\
\hline Manganese, $\mathrm{mg} / \mathrm{L}$ & 0.10 & 0.069 & 0.079 \\
\hline Mercury, mg/L & $<0.0002$ & $<0.0002$ & $<0.0002$ \\
\hline Methylene chloride, $\mu \mathrm{g} / \mathrm{L}$ & $<5$ & $<5$ & $<5$ \\
\hline N-nitroso-di-n-propylamine, $\mu \mathrm{g} / \mathrm{L}$ & $<10$ & $<10$ & $<10$ \\
\hline $\mathrm{N}$-nitrosodimethylamine, $\mu \mathrm{g} / \mathrm{L}$ & $<10$ & $<10$ & $<10$ \\
\hline $\mathrm{N}$-nitrosodiphenylamine, $\mu \mathrm{g} / \mathrm{L}$ & $<10$ & $<10$ & $<10$ \\
\hline Naphthalene, $\mu \mathrm{g} / \mathrm{L}$ & $<10$ & $<10$ & $<10$ \\
\hline Nickel, $\mathrm{mg} / \mathrm{L}$ & $<0.05$ & $<0.05$ & $<0.05$ \\
\hline Nitrate nitrogen, $\mathrm{mg} / \mathrm{L}$ & 0.50 & 0.20 & 0.38 \\
\hline Nitrobenzene, $\mu \mathrm{g} / \mathrm{L}$ & $<10$ & $<10$ & $<10$ \\
\hline Pentachlorophenol, $\mu \mathrm{g} / \mathrm{L}$ & $<50$ & $<50$ & $<50$ \\
\hline Phenanthrene, $\mu \mathrm{g} / \mathrm{L}$ & $<10$ & $<10$ & $<10$ \\
\hline Phenol, $\mu \mathrm{g} / \mathrm{L}$ & $<10$ & $<10$ & $<10$ \\
\hline Pyrene, $\mu \mathrm{g} / \mathrm{L}$ & $<10$ & $<10$ & $<10$ \\
\hline Sodium, mg/L & 4.3 & 3.6 & 4.0 \\
\hline Sulfate, $\mathrm{mg} / \mathrm{L}$ & 22.0 & 17.0 & 20.0 \\
\hline Suspended solids, mg/L & 28.0 & 9.0 & 22.0 \\
\hline Tetrachloroethene, $\mu \mathrm{g} / \mathrm{L}$ & $<5$ & $<5$ & $<5$ \\
\hline Toluene, $\mu \mathrm{g} / \mathrm{L}$ & $<5$ & $<5$ & $<5$ \\
\hline Trichloroethene, $\mu \mathrm{g} / \mathrm{L}$ & $<5$ & $<5$ & $<5$ \\
\hline Vinyl chloride, $\mu \mathrm{g} / \mathrm{L}$ & $<10$ & $<10$ & $<10$ \\
\hline Zinc, $\mathrm{mg} / \mathrm{L}$ & 0.033 & $<0.020$ & $<0.022$ \\
\hline bis(2-Chloroethoxy)methane, $\mu \mathrm{g} / \mathrm{L}$ & $<10$ & $<10$ & $<10$ \\
\hline bis(2-Chloroethyl)ether, $\mu \mathrm{g} / \mathrm{l}$ & $<10$ & $<10$ & $<10$ \\
\hline bis(2-Chloroisopropyl)ether, $\mu \mathrm{g} / \mathrm{L}$ & $<10$ & $<10$ & $<10$ \\
\hline bis(2-Ethylhexyl)phthalate, $\mu \mathrm{g} / \mathrm{L}$ & $<10$ & $<10$ & $<10$ \\
\hline cis-1,3-Dichloropropene, $\mu \mathrm{g} / \mathrm{L}$ & $<5$ & $<.5$ & $<5$ \\
\hline di-n-Octylphthalate, $\mu \mathrm{g} / \mathrm{L}$ & $<10$ & $<10$ & $<10$ \\
\hline $\mathrm{pH}$ & 8.5 & 7.6 & \\
\hline trans-1,2-Dichloroethene, $\mu \mathrm{g} / \mathrm{L}$ & $<5$ & $<5$ & $<5$ \\
\hline trans-1,3-Dichloropropene, $\mu \mathrm{g} / \mathrm{L}$ & $<5$ & $<5$ & $<5$ \\
\hline
\end{tabular}


Table 2.2.5. 1989 ORGDP concentrations at K-716

\begin{tabular}{|c|c|c|c|}
\hline \multirow[t]{2}{*}{ Parameter } & \multicolumn{3}{|c|}{ Concentration } \\
\hline & $\operatorname{Max}$ & Min & Av \\
\hline 1,1,1-Trichloroethane, $\mu \mathrm{g} / \mathrm{L}$ & $<5$ & $<5$ & $<5$ \\
\hline 1,1,2,2-Tetrachloroethane, $\mu \mathrm{g} / \mathrm{L}$ & $<5$ & $<5$ & $<5$ \\
\hline 1,1,2-Trichloroethane, $\mu \mathrm{g} / \mathrm{L}$ & $<5$ & $<5$ & $<5$ \\
\hline 1,1-Dichloroethane, $\mu \mathrm{g} / \mathrm{L}$ & $<5$ & $<5$ & $<5$ \\
\hline 1,1-Dichloroethene, $\mu \mathrm{g} / \mathrm{L}$ & $<5$ & $<5$ & $<5$ \\
\hline 1,2,4-Trichlorobenzene, $\mu \mathrm{g} / \mathrm{L}$ & $<10$ & $<10$ & $<10$ \\
\hline 1,2-Dichlorobenzene, $\mu \mathrm{g} / \mathrm{L}$ & $<10$ & $<10$ & $<10$ \\
\hline 1,2-Dichloroethane, $\mu \mathrm{g} / \mathrm{L}$ & $<5$ & $<5$ & $<5$ \\
\hline 1,2-Dichloropropane, $\mu \mathrm{g} / \mathrm{L}$ & $<5$ & $<5$ & $<5$ \\
\hline 1,3-Dichlorobenzene, $\mu \mathrm{g} / \mathrm{L}$ & $<10$ & $<10$ & $<10$ \\
\hline 1,4-Dichlorobenzene, $\mu \mathrm{g} / \mathrm{L}$ & $<10$ & $<10$ & $<10$ \\
\hline 2,4,6-Trichlorophenol, $\mu \mathrm{g} / \mathrm{L}$ & $<10$ & $<10$ & $<10$ \\
\hline 2,4-Dichlorophenol, $\mu \mathrm{g} / \mathrm{L}$ & $<10$ & $<10$ & $<10$ \\
\hline 2,4-Dimethylphenol, $\mu \mathrm{g} / \mathrm{L}$ & $<10$ & $<10$ & $<10$ \\
\hline 2,4-Dinitrophenol, $\mu \mathrm{g} / \mathrm{L}$ & $<10$ & $<10$ & $<10$ \\
\hline 2,4-Dinitrotoluene, $\mu \mathrm{g} / \mathrm{L}$ & $<10$ & $<10$ & $<10$ \\
\hline 2,6-Dinitrotoluene, $\mu \mathrm{g} / \mathrm{L}$ & $<10$ & $<10$ & $<10$ \\
\hline 2-Chloroethylvinyl ether, $\mu \mathrm{g} / \mathrm{L}$ & $<10$ & $<10$ & $<10$ \\
\hline 2-Chloronaphthalene, $\mu \mathrm{g} / \mathrm{L}$ & $<10$ & $<10$ & $<10$ \\
\hline 2-Chlorophenol, $\mu \mathrm{g} / \mathrm{L}$ & $<10$ & $<10$ & $<10$ \\
\hline 2-Nitrophenol, $\mu \mathrm{g} / \mathrm{L}$ & $<10$ & $<10$ & $<10$ \\
\hline 3,3'-Dichlorobenzidine, $\mu \mathrm{g} / \mathrm{L}$ & $<20$ & $<20$ & $<20$ \\
\hline 4,6-Dinitro-2-methylphenol, $\mu \mathrm{g} / \mathrm{L}$ & $<50$ & $<50$ & $<50$ \\
\hline 4-Bromophenyl-phenylether, $\mu \mathrm{g} / \mathrm{L}$ & $<10$ & $<10$ & $<10$ \\
\hline 4-Chloro-3-methylphenol, $\mu \mathrm{g} / \mathrm{L}$ & $<10$ & $<10$ & $<10$ \\
\hline 4.Cilorophenyl-phenylether, $\mu \mathrm{g} / \mathrm{L}$ & $<10$ & $<10$ & $<10$ \\
\hline 4-Nitrophenol, $\mu \mathrm{g} / \mathrm{L}$ & $<50$ & $<50$ & $<.50$ \\
\hline Acenaphthene, $\mu \mathrm{g} / \mathrm{L}$ & $<10$ & $<10$ & $<10$ \\
\hline Acenaphthylene, $\mu \mathrm{g} / \mathrm{L}$ & $<10$ & $<10$ & $<10$ \\
\hline Ammonia nitrogen, $\mathrm{mg} / \mathrm{L}$ & $<0.2$ & $<0.2$ & $<0.2$ \\
\hline Anthracene, $\mu \mathrm{g} / \mathrm{L}$ & $<10$ & $<10$ & $<10$ \\
\hline Arsenic, mg/L & $<0.005$ & $<0.005$ & $<0.005$ \\
\hline Benzene, $\mu \mathrm{g} / \mathrm{L}$ & $<5$ & $<5$ & $<5$ \\
\hline Benzidine, $\mu \mathrm{g} / \mathrm{L}$ & $<10$ & $<10$ & $<10$ \\
\hline Benzo(a)anthracene, $\mu \mathrm{g} / \mathrm{L}$ & $<10$ & $<10$ & $<10$ \\
\hline Benzo(a)pyrene, $\mu \mathrm{g} / \mathrm{L}$ & $<10$ & $<10$ & $<10$ \\
\hline Benzo(b)fluoranthene, $\mu \mathrm{g} / \mathrm{L}$ & $<10$ & $<10$ & $<10$ \\
\hline Benzo(g,h,i)perylene, $\mu \mathrm{g} / \mathrm{L}$ & $<10$ & $<10$ & $<10$ \\
\hline Benzo(k)fluoranthene, $\mu \mathrm{g} / \mathrm{L}$ & $<10$ & $<10$ & $<10$ \\
\hline Bromodichloromethane, $\mu \mathrm{g} / \mathrm{L}$ & $<5$ & $<5$ & $<5$ \\
\hline Bromoform, $\mu \mathrm{g} / \mathrm{L}$ & $<5$ & $<5$ & $<5$ \\
\hline Bromomethane, $\mu \mathrm{g} / \mathrm{L}$ & $<10$ & $<10$ & $<10$ \\
\hline Butylbenzylphthalate, $\mu \mathrm{g} / \mathrm{L}$ & $<10$ & $<10$ & $<10$ \\
\hline Cadmium, mg/L & $<0.002$ & $<0.002$ & $<0.002$ \\
\hline Carbon tetrachloride, $\mu \mathrm{g} / \mathrm{L}$ & $<5$ & $<5$ & $<5$ \\
\hline Chemical oxygen demand (COD), $\mathrm{mg} / \mathrm{L}$ & 14 & $<5$ & $<7$ \\
\hline Chlorobenzene, $\mu \mathrm{g} / \mathrm{L}$ & $<5$ & $<5$ & $<5$ \\
\hline Chloroethane, $\mu \mathrm{g} / \mathrm{L}$ & $<10$ & $<10$ & $<10$ \\
\hline Chloroform, $\mu \mathrm{g} / \mathrm{L}$ & $<5$ & $<5$ & $<5$ \\
\hline Chloromethane, $\mu \mathrm{g} / \mathrm{L}$ & $<10$ & $<10$ & $<10$ \\
\hline
\end{tabular}


Table 2.2.5 (Continued)

\begin{tabular}{|c|c|c|c|}
\hline & \multicolumn{3}{|c|}{ Concentration } \\
\hline & $\operatorname{Max}$ & $\mathrm{Min}$ & Av \\
\hline Chromium, mg/L & 0.018 & $<0.010$ & $<0.011$ \\
\hline Chrysene, $\mu \mathrm{g} / \mathrm{L}$ & $<10$ & $<10$ & $<10$ \\
\hline Copper, mg/L & 0.0086 & $<0.0040$ & $<0.0048$ \\
\hline Cyanide, $\mathrm{mg} / \mathrm{L}$ & $<0.1$ & $<0.1$ & $<0.1$ \\
\hline Di-n-butylphthalate, $\mu \mathrm{g} / \mathrm{L}$ & $<10$ & $<10$ & $<10$ \\
\hline Dibenz $(a, h)$ anthracene, $\mu \mathrm{g} / \mathrm{L}$ & $<10$ & $<10$ & $<10$ \\
\hline Dibromochloromethane, $\mu \mathrm{g} / \mathrm{L}$ & $<5$ & $<5$ & $<5$ \\
\hline Diethylphthalate, $\mu \mathrm{g} / \mathrm{L}$ & $<10$ & $<10$ & $<10$ \\
\hline Dimethylphthalate, $\mu \mathrm{g} / \mathrm{L}$ & $<10$ & $<10$ & $<10$ \\
\hline Dissolved solids, $\mathrm{mg} / \mathrm{L}$ & 196 & 74 & 131 \\
\hline Ethylbenzene, $\mu \mathrm{g} / \mathrm{L}$ & $<5$ & $<5$ & $<5$ \\
\hline Fluoranthene, $\mu \mathrm{g} / \mathrm{L}$ & $<10$ & $<10$ & $<10$ \\
\hline Fluorene, $\mu \mathrm{g} / \mathrm{L}$ & $<10$ & $<10$ & $<10$ \\
\hline Fluoride, $\mathrm{mg} / \mathrm{L}$ & $<0.20$ & $<0.10$ & $<0.14$ \\
\hline Hexachlorobenzene, $\mu \mathrm{g} / \mathrm{L}$ & $<10$ & $<10$ & $<10$ \\
\hline Hexachlorobutadiene, $\mu \mathrm{g} / \mathrm{L}$ & $<10$ & $<10$ & $<10$ \\
\hline Hexachlorocyclopentadiene, $\mu \mathrm{g} / \mathrm{L}$ & $<10$ & $<10$ & $<10$ \\
\hline Hexachloroetharie, $\mu \mathrm{g} / \mathrm{L}$ & $<10$ & $<10$ & $<10$ \\
\hline Indeno(1,2,3-cd)pyrene, $\mu \mathrm{g} / \mathrm{L}$ & $<10$ & $<10$ & $<10$ \\
\hline Isophorone, $\mu \mathrm{g} / \mathrm{L}$ & $<10$ & $<10$ & $<10$ \\
\hline Lead, $\mathrm{mg} / \mathrm{L}$ & 0.022 & $<0.0040$ & $<0.0063$ \\
\hline Manganese, $\mathrm{mg} / \mathrm{L}$. & 0.42 & 0.052 & 0.152 \\
\hline Mercury, mg/L & 0.0018 & $<0.0002$ & $<0.0016$ \\
\hline Methylene chloride, $\mu \mathrm{g} / \mathrm{L}$ & $<5$ & $<5$ & $<5$ \\
\hline $\mathrm{N}$-nitroso-di-n-propylamine, $\mu \mathrm{g} / \mathrm{L}$ & $<10$ & $<10$ & $<10$ \\
\hline $\mathrm{N}$-nitrosodimethylamine, $\mu \mathrm{g} / \mathrm{L}$ & $<10$ & $<10$ & $<10$ \\
\hline $\mathrm{N}$-nitrosodiphenylamine, $\mu \mathrm{g} / \mathrm{L}$ & $<10$ & $<10$ & $<10$ \\
\hline Naphthalene, $\mu \mathrm{g} / \mathrm{L}$ & $<10$ & $<10$ & $<10$ \\
\hline Nickel, mg/L & $<0.05$ & $<0,05$ & $<0.05$ \\
\hline Nitrate nitrogen, $\mathrm{mg} / \mathrm{L}$ & 0.80 & 0.30 & 0.54 \\
\hline Nitrobenzene, $\mu \mathrm{g} / \mathrm{L}$ & $<10$ & $<10$ & $<10$ \\
\hline Pentachlorophenol, $\mu \mathrm{g} / \mathrm{L}$ & $<50$ & $<50$ & $<50$ \\
\hline Phenanthrene, $\mu \mathrm{g} / \mathrm{L}$ & $<10$ & $<10$ & $<10$ \\
\hline Phenol, $\mu \mathrm{g} / \mathrm{L}$ & $<10$ & $<10$ & $<10$ \\
\hline Pyrene, $\mu \mathrm{g} / \mathrm{L}$ & $<10$ & $<10$ & $<10$ \\
\hline Sodium, $\mathrm{mg} / \mathrm{L}$ & 5.6 & 2.5 & 4.4 \\
\hline Sulfate, $\mathrm{mg} / \mathrm{L}$ & 38.0 & 20.0 & 29.4 \\
\hline Suspended solids, mg/L & 96.0 & 7.0 & 30.3 \\
\hline Tetrachloroethene, $\mu \mathrm{g} / \mathrm{L}$ & $<5$ & $<5$ & $<5$ \\
\hline Toluene, $\mu \mathrm{g} / \mathrm{L}$ & $<5$ & $<5$ & $<5$ \\
\hline Trichloroethene, $\mu \mathrm{g} / \mathrm{L}$ & $<5$ & $<5$ & $<5$ \\
\hline Vinyl chloride, $\mu \mathrm{g} / \mathrm{L}$ & $<10$ & $<10$ & $<10$ \\
\hline Zinc, $\mathrm{mg} / \mathrm{L}$ & 0.045 & $<0.020$ & $<0.024$ \\
\hline bis(2-Chloroethoxy)methane, $\mu \mathrm{g} / \mathrm{L}$ & $<10$ & $<10$ & $<10$ \\
\hline bis(2-Chloroethyl)ether, $\mu \mathrm{g} / \mathrm{L}$ & $<10$ & $<10$ & $<10$ \\
\hline bis(2-Chloroisopropyl)ether, $\mu \mathrm{g} / \mathrm{L}$ & $<10$ & $<10$ & $<10$ \\
\hline bis(2-Ethylhexyl)phthalate, $\mu \mathrm{g} / \mathrm{L}$ & 170 & $<10$ & $<33$ \\
\hline cis-1,3-Dichloropropene, $\mu \mathrm{g} / \mathrm{L}$ & $<5$ & $<5$ & $<5$ \\
\hline di-n-Octylphthalate, $\mu \mathrm{g} / \mathrm{L}$ & $<10$ & $<10$ & $<10$ \\
\hline $\mathrm{pH}$ & 8.4 & 7.4 & \\
\hline trans-1,2-Dichloroethene, $\mu \mathrm{g} / \mathrm{L}$ & $<5$ & $<5$ & $<5$ \\
\hline trans-1,3-Dichloropropene, $\mu \mathrm{g} / \mathrm{L}$ & $<5$ & $<5$ & $<5$ \\
\hline
\end{tabular}


Table 2.2.6. 1989 ORGDP concentrations at K-901 at 892

\begin{tabular}{|c|c|c|c|}
\hline \multirow{2}{*}{ Paranieter } & \multicolumn{3}{|c|}{$\begin{array}{l}\text { Concentration } \\
(\mathrm{mg} / \mathrm{L})\end{array}$} \\
\hline & $\operatorname{Max}$ & Min & Av \\
\hline Ammonia nitrogen, $\mathrm{mg} / \mathrm{L}$ & $<0.2$ & $<0.2$ & $<0.2$ \\
\hline Arsenic, $\mathrm{mg} / \mathrm{L}$ & $<0.005$ & $<0.005$ & $<0.005$ \\
\hline Cadmium, mg/L & $<0.002$ & $<0.002$ & $<0.002$ \\
\hline Chemical oxygen demand (COD), $\mathrm{mg} / \mathrm{L}$ & $<5$ & $<5$ & $<5$ \\
\hline Chromium, mg/L & $<0.010$ & $<0.010$ & $<0.010$ \\
\hline Copper, mg/L & $<0.0040$ & $<0.0040$ & $<0.0040$ \\
\hline Cyanide, $\mathrm{mg} / \mathrm{L}$ & 0.004 & 0.004 & 0.004 \\
\hline Dissolved solids, $\mathrm{mg} / \mathrm{L}$ & 114 & 114 & 114 \\
\hline Fluoride, $\mathrm{mg} / \mathrm{L}$ & $<0.10$ & $<0.10$ & $<0.10$ \\
\hline Lead, $\mathrm{mg} / \mathrm{L}$ & $<0.004$ & $<0.004$ & $<0.004$ \\
\hline Manganese, $\mathrm{mg} / \mathrm{L}$ & 0.084 & 0.084 & 0.084 \\
\hline Mercury, mg/L & $<0.0002$ & $<0.0002$ & $<0.0002$ \\
\hline Nickel, $\mathrm{mg} / \mathrm{L}$ & $<0.05$ & $<0.05$ & $<0.05$ \\
\hline Nitrate nitrogen, $\mathrm{mg} / \mathrm{L}$ & 0.41 & 0.41 & 0.41 \\
\hline Sodium, $\mathrm{mg} / \mathrm{L}$ & 3.9 & 3.9 & 3.9 \\
\hline Sulfate, $\mathrm{mg} / \mathrm{L}$ & 20.0 & 20.0 & 20.0 \\
\hline Suspended solids, $\mathrm{mg} / \mathrm{L}$ & 11.0 & 11.0 & 11.0 \\
\hline Zinc, $\mathrm{mg} / \mathrm{L}$ & 0.054 & 0.054 & 0.054 \\
\hline $\mathrm{pH}$ & 8.0 & 7.8 & \\
\hline
\end{tabular}


Table 2.2.7. 1989 ORGDP concentrations at K-1513

\begin{tabular}{|c|c|c|c|}
\hline \multirow{2}{*}{ Parameter } & \multicolumn{3}{|c|}{ Concentration } \\
\hline & Max & Min & Av \\
\hline 1,1,1-Trichloroethane, $\mu \mathrm{g} / \mathrm{L}$ & $<5$ & $<5$ & $<5$ \\
\hline 1,1,2,2-Tetrachloroethane, $\mu \mathrm{g} / \mathrm{L}$ & $<5$ & $<5$ & $<5$ \\
\hline 1,1,2-Trichloroethane, $\mu \mathrm{g} / \mathrm{L}$ & $<5$ & $<5$ & $<5$ \\
\hline 1,1-Dichloroethane, $\mu \mathrm{g} / \mathrm{L}$ & $<5$ & $<5$ & $<5$ \\
\hline 1,1-Dichloroethene, $\mu \mathrm{g} / \mathrm{L}$ & $<5$ & $<5$ & $<5$ \\
\hline 1,2,4-Trichlorobenzene, $\mu \mathrm{g} / \mathrm{L}$ & $<10$ & $<10$ & $<10$ \\
\hline 1,2-Dichlorobenzene, $\mu \mathrm{g} / \mathrm{L}$ & $<10$ & $<10$ & $<10$ \\
\hline 1,2-Dichloroethane, $\mu \mathrm{g} / \mathrm{L}$ & $<5$ & $<5$ & $<5$ \\
\hline 1,2-Dichloropropane, $\mu \mathrm{g} / \mathrm{L}$ & $<5$ & $<5$ & $<5$ \\
\hline 1,3-Dichlorobenzene, $\mu \mathrm{g} / \mathrm{L}$ & $<10$ & $<10$ & $<10$ \\
\hline 1,4-Dichlorobenzene, $\mu \mathrm{g} / \mathrm{L}$ & $<10$ & $<10$ & $<10$ \\
\hline 2,4,6-Trichlorophenol, $\mu \mathrm{g} / \mathrm{L}$ & $<10$ & $<10$ & $<10$ \\
\hline 2,4-Dichlorophenol, $\mu \mathrm{g} / \mathrm{L}$ & $<10$ & $<10$ & $<10$ \\
\hline 2,4-Dimethylphenol, $\mu \mathrm{g} / \mathrm{L}$ & $<10$ & $<10$ & $<10$ \\
\hline 2,4-Dinitrophenol, $\mu \mathrm{g} / \mathrm{L}$ & $<10$ & $<10$ & $<10$ \\
\hline 2,4-Dinitrotoluene, $\mu \mathrm{g} / \mathrm{L}$ & $<10$ & $<10$ & $<10$ \\
\hline 2,6-Dinitrotoluene, $\mu \mathrm{g} / \mathrm{L}$ & $<10$ & $<10$ & $<10$ \\
\hline 2-Chloroethylvinyl ether, $\mu \mathrm{g} / \mathrm{L}$ & $<10$ & $<10$ & $<10$ \\
\hline 2-Chloronaphthalene, $\mu \mathrm{g} / \mathrm{L}$ & $<10$ & $<10$ & $<10$ \\
\hline 2-Chlorophenol, $\mu \mathrm{g} / \mathrm{L}$ & $<10$ & $<10$ & $<10$ \\
\hline 2-Nitrophenol, $\mu \mathrm{g} / \mathrm{L}$ & $<10$ & $<10$ & $<10$ \\
\hline 3,3'-Dichlorobenzidine, $\mu \mathrm{g} / \mathrm{L}$ & $<20$ & $<20$ & $<20$ \\
\hline 4,6-Dinitro-2-methylphenol, $\mu \mathrm{g} / \mathrm{L}$ & $<50$ & $<50$ & $<50$ \\
\hline 4-Bromophenyl-phenylether, $\mu \mathrm{g} / \mathrm{L}$ & $<10$ & $<10$ & $<10$ \\
\hline 4-Chloro-3-methylphenol, $\mu \mathrm{g} / \mathrm{L}$ & $<10$ & $<10$ & $<10$ \\
\hline 4-Chlorophenyl-phenylether, $\mu \mathrm{g} / \mathrm{L}$ & $<10$ & $<10$ & $<10$ \\
\hline 4-Nitrophenol, $\mu \mathrm{g} / \mathrm{L}$ & $<50$ & $<50$ & $<50$ \\
\hline Acenaphthene, $\mu \mathrm{g} / \mathrm{L}$ & $<10$ & $<10$ & $<10$ \\
\hline Acenaphthylene, $\mu \mathrm{g} / \mathrm{L}$ & $<10$ & $<10$ & $<10$ \\
\hline Ammonia nitrogen, $\mathrm{mg} / \mathrm{L}$ & $<0.2$ & $<0.2$ & $<0.2$ \\
\hline Anthracene, $\mu \mathrm{g} / \mathrm{L}$ & $<10$ & $<10$ & $<10$ \\
\hline Arsenic, mg/L & $<0.005$ & $<0.005$ & $<0.005$ \\
\hline Benzene, $\mu \mathrm{g} / \mathrm{L}$ & $<5$ & $<5$ & $<5$ \\
\hline Benzidine, $\mu \mathrm{g} / \mathrm{L}$ & $<10$ & $<10$ & $<10$ \\
\hline Benzo(a)anthracene, $\mu \mathrm{g} / \mathrm{L}$ & $<10$ & $<10$ & $<10$ \\
\hline Benzo(a)pyrene, $\mu \mathrm{g} / \mathrm{L}$ & $<10$ & $<10$ & $<10$ \\
\hline Benzo(b)fluoranthene, $\mu \mathrm{g} / \mathrm{L}$ & $<10$ & $<10$ & $<10$ \\
\hline $\operatorname{Benzo}(\mathrm{g}, \mathrm{h}, \mathrm{i})$ perylene, $\mu \mathrm{g} / \mathrm{L}$ & $<10$ & $<10$ & $<10$ \\
\hline Benzo(k)fluoranthene, $\mu \mathrm{g} / \mathrm{L}$ & $<10$ & $<10$ & $<10$ \\
\hline Bromodichloromethane, $\mu \mathrm{g} / \mathrm{L}$ & $<5$ & $<5$ & $<5$ \\
\hline Bromoform, $\mu \mathrm{g} / \mathrm{L}$ & $<5$ & $\leqslant 5$ & $<5$ \\
\hline Bromomethane, $\mu \mathrm{g} / \mathrm{L}$ & $<10$ & $<10$ & $<10$ \\
\hline Butylbenzylphthalate, $\mu \mathrm{g} / \mathrm{L}$ & $<10$ & $<10$ & $<10$ \\
\hline Cadmium, mg/L & $<0.002$ & $<0.002$ & $<0.002$ \\
\hline Carbon tetrachloride, $\mu \mathrm{g} / \mathrm{L}$ & $<5$ & $<5$ & $<5$ \\
\hline Chemical oxygen demand (COD), $\mathrm{mg} / \mathrm{L}$ & 8,0 & $<5$ & $<5.3$ \\
\hline Chlorobenzene, $\mu \mathrm{g} / \mathrm{L}$ & $<5$ & $<5$ & $<5$ \\
\hline Chloroethane, $\mu \mathrm{g} / \mathrm{L}$ & $<10$ & $<10$ & $<10$ \\
\hline Chloroform, $\mu \mathrm{g} / \mathrm{L}$ & $<5$ & $<5$ & $<5$ \\
\hline Chloromethane, $\mu \mathrm{g} / \mathrm{L}$ & $<10$ & $<10$ & $<10$ \\
\hline
\end{tabular}


Table 2.2.7 (Continued)

\begin{tabular}{|c|c|c|c|}
\hline \multirow{2}{*}{ Parameter } & \multicolumn{3}{|c|}{ Concentration } \\
\hline & $\operatorname{Max}$ & Min & Av \\
\hline Chromium, $\mathrm{mg} / \mathrm{L}$ & $<0.010$ & $<0.010$ & $<0.010$ \\
\hline Chrysene, $\mu \mathrm{g} / \mathrm{L}$ & $<10$ & $<10$ & $<10$ \\
\hline Copper, mg/L & $<0.0071$ & $<0.0040$ & $<0.0043$ \\
\hline Cyanide, $\mathrm{mg} / \mathrm{L}$ & $<0.1$ & $<0.1$ & $<0.1$ \\
\hline Di-n-butylphthalate, $\mu \mathrm{g} / \mathrm{L}$ & $<10$ & $<10$ & $<10$ \\
\hline Dibenz(a,h)anthracene, $\mu \mathrm{g} / \mathrm{L}$ & $<10$ & $<10$ & $<10$ \\
\hline Dibromochloromethane, $\mu \mathrm{g} / \mathrm{L}$ & $<5$ & $<5$ & $<5$ \\
\hline Diethylphthalate, $\mu \mathrm{g} / \mathrm{L}$ & $<10$ & $<10$ & $<10$ \\
\hline Dimethylphthalate, $\mu \mathrm{g} / \mathrm{L}$ & $<10$ & $<10$ & $<10$ \\
\hline Dissolved solids, $\mathrm{mg} / \mathrm{L}$ & 194 & 118 & 160 \\
\hline Ethylbenzene, $\mu \mathrm{g} / \mathrm{L}$ & $<5$ & $<5$ & $<5$ \\
\hline Fluoranthene, $\mu \mathrm{g} / \mathrm{L}$ & $<10$ & $<10$ & $<10$ \\
\hline Fluorene, $\mu \mathrm{g} / \mathrm{L}$ & $<10$ & $<10$ & $<10$ \\
\hline Fluoride, $\mathrm{mg} / \mathrm{L}$ & 0.20 & $<0.10$ & $<0.11$ \\
\hline Hexachlorobenzene, $\mu \mathrm{g} / \mathrm{L}$ & $<10$ & $<10$ & $<10$ \\
\hline Hexachlorobutadiene, $\mu \mathrm{g} / \mathrm{L}$ & $<10$ & $<10$ & $<10$ \\
\hline Hexachlorocyclopentadiene, $\mu \mathrm{g} / \mathrm{L}$ & $<10$ & $<10$ & $<10$ \\
\hline Hexachloroethane, $\mu \mathrm{g} / \mathrm{L}$ & $<10$ & $<10$ & $<10$ \\
\hline Indeno(1,2,3-cd)pyrene, $\mu \mathrm{g} / \mathrm{L}$ & $<10$ & $<10$ & $<10$ \\
\hline Isophorone, $\mu \mathrm{g} / \mathrm{L}$ & $<10$ & $<10$ & $<10$ \\
\hline Lead, mg/L & 0.0055 & $<0.0040$ & $<0.0042$ \\
\hline Manganese, $\mathrm{mg} / \mathrm{L}$ & 0.059 & 0.016 & 0.035 \\
\hline Mercury, mg/L & $<0.0002$ & $<0.0002$ & $<0.0002$ \\
\hline Methylene chloride, $\mu \mathrm{g} / \mathrm{L}$ & $<5$ & $<5$ & $<5$ \\
\hline $\mathrm{N}$-nitroso-di-n-propylamine, $\mu \mathrm{g} / \mathrm{L}$ & $<10$ & $<10$ & $<10$ \\
\hline $\mathrm{N}$-nitrosodimethylamine, $\mu \mathrm{g} / \mathrm{L}$ & $<10$ & $<10$ & $<10$ \\
\hline $\mathrm{N}$-nitrosodiphenylamine, $\mu \mathrm{g} / \mathrm{L}$ & $<10$ & $<10$ & $<10$ \\
\hline Naphthalene, $\mu \mathrm{g} / \mathrm{L}$ & $<10$ & $<10$ & $<10$ \\
\hline Nickel, mg/L & $<0.05$ & $<0.05$ & $<0.05$ \\
\hline Nitrate nitrogen, $\mathrm{mg} / \mathrm{L}$ & 0.50 & 0.20 & 0.40 \\
\hline Nitrobenzene, $\mu \mathrm{g} / \mathrm{L}$ & $<10$ & $<10$ & $<10$ \\
\hline Pentachlorophenol, $\mu \mathrm{g} / \mathrm{L}$ & $<50$ & $<50$ & $<50$ \\
\hline Phenanthrene, $\mu \mathrm{g} / \mathrm{L}$ & $<10$ & $<10$ & $<10$ \\
\hline Phenol, $\mu \mathrm{g} / \mathrm{L}$ & $<10$ & $<10$ & $<10$ \\
\hline Pyrene, $\mu \mathrm{g} / \mathrm{L}$ & $<10$ & $<10$ & $<10$ \\
\hline Sodium, mg/L & 5.3 & 3.9 & 4.6 \\
\hline Sulfate, $\mathrm{mg} / \mathrm{L}$ & 27.0 & 18.0 & 20.7 \\
\hline Suspended solids, mg/L & 23.0 & 2.0 & 6.8 \\
\hline Tetrachloroethene, $\mu \mathrm{g} / \mathrm{L}$ & $<5$ & $<\varepsilon$ & $<5$ \\
\hline Toluene, $\mu \mathrm{g} / \mathrm{L}$ & $<5$ & $<5$ & $<5$ \\
\hline Trichloroethene, $\mu \mathrm{g} / \mathrm{L}$ & $<5$ & $<5$ & $<5$ \\
\hline Vinyl chloride, $\mu \mathrm{g} / \mathrm{L}$ & $<10$ & $<10$ & $<10$ \\
\hline Zinc, mg/L & $<0.020$ & $<0.020$ & $<0.020$ \\
\hline bis (2-Chloroethoxy)methane, $\mu \mathrm{g} / \mathrm{L}$ & $<10$ & $<10$ & $<10$ \\
\hline bis(2-Chloroethyl)ether, $\mu \mathrm{g} / \mathrm{L}$ & $<10$ & $<10$ & $<10$ \\
\hline bis(2-Chloroisopropyl)ether, $\mu \mathrm{g} / \mathrm{L}$ & $<10$ & $<10$ & $<10$ \\
\hline bis(2-Ethylhexyl)phthalate, $\mu \mathrm{g} / \mathrm{L}$ & 62 & $<5$ & $<15$ \\
\hline cis-1,3-Dichloropropene, $\mu \mathrm{g} / \mathrm{L}$ & $<5$ & $<5$ & $<5$ \\
\hline di-n-Octylphthalate, $\mu \mathrm{g} / \mathrm{L}$ & $<10$ & $<10$ & $<10$ \\
\hline $\mathrm{pH}$ & 8.7 & 7.5 & \\
\hline trans-1,2-Dichloroethene, $\mu \mathrm{g} / \mathrm{L}$ & $<.5$ & $<5$ & $<5$ \\
\hline trans-1,3m-Dichloropropene, $\mu \mathrm{g} / \mathrm{L}$ & $<5$ & $<5$ & $<5$ \\
\hline
\end{tabular}


Table 2.2.8. 1989 ORGDP concentrations at K-1710

\begin{tabular}{|c|c|c|c|}
\hline \multirow{2}{*}{ Parameter } & \multicolumn{3}{|c|}{ Concentration } \\
\hline & Max & Min & Av \\
\hline 1,1,1-Trichloroethane, $\mu \mathrm{g} / \mathrm{L}$ & $<5$ & $<5$ & $<5$ \\
\hline 1,1,2,2-Tetrachloroethane, $\mu \mathrm{g} / \mathrm{L}$ & $<5$ & $<5$ & $<5$ \\
\hline $1,1,2$-Trichloroethane, $\mu \mathrm{g} / \mathrm{L}$ & $<5$ & $<5$ & $<5$ \\
\hline 1,1-Dichloroethane, $\mu \mathrm{g} / \mathrm{L}$ & $<5$ & $<5$ & $<.5$ \\
\hline 1,1-Dichloroethene, $\mu \mathrm{g} / \mathrm{L}$ & $<5$ & $<5$ & $<5$ \\
\hline 1,2,4-Trichlorobenzene, $\mu \mathrm{g} / \mathrm{L}$ & $<10$ & $>10$ & $<10$ \\
\hline 1,2-Dichlorobenzene, $\mu \mathrm{g} / \mathrm{L}$ & $<10$ & $<10$ & $<10$ \\
\hline 1,2-Dichloroethane, $\mu \mathrm{g} / \mathrm{L}$ & $<5$ & $<5$ & $<5$ \\
\hline 1,2-Dichloropropane, $\mu \mathrm{g} / \mathrm{L}$ & $<5$ & $<5$ & $<5$ \\
\hline 1,3-Dichlorobenzene, $\mu \mathrm{g} / \mathrm{L}$ & $<10$ & $<10$ & $<10$ \\
\hline 1,4-Dichlorobenzene, $\mu \mathrm{g} / \mathrm{L}$ & $<10$ & $<10$ & $<10$ \\
\hline 2,4,6-Trichlorophenol, $\mu \mathrm{g} / \mathrm{L}$ & $<10$ & $<10$ & $<10$ \\
\hline 2,4-Dichiorophen l, $\mu \mathrm{g} / \mathrm{L}$ & $<10$ & $<10$ & $<10$ \\
\hline 2,4-Dimethylphenol, $\mu \mathrm{g} / \mathrm{L}$ & $<10$ & $<10$ & $<10$ \\
\hline 2,4-Dinitrophenol, $\mu \mathrm{g} / \mathrm{L}$ & $<10$ & $<10$ & $<10$ \\
\hline 2,4-Dinitrotoluene, $\mu \mathrm{g} / \mathrm{L}$ & $<10$ & $<10$ & $<10$ \\
\hline 2,6-Dinitrotoluene, $\mu \mathrm{g} / \mathrm{L}$ & $<10$ & $<10$ & $<10$ \\
\hline 2-Chloroethylvinyl ether, $\mu \mathrm{g} / \mathrm{L}$ & $<10$ & $<10$ & $<10$ \\
\hline 2-Chloronaphthalene, $\mu \mathrm{g} / \mathrm{L}$ & $<10$ & $<10$ & $<10$ \\
\hline 2-Chlorophenol, $\mu \mathrm{g} / \mathrm{L}$ & $<10$ & $<10$ & $<10$ \\
\hline 2-Nitrophencl, $\mu \mathrm{g} / \mathrm{L}$ & $<10$ & $<10$ & $<10$ \\
\hline 3,3'-Dichlorobenzidins, $\mu \mathrm{g} / \mathrm{L}$ & $<20$ & $<20$ & $<20$ \\
\hline 4,6-Dinitro-2-methylphenol, $\mu \mathrm{g} / \mathrm{L}$ & $<50$ & $<50$ & $<50$ \\
\hline 4-Bromopheny!-phenylether, $\mu \mathrm{g} / \mathrm{L}$ & $<10$ & $<10$ & $<10$ \\
\hline 4-Chloro-3-methylphenol, $\mu \mathrm{g} / \mathrm{L}$ & $<10$ & $<10$ & $<10$ \\
\hline 4-Chlorophenyl-phenylether, $\mu \mathrm{g} / \mathrm{L}$ & $<10$ & $<\mathrm{i} 0$ & $<10$ \\
\hline 4-Nitrophenol, $\mu \mathrm{g} / \mathrm{L}$ & $<50$ & $<50$ & $<50$ \\
\hline Acenaphthene, $\mu \mathrm{g} / \mathrm{L}$ & $<10$ & $<10$ & $<10$ \\
\hline Acenaphthylene, $\mu \mathrm{g} / \mathrm{L}$ & $<10$ & $<10$ & $<10$ \\
\hline Ammonia nitrogen, $\mathrm{mg} / \mathrm{L}$ & $<0.2$ & $<0.2$ & $<0.2$ \\
\hline Anthracene, $\mu \mathrm{g} / \mathrm{L}$ & $<10$ & $<10$ & $<10$ \\
\hline Arsenic, $\mathrm{mg} / \mathrm{L}$ & $<0.005$ & $<0.005$ & $<0.005$ \\
\hline Benzene, $\mu \mathrm{g} / \mathrm{L}$ & $<5$ & $<5$ & $<5$ \\
\hline Benzidine, $\mu \mathrm{g} / \mathrm{L}$ & $<10$ & $<10$ & $<10$ \\
\hline Benzo(a)anthracene, $\mu \mathrm{g} / \mathrm{L}$ & $<10$ & $<10$ & $<10$ \\
\hline Benzo(a)pyrene, $\mu \mathrm{g} / \mathrm{L}$ & $<10$ & $<10$ & $<10$ \\
\hline Benzo(b)fluor nthene, $\mu \mathrm{g} / \mathrm{L}$ & $<10$ & $<10$ & $<10$ \\
\hline Benzo $(g, h, i)$ perylene, $\mu \mathrm{g} / \mathrm{L}$ & $<10$ & $<10$ & $<10$ \\
\hline Benzo(k)fluoranthene, $\mu \mathrm{g} / \mathrm{L}$ & $<10$ & $<10$ & $<10$ \\
\hline Bromodichloromethane, $\mu \mathrm{g} / \mathrm{L}$ & $<5$ & $<5$ & $<5$ \\
\hline Bromoform, $\mu \mathrm{y} / \mathrm{L}$ & $<5$ & $<5$ & $<5$ \\
\hline Bromomethane, $\mu \mathrm{g} / \mathrm{L}$ & $<10$ & $<10$ & $<10$ \\
\hline Butylbenzylphthalate, $\mu \mathrm{g} / \mathrm{L}$ & $<10$ & $<10$ & $<10$ \\
\hline Cadmium, mg/L & $<0.002$ & $<0.002$ & $<0.002$ \\
\hline Carbon tetrachloride, $\mu \mathrm{g} / \mathrm{L}$ & $<5$ & $<5$ & $<5$ \\
\hline Chemical oxygen demand (COD), $\mathrm{mg} / \mathrm{L}$ & 18.0 & $<5$ & $<6.7$ \\
\hline Chlorobenzene, $\mu \mathrm{g} / \mathrm{L}$ & $<5$ & $<5$ & $<5$ \\
\hline Chloroethane, $\mu \mathrm{g} / \mathrm{L}$ & $<10$ & $<10$ & $<10$ \\
\hline Chloroform, $\mu_{\xi} / \mathrm{L}$ & $<5$ & $<5$ & $<5$ \\
\hline Chloromethane, $\mu \mathrm{g} / \mathrm{L}$ & $<10$ & $<10$ & $<10$ \\
\hline
\end{tabular}


Table 2.2.8 (Continued)

\begin{tabular}{|c|c|c|c|}
\hline \multirow{2}{*}{ Parameter } & \multicolumn{3}{|c|}{ Concentration } \\
\hline & $\operatorname{Max}$ & Min & Av \\
\hline Chromium, $\mathrm{mg}_{\mathrm{g}} / \mathrm{L}$ & $<0.010$ & $<0.010$ & $<0.010$ \\
\hline Chrysene, $\mu \mathrm{g} / \mathrm{L}$ & $<10$ & $<10$ & $<10$ \\
\hline Copper, mg/L & 0.0086 & $<0.0040$ & $<0.0049$ \\
\hline Cyanide, $\mathrm{mg} / \mathrm{L}$ & $<0.1$ & $<0.1$ & $<0.1$ \\
\hline Di-n-butylphthalate, $\mu \mathrm{g} / \mathrm{L}$ & $<10$ & $<10$ & $<10$ \\
\hline Dibenz(a,h)anthracene, $\mu \mathrm{g} / \mathrm{L}$ & $<10$ & $<10$ & $<10$ \\
\hline Dibromochloromethane, $\mu \mathrm{g} / \mathrm{L}$ & $<5$ & $<5$ & $<5$ \\
\hline Diethylphthalate, $\mu \mathrm{g} / \mathrm{L}$ & $<10$ & $<10$ & $<10$ \\
\hline Dimethylphthalate, $\mu \mathrm{g} / \mathrm{L}$ & $<10$ & $<10$ & $<10$ \\
\hline Dissolved solids, $\mathrm{mg} / \mathrm{L}$ & 258 & 62 & 157 \\
\hline Ethylbenzene, $\mu \mathrm{g} / \mathrm{L}$ & $<5$ & $<5$ & $<5$ \\
\hline Fluoranthene, $\mu \mathrm{g} / \mathrm{L}$ & $<10$ & $<10$ & $<10$ \\
\hline Fluorene, $\mu \mathrm{g} / \mathrm{L}$ & $<10$ & $<10$ & $<10$ \\
\hline Fluuride, $\mathrm{mg} / \mathrm{L}$ & 0.03 & $<0.10$ & $<0.17$ \\
\hline Hexachlorobenzene, $\mu \mathrm{g} / \mathrm{L}$ & $<10$ & $<10$ & $<10$ \\
\hline Hexachlorobutadiene, $\mu \mathrm{g} / \mathrm{L}$ & $<10$ & $<10$ & $<10$ \\
\hline Hexachlorocyclopentadiene, $\mu \mathrm{g} / \mathrm{L}$ & $<10$ & $<10$ & $<10$ \\
\hline Hexachloroethane, $\mu \mathrm{g} / \mathrm{L}$ & $<10$ & $<10$ & $<10$ \\
\hline Indeno(1,2,3-cd)pyrene, $\mu \mathrm{g} / \mathrm{L}$ & $<10$ & $<10$ & $<10$ \\
\hline Isophorone, $\mu \mathrm{g} / \mathrm{L}$ & $<10$ & $<10$ & $<10$ \\
\hline Lead, $\mathrm{mg} / \mathrm{L}$ & 0.0080 & $<0.0040$ & $<0.0045$ \\
\hline Manganese, $\mathrm{mg} / \mathrm{L}$ & 0.18 & 0.046 & 0.11 \\
\hline Mercury, $\mathrm{mg} / \mathrm{L}$ & $<0.0002$ & $<0.0002$ & $<0.0002$ \\
\hline Methylene chloride, $\mu \mathrm{g} / \mathrm{L}$ & $<5$ & $<5$ & $<5$ \\
\hline $\mathrm{N}$-nitroso-di-n-propylamine, $\mu \mathrm{g} / \mathrm{L}$ & $<10$ & $<10$ & $<10$ \\
\hline $\mathrm{N}$-nitrosodimethylamine, $\mu \mathrm{g} / \mathrm{L}$ & $<10$ & $<10$ & $<10$ \\
\hline $\mathrm{N}$-nitrosodiphenylamine, $\mu \mathrm{g} / \mathrm{L}$ & $<10$ & $<10$ & $<10$ \\
\hline Naphthialene, $\mu \mathrm{g} / \mathrm{L}$ & $<10$ & $<10$ & $<10$ \\
\hline Nickel, mg/L & $<0.05$ & $<0.05$ & $<0.05$ \\
\hline Nitrate nitrogen, $\mathrm{mg} / \mathrm{L}$ & 1.7 & $<0.20$ & $<0.71$ \\
\hline Nitrobenzene, $\mu \mathrm{g} / \mathrm{L}$ & $<10$ & $<10$ & $<10$ \\
\hline Pentachlorophenol, $\mu \mathrm{g} / \mathrm{L}$ & $<50$ & $<50$ & $<50$ \\
\hline Phenanthrene, $\mu \mathrm{g} / \mathrm{L}$ & $<10$ & $<10$ & $<10$ \\
\hline Phenol, $\mu \mathrm{g} / \mathrm{L}$ & $<10$ & $<10$ & $<10$ \\
\hline Pyrene, $\mu \mathrm{g} / \mathrm{L}$ & $<10$ & $<10$ & $<10$ \\
\hline Sodium, mg/L & 11.0 & 1.7 & 4.9 \\
\hline Sulfate, $\mathrm{mg} / \mathrm{L}$ & 42.0 & 20.0 & 33.6 \\
\hline Suspended solids, mg/L & 74.0 & 1.0 & 18.4 \\
\hline Tetrachloroethene, $\mu \mathrm{g} / \mathrm{L}$ & $<5$ & $<5$ & $<5$ \\
\hline Tuluene, $\mu \mathrm{g} / \mathrm{L}$ & $<5$ & $<5$ & $<5$ \\
\hline Trichloroethene, $\mu \mathrm{g} / \mathrm{L}$ & $<5$ & $<5$ & $<5$ \\
\hline Vinyl chloride, $\mu \mathrm{g} / \mathrm{L}$ & $<10$ & $<10$ & $<10$ \\
\hline Zinc, $\mathrm{mg} / \mathrm{L}$ & 0.021 & $<0.020$ & $<0.020$ \\
\hline bis(2-Chloroethoxy)methane, $\mu \mathrm{g} / \mathrm{L}$ & $<10$ & $<10$ & $<10$ \\
\hline bis(2-Chloroethy!)ether, $\mu \mathrm{g}$ /L & $<10$ & $<.10$ & $<10$ \\
\hline bis(2-Chloroisopropyl)ether, $\mu \mathrm{g} / \mathrm{L}$ & $<10$ & $<10$ & $<10$ \\
\hline bis(2-Ethylhexyl)phthalate, $\mu \mathrm{g} / \mathrm{L}$ & 100 & $<5$ & $<20$ \\
\hline cis-1,3-Dichloropropene, $\mu \mathrm{g} / \mathrm{L}$ & $<5$ & $<5$ & $<5$ \\
\hline di-n-Octylphthalate, $\mu \mathrm{g} / \mathrm{L}$ & $<10$ & $<10$ & $<10$ \\
\hline $\mathrm{pH}$ & 8.3 & 7.4 & \\
\hline trans-1,2-Dichloroethene, $\mu \mathrm{g} / \mathrm{L}$ & $<5$ & $<5$ & $<5$ \\
\hline trans-1,3-Dichloropropene, $\mu \mathrm{g} / \mathrm{L}$ & $<5$ & $<5$ & $<5$ \\
\hline
\end{tabular}


Table 2.2.9. 1989 ORGDP concentrations at K-1770

\begin{tabular}{|c|c|c|c|}
\hline \multirow[t]{2}{*}{ Parameter } & \multicolumn{3}{|c|}{ Concentration } \\
\hline & $\operatorname{Max}$ & Min & Av \\
\hline 1,1,1-Trichloroethane, $\mu \mathrm{g} / \mathrm{L}$ & $<5$ & $<5$ & $<5$ \\
\hline 1,1,2,2-Tetrachloroethane, $\mu \mathrm{g} / \mathrm{L}$ & $<5$ & $<5$ & $<5$ \\
\hline 1,1,2-Trichloroethane, $\mu \mathrm{g} / \mathrm{L}$ & $<5$ & $<5$ & $<5$ \\
\hline 1,1-Dichloroethane, $\mu \mathrm{g} / \mathrm{L}$ & $<5$ & $<5$ & $<5$ \\
\hline 1,1-Dichloroethene, $\mu \mathrm{g} / \mathrm{L}$ & $<5$ & $<5$ & $<5$ \\
\hline 1,2,4-Trichlorobenzene, $\mu \mathrm{g} / \mathrm{L}$ & $<10$ & $<10$ & $<10$ \\
\hline 1,2-Dichlorobenzene, $\mu \mathrm{g} / \mathrm{L}$ & $<10$ & $<10$ & $<10$ \\
\hline 1,2-Dichloroethane, $\mu \mathrm{g} / \mathrm{L}$ & $<5$ & $<5$ & $<5$ \\
\hline 1,2-Dichloropropane, $\mu \mathrm{g} / \mathrm{L}$ & $<5$ & $<5$ & $<5$ \\
\hline 1,3-Dichlorobenzene, $\mu \mathrm{g} / \mathrm{L}$ & $<10$ & $<10$ & $<10$ \\
\hline 1,4-Dichlorobenzene, $\mu \mathrm{g} / \mathrm{L}$ & $<10$ & $<10$ & $<10$ \\
\hline 2,4,6-Trichlorophenol, $\mu \mathrm{g} / \mathrm{L}$ & $<10$ & $<10$ & $<10$ \\
\hline 2,4-Dichlorophenol, $\mu \mathrm{g} / \mathrm{L}$ & $<10$ & $<10$ & $<10$ \\
\hline 2,4-Dimethylphenol, $\mu \mathrm{g} / \mathrm{L}$ & $<10$ & $<10$ & $<10$ \\
\hline 2,4-Dinitrophenol, $\mu \mathrm{g} / \mathrm{L}$ & $<10$ & $<10$ & $<10$ \\
\hline 2,4-Dinitrotoluene, $\mu \mathrm{g} / \mathrm{L}$ & $<10$ & $<10$ & $<10$ \\
\hline 2,6-Dinitrotoluene, $\mu \mathrm{g} / \mathrm{L}$ & $<10$ & $<10$ & $<10$ \\
\hline 2-Chloroethylvinyl ether, $\mu \mathrm{g} / \mathrm{L}$ & $<10$ & $<10$ & $<10$ \\
\hline 2-Chloronaphthalene, $\mu \mathrm{g} / \mathrm{L}$ & $<10$ & $<10$ & $<10$ \\
\hline 2-Chlorophenol, $\mu \mathrm{g} / \mathrm{L}$ & $<10$ & $<10$ & $<10$ \\
\hline 2-Nitrophenol, $\mu \mathrm{g} / \mathrm{L}$ & $<11)$ & $<10$ & $<10$ \\
\hline 3,3'-Dichlorobenzidine, $\mu \mathrm{g} / \mathrm{L}$ & $<20$ & $<20$ & $<20$ \\
\hline 4,6-Dinitro-2-methylphenol, $\mu \mathrm{g} / \mathrm{L}$ & $<50$ & $<50$ & $<50$ \\
\hline 4-Bromophenyl-phenylether, $\mu \mathrm{g} / \mathrm{L}$ & $<10$ & $<10$ & $<10$ \\
\hline 4-Chloro-3-methylphenol, $\mu \mathrm{g} / \mathrm{L}$ & $<10$ & $<10$ & $<10$ \\
\hline 4-Chlorophenyl-phenylether, $\mu \mathrm{g} / \mathrm{L}$ & $<10$ & $<10$ & $<10$ \\
\hline 4-Nitrophenol, $\mu \mathrm{g} / \mathrm{L}$ & $<50$ & $<50$ & $<50$ \\
\hline Acenaphthene, $\mu \mathrm{g} / \mathrm{L}$ & $<10$ & $<10$ & $<10$ \\
\hline Acenaphthylene, $\mu \mathrm{g} / \mathrm{L}$ & $<10$ & $<10$ & $<10$ \\
\hline Ammonia nitrogen, $\mathrm{mg} / \mathrm{L}$ & $<0.2$ & $<0.2$ & $<0.2$ \\
\hline Anthracene, $\mu \mathrm{g} / \mathrm{L}$ & $<10$ & $<10$ & $<10$ \\
\hline Arsenic, $\mathrm{mg} / \mathrm{L}$ & $<0.005$ & $<0.005$ & $<0.005$ \\
\hline Benzene, $\mu \mathrm{g} / \mathrm{L}$ & $<5$ & $<5$ & $<5$ \\
\hline Benzidine, $\mu \mathrm{g} / \mathrm{L}$ & $<10$ & $<10$ & $<10$ \\
\hline Benzo(a)anthracene, $\mu \mathrm{g} / \mathrm{L}$ & $<10$ & $<10$ & $<10$ \\
\hline Benzo(a)pyrene, $\mu \mathrm{g} / \mathrm{L}$ & $<10$ & $<10$ & $<10$ \\
\hline Benzo(b)fluoranthene, $\mu \mathrm{g} / \mathrm{L}$ & $<10$ & $<10$ & $<10$ \\
\hline Benzo(g,h,i)perylene, $\mu \mathrm{g} / \mathrm{L}$ & $<10$ & $<10$ & $<10$ \\
\hline Benzo(k)fluoranthene, $\mu \mathrm{g} / \mathrm{L}$ & $<10$ & $<10$ & $<10$ \\
\hline Bromodichloromethane, $\mu \mathrm{g} / \mathrm{L}$ & $<5$ & $<5$ & $<5$ \\
\hline Bromoform, $\mu \mathrm{g} / \mathrm{L}$ & $<5$ & $<5$ & $<5$ \\
\hline Bromomethane, $\mu \mathrm{g} / \mathrm{L}$ & $<10$ & $<10$ & $<10$ \\
\hline Butylbenzylphthalate, $\mu \mathrm{g} / \mathrm{L}$ & $<10$ & $<10$ & $<10$ \\
\hline Cadmium, mg/L & $<0.0038$ & $<0.0020$ & $<0.0022$ \\
\hline Carbon tetrachloride, $\mu \mathrm{g} / \mathrm{L}$ & $<5$ & $<5$ & $<5$ \\
\hline Chemical oxygen demand (COD), $\mathrm{mg} / \mathrm{L}$ & 38.0 & $<5$ & $<8.9$ \\
\hline Chlorobenzene, $\mu \mathrm{g} / \mathrm{L}$ & $<5$ & $<5$ & $<5$ \\
\hline Chloroethane, $\mu \mathrm{g} / \mathrm{L}$ & $<10$ & $<10$ & $<10$ \\
\hline Chloroform, $\mu \mathrm{g} / \mathrm{L}$ & $<5$ & $<5$ & $<5$ \\
\hline Chloromethane, $\mu \mathrm{g} / \mathrm{L}$ & $<10$ & $<10$ & $<10$ \\
\hline
\end{tabular}


Table 2.2.9 (Continued)

\begin{tabular}{|c|c|c|c|}
\hline \multirow[t]{2}{*}{ Parameter } & \multicolumn{3}{|c|}{ Concentration } \\
\hline & $\operatorname{Max}$ & Min & Av \\
\hline Chromium, mg/L & $<0.010$ & $<0.010$ & $<0.010$ \\
\hline Chrysene, $\mu \mathrm{g} / \mathrm{L}$ & $<10$ & $<10$ & $<10$ \\
\hline Copper, mg/L & $<0.0071$ & $<0.0040$ & $<0.0044$ \\
\hline Cyanide, $\mathrm{mg} / \mathrm{L}$ & $<0.1$ & $<0.1$ & $<0.1$ \\
\hline Di-n-butylphthalate, $\mu \mathrm{g} / \mathrm{L}$ & $<10$ & $<10$ & $<10$ \\
\hline $\operatorname{Dibenz}(\mathrm{a}, \mathrm{h})$ anthracene, $\mu \mathrm{g} / \mathrm{L}$ & $<10$ & $<10$ & $<10$ \\
\hline Dibromochloromethane, $\mu \mathrm{g} / \mathrm{L}$ & $<5$ & $<5$ & $<5$ \\
\hline Diethylphthalate, $\mu \mathrm{g} / \mathrm{L}$ & $<10$ & $<10$ & $<10$ \\
\hline Dimethylphthalate, $\mu \mathrm{g} / \mathrm{L}$ & $<10$ & $<10$ & $<10$ \\
\hline Dissolved solids, mg/L & 216 & 106 & 161 \\
\hline Ethylbenzene, $\mu \mathrm{g} / \mathrm{L}$ & $<5$ & $<5$ & $<5$ \\
\hline Fluoranthene, $\mu \mathrm{g} / \mathrm{L}$ & $<10$ & $<10$ & $<10$ \\
\hline Fluorene, $\mu \mathrm{g} / \mathrm{L}$ & $<10$ & $<10$ & $<10$ \\
\hline Fluoride, $\mathrm{mg} / \mathrm{L}$ & 0.30 & $<0.10$ & $<0.16$ \\
\hline Hexachlorobenzene, $\mu \mathrm{g} / \mathrm{L}$ & $<10$ & $<10$ & $<10$ \\
\hline Hexachlorobutadiene, $\mu \mathrm{g} / \mathrm{L}$ & $<10$ & $<10$ & $<10$ \\
\hline Hexachlorocyclopentadiene, $\mu \mathrm{g} / \mathrm{L}$ & $<10$ & $<10$ & $<10$ \\
\hline Hexachloroethane, $\mu \mathrm{g} / \mathrm{L}$ & $<10$ & $<10$ & $<10$ \\
\hline Indeno(1,2,3-cd)pyrene, $\mu \mathrm{g} / \mathrm{L}$ & $<10$ & $<10$ & $<10$ \\
\hline Isophorone, $\mu \mathrm{g} / \mathrm{L}$ & $<10$ & $<10$ & $<10$ \\
\hline Lead, $\mathrm{mg} / \mathrm{L}$ & 0.0094 & $<0.0040$ & $<0.0050$ \\
\hline Manganese, $\mathrm{mg} / \mathrm{L}$ & 0.42 & 0.018 & 0.11 \\
\hline Mercury, mg/L & $<0.0002$ & $<0.0002$ & $<0.0002$ \\
\hline Methylene chloride, $\mu \mathrm{g} / \mathrm{L}$ & $<5$ & $<5$ & $<5$ \\
\hline $\mathrm{N}$-nitroso-di-n-propylamine, $\mu \mathrm{g} / \mathrm{L}$ & $<10$ & $<10$ & $<10$ \\
\hline $\mathrm{N}$-nitrosodimethylamine, $\mu \mathrm{g} / \mathrm{L}$ & $<10$ & $<10$ & $<10$ \\
\hline $\mathrm{N}$-nitrosodiphenylamine, $\mu \mathrm{g} / \mathrm{L}$ & $<10$ & $<10$ & $<10$ \\
\hline Naphthalene, $\mu \mathrm{g} / \mathrm{L}$ & $<10$ & $<10$ & $<10$ \\
\hline Nickel, mg/L & $<0.05$ & $<0.05$ & $<0.05$ \\
\hline Nitrate nitrogen, $\mathrm{mg} / \mathrm{L}$ & 2.0 & $<0.20$ & $<0.51$ \\
\hline Nitrobenzene, $\mu \mathrm{g} / \mathrm{L}$ & $<10$ & $<10$ & $<10$ \\
\hline Pentachlorophenol, $\mu \mathrm{g} / \mathrm{L}$ & $<50$ & $<50$ & $<50$ \\
\hline Phenanthrene, $\mu \mathrm{g} / \mathrm{L}$ & $<10$ & $<10$ & $<10$ \\
\hline Phenol, $\mu \mathrm{g} / \mathrm{L}$ & $<10$ & $<10$ & $<10$ \\
\hline Pyrene, $\mu \mathrm{g} / \mathrm{L}$ & $<10$ & $<10$ & $<10$ \\
\hline Sodium, mg/L & 11.0 & 2.0 & 5.1 \\
\hline Sulfate, $\mathrm{mg} / \mathrm{L}$ & 47.0 & 20.0 & 25.8 \\
\hline Suspended solids, $\mathrm{mg} / \mathrm{L}$ & 143.0 & 3.0 & 23.6 \\
\hline Tetrachloroethene, $\mu \mathrm{g} / \mathrm{L}$ & $<5$ & $<5$ & $<5$ \\
\hline Toluene, $\mu \mathrm{g} / \mathrm{L}$ & $<5$ & $<5$ & $<5$ \\
\hline Trichloroethene, $\mu \mathrm{g} / \mathrm{L}$ & $<5$ & $<5$ & $<5$ \\
\hline Vinyl chloride, $\mu \mathrm{g} / \mathrm{L}$ & $<10$ & $<10$ & $<10$ \\
\hline Zinc, $\mathrm{mg} / \mathrm{L}$ & 0.040 & $<0.020$ & $<0.022$ \\
\hline bis(2-Chloroethoxy)methane, $\mu \mathrm{g} / \mathrm{L}$ & $<10$ & $<10$ & $<10$ \\
\hline bis(2-Chloroethyl)ether, $\mu \mathrm{g} / \mathrm{L}$ & $<10$ & $<10$ & $<10$ \\
\hline bis(2-Chloroisopropyl)ether, $\mu \mathrm{g} / \mathrm{L}$ & $<10$ & $<10$ & $<10$ \\
\hline bis(2-Ethylhexyl)phthalate, $\mu \mathrm{g} / \mathrm{L}$ & 370 & $<10$ & $<40$ \\
\hline cis-1,3-Dichloropropene, $\mu \mathrm{g} / \mathrm{L}$ & $<5$ & $<5$ & $<5$ \\
\hline di-n-Octylphthalate, $\mu \mathrm{g} / \mathrm{L}$ & $<10$ & $<10$ & $<10$ \\
\hline $\mathrm{pH}$ & 8.5 & 7.3 & \\
\hline trans-1,2-Dichloroethene, $\mu \mathrm{g} / \mathrm{L}$ & $<5$ & $<5$ & $<5$ \\
\hline trans-1,3-Dichloropropene, $\mu \mathrm{g} / \mathrm{L}$ & $<5$ & $<5$ & $<5$ \\
\hline
\end{tabular}


Table 2.2.10. 1989 ORGDP concentrations at Mitchell Branch

\begin{tabular}{|c|c|c|c|}
\hline \multirow[t]{2}{*}{ Parameter } & \multicolumn{3}{|c|}{ Concentration } \\
\hline & $\operatorname{Max}$ & $\mathrm{Min}$ & Av \\
\hline 1,1,1-Trichloroethane, $\mu \mathrm{g} / \mathrm{L}$ & $<5$ & $<5$ & $<5$ \\
\hline 1,1,2,2-Tetrachloroethane, $\mu \mathrm{g} / \mathrm{L}$ & $<, 5$ & $<5$ & $<5$ \\
\hline 1,1,2-Trichloroethane, $\mu \mathrm{g} / \mathrm{L}$ & $<s$ & $<5$ & $<5$ \\
\hline 1,1-Dichloroethane, $\mu \mathrm{g} / \mathrm{L}$ & $<5$ & $<5$ & $<5$ \\
\hline 1,1-Dichloroethene, $\mu \mathrm{g} / \mathrm{L}$ & $<5$ & $<5$ & $<5$ \\
\hline 1,2,4-Trichlorobenzene, $\mu \mathrm{g} / \mathrm{L}$ & $<10$ & $<10$ & $<10$ \\
\hline 1,2-Dichlorobenzene, $\mu \mathrm{g} / \mathrm{L}$ & $<10$ & $<10$ & $<10$ \\
\hline 1,2-Dichloroethane, $\mu \mathrm{g} / \mathrm{L}$ & $<5$ & $<5$ & $<5$ \\
\hline 1,2-Dichloropropane, $\mu \mathrm{g} / \mathrm{L}$ & $<5$ & $<5$ & $<5$ \\
\hline 1,3-Dichlorobenzene, $\mu \mathrm{g} / \mathrm{L}$ & $<10$ & $<10$ & $<10$ \\
\hline 1,4-Dichlorobenzene, $\mu \mathrm{g} / \mathrm{L}$ & $<10$ & $<10$ & $<10$ \\
\hline 2,4,6-Trichlorophenol, $\mu \mathrm{g} / \mathrm{L}$ & $<10$ & $<10$ & $<10$ \\
\hline 2,4-Dichlorophenol, $\mu \mathrm{g} / \mathrm{L}$ & $<10$ & $<10$ & $<10$ \\
\hline 2,4-Dimethylphenol, $\mu \mathrm{g} / \mathrm{L}$ & $<10$ & $<10$ & $<10$ \\
\hline 2,4-Dinitrophenol, $\mu \mathrm{g} / \mathrm{L}$ & $<10$ & $<10$ & $<10$ \\
\hline 2,4-Dinitrotoluene, $\mu \mathrm{g} / \mathrm{L}$ & $<10$ & $<10$ & $<10$ \\
\hline 2,6-Dinitrotoluene, $\mu \mathrm{g} / \mathrm{L}$ & $<10$ & $<10$ & $<10$ \\
\hline 2-Chloroethylvinyl ether, $\mu \mathrm{g} / \mathrm{L}$ & $<10$ & $<10$ & $<10$ \\
\hline 2-Chloronaphthalene, $\mu \mathrm{g} / \mathrm{L}$ & $<10$ & $<10$ & $<10$ \\
\hline 2-Chlorophenol, $\mu \mathrm{g} / \mathrm{L}$ & $<10$ & $<10$ & $<10$ \\
\hline 2-Nitrophenol, $\mu \mathrm{g} / \mathrm{L}$ & $<10$ & $<10$ & $<10$ \\
\hline 3,3'-Dichlorobenzidine, $\mu \mathrm{g} / \mathrm{L}$ & $<20$ & $<20$ & $<20$ \\
\hline 4,6-Dinitro-2-methylphenol, $\mu \mathrm{g} / \mathrm{L}$ & $<50$ & $<50$ & $<50$ \\
\hline 4-Bromophenyl-phenylether, $\mu \mathrm{g} / \mathrm{L}$ & $<10$ & $<10$ & $<10$ \\
\hline 4-Chloro-3-methylphenol, $\mu \mathrm{g} / \mathrm{L}$ & $<10$ & $<10$ & $<10$ \\
\hline 4-Chlorophenyl-phenylether, $\mu \mathrm{g} / \mathrm{L}$ & $<10$ & $<10$ & $<10$ \\
\hline 4-Nitrophenol, $\mu \mathrm{g} / \mathrm{L}$ & $<50$ & $<50$ & $<50$ \\
\hline Acenaphthene, $\mu \mathrm{g} / \mathrm{L}$ & $<10$ & $<10$ & $<10$ \\
\hline Acenaphthylene, $\mu \mathrm{g} / \mathrm{L}$ & $<10$ & $<10$ & $<10$ \\
\hline Ammonia nitrogen, $\mathrm{mg} / \mathrm{L}$ & $<0.2$ & $<0.2$ & $<0.2$ \\
\hline Anthracene, $\mu \mathrm{g} / \mathrm{L}$ & $<10$ & $<10$ & $<10$ \\
\hline Arsenic, $\mathrm{mg} / \mathrm{L}$ & $<0.005$ & $<0.005$ & $<0.005$ \\
\hline Benzene, $\mu \mathrm{g} / \mathrm{L}$ & $<5$ & $<5$ & $<5$ \\
\hline Benzidine, $\mu \mathrm{g} / \mathrm{L}$ & $<10$ & $<10$ & $<10$ \\
\hline Benzo(a)anthracene, $\mu \mathrm{g} / \mathrm{L}$ & $<10$ & $<10$ & $<10$ \\
\hline Benzo(a)pyrene, $\mu \mathrm{g} / \mathrm{L}$ & $<10$ & $<10$ & $<10$ \\
\hline Benzo(b)fluoranthene, $\mu \mathrm{g} / \mathrm{L}$ & $<10$ & $<10$ & $<10$ \\
\hline $\operatorname{Benzo}(\mathrm{g}, \mathrm{h}, \mathrm{i})$ perylene, $\mu \mathrm{g} / \mathrm{L}$ & $<10$ & $<10$ & $<10$ \\
\hline Benzo(k)fluoranthene, $\mu \mathrm{g} / \mathrm{L}$ & $<10$ & $<10$ & $<10$ \\
\hline Bromodichloromethane, $\mu \mathrm{g} / \mathrm{L}$ & $<5$ & $<5$ & $<5$ \\
\hline Bromoform, $\mu \mathrm{g} / \mathrm{L}$ & $<5$ & $<5$ & $<5$ \\
\hline Bromomethane, $\mu \mathrm{g} / \mathrm{L}$ & $<10$ & $<10$ & $<10$ \\
\hline Butylbenzylphthalate, $\mu \mathrm{g} / \mathrm{L}$ & $<10$ & $<10$ & $<10$ \\
\hline Cadmium, mg/L & $<0.002$ & $<0.002$ & $<0.002$ \\
\hline Carbon tetrachloride, $\mu \mathrm{g} / \mathrm{L}$ & $<5$ & $<5$ & $<5$ \\
\hline Chemical oxygen demand (COD), $\mathrm{mg} / \mathrm{L}$ & $<5$ & $<5$ & $<5$ \\
\hline Chlorobenzene, $\mu \mathrm{g} / \mathrm{L}$ & $<5$ & $<5$ & $<5$ \\
\hline Chloroethane, $\mu \mathrm{g} / \mathrm{L}$ & $<10$ & $<10$ & $<10$ \\
\hline Chloroform, $\mu \mathrm{g} / \mathrm{L}$ & $<5$ & $<5$ & $<5$ \\
\hline Chloromethane, $\mu \mathrm{g} / \mathrm{L}$ & $<10$ & $<10$ & $<10$ \\
\hline
\end{tabular}


Table 2.2.10 (Continued)

\begin{tabular}{|c|c|c|c|}
\hline \multirow[t]{2}{*}{ Parameter } & \multicolumn{3}{|c|}{ Concentration } \\
\hline & $\operatorname{Max}$ & Min & Av \\
\hline Chromium, mg/L & $<0.010$ & $<0.010$ & $<0.010$ \\
\hline Chrysene, $\mu \mathrm{g} / \mathrm{L}$ & $<10$ & $<10$ & $<10$ \\
\hline Copper, mg/L & $<0.004$ & $<0.004$ & $<0.004$ \\
\hline Cyanide, $\mathrm{mg} / \mathrm{L}$ & $<0.1$ & $<0.1$ & $<0.1$ \\
\hline Di-n-butylphthalate, $\mu \mathrm{g} / \mathrm{L}$ & $<10$ & $<10$ & $<10$ \\
\hline Dibenz(a,h)anthracene, $\mu \mathrm{g} / \mathrm{L}$ & $<10$ & $<10$ & $<10$ \\
\hline Dibromochloromethane, $\mu \mathrm{g} / \mathrm{L}$ & $<5$ & $<5$ & $<5$ \\
\hline Diethylphthalate, $\mu \mathrm{g} / \mathrm{L}$ & $<10$ & $<10$ & $<10$ \\
\hline Dimethylphthalate, $\mu \mathrm{g} / \mathrm{L}$ & $<10$ & $<10$ & $<10$ \\
\hline Dissolved solids, mg/L & 106 & 86 & 97 \\
\hline Ethylbenzene, $\mu \mathrm{g} / \mathrm{L}$ & $<5$ & $<5$ & $<5$ \\
\hline Fluoranthene, $\mu \mathrm{g} / \mathrm{L}$ & $<10$ & $<10$ & $<10$ \\
\hline Fluorene, $\mu \mathrm{g} / \mathrm{L}$ & $<10$ & $<10$ & $<10$ \\
\hline Fluoride, $\mathrm{mg} / \mathrm{L}$ & $<0.10$ & $<0.10$ & $<0.10$ \\
\hline Hexachlorobenzene, $\mu \mathrm{g} / \mathrm{L}$ & $<10$ & $<10$ & $<10$ \\
\hline Hexachlorobutadiene, $\mu \mathrm{g} / \mathrm{L}$ & $<10$ & $<10$ & $<10$ \\
\hline Hexachlorocyclopentadiene, $\mu \mathrm{g} / \mathrm{L}$ & $<10$ & $<10$ & $<10$ \\
\hline Hexachloroethane, $\mu \mathrm{g} / \mathrm{L}$ & $<10$ & $<10$ & $<10$ \\
\hline Indeno(1,2,3-cd)pyrene, $\mu \mathrm{g} / \mathrm{L}$ & $<10$ & $<10$ & $<10$ \\
\hline Isophorone, $\mu \mathrm{g} / \mathrm{L}$ & $<10$ & $<10$ & $<10$ \\
\hline Lead, mg/L & 0.0049 & $<0.0040$ & $<0.0043$ \\
\hline Manganese, $\mathrm{mg} / \mathrm{L}$ & 0.28 & 0.07 & 0.15 \\
\hline Mercury, mg/L & $<0.0002$ & $<0.0002$ & $<0.0002$ \\
\hline Methylene chloride, $\mu \mathrm{g} / \mathrm{L}$ & $<5$ & $<5$ & $<5$ \\
\hline $\mathrm{N}$-nitroso-di-n-propylamine, $\mu \mathrm{g} / \mathrm{L}$ & $<10$ & $<10$ & $<10$ \\
\hline $\mathrm{N}$-nitrosodimethylamine, $\mu \mathrm{g} / \mathrm{L}$ & $<10$ & $<10$ & $<10$ \\
\hline $\mathrm{N}$-nitrosodiphenylamine, $\mu \mathrm{g} / \mathrm{L}$ & $<10$ & $<10$ & $<10$ \\
\hline Naphthalene, $\mu \mathrm{g} / \mathrm{L}$ & $<10$ & $<10$ & $<10$ \\
\hline Nickel, mg/L & $<0.05$ & $<0.05$ & $<0.05$ \\
\hline Nitrate nitrogen, $\mathrm{mg} / \mathrm{L}$ & $<0.2$ & $<0.2$ & $<0.2$ \\
\hline Nitrobenzene, $\mu \mathrm{g} / \mathrm{L}$ & $<10$ & $<10$ & $<10$ \\
\hline Pentachlorophenol, $\mu \mathrm{g} / \mathrm{L}$ & $<50$ & $<50$ & $<50$ \\
\hline Phenanthrene, $\mu \mathrm{g} / \mathrm{L}$ & $<10$ & $<10$ & $<10$ \\
\hline Phenol, $\mu \mathrm{g} / \mathrm{L}$ & $<10$ & $<10$ & $<10$ \\
\hline Pyrene, $\mu \mathrm{g} / \mathrm{L}$ & $<10$ & $<10$ & $<10$ \\
\hline Sodium, $\mathrm{mg} / \mathrm{L}$ & 1.2 & 0.89 & 1.0 \\
\hline Sulfate, $\mathrm{mg} / \mathrm{L}$ & 4 & 2 & 3 \\
\hline Suspended solids, $\mathrm{mg} / \mathrm{L}$ & 13 & 4 & 8 \\
\hline Tetrachloroethene, $\mu \mathrm{g} / \mathrm{L}$ & $<5$ & $<5$ & $<5$ \\
\hline Toluene, $\mu \mathrm{g} / \mathrm{L}$ & $<5$ & $<5$ & $<5$ \\
\hline Trichloroethene, $\mu \mathrm{g} / \mathrm{L}$ & $<5$ & $<5$ & $<5$ \\
\hline Vinyl chloride, $\mu \mathrm{g} / \mathrm{L}$ & $<10$ & $<10$ & $<10$ \\
\hline Zinc, $\mathrm{mg} / \mathrm{L}$ & $<0.02$ & $<0.02$ & $<0.02$ \\
\hline bis(2-Chloroethoxy)methane, $\mu \mathrm{g} / \mathrm{L}$ & $<10$ & $<10$ & $<10$ \\
\hline bis(2-Chloroethyl)ether, $\mu \mathrm{g} / \mathrm{L}$ & $<10$ & $<10$ & $<10$ \\
\hline bis(2-Chloroisopropyl)ether, $\mu \mathrm{g} / \mathrm{L}$ & $<10$ & $<10$ & $<10$ \\
\hline bis(2-Ethylhexyl)phthalate, $\mu \mathrm{g} / \mathrm{L}$ & 370 & $<10$ & $<40$ \\
\hline cis-1,3-Dichloropropene, $\mu \mathrm{g} / \mathrm{L}$ & $<5$ & $<5$ & $<5$ \\
\hline di-n-Octylphthalate, $\mu \mathrm{g} / \mathrm{L}$ & $<10$ & $<10$ & $<10$ \\
\hline $\mathrm{pH}$ & 8.0 & 7.6 & \\
\hline trans-1,2-Dichloroethene, $\mu \mathrm{g} / \mathrm{L}$ & $<5$ & $<5$ & $<5$ \\
\hline $\operatorname{trans}-1,3-$ Dichloropropene, $\mu \mathrm{g} / \mathrm{L}$ & $<5$ & $<5$ & $<5$ \\
\hline
\end{tabular}


Table 2.2.11. NPDES-permitted outfalls

\begin{tabular}{|c|c|}
\hline $\begin{array}{l}\text { Outfall } \\
\text { number }\end{array}$ & Effluent description \\
\hline & $\begin{array}{c}\text { Oak Ridge Y-12 Plant } \\
\text { NPDES Permit Number TN } 0002968\end{array}$ \\
\hline 301 & Kerr Hollow Quarry \\
\hline 302 & Rogers Quarry \\
\hline 303 & New Hope Pond \\
\hline 304 & Bear Creek \\
\hline 305 & Leaking Burial Grounds-Oil Pond 1 \\
\hline 306 & Seepage from Burial Pit-Oil Pond 2 \\
\hline Category I & Uncontaminated precipitation runoff and/or groundwater \\
\hline Category II & $\begin{array}{l}\text { Cooling water, condensate, building area, and } \\
\text { foundation drains and/or precipitation } \\
\text { runoff contaminated by area sources of } \\
\text { pollution }\end{array}$ \\
\hline Category III & $\begin{array}{l}\text { Any of the Category I or II outfalls or process } \\
\text { wastewater requiring treatment at one of } \\
\text { the on-site Y }-12 \text { treatment facilities }\end{array}$ \\
\hline $401-422$ & $\begin{array}{l}\text { Category IV Discharges-Process wastewaters requiring } \\
\text { minimal treatment }\end{array}$ \\
\hline 623 & Steam Plant fly ash sluice water \\
\hline 501 & Central Pollution Control Facility \\
\hline 502 & West End Treatment Facility \\
\hline 503 & Steam Plant Wastewater Treatment Facility \\
\hline 504 & Plating Rinsewater Treatment Facility \\
\hline 508 & Experimental Mobile Wastewater Treatment Facility \\
\hline 506 & Building 9204-3 Sump Pump Oil Separator \\
\hline & $\begin{array}{l}\text { Oak Ridge National Laboratory } \\
\text { NPDES Permit Number TN 0002941 }\end{array}$ \\
\hline $\mathrm{X} 0 \mathrm{i}$ & ORNL sewage treatment plant \\
\hline $\mathrm{X} 02$ & Coal Yard Runoff Treatment Facility \\
\hline $\mathrm{X} 03$ & 1500 Area (Environmental Sciences) \\
\hline $\mathrm{X} 04$ & 2000 area \\
\hline $\mathrm{X} 06$ & 190 Ponds \\
\hline $\mathrm{X} 06 \mathrm{~A}$ & $1500 / 2000 / 190$ Ponds \\
\hline $\mathrm{X} 07$ & Process Waste Treatment Plant (3544) \\
\hline $\mathrm{X} 08$ & TRU ponds \\
\hline $\mathrm{X} 09$ & HFIR ponds \\
\hline X09A & TRU/HFIR ponds \\
\hline $\mathrm{X} 10$ & Oak Ridge Research Reactor resin regeneration facility (closed) \\
\hline $\mathrm{X} 11$ & Acid neutralization facility $(3518)$ \\
\hline $\mathrm{X} 13$ & Melton Branch (ambient station) \\
\hline $\mathrm{X} 14$ & White Oak Creek (ambient station) \\
\hline $\mathrm{X} 15$ & White Oak Dam \\
\hline VC7002 & $\begin{array}{l}\text { Vehicle cleaning facility }(7002) \\
\text { Cooling towers }\end{array}$ \\
\hline EF7002 & Equipment maintenance facility (7002) \\
\hline SP2519 & Steam plant boiler drainage $(2519)$ \\
\hline Category I & Storm drains \\
\hline Category II & $\begin{array}{l}\text { Parking lot drains, storage area drains, } \\
\text { once-through cooling water, cooling water blowdown, } \\
\text { condensate }\end{array}$ \\
\hline Category III & Process and/or laboratory drains \\
\hline
\end{tabular}


Table 2.2.11 (continued)

\begin{tabular}{|c|c|}
\hline $\begin{array}{l}\text { Outfall } \\
\text { number }\end{array}$ & Effluent description \\
\hline & $\begin{array}{l}\text { Oak Ridge Gaseous Diffusion Plant } \\
\text { NPDES Permit Number TN } 0002950\end{array}$ \\
\hline K-1700 & K-1407-E/F effluent, surface runoff, once-through cooling \\
\hline $\mathrm{K}-1203$ & Sanitary wastewaters, organic industrial wastewaters \\
\hline $\mathrm{K}-1007-\mathrm{B}$ & $\begin{array}{l}\text { Potable water from once-through cooling systems, firewater } \\
\text { from once-through systems, surface runoff }\end{array}$ \\
\hline K-901-A & $\begin{array}{l}\text { Lime-softening sludges from firewater makeup } \\
\text { treatment, surface runoff }\end{array}$ \\
\hline K-710-A & Sanitary wastewater (inactive) \\
\hline $\mathrm{K}-1515-\mathrm{C}$ & $\begin{array}{l}\text { Water from sludge and backwash systems } \\
\text { associated with the potable water plant, } \\
\text { surface runoff }\end{array}$ \\
\hline $\begin{array}{l}\mathrm{K}-1407-\mathrm{E} \\
\quad \text { and } \mathrm{K}-1407-\mathrm{F}\end{array}$ & Steam plant and coal yard effluent (since November 1988) \\
\hline $\mathrm{K}-1407-\mathrm{J}$ & $\begin{array}{l}\text { Central neutralization facility effluent } \\
\text { (since November 1988) }\end{array}$ \\
\hline
\end{tabular}


Table 2.2.12. Radionuclide concentrations in water from NPDES stations" at ORNL. in 1989

\begin{tabular}{|c|c|c|c|c|c|c|}
\hline \multirow{2}{*}{ Radionuclide } & \multicolumn{5}{|c|}{$\begin{array}{c}\text { Concentration } \\
(p(i / L)\end{array}$} & \multirow{2}{*}{$\begin{array}{l}\text { Percentage } \\
\text { of } \mathrm{DCG}^{\mathrm{C}}\end{array}$} \\
\hline & & Max & Min & Av & $\begin{array}{l}\text { Sid. } \\
\text { error }\end{array}$ & \\
\hline \multicolumn{7}{|c|}{ Sew'age Treatme'nt Plant (XOl) } \\
\hline${ }^{m} \mathrm{Co}$ & 12 & 54 & -30 & 0.4 & 6.4 & 0.13 \\
\hline${ }^{131} \mathrm{Cs}$ & 12 & 32 & -19 & 4.2 & 4.2 & 0.14 \\
\hline Gross alpha & 1 & -7.6 & -7.6 & -7.6 & $d$ & $d$ \\
\hline Gross beta & 12 & 4,900 & 59 & 610 & 390 & $d$ \\
\hline Total $\mathrm{Sr}^{\prime}$ & 12 & 180 & 12 & 97 & 15 & 9.7 \\
\hline \multicolumn{7}{|c|}{1.500 areda $(x(03)$} \\
\hline Gross alpha & 4 & 14 & 2.4 & 7.5 & 2.7 & $d$ \\
\hline Gross beta & 4 & 22 & 0.0 & 12 & 5.1 & $d$ \\
\hline \multicolumn{7}{|c|}{2000 area $(x(0)$} \\
\hline nco & 4 & 5.4 & -8.1 & -0.41 & 2.9 & $<0.001$ \\
\hline${ }^{137} \mathrm{Cs}$ & 4 & 35 & -19 & 0.068 & 12 & 0.0023 \\
\hline Gross beta & 4 & 59 & 0.0 & 26 & 12 & $d$ \\
\hline Total Sre & 4 & 11 & -0.27 & 4.1 & 2.6 & 0.41 \\
\hline \multicolumn{7}{|c|}{190 ponds $(\lambda(0)$} \\
\hline${ }^{\circ} \mathrm{CO}$ & 4 & 22 & -24 & -3.6 & 9.5 & $<0.001$ \\
\hline${ }^{137} \mathrm{Cs}$ & 4 & 41 & -2.7 & 12 & 9.7 & 0.39 \\
\hline Gross alpha & 4 & 30 & -2.7 & 13 & 6.7 & $d$ \\
\hline Gross beta & 4 & 46 & 32 & 38 & 2.9 & $d$ \\
\hline \multicolumn{7}{|c|}{190 ponds, 1500 . Area and 2000 Area (X06.A) } \\
\hline${ }^{\infty} \mathrm{Co}$ & 8 & 24 & -32 & -4.0 & 6.2 & $<0.001$ \\
\hline${ }^{137} \mathrm{Cs}$ & 8 & 24 & -8.1 & 10 & 3.5 & 0.34 \\
\hline Gross alpha & 8 & 16 & -5.4 & 5.4 & 2.1 & $d$ \\
\hline Gross beta & 8 & 81 & -11 & 27 & 11 & $d$ \\
\hline Total Sre & 8 & 17 & 2.4 & 8.1 & 1.6 & 0.81 \\
\hline \multicolumn{7}{|c|}{ Process Waste Treatment Plant (XO) } \\
\hline${ }^{n} \mathrm{Co}$ & 12 & 2.600 & 14 & 260 & 210 & 5.2 \\
\hline${ }^{1.37} \mathrm{Cs}$ & 12 & 2.700 & 57 & 1,900 & 220 & 63 \\
\hline Gross alpha & 12 & 140 & -3.0 & 54 & 12 & $d$ \\
\hline Gross beta & 12 & 3,200 & 380 & 1,800 & 210 & $d$ \\
\hline Total Sre & 12 & 3.50 & 15 & 110 & 33 & 11 \\
\hline \multicolumn{7}{|c|}{ TRL ponds $(X() 8)$} \\
\hline Gross beta & 1 & 130 & 130 & 130 & $d$ & $d$ \\
\hline \multicolumn{7}{|c|}{ HFIR ponds $\left(X^{\prime}(09)\right.$} \\
\hline${ }^{60} \mathrm{Co}$ & 1 & 2,700 & 2,700 & 2,700 & $d$ & 54 \\
\hline${ }^{137} \mathrm{Cs}$ & $i$ & -11 & -11 & -11 & $d$ & $<0.001$ \\
\hline Gross alpha & 1 & 5.7 & 5.7 & 5.7 & $d$ & $d$ \\
\hline Gross beta & 1 & $2,40 n$ & 2,400 & 2,400 & $d$ & $d$ \\
\hline${ }^{54} \mathrm{Mn}$ & 1 & -2.7 & -2.7 & -2.7 & $d$ & $<0.001$ \\
\hline
\end{tabular}


Table 2.2.12 (continued)

\begin{tabular}{|c|c|c|c|c|c|c|}
\hline \multirow{2}{*}{ Radionuclide } & \multirow{2}{*}{$\begin{array}{c}\text { Number of } \\
\text { samples }\end{array}$} & \multicolumn{4}{|c|}{$\begin{array}{l}\text { Concentration } \\
\quad(\mathrm{pCi} / \mathrm{L})\end{array}$} & \multirow{2}{*}{$\begin{array}{l}\text { Percentage } \\
\text { of } D_{C G}{ }^{c}\end{array}$} \\
\hline & & $\operatorname{Max}$ & Min & Av & $\begin{array}{l}\text { Standard } \\
\text { error }\end{array}$ & \\
\hline \multicolumn{7}{|c|}{ TRU/TURF and HFIR ponds (X09A) } \\
\hline${ }^{60} \mathrm{Co}$ & 8 & 5,100 & 32 & 1,500 & 670 & 30 \\
\hline${ }^{137} \mathrm{Cs}$ & 8 & 430 & -5.4 & 71 & 52 & 2.4 \\
\hline${ }^{152} \mathrm{Eu}$ & 2 & 680 & 350 & 510 & 160 & 2.6 \\
\hline${ }^{154} \mathrm{Eu}$ & 5 & 970 & 250 & 560 & 120 & 2.8 \\
\hline${ }^{155} \mathrm{Eu}$ & 6 & 510 & 89 & 270 & 66 & 0.27 \\
\hline Gross alpha & 8 & 19 & -1.9 & 8.0 & 2.9 & $d$ \\
\hline Gross beta & 8 & 3,500 & 14 & 1,400 & 410 & $d$ \\
\hline \multicolumn{7}{|c|}{ Acid Neutralization Facility $(X 11)$} \\
\hline Gross alpha & 12 & 43 & -5.4 & 9.2 & 3.6 & $d$ \\
\hline Gross beta & 12 & 84 & -22 & 28 & 9.5 & $d$ \\
\hline \multicolumn{7}{|c|}{ Melton Branch $1(X 13)$} \\
\hline${ }^{60} \mathrm{Co}$ & 12 & 86 & -2.7 & 25 & 6.9 & 0.51 \\
\hline${ }^{137} \mathrm{Cs}$ & 12 & 27 & -16 & -0.36 & 4.1 & $<0.001$ \\
\hline Total $\mathrm{Sr}^{e}$ & 12 & 650 & 190 & 410 & 45 & 41 \\
\hline${ }^{3} \mathrm{H}$ & 12 & $1,800,000$ & 350,000 & $1,300,000$ & 120,000 & 67 \\
\hline \multicolumn{7}{|c|}{ White Oak Creek (X14) } \\
\hline${ }^{60} \mathrm{Co}$ & 12 & 41 & -8.1 & 10 & 4.3 & 0.20 \\
\hline${ }^{137} \mathrm{Cs}$ & 12 & 210 & 30 & 76 & 15 & 2.5 \\
\hline Total $\mathrm{Sr}^{e}$ & 12 & 380 & 05 & 160 & 25 & 16 \\
\hline${ }^{3} \mathrm{H}$ & 12 & 150,000 & 7,800 & 73,000 & 11,000 & 3.6 \\
\hline \multicolumn{7}{|c|}{ White Oak Dam (X15) } \\
\hline${ }^{241} \mathrm{Am}$ & 18 & 1.6 & -0.23 & 0.40 & 0.097 & 1.3 \\
\hline${ }^{244} \mathrm{Cm}$ & 10 & 1.7 & -1.6 & 0.48 & 0.27 & 0.80 \\
\hline${ }^{60} \mathrm{Co}$ & 52 & 27 & -14 & 8.3 & 0.87 & 0.17 \\
\hline${ }^{137} \mathrm{Cs}$ & 52 & 320 & -11 & 69 & 9.3 & 2.3 \\
\hline Gross alpha & 30 & 33 & -38 & 9.5 & 2.3 & $d$ \\
\hline Gross beta & 40 & 930 & 210 & 450 & 23 & $d$ \\
\hline${ }^{238} \mathrm{Pu}$ & 18 & 0.51 & -0.068 & 0.077 & 0.029 & 0.19 \\
\hline${ }^{239} \mathrm{P}_{\mathrm{u}}$ & 18 & 0.76 & -0.30 & 0.11 & 0.058 & 0.35 \\
\hline Total $\mathrm{Sr}^{e}$ & 26 & 380 & 100 & 180 & 18 & 18 \\
\hline${ }^{3} \mathrm{H}$ & 26 & 430,000 & 76,000 & 260,000 & 22,000 & 13 \\
\hline
\end{tabular}

${ }^{a}$ See Fig. 2.2.9 in Vol. 1 for NPDES station locations.

${ }^{b}$ Standard error of the mean.

'Average concentration as a percentage of the derived concentration guide (DCG).

${ }^{d}$ Not applicable.

Total radioactive $\mathrm{Sr}\left({ }^{89} \mathrm{Sr}+{ }^{90} \mathrm{Sr}\right)$. 
Table 2.2.13, 1989 ORGDP radiological effluent at K-1203

\begin{tabular}{ccrrr}
\hline Radionuclide & $\begin{array}{c}\text { Emission } \\
\text { source }(\mathrm{Ci})\end{array}$ & $\begin{array}{c}\mathrm{DCG} \\
(\mathrm{pCi} / \mathrm{L})\end{array}$ & $\begin{array}{c}\text { Average } \\
\text { concentration } \\
(\mathrm{pCi} / \mathrm{L})\end{array}$ & $\begin{array}{r}\text { Percentage } \\
\text { of DCG }\end{array}$ \\
\hline${ }^{99} \mathrm{Tc}$ & $1.67 \times 10^{-2}$ & 100,000 & 24.9 & 0.03 \\
${ }^{234} \mathrm{U}$ & $2.35 \times 10^{-3}$ & 500 & 17.7 & 3.55 \\
${ }^{233} \mathrm{U}$ & $1.11 \times 10^{-4}$ & 600 & 0.17 & 0.03 \\
${ }^{236} \mathrm{U}$ & $2.85 \times 10^{-5}$ & 500 & 0.04 & $<0.01$ \\
${ }^{238} \mathrm{U}$ & $1.17 \times 10^{-3}$ & 600 & 2.55 & 0.42 \\
\hline
\end{tabular}

Table 2.2.14. 1989 ORGDP radiological effluent at K-1700

\begin{tabular}{llrcc}
\hline Radionuclide & $\begin{array}{c}\text { Emission } \\
\text { source }(\mathrm{Ci})\end{array}$ & $\begin{array}{c}\mathrm{DCG} \\
(\mathrm{pCi} / \mathrm{L})\end{array}$ & $\begin{array}{c}\text { Average } \\
\text { concentration } \\
(\mathrm{pCi} / \mathrm{L})\end{array}$ & $\begin{array}{c}\text { Percentage } \\
\text { of } \mathrm{DCG}\end{array}$ \\
\hline${ }^{237} \mathrm{~Np}$ & $1.02 \times 10^{-4}$ & 30 & 0.05 & 0.15 \\
${ }^{239} \mathrm{Pu}$ & $1.58 \times 10^{-4}$ & 30 & 0.07 & 0.24 \\
${ }^{99} \mathrm{Tc}$ & $2.13 \times 10^{-1}$ & 100,000 & 96.8 & 0.10 \\
${ }^{137} \mathrm{Cs}$ & 0 & 3,000 & 0 & $<0.01$ \\
${ }^{234} \mathrm{U}$ & $2.99 \times 10^{-2}$ & 500 & 32.8 & 6.5 \\
${ }^{235} \mathrm{U}$ & $1.76 \times 10^{-3}$ & 600 & 0.80 & 0.13 \\
${ }^{236} \mathrm{U}$ & $4.45 \times 10^{-4}$ & 500 & 0.20 & 0.04 \\
${ }^{238} \mathrm{U}$ & $1.38 \times 10^{-2}$ & 600 & 6.27 & 1.05
\end{tabular}


Table 2.2.15. 1989 ORGDP radiological effluent at K-1007-B

\begin{tabular}{lcccr}
\hline Radionuclide & $\begin{array}{c}\text { Emission } \\
\text { source }(\mathrm{Ci})\end{array}$ & $\begin{array}{c}\text { DCG } \\
(\mathrm{pCi} / \mathrm{L})\end{array}$ & $\begin{array}{c}\text { Average } \\
\text { concentration } \\
(\mathrm{pCi} / \mathrm{L})\end{array}$ & $\begin{array}{c}\text { Percentage } \\
\text { of DCG }\end{array}$ \\
\hline${ }^{237} \mathrm{~Np}$ & $9.12 \times 10^{-6}$ & 30 & $2.67 \times 10^{-3}$ & 0.01 \\
${ }^{239} \mathrm{Pu}$ & $1.32 \times 10^{-4}$ & 30 & $3.87 \times 10^{-2}$ & 0.13 \\
${ }^{99} \mathrm{Tc}$ & $-3.62 \times 10^{-1 a}$ & 100,000 & $-106^{a}$ & $<0.01$ \\
${ }^{137} \mathrm{Cs}$ & 0 & 3,000 & 0 & $<0.01$ \\
${ }^{234} \mathrm{U}$ & $4.54 \times 10^{-3}$ & 500 & 7.95 & 1.59 \\
${ }^{235} \mathrm{U}$ & $1.94 \times 10^{-4}$ & 600 & 0.06 & 0.01 \\
${ }^{236} \mathrm{U}$ & $5.71 \times 10^{-5}$ & 500 & 0.02 & $<0.01$ \\
${ }^{238} \mathrm{U}$ & $2.20 \times 10^{-3}$ & 600 & 0.65 & 0.11 \\
\hline
\end{tabular}

${ }^{a}$ Because of the intrinsic uncertainties associated with making radiation measurements, it is possible to subtract a background value from a sample result and obtain a negative number. Statistical summaries previously used detection limits to represent sample results even when samples were less than detection limits, which resulted in high biases. To remove these biases and to enable statistical summaries to be equally representative of all component values, recent changes in reporting methods include accepting all results at face value.

Table 2.2.16. 1989 ORGDP radiological effluent at K-901A

\begin{tabular}{clrrr}
\hline Radionuclide & \multicolumn{1}{c}{$\begin{array}{c}\text { Emission } \\
\text { source }(\mathrm{Ci})\end{array}$} & $\begin{array}{c}\text { DCG } \\
(\mathrm{pCi} / \mathrm{L})\end{array}$ & $\begin{array}{c}\text { Average } \\
\text { concentration } \\
(\mathrm{pCi} / \mathrm{L})\end{array}$ & $\begin{array}{c}\text { Percentage } \\
\text { of DCG }\end{array}$ \\
\hline${ }^{237} \mathrm{~Np}$ & $3.43 \times 10^{-5}$ & 30 & 0.04 & 0.13 \\
${ }^{239} \mathrm{Pu}$ & $1.07 \times 10^{-4}$ & 30 & 0.12 & 0.40 \\
${ }^{99} \mathrm{Tc}$ & $-5.93 \times 10^{-2 a}$ & 100,000 & $-66.8^{a}$ & $<0.01$ \\
${ }^{137} \mathrm{Cs}$ & 0 & 3,000 & 0 & $<0.01$ \\
${ }^{234} \mathrm{U}$ & $2.06 \times 10^{-3}$ & 500 & 0.40 & 0.08 \\
${ }^{235} \mathrm{U}$ & $9.70 \times 10^{-5}$ & 600 & 0.11 & 0.02 \\
${ }^{236} \mathrm{U}$ & $2.54 \times 10^{-5}$ & 500 & 0.03 & 0.01 \\
${ }^{238} \mathrm{U}$ & $1.05 \times 10^{-3}$ & 600 & 1.18 & 0.20 \\
\hline
\end{tabular}

${ }^{a}$ Because of the intrinsic uncertaintics associated with making radiation measurements, it is possible to subtract a background value from a sample result and obtain a negative number. Statistical summaries previously used detection limits to represent sample results even when samples were less than detection limits, which resulted in high biases. To remove these biases and to enable statistical summaries to be equally representative of all component values, recent changes in reporting methods include accepting all results at face value. 
Table 2.2.17. 1989 ORGDP radiological effluent at K-1407-J ${ }^{a}$

\begin{tabular}{lcccc}
\hline Radionuclide & $\begin{array}{c}\text { Emission source } \\
(\mathrm{Ci})\end{array}$ & $\begin{array}{c}\mathrm{DCG} \\
(\mathrm{pCi} / \mathrm{L})\end{array}$ & $\begin{array}{c}\text { Average } \\
\text { concentration } \\
(\mathrm{pCi} / \mathrm{L})\end{array}$ & $\begin{array}{c}\text { Percentage } \\
\text { of DCG }\end{array}$ \\
\hline${ }^{237} \mathrm{~Np}$ & $6.71 \times 10^{-6}$ & 30 & 0.64 & 2 \\
${ }^{239} \mathrm{Pu}$ & $3.42 \times 10^{-6}$ & 30 & 0.31 & 1 \\
${ }^{99} \mathrm{Tc}$ & $6.68 \times 10^{-3}$ & 100,000 & 526 & 0.53 \\
${ }^{137} \mathrm{Cs}$ & 0 & 3,000 & 0 & $<0.01$ \\
${ }^{234} \mathrm{U}$ & $5.91 \times 10^{-3}$ & 500 & 465 & 93 \\
${ }^{235} \mathrm{U}$ & $2.51 \times 10^{-4}$ & 600 & 19.8 & 3.3 \\
${ }^{236} \mathrm{U}$ & $5.04 \times 10^{-5}$ & 500 & 3.97 & 0.79 \\
${ }^{238} \mathrm{U}$ & $2.93 \times 10^{-3}$ & 600 & 231 & 38.5 \\
\hline
\end{tabular}

${ }^{\circ}$ Data are for September through December 1989 only. Beginning in September, the K-1407-J effluent was discharged through the CNF pipeline to Poplar Creek. Prior to that time discharge was to Mitchell Branch, and subsequently through K-1700, which is located downstream. During previous years the off-site discharge point was K-1700. 
Table 2.2.18. CY 1989 NPDES Permit Number TN 002968

\begin{tabular}{|c|c|c|c|c|c|}
\hline \multicolumn{6}{|c|}{ Discharge Point $=301^{a}$} \\
\hline \multirow{2}{*}{ Parameter } & \multirow{2}{*}{$\begin{array}{c}\text { No. } \\
\text { samples }\end{array}$} & \multicolumn{3}{|c|}{$\begin{array}{l}\text { Concentration } \\
(\mathrm{mg} / \mathrm{L})\end{array}$} & \multirow{2}{*}{$\begin{array}{l}\text { Std. } \\
\text { error }\end{array}$} \\
\hline & & $\operatorname{Max}$ & Min & Av & \\
\hline Total suspended solids & 14 & 20 & $<5$ & $<6$ & 0.3 \\
\hline Mercury & 14 & 0.0002 & $<0.0002$ & $<0.0002$ & 0.0000 \\
\hline Lithium & 14 & 0.188 & 0,017 & 0.068 & 0.004 \\
\hline Zirconium & 14 & $<0.002$ & $<0.002$ & $<0.002$ & 0.000 \\
\hline Potassium & 14 & 3.0 & 0.7 & 1.1 & 0.04 \\
\hline Sodium & 14 & 0.71 & 0.45 & 0.59 & 0.01 \\
\hline pH, standard units & 15 & 8.3 & 6.9 & $\mathrm{NA}^{b}$ & 0.02 \\
\hline Arsenic & 14 & $<0.04$ & $<0,04$ & $<0.04$ & 0.00 \\
\hline Cadmium & 14 & $<0.006$ & $<0,003$ & $<0.003$ & 0.000 \\
\hline Chromium & 14 & 0.013 & $<0.006$ & $<0.006$ & 0.00014 \\
\hline Copper & 14 & 0.007 & $<0.002$ & $<0.002$ & 0.0001 \\
\hline Iron & 14 & 0.44 & 0.02 & 0.13 & 0.01 \\
\hline Nickel & 14 & 0.010 & $<0.007$ & $<0.008$ & 0.0001 \\
\hline Selenium & 14 & $<0.002$ & $<0,002$ & $<0.002$ & 0.000 \\
\hline Zinc & 14 & 0.101 & $<0.003$ & $<0.024$ & 0.002 \\
\hline Lead & 14 & $<0.02$ & $<0.02$ & $<0.02$ & 0.00 \\
\hline Temperature, ${ }^{\circ} \mathrm{C}$ & 14 & 27.3 & 8.0 & 16.8 & 0.5 \\
\hline Flow, $\mathrm{Mgd}^{c}$ & 9 & 3.2880 & 0.0030 & 0.4810 & 0.1250 \\
\hline
\end{tabular}

${ }^{a}$ Y-1 2 Plant, Kerr Hollow Quarry.

${ }^{b} \mathrm{NA}=-\cdots$ not applicable.

'Flow during operations and/or discharging. 
Tatle 2.2.19. CY 1989 NPDES Permit Number TN 002968

\begin{tabular}{|c|c|c|c|c|c|}
\hline \multicolumn{6}{|c|}{ Discharge Point $=302^{\circ}$} \\
\hline \multirow{2}{*}{ Parameter } & \multirow{2}{*}{$\begin{array}{l}\text { No. } \\
\text { samples }\end{array}$} & \multicolumn{3}{|c|}{$\begin{array}{l}\text { Concentration } \\
(\mathrm{mg} / \mathrm{L})\end{array}$} & \multirow{2}{*}{$\begin{array}{l}\text { Std. } \\
\text { error }\end{array}$} \\
\hline & & $\operatorname{Max}$ & Min & Av & \\
\hline Total suspended solids & 52 & 40 & $<5$ & $<6$ & 1 \\
\hline Chemical oxygen demand (COD) & 52 & 16 & 4 & $<6$ & 0.30 \\
\hline Sulfate & 52 & 91 & 32 & 51 & 3 \\
\hline Oil and grease & 52 & 3 & $<2$ & $<2$ & 0.03 \\
\hline Settleable solids & 52 & 0.1 & $<0.1$ & $<0.1$ & 0.0 \\
\hline Selenium & 52 & 0.0180 & 0.0002 & $<0.0055$ & 0.0006 \\
\hline Mercury & 52 & 0.0004 & $<0.0002$ & $<0.0002$ & 0.000004 \\
\hline Arsenic & 52 & 0.18 & $<0.04$ & $<0.07$ & 0.01 \\
\hline Cadmium & 52 & 0.006 & $<0.003$ & $<0.003$ & 0.000 \\
\hline Chromium & 52 & 0.010 & $<0.006$ & $<0.006$ & 0.0002 \\
\hline Copper & 52 & 0.004 & $<0.002$ & $<0.002$ & 0.0001 \\
\hline Iron & 52 & 2.18 & 0.02 & 0.14 & 0.04 \\
\hline Nickel & 52 & 0.012 & $<0.007$ & $<0.008$ & 0.0002 \\
\hline Zinc & 52 & 0.059 & $<0.001$ & $<0.008$ & 0.001 \\
\hline Lead & 52 & 0.05 & $<0.02$ & $<0.02$ & 0.001 \\
\hline $\mathrm{pH}$, standard units & 52 & 9.1 & 6.9 & $\mathrm{NA}^{b}$ & 0.1 \\
\hline Temperature, ${ }^{\circ} \mathrm{C}$ & 52 & 29.6 & 7.5 & 17.5 & 1.0 \\
\hline Turbidity, NTU & 52 & 32.00 & 0.63 & 3.48 & 0.75 \\
\hline Flow, Mgdc & 365 & 9.02 & 0.14 & 1.07 & 0.06 \\
\hline
\end{tabular}

${ }^{\sigma} Y-12$ Plant, Rogers Quarry.

N.A $=$ not applicable.

Flow during uperations and/or discharging. 
Table 2.2.20. CY 1989 NPDES Permit Number TN 002968

Discharge Point $=303^{a}$

\begin{tabular}{|c|c|c|c|c|c|}
\hline \multirow{2}{*}{ Parameter } & \multirow{2}{*}{$\begin{array}{c}\text { No. } \\
\text { samples }\end{array}$} & \multicolumn{3}{|c|}{$\begin{array}{l}\text { Concentration } \\
(\mathrm{mg} / \mathrm{L})\end{array}$} & \multirow{2}{*}{$\begin{array}{l}\text { Std. } \\
\text { error }\end{array}$} \\
\hline & & $\operatorname{Max}$ & Min & $\mathrm{Av}$ & \\
\hline Ammonia, as $\mathrm{N}$ & 49 & 6.3 & $<0.2$ & $<1.8$ & 0.2 \\
\hline Chromium & 50 & 0.145 & $<0.006$ & $<0.009$ & 0.003 \\
\hline Fluoride & 49 & 0.74 & 0.28 & 0.54 & 0.02 \\
\hline Lithium & 50 & 0.039 & 0.006 & 0.014 & 0.001 \\
\hline Surfactants, as MBAS & 49 & 5.00 & $<0.05$ & $<0.25$ & 0.14 \\
\hline Dissolved solids & 49 & 610 & 100 & 373 & 20 \\
\hline Nickel & 50 & 0.131 & $<0.007$ & $<0.012$ & 0.002 \\
\hline Beryllium & 50 & 0.0027 & $<0.0001$ & $<0.0002$ & 0.0001 \\
\hline Residual chlorine & 53 & 0.16 & $<0.10$ & $<0.10$ & 0.00 \\
\hline Perchloroethylene & 22 & 0.010 & $<0.010$ & $<0.010$ & 0.000 \\
\hline Settleable solids & 53 & 0.1 & $<0.1$ & $<0.1$ & 0.0 \\
\hline Dissolved oxygen & 54 & 19.1 & 3.3 & 9.5 & 0.5 \\
\hline Oil and grease & 53 & 4 & $<2$ & $<2$ & 0 \\
\hline Suspended solids, total & 49 & 650 & $<5$ & $<34$ & 13 \\
\hline Zinc & 50 & 1.240 & 0.007 & 0.059 & 0.024 \\
\hline Nitrogen & 49 & 5.8 & 1.1 & 3.2 & 0.2 \\
\hline Cadmium & 50 & 0.01400 & $<0.00005$ & $<0.00225$ & 0.00030 \\
\hline Lead & 50 & 0.1200 & $<0.0005$ & $<0.0147$ & 0.0025 \\
\hline Copper & 50 & 0.360 & $<0.002$ & $<0.016$ & 0.007 \\
\hline Mercury & 49 & 0.1200 & $<0.0002$ & $<0.0038$ & 0.0024 \\
\hline Temperature, ${ }^{\circ} \mathrm{C}$ & 57 & 14.0 & 4.5 & 7.9 & 0.3 \\
\hline Biological oxygen demand & 0 & & & & \\
\hline Chemical oxygen demand & 0 & & & & \\
\hline $\mathrm{pH}$, standard units & 58 & 9.4 & 6.6 & $\mathrm{NA}^{b}$ & 0.1 \\
\hline Flow, Mgdc & 59 & 0.6429 & 0.0015 & 0.2272 & 0.0341 \\
\hline
\end{tabular}

${ }^{a}$ Y. 12 Plant, New Hope Pond.

${ }^{b} \mathrm{NA}=$ not applicable.

'Flow during operations and/or discharging. 
Table 2.2.21. CY 1989 NPDES Permit Number TN 002968

\begin{tabular}{|c|c|c|c|c|c|}
\hline \multicolumn{6}{|c|}{ Discharge Point $=304^{a}$} \\
\hline \multirow{2}{*}{ Parameter } & \multirow{2}{*}{$\begin{array}{c}\text { No. } \\
\text { samples }\end{array}$} & \multicolumn{3}{|c|}{$\begin{array}{l}\text { Concentration } \\
(\mathrm{mg} / \mathrm{L})\end{array}$} & \multirow{2}{*}{$\begin{array}{l}\text { Std. } \\
\text { error }\end{array}$} \\
\hline & & $\operatorname{Max}$ & Min & Av & \\
\hline Oil and grease & 52 & 5 & $<2$ & $<2$ & 0.08 \\
\hline Biological oxygen demand & 49 & 6 & $<5$ & $<5$ & 0.02 \\
\hline Chemical oxygen demand & 52 & 53 & $<5$ & $<11$ & 1 \\
\hline Dissolved solids & 52 & 300 & 94 & 196 & 0 \\
\hline Total suspended solids & 52 & 430 & $<5$ & $<50$ & 12 \\
\hline Nitrates, as $\mathbf{N}$ & 52 & 10.0 & 0.7 & 4.0 & 0.3 \\
\hline Conductivity, $\mu \mathrm{mhos} / \mathrm{cm}$ & 53 & 570.0 & $<0.5$ & $<334.3$ & 13.6 \\
\hline Dissolved oxygen & 76 & 14.5 & 7.1 & 9.0 & 0.2 \\
\hline Turbidity, NTU & 52 & 150.00 & 2.00 & 25.85 & 5.01 \\
\hline $\mathrm{pH}$, standard units & 82 & 8.3 & 6.8 & $\mathrm{NA}^{b}$ & 0.04 \\
\hline Flow, $\mathrm{Mgd}^{c}$ & 353 & 25.44 & 0.16 & 4.09 & 0.24 \\
\hline
\end{tabular}

${ }^{a}$ Y-12 Plant, Bear Creek.

${ }^{b} \mathrm{NA}=$ not applicable.

'Flow during operations and/or discharging.

Table 2.2.22. CY 1989 NPDES Permit Number TN 002968

Discharge Point $=305^{a}$

\begin{tabular}{lccccc}
\hline \multicolumn{1}{c}{ Parameter } & $\begin{array}{c}\text { No. } \\
\text { samples }\end{array}$ & & $\begin{array}{c}\text { Concentration } \\
(\mathrm{mg} / \mathrm{L})\end{array}$ & $\begin{array}{c}\text { Std. } \\
\text { error }\end{array}$ \\
\cline { 3 - 6 } & & Max & Min & Av & \\
\hline Oil and grease & 257 & 54 & $<2$ & $<2$ & 0.2 \\
Total suspended solids & 257 & 140 & $<1$ & $<11$ & 1 \\
Mercury & 257 & 0.0027 & $<0.0002$ & $<0.0002$ & 0.00001 \\
p!H, standard units & 252 & 9.1 & 6.6 & NA $^{b}$ & 0.02 \\
Beryllium & 257 & 0.0003 & $<0.0001$ & $<0.0001$ & 0.00 002 \\
Cadmium & 257 & $<0.006$ & $<0.003$ & $<0.004$ & 0.0001 \\
Lead & 257 & 0.29 & $<0.02$ & $<0.02$ & 0.001 \\
Silver & 257 & $<0.004$ & $<0.004$ & $<0.004$ & 0.000 \\
Flow, gal/dc & 235 & 152,381 & 0 & 54,878 & 2,381 \\
\hline
\end{tabular}

${ }^{a} \mathrm{Y}-12$ Plant, Oil Pond No. 1.

${ }^{b} \mathrm{NA}=$ not applicable

'Flow during operations and/or discharging. 
Table 2.2.23. CY 1989 NPDES Permit Number TN 002968

\begin{tabular}{lccccc}
\multicolumn{5}{c}{ Discharge Point $=306^{a}$} \\
\hline Parameter & $\begin{array}{c}\text { No. } \\
\text { samples }\end{array}$ & \multicolumn{3}{c}{$\begin{array}{c}\text { Concentration } \\
(\mathrm{mg} / \mathrm{L})\end{array}$} & $\begin{array}{c}\text { Std. } \\
\text { error }\end{array}$ \\
\cline { 3 - 6 } & & Max & Min & Av & \\
\hline Oil and grease & 90 & 70 & $<2$ & $<3$ & 1 \\
Total suspended solids & 90 & 61 & $<5$ & $<7$ & 1 \\
Mercury & 90 & 0.0002 & $<0.0002$ & $<0.0002$ & 0.0000 \\
pH (standard units) & 90 & 10.0 & 5.7 & $b$ & 0.1 \\
Cadmium & 90 & 0.049 & $<0.003$ & $<0.004$ & 0.001 \\
Nickel & 90 & 0.080 & $<0.007$ & $<0.008$ & 0.001 \\
Lead & 50 & 0.02 & $<0.02$ & $<0.02$ & 0.00 \\
Silver & 90 & 0.004 & $<0.004$ & $<0.004$ & 0.000 \\
Flow, gal/dc & 88 & 190,476 & 0 & 5,599 & 2,199 \\
\hline
\end{tabular}

${ }^{a} \mathrm{Y}-12$ Plant, Oil Pond No. 2.

${ }^{b}$ Not applicable.

${ }^{c}$ Flow during operations and/or discharging.

Table 2.2.24. CY 1989 NPDES Permit Number TN 002968

\begin{tabular}{|c|c|c|c|c|c|}
\hline \multicolumn{6}{|c|}{ Discharge Point $=307^{a}$} \\
\hline \multirow{2}{*}{ Parameter } & \multirow{2}{*}{$\begin{array}{l}\text { No. } \\
\text { samples }\end{array}$} & \multicolumn{3}{|c|}{$\begin{array}{l}\text { Concentration } \\
(\mathrm{mg} / \mathrm{L})\end{array}$} & \multirow{2}{*}{$\begin{array}{l}\text { Std. } \\
\text { error }\end{array}$} \\
\hline & & $\operatorname{Max}$ & Min & $A v$ & \\
\hline Oil and grease & 4 & 3 & $<2$ & $<2.3$ & 0.25 \\
\hline Total suspended solids & 4 & 103 & 28 & 52.8 & 17 \\
\hline $\mathrm{pH}$, standard units & 4 & 8.4 & 7.0 & $\mathrm{NA}^{b}$ & 0.3 \\
\hline Temperature, ${ }^{\circ} \mathrm{C}$ & 4 & 26.9 & 4.9 & 17.5 & 4.9 \\
\hline Flow, gal $/ \mathrm{d}^{c}$ & 1 & 7610 & 7610 & 7610 & 0.0 \\
\hline
\end{tabular}

"Y-12 Plant, West Borrow Area.

${ }^{b} \mathrm{NA}=$ not applicable.

'Flow during operations and/or discharging. 
Table 2.2.25. CY 1989 NPDES Permit Number TN 002968

\begin{tabular}{lccccc}
\multicolumn{7}{c}{ Discharge Point $=308^{a}$} \\
\hline \multicolumn{1}{c}{ Parameter } & $\begin{array}{c}\text { No. } \\
\text { samples }\end{array}$ & \multicolumn{3}{c}{$\begin{array}{c}\text { Concentration } \\
(\mathrm{mg} / \mathrm{L})\end{array}$} & $\begin{array}{c}\text { Std. } \\
\text { error }\end{array}$ \\
& & Max & Min & Av & \\
\hline Oil and grease & 4 & 3 & $<2$ & $<2.3$ & 0.25 \\
Total suspended solids & 4 & 84 & 7 & 39.0 & 16.6 \\
pH, standard units & 4 & 9.5 & 7.5 & $\mathrm{NA}^{b}$ & 0.4 \\
Temperature, ${ }^{\circ} \mathrm{C}$ & 4 & 26.9 & 4.5 & 16.9 & 4.9 \\
Flow, gal/d & 1 & 57,600 & 57,600 & 57,600 & 0.0 \\
\hline
\end{tabular}

${ }^{a}$ Y -12 Plant, East Borrow Area.

${ }^{b} \mathrm{NA}=$ not applicable.

${ }^{c}$ Flow during operations and/or discharging.

Table 2.2.26. CY 1989 NPDES Permit Number TN 002968

Discharge Point $=501^{\circ}$

\begin{tabular}{|c|c|c|c|c|c|}
\hline \multirow{2}{*}{ Parameter } & \multirow{2}{*}{$\begin{array}{l}\text { No. } \\
\text { samples }\end{array}$} & \multicolumn{3}{|c|}{$\begin{array}{l}\text { Concentration } \\
(\mathrm{mg} / \mathrm{L})\end{array}$} & \multirow{2}{*}{$\begin{array}{l}\text { Std. } \\
\text { error }\end{array}$} \\
\hline & & $\operatorname{Max}$ & Min & Av & \\
\hline Oil and grease & 45 & 5 & $<2$ & $<2$ & 0.07 \\
\hline Cyanide & 45 & 0.140 & $<0.002$ & $<0.011$ & 0.003 \\
\hline Copper & 45 & 0.025 & $<0.002$ & $<0.010$ & 0.001 \\
\hline Chromium & 45 & 0.024 & $<0.006$ & $<0.007$ & 0.001 \\
\hline Lead & 45 & 0.05 & $<0.02$ & $<0.02$ & 0.001 \\
\hline Nickel & 45 & 0.871 & $<0.010$ & $<0.297$ & 0.033 \\
\hline Temperature, ${ }^{\circ} \mathrm{C}$ & 45 & 29.0 & 17.2 & 23.2 & 0.5 \\
\hline Cadmium & 45 & 0.077 & $<0.003$ & $<0.006$ & 0.002 \\
\hline Zinc & 45 & 3.200 & 0.009 & 0.424 & 0.102 \\
\hline Total toxic organics & 44 & 0.270 & $<0.010$ & $<0.029$ & 0.010 \\
\hline Total suspended solids & 45 & 20 & $<5$ & $<8$ & 1 \\
\hline Silver & 45 & 0.008 & $<0.004$ & $<0.004$ & 0.0001 \\
\hline $\mathrm{pH}$, standard units & 46 & 8.8 & 6.2 & $\mathrm{NA}^{b}$ & 0.1 \\
\hline Color, NTU & 45 & 50.0 & $<5.0$ & $<24.4$ & 2.3 \\
\hline Sodium & 45 & 932.0 & 11.1 & 262.2 & 28.9 \\
\hline Nitrates, as $\mathrm{N}$ & 45 & 20.0 & $<0.1$ & $<1.0$ & 0.5 \\
\hline Surfactants, as MBAS & 45 & 0.24 & $<0.05$ & $<0.06$ & 0.004 \\
\hline Beryllium & 45 & 0.0003 & $<0.0001$ & $<0.0001$ & 0.000004 \\
\hline Phosphorus & 45 & 124.00 & 0.39 & 6.83 & 2.98 \\
\hline Chlorides & 45 & 430 & 48 & 194 & 12 \\
\hline Phenols & 45 & 0.360 & $<0.001$ & $<0.058$ & 0.012 \\
\hline Sulfates & 45 & 2900 & 1400 & 1959 & 66 \\
\hline Fluorides & 45 & 1.9 & 0.4 & 1.0 & 0.05 \\
\hline Aluminum & 45 & 0.45 & $<0.01$ & $<0.15$ & 0.02 \\
\hline Iron & 45 & 10.10 & 0.09 & 2.50 & 0.39 \\
\hline Mercury & 45 & 0.0002 & $<0.0002$ & $<0.0002$ & 0.0000 \\
\hline Flow, $\mathrm{gal} / \mathrm{d}^{c}$ & 45 & 15878 & 3782 & 11596 & 396 \\
\hline
\end{tabular}

${ }^{a}$ Y-12 Plani, Central Pollution Control Facility.

${ }^{b} \mathrm{NA}=$ not applicable.

${ }^{c}$ Flow during operations and/or discharging. 
Table 2.2.27. CY 1989 NPDES Permit Number TN 002968

Discharge Point $=502^{a}$

\begin{tabular}{|c|c|c|c|c|c|}
\hline \multirow{2}{*}{ Parameter } & \multirow{2}{*}{$\begin{array}{c}\text { No. } \\
\text { samples }\end{array}$} & \multicolumn{3}{|c|}{$\begin{array}{l}\text { Concentration } \\
(\mathrm{mg} / \mathrm{L})\end{array}$} & \multirow{2}{*}{$\begin{array}{l}\text { Std. } \\
\text { error }\end{array}$} \\
\hline & & $\operatorname{Max}$ & Min & Av & \\
\hline Silver & 64 & 0.011 & $<0.004$ & $<0.004$ & 0.0002 \\
\hline Cadmium & 64 & $<0.010$ & $<0.003$ & $<0.003$ & 0.0001 \\
\hline Cyanide & 66 & 1,900 & $<0.002$ & $<0.109$ & 0.042 \\
\hline Chromium & 64 & $<0.006$ & $<0.006$ & 0.006 & 0.000 \\
\hline Copper & 64 & 1.070 & 0.008 & 0.135 & 0.024 \\
\hline Nickel & 64 & 4.900 & 0.182 & 1.641 & 0.155 \\
\hline Lead & 64 & $<0.020$ & $<0.605$ & $<0.020$ & 0.0002 \\
\hline Zinc & 64 & 1.39 & 0.07 & 0.40 & 0.04 \\
\hline Total toxic organics & 15 & 0.520 & $<0.010$ & $<0.044$ & 0.034 \\
\hline Total suspended solids & 64 & 50 & $<5$ & $<10$ & 1 \\
\hline Oil and grease & 65 & 4 & $<2$ & $<2$ & 0.04 \\
\hline Temperature, ${ }^{\circ} \mathrm{C}$ & 64 & 22.0 & 10.1 & 15.9 & 0.3 \\
\hline pH, standard units & 64 & 8.7 & 7.3 & $b$ & 0.04 \\
\hline Arsenic & 64 & 0.09 & $<0.04$ & $<0.04$ & 0.001 \\
\hline Aluminum & 64 & 1.71 & 0.19 & 0.59 & 0.03 \\
\hline Mercury & 64 & 0.0004 & $<0.0002$ & $<0.0002$ & 0.000004 \\
\hline Beryllium & 64 & 0.0017 & $<0.0001$ & $<0.0003$ & 0.00004 \\
\hline Sulfate & 64 & 28000 & 2200 & 20903 & 500 \\
\hline Barium & 64 & 0.0659 & 0.0102 & 0.0327 & 0.0019 \\
\hline Nitrate, as $\mathrm{N}$ & 64 & $<0.1$ & $<0.1$ & $<0.1$ & 0.0 \\
\hline Fluoride & 64 & 110.0 & 1.3 & 63.8 & 2.7 \\
\hline Calcium & 64 & 28.30 & 3.16 & 15.66 & 0.76 \\
\hline Residual chlorine & 64 & 19.6 & $<0.1$ & $<0.4$ & 0.3 \\
\hline Phosphorus & 64 & $<0.06$ & $<0.06$ & $<0.06$ & 0.00 \\
\hline Iron & 64 & 1.24 & 0.10 & 0.38 & 0.03 \\
\hline Cobalt & 64 & 0.127 & 0.007 & 0.040 & 0.004 \\
\hline Magnesium & 64 & 70.90 & 7.23 & 29.56 & 2.11 \\
\hline Manganese & 64 & 0.24 & 0.01 & 0.08 & 0.01 \\
\hline Molybdenum & 64 & 1.240 & 0.164 & 0.506 & 0.032 \\
\hline Sodium & 64 & 12000 & 3050 & 8297 & 325 \\
\hline Chloride & 64 & 1300 & 790 & 1100 & 18 \\
\hline Potassium & 64 & 434.0 & 87.2 & 215.8 & 11.7 \\
\hline Flow, gal $/ \mathrm{d}^{c}$ & 77 & 28001 & 0 & 14720 & 1039 \\
\hline
\end{tabular}

${ }^{a}$ Y.12 Plant, West end treatment facility (WETF).

${ }^{b}$ Not applicable.

cFlow during operations and/or discharging. 
Table 2.2.28. CY 1989 NPDES Permit Number TN 002968

\begin{tabular}{|c|c|c|c|c|c|}
\hline \multicolumn{6}{|c|}{ Discharge Point $=503^{a}$} \\
\hline \multirow{2}{*}{ Parameter } & \multirow{2}{*}{$\begin{array}{c}\text { No. } \\
\text { samples }\end{array}$} & \multicolumn{3}{|c|}{$\begin{array}{l}\text { Concentration } \\
(\mathrm{mg} / \mathrm{L})\end{array}$} & \multirow{2}{*}{$\begin{array}{l}\text { Std. } \\
\text { error }\end{array}$} \\
\hline & & $\operatorname{Max}$ & Min & Av & \\
\hline Oil and grease & 150 & 16 & 1 & $<2$ & 0.12 \\
\hline Phenols & 149 & 0.033 & $<0.001$ & $<0.003$ & 0.0004 \\
\hline Mercury & 152 & 0.0176 & $<0.0002$ & $<0.0004$ & 0.0001 \\
\hline Selenium & 151 & 0.0180 & $<0.0004$ & $<0.0024$ & 0.0002 \\
\hline Chloride & 152 & 2500 & 18 & 298 & 25 \\
\hline Fluoride & 152 & 18.0 & 1.0 & 2.6 & 0.1 \\
\hline Total suspended solids & 152 & 28 & $<5$ & $<6$ & 0.2 \\
\hline Sulfate & 152 & 2800 & 56 & 1474 & 41 \\
\hline Sulfide & 152 & 3.6 & $<0.1$ & $<0.9$ & 0.03 \\
\hline Temperature, ${ }^{\circ} \mathrm{C}$ & 164 & 30.5 & 7.1 & 24.8 & 0.3 \\
\hline $\mathrm{pH}$, standard units & 166 & 9.2 & 6.2 & $b$ & 0.05 \\
\hline Aluminium & 152 & 3.41 & $<0.01$ & $<0.29$ & 0.03 \\
\hline Arsenic & 152 & 0.19 & $<0.04$ & $<0.04$ & 0.001 \\
\hline Barium & 152 & 0.2120 & 0.0167 & 0.0929 & 0.0033 \\
\hline Beryllium & 152 & 0.0072 & $<0.0001$ & $<0.0002$ & 0.00005 \\
\hline Boron & 152 & 8.140 & $<0.007$ & $<0.186$ & 0.070 \\
\hline Cadmium & 152 & 0.064 & $<0.003$ & $<0.004$ & 0.0004 \\
\hline Calcium & 152 & 1090.0 & 73.4 & 549.1 & 16.8 \\
\hline Cerium & 152 & 0.06 & $<0.02$ & $<0.02$ & 0.0003 \\
\hline Chromium & 152 & 0.075 & $<0.006$ & $<0.007$ & 0.0005 \\
\hline Cobalt & 152 & 0.079 & $<0.002$ & $<0.003$ & 0.001 \\
\hline Copper & 152 & 0.112 & $<0.002$ & $<0.004$ & 0.001 \\
\hline Gallium & 127 & $<0.01$ & $<0.01$ & $<0.01$ & 0.00 \\
\hline Iron & 152 & 2.33 & $<0.02$ & $<0.33$ & 0.02 \\
\hline Lanthanum & 152 & $<0.003$ & $<0.003$ & $<0.003$ & 0.000 \\
\hline Lead & 152 & 0.08 & $<0.02$ & $<0.02$ & 0.0004 \\
\hline Lithium & 152 & 0.279 & $<0.001$ & $<0.056$ & 0.003 \\
\hline Magnesium & 152 & 47.9 & 0.1 & 4.1 & 0.5 \\
\hline Manganese & 152 & 0.938 & $<0.001$ & $<0.010$ & 0.006 \\
\hline Molybdenum & 152 & 0.079 & $<0.006$ & $<0.007$ & 0.001 \\
\hline Nickel & 152 & 0.075 & $<0.007$ & $<0.008$ & 0.0005 \\
\hline Niobium & 152 & $<0.01$ & $<0.01$ & $<0.01$ & 0.00 \\
\hline Phosphorus & 152 & 2.30 & $<0.06$ & $<0.52$ & 0.03 \\
\hline Potassium & 152 & 22.6 & 1.6 & 7.3 & 0.3 \\
\hline Scandium & 151 & $<0.0004$ & $<0.0004$ & $<0.0004$ & 0.0000 \\
\hline Silver & 152 & 0.014 & $<0.004$ & $<0.004$ & 0.0001 \\
\hline Sodium & 152 & 3500.0 & 9.2 & 249.5 & 25.0 \\
\hline Strontium & 152 & 1.2 .10 & 0.120 & 0.560 & 0.017 \\
\hline Thorium & 152 & $<0.01$ & $<0.01$ & $<0.01$ & 0.00 \\
\hline Titanium & 151 & 0.071 & $<0.002$ & $<0.003$ & 0.0005 \\
\hline Vanadium & 152 & 0.150 & $<0.004$ & $<0.006$ & 0.001 \\
\hline Zinc & 152 & 0.109 & $<0.001$ & $<0.028$ & 0.001 \\
\hline Zirconium & 152 & 0.072 & $<0.002$ & $<0.002$ & 0.0005 \\
\hline Flow, gal/d $d^{c}$ & 362 & 507500 & 0 & 153701 & 4214 \\
\hline
\end{tabular}

${ }^{a}$ Y-12 Plant, Steam Plant Wastewater Treatment Facility.

${ }^{b}$ Not applicable.

'Flow during operations and/or discharging. 
Table 2.2.29. CY 1989 NPDES Permit Number TN 002968

\begin{tabular}{|c|c|c|c|c|c|}
\hline \multicolumn{6}{|c|}{ Discharge Point $=504^{a}$} \\
\hline \multirow{2}{*}{ Parameter } & \multirow{2}{*}{$\begin{array}{c}\text { No. } \\
\text { samples }\end{array}$} & \multicolumn{3}{|c|}{$\begin{array}{l}\text { Concentration } \\
(\mathrm{mg} / \mathrm{L})\end{array}$} & \multirow{2}{*}{$\begin{array}{l}\text { Std. } \\
\text { error }\end{array}$} \\
\hline & & $\operatorname{Max}$ & Min & Av & \\
\hline Silver & 22 & $<0.004$ & $<0.004$ & $<0.004$ & 0.000 \\
\hline Cadmium & 22 & $<0.0060$ & $<0.0005$ & $<0.0033$ & 0.0003 \\
\hline Cyanide & 22 & 0.044 & $<0.002$ & $<0.010$ & 0.003 \\
\hline Chromium & 22 & 0.018 & $<0.006$ & $<0.008$ & 0.001 \\
\hline Copper & 22 & 0.007 & $<0.002$ & $<0.003$ & 0.0004 \\
\hline Nickel & 22 & 1.400 & $<0.007$ & $<0.417$ & 0.070 \\
\hline Lead & 22 & $<0.020$ & $<0.001$ & $<0.019$ & 0.001 \\
\hline Zinc & 22 & 0.695 & 0.009 & 0.158 & 0.049 \\
\hline Total toxic organics & 22 & 0.290 & $<0.010$ & $<0.025$ & 0.013 \\
\hline Total suspended solids & 22 & 12 & $<5$ & $<5$ & 0.3 \\
\hline Oil and grease & 22 & 5 & $<2$ & $<2$ & 0.14 \\
\hline Temperature, ${ }^{\circ} \mathrm{C}$ & 23 & 28.9 & 15.4 & 22.7 & 0.8 \\
\hline $\mathrm{pH}$, standard units & 24 & 9.2 & 6.5 & $\mathbf{N A}^{b}$ & 0.1 \\
\hline Aluminum & 22 & 0.96 & 0.07 & 0.31 & 0.04 \\
\hline Mercury & 22 & 0.0003 & $<0.0002$ & $<0.0002$ & 0.000005 \\
\hline Beryllium & 22 & 0.0001 & $<0.0001$ & $<0.0001$ & 0.0000 \\
\hline Sulfate & 22 & 1000 & 40 & 199 & 43 \\
\hline Nitrate, as $\mathrm{N}$ & 22 & 26.0 & $<0.1$ & $<5.9$ & 1.4 \\
\hline Fluoride & 22 & 2.0 & 0.5 & 1.2 & 0.1 \\
\hline Phosphorus & 22 & 4.72 & 0.20 & 0.93 & 0.21 \\
\hline Iron & 22 & 7.67 & 0.11 & 3.42 & 0.47 \\
\hline Sodium & 22 & 466 & 14 & 67 & 20 \\
\hline Chloride & 22 & 62 & 12 & 29 & 3 \\
\hline Potassium & 22 & 39.2 & 1.0 & 12.9 & 2.2 \\
\hline Flow, gal/d $\mathrm{d}^{c}$ & 22 & 34390 & 8166 & 22011 & 1338 \\
\hline
\end{tabular}

${ }^{a}$ Y-12 Plant, Plating Rinsewater Treatment Facility (PRTF).

${ }^{b} \mathrm{NA}=$ not applisable.

'Flow during operations and/or discharging. 
Table 2.2.30. CY 1989 NPDES Permit Number TN 002968

\begin{tabular}{|c|c|c|c|c|c|}
\hline \multirow{2}{*}{ Parameter } & \multirow{2}{*}{$\begin{array}{c}\text { No. } \\
\text { samples }\end{array}$} & \multicolumn{3}{|c|}{$\begin{array}{c}\text { Concentration } \\
(\mathrm{mg} / \mathrm{L})\end{array}$} & \multirow{2}{*}{$\begin{array}{l}\text { Std. } \\
\text { error }\end{array}$} \\
\hline & & Max & Min & $\mathrm{Av}$ & \\
\hline Oil and grease & 31 & 3 & $<2$ & $<2$ & 0.04 \\
\hline Cyanide & 32 & 0.018 & $<0.002$ & $<0.006$ & 0.001 \\
\hline Chromium & 31 & 0.059 & $<0.006$ & $<0.008$ & 0.002 \\
\hline Copper & 31 & 0.185 & $<0.002$ & $<0.012$ & 0.006 \\
\hline Lead & 31 & $<0.1)_{2}$ & $<0.02$ & $<0.02$ & 0.00 \\
\hline Nickel & 31 & 1.250 & 0.110 & 0.450 & 0.044 \\
\hline Temperature, ${ }^{\circ} \mathrm{C}$ & 32 & 29.3 & 20.6 & 24.5 & 0.4 \\
\hline Cadmium & $3 !$ & 0.031 & $<0.003$ & $<0.005$ & 0.001 \\
\hline Zinc & 31 & 1.020 & 0.020 & 0.236 & 0.051 \\
\hline Total toxic organics & 31 & 0.100 & $<0.010$ & $<0.013$ & 0.003 \\
\hline Total suspended solids & 31 & 11 & $<5$ & $<6$ & 0.3 \\
\hline Silver & 31 & 0.129 & $<0.004$ & $<0.008$ & 0.004 \\
\hline $\mathrm{pH}$, standard units & 34 & 9.2 & 6.3 & $N^{b}$ & 0.1 \\
\hline Potassium & 31 & 299.0 & 10.5 & 89.3 & 11.7 \\
\hline Sodium & 31 & 6070.0 & 79.8 & 349.4 & 192.4 \\
\hline Nitrates & 30 & 12.0 & $<0.1$ & $<1.3$ & 0.5 \\
\hline Fluorides & 31 & 15.0 & 0.6 & 1.6 & 0.5 \\
\hline Aluminum & 31 & 0.88 & 0.08 & 0.30 & 0.04 \\
\hline Iron & 31 & 13.20 & 0.42 & 2.29 & 0.46 \\
\hline Mercury & 31 & 0.0005 & $<0.0002$ & $<0.0002$ & 0.00001 \\
\hline Beryllium & 31 & 0.0072 & $<0.0001$ & $<0.0003$ & 0.0002 \\
\hline Phosphorus & 31 & 190.00 & 0.33 & 7.32 & 6.09 \\
\hline Chlorides & 31 & 220 & 12 & 103 & 9 \\
\hline Sulfates & 31 & 2900 & 90 & 1044 & 111 \\
\hline Phenols & 32 & 0.410 & $<0.001$ & $<0.037$ & 0.014 \\
\hline Color & 31 & 75 & 1 & 29 & 4 \\
\hline Flow, gal/d $d^{c}$ & 30 & 37834 & 9002 & 24001 & 1509 \\
\hline
\end{tabular}

${ }^{a}$ Y-12 Plant, Central Pollution Control Facility/Plating Rinsewater Treatment Facility ${ }^{b} \mathrm{NA}=$ not applicable.

${ }^{c}$ Flow during operations and/or discharging.

Table 2.2.31. CY 1989 NPDES Permit Number TN 002968

Discharge Point $=506^{a}$

\begin{tabular}{|c|c|c|c|c|c|}
\hline \multirow{2}{*}{ Parameter } & \multirow{2}{*}{$\begin{array}{l}\text { No. } \\
\text { samples }\end{array}$} & \multicolumn{3}{|c|}{$\begin{array}{l}\text { Concentration } \\
(\mathrm{mg} / \mathrm{L})\end{array}$} & \multirow{2}{*}{$\begin{array}{l}\text { Std. } \\
\text { error }\end{array}$} \\
\hline & & Max & Min & $\mathrm{Av}$ & \\
\hline Oil and grease & 52 & 5 & $<2$ & $<2$ & 0 \\
\hline $\mathrm{pH}$, standard units & 53 & 9.2 & 7.0 & $b$ & 0.1 \\
\hline Temperature, ${ }^{\circ} \mathrm{C}$ & 52 & 39.8 & 20.3 & 27.1 & 0.4 \\
\hline Flow, gal/d $\mathrm{d}^{c}$ & 43 & 72,000 & 72,000 & 72,000 & 0 \\
\hline
\end{tabular}

${ }^{a} Y-12$ Plant, Building 9204-3 Sump Pump Oil Separator.

${ }^{b}$ Not applicable.

cFlow during operations and/or discharging. 
Table 2.2.32. CY 1989 NPDES Permit Number TN 002968

\begin{tabular}{|c|c|c|c|c|c|c|}
\hline \multicolumn{7}{|c|}{ Cooling towers ${ }^{a}$} \\
\hline \multirow{2}{*}{ Parameter } & \multirow{2}{*}{$\begin{array}{c}\text { No. } \\
\text { samples }\end{array}$} & \multirow{2}{*}{$\begin{array}{c}\text { Discharge } \\
\text { point }\end{array}$} & \multicolumn{3}{|c|}{$\begin{array}{l}\text { Concentration } \\
(\mathrm{mg} / \mathrm{L})\end{array}$} & \multirow{2}{*}{$\begin{array}{l}\text { Std. } \\
\text { error }\end{array}$} \\
\hline & & & $\operatorname{Max}$ & Min & Av & \\
\hline & & 602 & & & & \\
\hline Temperature, ${ }^{\circ} \mathrm{C}$ & 4 & & 30.0 & 23.0 & 26.0 & 1.6 \\
\hline pH, standard units & 4 & & 8.9 & 8.2 & $b$ & 0.2 \\
\hline Free chlorine & 4 & & 0.22 & $<0.10$ & $<0.15$ & 0.03 \\
\hline Chromium & 4 & & 0.008 & $<0.006$ & $<0.007$ & 0.0004 \\
\hline Copper & 4 & & 0.025 & 0.010 & 0.015 & 0.003 \\
\hline Zinc & 4 & & 0.139 & 0.083 & 0.114 & 0.012 \\
\hline \multirow[t]{2}{*}{ Flow, gal $/ \mathrm{d}^{c}$} & 4 & & 10800 & 8930 & 9828 & 403 \\
\hline & & 604 & & & & \\
\hline Temperature, ${ }^{\circ} \mathrm{C}$ & 4 & & 23.6 & 15.1 & 17.8 & 2.0 \\
\hline $\mathrm{pH}$, standard units & 4 & & 8.7 & 7.3 & $b$ & 0.3 \\
\hline Free chlorine & 4 & & 0.17 & $<0.10$ & $<0.12$ & 0.02 \\
\hline Chromium & 4 & & 0.013 & $<0.006$ & $<0.008$ & 0.002 \\
\hline Copper & 4 & & 0.010 & 0.005 & 0.007 & 0.001 \\
\hline Zinc & 4 & & 0.764 & 0.174 & 0.403 & 0.127 \\
\hline \multirow[t]{2}{*}{ Flow, gal/de } & 4 & & 10030 & 6640 & 8758 & 754 \\
\hline & & 610 & & & & \\
\hline Temperature, ${ }^{\circ} \mathrm{C}$ & 4 & & 30.0 & 16.4 & 25.3 & 3.1 \\
\hline $\mathrm{pH}$, standard units & 4 & & 8.8 & 8.3 & $b$ & 0.1 \\
\hline Free chlorine & 4 & & 0.43 & $<0.10$ & $<0.22$ & 0.08 \\
\hline Chromium & 4 & & 0.010 & 0.006 & 0.008 & 0.001 \\
\hline Copper & 4 & & 0.028 & 0.020 & 0.025 & 0.002 \\
\hline Zinc & 4 & & 0.239 & 0.117 & 0.150 & 0.030 \\
\hline \multirow[t]{2}{*}{ Flow, gal/dc } & 4 & & 28830 & 10320 & 18045 & 3896 \\
\hline & & 612 & & & & \\
\hline Temperature, ${ }^{\circ} \mathrm{C}$ & 2 & & 25.2 & 22.7 & 24.0 & 1.2 \\
\hline $\mathrm{pH}$, standard units & 2 & & 8.4 & 8.2 & $b$ & 0.1 \\
\hline Free chlorine & 2 & & 0.12 & $<0.10$ & $<0.11$ & 0.01 \\
\hline Chromium & 2 & & 0.006 & $<0.006$ & $<0.006$ & 0.000 \\
\hline Copper & 2 & & 0.021 & 0.014 & 0.018 & 0.003 \\
\hline Zinc & 2 & & 0.136 & 0.084 & 0.110 & 0.026 \\
\hline \multirow[t]{2}{*}{ Flow, gal $/ \mathrm{d}^{c}$} & 4 & & 89310 & 0 & 55970 & 20686 \\
\hline & & 613 & & & & \\
\hline Temperature, ${ }^{\circ} \mathrm{C}$ & 4 & & 27.1 & 21.4 & 24.6 & 1.4 \\
\hline $\mathrm{pH}$, standard units & 4 & & 8.8 & 8.3 & $b$ & 0.1 \\
\hline Free chlorine & 4 & & 0.40 & $<0.10$ & $<0.20$ & 0.07 \\
\hline Chromium & 4 & & 0.006 & $<0.006$ & $<0.006$ & 0.000 \\
\hline Copper & 4 & & 0.036 & 0.026 & 0.031 & 0.002 \\
\hline Zinc & 4 & & 0.148 & 0.072 & 0.109 & 0.019 \\
\hline Flow, gal/d ${ }^{c}$ & 4 & & 37880 & 14850 & 26770 & 4996 \\
\hline
\end{tabular}


Table 2.2.32 (continued)

\begin{tabular}{|c|c|c|c|c|c|c|}
\hline \multirow{2}{*}{ Parameter } & \multirow{2}{*}{$\begin{array}{c}\text { No, } \\
\text { samples }\end{array}$} & \multirow{2}{*}{$\begin{array}{c}\text { Discharge } \\
\text { point }\end{array}$} & \multicolumn{3}{|c|}{$\begin{array}{l}\text { Concentration } \\
(\mathrm{mg} / \mathrm{L})\end{array}$} & \multirow{2}{*}{$\begin{array}{l}\text { Std. } \\
\text { error }\end{array}$} \\
\hline & & & $\operatorname{Max}$ & Min & Av & \\
\hline & & 615 & & & & \\
\hline Temperature, ${ }^{\circ} \mathrm{C}$ & 4 & & 30.5 & 22.4 & 26.5 & 1.7 \\
\hline $\mathrm{pH}$, standard units & 4 & & 8.9 & 6.6 & $b$ & 0.6 \\
\hline Free chlorine & 4 & & 0.29 & $<0.10$ & $<0.16$ & 0.04 \\
\hline Chromiurn & 4 & & 0.060 & $<0.006$ & $<0.020$ & 0.013 \\
\hline Copper & 4 & & 0.103 & 0.010 & 0.035 & 0.023 \\
\hline Zinc & 4 & & 2.620 & 0.130 & 0.788 & 0.611 \\
\hline \multirow[t]{2}{*}{ Flow, gal $/ \mathrm{d}^{\mathrm{c}}$} & 4 & & 9 & 0 & 9 & 0 \\
\hline & & 617 & & & & \\
\hline Temperature, ${ }^{\circ} \mathrm{C}$ & 4 & & 25.6 & 17.0 & 21.3 & 2.3 \\
\hline $\mathrm{pH}$, standard units & 4 & & 8.8 & 8.2 & $b$ & 0.1 \\
\hline Free chlorine & 4 & & 0.32 & $<0.10$ & $<0.16$ & 0.05 \\
\hline Chromium & 4 & & 0.044 & $<0.006$ & $<0.017$ & 0.009 \\
\hline Copper & 4 & & 0.030 & 0.017 & 0.025 & 0.003 \\
\hline Zinc & 4 & & 0.175 & 0.080 & 0.115 & 0.021 \\
\hline \multirow[t]{2}{*}{ Flow, gal/d $\mathrm{d}^{\mathfrak{c}}$} & 4 & & 32750 & 12930 & 18980 & 4623 \\
\hline & & 618 & & & & \\
\hline Temperature, ${ }^{\circ} \mathrm{C}$ & 4 & & 29.9 & 23.2 & 25.5 & 1.5 \\
\hline $\mathrm{pH}$, standard units & 4 & & 8.8 & 8.2 & $b$ & 0.2 \\
\hline Free chlorine & 4 & & 0.34 & $<0.10$ & $<0.16$ & 0.06 \\
\hline Chromium & 4 & & 0.007 & $<0.006$ & $<0.007$ & 0.0003 \\
\hline Copper & 4 & & 0.124 & 0.026 & 0.067 & 0.022 \\
\hline Zinc & 4 & & 0.284 & 0.064 & 0.137 & 0.050 \\
\hline \multirow[t]{2}{*}{ Flow, gal/d $\mathrm{d}^{c}$} & 4 & & 22450 & 16110 & 19720 & 1361 \\
\hline & & 619 & & & & \\
\hline Temperature, ${ }^{\circ} \mathrm{C}$ & 4 & & 26.5 & 15.2 & 21.0 & 2.3 \\
\hline $\mathrm{pH}$, standard units & 4 & & 8.8 & 8.3 & $b$ & 0.1 \\
\hline Free chlorine & 4 & & 1.46 & $<0.10$ & $<0.55$ & 0.32 \\
\hline Chromium & 4 & & 0.033 & 0.012 & 0.018 & 0.005 \\
\hline Copper & 4 & & 0.033 & 0.011 & 0.018 & 0.005 \\
\hline Zinc & 4 & & 0.153 & 0.096 & 0.124 & 0.012 \\
\hline \multirow[t]{2}{*}{ Flow, gal/d $\mathrm{d}^{c}$} & 4 & & 15310 & 6230 & 9745 & 1946 \\
\hline & & 620 & & & & \\
\hline Temperature, ${ }^{\circ} \mathrm{C}$ & 4 & & 25.0 & 12.6 & 19.4 & 3.1 \\
\hline $\mathrm{pH}$, standard units & 4 & & 8.5 & 7.3 & $b$ & 0.3 \\
\hline Free chlorine & 4 & & 2.90 & 0.14 & 1.26 & 0.59 \\
\hline Chromium & 4 & & $<0.006$ & $<0.006$ & $<0.006$ & 0.000 \\
\hline Copper & 4 & & 0.020 & 0.005 & 0.012 & 0.003 \\
\hline Zinc & 4 & & 0.437 & 0.128 & 0.212 & 0.075 \\
\hline Flow, gal/dc & 4 & & 0 & 0 & 0 & 0 \\
\hline
\end{tabular}


Table 2.2.32 (continued)

\begin{tabular}{|c|c|c|c|c|c|c|}
\hline \multirow{2}{*}{ Parameter } & \multirow{2}{*}{$\begin{array}{c}\text { No. } \\
\text { samples }\end{array}$} & \multirow{2}{*}{$\begin{array}{c}\text { Discharge } \\
\text { point }\end{array}$} & \multicolumn{3}{|c|}{$\begin{array}{l}\text { Concentration } \\
(\mathrm{mg} / \mathrm{L})\end{array}$} & \multirow{2}{*}{$\begin{array}{l}\text { Std. } \\
\text { error }\end{array}$} \\
\hline & & & $\operatorname{Max}$ & Min & Av & \\
\hline & & 622 & & & & \\
\hline Temperature, ${ }^{\circ} \mathrm{C}$ & 4 & & 27.8 & 18.6 & 24.6 & 2.1 \\
\hline $\mathrm{pH}$, standard units & 4 & & 8.9 & 8.1 & $b$ & 0.2 \\
\hline Free chlorine & 4 & & 0.36 & $<0.10$ & $<0.19$ & 0.06 \\
\hline Chromium & 4 & & $<0.006$ & $<0.006$ & $<0.006$ & 0.000 \\
\hline Copper & 4 & & 0.027 & 0.009 & 0.018 & 0.004 \\
\hline Zinc & 4 & & 0.531 & 0.112 & 0.237 & 0.100 \\
\hline \multirow[t]{2}{*}{ Flow, gal $/ \mathrm{d}^{c}$} & 4 & & 49640 & 12960 & 30453 & 9859 \\
\hline & & 624 & & & & \\
\hline Temperature, ${ }^{\circ} \mathrm{C}$ & 2 & & 27.3 & 13.2 & 20.3 & 7.0 \\
\hline $\mathrm{pH}$, standard units & 2 & & 8.6 & 8.4 & $b$ & 0.1 \\
\hline Free chlorine & 2 & & 0.44 & $<0.10$ & $<0.27$ & 0.17 \\
\hline Chromium & 2 & & 0.006 & $<0.006$ & $<0.006$ & 0.000 \\
\hline Copper & 2 & & 0.015 & 0.012 & 0.014 & 0.002 \\
\hline Zinc & 2 & & 0.121 & 0.088 & 0.105 & 0.017 \\
\hline \multirow[t]{2}{*}{ Flow, gal $/ \mathrm{d}^{c}$} & 4 & & 53110 & 0 & 18750 & 12562 \\
\hline & & 626 & & & & \\
\hline Temperature, ${ }^{\circ} \mathrm{C}$ & 4 & & 28.6 & 14.2 & 21.5 & 3.3 \\
\hline $\mathrm{pH}$, standard units & 4 & & 8.9 & 7.1 & $b$ & 0.4 \\
\hline Free chlorine & 4 & & 0.34 & $<0.10$ & $<0.17$ & 0.06 \\
\hline Chromium & 4 & & 0.034 & 0.024 & 0.029 & 0.002 \\
\hline Copper & 4 & & 0.097 & 0.025 & 0.048 & 0.017 \\
\hline Zinc & 3 & & 0.642 & 0.055 & 0.275 & 0.185 \\
\hline \multirow[t]{2}{*}{ Flow, gal/d ${ }^{c}$} & 4 & & 48220 & 16400 & 25815 & 7504 \\
\hline & & 628 & & & & \\
\hline Temperature, ${ }^{\circ} \mathrm{C}$ & 2 & & 24.1 & 20.5 & 22.3 & 1.8 \\
\hline $\mathrm{pH}$, standard units & 2 & & 8.5 & 8.0 & $b$ & 0.3 \\
\hline Free chlorine & 2 & & 0.10 & $<0.10$ & $<0.10$ & 0.00 \\
\hline Chromium & 2 & & 0.032 & 0.010 & 0.021 & 0.011 \\
\hline Copper & 2 & & 0.037 & 0.013 & 0.025 & 0.012 \\
\hline Zinc & 2 & & 0.182 & 0.068 & 0.125 & 0.057 \\
\hline \multirow[t]{2}{*}{ Flow, gal $/ d^{c}$} & 4 & & 2740 & 0 & 685 & 685 \\
\hline & & 630 & & & & \\
\hline Temperature, ${ }^{\circ} \mathrm{C}$ & 3 & & 26.9 & 22.0 & 25.1 & 1.6 \\
\hline $\mathrm{pH}$, standard units & 3 & & 8.5 & 7.9 & $b$ & 0.2 \\
\hline Free chlorine & 3 & & 0.32 & $<0.10$ & $<0.19$ & 0.07 \\
\hline Chromium & 3 & & 0.026 & $<0.006$ & $<0.013$ & 0.007 \\
\hline Copper & 3 & & 0.021 & $<0.002$ & $<0.011$ & 0.005 \\
\hline Zinc & 3 & & 0.253 & 0.101 & 0.191 & 0.046 \\
\hline Flow, gal/d $d^{c}$ & 4 & & 15990 & 0 & 7572 & 4086 \\
\hline
\end{tabular}


Table 2.2.32 (continued)

\begin{tabular}{|c|c|c|c|c|c|c|}
\hline \multirow{2}{*}{ Parameter } & \multirow{2}{*}{$\begin{array}{c}\text { No. } \\
\text { samples }\end{array}$} & \multirow{2}{*}{$\begin{array}{c}\text { Discharge } \\
\text { point }\end{array}$} & \multicolumn{3}{|c|}{$\begin{array}{c}\text { Concentration } \\
(\mathrm{mg} / \mathrm{L})\end{array}$} & \multirow{2}{*}{$\begin{array}{l}\text { Std. } \\
\text { error }\end{array}$} \\
\hline & & & $\operatorname{Max}$ & $\operatorname{Min}$ & Av & \\
\hline & & 632 & & & & \\
\hline Temperature, ${ }^{\circ} \mathrm{C}$ & 1 & & 23.7 & 23.7 & 23.7 & 0.0 \\
\hline $\mathrm{pH}$, standard units & 1 & & 8.9 & 8.9 & $b$ & 0.0 \\
\hline Free chlorine & 1 & & 0.32 & 0.32 & 0.32 & 0.00 \\
\hline Chromium & 1 & & 0.006 & $<0.006$ & $<0.006$ & 0.000 \\
\hline Copper & 1 & & 0.038 & 0.038 & 0.038 & 0.000 \\
\hline Zinc & 1 & & 0.066 & 0.066 & 0.066 & 0.000 \\
\hline \multirow[t]{2}{*}{ Flow, gallons per dayc } & 4 & & 8690 & 0 & 3963 & 2309 \\
\hline & & 634 & & & & \\
\hline Temperature, ${ }^{\circ} \mathrm{C}$ & 2 & & 23.6 & 16.8 & 20.2 & 3.4 \\
\hline $\mathrm{pH}$, standard units & 2 & & 7.8 & 7.2 & $b$ & 0.3 \\
\hline Free chlorine & 2 & & 0.10 & $<0.10$ & $<0.10$ & 0.00 \\
\hline Chromium & 2 & & 0.045 & 0.010 & 0.028 & 0.017 \\
\hline Copper & 2 & & 0.102 & 0.028 & 0.065 & 0.037 \\
\hline Zinc & 2 & & 2.370 & 0.293 & 1.332 & 1.039 \\
\hline Flow, gallons per day ${ }^{c}$ & 4 & & 22870 & 0 & 11128 & 6429 \\
\hline
\end{tabular}

${ }^{a}$ Y.12 Plant.

${ }^{b}$ Not applicable.

'Flow during operations and/or discharging. 
Table 2.2.33. CY 1989 NPDES Permit Number TN 002968

\begin{tabular}{|c|c|c|c|c|c|}
\hline \multicolumn{6}{|c|}{ Discharge Point $=623^{a}$} \\
\hline \multirow{2}{*}{ Parameter } & \multirow{2}{*}{$\begin{array}{l}\text { No. } \\
\text { samples }\end{array}$} & \multicolumn{3}{|c|}{$\begin{array}{l}\text { Concentration } \\
(\mathrm{mg} / \mathrm{L})\end{array}$} & \multirow{2}{*}{$\begin{array}{l}\text { Std. } \\
\text { error }\end{array}$} \\
\hline & & $\operatorname{Max}$ & Min & Av & \\
\hline $\mathrm{pH}$, standard units & 53 & 8.70 & 7.4 & $\mathrm{NA}^{b}$ & 0.04 \\
\hline Flow, gal/Mgd ${ }^{c}$ & 19 & 3.31 & 3.31 & 3.31 & 0.00 \\
\hline
\end{tabular}

${ }^{a} \mathrm{Y}-12$ Plant, steam plant fly ash sluice water.

${ }^{b} \mathrm{NA}=$ not applicable.

${ }^{c}$ Flow during operations and/or discharging.

Table 2.2.34. CY 1989 Permit Number TN 0002968

Category I Outfalls ${ }^{a}$

\begin{tabular}{|c|c|c|c|c|c|c|c|c|c|}
\hline \multirow{2}{*}{$\begin{array}{c}\text { Outfall } \\
\text { no. }\end{array}$} & \multirow{2}{*}{$\begin{array}{c}\text { No. } \\
\text { samples }\end{array}$} & \multicolumn{3}{|c|}{$\underset{\text { (units) }}{\mathrm{pH}}$} & \multirow{2}{*}{$\begin{array}{l}\text { Std. } \\
\text { error }\end{array}$} & \multicolumn{3}{|c|}{$\begin{array}{l}\text { Flow }^{b} \\
(\mathrm{gal} / \mathrm{d})\end{array}$} & \multirow{2}{*}{$\begin{array}{l}\text { Std. } \\
\text { error }\end{array}$} \\
\hline & & $\operatorname{Max}$ & Min & Av & & $\operatorname{Max}$ & Min & $A v$ & \\
\hline 1 & 1 & 8.2 & 8.2 & $\mathrm{NA}^{c}$ & 0 & 17120 & 17120 & 17120 & 0 \\
\hline 3 & 1 & 6.9 & 6.9 & $N^{c}{ }^{c}$ & 0 & 5922 & 5922 & 5922 & 0 \\
\hline 6 & 1 & 7.6 & 7.6 & $\mathrm{NA}^{c}$ & 0 & 3600 & 3600 & 3600 & 0 \\
\hline 7 & 1 & 7.3 & 7.3 & $N A^{c}$ & 0 & 12500 & 12500 & 12500 & 0 \\
\hline 9 & 1 & 8.2 & 8.2 & $N A^{c}$ & 0 & 9511 & 9511 & 9511 & 0 \\
\hline 15 & 1 & 8.4 & 8.4 & $\mathrm{NA}^{c}$ & 0 & 1141 & 1141 & 1141 & 0 \\
\hline 17 & 1 & 7.9 & 7.9 & $\mathrm{NA}^{\circ}$ & 0 & 79088 & 79088 & 79088 & 0 \\
\hline 18 & 1 & 7,9 & 7.9 & $\mathrm{NA}^{c}$ & 0 & 2282 & 2282 & 2282 & 0 \\
\hline 19 & 1 & 7.8 & 7.8 & $\mathrm{NA}^{c}$ & 0 & 1140 & 1140 & 1140 & 0 \\
\hline 41 & 1 & 8.2 & 8.2 & $\mathrm{NA}^{c}$ & 0 & 3044 & 3044 & 3044 & 0 \\
\hline 44 & 1 & 8.2 & 8.2 & $N A^{c}$ & 0 & 11413 & 11413 & 11413 & 0 \\
\hline 45 & $i$ & 7.9 & 7.9 & $\mathrm{NA}^{c}$ & 0 & 190 & 190 & 190 & 0 \\
\hline 57 & 1 & 8.4 & 8.4 & $\mathrm{NA}^{c}$ & 0 & 4506 & 4506 & 4506 & 0 \\
\hline 170 & 1 & 4.3 & 4.3 & $\mathrm{NA}^{c}$ & 0 & 150218 & 150218 & 150218 & 0 \\
\hline 186 & 1 & 8.1 & 8.1 & $\mathrm{NA}^{c}$ & 0 & 11413 & 11413 & 11413 & 0 \\
\hline 193 & 1 & 7.6 & 7.6 & $\mathrm{NA}^{c}$ & 0 & 4150 & 4150 & 4150 & 0 \\
\hline 194 & $i$ & 8.1 & 8.1 & $\mathrm{NA}^{c}$ & 0 & 761 & 761 & 761 & 0 \\
\hline 198 & 1 & 8.2 & 8.2 & $N_{A}^{c}$ & 0 & 7910 & 7910 & 7910 & 0 \\
\hline 207 & 1 & 7.8 & 7.8 & $\mathrm{NA}^{c}$ & 0 & 1522 & 1522 & 1522 & 0 \\
\hline 221 & $i$ & 7.7 & 7.7 & $\mathrm{NA}^{c}$ & 0 & 133800 & 133800 & 133800 & 0 \\
\hline 236 & 1 & 8.2 & 8.2 & $\mathrm{NA}^{c}$ & 0 & 1141 & 1141 & 1141 & 0 \\
\hline
\end{tabular}

${ }^{a} \mathrm{Y}-12$ Plant, Category I outfalls.

${ }^{b}$ Flow during operations and/or discharging.

${ }^{c} \mathrm{NA}=$ not applicable. 

造总

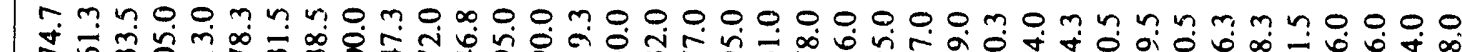

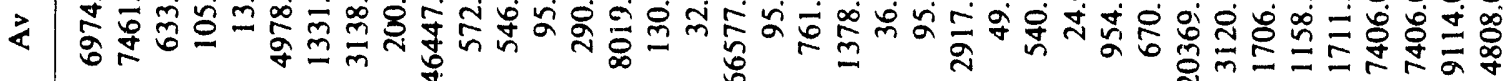

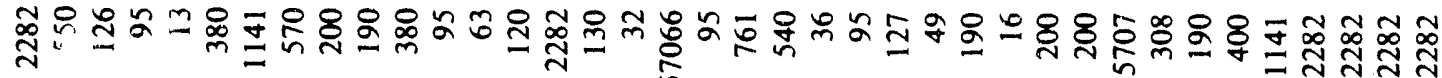

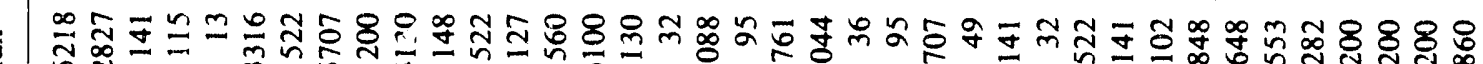

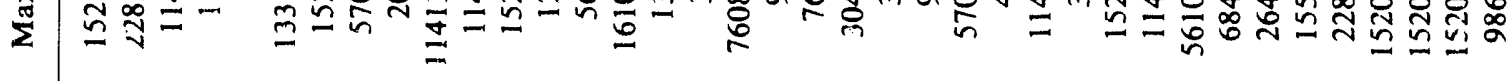

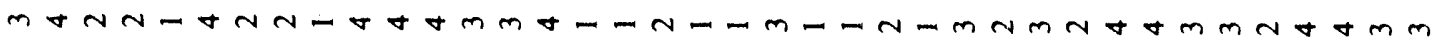

mं

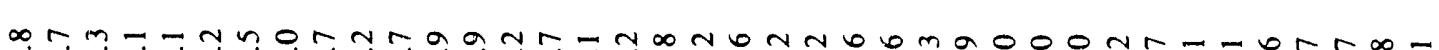

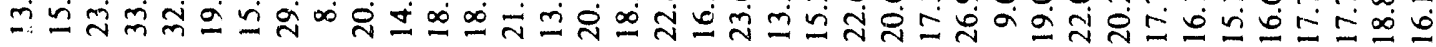

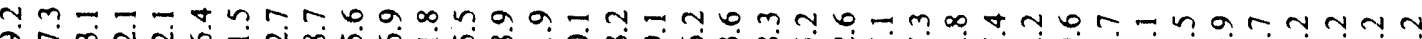

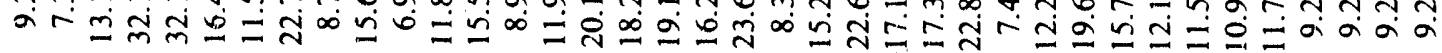

Z ৯

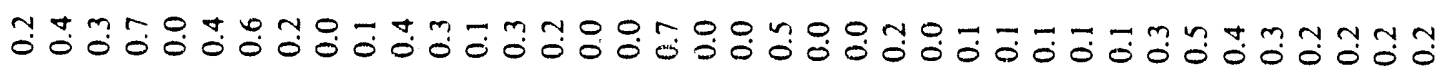

茫

m?ำ ำ$$
\stackrel{\times}{\Sigma}
$$

Ara n

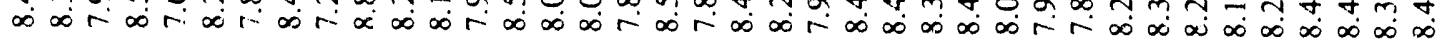

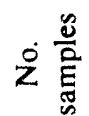
$r$

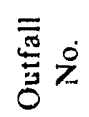
$\stackrel{0}{0}$

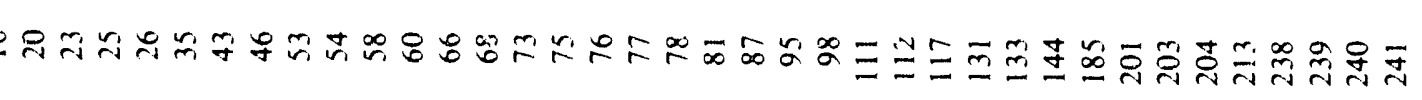




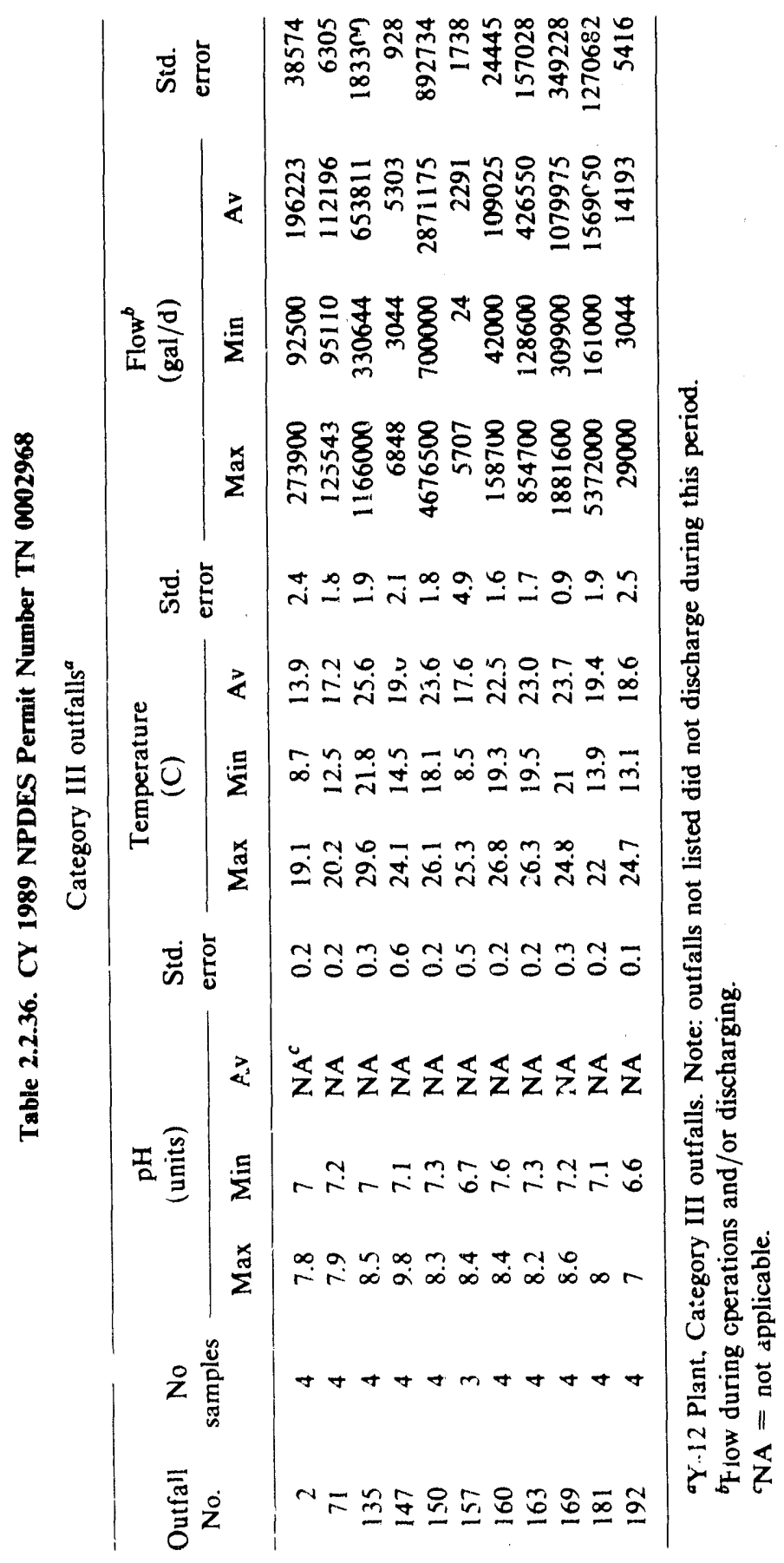


Table 2.2.37. CY 1989 NPDES Permit Number TN 002968

Category IV outfalls ${ }^{a}$

\begin{tabular}{|c|c|c|c|c|c|c|c|c|c|c|}
\hline \multirow{2}{*}{$\begin{array}{c}\text { Outfall } \\
\text { No. }\end{array}$} & \multirow{2}{*}{$\begin{array}{c}\text { No. } \\
\text { samples }\end{array}$} & \multicolumn{3}{|c|}{$\begin{array}{c}\mathrm{pH} \\
\text { (units) }\end{array}$} & \multirow{2}{*}{$\begin{array}{l}\text { Std. } \\
\text { error }\end{array}$} & \multicolumn{3}{|c|}{$\begin{array}{l}\text { Flow }^{b} \\
(\mathrm{gal} / \mathrm{d})\end{array}$} & \multirow{2}{*}{$\begin{array}{l}\text { No. } \\
\text { flows }\end{array}$} & \multirow{2}{*}{$\begin{array}{l}\text { Std. } \\
\text { error }\end{array}$} \\
\hline & & $\operatorname{Max}$ & Min & Av & & $\operatorname{Max}$ & Min & Av & & \\
\hline 401 & 5 & 8.2 & 7.6 & $\mathbf{N} A^{c}$ & 0.1 & & & & & \\
\hline 402 & 0 & & & & & & & & & $\mathrm{NF}^{d}$ \\
\hline 403 & 53 & 8.1 & 4.7 & NA & 0.1 & 26.4 & 26.4 & 26.4 & 1 & 0.0 \\
\hline 404 & 52 & 8.0 & 6.9 & NA & 0.04 & & & & & \\
\hline 405 & 47 & 8.5 & 6.8 & NA & 0.05 & 0.05 & 0.05 & 0.05 & 1 & 0.0 \\
\hline 406 & 16 & 8.7 & 6.5 & NA & 0.2 & 3.2 & 3.2 & 3.2 & 1 & 0.0 \\
\hline 407 & 1 & 7.7 & 7.7 & NA & 0.0 & & & & & \\
\hline 408 & 53 & 8.5 & 7.1 & NA & 0.04 & 2.6 & 2.6 & 2.6 & 1 & 0.0 \\
\hline 409 & 51 & 10.6 & 6.5 & NA & 0.1 & & & & & \\
\hline 410 & 22 & 8.5 & 6.9 & NA & 0.1 & & & & & \\
\hline 411 & 48 & 7.9 & 7.0 & $\mathbf{N A}$ & $0.0^{4}$ & & & & & \\
\hline 412 & 52 & 8.0 & 6.8 & NA & 0.04 & 1.1 & 1.1 & 1.1 & 1 & 0.0 \\
\hline 413 & 52 & 7.8 & 6.9 & NA & $\iota 03$ & 1.0 & 1.0 & 1.0 & 1 & 0.0 \\
\hline 414 & 53 & 8.0 & 6.8 & NA & 0.03 & 5.28 & 5.28 & 5.28 & 1 & 0.0 \\
\hline $41^{5}$ & 10 & 7.9 & 7.0 & NA & 0.1 & & & & & \\
\hline 416 & 0 & & & & & & & & & NF \\
\hline 417 & 0 & & & & & & & & & NF \\
\hline 418 & 17 & 7.8 & 6.8 & NA & 0.1 & 5.28 & 5.28 & 5.28 & 1 & 0.0 \\
\hline 419 & 3 & 7.6 & 7.4 & NA & 0.1 & & & & & \\
\hline 420 & 0 & & & & & & & & & $\mathrm{NF}$ \\
\hline 421 & 0 & & & & & & & & & $N F$ \\
\hline 422 & 1 & 7.0 & 7.0 & NA & 0.0 & & & & & \\
\hline
\end{tabular}

"Y-12 Plant.

${ }^{b}$ Flow during operations and/or discharging.

${ }^{\mathrm{N} A}=$ not applicable.

${ }^{d} \mathrm{NF}=$ no flow.

Table 2.7.38. CY 1989 NPDES Permit Number TN 002068

Miscellaneous Discharge Points ${ }^{a}$

\begin{tabular}{lccccccc}
\hline \multicolumn{1}{c}{ Parameter } & Cutfall & $\begin{array}{c}\text { No. } \\
\text { samples }\end{array}$ & & \multicolumn{3}{c}{$\begin{array}{c}\text { Concentration } \\
(\mathrm{mg} / \mathrm{L})\end{array}$} & $\begin{array}{c}\text { Std. } \\
\text { error }\end{array}$ \\
\hline $\begin{array}{l}\text { Total suspended solids } \\
\text { pH, standard units }\end{array}$ & 702 & 1 & 25 & 25 & 25 & 0 \\
$\begin{array}{l}\text { Total suspended solids } \\
\text { pH, standard units }\end{array}$ & 703 & 0 & NF & NF & NF & \\
$\begin{array}{l}\text { Total suspended solids } \\
\text { pH, standard units }\end{array}$ & 704 & 0 & NF & NF & NF & \\
\hline
\end{tabular}

${ }^{a} \mathrm{Y}-12$ Plant.

${ }^{b} \mathrm{NF}=$ no flow.

${ }^{\mathrm{NA}}=$ not applicahle 
Table 2.2.39. NPDES flows at ORNL in 1989

\begin{tabular}{lllll}
\hline Serial \# discharge & \multicolumn{1}{c}{ Effluent discharges } & \multicolumn{3}{c}{$\begin{array}{c}\text { Flow } \\
\left(\mathrm{L} \times 10^{6} / \mathrm{d}\right)\end{array}$} \\
\hline & Sewage treatment plant & Av & 0.88 & $(0.23)$ \\
X01 & & Max & 1.87 & $(0.49)$ \\
X02 & Coal yard runoff, final & Av & 0.11 & $(0.029)$ \\
& & Max & 0.94 & $(0.25)$ \\
X03 & 1500 area & Av & 0.077 & $(0.02)$ \\
X04 & 2000 area & Av & 0.055 & $(0.015)$ \\
X06 & 3539 and 3540 ponds & Av & 0.60 & $(0.16)$ \\
X06A & 3539 and 3540 ponds & Av & 0.72 & $(0.19)$ \\
X07 & 3544 Process Waste Plant & Av & 0.74 & $(0.2)$ \\
X08 & TRU waste basins & Max & 1.16 & $(0.31)$ \\
X09 & HFIR basins & Av & 0.0012 & $(0.00032)$ \\
X09A & TRU/TURF/HFIR storage tanks & Av & 0.023 & $(0.006)$ \\
X11 & 3518 Acid Neutralization Facility & Av & 0.009 & $(0.0024)$ \\
X13 & Melton Branch & Av & 13.4 & $(3.54)$ \\
X14 & White Oak Creek & Av 35.2 & $(9.29)$ \\
X15 & White Oak Lake Dam & Av 51.4 & $(13.6)$ \\
\hline
\end{tabular}

${ }^{a}$ Flow in millions of gallons per day is given in parentheses.

Table 2.2.40. ORNL Sewage Treatment Plant (X01), 1989

\begin{tabular}{|c|c|c|c|}
\hline Parameter & $\begin{array}{l}\text { Collection } \\
\text { frequency }\end{array}$ & Type & $\begin{array}{l}\text { Analysis } \\
\text { frequency }\end{array}$ \\
\hline Flow & Daily & Continuous & Daily \\
\hline $\mathrm{pH}$ & Weekly & Grab & Weekly \\
\hline Downstream pH & Weekly & Grab & Weekly \\
\hline $\mathrm{BOD}$ & $3 /$ week & 24-h composite & $3 /$ week \\
\hline TSS & $3 /$ week & 24-h composite & $3 /$ week \\
\hline Ammonia & $3 /$ week & 24-h composite & $3 /$ week \\
\hline Oil and grease & $3 /$ week & Grab & $3 /$ week \\
\hline DO & $5 /$ week & Grab & $5 /$ week \\
\hline Residual chlorine & $3 /$ week & Grab & $3 /$ week \\
\hline $\begin{array}{l}\text { Fecal coliform bacteria, } \\
\text { geometric mean }\end{array}$ & $3 /$ week & Grab & $3 /$ week \\
\hline Cyanide, total & Monthly & Grab & Monthly \\
\hline Copper, total & Monthly & 24-h composite & Monthly \\
\hline Mercury, toial & Monthly & 24-h composite & Monthly \\
\hline Silver, total & Monthly & 24-h composite & Monthly \\
\hline Zinc, total & Monthly & 24-h composite & Monthly \\
\hline Trichlorethylene & Monthly & Grab & Monthly \\
\hline Dichlorobromomethane & Monthly & Grab & Monthly \\
\hline Phenols, total & Monthly & Grab & Monthly \\
\hline
\end{tabular}


Table 2.2.41. ORNL coalyard runoff treatment facility (X02), 1989

\begin{tabular}{|c|c|c|c|}
\hline Parameter & $\begin{array}{l}\text { Collection } \\
\text { frequency }\end{array}$ & Type & $\begin{array}{l}\text { Analysis } \\
\text { frequency }\end{array}$ \\
\hline Flow & Daily & Continuous & Daily \\
\hline $\mathrm{pH}$ & Weekly & Grab & Weekly \\
\hline Temperature & Weekly & Grab & Weekly \\
\hline Downstream $\mathrm{pH}$ & Weekly & Grab & Weekly \\
\hline TSS & Weekly & 24-h composite & Weekly \\
\hline Oil and grease & Weekly & Grab & Weekly \\
\hline Chromium, total & Weekly & 24-h composite & Weekly \\
\hline Copper, total & Weekly & 24-h composite & Weekly \\
\hline Iron & Weekly & 24-h composite & Weekly \\
\hline Zinc, total & Weekly & 24-h composite & Weekly \\
\hline Sulfate & Monthly & 24-h composite & Monthly \\
\hline Arsenic, total & Weekly & 24-h composite & Weekly \\
\hline Cadmium, total & Weekly & 24-h composite & Weekly \\
\hline Lead, total & Weekly & 24-h cumposite & Weekly \\
\hline Manganese, total & Weekly & 24-h composite & Weekly \\
\hline Nickel, total & Weekly & 24-h composite & Weekly \\
\hline Selenium, total & Weekly & 24.h composite & Weekly \\
\hline Silver, total & Weekly & 24-h composite & Weekly \\
\hline
\end{tabular}

Table 2.2.42. ORNL 1500 area (X03), 1989"

\begin{tabular}{|c|c|c|c|}
\hline Parameter & $\begin{array}{l}\text { Collection } \\
\text { frequency }\end{array}$ & Type & $\begin{array}{l}\text { Analysis } \\
\text { frequency }\end{array}$ \\
\hline Flow & Monthly & Continuous & Monthly \\
\hline $\mathrm{pH}$ & Weekly & Grab & Weekly \\
\hline Downstream pH & Weekly & Grab & Weekly \\
\hline Temperature & $2 /$ month & Grab & $2 /$ month \\
\hline TSS & $2 /$ month & 24-h composite & $2 /$ month \\
\hline TOC & $2 /$ month & Grab & $2 /$ month \\
\hline Oil and grease & $2 /$ month & Grab & $2 /$ month \\
\hline Phosphorus, total & $2 /$ month & 24-h composite & $2 /$ month \\
\hline Arsenic, total & $2 /$ month & 24-h composite & $2 /$ month \\
\hline Cadmium, total & $2 /$ month & 24-h composite & $2 /$ month \\
\hline Chromium, total & $2 /$ month & 24-h composite & $2 /$ month \\
\hline Copper, total & $2 /$ month & 24-h composite & $2 /$ month \\
\hline Iron & $2 /$ month & 24-h composite & $2 /$ month \\
\hline Lead, total & $2 /$ month & 24-h composite & $2 /$ month \\
\hline Nickel, total & $2 /$ month & 24-h composite & $2 /$ month \\
\hline Zinc, total & $2 /$ month & 24-h composite & $2 /$ month \\
\hline
\end{tabular}

${ }^{a}$ Note: No discharge after May 1, 1989. 
Table 2.2.43. ORNL 2000 area (X04), 1989^

\begin{tabular}{|c|c|c|c|}
\hline Parameter & $\begin{array}{l}\text { Collection } \\
\text { frequency }\end{array}$ & Type & $\begin{array}{l}\text { Analysis } \\
\text { frequency }\end{array}$ \\
\hline flow & Mơnthly & Continuous & Monthly \\
\hline 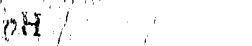 & Weekly & Grab & Weekly \\
\hline Doymistream pH & Weekly & Grab & Weekly \\
\hline Tomperature & $2 /$ month & Grab & $2 /$ month \\
\hline TSS & $2 /$ month & 24.h composite & $2 /$ month \\
\hline TOC & $2 /$ month & Grab & $2 /$ month \\
\hline Oil and grease & $2 /$ month & Grab & $2 /$ month \\
\hline Phosphorus, total & $2 /$ month & 24-h composite & $2 /$ month \\
\hline Arsenic, total & $2 /$ month & 24-h composite & $2 /$ month \\
\hline Cadmium, total & $2 /$ month & 24-h composite & $2 /$ month \\
\hline Chromium, total & $2 /$ month & 24-h composite & $2 /$ month \\
\hline Copper, total & $2 /$ month & 24-h composite & $2 /$ month \\
\hline Lead, total & $2 /$ month & 24-h composite & $2 /$ month \\
\hline Nickel, total & $2 /$ month & 24-h composite & $2 /$ month \\
\hline Silver, total & $2 /$ month & 24-h composite & $2 /$ month \\
\hline Zinc, total & $2 /$ month & 24-h composite & $2 /$ month \\
\hline
\end{tabular}

${ }^{a}$ Note: No discharge after May 1, 1989.

Table 2.2.44. ORNL 3539 and 3540 ponds (X06), 1989"

\begin{tabular}{|c|c|c|c|}
\hline Parameter & $\begin{array}{l}\text { Collection } \\
\text { frequency }\end{array}$ & Type & $\begin{array}{l}\text { Analysis } \\
\text { frequency }\end{array}$ \\
\hline Flow & Per batch & Total volume & Per batch \\
\hline $\mathrm{pH}$ & Weekly & Grab & Weekly \\
\hline Downstream pH & Weekly & Grab & Weekly \\
\hline Temperature & $2 /$ month & Grab & $2 /$ month \\
\hline TSS & $2 /$ month & 24-h composite & $2 /$ morith \\
\hline TOC & $2 /$ month & Grab & $2 /$ month \\
\hline Oil and grease & $2 /$ month & Grab & $2 /$ month \\
\hline Sulfate & $2 /$ month & 24-h composite & $2 /$ month \\
\hline Arsenic, total & $2 /$ month & 24-h composite & $2 /$ month \\
\hline Cadmium, total & $2 /$ month & 24-h composite & $2 /$ month \\
\hline Chromium, total & $2 /$ month & 24-h composite & $2 /$ month \\
\hline Copper, total & $2 /$ month & 24-h composite & $2 /$ month \\
\hline Lead, total & $2 /$ month & 24-h composite & $2 /$ month \\
\hline Nickel, total & $2 /$ month & 24-h composite & $2 / r$ onth \\
\hline Selenium, total & $2 /$ month & 24-h composite & $2 /$ month \\
\hline Zinc, total & $2 /$ month & 24-h composite & $2 /$ month \\
\hline
\end{tabular}

${ }^{a}$ Note: No discharge after May 1, 1989. 
Table 2.2.45. ORNL 3539 and 3540 ponds (X06A), 1989"

\begin{tabular}{llll}
\hline \multicolumn{1}{c}{ Parameter } & $\begin{array}{l}\text { Collection } \\
\text { frequency }\end{array}$ & \multicolumn{1}{c}{ Type } & $\begin{array}{c}\text { Analysis } \\
\text { frequency }\end{array}$ \\
\hline Flow & Weekly & Continuous & Weekly \\
pH & Weekly & Grab & Weekly \\
Downstream pH & Weekly & Grab & Weekly \\
Temperature & $2 /$ month & Grab & $2 /$ month \\
TSS & $2 /$ month & $24-$ h composite & $2 /$ month \\
TOC & $2 /$ month & Grab & $2 /$ month \\
Oil and grease & $2 /$ month & Grab & $2 /$ month \\
Sulfate & $2 /$ month & Grab & $2 /$ month \\
Phosphorous & $2 /$ month & Grab & $2 /$ month \\
Arsenic, total & $2 /$ month & Grab & $2 /$ month \\
Cadmium, total & $2 /$ month & Grab & $2 /$ month \\
Chromium, total & $2 /$ month & Grab & $2 /$ month \\
Copper, total & $2 /$ month & Grab & $2 /$ month \\
Iron, total & $2 /$ month & Grab & $2 /$ month \\
Lead, total & $2 /$ month & Grab & $2 /$ month \\
Mercury, total & $2 /$ month & Grab & $2 /$ month \\
Nickel, total & $2 /$ month & Grab & $2 /$ month \\
Selenium, total & $2 /$ month & Grab & $2 /$ month \\
Silver, total & $2 /$ month & Grab & $2 /$ month \\
Zinc, total & $2 /$ month & Grab & $2 /$ month \\
\hline
\end{tabular}

${ }^{a}$ ORNL 1500 Area, ORNL 2000 Area, and ORNL 3539 and 3540 ponds were cor' inad to form X06A, May 1, 1989.

Table 2.2.46. ORNL Process Waste T'reatment Plant (X07), 1989

\begin{tabular}{llll}
\hline \multicolumn{1}{c}{ Parameter } & $\begin{array}{l}\text { Collection } \\
\text { frequency }\end{array}$ & \multicolumn{1}{c}{ Type } & $\begin{array}{l}\text { Analysis } \\
\text { frequency }\end{array}$ \\
\hline Flow & Daily & Continuous & $\begin{array}{l}\text { Daily } \\
\text { Weekly }\end{array}$ \\
pH & Weekly & Grab & Weekly \\
Downstream pH & Weekly & Grab & $2 /$ month \\
Temperature & $2 /$ month & Grab & $2 /$ month \\
TSS & $2 /$ month & $24-h$ composite & $2 /$ month \\
TOC & $2 /$ month & Grab & $2 /$ month \\
TTO & $2 /$ month & Grab & $2 /$ month \\
Oil and grease & $2 /$ month & Grab & $2 /$ month \\
Nitrate, as N & $2 /$ month & $24-h$ composite & $2 /$ month \\
Sulfate & $2 /$ month & $24-h$ composite & $2 /$ month \\
Arsenic, total & $2 /$ month & $24-h$ composite & $2 /$ month \\
Cadmium, total & $2 /$ month & $24-h$ composite & $2 /$ month \\
Chromium, total & $2 /$ month & $24-h$ composite & $2 /$ month \\
Copper, total & $2 /$ month & $24-h$ composite & $2 /$ month \\
Lead, total & $2 /$ month & $24-h$ composite & $2 /$ month \\
Nickel, total & $2 /$ month & $24-h$ composite & $2 /$ month \\
Silver, total & $2 /$ month & $24-h$ composite & $2 /$ month \\
Zinc, total & $2 /$ month & $24-h$ composite & $2 /$ month \\
\hline
\end{tabular}


Table 2.2.47. ORNL TRU/TURF process waste basin (X08), 1989"

\begin{tabular}{llll}
\hline \multicolumn{1}{c}{ Parameter } & $\begin{array}{c}\text { Collection } \\
\text { frequency }\end{array}$ & \multicolumn{1}{c}{ Type } & $\begin{array}{c}\text { Analysis } \\
\text { frequency }\end{array}$ \\
\hline Flow & Per discharge & Total volume & $\begin{array}{l}\text { Per discharge } \\
\text { pH }\end{array}$ \\
Downstream pH & $1 /$ discharge & Grab & $1 /$ discharge \\
Temperature & $1 /$ discharge & Grab & $1 /$ discharge \\
TSS & $1 /$ discharge & Grab & $1 /$ discharge \\
TOC & $1 /$ discharge & Grab & $1 /$ discharge \\
Oil and grease & $1 /$ discharge & Grab & $1 /$ discharge \\
Nitrate, as N & $1 /$ discharge & Grab & $1 /$ discharge \\
Sulfate & $1 /$ discharge & Grab & $1 /$ discharge \\
Arsenic, total & $1 /$ discharge & Grab & $1 /$ discharge \\
Cadmium, total & $1 /$ discharge & Grab & $1 /$ discharge \\
Chromium, total & $1 /$ discharge & Grab & $1 /$ discharge \\
Copper, total & $1 /$ discharge & Grab & $1 /$ discharge \\
Lead, total & $1 /$ discharge & Grab & $1 /$ discharge \\
Nickel, total & $1 /$ discharge & Grab & $1 /$ discharge \\
Zinc, total & $1 /$ discharge & Grab & $1 /$ discharge \\
\hline
\end{tabular}

${ }^{a}$ Last discharge, May 26, 1989.

Table 2.2.48. ORNL HFIR process waste basin (X09), 1989

\begin{tabular}{|c|c|c|c|}
\hline Parameter & $\begin{array}{l}\text { Collection } \\
\text { frequency }\end{array}$ & Type & $\begin{array}{l}\text { Analysis } \\
\text { frequency }\end{array}$ \\
\hline Flow & Per discharge & Total volume & Per discharge \\
\hline $\mathrm{pH}$ & $1 /$ discharge & Grab & 1/discharge \\
\hline Downstream pH & 1/discharge & Grab & 1/discharge \\
\hline Temperature & 1/discharge & Grab & 1/discharge \\
\hline TSS & 1/discharge & Grab & $1 /$ discharge \\
\hline TOC & 1/discharge & Grab & 1/discharge \\
\hline Oil and grease & $1 /$ discharge & Grab & $1 /$ discharge \\
\hline Niti ite, as $\mathbf{N}$ & 1/discharge & Grab & 1/discharge \\
\hline Sulfate & 1/discharge & Grab & 1/discharge \\
\hline Arsenic, total & 1/discharge & Grab & $1 /$ discharge \\
\hline Cadmium, total & $1 /$ discharge & Grab & $1 /$ discharge \\
\hline Chromium, total & 1/discharge & Grab & 1/discharge \\
\hline Copper, total & 1/discharge & Grab & 1/discharge \\
\hline Lead, total & 1/discharge & Grab & $1 /$ discharge \\
\hline Nickel, total & $1 /$ discharge & Grab & 1/discharge \\
\hline Zinc, total & 1/discharge & Grab & 1/discharge \\
\hline
\end{tabular}

${ }^{a}$ Last discharge, May 26, 1989. 
Table 2.2.49. TRU/TURF/HFIR Storage Tanks (X09A)-ORR, 1989

\begin{tabular}{|c|c|c|c|}
\hline Parameter & $\begin{array}{l}\text { Collection } \\
\text { frequency }\end{array}$ & Type & $\begin{array}{l}\text { Analysis } \\
\text { frequency }\end{array}$ \\
\hline Flow & Per discharge & Volume & Per discharge \\
\hline $\mathrm{pH}$ & 1/discharge & Grab & $1 /$ discharge \\
\hline Downstream pH & 1/discharge & Grab & 1/discharge \\
\hline Temperature & 1/discharge & Grab & $1 /$ discharge \\
\hline TSS & 1/discharge & Grab & 1/discharge \\
\hline TOC & $1 /$ discharge & Grab & 1/discharge \\
\hline Oil and grease & 1/discharge & Grab & 1/discharge \\
\hline Nitrate, as $\mathrm{N}$ & 1/discharge & Grab & 1/discharge \\
\hline Sulfate & 1/discharge & Grab & 1/discharge \\
\hline Arsenic, total & 1/discharge & Grab & $1 /$ discharge \\
\hline Cadmium, total & 1/discharge & Grab & 1/discharge \\
\hline Chromium, total & $1 /$ discharge & Grab & 1/discharge \\
\hline Copper, total & $1 /$ discharge & Grab & 1/discharge \\
\hline Lead, total & $1 /$ discharge & Grab & 1/discharge \\
\hline Nickel, total & $1 /$ discharge & Grab & $1 /$ discharge \\
\hline Zinc, total & $1 /$ discharge & Grab & $1 /$ discharge \\
\hline
\end{tabular}

Table 2.2.50. ORNL 3518 Acid Neutralization Facility (X11), 1989

\begin{tabular}{|c|c|c|c|}
\hline Parameter & $\begin{array}{l}\text { Collection } \\
\text { frequency }\end{array}$ & Type & $\begin{array}{l}\text { Analysis } \\
\text { frequency }\end{array}$ \\
\hline Flow & Per discharge & Total volume & Per discharge \\
\hline $\mathrm{pH}$ & Weekly & Grab & Weekly \\
\hline Downstream pH & Weekly & Grab & Weekly \\
\hline Temperature & $2 /$ month & Grab & $2 /$ month \\
\hline TSS & $2 /$ month & Grab & $2 /$ month \\
\hline TOC & Weekly & Grab & Weekly \\
\hline Oil and grease & $2 /$ month & Grab & $2 /$ month \\
\hline Nitrate, as $\mathrm{N}$ & Weekly & Grab & Weekly \\
\hline Sulfate & Weekly & Grab & Weekly \\
\hline Phosphorus, total & $2 /$ month & Grab & $2 /$ month \\
\hline Arsenic, total & $2 /$ month & Grab & $2 /$ month \\
\hline Cadmium, total & $2 /$ month & Grab & $2 /$ month \\
\hline Chromium, total & $2 /$ month & Grab & $2 /$ month \\
\hline Copper, total & $2 /$ month & Grab & $2 /$ month \\
\hline Lead, total & $2 /$ month & Grab & $2 /$ month \\
\hline Nickel, total & $2 /$ month & Grab & $2 /$ month \\
\hline Zinc, total & $2 /$ month & Grab & $2 /$ month \\
\hline
\end{tabular}


Table 2.2.51. ORNL Melton Branch (X13), 1989

\begin{tabular}{|c|c|c|c|}
\hline Parameter & $\begin{array}{l}\text { Collection } \\
\text { frequency }\end{array}$ & Type & $\begin{array}{l}\text { Analysis } \\
\text { frequency }\end{array}$ \\
\hline Flow & Daily & Continuous & Daily \\
\hline TSS & Monthly & 24-h composite & Monthly \\
\hline Ammonia & Monthly & 24-h composite & Monthly \\
\hline BOD & Monthly & 24-h composite & Monthly \\
\hline TOC & Monthly & Grab & Monthly \\
\hline $\mathrm{pH}$ & Monthly & Grab & Monthly \\
\hline Fluoride & Monthly & 24-h composite & Monthly \\
\hline Nitrate & Monthly & 24-h composite & Monthly \\
\hline Phosphorus & Monthly & 24-h composite & Monthly \\
\hline Sulfate & Monthly & 24-h composite & Monthly \\
\hline Temperature & Monthly & Grab & Monthly \\
\hline Conductivity & Monthly & Grab & Monthly \\
\hline Turbidity & Monthly & Grab & Monthly \\
\hline Phenols, total & Monthly & Grab & Monthly \\
\hline DO & Weekly & Grab & Weekly \\
\hline TDS & Monthly & Grab & Monthly \\
\hline Oil and grease & Weekly & Grab & Weekly \\
\hline Residual chlorine & Weekly & Grab & Weekly \\
\hline Chloroform & Monthly & Grab & Monthly \\
\hline Trichloroethylene & Monthly & Grab & Monthly \\
\hline $\mathrm{PCB}$ & Monthly & 24-h composite & Monthly \\
\hline Aluminum, total & Monthly & 24-h composite & Monthly \\
\hline Arsenic, total & Monthly & 24-h composite & Monthly \\
\hline Cadmium, total & Monthly & 24-h composite & Monthly \\
\hline Chromium, total & Monthly & 24-h composite & Monthly \\
\hline Copper, total & Monthly & 24-h composite & Monthly \\
\hline Iron, total & Monthly & 24-h composite & Monthly \\
\hline Lead, total & Monthly & 24-h composite & Monthly \\
\hline Manganese, total & Monthly & 24-h composite & Monthly \\
\hline Mercury, total & Monthly & 24-h composite & Monthly \\
\hline Nickel, total & Monthly & 24-h composite & Monthly \\
\hline Silver, total & Monthly & 24-h composite & Monthly \\
\hline Zinc, total & Monthly & 24-h composite & Monthly \\
\hline
\end{tabular}


Table 2.2.52. ORNL White Oak Creek (X14), 1989

\begin{tabular}{|c|c|c|c|}
\hline Parameter & $\begin{array}{l}\text { Collection } \\
\text { frequency }\end{array}$ & Type & $\begin{array}{l}\text { Analysis } \\
\text { frequency }\end{array}$ \\
\hline Flow & Daily & Continuous & Daily \\
\hline TSS & Monthly & 24-h composite & Monthly \\
\hline Ammonia & Monthly & 24-h composite & Monthly \\
\hline BOD & Monthly & 24-h composite & Monthly \\
\hline TOC & Monthly & Grab & Monthly \\
\hline $\mathrm{pH}$ & Monthly & Grab & Monthly \\
\hline Fluoride & Monthly & 24-h composite & Monthly \\
\hline Nitrate & Monthly & 24-h composite & Monthly \\
\hline Phosphorus & Monthly & 24-h composite & Monthly \\
\hline Sulfate & Monthly & 24-h composite & Monthly \\
\hline Temperature & Monthly & Grab & Monthly \\
\hline Conductivity & Monthly & Grab & Monthly \\
\hline Turbidity & Monthly & Grab & Monthly \\
\hline Phenols, total & Monthly & Grab & Monthly \\
\hline DO & Weekly & Grab & Weekly \\
\hline TDS & Monthly & Grab & Monthly \\
\hline Oil and grease & Weekly & Grab & Weekly \\
\hline Residual chlorine & Weekly & Grab & Weekly \\
\hline Chloroform & Monthly & Grab & Monthly \\
\hline Trichloroethylene & Monthly & Grab & Monthly \\
\hline PCB & Monthly & 24-h composite & Monthly \\
\hline Aluminum, total & Monthìy & 24-h composite & Monthly \\
\hline Arsenic, total & Monthly & 24-h composite & Monthly \\
\hline Cadmium, total & Monthly & 24-h composite & Monthly \\
\hline Chromium, total & Monthly & 24-h composite & Monthly \\
\hline Copper, total & Monthly & 24-h composite & Monthly \\
\hline Iron, total & Monthly & 24-h composite & Monthly \\
\hline Lead, total & Monthly & 24-h composite & Monthly \\
\hline Manganese, total & Monthly & 24-h composite & Monthly \\
\hline Mercury, total & Monthly & 24-h composite & Monthly \\
\hline Nickel, total & Monthly & 24-h composite & Monthly \\
\hline Silver, total & Monthly & 24-h composite & Monthly \\
\hline Zinc, total & Monthly & 24-h composite & Monthly \\
\hline
\end{tabular}


Table 2.2.53. ORNL White Oak Lake (X15), 1989

\begin{tabular}{|c|c|c|c|}
\hline Parameter & $\begin{array}{l}\text { Collection } \\
\text { frequency }\end{array}$ & Type & $\begin{array}{l}\text { Analysis } \\
\text { frequency }\end{array}$ \\
\hline Flow & Daily & Continuous & Daily \\
\hline TSS & Monthly & 24-h composite & Monthly \\
\hline Ammonia & Monthly & 24-h composite & Monthly \\
\hline BOD & Monthly & 24-h composite & Monthly \\
\hline TOC & Monthly & Grab & Monthly \\
\hline $\mathrm{pH}$ & Monthly & Grab & Monthly \\
\hline Fluoride & Monthly & 24-h composite & Monthly \\
\hline Nitrate & Monthly & 24-h composite & Monthly \\
\hline Phosphorus & Monthly & 24-h composite & Monthly \\
\hline Sulfate & Monthly & 24-h composite & Monthly \\
\hline Temperature & Monthly & Grab & Monthly \\
\hline Conductivity & Monthly & Grab & Monthly \\
\hline Turbidity & Monthly & Grab & Monthly \\
\hline Phenols, total & Monthly & Grab & Monthly \\
\hline DO & Weekly & Grab & Weekly \\
\hline TDS & Monthly & Grab & Monthly \\
\hline Oil and grease & Weekly & Grab & Weekly \\
\hline Residual chlorine & Weekly & Grab & Weekly \\
\hline Chloroform & Monthly & Grab & Monthly \\
\hline Trichloroethylene & Monthly & Grab & Monthly \\
\hline PCB & Monthly & 24-h composite & Monthly \\
\hline Aluminum, total & Monthly & 24-h composite & Monthly \\
\hline Arsenic, total & Monthly & 24-h composite & Monthly \\
\hline Cadmium, total & Monthly & 24-h composite & Monthly \\
\hline Chromium, total & Monthly & 24-h composite & Monthly \\
\hline Copper, total & Monthly & 24-h composite & Monthly \\
\hline Iron, total & Monthly & 24-h composite & Monthly \\
\hline Lead, total & Monthly & 24-h composite & Monthly \\
\hline Manganese, total & Monthly & 24-h composite & Monthly \\
\hline Mercury, total & Monthly & 24-h composite & Monthly \\
\hline Nickel, total & Monthly & 24-h composite & Monthly \\
\hline Silver, total & Monthly & 24-h composite & Monthly \\
\hline Zinc, total & Monthly & 24-h composite & Monthly \\
\hline
\end{tabular}


Tabie 2.2.54. ORNL category I outfalls (storm drains), 1989

\begin{tabular}{lllc}
\multicolumn{1}{c}{ Parameter } & $\begin{array}{c}\text { Collection } \\
\text { frequency }\end{array}$ & Type & $\begin{array}{c}\text { Analysis } \\
\text { frequency }\end{array}$ \\
\hline Flow & Yearly & Instantaneous & Yearly \\
pH & Yearly & Grab & Yearly \\
Temperature & Yearly & Grab & Yearly \\
Oil and grease & Yearly & Grab & Yearly \\
TSS & Yearly & Grab & Yearly \\
\hline
\end{tabular}

Table 2.2.55. ORNL category II outfalls (parking lot drains, storage area drains, once-through water, condensate), 1989

\begin{tabular}{|c|c|c|c|}
\hline Parameter & $\begin{array}{l}\text { Collection } \\
\text { frequency }\end{array}$ & Type & $\begin{array}{l}\text { Analysis } \\
\text { frequency }\end{array}$ \\
\hline Flow & Quarterly & Instantaneous & Quarterly \\
\hline $\mathrm{pH}$ & Quarterly & Grab & Quarterly \\
\hline Temperature & Quarterly & Grab & Quarterly \\
\hline Oil and grease & Quarterly & Grab & Quarterly \\
\hline TSS & Quarterly & Grab & Quarterly \\
\hline
\end{tabular}

Table 2.2.56. ORNL category III outfalls (process and/or lab drains), 1989

\begin{tabular}{llcc}
\hline Parameter & $\begin{array}{l}\text { Collection } \\
\text { frequency }\end{array}$ & Type & $\begin{array}{c}\text { Analysis } \\
\text { frequency }\end{array}$ \\
\hline $\begin{array}{l}\text { Flow } \\
\mathrm{pH}\end{array}$ & $\begin{array}{l}\text { Quarterly } \\
\text { Quarterly }\end{array}$ & $\begin{array}{l}\text { Instantaneous } \\
\text { Instantaneous }\end{array}$ & $\begin{array}{l}\text { Quarterly } \\
\text { Quarterly }\end{array}$ \\
\hline
\end{tabular}

Table 2.2.57. ORNL paint facility (PF7007), 1989

\begin{tabular}{|c|c|c|c|}
\hline Parameter & $\begin{array}{l}\text { Collection } \\
\text { frequency }\end{array}$ & Type & $\begin{array}{l}\text { Analysis } \\
\text { frequency }\end{array}$ \\
\hline Flow & Per discharge & Volume & Per discharge \\
\hline $\mathrm{pH}$ & $1 /$ month & Grab & Per discharge \\
\hline Oil and grease & $1 /$ munth & Grab & Per discharge \\
\hline TSS & $1 /$ month & Grab & Per discharge \\
\hline Phenols, total & $1 /$ quarter & Grab & Per discharge \\
\hline
\end{tabular}

Note: No discharges during 1989. 
Table 2.2.58. ORNL steam plant (SP2519), 1989

\begin{tabular}{|c|c|c|c|}
\hline Parameter & $\begin{array}{l}\text { Collection } \\
\text { frequency }\end{array}$ & Type & $\begin{array}{l}\text { Analysis } \\
\text { frequency }\end{array}$ \\
\hline Flow & Quarterly & $a$ & Quarte \\
\hline Temperature & Quarterly & Grab & Quarterly \\
\hline $\mathrm{pH}$ & Quarterly & Grab & Quarterly \\
\hline
\end{tabular}

${ }^{a}$ Not applicuble.

Table 2.2.59. ORNL vehicle cleaning facilities (VC7002), 1989

\begin{tabular}{llll}
\multicolumn{1}{c}{ Parameter } & $\begin{array}{l}\text { Collection } \\
\text { frequency }\end{array}$ & Type & $\begin{array}{c}\text { Analysis } \\
\text { frequency }\end{array}$ \\
\hline Flow & $\begin{array}{l}\text { Daily } \\
\text { pH }\end{array}$ & Continuous & $\begin{array}{l}\text { Daily } \\
\text { Oil and grease }\end{array}$ \\
TSS & $1 /$ month & Grab & $1 /$ month \\
Phenols, total & $1 /$ month & Grab & $1 /$ month \\
BOD & $1 /$ month & Grab & $1 /$ month \\
Fecal coliform bacteria & $1 /$ month & Grab & $1 /$ month \\
& $1 /$ month & Grab & $1 /$ month \\
\hline
\end{tabular}

Table 2.2.60. ORNL equipment maintenance facility (EF7002), 1989

\begin{tabular}{clcl} 
Parameter & $\begin{array}{l}\text { Collection } \\
\text { frequency }\end{array}$ & Type & $\begin{array}{c}\text { Analysis } \\
\text { frequency }\end{array}$ \\
\hline $\begin{array}{llll}\text { Oil and grease } \\
\text { pH }\end{array}$ & $\begin{array}{l}\text { Quarterly } \\
\text { Quarterly }\end{array}$ & $\begin{array}{l}\text { Grab } \\
\text { Grab }\end{array}$ & $\begin{array}{l}\text { Quarterly } \\
\text { Quarterly }\end{array}$ \\
\hline
\end{tabular}

Table 2.2.61. ORNL cooling systems (cooling tower blowdown), 1989

\begin{tabular}{llll}
\multicolumn{1}{c}{ Parameter } & $\begin{array}{c}\text { Collection } \\
\text { frequency }\end{array}$ & Type & $\begin{array}{c}\text { Analysis } \\
\text { frequency }\end{array}$ \\
\hline Flow & Quarterly & $a$ & Quarterly \\
Chromium, total & Quarterly & Grab & Quarterly \\
Zinc, total & Quarterly & Grab & Quarterly \\
Copper, total & Quarterly & Grab & Quarterly \\
Temperature & Quarterly & Grab & Quarterly \\
Residual chlorine & During & Grab & $\begin{array}{c}\text { During } \\
\text { addition }\end{array}$ \\
& addition & &
\end{tabular}

${ }^{a}$ Not applicable. 
Table 2.2.62. NPDES Permit Number TN 0002941, 1989 Discharge point $\mathrm{X} 01$ at $\mathrm{ORNL}$

\begin{tabular}{|c|c|c|c|c|c|}
\hline \multirow{2}{*}{ Parameter } & \multirow{2}{*}{$\begin{array}{c}\text { No. of } \\
\text { samples }\end{array}$} & \multicolumn{4}{|c|}{$\begin{array}{l}\text { Concentration } \\
(\mathrm{mg} / \mathrm{L})\end{array}$} \\
\hline & & $\operatorname{Max}^{a}$ & $\operatorname{Min}^{a}$ & $A v^{a}$ & $\begin{array}{l}\text { Std. } \\
\text { error }\end{array}$ \\
\hline Ammonia, as $\mathbf{N}$ & 156 & 1.3 & 0.0060 & 0.081 & 0.011 \\
\hline BOD & 156 & $<5.0$ & $<5.0$ & $<5.0$ & 0 \\
\hline Bromodichloromethane & 11 & $<0.0050$ & $\mathrm{~J} 0.00090$ & $\mathrm{~J} 0.0022$ & 0.00045 \\
\hline Chlorine, total residual & 156 & 0.49 & $<0.010$ & $<0.28$ & 0.010 \\
\hline Copper, total & 12 & 0.060 & $<0.0040$ & $<0.016$ & 0.0043 \\
\hline Cyanide, total & 12 & $<0.0020$ & $<0.0020$ & $<0.0020$ & 0 \\
\hline Downstream $\mathrm{pH}$, standard units & 52 & 8.1 & 6.7 & $b$ & $b$ \\
\hline Fecal coliform, col./100 mL & 157 & 190 & $<1.0$ & $<1.4^{c}$ & 1.1 \\
\hline Flow, Mgd & 251 & 0.49 & 0.096 & 0.23 & 0.0040 \\
\hline Mercury, total & 12 & 0.00099 & $<0.000050$ & $<0.00017$ & 0.000077 \\
\hline Oil and grease & 156 & 160 & $<2.0$ & $<3.3$ & 1.0 \\
\hline Oxygen, dissolved & 249 & 14 & 6.0 & 8.2 & 0.090 \\
\hline $\mathrm{pH}$, standard units & 52 & 8.0 & 6.6 & $b$ & $b$ \\
\hline Recoverable phenolics, total & 12 & 0.0020 & $<0.0010$ & $<0.0011$ & 0.000083 \\
\hline Silver, total & 12 & $<0.0060$ & $<0.0050$ & $<0.0052$ & 0.00012 \\
\hline TSS & 156 & 73 & $<2.0$ & $<6.4$ & 0.61 \\
\hline Trichloroethene & 11 & $<0.0050$ & $\mathrm{~J} 0.00080$ & $\mathrm{~J} 0.0046$ & 0.00038 \\
\hline Zinc, total & 12 & 0.11 & 0.025 & 0.069 & 0.0056 \\
\hline
\end{tabular}

${ }^{\circ}<$ = Undetected; $\mathrm{J}=$ below detection limit, but estimated.

${ }^{b}$ Not applicable.

'Geometric mean. 
Table 2.2.03. NPDES Permit Number TN 0002941, 1989

Discharge point X02 at ORNL

\begin{tabular}{|c|c|c|c|c|c|}
\hline \multirow{2}{*}{ Parameter } & \multirow{2}{*}{$\begin{array}{l}\text { No. of } \\
\text { sampies }\end{array}$} & \multicolumn{4}{|c|}{$\begin{array}{l}\text { Concentration } \\
(\mathrm{mg} / \mathrm{L})\end{array}$} \\
\hline & & $\operatorname{Max}^{a}$ & $\operatorname{Min}^{a}$ & $A v^{\circ}$ & $\begin{array}{l}\text { Std. } \\
\text { error }\end{array}$ \\
\hline Arsenic, total & 52 & 0.29 & 0.033 & 0.13 & 0.0089 \\
\hline Cadmium, total & 52 & 0.017 & $<0.0020$ & $<0.0048$ & 0.00063 \\
\hline Chromium, total & 52 & 0.041 & $<0.0030$ & $<0.012$ & 0.0013 \\
\hline Copper, total & 52 & 0.067 & $<0.0040$ & $<0.012$ & 0.0013 \\
\hline Downstream $\mathrm{pH}$, standard units & 247 & 8.9 & 6.4 & $b$ & $b$ \\
\hline Flow, Mgd & 249 & 0.25 & 0 & 0.029 & 0.0024 \\
\hline Iron, total & 52 & 4.7 & $<0.010$ & $<0.21$ & 0.089 \\
\hline Lead, total & 52 & $<0.050$ & $<0.030$ & $<0.036$ & 0.0013 \\
\hline Manganese, total & 52 & 0.097 & $<0.0020$ & $<0.024$ & 0.0028 \\
\hline Nickel, total & 52 & 0.021 & $<0.0050$ & $<0.010$ & 0.00081 \\
\hline Oil and grease & 52 & 120 & $<2.0$ & $<5.4$ & 2.4 \\
\hline $\mathrm{pH}$, standard units & 247 & 11 & 3.1 & $b$ & $b$ \\
\hline Selenium, total & 52 & $<0.080$ & $<0.030$ & $<0.066$ & 0.0023 \\
\hline Silver, total & 52 & 0.021 & $<0.0040$ & $<0.0058$ & 0.00032 \\
\hline Sulfate, as $\mathrm{SO}_{4}$ & 12 & 1900 & 580 & 1100 & 110 \\
\hline TSS & 52 & 24 & $<2.0$ & $<6.7$ & 0.62 \\
\hline Temperature, ${ }^{\circ} \mathrm{C}$ & 247 & 30 & 3.0 & 17 & 0.45 \\
\hline Zinc, total & 52 & 0.16 & $<0.0080$ & $<0.026$ & 0.0037 \\
\hline
\end{tabular}

${ }^{a}<=$ Undetected.

${ }^{b}$ Not applicable. 
Table 2.2.64. NPDES Permit Number TN 0002941, 1989 Discharge point X03 at ORNL

\begin{tabular}{|c|c|c|c|c|c|}
\hline \multirow{2}{*}{ Parameter } & \multirow{2}{*}{$\begin{array}{l}\text { No. of } \\
\text { samples }\end{array}$} & \multicolumn{4}{|c|}{$\begin{array}{l}\text { Concentration } \\
(\mathrm{mg} / \mathrm{L})\end{array}$} \\
\hline & & $\operatorname{Max}^{a}$ & $\operatorname{Min}^{a}$ & $A v^{a}$ & $\begin{array}{l}\text { Std. } \\
\text { error }\end{array}$ \\
\hline Arsenic, total & 8 & $<0.060$ & $<0.050$ & $<0.056$ & 0.0018 \\
\hline Cadmium, total & 8 & 0.016 & $<0.0020$ & $<0.0062$ & 0.0019 \\
\hline Chromium, total & 8 & 0.050 & $<0.0050$ & $<0.020$ & 0.0062 \\
\hline Copper, total & 8 & 0.020 & 0.0050 & 0.011 & 0.0015 \\
\hline Downstream pH, standard units & 17 & 7.9 & 5.8 & $b$ & $b$ \\
\hline Flow, Myd & 4 & 0.041 & 0.0094 & 0.020 & 0.0073 \\
\hline Iron, total & 8 & 0.19 & $<0.3040$ & $<0.065$ & 0.022 \\
\hline Lead, total & 8 & $<0.055$ & $<0.050$ & $<0.051$ & 0.00063 \\
\hline Nickel, total & 8 & 0.033 & $<0.0050$ & $<0.012$ & 0.0034 \\
\hline Oil and grease & 8 & 5.0 & $<2.0$ & $<2.6$ & 0.42 \\
\hline Organic carbon, total & 8 & 4.5 & 3.3 & 3.9 & 0.14 \\
\hline $\mathrm{pH}$, standard units & 17 & 8.1 & 3.4 & $b$ & $b$ \\
\hline Phosphorus, total & 8 & 0.68 & 0.30 & 0.49 & 0.049 \\
\hline TSS & 8 & $<5.0$ & $<5.0$ & $<5.0$ & 0 \\
\hline Temperature, ${ }^{\circ} \mathrm{C}$ & 17 & 23 & 11 & 16 & 0.71 \\
\hline Zinc, total & 8 & 0.18 & 0.040 & 0.11 & 0.015 \\
\hline
\end{tabular}

${ }^{a}<$ = Undetected.

${ }^{b}$ Not applicable. 
Table 2.2.65. NPDES Permit Number TN 0002941, 1989 Discharge point X04 at ORNL

\begin{tabular}{|c|c|c|c|c|c|}
\hline \multirow{2}{*}{ Parameter } & \multirow{2}{*}{$\begin{array}{l}\text { No. of } \\
\text { samples }\end{array}$} & \multicolumn{4}{|c|}{$\begin{array}{l}\text { Concentration } \\
(\mathrm{mg} / \mathrm{L})\end{array}$} \\
\hline & & $\operatorname{Max}^{a}$ & $\operatorname{Min}^{a}$ & $A v^{a}$ & $\begin{array}{l}\text { Std. } \\
\text { error }\end{array}$ \\
\hline Arsenic, total & 8 & 0.060 & $<0.050$ & $<0.059$ & 0.0013 \\
\hline Cadmium, total & 8 & 0.018 & $<0.0020$ & $<0.0068$ & 0.0022 \\
\hline Chromium, total & 8 & 0.020 & $<0.0050$ & $<0.011$ & 0.0018 \\
\hline Copper, total & 8 & 0.016 & $<0.0040$ & $<0.010$ & 0.0012 \\
\hline Downstream $\mathrm{pH}$, standard units & 17 & 8.0 & 6.9 & $b$ & $b$ \\
\hline Flow, Mgd & 3 & 0.034 & 0.00049 & 0.015 & 0.010 \\
\hline Lead, total & 8 & 0.23 & $<0.050$ & $<0.073$ & 0.023 \\
\hline Nickel, total & 8 & 0.017 & $<0.0050$ & $<0.0090$ & 0.0017 \\
\hline Oil and grease & 8 & 10 & $<2.0$ & $<4.6$ & 1.2 \\
\hline Organic carbon, total & 8 & 2.2 & 1.2 & 1.6 & 0.11 \\
\hline $\mathrm{pH}$, standard units & 17 & 8.2 & 6.1 & $b$ & $b$ \\
\hline Phosphorus, total & 8 & 0.50 & 0.20 & 0.33 & 0.037 \\
\hline Silver, total & 8 & 0.029 & $<0.0050$ & $<0.0085$ & 0.0029 \\
\hline TSS & 8 & $<5.0$ & $<5.0$ & $<5.0$ & 0 \\
\hline Temperature, ${ }^{\circ} \mathrm{C}$ & 17 & $2 \mathrm{I}$ & 9.8 & 14 & 0.64 \\
\hline Zinc, total & 8 & 0.17 & 0.025 & 0.11 & 0.018 \\
\hline
\end{tabular}

$a_{<}=$Undetected.

${ }^{b}$ Not applicable.

Table 2.2.66. NPDES Permit No. TN 0002941, 1989 Discharge point X06 at ORNL

\begin{tabular}{|c|c|c|c|c|c|}
\hline \multirow{2}{*}{ Parameter } & \multirow{2}{*}{$\begin{array}{l}\text { No. of } \\
\text { samples }\end{array}$} & \multicolumn{4}{|c|}{$\begin{array}{l}\text { Concentration } \\
(\mathrm{mg} / \mathrm{L})\end{array}$} \\
\hline & & $\operatorname{Max}^{a}$ & $\operatorname{Min}^{a}$ & $A v^{a}$ & $\begin{array}{l}\text { Std. } \\
\text { error }\end{array}$ \\
\hline Arsenic, total & 8 & $<0.060$ & 0.040 & 0.055 & $0.002 \%$ \\
\hline Cadmium, tutal & 8 & 0.022 & $<0.0020$ & $<0.0074$ & 0.0026 \\
\hline Chromium, total & 8 & 0.11 & $<0.010$ & $<0.03$ & 0.012 \\
\hline Copper, to al & 8 & 0.15 & $<0.010$ & $<0.043$ & 0.016 \\
\hline Downstrea $\mathrm{n}$ pH, standard units & 17 & 8.2 & 6.5 & $b$ & $b$ \\
\hline Flow, Mgd & 4 & 0.17 & 0.15 & 0.16 & 0.0039 \\
\hline Lead, total & 8 & 0.13 & $<0.050$ & $<0.069$ & 0.012 \\
\hline Nickel, total & 8 & 0.017 & $<0.0050$ & $<0.012$ & 0.0016 \\
\hline Oil and grease & 8 & 3.0 & $<2.0$ & $<2.1$ & 0.13 \\
\hline Organic carbon, total & 8 & 6.3 & 2.6 & 4.3 & 0.50 \\
\hline $\mathrm{pH}$, standard units & 17 & 8.1 & 6.2 & $b$ & $b$ \\
\hline Selenium, total & 8 & $<0.060$ & $<0.030$ & $<0.054$ & 0.0038 \\
\hline Sulfate, as $\mathrm{SO}_{4}$ & 8 & 35 & 22 & 30 & 1.9 \\
\hline TSS & 8 & 22 & $<5.0$ & $<7.1$ & 2.1 \\
\hline Temperature, ${ }^{\circ} \mathrm{C}$ & 17 & 21 & 11 & i5 & 0.68 \\
\hline Zinc, total & 8 & 0.13 & 0.059 & 0.10 & 0.010 \\
\hline
\end{tabular}

${ }^{a}<=$ Undetected

${ }^{b}$ Not applicatle. 
Table 2.2.67. NPDES Permit Number TN 0002941, 1989 Discharge point X06A at ORNL

\begin{tabular}{|c|c|c|c|c|c|}
\hline \multirow{2}{*}{ Parameter } & \multirow{2}{*}{$\begin{array}{l}\text { No. of } \\
\text { samples }\end{array}$} & \multicolumn{4}{|c|}{$\begin{array}{l}\text { Concentration } \\
(\mathrm{mg} / \mathrm{L})\end{array}$} \\
\hline & & $\operatorname{Max}^{a}$ & $\mathrm{Min}^{a}$ & $A v^{a}$ & $\begin{array}{l}\text { Std. } \\
\text { error }\end{array}$ \\
\hline Arsenic, total & 16 & $<0.050$ & $<0.050$ & $<0.050$ & 0 \\
\hline Cadmium, total & 16 & 0.069 & $<0.0020$ & $<0.0077$ & 0.0042 \\
\hline Chromium, total & 16 & 0.025 & $<0.0030$ & $<0.0087$ & 0.0018 \\
\hline Copper, total & 16 & 0.095 & 0.017 & 0.054 & 0.0065 \\
\hline Downstream $\mathrm{pH}$, standard units & 35 & 8.3 & 6.9 & $b$ & $b$ \\
\hline Flow, Mgd & 13 & 0.23 & 0.12 & 0.19 & 0.0077 \\
\hline Iron, total & 16 & 1.5 & 0.043 & 0.20 & 0.089 \\
\hline Lead, total & 16 & 0.088 & $<0.030$ & $<0.03 .4$ & 0.0036 \\
\hline Mercury, total & 16 & 0.0035 & 0.00050 & 0.0017 & 0.00021 \\
\hline Nickel, total & 16 & $<0.020$ & $<0.0060$ & $<0.0087$ & 0.0014 \\
\hline Oil and grease & 16 & 5.0 & $<2.0$ & $<2.3$ & 0.19 \\
\hline Organic carbon, total & 16 & 10 & 2.0 & 4.8 & 0.51 \\
\hline $\mathrm{pH}$, standard units & 35 & 8.6 & 6.5 & $b$ & $b$ \\
\hline Phosphorus, total & 16 & 0.60 & 0.30 & 0.43 & 0.024 \\
\hline Selenium, total & 16 & $<0.080$ & $<0.040$ & $<0.073$ & 0.0040 \\
\hline Silver, total & 16 & 0.036 & $<0.0050$ & $<0.0096$ & 0.0020 \\
\hline Sulfate, as $\mathrm{SO}_{4}$ & 16 & 30 & 23 & 26 & 0.45 \\
\hline TSS & 16 & $<5.0$ & $<5.0$ & $<5.0$ & 0 \\
\hline Temperature, ${ }^{\circ} \mathrm{C}$ & 35 & 25 & 12 & 20 & 0.59 \\
\hline Zinc, total & 16 & 1.0 & 0.060 & 0.17 & 0.056 \\
\hline
\end{tabular}

${ }^{0}<$ = Undetected

${ }^{b}$ Not applicable. 
Table 2.2.68. NPDES Permit Number TN 0002941, 1989 Discharge point X07 at ORNL

\begin{tabular}{|c|c|c|c|c|c|}
\hline \multirow{2}{*}{ Parameter } & \multirow{2}{*}{$\begin{array}{c}\text { No. of } \\
\text { samples }\end{array}$} & \multicolumn{4}{|c|}{$\begin{array}{l}\text { Concentration } \\
(\mathrm{mg} / \mathrm{L})\end{array}$} \\
\hline & & $\operatorname{Max}^{a}$ & $\operatorname{Min}^{a}$ & $A v^{a}$ & $\begin{array}{l}\text { Std. } \\
\text { error }\end{array}$ \\
\hline Arsenic, total & 24 & $<0.060$ & $<0.050$ & $<0.052$ & 0.00085 \\
\hline Cadmium, total & 24 & 0.018 & $<0.0020$ & $<0.0044$ & 0.00087 \\
\hline Chromium, total & 24 & 0.030 & $<0.0030$ & $<0.0083$ & 0.0014 \\
\hline Copper, total & 24 & 0.019 & $<0.0040$ & $<0.0094$ & 0.00060 \\
\hline Downstream $\mathrm{pH}$, standard units & 52 & 8.2 & 6.3 & $b$ & $b$ \\
\hline Flow, Mgd & 249 & $0.3 i$ & 0.0063 & 0.20 & 0.0035 \\
\hline Lead, total & 24 & $<0.050$ & $<0.030$ & $<0.037$ & 0.0020 \\
\hline Nickel, total & 24 & 0.029 & $<0.0050$ & $<0.0099$ & 0.0013 \\
\hline Nitrate & 24 & 37 & $<5.0$ & $<7.3$ & 1.3 \\
\hline Oil and grease & 24 & 3.0 & $<2.0$ & $<2.0$ & 0.042 \\
\hline Organic carbon, total & 24 & 2.4 & 0.40 & 1.5 & 0.091 \\
\hline $\mathrm{pH}$, standard units & 52 & 8.8 & 6.2 & $b$ & $b$ \\
\hline Silver, total & 24 & $<0.0060$ & $<0.0050$ & $<0.0051$ & 0.000069 \\
\hline Sulfate, as $\mathrm{SO}_{4}$ & 24 & 770 & 100 & 290 & 30 \\
\hline TSS & 24 & 11 & $<5.0$ & $<5.3$ & 0.26 \\
\hline Temperature, ${ }^{\circ} \mathrm{C}$ & 52 & 28 & 9.2 & 19 & 0.76 \\
\hline Total toxic organics & 24 & 0.091 & 0 & 0.017 & 0.0041 \\
\hline Zinc, total & 24 & 0.027 & 0.0040 & 0.013 & 0.0014 \\
\hline
\end{tabular}

${ }^{a}<=$ Undetected.

${ }^{b}$ Not applicable. 
Table 2.2.69. NPDES Permit Number TN 0002941, 1989 Discharge point X'08 at ORNL

\begin{tabular}{|c|c|c|c|c|c|}
\hline \multirow{2}{*}{ Parameter } & \multirow{2}{*}{$\begin{array}{l}\text { No. of } \\
\text { samples }\end{array}$} & \multicolumn{4}{|c|}{$\begin{array}{l}\text { Concentration } \\
(\mathrm{mg} / \mathrm{L})\end{array}$} \\
\hline & & $\operatorname{Max}^{a}$ & $\operatorname{Min}^{a}$ & $A v^{a}$ & $\begin{array}{l}\text { Sid. } \\
\text { error }\end{array}$ \\
\hline Arsenic, total & 1 & $<0.050$ & $<0.050$ & $<0.050$ & $b$ \\
\hline Cadmium, total & 1 & $<0.0020$ & $<0.0020$ & $<0.0020$ & $\dot{b}$ \\
\hline Chromium, total & 1 & 0.12 & 0.12 & 0.12 & $b$ \\
\hline Copper, total & 1 & 0.092 & 0.092 & 0.092 & $b$ \\
\hline Downstream $\mathrm{pH}$, standard units & 1 & 7.7 & 7.7 & $b$ & $b$ \\
\hline Flow, Mgd & 1 & 0.00032 & 0.00032 & 0.00032 & $b$ \\
\hline Lead, total & 1 & $<0.030$ & $<0.030$ & $<0.030$ & $b$ \\
\hline Nickel, total & 1 & 0.018 & 0.018 & 0.018 & $b$ \\
\hline Nitrate & 1 & $<0.50$ & $<0.50$ & $<0.50$ & $b$ \\
\hline Oil and grease & 1 & 3.0 & 3.0 & 3.0 & $b$ \\
\hline Organic carbon, total & 1 & 80 & 80 & 80 & $b$ \\
\hline $\mathrm{pH}$, standard units & 1 & 7.6 & 7.6 & $b$ & $b$ \\
\hline Sulfate, as $\mathrm{SO}_{4}$ & 1 & 10 & 10 & 10 & $b$ \\
\hline TSS & 1 & 38 & 38 & 38 & $b$ \\
\hline Temperature, ${ }^{\circ} \mathrm{C}$ & 1 & 28 & 28 & 28 & $b$ \\
\hline Zinc, total & 1 & 0.77 & 0.77 & 0.77 & $b$ \\
\hline
\end{tabular}

${ }^{a}<=$ Undetected.

${ }^{b}$ Noi applicable.

Table 2.2.70. NPDES Permit Number TN 0002941, 1989 Discharge point X09 at ORNL

\begin{tabular}{|c|c|c|c|c|c|}
\hline \multirow{2}{*}{ Parameter } & \multirow{2}{*}{$\begin{array}{c}\text { No. of } \\
\text { samples }\end{array}$} & \multicolumn{4}{|c|}{$\begin{array}{c}\text { Concentration } \\
(\mathrm{mg} / \mathrm{L})\end{array}$} \\
\hline & & $\operatorname{Max}^{a}$ & $\operatorname{Min}^{\circ}$ & $A v^{a}$ & $\begin{array}{l}\text { Std. } \\
\text { error }\end{array}$ \\
\hline Arsenic, total & 9 & 0.096 & $<0.050$ & $<0.062$ & 0.0045 \\
\hline Cadmium, total & 9 & 0.018 & $<0.0020$ & $<0.0060$ & 0.0020 \\
\hline Chromium, total & 9 & 0.078 & $<0.0030$ & $<0.020$ & 0.0083 \\
\hline Copper, total & 9 & 0.055 & $<0.010$ & $<0.028$ & 0.0059 \\
\hline Downstream $\mathrm{pH}$, standard units & 6 & 8.3 & 7.3 & $b$ & $b$ \\
\hline Flow, Mgd & 9 & 0.0097 & 0.0032 & 0.0060 & 0.00065 \\
\hline Lead, total & 9 & $<0.050$ & $<0.030$ & $<0.046$ & 0.0029 \\
\hline Nickel, total & 9 & 0.018 & $<0.0050$ & $<0.0098$ & 0.0017 \\
\hline Nitrate & 9 & 7.9 & $<5.0$ & $<5.4$ & 0.32 \\
\hline Oil and grease & 9 & 24 & $<2.0$ & $<6.0$ & 2.3 \\
\hline Organic carbon, total & 9 & 8.1 & 2.1 & 4.4 & 0.55 \\
\hline $\mathrm{pH}$, standard units & 9 & 8.6 & 7.3 & $b$ & $b$ \\
\hline Sulfate, as $\mathrm{SO}_{4}$ & 9 & 110 & 20 & 55 & 9.5 \\
\hline TSS & 9 & 14 & $<5.0$ & $<7.6$ & 1.1 \\
\hline Temperature, ${ }^{\circ} \mathrm{C}$ & 9 & 24 & 5.4 & 14 & 2.2 \\
\hline Zinc, total & 9 & 0.11 & $<0.0080$ & $<0.048$ & 0.0096 \\
\hline
\end{tabular}

${ }^{a}<=$ Undetected.

${ }^{b}$ Not applicable. 
Table 2.2.71. NPDES Permit Number TN 0002941, 1989 Discharge point X09A at ORNL

\begin{tabular}{|c|c|c|c|c|c|}
\hline \multirow{2}{*}{ Parameter } & \multirow{2}{*}{$\begin{array}{c}\text { No. of } \\
\text { samples }\end{array}$} & \multicolumn{4}{|c|}{$\begin{array}{l}\text { Concentration } \\
(\mathrm{mg} / \mathrm{L})\end{array}$} \\
\hline & & $\operatorname{Max}^{a}$ & $\operatorname{Min}^{a}$ & $A v^{a}$ & $\begin{array}{l}\text { Std. } \\
\text { error }\end{array}$ \\
\hline Arsenic, total & 35 & 0.080 & $<0.050$ & $<0.051$ & 0.00087 \\
\hline Cadmium, total & 35 & 0.014 & $<0.0020$ & $<0.0039$ & 0.00059 \\
\hline Chromium, total & 35 & 0.031 & $<0.0030$ & $<0.0093$ & 0.0012 \\
\hline Copper, total & 35 & 0.27 & $<0.0080$ & $<0.10$ & 0.0091 \\
\hline Downstream $\mathrm{pH}$, standard units & 35 & 9.6 & 6.5 & $b$ & $b$ \\
\hline Flow, Mgd & 35 & 0.0037 & 0.0011 & 0.0024 & 0.000090 \\
\hline Lead, total & 35 & 0.041 & $<0.030$ & $<0.030$ & 0.00032 \\
\hline Nickel, total & 35 & $<.0 .020$ & $<0.0060$ & $<0.0090$ & 0.00095 \\
\hline Nitrate & 35 & 11 & 0.80 & 5.1 & 0.32 \\
\hline Oil and grease & 35 & 5.0 & $<2.0$ & $<2.1$ & 0.10 \\
\hline Organic carbon, total & 35 & 5.4 & 1.1 & 2.5 & 0.17 \\
\hline $\mathrm{pH}$, standard units & 35 & 11 & 6.5 & $b$ & $b$ \\
\hline Sulfate, as $\mathrm{SO}_{4}$ & 35 & 240 & 22 & 47 & 7.8 \\
\hline TSS & 35 & 51 & 2.0 & 8.3 & 1.8 \\
\hline Temperature, ${ }^{\circ} \mathrm{C}$ & 35 & 32 & 13 & 24 & 0.94 \\
\hline Zinc, total & 35 & 0.27 & 0.025 & 0.12 & 0.0087 \\
\hline
\end{tabular}

${ }^{a}<=$ Undetected.

${ }^{b}$ Not applicable.

Table 2.2.72. NPDES Permit Number TN 0002941, 1989 Discharge point $\mathrm{X} 11$ at $\mathrm{ORNL}$

\begin{tabular}{|c|c|c|c|c|c|}
\hline \multirow{2}{*}{ Parameter } & \multirow{2}{*}{$\begin{array}{c}\text { No. of } \\
\text { samples }\end{array}$} & \multicolumn{4}{|c|}{$\begin{array}{l}\text { Concentration } \\
(\mathrm{mg} / \mathrm{L})\end{array}$} \\
\hline & & $\operatorname{Max}^{a}$ & $\operatorname{Min}^{a}$ & $A v^{a}$ & $\begin{array}{l}\text { Std. } \\
\text { error }\end{array}$ \\
\hline Arsenic, total & 24 & 0.22 & $<0.050$ & $<0.11$ & 0.012 \\
\hline Cadmium, total & 24 & 0.016 & $<0.0020$ & $<0.0045$ & 0.00086 \\
\hline Chromium, total & 24 & 0.085 & $<0.0030$ & $<0.025$ & 0.0044 \\
\hline Copper, total & 24 & 0.077 & $<0.010$ & $<0.019$ & 0.0030 \\
\hline Downstream $\mathrm{pH}$, standard units & 52 & 9.0 & 6.5 & $b$ & $b$ \\
\hline Flow, Mgd & 12 & 0.29 & 0.014 & 0.047 & 0.022 \\
\hline Lead, total & 24 & $<0.050$ & $<0.030$ & $<0.037$ & 0.0020 \\
\hline Nickel, total & 24 & 0.047 & $<0.0050$ & $<0.013$ & 0.0019 \\
\hline Nitrate & 52 & $<50$ & 2.5 & 6.1 & 0.88 \\
\hline Oil and grease & 24 & 41 & $<2.0$ & $<3.8$ & 1.6 \\
\hline Organic carbon, total & 52 & B.5 & 0.70 & 4.5 & 0.29 \\
\hline $\mathrm{pH}$, standard units & 52 & 8.8 & 6.2 & $b$ & $b$ \\
\hline Phosphorus, total & 24 & 5.9 & 0.60 & 3.4 & 0.36 \\
\hline Sulfate, as $\mathrm{SO}_{4}$ & 52 & 2800 & 620 & 1700 & 87 \\
\hline TSS & 24 & 46 & $<5.0$ & $<19$ & 2.3 \\
\hline Temperature, ${ }^{\circ} \mathrm{C}$ & 52 & 26 & 13 & 19 & 0.50 \\
\hline Zinc, total & 24 & 1.2 & 0.26 & 0.72 & 0.054 \\
\hline
\end{tabular}

${ }^{a}<=$ Undetected.

${ }^{b}$ Not applicable. 
Table 2.2.73. NPDES Permit Number TN 0002941, 1989 Discharge point X13 at ORNL

\begin{tabular}{|c|c|c|c|c|c|}
\hline \multirow{2}{*}{ Paran eter } & \multirow{2}{*}{$\begin{array}{c}\text { No. of } \\
\text { samples }\end{array}$} & \multicolumn{4}{|c|}{$\begin{array}{l}\text { Concentration } \\
(\mathrm{mg} / \mathrm{L})\end{array}$} \\
\hline & & $\operatorname{Max}^{a}$ & $\operatorname{Min}^{a}$ & $A v^{a}$ & $\begin{array}{l}\text { Std. } \\
\text { error }\end{array}$ \\
\hline Aluminum, total & 12 & 7.1 & $<0.050$ & $<1.1$ & 0.56 \\
\hline Ammonia, as $\mathrm{N}$ & 12 & 0.070 & 0.0090 & 0.030 & 0.0045 \\
\hline Arsenic, total & 12 & $<0.060$ & $<0.050$ & $<0.052$ & 0.0011 \\
\hline BOD & 12 & $<5.0$ & $<5.0$ & $<5.0$ & 0 \\
\hline Cadmium, total & 12 & $<0.0020$ & $<0.0020$ & $<0.0020$ & 0 \\
\hline Chlorine, total residual & 52 & $<0.010$ & $<0.010$ & $<0.010$ & 0 \\
\hline Chloroform & 11 & $<0.025$ & $\mathrm{~J} 0.00050$ & $\mathrm{~J} 0.0056$ & 0.0020 \\
\hline Chromium, total & 12 & 0.027 & $<0.0030$ & $<0.012$ & 0.0022 \\
\hline Conductivity, $\mathrm{mS} / \mathrm{cm}$ & 12 & 1.4 & 0.10 & 0.61 & 0.14 \\
\hline Copper, total & 12 & 0.23 & $<0.0040$ & $<0.029$ & 0.018 \\
\hline Dissolved solids, total & 12 & 280 & 120 & 190 & 14 \\
\hline Flow, Mgd & 249 & 73 & 0.40 & 3.5 & 0.57 \\
\hline Fluoride, total & 12 & 1.0 & $<1.0$ & $<1.0$ & 0 \\
\hline Iron, total & 12 & 10 & 0.12 & 1.1 & 0.81 \\
\hline Lead, total & 12 & 0.010 & $<0.0040$ & $<0.0046$ & 0.00050 \\
\hline Manganese, total & 12 & 2.1 & $<0.0020$ & $<0.27$ & 0.17 \\
\hline Mercury, total & 12 & 0.00010 & $<0.000050$ & $<0.000056$ & 0.0000043 \\
\hline Nickel, total & 12 & $<0.020$ & $<0.0060$ & $<0.6094$ & 0.0015 \\
\hline Nitrate & 12 & $<5.0$ & $<5.0$ & $<5.0$ & 0 \\
\hline Oil and grease & 52 & 88 & $<2.0$ & $<7.0$ & 2.3 \\
\hline Organic carbon, total & 12 & 5.1 & 1.6 & 2.9 & 0.29 \\
\hline Oxygen, dissolved & 52 & 15 & 5.1 & 9.3 & 0.30 \\
\hline PCB's, total & 11 & $<0.00050$ & $<0.00050$ & $<0.00050$ & 0 \\
\hline $\mathrm{pH}$, standard units & 12 & 8.0 & 6.5 & $b$ & $b$ \\
\hline Phosphorus, total & 12 & 0.60 & 0.10 & 0.19 & 0.043 \\
\hline Recoverable phenolics, total & 12 & 0.0030 & $<0.0010$ & $<0.0015$ & 0.00026 \\
\hline Silver, total & 12 & $<0.0050$ & $<0.0050$ & $<0.0050$ & 0 \\
\hline Sulfate, as $\mathrm{SO}_{4}$ & 12 & 27 & 2 & 21 & 1.3 \\
\hline TSS & 12 & 390 & $<5.0$ & $<45$ & 32 \\
\hline Temperature, ${ }^{\circ} \mathrm{C}$ & 64 & 27 & 1.6 & 15 & 0.78 \\
\hline Trichloroethene & 11 & $<0.025$ & $\mathrm{~J} 0.00030$ & $\mathrm{~J} 0.0046$ & 0.0021 \\
\hline Turbidity, NTU & 12 & 220 & 10 & 92 & 17 \\
\hline Zinc, total & 12 & 0.16 & $<0.0080$ & $<0.028$ & 0.012 \\
\hline
\end{tabular}

${ }^{a}<=$ Undetected; $\mathrm{J}=$ below detection limit, but estimated.

${ }^{b}$ Not applicable. 
Table 2.2.74. NPDES Permit Number TN 0002941, 1989 Discharge point X14 at ORNL

\begin{tabular}{|c|c|c|c|c|c|}
\hline \multirow{2}{*}{ Parameter } & \multirow{2}{*}{$\begin{array}{l}\text { No. of } \\
\text { samples }\end{array}$} & \multicolumn{4}{|c|}{$\begin{array}{c}\text { Concentration } \\
(\mathrm{mg} / \mathrm{L})\end{array}$} \\
\hline & & $\operatorname{Max}^{a}$ & $\operatorname{Min}^{a}$ & $A v^{a}$ & $\begin{array}{c}\text { Std. } \\
\text { error }\end{array}$ \\
\hline Aluminum, total & 12 & 3.2 & $<0.050$ & $<0.72$ & 0.27 \\
\hline Ammonia, as $\mathbf{N}$ & 12 & 0.10 & 0.018 & 0.041 & 0.0071 \\
\hline Arsenic, total & 12 & $<0.060$ & $<0.050$ & $<0.052$ & 0.0011 \\
\hline BOD & 12 & $>34$ & $<5.0$ & $<7.4$ & 2.4 \\
\hline Cadmium, total & 12 & $<0.0020$ & $<0.0020$ & $<0.0020$ & 0 \\
\hline Chlorine, total residual & 52 & $<0.010$ & $<0.010$ & $<0.010$ & 0 \\
\hline Chloroform & 11 & $<0.025$ & $\mathrm{~J} 0.00060$ & $\mathrm{~J} 0.0055$ & 0.0020 \\
\hline Chromium, total & 12 & 0.035 & $<0.0030$ & $<0.013$ & 0.0026 \\
\hline Conductivity, $\mathrm{mS} / \mathrm{cm}$ & 12 & 1.8 & 0.20 & 0.79 & 0.15 \\
\hline Copper, total & 12 & 0.15 & $<0.0040$ & $<0.024$ & 0.012 \\
\hline Dissolved solids, total & 12 & 300 & 130 & 210 & 15 \\
\hline Flow, Mgd & 249 & 81 & 0.98 & 9.3 & 0.51 \\
\hline Fluoride, total & 12 & 1.0 & $<1.0$ & $<1.0$ & 0 \\
\hline Iron, total & 12 & 4.1 & 0.088 & 0.66 & 0.32 \\
\hline Lead, total & 12 & 0.0080 & $<0.0040$ & $<0.0043$ & 0.00033 \\
\hline Manganese, total & 12 & 0.26 & 0.017 & 0.067 & 0.020 \\
\hline Mercury, total & 12 & 0.00012 & $<0.000050$ & $<0.000076$ & 0.0000076 \\
\hline Nickel, total & 12 & $<0.020$ & $<0.0050$ & $<0.0093$ & 0.0015 \\
\hline Nitrate & 12 & $<5.0$ & $<5.0$ & $<5.0$ & 0 \\
\hline Oil and grease & 52 & 42 & $<2.0$ & $<3.9$ & 0.85 \\
\hline Organic carbon, total & 12 & 6.9 & 1.3 & 2.7 & 0.42 \\
\hline Oxygen, dissolved & 52 & 19 & 6.0 & 9.1 & 0.34 \\
\hline PCBs, total & 11 & $<0.00050$ & $<0.00050$ & $<0.00050$ & 0 \\
\hline $\mathrm{pH}$, standard units & 12 & 8.5 & 6.5 & $b$ & $b$ \\
\hline Phosphorus, total & 12 & 0.40 & 0.10 & 0.26 & 0.036 \\
\hline Recoverable phenolics, total & 12 & 0.0040 & $<0.0010$ & $<0.0013$ & 0.00025 \\
\hline Silver, total & 12 & $<0.0050$ & $<0.0050$ & $<0.0050$ & 0 \\
\hline Sulfate, as $\mathrm{SO}_{4}$ & 12 & 67 & 18 & 40 & 4.1 \\
\hline TSS & 12 & 130 & $<5.0$ & $<19$ & 10 \\
\hline Temperature, ${ }^{\circ} \mathrm{C}$ & 64 & 26 & 7.1 & 16 & 0.65 \\
\hline Trichloroethene & 11 & $<0.025$ & $\mathrm{~J} 0.00060$ & $\mathrm{~J} 0.0060$ & 0.0020 \\
\hline Turbidity, NTU & 12 & 270 & 16 & 100 & 26 \\
\hline Zinc, total & 12 & 0.13 & $<0.0080$ & $<0.047$ & 0.0088 \\
\hline
\end{tabular}

${ }^{a}<=$ Undetected; $\mathbf{J}=$ below detection limit, but estimated.

${ }^{b}$ Not applicable. 
Table 2.2.75. NPDES Permit Number TN 0002941, 1989 Discharge point X15 at ORNL

\begin{tabular}{|c|c|c|c|c|c|}
\hline \multirow{2}{*}{ Parameter } & \multirow{2}{*}{$\begin{array}{c}\text { No. of } \\
\text { samples }\end{array}$} & \multicolumn{4}{|c|}{$\begin{array}{l}\text { Concentration } \\
(\mathrm{mg} / \mathrm{L})\end{array}$} \\
\hline & & $\operatorname{Max}^{a}$ & $\operatorname{Min}^{a}$ & $A v^{a}$ & $\begin{array}{l}\text { Std. } \\
\text { error }\end{array}$ \\
\hline Aluminum, total & 12 & 2.8 & $<0.050$ & $<0.86$ & 0.22 \\
\hline Ammonia, as $\mathbf{N}$ & 12 & 0.16 & 0.011 & 0.046 & 0.012 \\
\hline Arsenic, total & 12 & 0.090 & $<0.050$ & $<0.055$ & 0.0034 \\
\hline BOD & 12 & $>34$ & $<5.0$ & $<7.4$ & 2.4 \\
\hline Cadmium, total & 12 & $<0.0020$ & $<0.0020$ & $<0.0020$ & 0 \\
\hline Chlorine, total residual & 52 & $<0.010$ & $<0.010$ & $<0.010$ & 0 \\
\hline Chloroform & 11 & $<0.025$ & $\mathrm{~J} 0.0010$ & $\mathrm{~J} 0.0041$ & 0.0021 \\
\hline Chromium, total & 12 & 0.028 & $<0.0030$ & $<0.016$ & 0.0025 \\
\hline Conductivity, $\mathrm{mS} / \mathrm{cm}$ & 12 & 1.7 & 0.23 & 0.87 & 0.14 \\
\hline Copper, total & 12 & 0.13 & 0.0050 & 0.019 & 0.010 \\
\hline Dissolved solids, total & 12 & 240 & 140 & 200 & 9.4 \\
\hline Flow, Mgd & 249 & 150 & 4.2 & 14 & 1.0 \\
\hline Fluoride, total & 12 & 1.0 & $<1.0$ & $<1.0$ & 0 \\
\hline Iron, total & 12 & 2.3 & 0.20 & 0.67 & 0.16 \\
\hline Lead, total & 12 & 0.0040 & $<0.0040$ & $<0.0040$ & 0 \\
\hline Manganese, total & 12 & 0.10 & $<0.0020$ & $<0.060$ & 0.0081 \\
\hline Mercury, total & 12 & 0.00011 & $<0.000050$ & $<0.000063$ & 0.0000058 \\
\hline Nickel, total & 12 & $<0.020$ & $<0.0050$ & $<0.010$ & 0.0017 \\
\hline Nitrate & 12 & $<5.0$ & $<5.0$ & $<5.0$ & 0 \\
\hline Oil and grease & 52 & $>200$ & $<2.0$ & $<10$ & 4.3 \\
\hline Organic carbon, total & 12 & 64 & 1.7 & 2.9 & 0.37 \\
\hline Oxygen, dissolved & 52 & 14 & 4.0 & 8.5 & 0.29 \\
\hline PCBs, total & 11 & $<0.00050$ & $<0.00050$ & $<0.00050$ & 0 \\
\hline $\mathrm{pH}$, standard units & 12 & 8.9 & 6.7 & $b$ & $b$ \\
\hline Phosphorus, total & 12 & 0.50 & 0.10 & 0.23 & 0.031 \\
\hline Silver, total & 12 & $<0.0050$ & $<0.0050$ & $<0.0050$ & 0 \\
\hline Sulfate, as $\mathrm{SO}_{4}$ & 12 & 48 & 12 & 34 & 2.9 \\
\hline TSS & 12 & 37 & $<5.0$ & $<13$ & 3.2 \\
\hline Temperature, ${ }^{\circ} \mathrm{C}$ & 64 & 28 & 3.9 & 17 & 0.80 \\
\hline Trichloroethene & 11 & $<0.025$ & $\mathrm{~J} 0.00070$ & $\mathrm{~J} 0.0064$ & 0.0019 \\
\hline Turbidity, NTU & 12 & 240 & 10 & 74 & 23 \\
\hline Zinc, total & 12 & 0.040 & $<0.0080$ & $<0.024$ & 0.0036 \\
\hline
\end{tabular}

${ }^{a}<=$ Undetected; $\mathbf{J}=$ below detection limit, but estimated.

${ }^{b}$ Not applicable. 
Table 2.2.76. NPDES Permit Number TN 0002941, 1989 Discharge point EF7002 at ORNL

\begin{tabular}{|c|c|c|c|c|c|}
\hline \multirow{2}{*}{ Parameter } & \multirow{2}{*}{$\begin{array}{l}\text { No. of } \\
s \text { :mples }\end{array}$} & \multicolumn{4}{|c|}{$\begin{array}{l}\text { Concentration } \\
(\mathrm{mg} / \mathrm{L})\end{array}$} \\
\hline & & $\operatorname{Max}$ & Min & Av & $\begin{array}{l}\text { Std. } \\
\text { error }\end{array}$ \\
\hline Oil and grease & 4 & 310 & 9.0 & 100 & 72 \\
\hline $\mathrm{pH}_{i}$ stardard units & 4 & 7.8 & 6.2 & $a$ & $a$ \\
\hline
\end{tabular}

${ }^{a}$ Not applicable.

Table 2.2.77. NPDES Permit Number TN 0002941, 1989 Discharge point SP2519 at ORNL

\begin{tabular}{|c|c|c|c|c|c|}
\hline \multirow{2}{*}{ Parameter } & \multirow{2}{*}{$\begin{array}{l}\text { No. of } \\
\text { samples }\end{array}$} & \multicolumn{4}{|c|}{$\begin{array}{l}\text { Concentration } \\
(\mathrm{mg} / \mathrm{L})\end{array}$} \\
\hline & & $\operatorname{Max}$ & Min & Av & $\begin{array}{l}\text { Std. } \\
\text { error }\end{array}$ \\
\hline Flow, Mgd & 4 & 0.011 & 0.000046 & 0.0051 & 0.0029 \\
\hline $\mathrm{pH}$, standard units & 4 & 10 & 7.7 & $a$ & $a$ \\
\hline Temperature, ${ }^{\circ} \mathrm{C}$ & 4 & 49 & 21 & 31 & 6.1 \\
\hline
\end{tabular}

${ }^{a}$ Not applicable.

'Table 2.2.78. NPDES Permit Number TN 0002941, 1989 Discharge point VC7002 at ORNL

\begin{tabular}{|c|c|c|c|c|c|}
\hline \multirow{2}{*}{ Parameter } & \multirow{2}{*}{$\begin{array}{c}\text { No. of } \\
\text { samples }\end{array}$} & \multicolumn{4}{|c|}{$\begin{array}{c}\text { Concentration }{ }^{a} \\
(\mathrm{mg} / \mathrm{L})\end{array}$} \\
\hline & & Max & Min & Av & $\begin{array}{l}\text { Std. } \\
\text { error }\end{array}$ \\
\hline BOD & 11 & $>400$ & $<5.0$ & $<99$ & 41 \\
\hline Fecal coliform, col. $/ 100 \mathrm{~mL}$ & 11 & $>80000$ & $<1.0$ & $<8500$ & 7200 \\
\hline Flow, Mgd & 167 & 0.00044 & 0 & 0.000072 & 0.0000066 \\
\hline Oil and grease & 11 & 1200 & $<2.0$ & $<230$ & 110 \\
\hline $\mathrm{pH}$, standard units & 12 & 11 & 5.3 & $b$ & $b$ \\
\hline Recoverable phenolics, total & 11 & 7.5 & $<0.0010$ & $<0.76$ & 0.68 \\
\hline TSS & 11 & 24000 & $<5.0$ & $<5000$ & 2800 \\
\hline
\end{tabular}

${ }^{a}<=$ Undetected.

${ }^{b}$ Not applicable. 
Table 2.2.79. NPDES Permit Number TN 0002941, 1989 Cooling systems at ORNL

\begin{tabular}{|c|c|c|c|c|c|}
\hline \multirow{2}{*}{ Parameter } & \multirow{2}{*}{$\begin{array}{l}\text { No. of } \\
\text { samr }^{-} 8\end{array}$} & \multicolumn{4}{|c|}{$\begin{array}{c}\text { Conceritration } \\
(\mathrm{mg} / \mathrm{L})\end{array}$} \\
\hline & & $\operatorname{Max}$ & Min & Av & $\begin{array}{l}\text { Std. } \\
\text { error }\end{array}$ \\
\hline Chlorine, total residual & 54 & 3.6 & $<0.010$ & $<0.19$ & 0.073 \\
\hline Chromium, tota! & 54 & 0.49 & $<0.0030$ & $<0.029$ & 0.0091 \\
\hline Copper, total & 54 & 2.4 & $<0.0040$ & $<0.21$ & 0.063 \\
\hline Downstream $\mathrm{pH}$, standard units & 43 & 9.0 & 7.4 & $b$ & $b$ \\
\hline Flow, Mgd & 54 & 0.18 & 0.0010 & 0.019 & 0.0063 \\
\hline $\mathrm{pH}$, standard units & 54 & 9.0 & 7.5 & $b$ & $b$ \\
\hline Temperature, ${ }^{\circ} \mathrm{C}$ & 54 & 33 & 9.9 & 23 & 0.63 \\
\hline Zinc, total & 54 & 10 & $0.0: 1$ & 0.72 & 0.19 \\
\hline
\end{tabular}

$0<$ - Undetecter

${ }^{6}$ Not applicable.

Table 2.2.80. NPDES Permit Number TN 0002941, 1989 Category I outfalls at ORNL

\begin{tabular}{|c|c|c|c|c|c|}
\hline \multirow{2}{*}{ Parameter } & \multirow{2}{*}{$\begin{array}{l}\text { No. of } \\
\text { samples }\end{array}$} & \multicolumn{4}{|c|}{$\begin{array}{c}\text { Concentration }{ }^{a} \\
(\mathrm{mg} / \mathrm{L})\end{array}$} \\
\hline & & Max & Min & Av & $\begin{array}{l}\text { Std. } \\
\text { error }\end{array}$ \\
\hline Downstream pH, standard units & 25 & 8.8 & 6.9 & $b$ & $b$ \\
\hline Flow, Mgd & 25 & 0.11 & 0.00014 & 0.012 & 0.0055 \\
\hline Oil and grease & 25 & 210 & $<2.0$ & $<19$ & 8.5 \\
\hline $\mathrm{pH}$, standard units & 25 & 8.5 & 3.3 & $b$ & $b$ \\
\hline TSS & 25 & 3700 & $<5.0$ & $<300$ & 160 \\
\hline Temperature, ${ }^{\circ} \mathrm{C}$ & 25 & 38 & 15 & 21 & 0.81 \\
\hline
\end{tabular}

${ }^{a}<=$ Undelected.

'Not applicable. 
Table 2.2.81. NPDES Permit Number TN 0002941, 1989 Category II outfalls at ORNL

\begin{tabular}{|c|c|c|c|c|c|}
\hline \multirow{2}{*}{ Parameter } & \multirow{2}{*}{$\begin{array}{l}\text { No. of } \\
\text { samples }\end{array}$} & \multicolumn{4}{|c|}{$\begin{array}{c}\text { Concentration }^{a} \\
(\mathrm{mg} / \mathrm{L})\end{array}$} \\
\hline & & $\operatorname{Max}$ & Min & Av & $\begin{array}{l}\text { Std. } \\
\text { error }\end{array}$ \\
\hline Downstream $\mathrm{pH}$, standard units & 147 & 8.3 & 6.8 & $b$ & $b$ \\
\hline Flow, Mgd & 147 & 0.26 & 0.000029 & 0.029 & 0.0036 \\
\hline Oil and grease & 147 & 150 & $<2.0$ & $<9.0$ & 1.7 \\
\hline $\mathrm{pH}$, standard units & 147 & 8.5 & 6.2 & $b$ & $b$ \\
\hline TSS & 147 & 1100 & $<5.0$ & $<32$ & 10 \\
\hline Temperature, ${ }^{\circ} \mathrm{C}$ & 147 & 57 & 5.2 & 18 & 0.65 \\
\hline
\end{tabular}

${ }^{\circ}<=$ Undetected.

${ }^{b}$ Not applicable.

Tuble 2.2.82. NPDES Permit Number TN 0002941, 1989 Category III outfalls at ORNL

\begin{tabular}{|c|c|c|c|c|c|}
\hline \multirow{2}{*}{ Parameter } & \multirow{2}{*}{$\begin{array}{l}\text { No. of } \\
\text { samples }\end{array}$} & \multicolumn{4}{|c|}{$\begin{array}{l}\text { Concentration } \\
(\mathrm{mg} / \mathrm{L})\end{array}$} \\
\hline & & $\operatorname{Max}$ & $\operatorname{Min}$ & Av & $\begin{array}{l}\text { Std. } \\
\text { error }\end{array}$ \\
\hline Flow, Mgd & 85 & 0.32 & 0.00014 & 0.027 & 0.0060 \\
\hline $\mathrm{pH}$, standard units & 85 & 8.4 & 4.6 & $a$ & $a$ \\
\hline
\end{tabular}

"Not applicable. 
Table 2.2.83. 1989 ORNL gross beta concentrations at category I and II outfalls.

\begin{tabular}{|c|c|c|}
\hline Station & Date & Concentration $(\mathrm{pCi} / \mathrm{L})$ \\
\hline \multicolumn{3}{|c|}{ Category l Outfalls } \\
\hline 102 & $29 \mathrm{Sep}$ & 2.7 \\
\hline 103 & $29 \mathrm{Sep}$ & 300 \\
\hline 104 & 29 Sep & -11 \\
\hline 106 & 29 Sep & 35 \\
\hline 108 & $29 \mathrm{Sep}$ & -11 \\
\hline 109 & 29 Sep & 22 \\
\hline 110 & $29 \mathrm{Sep}$ & 27 \\
\hline 111 & $29 \mathrm{Sep}$ & -22 \\
\hline 113 & $29 \mathrm{Sep}$ & -2.7 \\
\hline 114 & 29 Sep & 22 \\
\hline 116 & $30 \mathrm{Sep}$ & 22 \\
\hline 141 & $29 \mathrm{Sep}$ & 240 \\
\hline 142 & 29 Sep & 35 \\
\hline 143 & 29 Sep & 24 \\
\hline 144 & $29 \mathrm{Sep}$ & 2.7 \\
\hline 161 & $30 \mathrm{Sep}$ & -19 \\
\hline 162 & $30 \mathrm{Sep}$ & -22 \\
\hline 164 & $30 \mathrm{Sep}$ & 43 \\
\hline 165 & 30 Sep & 26000 \\
\hline 168 & $30 \mathrm{Sep}$ & 54 \\
\hline 169 & $30 \mathrm{Sep}$ & -14 \\
\hline 171 & 30 Sep & -8.1 \\
\hline 172 & $30 \mathrm{Sep}$ & 11 \\
\hline 173 & $30 \mathrm{Sep}$ & 11 \\
\hline 191 & $29 \mathrm{Sep}$ & 24 \\
\hline \multicolumn{3}{|c|}{ Category II Outfalls } \\
\hline \multirow[t]{2}{*}{204} & 22 Sep & 810 \\
\hline & $27 \mathrm{Nov}$ & 4100 \\
\hline \multirow[t]{2}{*}{205} & 11 Sep & 27 \\
\hline & 27 Nov & 18 \\
\hline 206 & $22 \mathrm{Sep}$ & 41 \\
\hline \multirow[t]{2}{*}{207} & $11 \mathrm{Sep}$ & 140 \\
\hline & 27 Nov & 180 \\
\hline 208 & $22 \mathrm{Sep}$ & 6.5 \\
\hline 209 & $22 \mathrm{Sep}$ & 11 \\
\hline \multirow[t]{2}{*}{210} & $11 \mathrm{Sep}$ & 2.4 \\
\hline & 27 Nov & 3.5 \\
\hline \multirow[t]{2}{*}{211} & $11 \mathrm{Sep}$ & 1.1 \\
\hline & $08 \mathrm{Dec}$ & 11 \\
\hline 212 & 27 Nov & 4.6 \\
\hline 214 & $22 \mathrm{Sep}$ & 11 \\
\hline 216 & $22 \mathrm{Sep}$ & 9.2 \\
\hline \multirow[t]{2}{*}{217} & $11 \mathrm{Sep}$ & 3.2 \\
\hline & 27 Nov & 7.6 \\
\hline \multirow[t]{2}{*}{218} & 11 Sep & 6.2 \\
\hline & 27 Nov & 2.7 \\
\hline \multirow[t]{2}{*}{219} & 11 Sep & 1.9 \\
\hline & $27 \mathrm{Nov}$ & 6.8 \\
\hline 222 & $15 \mathrm{Sep}$ & 10 \\
\hline
\end{tabular}


Table 2.2 .83 (continued)

\begin{tabular}{|c|c|c|}
\hline Station & Date & Concentration $(\mathrm{pCi} / \mathrm{L})$ \\
\hline \multirow[t]{2}{*}{223} & $11 \mathrm{Sep}$ & 4.3 \\
\hline & $15 \mathrm{Sep}$ & 5.1 \\
\hline 224 & 22 Sep & 0.81 \\
\hline \multirow[t]{2}{*}{226} & $11 \mathrm{Sep}$ & 1.9 \\
\hline & $27 \mathrm{Nov}$ & -0.81 \\
\hline \multirow[t]{2}{*}{227} & 11 Sep & 2.4 \\
\hline & 27 Nov & 5.1 \\
\hline 230 & $15 \mathrm{Sep}$ & 6.5 \\
\hline \multirow[t]{2}{*}{231} & $15 \mathrm{Sep}$ & 2.7 \\
\hline & $27 \mathrm{Nov}$ & 2.7 \\
\hline 232 & 22 Sep & 6.5 \\
\hline \multirow[t]{2}{*}{233} & $15 \mathrm{Sep}$ & 2.2 \\
\hline & 27 Nov & 4.9 \\
\hline \multirow[t]{2}{*}{234} & $15 \mathrm{Sep}$ & 12 \\
\hline & 27 Nov & 4.3 \\
\hline 241 & 22 Sep & 4.3 \\
\hline 242 & $22 \mathrm{Sep}$ & 2.2 \\
\hline \multirow[t]{2}{*}{243} & 22 Sep & 3.8 \\
\hline & $08 \mathrm{Dec}$ & -16 \\
\hline \multirow[t]{2}{*}{244} & 22 Sep & 5.9 \\
\hline & $08 \mathrm{Dec}$ & 22 \\
\hline 245 & 22 Sep & 3.0 \\
\hline \multirow[t]{2}{*}{247} & $15 \mathrm{Sep}$ & 14 \\
\hline & $27 \mathrm{Nov}$ & 5.9 \\
\hline \multirow[t]{2}{*}{248} & $22 \mathrm{Sep}$ & 0.5 \\
\hline & $08 \mathrm{Dec}$ & -51 \\
\hline \multirow[t]{2}{*}{249} & $15 \mathrm{Sep}$ & 3.5 \\
\hline & 27 Nov & 8.4 \\
\hline \multirow[t]{2}{*}{250} & $15 \mathrm{Sep}$ & 3.0 \\
\hline & 27 Nov & 3.0 \\
\hline \multirow[t]{2}{*}{261} & 22 Sep & 1.6 \\
\hline & 27 Nov & 23 \\
\hline \multirow[t]{2}{*}{262} & 22 Sep & 1.1 \\
\hline & 27 Nov & 3.0 \\
\hline \multirow[t]{2}{*}{265} & 22 Sep & 54 \\
\hline & 27 Nov & 76 \\
\hline 266 & 22 Sep & 3.2 \\
\hline \multirow[t]{2}{*}{267} & 22 Sep & 7.6 \\
\hline & 27 Nov & 4.6 \\
\hline \multirow[t]{2}{*}{268} & 11 Sep & 5.4 \\
\hline & 27 Nov & 4.9 \\
\hline \multirow[t]{2}{*}{281} & 15 Sep & 11 \\
\hline & 27 Nov & 11 \\
\hline \multirow[t]{2}{*}{282} & $15 \mathrm{Sep}$ & 250 \\
\hline & $27 \mathrm{Nov}$ & 190 \\
\hline \multirow[t]{2}{*}{283} & 22 Sep & 1.9 \\
\hline & 27 Nov & 81 \\
\hline \multirow[t]{2}{*}{284} & $22 \mathrm{Sep}$ & 7.0 \\
\hline & 27 Nov & 13 \\
\hline 285 & $21 \mathrm{Feb}$ & 35 \\
\hline & $22 \mathrm{Sep}$ & 16 \\
\hline 291 & $15 \mathrm{Sep}$ & 0.97 \\
\hline & $08 \mathrm{Dec}$ & 24 \\
\hline
\end{tabular}


Table 2.2.84. 1989 mercury concentrations in ORNL area surface water"

\begin{tabular}{|c|c|c|c|c|c|c|}
\hline \multirow{2}{*}{ Station } & \multirow{2}{*}{$\begin{array}{c}\text { Number of } \\
\text { samples }\end{array}$} & \multicolumn{4}{|c|}{ Concentration $(\mu \mathrm{g} / \mathrm{L})$} & \multirow{2}{*}{$\begin{array}{c}\text { Percentage } \\
\text { TWQ }^{b}\end{array}$} \\
\hline & & $\operatorname{Max}$ & Min & Av & Std. error & \\
\hline \multicolumn{7}{|c|}{ First Creek } \\
\hline 141 & 6 & $<0.050$ & $<0.050$ & $<0.050$ & 0 & $<2.1$ \\
\hline 142 & 6 & $<0.050$ & $<0.050$ & $<0.050$ & 0 & $<2.1$ \\
\hline 143 & 6 & $<0.050$ & $<0.050$ & $<0.050$ & 0 & $<2.1$ \\
\hline 241 & 6 & $<0.050$ & $<0.050$ & $<0.050$ & 0 & $<2.1$ \\
\hline 243 & 6 & $<0.050$ & $<0.050$ & $<0.050$ & 0 & $<2.1$ \\
\hline 244 & 6 & $<0.050$ & $<0.050$ & $<0.050$ & 0 & $<2.1$ \\
\hline 246 & 6 & 0.060 & $<0.050$ & $<0.052$ & 0.0017 & $<2.2$ \\
\hline 247 & 6 & $<0.050$ & $<0.050$ & $<0.050$ & 0 & $<2.1$ \\
\hline 248 & 6 & $<0.050$ & $<0.050$ & $<0.050$ & 0 & $<2.1$ \\
\hline 341 & 6 & 0.39 & $<0.050$ & $<0.21$ & 0.071 & $<8.7$ \\
\hline 342 & 6 & $<0.050$ & $<0.050$ & $<0.050$ & 0 & $<2.1$ \\
\hline 343 & 6 & $<0.050$ & $<0.050$ & $<0.050$ & 0 & $<2.1$ \\
\hline 344 & 3 & $<0.050$ & $<0.050$ & $<0.050$ & 0 & $<2.1$ \\
\hline $\mathrm{X} 12$ & 6 & $<0.050$ & $<0.050$ & $<0.050$ & 0 & $<2.1$ \\
\hline \multicolumn{7}{|l|}{ Stream } \\
\hline summary & 81 & 0.39 & $<0.050$ & $<0.062$ & 0.0067 & 2.6 \\
\hline \multicolumn{7}{|c|}{ Fifth Creek } \\
\hline 161 & 6 & $<0.050$ & $<0.050$ & $<0.050$ & 0 & $<2.1$ \\
\hline 162 & 6 & $<0.050$ & $<0.050$ & $<0.050$ & 0 & $<2.1$ \\
\hline 163 & 6 & $<0.050$ & $<0.050$ & $<0.050$ & 0 & $<2.1$ \\
\hline 164 & 6 & $<0.050$ & $<0.050$ & $<0.050$ & 0 & $<2.1$ \\
\hline 261 & 6 & 0.50 & $<0.050$ & $<0.19$ & 0.073 & $<8.0$ \\
\hline 262 & 6 & $<0.050$ & $<0.050$ & $<0.050$ & 0 & $<2.1$ \\
\hline 265 & 6 & $<0.050$ & $<0.050$ & $<0.050$ & 0 & $<2.1$ \\
\hline 268 & 6 & $<0.050$ & $<0.050$ & $<0.050$ & 0 & $<2.1$ \\
\hline 361 & 6 & $<0.050$ & $<0.050$ & $<0.050$ & 0 & $<2.1$ \\
\hline 362 & 6 & $<0.050$ & $<0.050$ & $<0.050$ & 0 & $<2.1$ \\
\hline 363 & 6 & 0.60 & 0.10 & 0.30 & 0.099 & 13 \\
\hline 364 & 6 & $<0.050$ & $<0.050$ & $<0.050$ & 0 & $<2.1$ \\
\hline 365 & 6 & $<0.050$ & $<0.050$ & $<0.050$ & 0 & $<2.1$ \\
\hline 366 & 6 & $<0.050$ & $<0.050$ & $<0.050$ & 0 & $<2.1$ \\
\hline 367 & 6 & 3.7 & $<0.050$ & $<1.5$ & 0.68 & $<64$ \\
\hline 368 & 6 & $<0.050$ & $<0.050$ & $<0.050$ & 0 & $<2.1$ \\
\hline$\times 10$ & 3 & $<0.050$ & $<0.050$ & $<0.050$ & 0 & $<2,1$ \\
\hline \multicolumn{7}{|l|}{ Stream } \\
\hline summary & 99 & 3.7 & $<0.050$ & $<0.16$ & 0.053 & 6.8 \\
\hline \multicolumn{7}{|c|}{ Melton Branch } \\
\hline 181 & 6 & $<0.050$ & $<0.050$ & $<0.050$ & 0 & $<2.1$ \\
\hline 281 & 6 & $<0.050$ & $<0.050$ & $<0.050$ & 0 & $<2.1$ \\
\hline 283 & 6 & $<0.050$ & $<0.050$ & $<0.050$ & 0 & $<2.1$ \\
\hline 381 & 6 & $<0.050$ & $<0.050$ & $<0.050$ & 0 & $<2.1$ \\
\hline 382 & 6 & $<0.050$ & $<0.050$ & $<0.050$ & 0 & $<2.1$ \\
\hline 383 & 6 & $<0.050$ & $<0.050$ & $<0.050$ & 0 & $<2.1$ \\
\hline 384 & 6 & $<0.050$ & $<0.050$ & $<0.050$ & 0 & $<2.1$ \\
\hline 385 & 3 & $<0.050$ & $<0.050$ & $<0.050$ & 0 & $<2.1$ \\
\hline 386 & 6 & $<0.050$ & $<0.050$ & $<0.050$ & 0 & $<2.1$ \\
\hline
\end{tabular}


Table 2.2.84 (continued)

\begin{tabular}{|c|c|c|c|c|c|c|}
\hline \multirow{2}{*}{ Station } & \multirow{2}{*}{$\begin{array}{c}\text { Number of } \\
\text { samples }\end{array}$} & \multicolumn{4}{|c|}{ Concentration $(\mu \mathrm{g} / \mathrm{L})$} & \multirow{2}{*}{$\begin{array}{c}\text { Percentage } \\
\text { TWQ }^{b}\end{array}$} \\
\hline & & $\operatorname{Max}$ & Min & Av & Std. error & \\
\hline HDWTR & 6 & $<0.050$ & $<0.050$ & $<0.050$ & 0 & $<2.1$ \\
\hline MBS & 6 & $<0.050$ & $<0.050$ & $<0.050$ & 0 & $<2.1$ \\
\hline MHD & 6 & $<0.050$ & $<0.050$ & $<0.050$ & 0 & $<2.1$ \\
\hline X08 & 3 & $<0.050$ & $<0.050$ & $<0.050$ & 0 & $<2.1$ \\
\hline $\mathrm{X} 09$ & 6 & $<0.050$ & $<0.050$ & $<0.050$ & 0 & $<2.1$ \\
\hline \multicolumn{7}{|l|}{ Stream } \\
\hline summary & 78 & $<0.050$ & $<0, n \leq 0$ & $<0.050$ & 0 & 2.1 \\
\hline \multicolumn{7}{|c|}{ Northwest Tributary } \\
\hline $\mathrm{X} 03$ & 3 & $<0.050$ & $<0.050$ & $<0.050$ & 0 & $<2.1$ \\
\hline \multicolumn{7}{|l|}{ Stream } \\
\hline summary & 3 & $<0.050$ & $<0.050$ & $<0.050$ & 0 & 2.1 \\
\hline \multicolumn{7}{|c|}{ White Oak Creek } \\
\hline 101 & 6 & 0.20 & $<0.050$ & $<0.092$ & 0.024 & $<3.8$ \\
\hline 103 & 6 & 0.070 & $<0.050$ & $<0.057$ & 0.0033 & $<2.4$ \\
\hline 106 & 6 & 0.070 & $<0.050$ & $<0.055$ & 0.0034 & $<2.3$ \\
\hline 109 & 6 & $<0.050$ & $<0.050$ & $<0.050$ & 0 & $<2.1$ \\
\hline 116 & 6 & $<0.050$ & $<0.050$ & $<0.050$ & 0 & $<2.1$ \\
\hline 202 & 6 & 0.70 & $<0.050$ & $<0.33$ & 0.13 & $<14$ \\
\hline 204 & 6 & 0.11 & $<0.050$ & $<0.072$ & 0.010 & $<3.0$ \\
\hline 206 & 6 & 1.0 & 0.060 & 0.39 & 0.17 & 16 \\
\hline 207 & 6 & 0.20 & $<0.050$ & $<0.10$ & 0.031 & $<4.3$ \\
\hline 208 & 6 & $<0.050$ & $<0.050$ & $<0.050$ & 0 & $<2.1$ \\
\hline 209 & 6 & 0.15 & $<0.050$ & $<0 .\ulcorner 98$ & 0.022 & $<4.1$ \\
\hline 210 & 6 & 0.060 & $<0.050$ & $<0.052$ & 0.0017 & $<2.2$ \\
\hline 216 & 6 & $<0.050$ & $<0.050$ & $<0.050$ & 0 & $<2.1$ \\
\hline 217 & 6 & $<0.050$ & $<0.050$ & $<0.050$ & 0 & $<2.1$ \\
\hline 218 & 6 & $<0.050$ & $<0.050$ & $<0.050$ & 0 & $<2.1$ \\
\hline 222 & 6 & $<0.050$ & $<0.050$ & $<0.050$ & 0 & $<2.1$ \\
\hline 223 & 6 & $<0.050$ & $<0.050$ & $<0.050$ & 0 & $<2.1$ \\
\hline 230 & 6 & $<0.050$ & $<0.050$ & $<0.050$ & 0 & $<2.1$ \\
\hline 232 & 6 & $<0.050$ & $<0.050$ & $<0.050$ & 0 & $<2.1$ \\
\hline 233 & 6 & $<0.050$ & $<0.050$ & $<0.050$ & 0 & $<2.1$ \\
\hline 234 & 6 & $<0.050$ & $<0.050$ & $<0.050$ & 0 & $<2.1$ \\
\hline 301 & 6 & 0.10 & 0.050 & 0.062 & 0.0079 & 2.6 \\
\hline 302 & 6 & 0.27 & 0.050 & 0.15 & 0.045 & 6.3 \\
\hline 303 & 6 & 0.24 & $<0.050$ & $<0.10$ & 0.031 & $<4.2$ \\
\hline 304 & 6 & 3.3 & 0.38 & 1.2 & 0.50 & 49 \\
\hline 305 & 6 & 0.10 & 0.050 & 0.065 & 0.0096 & 2.7 \\
\hline 306 & 6 & 0.080 & $<0.050$ & $<0.065$ & 0.0067 & $<2.7$ \\
\hline 307 & 6 & $<0.050$ & $<0.050$ & $<0.050$ & 0 & $<2.1$ \\
\hline 308 & 6 & 0.10 & $<0.050$ & $<0.068$ & 0.0087 & $<2.8$ \\
\hline 309 & 6 & 0.70 & 0.090 & 0.35 & 0.12 & 15 \\
\hline 310 & 6 & 0.060 & $<0.050$ & $<0.052$ & 0.0017 & $<2.2$ \\
\hline 311 & 6 & $<0.050$ & $<0.050$ & $<0.050$ & 0 & $<2.1$ \\
\hline 312 & 6 & $<0.050$ & $<0.050$ & $<0.050$ & 0 & $<2.1$ \\
\hline 313 & 6 & $<0.050$ & $<0.050$ & $<0.050$ & 0 & $<2.1$ \\
\hline 314 & 6 & $<0.050$ & $<0.050$ & $<0.050$ & 0 & $<2.1$ \\
\hline 7500 & 6 & $<0.050$ & $<0.050$ & $<0.050$ & 0 & $<2.1$ \\
\hline
\end{tabular}


Table 2.2.84 (continued)

\begin{tabular}{|c|c|c|c|c|c|c|}
\hline \multirow{2}{*}{ Station } & \multirow{2}{*}{$\begin{array}{c}\text { Number of } \\
\text { samples }\end{array}$} & \multicolumn{4}{|c|}{ Concentration $(\mu \mathrm{g} / \mathrm{L})$} & \multirow{2}{*}{$\begin{array}{c}\text { Percentage } \\
\text { TWQ }^{b}\end{array}$} \\
\hline & & $\operatorname{Max}$ & Min & Av & Std. error & \\
\hline FLUME & 6 & 0.10 & $<0.050$ & $<0.075$ & 0.011 & $<3.1$ \\
\hline HDW & 6 & $<0.050$ & $<0.050$ & $<0.050$ & 0 & $<2.1$ \\
\hline LSC & 6 & 0.080 & $<0.050$ & $<0.05 \overline{5}$ & 0.0050 & $<2.3$ \\
\hline WOD & 6 & $<0.050$ & $<0.050$ & $<0.050$ & 0 & $<2.1$ \\
\hline X01 & 6 & $<0.050$ & $<0.050$ & $<0.050$ & 0 & $<2.1$ \\
\hline $\mathrm{X} 02$ & 6 & 0.090 & $<0.050$ & $<0.065$ & 0.0072 & $<2.7$ \\
\hline $\mathrm{X} 03$ & 3 & $<0.050$ & $<0.050$ & $<0.050$ & 0 & $<2.1$ \\
\hline X04 & 6 & 0.10 & $<0.050$ & $<0.075$ & 0.011 & $<3,1$ \\
\hline $\mathrm{X} 06$ & 6 & 0.80 & $<0.050$ & $<0.43$ & 0.17 & $<18$ \\
\hline X07 & 6 & 0.14 & $<0.050$ & $<0.093$ & 0.019 & $<3.9$ \\
\hline $\mathrm{X} \| 1$ & 6 & 0.10 & 0.050 & 0.075 & 0.011 & 3.1 \\
\hline \multicolumn{7}{|l|}{ Stream } \\
\hline summary & 279 & 3.3 & $<0.050$ & $<0.11$ & 0.016 & 4.7 \\
\hline \multicolumn{7}{|l|}{ Overall } \\
\hline summary & 540 & 3.7 & $<0.050$ & $<0.11$ & 0.013 & 4.4 \\
\hline
\end{tabular}

${ }^{a}$ See Figs. 2.2.9-2.2.11 in Vol. 1.

${ }^{b}$ Percentage of proposed Tennessee Water Quality Standards for the protection of fish and aquatic life. 
Table 2.2.85. 1989 NPDES Permit Number TN 0002950 Discharge Point K-1700 at ORGDP

\begin{tabular}{|c|c|c|c|c|c|}
\hline \multirow{2}{*}{ Parameter } & \multirow{2}{*}{$\begin{array}{c}\text { No. } \\
\text { samples }\end{array}$} & \multicolumn{3}{|c|}{ Concentration } & \multirow{2}{*}{$\begin{array}{l}\text { Sitd. } \\
\text { error }\end{array}$} \\
\hline & & $\operatorname{Max}$ & Min & Av & \\
\hline 1,1,1-Trichloroethane, $\mu \mathrm{g} / \mathrm{L}$ & 117 & 39 & $<5$ & $<5$ & 3.78 \\
\hline $1,1,2,2$-Tetrachloroethane, $\mu \mathrm{g} / \mathrm{L}$ & 117 & $<5$ & $<5$ & $<5$ & 0 \\
\hline 1,1,2-Trichloroethane, $\mu \mathrm{g} / \mathrm{L}$ & 117 & $<5$ & $<5$ & $<5$ & 0 \\
\hline 1,1-Dichloroethane, $\mu \mathrm{g} / \mathrm{L}$ & 117 & 6 & $<5$ & $<5$ & 1.43 \\
\hline 1,1-Dichloroethene, $\mu \mathrm{g} / \mathrm{L}$ & 117 & 14 & $<5$ & $<5$ & 1.71 \\
\hline 1,2-Dichloroethane, $\mu \mathrm{g} / \mathrm{L}$ & 117 & $<5$ & $<5$ & $<5$ & 0 \\
\hline 1,2-Dichloropropane, $\mu \mathrm{g} / \mathrm{L}$ & 117 & $<5$ & $<5$ & $<5$ & 0 \\
\hline 2-Butanone, $\mu \mathrm{g} / \mathrm{L}$ & 1 & $<10$ & $<10$ & $<10$ & 0 \\
\hline 2-Chloroethylvinyl ether, $\mu \mathrm{g} / \mathrm{L}$ & 116 & $<10$ & $<10$ & $<10$ & 0 \\
\hline 2-Hexanone, $\mu \mathrm{g} / \mathrm{L}$ & 1 & $<10$ & $<10$ & $<10$ & 0 \\
\hline 4-Methyl-2-pentatone, $\mu \mathrm{g} / \mathrm{L}$ & 1 & $<10$ & $<10$ & $<10$ & 0 \\
\hline Acetone, $\mu \mathrm{g} / \mathrm{L}$ & 1 & $<10$ & $<10$ & $<1 \mathrm{C}$ & 0 \\
\hline Aluminum, $\mathrm{mg} / \mathrm{L}$ & 114 & 9.4 & $<0.1$ & $<0.660$ & 1.30 \\
\hline Arsenic, $\mathrm{mg} / \mathrm{L}$ & 1 & $<0.005$ & $<0.005$ & $<0.005$ & 0 \\
\hline Barium, $\mathrm{ng} / \mathrm{L}$ & 1 & $<0.1$ & $<0.1$ & $<0.1$ & 0 \\
\hline Benzene, $\mu \mathrm{g} / \mathrm{L}$ & 117 & 5 & $<5$ & $<5$ & 0.57 \\
\hline Beryllium, mg/L & 115 & $<0.001$ & $<0.0003$ & $<0.001$ & 0 \\
\hline Boron, $\mathrm{mg} / \mathrm{L}$ & 1 & 0.072 & 0.072 & 0.072 & 0 \\
\hline Bromodichloromethane, $\mu \mathrm{g} / \mathrm{L}$ & 117 & 5 & $<5$ & $<5$ & 0.77 \\
\hline Bromoform, $\mu \mathrm{g} / \mathrm{L}$ & 117 & $<5$ & $<5$ & $<5$ & 0 \\
\hline Bromomethane, $\mu \mathrm{g} / \mathrm{L}$ & 117 & $<10$ & $<10$ & $<10$ & 0 \\
\hline Cadmium, mg/L & 115 & 0.003 & $<0.002$ & $<0.002$ & 0 \\
\hline Calcium, $\mathrm{mg} / \mathrm{L}$ & 1 & 36 & 36 & 36 & 0 \\
\hline Carbon disulfide, $\mu \mathrm{g} / \mathrm{L}$ & 1 & $<5$ & $<5$ & $<5$ & 0 \\
\hline Carion tetrachloride, $\mu \mathrm{g} / \mathrm{L}$ & 117 & $<5$ & $<5$ & $<5$ & 0 \\
\hline Chemical oxygen demand, $\mathrm{mg} / \mathrm{L}$ & 229 & 159 & $<4$ & $<7.449$ & 10.57 \\
\hline Chlorobenzene, $\mu \mathrm{g} / \mathrm{L}$ & 117 & 5 & $<5$ & $<5$ & 0.18 \\
\hline Chloroethane, $\mu \mathrm{g} / \mathrm{L}$ & 117 & $<10$ & $<10$ & $<10$ & 0 \\
\hline Chloroform, $\mu \mathrm{g} / \mathrm{L}$ & 117 & 10 & $<5$ & $<5$ & 1.72 \\
\hline Chloromethane, $\mu \mathrm{g} / \mathrm{L}$ & 117 & $<10$ & $<10$ & $<10$ & 0 \\
\hline Chromium, mg/L & 115 & 0.025 & $<0.01$ & $<0.010$ & 0 \\
\hline Cis-1,3-dichloropropene, $\mu \mathrm{g} / \mathrm{L}$ & 117 & $<5$ & $<5$ & $<5$ & 0 \\
\hline Cobalt, $\mathrm{mg} / \mathrm{L}$ & 1 & $<0.1$ & $<0.1$ & $<0.1$ & 0 \\
\hline Copper, $\mathrm{mg} / \mathrm{L}$ & 1 & 0.011 & 0.011 & 0.011 & 0 \\
\hline Dibromochloromethane, $\mu \mathrm{g} / \mathrm{L}$ & 117 & $<5$ & $<5$ & $<5$ & 0 \\
\hline Dissolved solids, $\mathrm{mg} / \mathrm{L}$ & 115 & 996 & 80 & 377.339 & 194.50 \\
\hline Ethyl benzene, $\mu \mathrm{g} / \mathrm{L}$ & 117 & $<5$ & $<5$ & $<5$ & 0 \\
\hline Fluoride, $\mathrm{mg} / \mathrm{L}$ & 113 & 1.7 & 0.1 & 0.388 & 0.34 \\
\hline Iron, $\mathrm{mg} / \mathrm{L}$ & 2 & 3.2 & 0.84 & 2.020 & 1.67 \\
\hline Lead, $\mathrm{mg} / \mathrm{L}$ & 115 & 0.4 & $<0.004$ & $<0.008$ & 0.04 \\
\hline Lithium, mg/L & 1 & 0.0054 & 0.0054 & 0.005 & 0 \\
\hline Magnnesium, mg/L & 1 & 5.9 & 5.9 & 5.9 & 0 \\
\hline Manganese, $\mathrm{mg} / \mathrm{L}$ & 1 & 0.093 & 0.093 & 0.093 & 0 \\
\hline MBAS, $\mathrm{mg} / \mathrm{L}$ & 1 & $<0.2$ & $<0.2$ & $<0.2$ & 0 \\
\hline Mercury, $\mathrm{mg} / \mathrm{L}$ & 115 & $<0.00065$ & $<0.0002$ & $<0.001$ & 0 \\
\hline Methylene chloride, $\mu \mathrm{g} / \mathrm{L}$ & 117 & 5 & $<5$ & $<5$ & 1.23 \\
\hline Molybdenum, mg/L & 1 & $<0.01$ & $<0.01$ & $<0.01$ & 0 \\
\hline Nickel, mg/L & 1 & $<0.05$ & $<0.05$ & $<0.05$ & 0 \\
\hline Niobium, mg/L & 1 & $<0.007$ & $<0.007$ & $<0.007$ & 0 \\
\hline
\end{tabular}


Table 2.2.85 (Continued)

\begin{tabular}{|c|c|c|c|c|c|}
\hline \multirow{2}{*}{ Parameter } & \multirow{2}{*}{$\begin{array}{c}\text { No. } \\
\text { samples }\end{array}$} & \multicolumn{3}{|c|}{ Concentration } & \multirow{2}{*}{$\begin{array}{l}\text { Std. } \\
\text { error }\end{array}$} \\
\hline & & $\operatorname{Max}$ & Min & Av & \\
\hline Nitrate nitrogen, $\mathrm{mg} / \mathrm{L}$ & 113 & 4 & 0.2 & 0.476 & 0.48 \\
\hline Oil and grease, $\mathrm{mg} / \mathrm{L}$ & 117 & $<2$ & $<2$ & $<2$ & 0 \\
\hline pH, units & 388 & 8.6 & 2.8 & $\mathrm{NA}^{a}$ & NA \\
\hline Phosphorus, mg/L & 1 & $<0.2$ & $<0.2$ & $<0.2$ & 0 \\
\hline Potassium, mg/L & 1 & 3.6 & 3.6 & 3.6 & 0 \\
\hline Selenium, mg/L & 115 & $<0.005$ & $<0.004$ & $<0.005$ & 0 \\
\hline Silicon, mg/L & 1 & 6.1 & 6.1 & 6.1 & 0 \\
\hline Silver, $\mathrm{mg} / \mathrm{L}$ & 115 & $<0.01$ & $<0.006$ & $<0.010$ & 0 \\
\hline Sodium, mg/L & 1 & 8.7 & 8.7 & 8.7 & 0 \\
\hline Strontium, mg/L & 1 & 0.082 & 0.082 & 0.082 & 0 \\
\hline Styrene, $\mu \mathrm{g} / \mathrm{L}$ & 1 & $<5$ & $<5$ & 5 & 0 \\
\hline Suspended solids, $\mathrm{mg} / \mathrm{L}$ & 219 & 50 & $<1$ & $<6.995$ & 8.19 \\
\hline Temperature, ${ }^{\circ} \mathrm{C}$ & 388 & 30.3 & 5 & 15.854 & 5.42 \\
\hline Tetrachloroethene, $\mu \mathrm{g} / \mathrm{L}$ & 117 & 98 & $<5$ & $<5$ & 9.81 \\
\hline Thorium, mg/L & 1 & $<0.2$ & $<0.2$ & $<0.2$ & 0 \\
\hline Titanium, mg/L & 1 & 0.057 & 0.057 & 0.057 & 0 \\
\hline Toluene, $\mu \mathrm{g} / \mathrm{L}$ & 117 & 5 & $<5$ & $<5$ & 1.75 \\
\hline Total xylenes, $\mu \mathrm{g} / \mathrm{L}$ & 1 & $<5$ & $<5$ & $<5$ & 0 \\
\hline Trans-1,2-dichloroethene, $\mu \mathrm{g} / \mathrm{L}$ & 116 & 50 & $<2$ & $<28.353$ & 12.46 \\
\hline Trans-1,3-dichloropropene, $\mu \mathrm{g} / \mathrm{L}$ & 117 & 32 & $<5$ & $<5.230$ & 2.50 \\
\hline Trichloroethene, $\mu \mathrm{g} / \mathrm{L}$ & 117 & 72 & $<2$ & $<33.752$ & 14.05 \\
\hline Turbidity, NTU & 228 & 1400 & 1.1 & 18.843 & 93.60 \\
\hline Vanadium, $\mathrm{mg} / \mathrm{L}$ & 1 & $<0.5$ & $<0.5$ & $<0.5$ & 0 \\
\hline Vinyl acetate, $\mu \mathrm{g} / \mathrm{L}$ & 1 & $<10$ & $<10$ & $<10$ & 0 \\
\hline Vinyl chloride, $\mu \mathrm{g} / \mathrm{L}$ & 117 & 10 & $<1$ & $<6.598$ & 2.67 \\
\hline Zinc, $\mathrm{mg} / \mathrm{L}$ & 115 & 0.075 & $<0.004$ & $<0.022$ & 0.01 \\
\hline
\end{tabular}

${ }^{a} \mathrm{NA}=$ not applicable. 
Table 2.2.86. 1989 NPDES Permit Number TN 002950

Discharge Point K-1203 at ORGDP

\begin{tabular}{|c|c|c|c|c|c|}
\hline \multirow{2}{*}{ Parameter } & \multirow{2}{*}{$\begin{array}{c}\text { No. } \\
\text { samples }\end{array}$} & \multicolumn{3}{|c|}{ Concentration } & \multirow{2}{*}{$\begin{array}{l}\text { Std. } \\
\text { error }\end{array}$} \\
\hline & & $\operatorname{Max}$ & $\operatorname{Min}$ & Av & \\
\hline Ammonia nitrogen, $\mathrm{mg} / \mathrm{L}$ & 166 & 0.31 & $<0.2$ & $<0.201$ & 0.01 \\
\hline Biological oxygen demand (BOD), $\mathrm{mg} / \mathrm{L}$ & 171 & 16 & $<1.7$ & $<5.105$ & 1.29 \\
\hline Chemical oxygen demand (COD), $\mathrm{mg} / \mathrm{L}$ & 224 & 300 & $<5$ & $<13.906$ & 29.87 \\
\hline Chlorine, $\mathrm{mg} / \mathrm{L}$ & 375 & 0.51 & 0.02 & 0.075 & 0.04 \\
\hline Dissolved oxygen, $\mathrm{mg} / \mathrm{L}$ & 383 & 18 & 5 & 8.955 & 1.32 \\
\hline Dissolved solids, $\mathrm{mg} / \mathrm{L}$ & 54 & 268 & 30 & 221.814 & 31.71 \\
\hline Fecal coliform, col $/ 100 \mathrm{~mL}$ & 167 & 620 & $<1$ & $<10.149$ & 54.70 \\
\hline $\mathrm{pH}$, units & 383 & 8.5 & 7.1 & $\mathrm{NA}^{a}$ & NA \\
\hline Settleable solids, $\mathrm{mg} / \mathrm{L}$ & 278 & 0.6 & $<0.1$ & $<0.116$ & 0.05 \\
\hline Suspended solids, $\mathrm{mg} / \mathrm{L}$ & 168 & 28 & $<1$ & $<6.119$ & 4.34 \\
\hline Temperature, ${ }^{\circ} \mathrm{C}$ & 383 & 27.8 & 4.4 & 18.650 & 4.73 \\
\hline Total organic carbon, $\mathrm{mg} / \mathrm{L}$ & 54 & 24 & 1 & 5.518 & 4.11 \\
\hline
\end{tabular}

${ }^{a} \mathrm{NA}=$ not applicable.

Table 2.2.87. 1989 NPDES Permit Number TN 0002950

Discharge Point K-1007-B at ORGDP

\begin{tabular}{|c|c|c|c|c|c|}
\hline \multirow{2}{*}{ Parameter } & \multirow{2}{*}{$\begin{array}{c}\text { No. } \\
\text { samples }\end{array}$} & \multicolumn{3}{|c|}{ Concentration } & \multirow{2}{*}{$\begin{array}{l}\text { Std. } \\
\text { error }\end{array}$} \\
\hline & & Max & Min & Av & \\
\hline Chemical oxygen demand (COD), $\mathrm{mg} / \mathrm{L}$ & 114 & 24 & $<4$ & $<8.210$ & 4.08 \\
\hline Chromium, $\mathrm{mg} / \mathrm{L}$ & 58 & $<0.01$ & $<0.01$ & $<0.01$ & 0 \\
\hline Dissolved oxygen, $\mathrm{mg} / \mathrm{L}$ & 383 & 16 & 5 & 9.362 & 2.17 \\
\hline Fluoride, $\mathrm{mg} / \mathrm{L}$ & 58 & 0.2 & $<0.1$ & $<0.149$ & 0.05 \\
\hline Oil and grease, $\mathrm{mg} / \mathrm{L}$ & 56 & $<2$ & $<2$ & $<2.000$ & 0 \\
\hline $\mathrm{pH}$, units & 385 & 9.1 & 7.1 & $\mathrm{NA}^{a}$ & NA \\
\hline Suspended solids, $\mathrm{mg} / \mathrm{L}$ & 113 & 15 & $<1$ & $<6.106$ & 2.42 \\
\hline Temperature, ${ }^{\circ} \mathrm{C}$ & 385 & 30.8 & 2.5 & 17.143 & 8.05 \\
\hline
\end{tabular}

${ }^{a} \mathrm{NA}=$ not applicable. 
Table 2.2.88. 1989 NPDES Permit Number TN 0002950 Discharge Point K-901A at ORGDP

\begin{tabular}{|c|c|c|c|c|c|}
\hline \multirow{2}{*}{ Parameter } & \multirow{2}{*}{$\begin{array}{c}\text { No. } \\
\text { samples }\end{array}$} & \multicolumn{3}{|c|}{ Concentration } & \multirow{2}{*}{$\begin{array}{l}\text { Std. } \\
\text { error }\end{array}$} \\
\hline & & $\operatorname{Max}$ & Min & Av & \\
\hline Chemical oxygen demand (COD), $\mathrm{mg} / \mathrm{L}$ & 114 & 20 & $<4$ & $<6.903$ & 3.14 \\
\hline Chromium, $\mathrm{mg} / \mathrm{L}$ & 61 & 0.1 & $<0.01$ & $<0.015$ & 0.01 \\
\hline Dissolved oxygen, $\mathrm{mg} / \mathrm{L}$ & 372 & 17 & 3.8 & 8.048 & 2.44 \\
\hline Fluoride, $\mathrm{mg} / \mathrm{L}$ & 58 & 0.2 & $<0.1$ & $<0.128$ & 0.04 \\
\hline Oil and grease, $\mathrm{mg} / \mathrm{L}$ & 59 & 5.3 & $<2$ & $<2.055$ & 0.43 \\
\hline $\mathrm{pH}$, units & 372 & 8.7 & 6.5 & $\mathrm{NA}^{a}$ & NA \\
\hline Suspended solids, $\mathrm{mg} / \mathrm{L}$ & 113 & 22 & 1 & 7.477 & 4.10 \\
\hline Temperature, ${ }^{\circ} \mathrm{C}$ & 372 & 28.9 & 1.4 & 16.294 & 7.42 \\
\hline Turbidity, NTU & 113 & 26 & 2.7 & 11.252 & 5.24 \\
\hline
\end{tabular}

${ }^{a} \mathrm{NA}=$ not applicable.

Table 2.2.89. 1989 NPDES Permit Number TN 0002950 Discharge Point K-1515 at OR.GDP

\begin{tabular}{|c|c|c|c|c|c|}
\hline \multirow{2}{*}{ Parameter } & \multirow{2}{*}{$\begin{array}{l}\text { No. } \\
\text { samples }\end{array}$} & \multicolumn{3}{|c|}{ Concentration } & \multirow{2}{*}{$\begin{array}{l}\text { Std. } \\
\text { error }\end{array}$} \\
\hline & & $\operatorname{Max}$ & Min & Av & \\
\hline Aluminum, $\mathrm{mg} / \mathrm{L}$ & 52 & 0.76 & $<0.1$ & $<0.385$ & 0.15 \\
\hline Chemical oxygen demand (COD), $\mathrm{mg} / \mathrm{L}$ & 52 & 19 & $<4$ & $<6.173$ & 2.46 \\
\hline $\mathrm{pH}$, units & 361 & 9 & 6.3 & $\mathrm{NA}^{a}$ & $\mathrm{NA}$ \\
\hline Sulfate, $\mathrm{mg} / \mathrm{L}$ & 52 & 26 & 7 & 19.038 & 4.48 \\
\hline Suspended solids, $\mathrm{mg} / \mathrm{L}$ & 51 & 10 & $<1$ & $<4.039$ & 2.13 \\
\hline Temperature, ${ }^{\circ} \mathrm{C}$ & 359 & 30.3 & 0.5 & 15.692 & 7.38 \\
\hline Total residual chlorine, $\mathrm{mg} / \mathrm{L}$ & 64 & 2.08 & $<0.01$ & $<0.179$ & 0.43 \\
\hline
\end{tabular}

${ }^{a}$ Not applicable. 
Table 2.2.90. 1989 N.'DES Permit Number TN 0002950

Discharge Point K-1407-J at ORGDP

\begin{tabular}{|c|c|c|c|c|c|}
\hline \multirow{2}{*}{ Parameter } & \multirow{2}{*}{$\begin{array}{c}\text { No. } \\
\text { samples }\end{array}$} & \multicolumn{3}{|c|}{ Concentration } & \multirow{2}{*}{$\begin{array}{l}\text { Std. } \\
\text { error }\end{array}$} \\
\hline & & Max & Min & Av & \\
\hline Total toxic organics (TTO), $\mu \mathrm{g} / \mathrm{L}$ & 11 & 2123 & $<5$ & $<145$ & \\
\hline 1,1,1-Trichloroethane, $\mu \mathrm{g} / \mathrm{L}$ & 119 & 140 & $<0.2$ & $<9.048$ & 16.46 \\
\hline $1,1,2,2$-Tetrachloroethane, $\mu \mathrm{g} / \mathrm{L}$ & 119 & 5 & $<.5$ & $<5$ & 0 \\
\hline $1,1,2$-Trichloroethane, $\mu \mathrm{g} / \mathrm{L}$ & 119 & $<5$ & $<5$ & $<5$ & 0 . \\
\hline 1,1-Dichloroethane, $\mu \mathrm{g} / \mathrm{L}$ & 119 & 36 & $<5$ & $<5.259$ & 3.48 \\
\hline 1,1 -Dichloroethene, $\mu \mathrm{g} / \mathrm{L}$ & 119 & 100 & $<5$ & $<5.864$ & 9.07 \\
\hline 1,2 4-Trichlorobenzene, $\mu \mathrm{g} / \mathrm{L}$ & 45 & 12 & $<5$ & $<9.022$ & 2.28 \\
\hline 1,2-Dichlorobenzene, $\mu \mathrm{g} / \mathrm{L}$ & 45 & $<12$ & $<5$ & $<9.088$ & 2.11 \\
\hline 1,2-Dichloroethane, $\mu \mathrm{g} / \mathrm{L}$ & 119 & $<5$ & $<5$ & $<5$ & 0 \\
\hline 1,2-Dichloropropane, $\mu \mathrm{g} / \mathrm{L}$ & 119 & $<5$ & $<5$ & $<5$ & 0 \\
\hline 1,3-Dichlorobenzene, $\mu \mathrm{g} / \mathrm{L}$ & 45 & $<12$ & $<5$ & $<9.088$ & 2.11 \\
\hline 1,4-Dichlorobenzene, $\mu \mathrm{g} / \mathrm{L}$ & 45 & $<12$ & $<5$ & $<9.088$ & 2.11 \\
\hline $2,4,5$-Trichlorophenol, $\mu \mathrm{g} / \mathrm{L}$ & 1 & $<50$ & $<50$ & $<50$ & 0 \\
\hline $2,4,6$-Trichlorophenol, $\mu \mathrm{g} / \mathrm{L}$ & 45 & $<12$ & $<5$ & $<9.088$ & 2.11 \\
\hline 2,4-Dichlorophenol, $\mu \mathrm{g} / \mathrm{L}$ & 45 & $<12$ & $<5$ & $<9.088$ & 2.11 \\
\hline 2,4-Dimethylphenol, $\mu \mathrm{g} / \mathrm{L}$ & 45 & $<12$ & $<5$ & $<9.088$ & 2.11 \\
\hline 2,4-Dinitrophenol, $\mu \mathrm{g} / \mathrm{L}$ & 45 & $<50$ & $<5$ & $<9.977$ & 6.45 \\
\hline 2,4-Dinitrotoluene, $\mu \mathrm{g} / \mathrm{L}$ & 45 & $<12$ & $<5$ & $<9.083$ & 2.11 \\
\hline 2,6-Dinitrotoluene, $\mu \mathrm{g} / \mathrm{L}$ & 45 & $<12$ & $<5$ & $<9.088$ & 2.11 \\
\hline 2-Chloroethylvinyl ether, $\mu \mathrm{g} / \mathrm{L}$ & 119 & $<10$ & $<10$ & $<10$ & 0 \\
\hline 2-Chloronaphthalene, $\mu \mathrm{g} / \mathrm{L}$ & 45 & $<12$ & $<5$ & $<9.088$ & 2.11 \\
\hline 2-Chlorophenol, $\mu \mathrm{g} / \mathrm{L}$ & 45 & $<12$ & $<5$ & $<9.088$ & 2.11 \\
\hline 2-Methylnaphthalene, $\mu \mathrm{g} / \mathrm{L}$ & 1 & $<10$ & $<10$ & $<10$ & 0 \\
\hline 2-Methylphenol, $\mu \mathrm{g} / \mathrm{L}$ & 1 & $<10$ & $<10$ & $<10$ & 0 \\
\hline 2-Nitroaniline, $\mu \mathrm{g} / \mathrm{L}$ & $i$ & $<50$ & $<50$ & $<50$ & 0 \\
\hline 2-Nitrophenol, $\mu \mathrm{g} / \mathrm{L}$ & 45 & $<12$ & $<5$ & $<9.088$ & 2.11 \\
\hline 3,3'-Dichlorobenzidine, $\mu \mathrm{g} / \mathrm{L}$ & 45 & $<24$ & $<10$ & $<18.222$ & 4.13 \\
\hline 3-Nitroaniline, $\mu \mathrm{g} / \mathrm{L}$ & 1 & $<50$ & $<50$ & $<50$ & 0 \\
\hline 4,6-Dinitro-2-methylphenol, $\mu \mathrm{g} / \mathrm{L}$ & 45 & $<61$ & $<25$ & $<45.555$ & 10.48 \\
\hline 4-Biomophenyl-phenylether, $\mu \mathrm{g} / \mathrm{L}$ & 45 & $<12$ & $<5$ & $<9.088$ & 2.11 \\
\hline 4-Chloro-3-methylphenol, $\mu \mathrm{g} / \mathrm{L}$ & 45 & $<12$ & $<5$ & $<9.088$ & 2.11 \\
\hline 4-Chloroaniline, $\mu \mathrm{g} / \mathrm{L}$ & 1 & $<10$ & $<10$ & $<10$ & 0 \\
\hline 4-Chlorophenyl-phenylether, $\mu \mathrm{g} / \mathrm{L}$ & 45 & $<12$ & $<5$ & $<9.088$ & 2.11 \\
\hline 4-Methylphenol, $\mu \mathrm{g} / \mathrm{L}$ & 1 & $<10$ & $<10$ & $<10$ & 0 \\
\hline 4-Nitroaniline, $\mu \mathrm{g} / \mathrm{L}$ & $i$ & $<50$ & $<50$ & $<50$ & 0 \\
\hline 4-Nitrophenol, $\mu \mathrm{g} / \mathrm{L}$ & 45 & $<61$ & $<25$ & $<45.555$ & 10.48 \\
\hline Acenaphthene, $\mu \mathrm{g} / \mathrm{L}$ & 45 & $<12$ & $<5$ & $<9.088$ & 2.11 \\
\hline Acenaphthylene, $\mu \mathrm{g} / \mathrm{L}$ & 45 & $<12$ & $<5$ & $<9.088$ & 2.11 \\
\hline Aluminum, $\mathrm{mg} / \mathrm{L}$ & 51 & 15 & $<0.1$ & $<0.675$ & 2.08 \\
\hline Ammonia nitrogen, $\mathrm{mg} / \mathrm{L}$ & 32 & 2.79 & $<0.2$ & $<0.350$ & 0.50 \\
\hline Anthracene, $\mu \mathrm{g} / \mathrm{L}$ & 45 & $<12$ & $<5$ & $<9.088$ & 2.11 \\
\hline Antimony, $\mathrm{mg} / \mathrm{L}$ & 50 & $<0.05$ & $<0.05$ & $<0.050$ & 0 \\
\hline Arsenic, $\mathrm{mg} / \mathrm{L}$ & 47 & 0.15 & $<0.005$ & $<0.009$ & 0.02 \\
\hline Barium, mg/L & 51 & 0.14 & $<0.1$ & $<0.101$ & 0.01 \\
\hline Benzene, $\mu \mathrm{g} / \mathrm{L}$ & 119 & $<5$ & $<5$ & $<5$ & 0 . \\
\hline Benzidine, $\mu \mathrm{g} / \mathrm{L}$ & 44 & $<12$ & $<5$ & $<9.068$ & 2.13 \\
\hline Benzo(a)anthracene, $\mu \mathrm{g} / \mathrm{L}$ & 45 & $<12$ & $<5$ & $<9.088$ & 2.11 \\
\hline Benzo(a)pyrene, $\mu \mathrm{g} / \mathrm{L}$ & 45 & $<12$ & $<5$ & $<9.088$ & 2.11 \\
\hline Benzo(b)fluoranthene, $\mu \mathrm{g} / \mathrm{L}$ & 45 & $<12$ & $<5$ & $<9.088$ & 2.11 \\
\hline Benzo(g,h,i)perylene, $\mu \mathrm{g} / \mathrm{L}$ & 45 & 15 & $<5$ & $<9.311$ & 2.19 \\
\hline Benzo(k)fluoranthene, $\mu \mathrm{g} / \mathrm{L}$ & 45 & $<12$ & $<5$ & $<9.088$ & 2.11 \\
\hline Benzoic acid, $\mu \mathrm{g} / \mathrm{L}$ & 1 & $<50$ & $<50$ & $<50$ & 0 \\
\hline
\end{tabular}


Tabi: 2.2 .90 (continued)

\begin{tabular}{|c|c|c|c|c|c|}
\hline \multirow{2}{*}{ Parameter } & \multirow{2}{*}{$\begin{array}{c}\text { No. } \\
\text { samples }\end{array}$} & \multicolumn{3}{|c|}{ Concentration } & \multirow{2}{*}{$\begin{array}{l}\text { Std. } \\
\text { error }\end{array}$} \\
\hline & & $\operatorname{Max}$ & Min & Av & \\
\hline Benzyl alcohol, $\mu \mathrm{g} / \mathrm{L}$ & 1 & $<10$ & $<10$ & $<10$ & 0 \\
\hline Beryllium, $\mathrm{mg} / \mathrm{L}$ & 51 & 0.0016 & $<0.001$ & $<0.001$ & 0 \\
\hline Bis(2-chloroethoxy)methane, $\mu \mathrm{g} / \mathrm{L}$ & 45 & $<12$ & $<5$ & $<9.088$ & 2.11 \\
\hline Bis(2-chloroethyl)ether, $\mu \mathrm{g} / \mathrm{L}$ & 45 & $<12$ & $<5$ & $<9.088$ & 2.11 \\
\hline Bis(2-chloroisopropyl)ether, $\mu \mathrm{g} / \mathrm{L}$ & 45 & $<12$ & $<5$ & $<9.088$ & 2.11 \\
\hline Bis(2-ethylhexyl)phthalate, $\mu \mathrm{g} / \mathrm{L}$ & 45 & 210 & $<2$ & $<25.977$ & 43.63 \\
\hline Boron, $n . g / L$ & 51 & 1.1 & $<0.004$ & $<0.217$ & 0.23 \\
\hline Bromide, $\mathrm{mg} / \mathrm{L}$ & 24 & $<2$ & $<2$ & $<2$ & 0 \\
\hline Bromodichloromethane, $u \mathrm{~g} / \mathrm{L}$ & 119 & 5 & $<0.5$ & $<4.856$ & 0.77 \\
\hline Bromoform, $\mu \mathrm{g} /{ }^{\top}$ & 119 & $<5$ & $<5$ & $<5$ & 0 \\
\hline Bromomethane, $\mu \mathrm{g} / \mathrm{L}$ & 119 & $<10$ & $<10$ & $<10$ & 0 \\
\hline Butylbenzylphthalate. $u \mathrm{~g} / \mathrm{L}$ & 45 & 12 & $<1$ & $<8.844$ & 2.54 \\
\hline Cadmium, mg/L & 51 & 0.0086 & $<0.002$ & $<0.002$ & 0 \\
\hline Calcium, mg/L & 11 & 190 & 68 & 109.090 & 46.38 \\
\hline Carbon tetrachloride, $\mu \mathrm{g} / \mathrm{L}$ & 119 & 5 & $<1$ & $<4.840$ & 0.71 \\
\hline Chemical oxygen demand (COD), $\mathrm{mg} / \mathrm{L}$ & 84 & 157 & $<5$ & $<19.416$ & 20.79 \\
\hline Chloride, $\mathrm{mg} / \mathrm{L}$ & 25 & 1250 & 56 & 396.760 & 336.50 \\
\hline Chlorobenzene, $\mu \mathrm{g} / \mathrm{L}$ & 119 & 5 & $<2$ & $<4.974$ & 0.28 \\
\hline Chloroethane, $\mu \mathrm{g} / \mathrm{L}$ & 119 & $<10$ & $<10$ & $<10$ & 0 \\
\hline Chloroform, $\mu \mathrm{g} / \mathrm{L}$ & 119 & 11 & $<5$ & $<5$ & 1.75 \\
\hline Chloromethane, $\mu \mathrm{g} / \mathrm{L}$ & 119 & $<10$ & $<10$ & $<10$ & 0 \\
\hline Chromium, mg/L & 51 & 0.44 & $<0.01$ & $<0.022$ & 0.06 \\
\hline Chrysene, $\mu \mathrm{g} / \mathrm{L}$ & 45 & $<12$ & $<5$ & $<9.088$ & 2.11 \\
\hline Cis-1,3-dichloropropene, $\mu \mathrm{g} / \mathrm{L}$ & 119 & $<5$ & $<5$ & $<5$ & 0 \\
\hline Cobalt, $\mathrm{mg} / \mathrm{L}$ & 51 & $<0.1$ & $<0.1$ & $<0.100$ & 0 \\
\hline Copper, mg/L & 51 & 0.49 & $<0.004$ & $<0.034$ & 0.07 \\
\hline Cyanide, $\mathrm{mg} / \mathrm{L}$ & 38 & 0.68 & $<0.002$ & $<0.088$ & 0.10 \\
\hline Di-n-butylphthalate, $\mu \mathrm{g} / \mathrm{L}$ & 45 & $<12$ & $<5$ & $<9.088$ & 2.11 \\
\hline Di-n-octylphthalate, $\mu \mathrm{g} / \mathrm{L}$ & 45 & 12 & $<5$ & $<8.426$ & 3.17 \\
\hline $\operatorname{Dibenz}(\mathrm{a}, \mathrm{h})$ anthracene, $\mu \mathrm{g} / \mathrm{L}$ & 45 & 14 & $<5$ & $<9.288$ & 2.14 \\
\hline Dibenzofuran, $\mu \mathrm{g} / \mathrm{L}$ & 1 & $<10$ & $<10$ & $<10$ & 0 \\
\hline Dibromochloromethane, $\mu \mathrm{g} / \mathrm{L}$ & 119 & $<5$ & $<5$ & $<5$ & 0 \\
\hline Diethylphthalate, $\mu \mathrm{g} / \mathrm{L}$ & 45 & 12 & $<5$ & $<8.835$ & 2.54 \\
\hline Dimethylphthalate, $\mu \mathrm{g} / \mathrm{L}$ & 45 & $<12$ & $<5$ & $<9.088$ & 2.11 \\
\hline Dissolved solids, mg/L & 82 & 4096 & 576 & 1582.487 & 844.54 \\
\hline Ethylbenzene, $\mu \mathrm{g} / \mathrm{L}$ & 119 & 5 & $<2$ & $<4.974$ & 0.28 \\
\hline Fluoranthene, $\mu \mathrm{g} / \mathrm{L}$ & 45 & 12 & $<0.5$ & $<8.988$ & 2.39 \\
\hline Fluorene, $\mu \mathrm{g} / \mathrm{L}$ & 45 & $<12$ & $<5$ & $<9.088$ & 2.11 \\
\hline Fluoride, $\mathrm{mg} / \mathrm{L}$ & 79 & 95 & 0.5 & 9.873 & 10.97 \\
\hline Hexachlorobenzene, $\mu \mathrm{g} / \mathrm{L}$ & 45 & $<12$ & $<5$ & $<9.088$ & 2.11 \\
\hline Hexachlorobutadiene, $\mu \mathrm{g} / \mathrm{L}$ & 45 & $<12$ & $<5$ & $<9.088$ & 2.11 \\
\hline Hexachlorocyclopentadiene, $\mu \mathrm{g} / \mathrm{L}$ & 45 & $<12$ & $<5$ & $<9.088$ & 2.11 \\
\hline Hexachloroethane, $\mu \mathrm{g} / \mathrm{L}$ & 45 & $<12$ & $<5$ & $<9.088$ & 2.11 \\
\hline Indeno(1,2,3-cd)pyrene, $\mu \mathrm{g} / \mathrm{L}$ & 45 & 14 & $<5$ & $<9.355$ & 2.05 \\
\hline Iron, $\mathrm{mg} / \mathrm{L}$ & 51 & 42 & 0.17 & 1.994 & 5.87 \\
\hline Isophorone, $\mu \mathrm{g} / \mathrm{L}$ & 45 & $<12$ & $<5$ & $<9.088$ & 2.11 \\
\hline Kjeldahl nitrogen, $\mathrm{mg} / \mathrm{L}$ & 31 & 3.6 & $<0.05$ & $<1.036$ & 0.80 \\
\hline Lead, $\mathrm{mg} / \mathrm{L}$ & 51 & 0.129 & $<0.004$ & $<0.011$ & 0.02 \\
\hline Lithium, mg/L & 11 & 0.027 & 0.0093 & 0.016 & 0.01 \\
\hline Magnesium, mg/L & 51 & 30 & 7.7 & 16.117 & 5.54 \\
\hline Manganese, mg/L & 51 & 0.61 & 0.028 & 0.161 & 0.15 \\
\hline MBAS, mg/L & 25 & $<0.2$ & $<0.2$ & $<0.200$ & 0 \\
\hline Mercury, mg/L & 51 & 0.059 & $<0.0002$ & $<0.001$ & 0.01 \\
\hline
\end{tabular}


Oak Ridge Environmental Report, Vol, 2-1989

Table 2.2.59 (continued)

\begin{tabular}{|c|c|c|c|c|c|}
\hline \multirow{2}{*}{ Parameter } & \multirow{2}{*}{$\begin{array}{c}\text { No. } \\
\text { samples }\end{array}$} & \multicolumn{3}{|c|}{ Concentration } & \multirow{2}{*}{$\begin{array}{l}\text { Std. } \\
\text { error }\end{array}$} \\
\hline & & $\operatorname{Max}$ & Min & Av & \\
\hline Methywale chloride, $\mu \mathrm{g} / \mathrm{L}$ & 119 & 8 & $<0.7$ & $<4.555$ & 1.30 \\
\hline Molybdenum, mg/L & 51 & 0.04 & $<0.01$ & $<0.012$ & 0.01 \\
\hline $\mathrm{N}$-nitroso-di-N-propylamine, $\mu \mathrm{g} / \mathrm{L}$ & 45 & $<12$ & $<5$ & $<9.088$ & 2.11 \\
\hline $\mathrm{N}$-nitrosodimethylamine, $\mu \mathrm{g} / \mathrm{L}$ & 44 & $<12$ & $<5$ & $<9.068$ & 2.13 \\
\hline $\mathrm{N}$-nitrosodiphenylamine, $\mu \mathrm{g} / \mathrm{L}$ & 45 & $<12$ & $<5$ & $<9.088$ & 2.11 \\
\hline Naphthalene, $\mu \mathrm{g} / \mathrm{L}$ & 45 & $<12$ & $<5$ & $<9.088$ & 2.11 \\
\hline Nickel, mg/L & 51 & 2.6 & $<0.05$ & $<0.240$ & 0.38 \\
\hline Niobium, mg/L & 11 & 0.016 & $<0.007$ & $<0.008$ & 0 \\
\hline Nitrate nitrogen, $\mathrm{mg} / \mathrm{L}$ & 56 & 1390 & $<0.2$ & $<26.057$ & 185.59 \\
\hline Nitrite nitrogen, $\mathrm{mg} / \mathrm{L}$ & 2 & 1.4 & 0.9 & 1.150 & 0.35 \\
\hline Nitrobenzene, $\mu \mathrm{g} / \mathrm{L}$ & 45 & $<12$ & $<5$ & $<9.088$ & 2.11 \\
\hline Oil and grease, $\mathrm{mg} / \mathrm{L}$ & 60 & $<2$ & $<2$ & $<2$ & 0 \\
\hline PCB (Aroclor-1016), $\mu \mathrm{g} / \mathrm{L}$ & 36 & $<0.5$ & $<0.5$ & $<0.5$ & 0 \\
\hline PCB (Aroclor-1221), $\mu \mathrm{g} / \mathrm{L}$ & 36 & $<0.5$ & $<0.5$ & $<0.5$ & 0 \\
\hline PCB (Aroclor-1232), $\mu \mathrm{g} / \mathrm{L}$ & 36 & $<0.5$ & $<0.5$ & $<0.5$ & $\mathbf{0}$ \\
\hline PCB (Aroclor-1242), $\mu \mathrm{g} / \mathrm{L}$ & 36 & $<0.5$ & $<0.5$ & $<0.5$ & 0 \\
\hline PCB (Aroclor-1248), $\mu \mathrm{g} / \mathrm{L}$ & 36 & $<0.5$ & $<0.5$ & $<0.5$ & 0 \\
\hline PCB (Aroclor-1254), $\mu \mathrm{g} / \mathrm{L}$ & 36 & $<1$ & $<1$ & $<1$ & 0 \\
\hline PCB (Aroclor -1260$), \mu \mathrm{g} / \mathrm{L}$ & 36 & $<1$ & $<1$ & $<1$ & 0 \\
\hline Pentachlorophenol, $\mu \mathrm{g} / \mathrm{L}$ & 45 & $<61$ & $<25$ & $<45.555$ & : 0.48 \\
\hline $\mathrm{pH}$ & 215 & 9.3 & 6.2 & $<7.969$ & 0.48 \\
\hline Phenanthrene, $\mu \mathrm{g} / \mathrm{L}$ & 45 & 12 & $<1$ & $<8.688$ & 2.69 \\
\hline Phenol, $\mu \mathrm{g} / \mathrm{L}$ & 45 & $<12$ & $<5$ & $<9.088$ & 2.11 \\
\hline Phenols, mg/L & 37 & 0.1 & $<0.001$ & $<0.019$ & 0.02 \\
\hline Phosphate (total) & 5 & 13 & 2.4 & 5.720 & 4.28 \\
\hline Phosphorus, mg/L & 46 & 9.3 & $<0.2$ & $<1.025$ & 1.75 \\
\hline Potassium, mg/l & 11 & 23 & 5.6 & 10.436 & 5.54 \\
\hline Pyrene, $\mu \mathrm{g} / \mathrm{L}$ & 45 & 12 & $<0.4$ & $<8.986$ & 2.40 \\
\hline Selenium, mg/L & 50 & $<0.005$ & $<0.005$ & $<0.005$ & 0 \\
\hline Silicon, mg/L & 11 & 12 & 0.79 & 4.471 & 3.23 \\
\hline Silver, mg/L & 49 & 0.01 & $<0.01$ & $<0.010$ & 0 \\
\hline Sodium, mg/L & 11 & 910 & 59 & 376.272 & 235.48 \\
\hline Strontium, mg/L & 11 & 0.29 & 0.1 & 0.162 & 0.06 \\
\hline Sulfate, $\mathrm{mg} / \mathrm{L}$ & 23 & 1500 & 267 & 548.869 & 324.61 \\
\hline Sulfide, $\mathrm{mg} / \mathrm{L}$ & 28 & $<2$ & $<1$ & $<1.821$ & 0.29 \\
\hline Sulfite, $\mathrm{mg} / \mathrm{L}$ & 24 & $<2$ & $<2$ & $<2$ & 0 \\
\hline Suspended solids, $\mathrm{mg} / \mathrm{L}$ & 83 & 359 & 2 & 19.084 & 42.15 \\
\hline Temperature, ${ }^{\circ} \mathrm{C}$ & 212 & 31.9 & 1.8 & 14.886 & 7.09 \\
\hline Tetrachloroethene, $\mu \mathrm{g} / \mathrm{L}$ & 119 & 240 & $<5$ & $<13.737$ & 25.08 \\
\hline Thallium, mg/L & 47 & $<0.01$ & $<0.01$ & $<0.010$ & 0 \\
\hline Thu ium, mg/L & 10 & $<0.2$ & $<0.2$ & $<0.2$ & 0 \\
\hline Tin, $\mathrm{mg} / \mathrm{L}$ & 56 & 0.8 & $<0.01$ & $<0.092$ & 0.21 \\
\hline Titanium, mg/L & 51 & 0.22 & $<0.003$ & $<0.015$ & 0.03 \\
\hline Toluene, $\mu \mathrm{g} / \mathrm{L}$ & 119 & 5 & $<0.4$ & $<3.788$ & 1.93 \\
\hline Total organic carbon (TOC), $\mathrm{mg} / \mathrm{L}$ & 49 & 20 & 2 & 8.346 & 3.98 \\
\hline Total phosphate, mg/L & 28 & 8.2 & 0.6 & 2.264 & 1.80 \\
\hline Total residual chlorine, $\mathrm{mg} / \mathrm{L}$ & 29 & 0.4 & $<0.01$ & $<0.056$ & 0.10 \\
\hline Trans-1 2-dichloroethene, $\mu \mathrm{g} / \mathrm{L}$ & 119 & 200 & $<5$ & $<13.030$ & 23.50 \\
\hline Trans-1 3-dichloropropene, $\mu \mathrm{g} / \mathrm{L}$ & 119 & $<5$ & $<5$ & $<5$ & 0 \\
\hline Trichloroethene, $\mu \mathrm{g} / \mathrm{L}$ & 119 & 1400 & $<5$ & $<47.866$ & 145.84 \\
\hline Uranium, mg/L & 47 & 2.93 & 0.035 & 0.549 & 0.55 \\
\hline Vanadium, mg/L & 14 & 11 & $<0.5$ & $<0.5$ & 0 \\
\hline Vinyl chloride, $\mu \mathrm{g} / \mathrm{L}$ & 119 & 12 & $<2$ & $<9.823$ & 1.18 \\
\hline Zinc, $\mathrm{mg} / \mathrm{L}$ & 51 & 0.36 & $<0.02$ & $<0.055$ & 0.05 \\
\hline Zirconium, mg/L & 11 & $<0.005$ & $<0.005$ & $<0.005$ & 0 \\
\hline
\end{tabular}


Table 2.2.91. 1989 NPDES Permit Number TN 0002950 Discharge Point K-1407-E/F at ORGDP

\begin{tabular}{|c|c|c|c|c|c|}
\hline \multirow{2}{*}{ Parameter } & \multirow{2}{*}{$\begin{array}{c}\text { No. } \\
\text { samples }\end{array}$} & \multicolumn{3}{|c|}{ Concentration } & \multirow{2}{*}{$\begin{array}{l}\text { Std. } \\
\text { error }\end{array}$} \\
\hline & & Max & Min & Av & \\
\hline Aluminum, $\mathrm{mg} / \mathrm{L}$ & 3 & 0.55 & $<0.1$ & $<0.250$ & 0.26 \\
\hline Arsenic, $\mathrm{mg} / \mathrm{L}$ & 47 & $<0.005$ & $<0.005$ & $<0.005$ & 0 \\
\hline Barium, mg/L & 3 & $<0.1$ & $<0.1$ & $<0.1$ & 0 \\
\hline Beryllium, mg/L & 3 & $<0.001$ & $<0.001$ & $<0.001$ & 0 \\
\hline Boron, $\mathrm{mg} / \mathrm{L}$ & 3 & 0.13 & 0.0068 & 0.064 & 0.06 \\
\hline Cadmium, mg/L & 50 & 0.003 & $<0.002$ & $<0.002$ & 0 \\
\hline Calcium, mg/L & 3 & 220 & 670 & 129.0 & 80.52 \\
\hline Chromium, mg/L & 50 & 0.1 & $<0.01$ & $<0.011$ & 0.01 \\
\hline Cobalt, $\mathrm{mg} / \mathrm{L}$ & 3 & $<0.1$ & $<0.1$ & $<0.1$ & 0 \\
\hline Copper, mg/L & 50 & 0.064 & $<0.004$ & $<0.018$ & 0.01 \\
\hline Iron, $\mathrm{mg} / \mathrm{L}$ & 53 & 4.7 & $<0.05$ & $<0.705$ & 0.73 \\
\hline Lead, mg/L & 50 & 0.05 & $<0.004$ & $<0.007$ & 0.01 \\
\hline Lithium, mg/L & 3 & 0.014 & 0.011 & 0.012 & 0 \\
\hline Magnesium, mg/L & 3 & 24 & 12 & 16.0 & 6.93 \\
\hline Manganese, mg/L & 50 & 0.14 & $<0.03$ & $<0.080$ & 0.03 \\
\hline Molybdenum, mg/L & 3 & $<0.01$ & $<0.01$ & $<0.01$ & 0 \\
\hline Nickel, mg/L & 50 & 6.3 & $<0.05$ & $<0.240$ & 0.90 \\
\hline Niobium, mg/L & 3 & $<0.007$ & $<0.007$ & $<0.007$ & 0 \\
\hline Oil and grease, $\mathrm{mg} / \mathrm{L}$ & 49 & 2.7 & $<2$ & $<2.014$ & 0.10 \\
\hline PCB (aroclor -1016$), \mu \mathrm{g} / \mathrm{L}$ & 49 & $<0.5$ & $<0.5$ & $<0.5$ & 0 \\
\hline PCB (aroclor-1221), $\mu \mathrm{g} / \mathrm{L}$ & 49 & $<0.5$ & $<0.5$ & $<0.5$ & 0 \\
\hline PCB (aroclor-1232), $\mu \mathrm{g} / \mathrm{L}$ & 49 & $<0.5$ & $<0.5$ & $<0.5$ & 0 \\
\hline PCB (aroclor-1242), $\mu \mathrm{g} / \mathrm{L}$ & 49 & $<0.5$ & $<0.5$ & $<0.5$ & 0 \\
\hline PCB (aroclor -1248$), \mu \mathrm{g} / \mathrm{L}$ & 49 & $<0.5$ & $<0.5$ & $<0.5$ & 0 \\
\hline PCB (aroclor-1254), $\mu \mathrm{g} / \mathrm{L}$ & 49 & $<1$ & $<1$ & $<i$ & 0 \\
\hline PCB (aroclor-1260), $\mu \mathrm{g} / \mathrm{L}$ & 49 & $<1$ & $<1$ & $<1$ & 0 \\
\hline pH, units & 636 & 11.5 & 4.6 & $\mathrm{NA}^{a}$ & NA \\
\hline Phosphorus, mg/ & 3 & 18 & $<0.2$ & $<6.133$ & 10.28 \\
\hline Potassium, mg/L & 3 & 8.1 & 4.7 & 6.066 & 1.80 \\
\hline Selenium, mg/L & 41 & $<0.405$ & $<0.005$ & $<0.005$ & 0 \\
\hline Siticon, mg/L & 3 & 3.6 & 2.4 & 3.066 & 0.61 \\
\hline Silver, $\mathrm{mg} / \mathrm{L}$ & 50 & 0.1 & $<0.01$ & $<0.011$ & 0.01 \\
\hline Sodium, mg/L & 3 & 370 & 20 & 155.666 & 186.98 \\
\hline Strontium, mg/L & 3 & 0.2 & 0.098 & 0.132 & 0.06 \\
\hline Sulfate, $\mathrm{mg} / \mathrm{L}$ & 19 & 1710 & 22 & 606.631 & 352.27 \\
\hline Suspended solids, $\mathrm{mg} / \mathrm{L}$ & 51 & 65 & $<1$ & $<11.039$ & 12.54 \\
\hline Temperature, ${ }^{\circ} \mathrm{C}$ & 657 & 33.3 & 0.5 & 17.922 & 6.96 \\
\hline Thorium, mg/L & 3 & $<0.2$ & $<0.2$ & $<0.2$ & 0 \\
\hline Titanium, mg/L & 3 & 0.018 & $<0.003$ & $<0.008$ & C.01 \\
\hline Turbidity, NTU & 2 & 3.3 & 1.6 & 2.450 & 1.20 \\
\hline Vanadium, mg/L & 3 & $<0.5$ & $<0.5$ & $<0.5$ & 0 \\
\hline Zinc, $\mathrm{mg} / \mathrm{L}$ & 50 & 0.32 & $<0.02$ & $<0.032$ & 0.05 \\
\hline Zirconium, mg/L & 3 & $<0.005$ & $<0.005$ & $<0.005$ & 0 \\
\hline
\end{tabular}

${ }^{\circ} \mathrm{NA}=$ not applicable. 
Table 2.2.92. ORGDP K-1407-J pond toxicity endpoints during 1989

\begin{tabular}{lcclcc}
\hline \multirow{3}{*}{ Month } & \multicolumn{2}{c}{ Fathead minnows } & & \multicolumn{2}{c}{ Ceriodaphnia } \\
\cline { 2 - 3 } \cline { 6 - 6 } & $\begin{array}{c}\text { Survival } \\
\text { NOEC } \\
(\%)\end{array}$ & $\begin{array}{c}\text { Growth } \\
\text { NOEC } \\
(\%)\end{array}$ & & $\begin{array}{c}\text { Survival } \\
\text { NOEC } \\
(\%)\end{array}$ & $\begin{array}{c}\text { Reproduction } \\
\text { NOEC } \\
(\%)\end{array}$ \\
\hline February & 100 & 100 & & 12 & 12 \\
March & $\geqslant 60^{b}$ & $\geqslant 60^{b}$ & & $\geqslant 60^{b}$ & $\geqslant 63^{b}$ \\
April & 100 & 100 & & 50 & 50 \\
June & 100 & 100 & & 12 & 12 \\
August & 50 & 50 & & 25 & 12 \\
Ortober & 100 & 100 & & 6 & 6 \\
November & 100 & 100 & & $<6^{c}$ & $<6^{c}$ \\
\hline
\end{tabular}

${ }^{a} \mathrm{NOEC}=$ no observed effect concentration.

${ }^{b} 60 \%$ was highest concentration tested.

${ }^{\circ} 6 \%$ was lowest concentration tested.

Table 2.2.93. ORGDP K-1407-E and K-1407-F pond toxicity endpoints during 1989

\begin{tabular}{lccccc}
\hline \multirow{3}{*}{ Munth } & \multicolumn{2}{c}{ Fathead minnows } & & \multicolumn{2}{c}{ Ceriodaphnia } \\
\cline { 2 - 3 } & $\begin{array}{c}\text { Survival } \\
\text { NOEC }^{a} \\
(\%)\end{array}$ & $\begin{array}{c}\text { Growth } \\
\text { NOEC } \\
(\%)\end{array}$ & & $\begin{array}{c}\text { Survival } \\
\text { NOEC } \\
(\%)\end{array}$ & $\begin{array}{c}\text { Reproduction } \\
\text { NOEC } \\
(\%)\end{array}$ \\
\hline February & 100 & 100 & & 50 & 50 \\
April & 100 & 100 & & $<25^{b}$ & $<25^{b}$ \\
June & 100 & 100 & & 100 & 100 \\
August & 100 & 100 & & 25 & 25 \\
October & 100 & 100 & & 12 & 12 \\
November & 100 & 100 & & $<6^{c}$ & $<b^{c}$ \\
\hline
\end{tabular}

${ }^{a} \mathrm{NOEC}=$ no observed effect concentration.

${ }^{\circ} 25 \%$ was lowest concentration tested.

${ }^{b} 6 \%$ was lowest concentration tested. 
Table 2.2.94. ORR 1989 concentration of PCB in sedin ant

\begin{tabular}{|c|c|c|c|c|c|c|}
\hline \multirow{2}{*}{ Location $^{a}$} & \multirow{2}{*}{ Analysi: } & \multirow{2}{*}{$\begin{array}{c}\text { Number of } \\
\text { samples }\end{array}$} & \multicolumn{4}{|c|}{ Concentraticn $(\mu \mathrm{g} / \mathrm{kg})$} \\
\hline & & & $\operatorname{Max}$ & Min & Av & Std. error ${ }^{b}$ \\
\hline \multirow[t]{7}{*}{ WOC 06} & Aroclor-1016 & 3 & $<250$ & $<200$ & $<230$ & 14 \\
\hline & Aroclor -1221 & 3 & $<250$ & $<200$ & $<230$ & 14 \\
\hline & Aroclor -1232 & 3 & $<250$ & $<200$ & $<230$ & 14 \\
\hline & Aroclor -1242 & 3 & $<250$ & $<200$ & $<230$ & 14 \\
\hline & Aroclor- 1248 & 3 & $<250$ & $<200$ & $<250$ & 14 \\
\hline & Aroclor- 1254 & 3 & 3400 & -68 & -1300 & 1068 \\
\hline & Aroclor -1260 & 3 & 1900 & $<400$ & $<930$ & 503 \\
\hline \multirow[t]{7}{*}{ WOC 10} & Aroclor -1016 & 3 & $<1500$ & $<140$ & $<600$ & 429 \\
\hline & Aroclor -1221 & 3 & $<1500$ & $<140$ & $<600$ & 429 \\
\hline & Aroclor -1232 & 3 & $<1500$ & $<140$ & $<600$ & 429 \\
\hline & Aroclor -1242 & 3 & $<1500$ & $<140$ & $<600$ & 429 \\
\hline & Aroclor -1248 & 3 & $<1500$ & $<140$ & $<600$ & 429 \\
\hline & Aroclor -1254 & 3 & 500 & $<34$ & $<320$ & 144 \\
\hline & Aroclor -1260 & 3 & $<2900$ & 340 & $<1200$ & 849 \\
\hline \multirow[t]{7}{*}{ WOC 14} & Aroclor-1016 & 3 & $<2600$ & $<180$ & $<1000$ & 816 \\
\hline & Aroclor -1221 & 3 & $<2600$ & $<180$ & $<1000$ & 816 \\
\hline & Aroclor- 1232 & 3 & $<2600$ & $<180$ & $<1000$ & 816 \\
\hline & Aroclor -1242 & 3 & $<2600$ & $<180$ & $<1000$ & 816 \\
\hline & Aroclor -1248 & 3 & $<2600$ & $<180$ & $<1000$ & 816 \\
\hline & Aroclor-1254 & 3 & $<400$ & $\sim 28$ & $\sim 270$ & 119 \\
\hline & Aroclor -1260 & 3 & $<5300$ & $<370$ & $<2000$ & 1632 \\
\hline \multirow[t]{7}{*}{ WOD 13} & Aroclor-1016 & 3 & $<1200$ & $<130$ & $<510$ & 349 \\
\hline & Aroclor-1221 & 3 & $<1200$ & $<130$ & $<510$ & 349 \\
\hline & Aroclor- 1232 & 3 & $<1200$ & $<130$ & $<510$ & 349 \\
\hline & Aroclor- 1242 & 3 & $<1200$ & $<130$ & $<510$ & 349 \\
\hline & Aroclor -1248 & 3 & $<1200$ & $<130$ & $<510$ & 349 \\
\hline & Aroclor- 1254 & 3 & $<400$ & -190 & $\sim 280$ & 62 \\
\hline & Aroclor -1260 & 3 & $<400$ & -94 & $\sim 250$ & 88 \\
\hline \multirow[t]{7}{*}{ MB 07} & Arocle r-1016 & 3 & $<200$ & $<110$ & $<150$ & 26 \\
\hline & Aroclor -1221 & 3 & $<200$ & $<110$ & $<150$ & 26 \\
\hline & Aroclor-1232 & 3 & $<200$ & $<110$ & $<150$ & 26 \\
\hline & Aroclor-1242 & 3 & $<200$ & $<110$ & $<150$ & 26 \\
\hline & Aroclor -1248 & 3 & $<200$ & $<110$ & $<150$ & 26 \\
\hline & Aroclor -1254 & 3 & $<400$ & -13 & $\sim 220$ & 113 \\
\hline & Aroclor -1260 & 3 & $<400$ & $<220$ & $<290$ & 53 \\
\hline \multirow[t]{7}{*}{ CR 08} & Aroclor-1016 & 3 & $<1500$ & $<160$ & $<610$ & 429 \\
\hline & Aroclor- 1221 & 3 & $<1500$ & $<160$ & $<610$ & 429 \\
\hline & Aroclor- 1232 & 3 & $<1500$ & $<160$ & $<610$ & 429 \\
\hline & Aroclor-1242 & 3 & $<1500$ & $<160$ & $<610$ & 429 \\
\hline & Aroclor- 1248 & 3 & $<1500$ & $<160$ & $<610$ & 429 \\
\hline & Aroclor- 1254 & 3 & $<2900$ & $<310$ & $<1200$ & 859 \\
\hline & Arocior -1200 & 3 & $<2900$ & $<310$ & $<1200$ & 859 \\
\hline \multirow[t]{7}{*}{ CR 09} & Aroclor-1016 & 3 & $<200$ & $<130$ & $<160$ & 22 \\
\hline & Aroclor-1221 & 3 & $<200$ & $<130$ & $<160$ & 22 \\
\hline & Aroclor- 1232 & 3 & $<200$ & $<130$ & $<160$ & 22 \\
\hline & Aroclor -1242 & 3 & $<200$ & $<130$ & $<160$ & 22 \\
\hline & Aroclor- 1248 & 3 & $<200$ & $<130$ & $<160$ & 22 \\
\hline & Aroclor- 1254 & 3 & $<400$ & -24 & -240 & 110 \\
\hline & Aroclor -1260 & 3 & $<400$ & $<250$ & $<310$ & 44 \\
\hline
\end{tabular}


Table 2.2.94 (continued)

\begin{tabular}{|c|c|c|c|c|c|c|}
\hline \multirow{2}{*}{ Location $^{a}$} & \multirow{2}{*}{ Analysis } & \multirow{2}{*}{$\begin{array}{c}\text { Number of } \\
\text { samples }\end{array}$} & \multicolumn{4}{|c|}{ Concentration $(\mu \mathrm{g} / \mathrm{kg})$} \\
\hline & & & $\operatorname{Max}$ & Min & Av & Std. error ${ }^{b}$ \\
\hline \multirow[t]{7}{*}{ CR 11} & Aroclor-1016 & 3 & $<200$ & $<81$ & $<120$ & 37 \\
\hline & Aroclor- 1221 & 3 & $<200$ & $<81$ & $<120$ & 37 \\
\hline & Aroclor -1232 & 3 & $<200$ & $<81$ & $<120$ & 37 \\
\hline & Aroclor -1242 & 3 & $<200$ & $<81$ & $<120$ & 37 \\
\hline & Aroclor -1248 & 3 & $<200$ & $<81$ & $<120$ & 37 \\
\hline & Aroclor -1254 & 3 & $<400$ & $<160$ & $<250$ & 75 \\
\hline & Aroclor -1260 & 3 & $<400$ & $<160$ & $<250$ & 75 \\
\hline \multirow[t]{7}{*}{ CR 12} & Aroclor-1016 & 3 & $<1200$ & $<200$ & $<870$ & 333 \\
\hline & Aroclor-1221 & 3 & $<1200$ & $<200$ & $<870$ & 333 \\
\hline & Aroclor -1232 & 3 & $<1200$ & $<200$ & $<870$ & 333 \\
\hline & Aroclor -1242 & 3 & $<1200$ & $<200$ & $<870$ & 333 \\
\hline & Aroclor- 1248 & 3 & $<1200$ & $<200$ & $<870$ & 333 \\
\hline & Aroclor -1254 & 3 & -460 & -68 & -310 & 122 \\
\hline & Aroclor -1260 & 3 & $<2400$ & $<400$ & $<1700$ & 667 \\
\hline
\end{tabular}

${ }^{a}$ See Fig. 2.2 .9 in Vol. 1.

${ }^{b}$ Standard error about the average. 
2.3 GROUNDWATER 


\section{REFERENCES}

The following references are referred to in

Tables 2.3.1-2.3.11.

1. RCRA 40 CFR Pt. 265 Appendix 3.

2. Safe Drinking Water Act--National Primary Drinking Water Regulations, 40 CFR Pt. 141, as amended.

3. Safe Drinking Water Act-National Secondary Drinking Water Regulations, 40 CFR Pt. 143, as amended.
4. State of Tennessee Hazardous Waste Regulations TN 1200-1-11-05. Appendix 05/B.

5. DOE Order 5400.5. Derived Concentration Guides (DCGs) for Air and Water.

6. National Primary Drinking Water Regulations; Synthetic Organic Chemicals, US EPA, Federal Register, July 8, 1987, pp. 25690-25717.

Table 2.3.1. Primary drinking water parameters monitored in ,roundwater during 1989

\begin{tabular}{|c|c|c|}
\hline Parameter & Reference $^{a}$ & $\begin{array}{l}\text { Applicable } \\
\text { standards }^{b} \\
(\mathrm{mg} / \mathrm{L})\end{array}$ \\
\hline As & $1,2,4$ & 0.05 \\
\hline $\mathrm{Ba}$ & $1,2,4$ & 1.0 \\
\hline $\mathrm{Cd}$ & $1,2,4$ & 0.010 \\
\hline $\mathrm{Cr}$ & $1,2,4$ & 0.05 \\
\hline $\mathrm{F}$ & $1,2,3,4$ & $4.0,2.0^{c}, 1.4-2.4^{d}$ \\
\hline $\mathrm{Pb}$ & $1,2,4$ & 0.05 \\
\hline Nitrate & $1,2,4$ & 10 \\
\hline $\mathrm{Hg}$ & $1,2,4$ & 0.002 \\
\hline $\mathrm{Se}$ & $1,2,4$ & 0.01 \\
\hline $\mathrm{Ag}$ & $1,2,4$ & 0.05 \\
\hline Endrin & $1,2,4$ & 0.0002 \\
\hline Lindane & $1,2,4$ & 0.004 \\
\hline Methoxychlor & $1,2,4$ & 0.1 \\
\hline Toxaphene & $1,2,4$ & 0.005 \\
\hline $2,4-D$ & $1,2,4$ & 0.1 \\
\hline 2,4,5-TP Silvex & $1,2,4$ & 0.01 \\
\hline${ }^{226} \mathrm{Ra}$ and ${ }^{228} \mathrm{Ra}(\mathrm{pCi} / \mathrm{L})$ & $1,2,4$ & 5 \\
\hline Gross alpha $(\mathrm{pCi} / \mathrm{L})$ & $1,2,4$ & 15 \\
\hline Gross beta (mrem/year) & $1,2,4$ & 4 \\
\hline $\begin{array}{l}\text { Coliform bacteria } \\
(\text { col. } / 100 \mathrm{~mL})\end{array}$ & $1,2,4$ & $1^{b}$ \\
\hline
\end{tabular}

${ }^{a}$ References for applicable standards precede this table.

${ }^{6}$ Maximum contaminant level.

'Secondary maximum contaminant level.

${ }^{d}$ RCRA 40 CFR. Pt. 265 Appendix B and State of Tennessee Hazardous Waste Regulations. 
Table 2.3.2. Parameters establishing groundwater quality monitored during $\mathbf{1 9 8 9}$

\begin{tabular}{lcc}
\hline Parameter & Reference & $\begin{array}{c}\text { Applicable } \\
\text { standards } \\
(\mathrm{mg} / \mathrm{L})\end{array}$ \\
\hline Chloride & 3 & 250 \\
$\mathrm{Fe}$ & 3 & 0.3 \\
$\mathrm{Mn}$ & 3 & 0.05 \\
Phenols & & None \\
$\mathrm{Na}$ & 3 & None \\
Sulfate & 3 & 250 \\
\hline
\end{tabular}

${ }^{a}$ Secondary maximum contaminant level.

Table 2.3.3. Indicator parameters monitored in groundwater semiannually in $\mathbf{1 9 8 9}$

\begin{tabular}{lcl}
\hline \multicolumn{1}{c}{ Parameter } & Reference & $\begin{array}{l}\text { Applicable } \\
\text { standards }\end{array}$ \\
\hline Total organic carbon $(\mathrm{mg} / \mathrm{L})$ & & None \\
Total organic hatogen $(\mathrm{mg} / \mathrm{L})$ & & None \\
Specific conductance $(\mathrm{mS} / \mathrm{cm})$ & 3 & $\begin{array}{l}\text { None } \\
\text { pH }\end{array}$ \\
\hline
\end{tabular}

${ }^{a}$ Secondary maximum contaminant level. 
Table 2.3.4. Typical inductively coupled argon plasma (ICAP) metals scan of groundwater (results used for metals analysis and site characterization studies)

\begin{tabular}{|c|c|c|}
\hline Parameter & Reference & $\begin{array}{c}\text { Applicable } \\
\text { standards } \\
(\mathrm{mg} / \mathrm{L})\end{array}$ \\
\hline All & & None \\
\hline $\mathrm{Sb}$ & & None \\
\hline $\mathrm{Ba}$ & $1,2,4$ & $1.0^{a}$ \\
\hline $\mathrm{Be}$ & & None \\
\hline B & & None \\
\hline $\mathrm{Cd}$ & $1,2,4$ & $0.01^{a}$ \\
\hline $\mathrm{Ca}$ & & None \\
\hline $\mathrm{Cr}$ & $1,2,4$ & $0.05^{a}$ \\
\hline $\mathrm{Co}$ & & None \\
\hline $\mathrm{Cu}$ & 3 & $1.0^{b}$ \\
\hline $\mathrm{Pb}$ & 3 & $0.3^{b}$ \\
\hline $\mathrm{Li}$ & & None \\
\hline $\mathrm{Mg}$ & & None \\
\hline $\mathrm{Mn}$ & 3 & $0.05^{b}$ \\
\hline Mo & & None \\
\hline $\mathrm{Ni}$ & & None \\
\hline $\mathrm{Nb}$ & & None \\
\hline $\mathrm{P}$ & & None \\
\hline K & & None \\
\hline $\mathrm{Si}$ & & None \\
\hline $\mathrm{Ag}$ & $1,2,4$ & $0.05^{a}$ \\
\hline $\mathrm{Na}$ & & None \\
\hline $\mathrm{Sr}$ & & None \\
\hline $\mathrm{Th}$ & & None \\
\hline $\mathrm{Ti}$ & & None \\
\hline $\mathrm{V}$ & & None \\
\hline $\mathrm{Zn}$ & 3 & $5.0^{b}$ \\
\hline $\mathrm{Zr}$ & & None \\
\hline
\end{tabular}

${ }^{a}$ Maximum contaminant level.

${ }^{b}$ Secondary maximum contaminant level. 
Table 2.3.5. Typical metals sought in groundwater by atomic absorption (AA) spectroscopy (results used to fulfill required monitoring and in characterization studies)

\begin{tabular}{lll}
\hline Parameter & Reference & \multicolumn{1}{c}{$\begin{array}{c}\text { Applicable } \\
\text { standards } \\
(\mathrm{mg} / \mathrm{L})\end{array}$} \\
\hline $\mathrm{Sb}$ & $1,2,4$, & None \\
$\mathrm{As}$ & $1,2,4$ & 0.05 \\
$\mathrm{Ba}$ & $1,2,4$ & 1.00 \\
$\mathrm{Be}$ & $1,2,4$ & None \\
$\mathrm{Cd}$ & 3 & 0.010 \\
$\mathrm{Cr}$ & $1,2,4$ & 0.05 \\
$\mathrm{Cu}$ & $1,2,4$ & 1 \\
$\mathrm{~Pb}$ & $1,2,4$ & 0.05 \\
$\mathrm{Hg}$ & $1,2,4$ & 0.002 \\
$\mathrm{Ni}$ & & None \\
$\mathrm{Se}$ & 3 & 0.01 \\
$\mathrm{Ag}$ & $\mathrm{Tl}$ & 0.05 \\
$\mathrm{Zn}$ & None \\
\hline
\end{tabular}

a Maximum contaminant level.

${ }^{b}$ Secondary maximum contaminant level.

Table 2.3.6. Typical anions sought in groundwater

Results used for required monitoring and in characterization studies

\begin{tabular}{lcc}
\hline Parameter & Reference & \multicolumn{1}{c}{$\begin{array}{c}\text { Applicable } \\
\text { standards } \\
(\mathrm{mg} / \mathrm{L})\end{array}$} \\
\hline $\begin{array}{l}\text { Chloride } \\
\text { Fluoride }\end{array}$ & 3 & $250^{a}$ \\
Nitrate & $1,2,4$ & $4.0^{a}, 2.0^{b}, 1.4-2.4^{c}$ \\
Nitrite & \multicolumn{1}{c}{$10^{b}$} \\
Phosphate & \multicolumn{1}{c}{$1^{d}$} \\
Sulfate & 3 & $\begin{array}{c}\text { None } \\
250^{b}\end{array}$ \\
\hline
\end{tabular}

${ }^{a}$ Maximum contaminant level.

${ }^{b}$ Secondary maximum contaminant level.

'State of Tennessee Hazardous Waste Regulations, TN 1200-1-11.05, Appendix 0.05/B.

${ }^{d}$ Proposed by Y-12 Environmental Management Department for required monitoring and compliance limit. 
Table 2.3.7. Volatile organics (hazardous substance list) sought in groundwater

\begin{tabular}{|c|c|c|c|}
\hline Parameter & Reference & $\begin{array}{l}\text { Chemical } \\
\text { Abstracts } \\
\text { Service No. }\end{array}$ & $\begin{array}{c}\text { Applicable } \\
\text { standards } \\
(\mathrm{mg} / \mathrm{L})\end{array}$ \\
\hline Chloromethane & & $74-87-3$ & None \\
\hline Bromomethane & & $74-83-9$ & None \\
\hline Vinyl chloride & 6 & $75-01-4$ & 0.002 \\
\hline Chlorocthane & & $75-00-3$ & None \\
\hline Methylene chloride & & $75-09-2$ & None \\
\hline Acetone & & $67-64-1$ & None \\
\hline Carbon disulfide & & $75-15-0$ & None \\
\hline 1,1-dichloroethene & 6 & $75-35-4$ & 0.007 \\
\hline 1,1-dichloroethane & & $75-35-3$ & None \\
\hline 1,2-dichloroethene (total) & & $540-59-0$ & None \\
\hline Chloroform & & $67-66-3$ & None \\
\hline 1,2-dichloroethane & 6 & $10706-2$ & 0.005 \\
\hline 2-butanone & & $78-93-3$ & None \\
\hline 1,1,1-trichloroethane & 6 & $71 \cdot 55-6$ & 0.20 \\
\hline Carbon tetrachloride & 6 & $56-23-5$ & 0.005 \\
\hline Vinyl acetate & & $108-05-4$ & None \\
\hline Bromodichloromethane & & $75-27-4$ & None \\
\hline 1,1,2,2-tetrachloroethane & & $79-34-5$ & None \\
\hline 1,2-dichloropropane & & $78-87-5$ & None \\
\hline Cis-1,3,-dichloropropene & & $10061-01-5$ & None \\
\hline Trichloroethene & 6 & $79-01-6$ & 0.005 \\
\hline Dibromochloromethane & & $124-48-1$ & None \\
\hline 1,1,2-trichloroethane & & $79-00-5$ & None \\
\hline Benzene & 6 & $71-43-2$ & 0.005 \\
\hline trans-1,3-dichloropropene & & $10061-02-6$ & None \\
\hline Bromoform & & $75-25-2$ & None \\
\hline 2-hexanone & & $591-78-6$ & None \\
\hline 4-methyl-2-pentanone & & $108-10-1$ & None \\
\hline Tetrachloroethene & & $127-18-4$ & None \\
\hline Toluene & & $108-88-3$ & None \\
\hline Chlorobenzene & & $108-90-7$ & None \\
\hline Ethyl benzene & & $100-41-4$ & None \\
\hline Styrene & & $100-42.5$ & None \\
\hline Xylenes (total) & & $133-02-7$ & None \\
\hline
\end{tabular}

${ }^{a}$ Maximum contaminant level effective $7 / 8 / 87$. 
Table 2.3.8. Pesticides and polychlorinated biphenyls (hazardous substance list) sought in groundwater

\begin{tabular}{|c|c|c|c|}
\hline Parameter & Reference & $\begin{array}{c}\text { Chemical } \\
\text { Abstracts } \\
\text { Service No. }\end{array}$ & $\begin{array}{c}\text { Applicable } \\
\text { standards } \\
(\mu \mathrm{g} / \mathrm{L})\end{array}$ \\
\hline Alpha-BHC & & $319-84-6$ & None \\
\hline Beta-BHC & & $319.85-7$ & None \\
\hline Delta-BHC & & $319-86-8$ & None \\
\hline Gamma-BHC (Lindane) & & $58-89-9$ & None \\
\hline Heptachlor & & $76-44-8$ & None \\
\hline Aldrin & & $309-00-2$ & None \\
\hline Heptachlor epoxide & & $1024-57-3$ & None \\
\hline Endrin & & $72-20-8$ & None \\
\hline Dieldrin & & $60-57-1$ & None \\
\hline $4,4^{\prime}-\mathrm{DDE}$ & & $72-55-9$ & None \\
\hline Endosulfan I & & $959-98-8$ & 0.0002 \\
\hline Endosulfan II & & $33213-65.9$ & None \\
\hline $4,4^{\prime} \cdot \mathrm{DDD}$ & & $72-54-8$ & None \\
\hline Endosulfan sulfate & & $1031-07-8$ & None \\
\hline 4,4'-DDT & & $50-29-3$ & None \\
\hline Endrin ketone & & $53494-70.5$ & None \\
\hline Methoxychlor & & $72-43-5$ & 0.1 \\
\hline Alpha-chlordane & & $5103-71-9$ & None \\
\hline Gamma-chlordane & & $5103-74-2$ & None \\
\hline Toxaphene & & $8001-35-2$ & 0.005 \\
\hline Aroclor-1016 & & $12674-11-2$ & None \\
\hline Aroclor -1221 & & $11104-28-2$ & None \\
\hline Aroclor -1232 & & $11141-16-5$ & None \\
\hline Aroclor- 1242 & & $53469-21-9$ & None \\
\hline Aroclor -1248 & & $12672-29-6$ & None \\
\hline Aroclor -1254 & & $11097-69-1$ & None \\
\hline Aroclor -1260 & & $11096-82-5$ & None \\
\hline
\end{tabular}


Table 2.3.9. Base/neutral/acid extractable organics (hazardous substance list) sought in groundwater

\begin{tabular}{|c|c|c|c|}
\hline Parameter & Reference & $\begin{array}{l}\text { Chemical } \\
\text { Abstracts } \\
\text { Service No. }\end{array}$ & $\begin{array}{c}\text { Applicable } \\
\text { standards } \\
(\mathrm{mg} / \mathrm{L})\end{array}$ \\
\hline Phenol & & $108-95-2$ & None \\
\hline bis(2-chloroethyl) ether & & $111-44-4$ & None \\
\hline 2-chlorophenol & & $95-57.8$ & None \\
\hline 1,3-dichlorobenzene & & $541-73-1$ & None \\
\hline 1,4-dichlorobenzene & 6 & $106-46-7$ & 0.075 \\
\hline Benzyl alcohol & & $100-51-6$ & None \\
\hline 1,2-dichlorobenzene & & $95-50-1$ & None \\
\hline 2-rnethylphenol & & $95-48-7$ & None \\
\hline bis(2-chloroisopropyl)ether & & $39638-32-9$ & None \\
\hline 4-methylphenol & & $106-44-5$ & None \\
\hline N-Nitroso-di-n-propylamine & & $621-64-7$ & None \\
\hline Hexachloroethane & & $67-72 .-1$ & None \\
\hline Nitrobenzene & & $98-95-3$ & None \\
\hline Isophorone & & $78-59-1$ & None \\
\hline 2-nitrophenol & & 88.75 .5 & None \\
\hline 2,4-dimethylphenol & & $105-67-9$ & None \\
\hline Benzoic acid & & $65-85-0$ & None \\
\hline bis(2-chloroethoxy) methane & & 111.91 .1 & None \\
\hline 2,4-dichlorophenol & & $120-83-2$ & None \\
\hline 1,2,4-trichlorobenzene & & $120-82-1$ & None \\
\hline Naphthalene & & $91-20-3$ & None \\
\hline 4-chloroaniline & & $106.47-8$ & None \\
\hline Hexachlorobutadiene & & $87-68-3$ & None \\
\hline $\begin{array}{l}\text { 4-chloro-3-methylphenol } \\
\text { (para-chloro-meta-cresol) }\end{array}$ & & $59-50-7$ & None \\
\hline 2-methylnaphthalene & & $91-57-6$ & None \\
\hline Hexachlorocyclopentadiene & & $77-47-4$ & None \\
\hline 2,4,6-trichlorophenol & & $88-06-2$ & None \\
\hline 2,4,5-trichlorophenol & & $95.95-4$ & None \\
\hline 2-chloronaphthalene & & $91-58-7$ & None \\
\hline 2-nitroaniline & & $88-74-4$ & None \\
\hline Dimethyl phthalate & & $131-11-3$ & None: \\
\hline Acenaphthylene & & $208-96-8$ & None \\
\hline 2,6-dinitrotoluene & & $606-20-2$ & None \\
\hline 3-nitroaniline & & $99-09.2$ & None \\
\hline Acenaphthene & & $83-32-9$ & None \\
\hline 2,4-dinitrophenol & & $51-28-5$ & None \\
\hline 4-nitrophenol & & $100-02-7$ & None \\
\hline Dibenzofuran & & $132-64-9$ & None \\
\hline 2,4-dinitrotoluene & & $121-14-2$ & None \\
\hline Diethylphthalate & & $84-66-2$ & None \\
\hline 4-chlorophenyl phenyl ether & & $7005-72-3$ & None \\
\hline Fluorene & & $86-73-7$ & None \\
\hline 4-nitroaniline & & $100-01-6$ & None \\
\hline 4,6-dinitro-2-methylphenol & & $534.52 \cdot 1$ & None \\
\hline $\mathrm{N}$-nitrosodiphenylamint & & $86-30-6$ & None \\
\hline 4-bromophenyl phenyl ether & & $101-55-3$ & None \\
\hline fexachlorobenzene & & $118-74-1$ & None \\
\hline Pentachlorophenol & & $87-86-5$ & None \\
\hline Phenanthrene & & $85-01.8$ & None \\
\hline Anthracene & & $120-12-7$ & None \\
\hline Di-n-butylphthalate & & $84-74-2$ & None \\
\hline Fluoranthene & & $206-44-0$ & None \\
\hline Pyrene & & $129-00-0$ & None \\
\hline
\end{tabular}


Table 2.3.9 (continued)

\begin{tabular}{|c|c|c|c|}
\hline Parameter & Reference & $\begin{array}{l}\text { Chemical } \\
\text { Abstracts } \\
\text { Service No. }\end{array}$ & $\begin{array}{c}\text { Applicable } \\
\text { standards } \\
(\mathrm{mg} / \mathrm{L})\end{array}$ \\
\hline Butyl benzyl phthalate & & $85-68-7$ & None \\
\hline 3,3'-dichlorobenzidine & & $91-94-1$ & None \\
\hline Benzo[a]anthracene & & $56-55-3$ & None \\
\hline Chrysene & & $218-01-9$ & None \\
\hline bis(2-ethylhexyl)phthalate & & $117.81-7$ & None \\
\hline Di-n-octyl phthalate & & $117-84-0$ & None \\
\hline Benzo[ $b$ ]fluoranthene & & $205-99-2$ & None \\
\hline Benzo $[k]$ fluoranthene & & $207-08-9$ & None \\
\hline Benzo $[a]$ pyrene & & $50-32-8$ & None \\
\hline Indeno(1,2,3-cd)pyrene & & $193-39-5$ & None \\
\hline Dibenz $[a, h]$ anthracene & & $53-70-3$ & None \\
\hline Benzo $[g, h, i]$ perylene & & $191-24-2$ & None \\
\hline
\end{tabular}

${ }^{a}$ Maximum contaminant level effective 7/8/87. 
Table 2.3.10. Radionuclides and radioactive metals sought in groundwater

\begin{tabular}{|c|c|c|}
\hline Parameter & Reference & $\begin{array}{c}\text { Applicable } \\
\text { standards }^{a} \\
(\mathrm{pCi} / \mathrm{L})\end{array}$ \\
\hline Gross alpha radiation & $1,2,4$ & 15 \\
\hline Gross beta radiation (mrem/yr) & $1,2,4$ & $4^{b}$ \\
\hline Gross gamma radiation & & None \\
\hline${ }^{226} \mathrm{Ra}$ and ${ }^{228} \mathrm{Ra}$ & $1,2,4$ & 5 \\
\hline${ }^{137} \mathrm{Cs}$ & 5 & 3,000 \\
\hline${ }^{90} \mathrm{Sr}$ & 5,2 & 1,$000 ; 8.0$ \\
\hline${ }^{60} \mathrm{Co}$ & 5 & 10,000 \\
\hline Tritium & 5,2 & $2,000,000 ; 20,000$ \\
\hline${ }^{99} \mathrm{Tc}$ & 5 & 100,000 \\
\hline${ }^{239} \mathrm{Pu}$ & 5 & 30 \\
\hline${ }^{235} \mathrm{U}$ & 5 & 600 \\
\hline Total uranium $(\mathrm{mg} / \mathrm{L})$ & & None \\
\hline
\end{tabular}

${ }^{a}$ Maximum contaminant level.

${ }^{b}$ Maximum contaminant level in the absence of ${ }^{90} \mathrm{Sr}$ and alpha emitters $=1,000 \mathrm{pCi} / \mathrm{L}$.

Table 2.3.11. Other typical parameters that may be included in groundwater studies

\begin{tabular}{lcc}
\hline \multicolumn{1}{c}{ Parameter } & Reference & $\begin{array}{c}\text { Applicable } \\
\text { standards } \\
(\mathrm{mg} / \mathrm{L})\end{array}$ \\
\hline Alkalinity $\left(\mathrm{CO}_{3}\right)$ & None \\
Alkalinity $\left(\mathrm{HCO}_{3}\right)$ & None \\
Total phosphorus & None \\
Solids: & & None \\
$\quad$ Total & None \\
$\quad$ Suspended & 500 \\
Dissolved & 3 & 5 \\
Turbidity (JTU) & 2 & None \\
Total Kjeldahl nitrogen & & None \\
Ammonia (N) & & None \\
Chemical oxygen demand & & None \\
MBAS & & \\
\hline
\end{tabular}


Table 2.3.12. Constituents in groundwater at the Y-12 Plant site, 1989

\begin{tabular}{|c|c|c|c|c|c|c|c|}
\hline \multirow{2}{*}{ Parameter } & \multirow{2}{*}{$\begin{array}{l}\text { Nuniber } \\
\text { detected }\end{array}$} & \multirow{2}{*}{$\begin{array}{c}\text { Number of } \\
\text { samples }\end{array}$} & \multicolumn{3}{|c|}{ Values above detection limit } & \multirow{2}{*}{$\begin{array}{l}\text { Reference } \\
\text { value }\end{array}$} & \multirow{2}{*}{$\begin{array}{l}\text { Number of values } \\
\text { exseeding reference }\end{array}$} \\
\hline & & & $\operatorname{Max}$ & $\operatorname{Min}$ & Av & & \\
\hline \multicolumn{8}{|c|}{ Beta.4 Security Pit } \\
\hline Dissolved solids, $\mathrm{mg} / \mathrm{L}$ & $\mathrm{NA}^{a}$ & 13 & 540 & 146 & 321 & 500 & 2 \\
\hline Iron, total, $\mathrm{mg} / \mathrm{L}$ & 6 & 6 & 44 & 0.14 & 24 & 0.3 & 5 \\
\hline Manganese, total, $\mathrm{mg} / \mathrm{L}$ & 6 & 6 & 3.9 & 0.048 & 2.1 & 0.05 & 5 \\
\hline $\mathrm{pH}$ & NA & 52 & 7.8 & 5.8 & NA & $6.5 / 8.5$ & 16 \\
\hline Selenium, total, $\mathrm{mg} / \mathrm{L}$ & 2 & 6 & 0.065 & $<0.05$ & 0.06 & 0.01 & 2 \\
\hline Turbidity, NTU & NA & 13 & 2,600 & 2.6 & $\$ 90$ & 5 & 12 \\
\hline \multicolumn{8}{|c|}{ Burial Grounds } \\
\hline Acetone, $\mu \mathrm{g} / \mathrm{L}$ & 16 & 119 & 130 & $<10$ & 30 & $\mathrm{NR}^{b}$ & NA \\
\hline Barium, total, $\mathrm{mg} / \mathrm{L}$ & 21 & 27 & 6.8 & $<0.1$ & 0.60 & 1 & 1 \\
\hline Benzrae. $\mu \mathrm{g} / \mathrm{L}$ & 4 & 119 & 69 & $<5$ & 42 & 5 & 4 \\
\hline Bromolorm, $\mu \mathrm{g} / \mathrm{L}$ & 1 & 119 & 11 & $<5$ & 11 & NR & NA \\
\hline Cadinium, total, $\mathrm{mg} / \mathrm{L}$ & 18 & 117 & 0.02 & $<0.002$ & 0.0054 & 0.01 & 1 \\
\hline Chioride, $\mathrm{mg} / \mathrm{L}$ & 118 & 119 & 3,300 & $<1$ & 190 & 250 & 12 \\
\hline Chloroform, $\mu \mathrm{g} / \mathrm{L}$ & 4 & 119 & 54 & $<5$ & 26 & NR & NA \\
\hline Chromium, tctal, mg/L & 15 & 119 & 0.16 & $<0.01$ & 0.062 & 0.05 & 7 \\
\hline Dissolved solids, mg/L & NA & 119 & 4,790 & 38 & 566 & 500 & 21 \\
\hline Gross alpha, $\mathrm{pCi} / \mathrm{L}$ & NA & 119 & 180 & -5 & 4.1 & 15 & 5 \\
\hline Gross beta, pCi/L & NA & 119 & 450 & -100 & 33 & 50 & 14 \\
\hline Lead, total, $\mathrm{mg} / \mathrm{L}$ & 64 & 119 & 1.5 & $<0.004$ & 0.047 & 0.05 & 4 \\
\hline Methylene chloride, $\mu \mathrm{g} / \mathrm{L}$ & 4 & 119 & 52 & $<5$ & 21 & NR & NA \\
\hline Nitrate-N, mg/L & 25 & 119 & 32 & $<20$ & 3 & 10 & 1 \\
\hline $\mathrm{pH}$ & NA & 476 & 11 & 5.1 & NA & $6.5 / 8.5$ & 268 \\
\hline Strontium, $\mathrm{pCi} / \mathrm{L}$ & NA & 8 & 77 & -42 & 8.1 & 8 & 2 \\
\hline Tetrachloroethene, $\mu \mathrm{g} / \mathrm{L}$ & 21 & 119 & 1,500 & $<5$ & 570 & NR & NA \\
\hline Toluene, $\mu \mathrm{g} / \mathrm{L}$ & 2 & 119 & 12 & $<5$ & 9 & NR & NA \\
\hline Trichloroethene, $\mu \mathrm{g} / \mathrm{L}$ & 15 & 119 & 660 & $<5$ & 164 & 5 & 14 \\
\hline Turbidity, NTU & NA & 118 & 3,000 & 0.2 & 102 & 5 & 71 \\
\hline Vinyl chloride, $\mu \mathrm{g} / \mathrm{L}$ & 7 & 119 & 510 & $<10$ & 170 & 2 & $\%$ \\
\hline Xylenes, $\mu \mathrm{g} / \mathrm{L}$ & 5 & 119 & 20 & $<5$ & 13.8 & NR & NA \\
\hline 1,1-Dichloroethane, $\mu \mathrm{g} / \mathrm{L}$ & 13 & 119 & 720 & $<5$ & 101 & NR & NA \\
\hline 1,1-Dichloroethene, $\mu \mathrm{g} / \mathrm{L}$ & 4 & 119 & 100 & $<5$ & 68.8 & $?$ & 4 \\
\hline 1,1,1-Trichloroethane, $\mu \mathrm{g} / \mathrm{L}$ & 4 & 119 & 540 & $<5$ & 260 & 200 & 2 \\
\hline 1,2-Dichlorocthane, $\mu \mathrm{g} / \mathrm{L}$ & 1 & 119 & 7 & $<5$ & 7 & 5 & 1 \\
\hline 1,2-Dichloroethene, total, $\mu \mathrm{g} / \mathrm{L}$ & 10 & 119 & 2,000 & $<5$ & 430 & NR & NA \\
\hline 2-Butanone, $\mu \mathrm{B} / \mathrm{L}$ & 3 & 119 & 20 & $<10$ & 17.7 & NR & NA \\
\hline $228 \mathrm{Radium}, \mathrm{pCi} / \mathrm{L}$ & NA & 7 & 27 & -1.6 & 7.49 & 5 & 4 \\
\hline 4-Methyl-2-pentanone, $\mu \mathrm{g} / \mathrm{L}$ & 1 & 119 & 22 & $<10$ & 22 & NR & N.A \\
\hline
\end{tabular}

Barium, Total $\mathrm{mg} / \mathrm{L}$

Bis(2-ethylhexyl)phthalate, $\mu \mathrm{g} / \mathrm{L}$

Chromium, total, mg/L

Dissolved solids, $\mathrm{mg} / \mathrm{L}$.

Gross alpha, $\mathrm{pCi} / \mathrm{L}$

Gross beta, $\mathrm{pCi} / \mathrm{L}$

Iron, tctal, $\mathrm{mg} / \mathrm{L}$

Lead, total, $\mathrm{mg} / \mathrm{L}$

Manganese, total, mg/L

Nitrate-N, mg/L

$\mathrm{pH}$

Radium,, $\mathrm{Ci} / \mathrm{L}$

Turbidity, NTU
Burial Grounds-LLWDDD Lysimeter Demonstration Site

$\begin{array}{rcccc}9 & 9 & 1.6 & 0.18 & 0.56 \\ 1 & 6 & 12 & <10 & 12 \\ 2 & 9 & 0.071 & <0.01 & 0.049 \\ \text { NA } & 9 & 3.770 & 176 & 850 \\ \text { NA } & 9 & 43 & -2.8 & 6.4 \\ \text { NA } & 9 & 79 & -5.5 & 12 \\ 9 & 9 & 73 & 0.0082 & 18 \\ 3 & 9 & 0.09 & <0.004 & 0.038 \\ 9 & 9 & 6.5 & 0.0038 & 1.3 \\ 2 & 9 & 790 & <0.2 & 390 \\ \text { NA } & 36 & 7.8 & 5.8 & \text { NA } \\ \text { NA } & 9 & 38 & 0.11 & 7.0 \\ \text { NA } & 9 & 930 & 1.4 & 140\end{array}$


Table 2.3.12 (continued)

\begin{tabular}{|c|c|c|c|c|c|c|c|}
\hline \multirow{2}{*}{ Parameter } & \multirow{2}{*}{$\begin{array}{l}\text { Number } \\
\text { detected }\end{array}$} & \multirow{2}{*}{$\begin{array}{l}\text { Number of } \\
\text { samples }\end{array}$} & \multicolumn{3}{|c|}{ Values above detection limit } & \multirow{2}{*}{$\begin{array}{c}\text { Reference } \\
\text { value }\end{array}$} & \multirow{2}{*}{$\begin{array}{l}\text { Number of values } \\
\text { exceeding reference }\end{array}$} \\
\hline & & & $\operatorname{Max}$ & Min & Ar & & \\
\hline \multicolumn{8}{|c|}{ Burial Grounds-LLWDDD Packaging } \\
\hline Chromium, total, mg/L & 3 & 15 & 0.51 & $<0.01$ & 0.18 & 0.05 & 1 \\
\hline Coliform, col./100 mL & 1 & 15 & 8 & 0 & 8 & 1 & 1 \\
\hline Dissolved solids, $\mathrm{mg} / \mathrm{L}$ & NA & 15 & 606 & 102 & 266 & 500 & 3 \\
\hline Fluoride, $\mathrm{mg} / \mathrm{L}$ & 11 & 15 & 4 & $<0.1$ & 1 & 4 & 2 \\
\hline Gross alpha, pCi/L & NA & 15 & 41 & -2 & 2.8 & 15 & 1 \\
\hline Iron, total, mg/L & 15 & 15 & 33 & 0.049 & 4.2 & 0.3 & 10 \\
\hline Lead, total, mg/L & 7 & 15 & 0.058 & $<0.004$ & 0.019 & 0.05 & 1 \\
\hline Manganese, total, $\mathrm{mg} / \mathrm{L}$ & 15 & 15 & 2.1 & 0.0035 & 0.38 & 0.05 & 8 \\
\hline $\mathrm{pH}$ & NA & 60 & 9.1 & 5.4 & NA & $6.5 / 8.5$ & 28 \\
\hline Tetrachloroethene, $\mu \mathrm{g} / \mathrm{L}$ & 8 & 15 & 2100 & $<5$ & 730 & NR & NA \\
\hline Trichloroethene, $\mu \mathrm{g} / \mathrm{L}$ & 3 & 15 & 29 & $<5$ & 26 & 5 & 3 \\
\hline Turbidity, NTU & NA & 15 & 100 & 1.3 & 18 & 5 & 5 \\
\hline \multicolumn{8}{|c|}{ Chestnut Ridge Security Pit } \\
\hline Acetone, $\mu \mathrm{g} / \mathrm{L}$ & 3 & 41 & 20 & $<10$ & 14 & NR & NA \\
\hline Chromium, total, $\mathrm{mg} / \mathrm{L}$ & 6 & 41 & 0.22 & $<0.01$ & 0.079 & 0.05 & 2 \\
\hline Freon, $\mu \mathrm{g} / \mathrm{L}$ & 5 & 7 & 230 & $<10$ & 74 & NR & NA \\
\hline Gross beta, $\mathrm{pCi} / \mathrm{L}$ & NA & 41 & 140 & -24 & 20 & 50 & 6 \\
\hline Lead, total, mg/L & 25 & 41 & 0.31 & $<0.004$ & 0.028 & 0.05 & 2 \\
\hline $\mathrm{pH}$ & NA & 164 & 8.5 & 6.1 & NA & $6.5 / 8.5$ & 25 \\
\hline Tetrachloroethene, $\mu \mathrm{g} / \mathrm{L}$ & 25 & 41 & 140 & $<5$ & 35 & NR & NA \\
\hline Trichlorofluoromethane, $\mu \mathrm{g} / \mathrm{L}$ & 8 & 11 & 220 & $<10$ & 51 & NR & NA \\
\hline Turbidity, NTU & NA & 31 & 130 & 0.41 & 11 & 5 & 10 \\
\hline 1,1-Dichloroethane, $\mu \mathrm{g} / \mathrm{L}$ & 21 & 41 & 200 & $<5$ & 37 & NR & NA \\
\hline 1,1-Dichioroethene, $\mu \mathrm{g} / \mathrm{L}$ & 10 & 41 & 98 & $<5$ & 52 & 7 & 9 \\
\hline 1,1,1.Trichloroethane, $\mu \mathrm{g} / \mathrm{L}$ & 25 & 41 & 630 & $<5$ & 160 & 200 & 6 \\
\hline 1,2-Dichloroethane, $\mu \mathrm{g} / \mathrm{L}$ & 1 & $4 i$ & 9 & $<5$ & 9 & 5 & 1 \\
\hline 1,2-Dichloroethene, total, $\mu \mathrm{g} / \mathrm{L}$ & 3 & 41 & 15 & $<5$ & 13 & NR & NA \\
\hline \multicolumn{8}{|c|}{ Chestmut Ridge Sediment Disposal Basin } \\
\hline Acetone, $\mu \mathrm{g} / \mathrm{L}$ & 1 & 26 & 23 & $<10$ & 23 & NR & NA \\
\hline Chromium, total, $\mathrm{mg} / \mathrm{L}$ & 5 & 26 & 0.071 & $<0.01$ & 0.03 & 0.05 & 1 \\
\hline Gross alpha, pCi/L & NA & 26 & 120 & -2 & 12 & 15 & 3 \\
\hline Gross beta, pCi/L & NA & 26 & 94 & -4.3 & 19.0 & 50 & 2 \\
\hline Iron, total, mg/L & 25 & 25 & 120 & 0.045 & 8.1 & 0.3 & 18 \\
\hline Lead, total, mg/L & 14 & 26 & 0.15 & $<0.004$ & 0.023 & 0.05 & 1 \\
\hline Manganese, total, $\mathrm{mg} / \mathrm{L}$ & 23 & 25 & 4.2 & $<0.001$ & 0.29 & 0.05 & 10 \\
\hline $\mathrm{pH}$ & NA & 104 & 8.5 & 5.6 & NA & $6.5 / 8.5$ & 35 \\
\hline Tetrachloroethene, $\mu \mathrm{g} / \mathrm{L}$ & 1 & 26 & 6 & $<5$ & 6 & NR & NA \\
\hline Toluene, $\mu g / L$ & 1 & 26 & 6 & $<5$ & 6 & NR & NA \\
\hline Turbidity, NTU & NA & 18 & 1100 & 0.7 & 89 & 5 & 12 \\
\hline Xylenes, $\mu \mathrm{g} / \mathrm{L}$ & 1 & 26 & 7 & $<5$ & 7 & NR & NA \\
\hline \multicolumn{8}{|c|}{ East Chestnut Ridge Waste Pile } \\
\hline Arsenic, total, $\mathrm{mg} / \mathrm{L}$ & 1 & 8 & 0.089 & $<0.5$ & 0.089 & 0.05 & 1 \\
\hline Iron, total, $\mathrm{mg} / \mathrm{L}$ & 5 & 8 & 1.3 & $<0.04$ & 0.46 & 0.3 & 2 \\
\hline Ledd, Intal, $\mathrm{mg} / \mathrm{L}$ & 4 & 8 & 0.83 & $<0.5$ & 0.59 & 0.05 & 4 \\
\hline $\mathrm{pH}$ & NA & 32 & 7.9 & 6.4 & NA. & $6.5 / 8.5$ & 1 \\
\hline Silver, total, $\mathrm{mg} / \mathrm{L}$ & 3 & 8 & 0.094 & $<0.06$ & 0.074 & 0.05 & 3 \\
\hline Turbidity, NTU & NA & 8 & 17 & 0.5 & 3.2 & 5 & 2 \\
\hline
\end{tabular}


Table 2.3.12 (continued)

\begin{tabular}{|c|c|c|c|c|c|c|c|}
\hline \multirow{2}{*}{ Parameter } & \multirow{2}{*}{$\begin{array}{l}\text { Number } \\
\text { detected }\end{array}$} & \multirow{2}{*}{$\begin{array}{c}\text { Number of } \\
\text { samples }\end{array}$} & \multicolumn{3}{|c|}{ Values above detection limit } & \multirow{2}{*}{$\begin{array}{c}\text { Reference } \\
\text { value }\end{array}$} & \multirow{2}{*}{$\begin{array}{l}\text { Number of values } \\
\text { exceeding reference }\end{array}$} \\
\hline & & & $\operatorname{Max}$ & Min & Av & & \\
\hline \multicolumn{8}{|c|}{ Fly Ash Pond } \\
\hline Bis(2-ethylhexyl)phthalate, $\mu \mathrm{g} / \mathrm{L}$ & 4 & 11 & 370 & $<10$ & 200 & NR & NA \\
\hline Coliform, col. $/ 100 \mathrm{~mL}$ & 2 & 16 & 13 & 0 & 8 & 1 & 2 \\
\hline Iron, total, $\mathrm{mg} / \mathrm{L}$ & 16 & 16 & 1.3 & 0.0043 & 0.24 & 0.3 & 3 \\
\hline PCB-(Aroclor)-1254, $\mu \mathrm{g} / \mathrm{L}$ & i & 16 & 33 & $<1$ & 33 & NR & NA \\
\hline $\mathrm{pH}$ & NA & 64 & 8 & 5.7 & NA & $6.5 / 8.5$ & 31 \\
\hline Turbidity, NTU & NA & 16 & 26 & 1.4 & .3 & 5 & 4 \\
\hline 2-Butanone, $\mu \mathrm{g} / \mathrm{L}$ & 2 & 12 & 11 & $<10$ & 11 & NR & NA \\
\hline \multicolumn{8}{|c|}{ Industria! Landfill III } \\
\hline Acetone, $\mu \mathrm{g} / \mathrm{L}$ & 1 & 21 & 11 & $<10$ & 11 & NR & NA \\
\hline Coliform, col./100 mL & 6 & 28 & 136 & 0 & 30 & 1 & 5 \\
\hline Fluoride, $\mathrm{mg} / \mathrm{L}$ & 6 & 28 & 7 & $<0.1$ & 1 & 4 & 1 \\
\hline Iron, total, mg/L & 28 & 28 & 60 & 0.0072 & 5.3 & 0.3 & 17 \\
\hline Lead, total, $\mathrm{mg} / \mathrm{L}$ & 15 & 28 & 0.11 & $<0.004$ & 0.027 & 0.05 & 2 \\
\hline Manganese, total, mg/L & 25 & 28 & 0.79 & $<0.001$ & 0.11 & 0.05 & 9 \\
\hline $\mathrm{pH}$ & NA & 112 & 8.1 & 6 & NA & $6.5 / 8.5$ & 38 \\
\hline Radium, pCi/L & NA & 28 & 7.3 & -0.35 & 1.1 & 5 & 1 \\
\hline Turbidity, NTU & NA & 28 & 1300 & 0.4 & 140 & 5 & 15 \\
\hline \multicolumn{8}{|c|}{ Industrial Landfill IV } \\
\hline Coliform, col. $/ 100 \mathrm{~mL}$ & 2 & 13 & 15 & 0 & 10 & 1 & 2 \\
\hline Iron, total, mg/L & 13 & 13 & 45 & 0.021 & 5.06 & 0.3 & 5 \\
\hline Lead, total, mg/L & 5 & 13 & 0.063 & $<0.004$ & 0.025 & 0.05 & 1 \\
\hline Manganese, total, $\mathrm{mg} / \mathrm{L}$ & 10 & 13 & 0.53 & $<0.001$ & 0.09 & 0.05 & 2 \\
\hline $\mathrm{pH}$ & NA & 52 & 8.2 & 6 & NA & $6.5 / 8.5$ & 8 \\
\hline Radium, $\mathrm{pCi} / \mathrm{L}$ & NA & 13 & 5.1 & -0.32 & 1.0 & 5 & 1 \\
\hline Turbidity, NTU & NA & 13 & 450 & 1.9 & 57 & 5 & 8 \\
\hline \multicolumn{8}{|c|}{ Kerr Hollow Quarry } \\
\hline Arsenic, total, mg/L & 3 & 7 & 0.11 & $<0.05$ & 0.073 & 0.05 & 3 \\
\hline Dissolved solids, mg/L & NA & 21 & 2,220 & 132 & 311 & 500 & 1 \\
\hline Iron, total, mg/L & 6 & 7 & 17 & $<0.004$ & 4.9 & 0.3 & 3 \\
\hline Manganese, total, $\mathrm{mg} / \mathrm{L}$ & 5 & 7 & 0.088 & $<0.001$ & 0.041 & 0.05 & 2 \\
\hline $\mathrm{pH}$ & NA & 84 & 7.4 & 6.3 & NA & $6.5 / 8.5$ & 8 \\
\hline Turbidity, NTU & NA & 14 & 160 & 0.3 & 29 & 5 & 9 \\
\hline \multicolumn{8}{|c|}{ New Hope Pond } \\
\hline Acetone, $\mu g / \mathrm{L}$ & 1 & 59 & 23 & $<10$ & 23 & NR & NA \\
\hline Carbon disulfide, $\mu \mathrm{g} / \mathrm{L}$ & 1 & 59 & 190 & $<5$ & 190 & NR & NA \\
\hline Carbon tetrachloride, $\mu \mathrm{g} / \mathrm{L}$ & 35 & 59 & 10,000 & $<5$ & 1,600 & 5 & 35 \\
\hline Chloride, $\mathrm{mg} / \mathrm{L}$ & 59 & 59 & 430 & 2 & 45 & 250 & 2 \\
\hline Chloroform, $\mu \mathrm{g} / \mathrm{L}$ & 26 & 59 & 1,000 & $<5$ & 160 & NR & NA \\
\hline Chromium, total, mg/L & 7 & 59 & 0.18 & $<0.01$ & 0.066 & 0.05 & 2 \\
\hline Dissolved solids, $\mathrm{mg} / \mathrm{L}$ & NA & 59 & 780 & 168 & 369 & 500 & 9 \\
\hline Gross alpha, $\mathrm{pCi} / \mathrm{L}$ & NA & 59 & 790 & -2 & 30 & 15 & 6 \\
\hline Gross beta, pCi/L & NA & 59 & 1,100 & -3 & 43 & 50 & 5 \\
\hline Lead, total, mg/L & 29 & 59 & 0.24 & $<0.004$ & 0.023 & 0.05 & 2 \\
\hline Methylene chloride, $\mu \mathrm{g} / \mathrm{L}$ & 1 & 59 & 7 & $<5$ & 7 & NR & $\mathrm{NA}$ \\
\hline $\mathrm{pH}$ & NA & 236 & 9.1 & 6.1 & NA & $6.5 / 8.5$ & 51 \\
\hline Tetrachloroethene, $\mu \mathrm{g} / \mathrm{L}$ & 24 & 59 & 710 & $<5$ & 160 & NR & NA \\
\hline
\end{tabular}


Table 2.3.12 (continued)

\begin{tabular}{|c|c|c|c|c|c|c|c|}
\hline \multirow{2}{*}{ Parameter } & \multirow{2}{*}{$\begin{array}{l}\text { Number } \\
\text { detected }\end{array}$} & \multirow{2}{*}{$\begin{array}{c}\text { Number of } \\
\text { samples }\end{array}$} & \multicolumn{3}{|c|}{ Values above detection limit } & \multirow{2}{*}{$\begin{array}{l}\text { Reference } \\
\text { value }\end{array}$} & \multirow{2}{*}{$\begin{array}{l}\text { Number of values } \\
\text { exceeding reference }\end{array}$} \\
\hline & & & $\operatorname{Max}$ & Min & Av & & \\
\hline Trichloroethene, $\mu \mathrm{g} / \mathrm{L}$ & 14 & 59 & 150 & $<5$ & 64 & 5 & 14 \\
\hline Turbidity, NTU & NA & 43 & 280 & 0.5 & 17 & 5 & 24 \\
\hline 1,2-Dichloroethene, total, $\mu \mathrm{g} / \mathrm{L}$ & 10 & 59 & 110 & $<5$ & 52 & NR & NA \\
\hline \multicolumn{8}{|c|}{ Oil Landfarm } \\
\hline Acetone, $\mu \mathrm{g} / \mathrm{L}$ & 8 & 90 & 51 & $<10$ & 23 & NR & NA \\
\hline Barium, total, mg/L. & 24 & 25 & 1.1 & $<0.1$ & 0.31 & 1 & 1 \\
\hline Benzene, $\mu \mathrm{g} / \mathrm{L}$ & 5 & 90 & 41 & $<5$ & 18 & 5 & 5 \\
\hline Cadmium, total, mg/L & 7 & 25 & 0.019 & $<0.002$ & 0.0064 & 0.01 & 2 \\
\hline Carbon tetrachloride, $\mu \mathrm{g} / \mathrm{L}$ & 8 & 90 & 9 & $<5$ & 6 & 5 & 4 \\
\hline Chlorobenzene, $\mu \mathrm{g} / \mathrm{L}$ & 2 & 90 & 6 & $<5$ & 6 & NR & NA \\
\hline Chloroform, $\mu \mathrm{g} / \mathrm{L}$ & 5 & 90 & 33 & $<5$ & 16 & NR & NA \\
\hline Chromium, total, $\mathrm{mg} / \mathrm{L}$ & 21 & 90 & 0.11 & $<0.01$ & 0.034 & 0.05 & 4 \\
\hline Dissolved solids, $\mathrm{mg} / \mathrm{L}$ & NA & 90 & 1.210 & 98 & 444 & 500 & 33 \\
\hline Gross alpha, $\mathrm{pCi} / \mathrm{L}$ & NA & 90 & 69 & -26 & 4.8 & 15 & 6 \\
\hline Gross beta, $\mathrm{pCi} / \mathrm{L}$ & NA & 90 & 970 & -35 & 39 & 50 & 11 \\
\hline Lead, total, $\mathrm{mg} / \mathrm{L}$ & 47 & 90 & 0.64 & $\Upsilon 0.004$ & 0.035 & 0.05 & 6 \\
\hline Nitrate-N, $\mathrm{mg} / \mathrm{L}$ & 60 & 90 & 210 & $<0.2$ & 21 & 10 & 22 \\
\hline $\mathrm{pH}$ & NA & 360 & 12.1 & 5.1 & NA & $6.5 / 8.5$ & 103 \\
\hline Strontium, $\mathrm{pCi} / \mathrm{L}$ & NA & 18 & 98 & -61 & 5.7 & 8 & 6 \\
\hline Tetrachloroethene, $\mu \mathrm{g} / \mathrm{L}$ & 11 & 90 & 360 & $<5$ & 78 & NR & NA \\
\hline Trichloroethene, $\mu \mathrm{g} / \mathrm{L}$ & 55 & 90 & 400 & $<5$ & 81 & 5 & 55 \\
\hline Turbidity, NTU & NA & 68 & 950 & 0.35 & 64 & 5 & 42 \\
\hline Vinyl chloride, $\mu \mathrm{g} / \mathrm{L}$ & 4 & 90 & 35 & $<10$ & 23 & 2 & 4 \\
\hline 1,1-Dichloroethane, $\mu \mathrm{g} / \mathrm{L}$ & 23 & 90 & 14 & $<5$ & 8 & NR & NA \\
\hline 1.1-Dichloroethene, $\mu \mathrm{g} / \mathrm{L}$ & 13 & 90 & 25 & $<5$ & 11 & 7 & 4 \\
\hline 1,2-Dichloroethane, $\mu \mathrm{g} / \mathrm{L}$ & 2 & 90 & 11 & $<5$ & 9 & 5 & 2 \\
\hline 1,2-Dichloroethene, total, $\mu \mathrm{g} / \mathrm{L}$ & 44 & 90 & 190 & $<5$ & 30 & NR & NA \\
\hline $228 \mathrm{Radium}, \mathrm{pCi} / \mathrm{L}$ & NA & 19 & 51 & -5.4 & 5.7 & 5 & 5 \\
\hline \multicolumn{8}{|c|}{ Roger's Quarry } \\
\hline Coliform, col. $/ 100 \mathrm{~mL}$ & 2 & 4 & 17 & 0 & 10 & 1 & 2 \\
\hline Dissolved solids, $\mathrm{mg} / \mathrm{L}$ & NA & 4 & 584 & 260 & 472 & 500 & 3 \\
\hline Iron, total, $\mathrm{mg} / \mathrm{L}$ & 4 & 4 & 4 & 0.058 & 1.3 & 0.3 & 2 \\
\hline Manganese, total, $\mathrm{mg} / \mathrm{L}$ & 4 & 4 & 0.46 & 0.0013 & 0.15 & 0.05 & 2 \\
\hline Nitrate- $\mathrm{N}, \mathrm{mg} / \mathrm{L}$ & 1 & 4 & 37 & $<0.1$ & 37 & 10 & 1 \\
\hline Turbidity, NTU & NA & 4 & 24 & 7.6 & 13 & 5 & 4 \\
\hline \multicolumn{8}{|c|}{ Rust Spoil Area } \\
\hline Acetone, $\mu \mathrm{g} / \mathrm{L}$ & 5 & 18 & 40 & $<10$ & 22 & NR & NA \\
\hline Cadmium, total, $\mathrm{mg} / \mathrm{L}$ & 2 & 18 & 0.016 & $<0.002$ & 0.011 & 0.01 & 1 \\
\hline Chloroform, $\mu \mathrm{g} / \mathrm{L}$ & 3 & 18 & 6 & $<5$ & 6 & NR & NA \\
\hline Coliform, col./100 mL & 6 & 18 & 30 & 0 & 7 & 1 & 4 \\
\hline Dissolved solids, $\mathrm{mg} / \mathrm{L}$ & NA & 18 & 768 & 194 & 543 & 500 & 11 \\
\hline Gross alpha, $\mathrm{pCi} / \mathrm{L}$ & NA & 18 & 18 & -1 & 3.6 & 15 & 1 \\
\hline Gross beta, $\mathrm{pCi} / \mathrm{L}$ & NA & 18 & 57 & -1 & 30 & 50 & 1 \\
\hline Iron, total, $\mathrm{mg} / \mathrm{L}$ & 17 & 18 & 20 & $<0.004$ & 3.5 & 0.3 & 10 \\
\hline Lead, total, $\mathrm{mg} / \mathrm{L}$ & 9 & 18 & 0.11 & $<0.004$ & 0.024 & 0.05 & 1 \\
\hline Manganese, total, mg/L & 18 & 18 & 2.9 & 0.0021 & 0.59 & 0.05 & 10 \\
\hline Nitrate- $N, m_{g} / L$ & 18 & 18 & 24 & 0.4 & 12 & 10 & 12 \\
\hline $\mathrm{pH}$ & NA & 72 & 12.7 & 6.2 & NA & $6.5 / 8.5$ & 32 \\
\hline Trichloroethene, $\mu \mathrm{g} / \mathrm{L}$ & 18 & 18 & 120 & 21 & 49 & 5 & 18 \\
\hline Turbidity, NTU & NA & 18 & 170 & 0.5 & 28 & 5 & 9 \\
\hline 1,2-Dichloroethene, total, $\mu \mathrm{g} / \mathrm{L}$ & 12 & 18 & 200 & $<5$ & 40 & NR & NA \\
\hline
\end{tabular}


Table 2.3.12 (continued)

\begin{tabular}{|c|c|c|c|c|c|c|c|}
\hline \multirow{2}{*}{ Parameter } & \multirow{2}{*}{$\begin{array}{l}\text { Number } \\
\text { detected }\end{array}$} & \multirow{2}{*}{$\begin{array}{c}\text { Number of } \\
\text { samples }\end{array}$} & \multicolumn{3}{|c|}{ Values above detection limit } & \multirow{2}{*}{$\begin{array}{c}\text { Reference } \\
\text { value }\end{array}$} & \multirow{2}{*}{$\begin{array}{l}\text { Number of values } \\
\text { exceeding reference }\end{array}$} \\
\hline & & & $\operatorname{Max}$ & Min & Av & & \\
\hline \multicolumn{8}{|c|}{ S.2 Pond Site } \\
\hline Cadmium, total, $\mathrm{mg} / \mathrm{L}$ & 5 & 5 & 5.1 & 0.23 & 1.2 & 0.01 & 5 \\
\hline Carbon tetrachloride, $\mu \mathrm{g} / \mathrm{L}$ & 5 & 5 & 39 & 19 & 26 & 5 & 5 \\
\hline Chloroform, $\mu \mathrm{g} / \mathrm{L}$ & 5 & 5 & 42 & 9 & 16 & NR & NA \\
\hline Copper, total, mg/L & 2 & 2 & 120 & 1.5 & 61 & 1 & 2 \\
\hline Dissolved solids, $\mathrm{mg} / \mathrm{L}$ & NA & 5 & 2,880 & 728 & 1,210 & 500 & 5 \\
\hline Gross alpha, $\mathrm{pCi} / \mathrm{L}$ & NA & 5 & 32 & 0.03 & 11 & 15 & 1 \\
\hline Iron, total, mg/L & 2 & 2 & 4.5 & 0.33 & 2.4 & 0.3 & 2 \\
\hline Lead, total, mg/L & 3 & 5 & 0.099 & $<0.004$ & 0.038 & 0.05 & 1 \\
\hline Manganese, total, $\mathrm{mg} / \mathrm{L}$ & 2 & 2 & 45 & 9.1 & 27 & 0.05 & 2 \\
\hline Mercury, total, mg/L & 3 & 5 & 0.017 & $<0.0002$ & 0.0058 & 0.002 & 1 \\
\hline Nitrate- $\mathrm{N}, \mathrm{mg} / \mathrm{L}$ & 5 & 5 & 510 & 91 & 180 & 10 & 5 \\
\hline $\mathrm{pH}$ & NA & 2.0 & 6.2 & 5.2 & NA & $6.5 / 8.5$ & 20 \\
\hline Radium, pCi/L & $\mathrm{NA}$ & 2 & 30 & 4.3 & 17 & 5 & 1 \\
\hline Strontium, $\mathrm{pCi} / \mathrm{L}$ & NA & 2 & 8.0 & 5.6 & 6.8 & 8 & 1 \\
\hline Tetrachlorocthene, $\mu \mathrm{g} / \mathrm{L}$ & 5 & 5 & 760 & 200 & 440 & NR & NA \\
\hline Trichloroethene, $\mu \mathrm{g} / \mathrm{L}$ & 5 & 5 & 470 & 130 & 220 & 5 & 5 \\
\hline Turbidity, NTU & NA & 5 & 36 & 7.1 & 20 & 5 & 5 \\
\hline Zinc, total, $\mathrm{mg} / \mathrm{L}$ & 2 & 2 & 6.9 & 0.25 & 3.6 & 5 & 1 \\
\hline 1,2-Dichloroethene, totai, $\mu \mathrm{g} / \mathrm{L}$ & 3 & 5 & 60 & $<5$ & 25 & NR & NA \\
\hline \multicolumn{8}{|c|}{ S-3 Ponds Site } \\
\hline Acetone, $\mu \mathrm{g} / \mathrm{L}$ & 18 & 124 & 390 & $<10$ & 131 & NR & NA \\
\hline Barium, total, $\mathrm{mg} / \mathrm{L}$ & 116 & 124 & 170 & $<0.1$ & 20 & 1 & 62 \\
\hline Bromoform, $\mu \mathrm{g} / \mathrm{L}$ & 2 & 124 & 9 & $<5$ & 9 & NR & NA \\
\hline Cadmium, total, $\mathrm{mg} / \mathrm{L}$ & 47 & 124 & 4.9 & $<0.002$ & 0.57 & 0.01 & 34 \\
\hline Chloride, $\mathrm{mg} / \mathrm{L}$ & 109 & 124 & 460 & $<100$ & 74 & 250 & 8 \\
\hline Chloroform, $\mu \mathrm{g} / \mathrm{L}$ & 22 & 124 & 30 & $<5$ & 18 & NR & NA \\
\hline Chlorumethane, $\mu \mathrm{g} / \mathrm{L}$ & 1 & 124 & 10 & $<10$ & 10 & NR & NA \\
\hline Chromium, total, mg/L & 22 & 124 & 0.42 & $<0.02$ & 0.067 & 0.05 & 6 \\
\hline Dissolved solids, $\mathrm{mg} / \mathrm{L}$ & NA & 124 & 79,400 & 18 & 11,000 & 500 & 85 \\
\hline Fluoride, mg/L & 52 & 123 & 36 & $<0.1$ & 5 & 4 & 18 \\
\hline Gross alpha, $\mathrm{pCi} / \mathrm{L}$ & NA & 124 & 14,000 & -170 & 250 & 15 & 40 \\
\hline Gross beta, pCi/L & NA & 124 & 38,000 & -710 & 2,000 & 50 & 60 \\
\hline Lead, total, mg/L & $\$ 5$ & 124 & 0.15 & $<0.01$ & 0.015 & 0.05 & 2 \\
\hline Mercury, total, mg/L. & 22 & 124 & 0.16 & $<0.0002$ & 0.025 & 0.002 & 12 \\
\hline Methylene chloride, $\mu \mathrm{g} / \mathrm{L}$ & 24 & 124 & 87 & $<5$ & 29 & NR & NA \\
\hline Nitrate- $\mathrm{N}, \mathrm{mg} / \mathrm{L}$ & 101 & 124 & 18,000 & $<2$ & 2,1500 & 10 & 80 \\
\hline $\mathrm{pH}$ & NA & 492 & 10 & 3.4 & NA & $6.5 / 8.5$ & 320 \\
\hline Radium, pCi/L & NA & 47 & 81 & -0.38 & 12 & 5 & 20 \\
\hline Strontium, $\mathrm{pCi} / \mathrm{L}$ & NA & 43 & 302 & -37 & 40 & 8 & 23 \\
\hline Sulfate, $\mathrm{mg} / \mathrm{L}$ & 83 & 124 & 760 & $<100$ & 78 & 250 & 6 \\
\hline Tetrachloroethene, $\mu g / L$ & 25 & 124 & 2,300 & $<5$ & 230 & NR & NA \\
\hline Toluene, $\mu \mathrm{g} / \mathrm{L}$ & 2 & 124 & 14 & $<5$ & 14 & NR & NA \\
\hline Trichloroethene, $\mu \mathrm{g} / \mathrm{L}$ & 10 & 124 & 11 & $<5$ & 8 & 5 & 9 \\
\hline Turbidity, NTU & NA & 90 & 170 & 0.3 & 17 & 5 & 44 \\
\hline Xvlenes, $\mu \mathrm{g} / \mathrm{L}$ & 4 & 124 & 16 & $<5$ & 12 & NR & NA \\
\hline 1,1-Dichloroethane, $\mu \mathrm{g} / \mathrm{L}$ & 1 & 124 & 5 & $<5$ & 5 & NR & NA \\
\hline 1,2-Dichloroethene, total, $\mu_{\mathrm{B}} / \mathrm{L}$ & 2 & 124 & 9 & $<5$ & 8 & NR & NA \\
\hline 2-Butanone, $\mu g / L$ & 5 & 124 & 66 & $<10$ & 33 & NR & NA \\
\hline Radium-228, pCi/L & NA & 40 & 120 & -4.9 & 36 & 5 & 27 \\
\hline 4-Methyl-2-pentanone, $\mu \mathrm{g} / \mathrm{L}$ & 2 & 124 & 12 & $<10$ & 12 & NR & NA \\
\hline
\end{tabular}


Table 2.3.12 (continued)

\begin{tabular}{|c|c|c|c|c|c|c|c|}
\hline \multirow{2}{*}{ Parameter } & \multirow{2}{*}{$\begin{array}{l}\text { Number } \\
\text { detected }\end{array}$} & \multirow{2}{*}{$\begin{array}{l}\text { Number of } \\
\text { samples }\end{array}$} & \multicolumn{3}{|c|}{ Values above detection limit } & \multirow{2}{*}{$\begin{array}{l}\text { Reference } \\
\text { value }\end{array}$} & \multirow{2}{*}{$\begin{array}{l}\text { Number of values } \\
\text { exceeding reference }\end{array}$} \\
\hline & & & Max & Min & Av & & \\
\hline \multicolumn{8}{|c|}{ Sı 3 Ponds Site Salvage Yard/OSDS } \\
\hline Acetone, $\mu \mathrm{g} / \mathrm{L}$ & 3 & 28 & 22 & $<10$ & 18 & NR & NA \\
\hline Arsenic, total, mg/L & 2 & 28 & 0.063 & $<0.05$ & 0.06 & 0.05 & 2 \\
\hline Dissolved soids, $\mathrm{mg} / \mathrm{L}$ & $\mathrm{NA}$ & 28 & 704 & 70 & 257 & 500 & 4 \\
\hline Ethylbenzene, $\mu \mathrm{g} / \mathrm{L}$ & 1 & 28 & 5 & $<5$ & 5 & NR & NA. \\
\hline Iron, total, $\mathrm{mg} / \mathrm{L}$ & 27 & 28 & 17 & $<0.004$ & 1.9 & 0.3 & 20 \\
\hline Lead, total, $\mathrm{mg} / \mathrm{L}$ & 10 & 28 & 0.4 & $<0.004$ & 0.049 & 0.05 & 1 \\
\hline Manganese, total, mg/L & 28 & 28 & 0.99 & 0.0092 & 0.15 & 0.05 & 14 \\
\hline Nitrate- $N, \mathrm{mg} / \mathrm{L}$ & 13 & 28 & 1,600 & $<0.2$ & 240 & 10 & 2 \\
\hline $\mathrm{pH}$ & NA & 112 & 7.8 & 5.1 & NA & $6.5 / 8.5$ & 76 \\
\hline Sulfate, $\mathrm{mg} / \mathrm{L}$ & 28 & 28 & 1,300 & 5 & 79 & 250 & 1 \\
\hline Tetrachloroethene, $\mu \mathrm{g} / \mathrm{L}$ & 8 & 28 & 280 & $<5$ & 110 & NR & NA \\
\hline Trichloroethene, $\mu \mathrm{g} / \mathrm{L}$ & 5 & 28 & 27 & $<5$ & 20 & 5 & 5 \\
\hline Turbidity, NTU & NA & 28 & 190 & 0.8 & 20 & 5 & 21 \\
\hline 1,1-Dichloroethene, $\mu \mathrm{g} / \mathrm{L}$ & 4 & 28 & 31 & $<5$ & 25 & 7 & 4 \\
\hline 1,2-Dichloroethene, total, $\mu \mathrm{g} / \mathrm{L}$ & 4 & 28 & 18 & $<5$ & 14 & NR & NA \\
\hline 2-Butanone, $\mu \mathrm{g} / \mathrm{L}$ & 1 & 28 & 10 & $<10$ & 10 & NR & $\mathrm{NA}$ \\
\hline \multicolumn{8}{|c|}{ Sanitary Landfill II } \\
\hline Acetone, $\mu \mathrm{g} / \mathrm{L}$ & 1 & 10 & 26 & $<10$ & 26 & NR & NA \\
\hline Arsenic, total, mg/L & 4 & 12 & 0.12 & $<0.040$ & 0.080 & 0.05 & 3 \\
\hline Cadmium, total, mg/L & 4 & 6 & 0.023 & $<0.00050$ & 0.0064 & 0.01 & 1 \\
\hline Carbon tetrachloride, $\mu \mathrm{g} / \mathrm{L}$ & 1 & 10 & 16 & $<10$ & 16 & 5 & 1 \\
\hline Chromium, total, mg/L & 3 & 6 & 0.082 & $<0.025$ & 0.048 & 0.05 & 2 \\
\hline Gross alpha, $\mathrm{pCi} / \mathrm{L}$ & NA & 5 & 74 & 6.5 & 29 & 15 & 2 \\
\hline Gross beta, $\mathrm{pCi} / \mathrm{L}$ & $\mathrm{NA}$ & 5 & 84 & -1.7 & 26 & 50 & 1 \\
\hline Iron, total, mg/L & 5 & 6 & 19 & $<0.15$ & 4.7 & 0.3 & 3 \\
\hline Lead, total, $\mathrm{mg} / \mathrm{L}$ & 6 & 6 & 0.16 & 0.0010 & 0.033 & 0.05 & 1 \\
\hline Manganese, total, $\mathrm{mg} / \mathrm{L}$ & 5 & 6 & 0.92 & $<0.010$ & 0.30 & 0.05 & 3 \\
\hline Mercury, total, mg/L & 2. & 6 & 0.0022 & $<0.00020$ & 0.0015 & 0.002 & 1 \\
\hline Tetrachlorocthene, $\mu \mathrm{g} / \mathrm{L}$ & 2 & 10 & 13 & $<10$ & 10 & NR & NA \\
\hline 1,1-Dichloroethane, $\mu \mathrm{g} / \mathrm{L}$ & 3 & 10 & 49 & $<10$ & 37 & NR & NA \\
\hline 226 Radium, pCi/L & 1 & 6 & 9.2 & $<1.0$ & 9.2 & 5 & 1 \\
\hline \multicolumn{8}{|c|}{ United Nuclear Site } \\
\hline Arsenic, total, mg/L & 3 & 15 & 0.2 & $<0.05$ & 0.11 & 0.05 & 3 \\
\hline Cadmium, total, mg/L & 3 & 20 & 0.02 & $<0.003$ & 0.01 & 0.01 & 1 \\
\hline Chromium, total, $\mathrm{mg} / \mathrm{L}$ & 3 & 20 & 0.2 & $<0.01$ & 0.076 & 0.05 & 1 \\
\hline Gross beta, $\mathrm{pCi} / \mathrm{L}$ & NA & 15 & 190 & 0.2 & 27 & 50 & 2 \\
\hline Iton, total, mg/L & 19 & 20 & 370 & $<0.004$ & 21 & 0.3 & 6 \\
\hline Lead, total, mg/L & 1 & 15 & 0.99 & $<0.05$ & 0.99 & 0.05 & 1 \\
\hline Manganese, total, $\mathrm{mg} / \mathrm{L}$ & 15 & 20 & 14 & $<0.001$ & 0.98 & 0.05 & 3 \\
\hline $\mathrm{pH}$ & NA & 80 & 8.1 & 6.3 & NA & $6.5 / 8.5$ & 17 \\
\hline Radium, nCi/L & NA & 20 & 19 & -0.27 & 1.7 & 5 & 1 \\
\hline Seleniurn, total, $\mathrm{mg} / \mathrm{L}$ & 1 & 15 & 0.2 & $<0.05$ & 0.2 & 0.01 & 1 \\
\hline Turbidity, NTU & NA & 15 & 1,900 & 0.5 & 140 & 5 & 6 \\
\hline
\end{tabular}


Table 2.3.12 (continued)

\begin{tabular}{|c|c|c|c|c|c|c|c|}
\hline \multirow{2}{*}{ Parameter } & \multirow{2}{*}{$\begin{array}{l}\text { Number } \\
\text { detected }\end{array}$} & \multirow{2}{*}{$\begin{array}{l}\text { Number of } \\
\text { samples }\end{array}$} & \multicolumn{3}{|c|}{ Values above detection limit } & \multirow{2}{*}{$\begin{array}{c}\text { Reference } \\
\text { value }\end{array}$} & \multirow{2}{*}{$\begin{array}{l}\text { Number of values } \\
\text { exceeding reference }\end{array}$} \\
\hline & & & Max & Min & Av & & \\
\hline \multicolumn{8}{|c|}{ Waste Coolant Facility } \\
\hline Acetone, $\mu \mathrm{g} / \mathrm{L}$ & 9 & 24 & 560 & $<10$ & 260 & NR & NA \\
\hline Bis(2-ethylhexyl)phthalate, $\mu \mathrm{g} / \mathrm{L}$ & 5 & 24 & 770 & $<10$ & 190 & NR & NA \\
\hline Cadmium, total, $\mathrm{mg} / \mathrm{L}$ & $!$ & 24 & 0.049 & $<0,002$ & 0.049 & 0.01 & 1 \\
\hline Chloroform, $\mu \mathrm{g} / \mathrm{L}$ & 1 & 24 & 5 & $<5$ & 5 & NR & NA \\
\hline Coliform, col. $/ 100 \mathrm{~mL}$ & 5 & 23 & 13 & 0 & 4 & 1 & 2 \\
\hline Iron, total, $\mathrm{mg} / \mathrm{L}$ & 24 & 24 & 9.2 & 0.039 & 2.3 & 0.3 & 18 \\
\hline Manganese, total, mg/L & 24 & 24 & 2.2 & 0.004 & 0.3 & 0.05 & 19 \\
\hline Methylene chloride, $\mu \mathrm{g} / \mathrm{L}$ & 1 & 24 & 6 & $<5$ & 6 & NR & NA \\
\hline Naphthalene, $\mu \mathrm{g} / \mathrm{L}$ & 1 & 24 & 12 & $<10$ & 12 & NR & NA \\
\hline $\mathrm{pH}$ & NA & 96 & 7.3 & 5.4 & NA & $6.5 / 8.5$ & 62 \\
\hline Tetrachloroethene, $\mu \mathrm{g} / \mathrm{L}$ & 18 & 24 & 2,100 & $<5$ & 690 & NR & NA \\
\hline Trichloroethene, $\mu \mathrm{g} / \mathrm{L}$ & 18 & 24 & 2,000 & $<5$ & 520 & 5 & 18 \\
\hline Turbidity, NTU & NA & 24 & 110 & 0.49 & 33 & 5 & 21 \\
\hline Vinyl chloride, $\mu \mathrm{g} / \mathrm{L}$ & 11 & 24 & 440 & $<10$ & 190 & 2 & 11 \\
\hline 1,1-Dichloroethane, $\mu \mathrm{g} / \mathrm{L}$ & 12 & 24 & 160 & $<5$ & 70 & NR & NA \\
\hline 1,1-Dichlorocthene, $\mu \mathrm{g} / \mathrm{L}$ & 12 & 24 & 420 & $<5$ & 230 & 7 & 12 \\
\hline 1,1,1-Trichloroethane, $\mu \mathrm{g} / \mathrm{L}$ & 12 & 24 & 550 & $<5$ & 190 & 200 & 3 \\
\hline 1,2-Dichloroethene, total, $\mu \mathrm{g} / \mathrm{L}$ & 16 & 24 & 15,000 & $<5$ & 3.700 & NR & NA \\
\hline 2-Butanone, $\mu \mathrm{g} / \mathrm{L}$ & 3 & 24 & 580 & $<10$ & 370 & NR & NA \\
\hline 4-Methyl-2-pentanone, $\mu \mathrm{g} / \mathrm{L}$ & 3 & 24 & 140 & $<10$ & 82 & NR & NA \\
\hline \multicolumn{8}{|c|}{ 9754-2 Fuel Facility } \\
\hline Bis(2-ethylhexyl)phthalate, $\mu \mathrm{g} / \mathrm{L}$ & 1 & 15 & 15 & $<10$ & 15 & NR & NA \\
\hline Cadmium, total, $\mathrm{mg} / \mathrm{L}$ & 6 & 15 & 0.042 & $<0.002$ & 0.015 & 0.01 & 2 \\
\hline Chloride, $\mathrm{mg} / \mathrm{L}$ & 15 & 15 & 310 & 3.0 & 71 & 250 & 2 \\
\hline Chromium, total, mg/L & 14 & 15 & 5.4 & $<0.01$ & 0.84 & 0.05 & 8 \\
\hline Coliform, col./100 mL & 4 & 15 & 19 & 0 & 8 & 1 & 4 \\
\hline Di-n-octylphthalate, $\mu \mathrm{g} / \mathrm{L}$ & 1 & 15 & 29 & $<10$ & 29 & NR & NA \\
\hline Dissolved sclids, mg/L & NA & 15 & 1,180 & 68 & 423 & 500 & 5 \\
\hline Gross alpina, $\mathrm{pCi} / \mathrm{L}$ & NA & 15 & 24 & -2 & 3.6 & 15 & 1 \\
\hline Gross bet:, $\mathrm{pCi} / \mathrm{L}$ & NA & 15 & 130 & -6.1 & 13 & 50 & 1 \\
\hline Iron, total, mg/Ll & 15 & 15 & 84 & 0.45 & 12 & 0.3 & 15 \\
\hline Lead, total, $\mathrm{mg} / \mathrm{L}$ & 9 & 15 & 0.064 & $<0.004$ & 0.018 & 0.05 & 1 \\
\hline Manganese, total, $\mathrm{mg} / \mathrm{L}$ & 15 & 15 & 3.1 & 0.2 & 1.3 & 0.05 & 15 \\
\hline Mercury, total, mg/L & 5 & 15 & 0.0034 & $<0.0002$ & 0.0016 & 0.002 & 2 \\
\hline Methylene chloride, $\mu \mathrm{g} / \mathrm{L}$ & 1 & 15 & 59 & $<5$ & 59 & NR & NA \\
\hline $\mathrm{pH}$ & NA & 60 & 7.2 & 5.9 & NA & $6.5 / 8.5$ & 21 \\
\hline Turbidity, NTU & NA & 15 & 1400 & 9.6 & 240 & 5 & 15 \\
\hline
\end{tabular}

${ }^{a} \mathrm{NA}=$ not applicable

${ }^{b} \mathrm{NR}=$ no reference. 
Table 2.3.13. Constituents in the WAG 1 groundwater at ORNL, January-December 1989

\begin{tabular}{|c|c|c|c|c|c|c|}
\hline \multirow{2}{*}{ Parameter } & \multirow{2}{*}{$\begin{array}{l}\text { No. detected/ } \\
\text { No. of samples }\end{array}$} & \multicolumn{2}{|c|}{ Concentration $^{b}$} & \multirow{2}{*}{$\begin{array}{c}\text { Average of values } \\
\text { above the } \\
\text { detection limit }\end{array}$} & \multirow{2}{*}{$\begin{array}{l}\text { Reference } \\
\text { value }^{c}\end{array}$} & \multirow{2}{*}{$\begin{array}{l}\text { No. of values } \\
\text { exceeding } \\
\text { reference }\end{array}$} \\
\hline & & $\operatorname{Max}$ & Min & & & \\
\hline \multicolumn{7}{|c|}{ Perimeter wells } \\
\hline \multicolumn{7}{|l|}{ Anions, mg/L } \\
\hline Fluoride, total & $18 / 55$ & 4.3 & $1.0 \mathrm{U}$ & 1.7 & 1.4 & 6 \\
\hline \multicolumn{7}{|l|}{ Field data } \\
\hline pH, standard units & $371 / 371$ & 9.3 & 6.2 & 7.2 & $d$ & $e$ \\
\hline Conductivity, $\mathrm{mS} / \mathrm{cm}$ & $371 / 371$ & 1.4 & 0.040 & 0.55 & $d$ & $e$ \\
\hline Temperature, ${ }^{\circ} \mathrm{C}$ & $371 / 371$ & 40 & 12 & 18 & $d$ & $e$ \\
\hline \multicolumn{7}{|l|}{ Metals, mg/L } \\
\hline Aluminum, dissolved & $26 / 38$ & $2.0 \mathrm{U}$ & $0.050 \mathrm{U}$ & 0.32 & $d$ & $e$ \\
\hline Aluminum, total & $37 / 55$ & $2.0 \mathrm{U}$ & $0.050 \mathrm{U}$ & 0.58 & $d$ & $e$ \\
\hline Antimony, dissolved & $5 / 38$ & 0.11 & $0.040 \mathrm{U}$ & 0.065 & $d$ & $e$ \\
\hline Barium, total & $38 / 55$ & 2.3 & 0.0049 & 0.18 & 1.0 & 1 \\
\hline Beryllium, dissolved & $5 / 38$ & 0.030 & $0.00040 \mathrm{U}$ & 0.029 & $d$ & $e$ \\
\hline Beryllium, total & $12 / 55$ & 0.030 & $0.00030 \mathrm{U}$ & 0.029 & $d$ & $e$ \\
\hline Boron, dissolved & $8 / 38$ & 0.91 & $0.080 \mathrm{U}$ & 0.63 & $d$ & $e$ \\
\hline Boron, total & $12 / 55$ & 0.99 & $0.080 \mathrm{U}$ & 0.60 & $d$ & $e$ \\
\hline Cadmiurn, dissolved & $6 / 38$ & 0.011 & $0.0020 U$ & 0.0091 & 0.010 & 1 \\
\hline Cadmiurn, total & $13 / 55$ & 0.045 & $0.0020 \mathrm{U}$ & 0.012 & 0.010 & 2 \\
\hline Calcium, dissolved & $38 / 38$ & 190 & 0.96 & 89 & $d$ & $e$ \\
\hline Calcium, total & $55 / 55$ & 170 & 0.54 & 85 & $d$ & $e$ \\
\hline Chromium, total & $27 / 55$ & 0.11 & $0.0030 \mathrm{U}$ & 0.021 & 0.050 & 1 \\
\hline Cobalt, dissolved & $12 / 38$ & 0.0074 & $0.0030 \mathrm{U}$ & 0.0045 & $d$ & $e$ \\
\hline Cobalt, total & $12 / 55$ & 0.014 & $0.0030 \mathrm{U}$ & 0.0054 & $d$ & $e$ \\
\hline Copper, dissolved & $4 / 38$ & $0.010 \mathrm{U}$ & $0.0080 \mathrm{U}$ & 0.0089 & $d$ & $e$ \\
\hline Iron, dissolved & $24 / 38$ & 14 & $0.010 \mathrm{U}$ & 2.9 & 0.30 & 12 \\
\hline Iron, total & $49 / 55$ & 16 & $0.010 \mathrm{U}$ & 2.4 & 0.30 & 34 \\
\hline Lithium, total & $2 / 53$ & $15 \mathrm{U}$ & $0.20 \mathrm{U}$ & 0.28 & $d$ & $e$ \\
\hline Magnesium, dissolved & $38 / 38$ & 31 & 0.44 & 14 & $d$ & $e$ \\
\hline Magnesium, total & $55 / 55$ & 27 & 0.43 & 12 & $d$ & $e$ \\
\hline Manganese, dissolved & $29 / 38$ & 8.6 & $0.0020 \mathrm{U}$ & 1.9 & 0.050 & 20 \\
\hline Manganese, total & $52 / 55$ & 7.8 & $0.0020 \mathrm{U}$ & 1.3 & 0.050 & 29 \\
\hline Nickel, dissolved & $4 / 38$ & 0.036 & $0.0060 \mathrm{U}$ & 0.023 & $d$ & $e$ \\
\hline Nickel, total & $18 / 55$ & 0.052 & $0.0050 \mathrm{U}$ & 0.016 & $d$ & $\boldsymbol{e}$ \\
\hline Phosphorus, dissolved & $1 / 38$ & 0.65 & $0.30 \mathrm{U}$ & 0.65 & $d$ & $e$ \\
\hline Silicon, dissolved & $38 / 38$ & 8.8 & 2.9 & 5.0 & $d$ & $e$ \\
\hline Silicon, total & $55 / 55$ & 7.7 & 2.3 & 4.8 & $d$ & $e$ \\
\hline Sodium, dissolved & $35 / 38$ & 310 & $2.0 \mathrm{U}$ & 56 & $d$ & $e$ \\
\hline Sodium, total & $52 / 55$ & 340 & $2.0 \mathrm{U}$ & 53 & $d$ & $e$ \\
\hline Strontium, dissolved & $38 / 38$ & 2.6 & 0.075 & 0.57 & $d$ & $e$ \\
\hline Strontium, total & $38 / 38$ & 2.7 & 0.068 & 0.56 & $d$ & $e$ \\
\hline Titanium, total & $17 / 55$ & 0.033 & $0.020 \mathrm{U}$ & 0.022 & $d$ & $e$ \\
\hline Vanadium, dissolved & $4 / 38$ & $0.0040 \mathrm{U}$ & $0.0030 \mathrm{U}$ & 0.0036 & $d$ & $e$ \\
\hline Vanadium, total & $22 / 55$ & 0.014 & $0.0030 \mathrm{U}$ & 0.0095 & $d$ & $e$ \\
\hline Zinc, dissolved & $5 / 38$ & 0.014 & $0.0050 \mathrm{U}$ & 0.011 & $d$ & $e$ \\
\hline \multicolumn{7}{|l|}{ Other } \\
\hline Fecal coliform, col. $/ 100 \mathrm{~mL}$ & $1 / 17$ & 100 & $1.0 \mathrm{U}$ & 100 & 1.0 & 1 \\
\hline Turbidity, NTU & $17 / 17$ & 50 & 0.28 & 9.0 & 1.0 & 10 \\
\hline \multicolumn{7}{|l|}{ Radionuclides, $\mathrm{pCi} / \mathrm{L}$. } \\
\hline Gross alpha & $55 / 55$ & 180 & -0.81 & 9.2 & 15 & 4 \\
\hline Gross beta & $55 / 55$ & 11000 & -22 & 608 & 50 & \\
\hline Radioactive strontium, total & $55 / 55$ & 6200 & -3.0 & 284 & 8.1 & 12 \\
\hline Tritium & $55 / 55$ & 38000 & -780 & 4231 & 20000 & 3 \\
\hline
\end{tabular}


Table 2.3.13 (continued)

\begin{tabular}{|c|c|c|c|c|c|c|}
\hline \multirow{2}{*}{ Parameter } & \multirow{2}{*}{$\begin{array}{l}\text { No. detected/ } \\
\text { No. of samples }\end{array}$} & \multicolumn{2}{|c|}{ Concentration $^{b}$} & \multirow{2}{*}{$\begin{array}{c}\text { Average of values } \\
\text { above the } \\
\text { detection limit }\end{array}$} & \multirow{2}{*}{$\begin{array}{c}\text { Reference } \\
\text { value }^{c}\end{array}$} & \multirow{2}{*}{$\begin{array}{c}\text { No. of values } \\
\text { exceeding } \\
\text { reference }\end{array}$} \\
\hline & & $\operatorname{Max}$ & Min & & & \\
\hline \multicolumn{7}{|l|}{ Semivolatiles, mg/L } \\
\hline Di-n-octylphthalate & $1 / 38$ & 0.014 & $0.010 \mathrm{U}$ & 0.014 & $d$ & $e$ \\
\hline Organic carbon, total & $64 / 68$ & 3.5 & $0.50 \mathrm{U}$ & 1.3 & $d$ & $e$ \\
\hline Organic calides, total & $31 / 68$ & 0.090 & $0.0050 \mathrm{U}$ & 0.030 & $d$ & $e$ \\
\hline \multicolumn{7}{|l|}{ Volatile organics, mg/L } \\
\hline Chloroform & $2 / 38$ & 0.011 & $0.0030 \mathrm{~J}$ & 0.0090 & $d$ & $e$ \\
\hline Methylene chloride & $6 / 38$ & $0.0050 \mathrm{~B}$ & $0.0010 \mathrm{~J}$ & 0.0050 & $d$ & $e$ \\
\hline Trichloroethene & $2 / 38$ & 0.011 & $0.0030 \mathrm{~J}$ & 0.0080 & 0.0050 & 1 \\
\hline Vinyl chloride & $1 / 38$ & 0.028 & $0.0060 \mathrm{~J}$ & 0.028 & 0.0020 & 38 \\
\hline 1,2-Dichloroethene & $2 / 38$ & 0.011 & $0.0050 \mathrm{U}$ & 0.010 & $d$ & $e$ \\
\hline \multicolumn{7}{|c|}{ Upgradient wells } \\
\hline Cyanide, total & $2 / 12$ & 0.0030 & $0.0020 \mathrm{U}$ & 0.0025 & $d$ & $e$ \\
\hline \multicolumn{7}{|l|}{ Field data } \\
\hline $\mathrm{pH}$, standard units & $126 / 126$ & 12 & 6.7 & 7.4 & $d$ & $e$ \\
\hline Conductivity, $\mathrm{mS} / \mathrm{cm}$ & $126 / 126$ & 0.66 & 0.17 & 0.39 & $d$ & $e$ \\
\hline Temperature, ${ }^{\circ} \mathrm{C}$ & $126 / 126$ & 21 & 13 & 16 & $d$ & $e$ \\
\hline \multicolumn{7}{|l|}{ Metals, $\mathrm{mg} / \mathrm{L}$} \\
\hline Aluminum, dissolved & $8 / 12$ & $2.0 \mathrm{U}$ & $0.050 \mathrm{U}$ & 0.36 & $d$ & $e$ \\
\hline Aluminum, total & $13 / 18$ & 12 & $0.050 \mathrm{U}$ & 1.8 & $d$ & $e$ \\
\hline Antimony, total & $1 / 18$ & 0.17 & $0.030 \mathrm{U}$ & 0.17 & $d$ & $e$ \\
\hline Beryllium, dissolved & $1 / 12$ & 0.030 & $0.00040 \mathrm{U}$ & 0.030 & $d$ & $e$ \\
\hline Beryllium, total & $4 / 18$ & 0.031 & $0.00030 \mathrm{U}$ & 0.017 & $d$ & $e$ \\
\hline Boron, dissolved & $6 / 1.2$ & 0.36 & $0.080 \mathrm{U}$ & 0.20 & $d$ & $e$ \\
\hline Boron, total & $6 / 18$ & 0.33 & $0.080 \mathrm{U}$ & 0.20 & $d$ & $e$ \\
\hline Cadmium, total & $3 / 18$ & 0.011 & $0.0020 \mathrm{U}$ & 0.00993333333 & 0.010 & 1 \\
\hline Calsium, dissolved & $12 / 12$ & 140 & 42 & 84 & $d$ & $e$ \\
\hline Calcium, total & $18 / 18$ & 130 & 41 & 85 & $d$ & $e$ \\
\hline Cobalt, dissolved & $4 / 12$ & 0.0084 & $0.0030 \mathrm{U}$ & 0.0058 & $d$ & $e$ \\
\hline Cobalt, total & $5 / 18$ & 0.0073 & $0.0030 \mathrm{U}$ & 0.0051 & $d$ & $e$ \\
\hline Copper, dissolved & $2 / 12$ & 0.021 & $0.0080 \mathrm{U}$ & 0.014 & $d$ & $e$ \\
\hline Iron, total & $18 / 18$ & 6.2 & 0.011 & 0.83 & 0.30 & 9 \\
\hline Magnesium, dissolved & $12 / 12$ & 32 & 9.9 & 23 & $d$ & $e$ \\
\hline Magnesium, total & $18 / 18$ & 30 & 6.2 & 18 & $d$ & $e$ \\
\hline Manganese, total & $18 / 18$ & 0.16 & 0.0029 & 0.038 & 0.050 & 4 \\
\hline Nickel, dissolved & $1 / 12$ & 0.0073 & $0.0060 \mathrm{U}$ & 0.0073 & $d$ & $e$ \\
\hline Nickel, total & $7 / 18$ & 0.028 & $0.0050 \mathrm{U}$ & 0.011 & $d$ & $e$ \\
\hline Silicon, dissolved & $12 / 12$ & 7.3 & 2.2 & 4.6 & $d$ & $e$ \\
\hline Silicon, total & $18 / 18$ & 8.6 & 0.99 & 5.4 & $d$ & $e$ \\
\hline Sodium, dissolved & $9 / 12$ & 28 & $2.0 \mathrm{U}$ & 14 & $d$ & $e$ \\
\hline Sodium, total & $14 / 18$ & 29 & $2.0 \mathrm{U}$ & 13 & $d$ & $e$ \\
\hline Strontium, dissolved & $12 / 12$ & 1.8 & 0.15 & 0.62 & $d$ & $e$ \\
\hline Strontium, total & $12 / 12$ & 1.7 & 0.17 & 0.60 & $d$ & $e$ \\
\hline Titanium, total & $6 / 18$ & 0.086 & $0.020 \mathrm{U}$ & 0.041 & $d$ & $e$ \\
\hline Vanadium, total & $7 / 18$ & 0.014 & $0.0030 \mathrm{U}$ & 0.0092 & $d$ & $e$ \\
\hline Zinc, dissolved & $4 / 12$ & 0.023 & $0.0050 \mathrm{U}$ & 0.017 & $d$ & $e$ \\
\hline \multicolumn{7}{|l|}{ Other } \\
\hline Fecal coliform, col. $/ 100 \mathrm{~mL}$ & $1 / 6$ & 20 & $1.0 \mathrm{U}$ & 20 & 1.0 & 1 \\
\hline Turbidity, NTU & $6 / 6$ & 43 & 0.090 & 8.9 & 1.0 & 2 \\
\hline \multicolumn{7}{|l|}{ Radionuclides, $\mathrm{pCi} / \mathrm{L}$} \\
\hline Gross beta & $18 / 18$ & 6.5 & -8.1 & 2.2 & 50 & \\
\hline
\end{tabular}


Table 2.3.13 (continued)

\begin{tabular}{|c|c|c|c|c|c|c|}
\hline \multirow{2}{*}{ Parameter } & \multirow{2}{*}{$\begin{array}{l}\text { No. detected/ } \\
\text { No: of samples }\end{array}$} & \multicolumn{2}{|c|}{ Concentration ${ }^{b}$} & \multirow{2}{*}{$\begin{array}{l}\text { Average of values } \\
\text { above the } \\
\text { detection limit }\end{array}$} & \multirow{2}{*}{$\begin{array}{l}\text { Reference } \\
\text { value }^{c}\end{array}$} & \multirow{2}{*}{$\begin{array}{l}\text { No. of values } \\
\text { exceeding } \\
\text { reference }\end{array}$} \\
\hline & & $\operatorname{Max}$ & Min & & & \\
\hline \multicolumn{7}{|l|}{ Semivolatiles, mg/L } \\
\hline Organic carbon, total & $16 / 24$ & 1.0 & $0.50 \mathrm{U}$ & 0.85 & $d$ & $e$ \\
\hline Organic halides, total & $3 / 24$ & 0.058 & $0.0050 \mathrm{U}$ & 0.030 & $d$ & $e$ \\
\hline \multicolumn{7}{|l|}{ Volatile organics, $\mathrm{mg} / \mathrm{L}$} \\
\hline Chloroform & $1 / 12$ & 0.014 & $0.0050 \mathrm{U}$ & 0.014 & $d$ & $e$ \\
\hline Methylene chloride & $1 / 12$ & $0.0050 \mathrm{~B}$ & $0.0010 \mathrm{~J}$ & 0.0050 & $d$ & $e$ \\
\hline
\end{tabular}

${ }^{a}$ See Fig. 2.3.6 in Vol. 1.

${ }^{b}$ Data qualifiers (organics): $\mathrm{U}=$ Undetected; $\mathbf{B}=$ Present in blank; $\mathbf{J}=$ Below detection limit, but estimated; $\mathbf{E}=$ Concentration exceeds the calibration range of the instrument; (inorganics): $U=$ Undetected; $B=$ Value $<$ Contract required detection limit $>$ Instrument detection limil; $\mathrm{E}=\mathrm{Value}$ is estimated because of the presence of interference.

'See Table 2.3.1 in Volume 2 for more information.

${ }^{d}$ No reference.

'Not applicable. 
Table 2.3.14. Constituents in the WAG 6 groundwater at ORNL, January-December 1989

\begin{tabular}{|c|c|c|c|c|c|c|}
\hline \multirow{2}{*}{ Parameter } & \multirow{2}{*}{$\begin{array}{l}\text { No. detected/ } \\
\text { No. of samples }\end{array}$} & \multicolumn{2}{|c|}{ Concentration ${ }^{b}$} & \multirow{2}{*}{$\begin{array}{l}\text { Average of values } \\
\text { above the } \\
\text { detection limit }\end{array}$} & \multirow{2}{*}{$\begin{array}{l}\text { Reference } \\
\text { value }^{c}\end{array}$} & \multirow{2}{*}{$\begin{array}{l}\text { No. of values } \\
\text { exceeding } \\
\text { reference }\end{array}$} \\
\hline & & $\operatorname{Max}$ & Min & & & \\
\hline \multicolumn{7}{|c|}{ Site characterization wells } \\
\hline \multicolumn{7}{|l|}{ Field data } \\
\hline $\mathrm{pH}$, standard units & $56 / 56$ & 7.1 & 6.1 & $e$ & $d$ & $e$ \\
\hline Conductivity, $\mathrm{mS} / \mathrm{cm}$ & $56 / 56$ & 0.88 & 0.26 & 0.53 & $d$ & $e$ \\
\hline Temperature, ${ }^{\circ} \mathrm{C}$ & $56 / 56$ & 16 & 12 & 14 & $d$ & $e$ \\
\hline \multicolumn{7}{|l|}{ Metals, $\mathrm{mg} / \mathrm{L}$} \\
\hline Aluminum, total & $8 / 8$ & 0.94 & 0.52 & 0.73 & $d$ & $e$ \\
\hline Barium, dissolved & $2 / 8$ & 2.7 & $1.0 \mathrm{U}$ & 1.9 & 1.0 & 2 \\
\hline Barium, total & $2 / 8$ & 2.6 & $1.0 \mathrm{U}$ & 1.8 & 1.0 & 2 \\
\hline Calcium, total & $8 / 8$ & 140 & 51 & 97 & $d$ & $e$ \\
\hline Cobalt, total & $1 / 8$ & 0.013 & $0.0030 \mathrm{U}$ & 0.013 & $d$ & $e$ \\
\hline Iron, dissolved & $5 / 8$ & 39 & $0.050 \mathrm{U}$ & 8.6 & 0.30 & 4 \\
\hline Iron, total & $8 / 8$ & 40 & 0.25 & 6.1 & 0.30 & 7 \\
\hline Magnesium, total & $8 / 8$ & 8.8 & 8.1 & 8.5 & $d$ & $e$ \\
\hline Manganese, dissolved & $8 / 8$ & 12 & 0.010 & 1.8 & 0.050 & 7 \\
\hline Manganese, total & $8 / 8$ & 13 & 0.020 & 1.8 & 0.050 & 7 \\
\hline Nickel, total & $8 / 8$ & 0.028 & 0.0059 & 0.014 & $d$ & $e$ \\
\hline Silicon, total & $8 / 8$ & 13 & 5.4 & 9.6 & $d$ & $e$ \\
\hline Sodium, dissolved & $8 / 8$ & 13 & 5.9 & 8.1 & $d$ & $e$ \\
\hline Sodium, total & $8 / 8$ & 13 & 5.6 & 8.0 & $d$ & $e$ \\
\hline Titanium, total & $8 / 8$ & 0.031 & 0.024 & 0.028 & $d$ & $e$ \\
\hline Vanadium, total & $8 / 8$ & 0.014 & 0.0082 & 0.010 & $d$ & $e$ \\
\hline \multicolumn{7}{|l|}{ Other } \\
\hline Alkalinity, as $\mathrm{CaCO}_{3}$ & $8 / 8$ & 610 & 150 & 342 & $d$ & $e$ \\
\hline Turbidity, NTU & $8 / 8$ & 62 & 0.53 & 9.7 & 1.0 & 6 \\
\hline \multicolumn{7}{|l|}{ Radionuclides, $\mathrm{pCi} / \mathrm{L}$} \\
\hline Gross alpha & $8 / 8$ & 0.78 & -0.27 & 0.43 & 15 & 0 \\
\hline Gross beta & $8 / 8$ & 30 & 0.81 & 5.9 & 50 & \\
\hline Tritium & $8 / 8$ & $2,000,000$ & 3,000 & 417,939 & 20,000 & 7 \\
\hline \multicolumn{7}{|l|}{ Semivolatiles, $\mathrm{mg} / \mathrm{L}$} \\
\hline Naphthalene & $1 / 8$ & $0.45 \mathrm{E}$ & $0.011 \mathrm{U}$ & 0.45 & $d$ & $e$ \\
\hline Organic carbon, total & $32 / 32$ & 10 & 0.60 & 3.4 & $d$ & $e$ \\
\hline Organic halides, total & $13 / 32$ & 2.1 & $0.0050 \mathrm{U}$ & 0.60 & $d$ & $e$ \\
\hline Recoverable phenolics, total & $1 / 8$ & 0.029 & $0.0010 \mathrm{U}$ & 0.029 & $d$ & $e$ \\
\hline 2-Methylphenol & $1 / 8$ & 0.022 & $0.011 \mathrm{U}$ & 0.022 & $d$ & $e$ \\
\hline \multicolumn{7}{|l|}{ Volatile organics, $\mathrm{mg} / \mathrm{L}$} \\
\hline Acetone & $1 / 8$ & $0.024 \mathrm{~B}$ & $0.0020 \mathrm{~J}$ & 0.024 & $d$ & $e$ \\
\hline Benzene & $3 / 8$ & 0.096 & $0.0050 \mathrm{U}$ & 0.041 & 0.0050 & 3 \\
\hline Ethylbenzene & $1 / 8$ & $0.70 \mathrm{E}$ & $0.0050 \mathrm{U}$ & 0.70 & $d$ & $e$ \\
\hline Toluene & $1 / 8$ & $1.9 \mathrm{E}$ & $0.0050 \mathrm{U}$ & 1.9 & $d$ & $e$ \\
\hline Trichloroethene & $1 / 8$ & $1.1 \mathrm{E}$ & $0.0050 \mathrm{U}$ & 1.1 & 0.0050 & 1 \\
\hline Vinyl chloride & $1 / 8$ & 0.063 & $0.010 \mathrm{U}$ & 0.063 & 0.0020 & 8 \\
\hline Xylene, total & $2 / 8$ & $3.8 \mathrm{E}$ & $0.0050 \mathrm{U}$ & 1.9 & $d$ & $e$ \\
\hline 1,1-Dichloroethane & $2 / 8$ & 0.017 & $0.0050 \mathrm{U}$ & 0.016 & $d$ & $e$ \\
\hline 1,2-Dichloroethene & $3 / 8$ & $0.26 \mathrm{E}$ & $0.0050 \mathrm{U}$ & 0.093 & $d$ & $e$ \\
\hline \multicolumn{7}{|c|}{ Perimeter wells } \\
\hline \multicolumn{7}{|l|}{ Anions, mg/L } \\
\hline Phosphate & $1 / 30$ & 5.0 & $5.0 \mathrm{U}$ & 5.0 & $d$ & $e$ \\
\hline \multicolumn{7}{|l|}{ Field data } \\
\hline $\mathrm{pH}$, standard units & $210 / 210$ & 7.8 & 4.3 & $e$ & $d$ & $e$ \\
\hline Conductivity, $\mathrm{mS} / \mathrm{cm}$ & $210 / 210$ & 0.82 & 0.010 & 0.30 & $d$ & $e$ \\
\hline Temperature, ${ }^{\circ} \mathrm{C}$ & $210 / 210$ & 17 & 9.5 & 14 & $d$ & $e$ \\
\hline
\end{tabular}


Table 2.3.14 (continued)

\begin{tabular}{|c|c|c|c|c|c|c|}
\hline \multirow{2}{*}{ Parameter } & \multirow{2}{*}{$\begin{array}{l}\text { No. detected/ } \\
\text { No. of samples }\end{array}$} & \multicolumn{2}{|c|}{ Concentration ${ }^{b}$} & \multirow{2}{*}{$\begin{array}{l}\text { Average of values } \\
\text { above the } \\
\text { detection limit }\end{array}$} & \multirow{2}{*}{$\begin{array}{l}\text { Reference } \\
\text { value }^{c}\end{array}$} & \multirow{2}{*}{$\begin{array}{l}\text { No. of values } \\
\text { exceeding } \\
\text { reference }\end{array}$} \\
\hline & & $\operatorname{Max}$ & Min & & & \\
\hline \multicolumn{7}{|l|}{ Metals, mg/L } \\
\hline Aluminum, total & $24 / 30$ & 0.89 & $0.050 \mathrm{U}$ & 0.48 & $d$ & $\boldsymbol{e}$ \\
\hline Beryllium, total & $5 / 30$ & 0.025 & $0.00030 \mathrm{U}$ & 0.025 & $d$ & $\boldsymbol{e}$ \\
\hline Calcium, total & $29 / 30$ & 180 & $0.10 \mathrm{U}$ & 65 & $d$ & $e$ \\
\hline Cobalt, total & $5 / 30$ & 0.0048 & $0.0030 \mathrm{U}$ & 0.0041 & $d$ & $e$ \\
\hline Iron, total & $28 / 30$ & 4.8 & $0.050 \mathrm{U}$ & 0.96 & 0.30 & 25 \\
\hline Magnesium, total & $29 / 30$ & 29 & $0.010 \mathrm{U}$ & 8.6 & $d$ & $e$ \\
\hline Manganese, dissolved & $21 / 30$ & 0.10 & $0.010 \mathrm{U}$ & 0.028 & 0.050 & 3 \\
\hline Manganese, total & $25 / 30$ & 0.11 & $0.010 \mathrm{U}$ & 0.052 & 0.050 & 11 \\
\hline Nickel, total & $20 / 30$ & 0.028 & $0.0050 \mathrm{U}$ & 0.010 & $d$ & $e$ \\
\hline Silicon, total & $29 / 30$ & 14 & $0.20 \mathrm{U}$ & 8.0 & $d$ & $\boldsymbol{e}$ \\
\hline Sodium, dissolved & $30 / 30$ & 46 & 1.1 & 9.7 & $d$ & $e$ \\
\hline Sodium, total & $30 / 30$ & 49 & 1.0 & 9.5 & $d$ & $e$ \\
\hline Strontium, total & $12 / 15$ & 1.2 & $0.0050 \mathrm{U}$ & 0.41 & $d$ & $e$ \\
\hline Titanium, total & $15 / 30$ & 0.033 & $0.020 \mathrm{U}$ & 0.027 & $d$ & $\boldsymbol{e}$ \\
\hline Vanadium, total & $15 / 30$ & 0.012 & $0.0040 \mathrm{U}$ & 0.010 & $d$ & $e$ \\
\hline \multicolumn{7}{|l|}{ Other } \\
\hline Alkalinity, as $\mathrm{CaCO}_{3}$ & $30 / 30$ & 440 & 4.0 & 195 & $d$ & $\boldsymbol{e}$ \\
\hline Fecal coliform, col./100 mL & $1 / 30$ & 50 & $1.0 \mathrm{U}$ & 50 & 1.0 & 1 \\
\hline Turbidity, NTU & $30 / 30$ & 54 & 0.055 & 6.2 & 1.0 & 19 \\
\hline \multicolumn{7}{|l|}{ Radionuclides, $\mathrm{pCi} / \mathrm{L}$} \\
\hline Gross alpha & $30 / 30$ & 2.5 & -0.027 & 3.9 & 15 & 0 \\
\hline Gross beta & $30 / 30$ & 180 & -0.054 & 15 & 50 & \\
\hline Tritium & $30 / 30$ & $1,200,000$ & $-1,400$ & 128,063 & 20,000 & 14 \\
\hline \multicolumn{7}{|l|}{ Semivolatiles, mg/L } \\
\hline Di-n-butylphthalate & $1 / 17$ & 0.019 & $0.011 \mathrm{U}$ & 0.019 & $d$ & $e$ \\
\hline Diethyl phthalate & $1 / 17$ & 0.048 & $0.0030 \mathrm{~J}$ & 0.048 & $d$ & $\boldsymbol{e}$ \\
\hline Organic carbon, total & $64 / 120$ & 4.7 & $0.50 \mathrm{U}$ & 1.2 & $d$ & $\boldsymbol{e}$ \\
\hline Organic nalides, total & $32 / 120$ & 0.59 & $0.0050 \mathrm{U}$ & 0.12 & $d$ & $e$ \\
\hline Recoverable phenolics, total & $1 / 30$ & 0.0010 & $0.0010 \mathrm{U}$ & 0.0010 & $d$ & $\boldsymbol{e}$ \\
\hline \multicolumn{7}{|l|}{ Volatile organics, $\mathrm{mg} / \mathrm{L}$} \\
\hline Carbon tetrachloride & $2 / 30$ & 0.086 & $0.00050 \mathrm{~J}$ & 0.084 & 0.0050 & 2 \\
\hline Chloroform & $3 / 30$ & 0.097 & $0.0020 \mathrm{~J}$ & 0.055 & $d$ & $e$ \\
\hline Chloromethane & $1 / 30$ & 0.015 & $0.0040 \mathrm{~J}$ & 0.015 & $d$ & $\boldsymbol{e}$ \\
\hline Toluene & $1 / 30$ & $0.0070 \mathrm{~B}$ & $0.0020 \mathrm{~J}$ & 0.0070 & $d$ & $\boldsymbol{e}$ \\
\hline Trichloroethene & $3 / 30$ & 0.49 & $0.0010 \mathrm{~J}$ & 0.28 & 0.0050 & 3 \\
\hline Xylene, total & $1 / 30$ & 0.011 & $0.0050 \mathrm{U}$ & 0.011 & $d$ & $e$ \\
\hline 1,1-Dichloroethane & $1 / 30$ & 0.0090 & $0.0030 \mathrm{~J}$ & 0.0090 & $d$ & $e$ \\
\hline 1,2-Dichloroethane & $2 / 30$ & 0.044 & $0.0050 \mathrm{U}$ & 0.031 & 0.0050 & 2 \\
\hline 1,2-Dichloroethene & $3 / 30$ & 0.019 & $0.0050 \mathrm{U}$ & 0.012 & $d$ & $e$ \\
\hline \multicolumn{7}{|c|}{ Upgradient wells } \\
\hline \multicolumn{7}{|l|}{ Anions, $\mathrm{mg} / \mathrm{L}$} \\
\hline Sulfate, as $\mathrm{SO}_{4}$ & $10 / 14$ & 330 & $5.0 \mathrm{U}$ & 93 & 250 & 2 \\
\hline \multicolumn{7}{|l|}{ Field data } \\
\hline $\mathrm{pH}$, standard units & $98 / 98$ & 8.5 & 4.7 & $e$ & $d$ & $e$ \\
\hline Conductivity, $\mathrm{mS} / \mathrm{cm}$ & $98 / 98$ & 0.97 & 0.020 & 0.37 & $d$ & $e$ \\
\hline Temperature, ${ }^{\circ} \mathrm{C}$ & $98 / 98$ & 18 & 12 & 14 & $d$ & $e$ \\
\hline
\end{tabular}


Table 2.3.14 (continued)

\begin{tabular}{|c|c|c|c|c|c|c|}
\hline \multirow{2}{*}{ Parameter } & \multirow{2}{*}{$\begin{array}{l}\text { No. detected/ } \\
\text { No. of samples }\end{array}$} & \multicolumn{2}{|c|}{ Concentration $^{b}$} & \multirow{2}{*}{$\begin{array}{c}\text { Average of values } \\
\text { above the } \\
\text { detection limit }\end{array}$} & \multirow{2}{*}{$\begin{array}{l}\text { Reference } \\
\text { value }^{c}\end{array}$} & \multirow{2}{*}{$\begin{array}{l}\text { No. of value } \\
\text { exceeding } \\
\text { reference }\end{array}$} \\
\hline & & $\operatorname{Max}$ & Min & & & \\
\hline \multicolumn{7}{|l|}{ Metals, mg/L } \\
\hline Aluminum, total & $13 / 14$ & 0.66 & $0.050 \mathrm{U}$ & 0.38 & $d$ & $e$ \\
\hline Beryllium, total & $10 / 14$ & 0.025 & $0.00030 \mathrm{U}$ & 0.015 & $d$ & $e$ \\
\hline Calcium, total & $12 / 14$ & 170 & $0.10 \mathrm{U}$ & 91 & $d$ & $e$ \\
\hline Cobalt, total & $1 / 14$ & 0.0031 & $0.0030 \mathrm{U}$ & 0.0031 & $d$ & $e$ \\
\hline Iron, dissolved & $3 / 14$ & 0.63 & $0.050 \mathrm{U}$ & 0.38 & 0.30 & 2 \\
\hline Iron, total & $12 / 14$ & 1.7 & $0.050 \mathrm{U}$ & 0.62 & 0.30 & 7 \\
\hline Magnesium, total & $14 / 14$ & 54 & 0.99 & 12 & $d$ & $e$ \\
\hline Manganese, dissolved & $9 / 14$ & 0.060 & $0.010 \mathrm{U}$ & 0.023 & 0.050 & 2 \\
\hline Manganese, total & $10 / 14$ & 0.13 & $0.010 \mathrm{U}$ & 0.034 & 0.050 & 1 \\
\hline Nickel, total & $6 / 14$ & 0.020 & $0.0050 \mathrm{U}$ & 0.013 & $d$ & $e$ \\
\hline Silicon, total & $14 / 14$ & 14 & 4.2 & 8.0 & $d$ & $e$ \\
\hline Sodium, dissolved & $14 / 14$ & 18 & 0.68 & 7.4 & $d$ & $e$ \\
\hline Sodium, total & $14 / 14$ & 19 & 0.69 & 7.3 & $d$ & $e$ \\
\hline Strontium, total & $11 / 12$ & 0.40 & $0.0050 \mathrm{U}$ & 0.19 & $d$ & $e$ \\
\hline Titanium, total & $2 / 14$ & 0.027 & $0.020 \mathrm{U}$ & 0.025 & $d$ & $e$ \\
\hline Vanadium, total & $2 / 9$ & 0.011 & $0.0040 \mathrm{U}$ & 0.010 & $d$ & $e$ \\
\hline \multicolumn{7}{|l|}{ Other } \\
\hline Alkalinity, as $\mathrm{CaCO}_{3}$ & $14 / 14$ & 430 & 7.0 & 198 & $d$ & $e$ \\
\hline Turbidity, NTU & $14 / 14$ & 120 & 0.075 & 11 & 1.0 & 7 \\
\hline \multicolumn{7}{|l|}{ Radionuclides, pCi/L } \\
\hline Gross alpha & $14 / 14$ & 2.4 & -0.15 & 0.46 & 15 & 0 \\
\hline Gross beta & $14 / 14$ & 8.4 & 0.78 & 2.3 & 50 & \\
\hline \multicolumn{7}{|l|}{ Semivolatiles, mg/L } \\
\hline Organic carbon, total & $32 / 56$ & 1.2 & 0.40 & 0.85 & $d$ & $e$ \\
\hline Organic halides, total & $2 / 56$ & 0.0080 & $0.0050 \mathrm{U}$ & 0.0075 & $d$ & $e$ \\
\hline \multicolumn{7}{|l|}{ Volatile organics, $\mathrm{mg} / \mathrm{L}$} \\
\hline Acetone & $2 / 14$ & $0.093 \mathrm{~B}$ & $0.0030 \mathrm{~J}$ & 0.053 & $\vec{a}$ & $e$ \\
\hline
\end{tabular}

${ }^{a}$ See Fig. 2.3.6 in Vol. 1.

${ }^{b}$ Data qualifiers (organics): $\mathrm{U}=$ Undetected; $\mathrm{B}=$ Present in blank; $\mathrm{J}=$ Below detection limit, but estimated; $\mathrm{E}=$ Concentration exceeds the calibration range of the instrument; (inorganics): $U=$ Undetected; $B=$ Value $<$ Contract required detection limit $>$ Instrument detection limit; $E=$ Value is estimated because of the presence of interference.

'See Table 2.3.1 in Volume 2 for more information.

${ }^{d}$ No reference.

Not applicable. 
Table 2.3.15. ORGDP groundwater monitoring for 1989

\begin{tabular}{|c|c|c|c|c|c|c|c|}
\hline \multirow[t]{2}{*}{ Parameter } & \multirow{2}{*}{$\begin{array}{l}\text { Number of } \\
\text { samples }\end{array}$} & \multicolumn{2}{|c|}{$\begin{array}{l}\text { Samples above } \\
\text { detection limit }\end{array}$} & \multirow{2}{*}{$\operatorname{Min}^{b}$} & \multirow{2}{*}{$\operatorname{Max}^{b}$} & \multirow{2}{*}{$\begin{array}{c}\text { Reference } \\
\text { value }\end{array}$} & \multirow{2}{*}{$\begin{array}{c}\text { No. of } \\
\text { samples } \\
>\text { reference }\end{array}$} \\
\hline & & No. & $\mathrm{Av}^{a}$ & & & & \\
\hline \multicolumn{8}{|c|}{$K-. .77 / 29$} \\
\hline Benzene, $\mathrm{mg} / \mathrm{L}$ & 25 & 1 & 0.006 & $<0.005$ & $<0.01$ & 0.005 & 1 \\
\hline Cadmium, mg/L & 50 & 10 & 0.0055 & $<0.003$ & $<0.013$ & 0.01 & 1 \\
\hline Carbon tetrachloride, $\mathrm{mg} / \mathrm{L}$ & 25 & 8 & 0.0044 & $<0.0006$ & $<0.011$ & 0.005 & 2 \\
\hline Chromium (total), mg/L & 50 & 35 & 0.072 & $<0.01$ & 0.46 & 0.05 & 8 \\
\hline Iron, mg/L & 50 & 39 & 4.89 & $<0.004$ & 31 & 0.3 & 21 \\
\hline Lead, $\mathrm{mg} / \mathrm{L}$ & 100 & 22 & 0.014 & $<0.004$ & $<0.055$ & 0.05 & 1 \\
\hline Manganese, $\mathrm{mg} / \mathrm{L}$ & 50 & 48 & 1.34 & $<0.001$ & 12.0 & 0.05 & 24 \\
\hline Total coliform bacteria ( $\mathrm{COL}$ ) & 11 & 2 & 21 & $<1.0$ & $<22$ & 1 & 2 \\
\hline Trichloroethene, $\mathrm{mg} / \mathrm{L}$ & 25 & 22 & 0.1 & $<0.005$ & $<0.41$ & 0.005 & 22 \\
\hline Turbidity, NTU & 25 & 25 & 112 & 0.7 & 800 & 5 & 21 \\
\hline Vinyl chloride, $\mathrm{mg} / \mathrm{L}$ & 25 & 2 & 0.011 & $<0.002$ & $<0.02$ & 0.002 & 1 \\
\hline \multicolumn{8}{|c|}{$K-31$} \\
\hline Iron, mg/L & 19 & 9 & 2.59 & $<0.004$ & 16.0 & 0.3 & 6 \\
\hline Manganese, $\mathrm{mg} / \mathrm{L}$ & 19 & 18 & 0.16 & $<0.001$ & 0.43 & 0.05 & 12 \\
\hline Total coliform bacteria (COL) & 4 & 2 & 259 & $<1.0$ & $<262$ & 1 & 2 \\
\hline Turbidity, NTU & 9 & 9 & 37 & 0.78 & 200 & 5 & 7 \\
\hline \multicolumn{8}{|c|}{$K-33$} \\
\hline Iron, mg/L & 20 & 16 & 3.91 & $<0.004$ & 9.1 & 0.3 & 12 \\
\hline Manganese, $\mathrm{mg} / \mathrm{L}$ & 20 & 18 & 4.76 & $<0.001$ & 9.9 & 0.05 & 14 \\
\hline Total coliform bacteria (COL) & 1 & 1 & 8.0 & 8.0 & 8.0 & 1 & 1 \\
\hline Trichloroethene, $\mathrm{mg} / \mathrm{L}$ & 10 & 2 & 0.05 & $<0.005$ & $<0.052$ & 0.005 & 2 \\
\hline Turbidity, NTU & 10 & 10 & 260 & 1.3 & 1600 & 5 & 8 \\
\hline \multicolumn{8}{|c|}{$K-720$} \\
\hline Iron, mg/L & 12 & 12 & 28 & 0.055 & 56 & 0.3 & 11 \\
\hline Lead, $\mathrm{mg} / \mathrm{L}$ & 24 & 7 & 0.046 & $<0.004$ & $<0.13$ & 0.05 & 2 \\
\hline Manganese, mg/L & 12 & 12 & 50 & 0.21 & 100 & 0.05 & 12 \\
\hline Nitrate, $\mathrm{mg} / \mathrm{L}$ & 6 & 2 & 32 & $<0.2$ & 64 & 10 & 1 \\
\hline Sulfate, $\mathrm{mg} / \mathrm{L}$ & 6 & 6 & 1039 & 4.0 & 1820 & 250 & 5 \\
\hline Turbidity, NTU & 6 & 6 & 150 & 33 & 400 & 5 & 6 \\
\hline \multicolumn{8}{|c|}{$K-770$} \\
\hline Iron, mg/L & 27 & 19 & 1.95 & $<0.004$ & 4.6 & 0.3 & 16 \\
\hline Manganese, $\mathrm{mg} / \mathrm{L}$ & 27 & 27 & 0.61 & 0.031 & 1.5 & 0.05 & 25 \\
\hline Turbidity, NTU & 11 & 11 & 20 & 3.5 & 80 & 5 & 8 \\
\hline \multicolumn{8}{|c|}{$K-802-B$ and $H$} \\
\hline Carbon tetrachloride, $\mathrm{mg} / \mathrm{L}$ & 10 & 2 & 0.005 & $<0.004$ & $<0.006$ & 0.005 & 1 \\
\hline Iron, mg/L & 21 & 15 & 0.62 & $<0.004$ & 7.8 & 0.3 & 3 \\
\hline Lead, $\mathrm{mg} / \mathrm{L}$ & 42 & 3 & 0.023 & $<0.004$ & $<0.058$ & 0.05 & 1 \\
\hline Manganese, $\mathrm{mg} / \mathrm{L}$ & 21 & 19 & 0.02 & $<0.001$ & 0.13 & 0.05 & 2 \\
\hline Total coliform bacteria (COL) & 4 & 2 & 32 & $<1.0$ & 34 & 1 & 2 \\
\hline Trichloroethene, $\mathrm{mg} / \mathrm{L}$ & 10 & 2 & 0.016 & $<0.005$ & $<0.018$ & 0.005 & 2 \\
\hline Turbidity, NTU & 10 & 10 & 355 & 0.7 & 2800 & 5 & 3 \\
\hline
\end{tabular}


Table 2.3.15 (Continued)

\begin{tabular}{|c|c|c|c|c|c|c|c|}
\hline \multirow[t]{2}{*}{ Parameter } & \multirow{2}{*}{$\begin{array}{l}\text { Number of } \\
\text { samples }\end{array}$} & \multicolumn{2}{|c|}{$\begin{array}{l}\text { Samples above } \\
\text { detection limit }\end{array}$} & \multirow{2}{*}{$\operatorname{Min}^{b}$} & \multirow{2}{*}{$\operatorname{Max}^{b}$} & \multirow{2}{*}{$\begin{array}{c}\text { Reference } \\
\text { value }\end{array}$} & \multirow{2}{*}{$\begin{array}{c}\text { No. of } \\
\text { samples } \\
>\text { reference }\end{array}$} \\
\hline & & No. & $A v^{a}$ & & & & \\
\hline \multicolumn{8}{|c|}{$K-832-H$} \\
\hline Cadmium, $\mathrm{mg} / \mathrm{L}$ & 16 & 5 & 0.01 & $<0.003$ & 0.027 & 0.01 & 1 \\
\hline Iron, $\mathrm{mg} / \mathrm{L}$ & 16 & 12 & 0.88 & $<0.004$ & 5.5 & 0.3 & 4 \\
\hline Lead, mg/L & 32 & 6 & 0.037 & $<0.0554$ & $<0.089$ & 0.05 & 3 \\
\hline Manganese, mg/L & 16 & 16 & 2.61 & 0.006 & 15.0 & 0.05 & 14 \\
\hline Trichloi sethene, $\mathrm{mg} / \mathrm{L}$ & 8 & 2 & 0.0075 & $<0.002$ & $<0.013$ & 0.005 & 1 \\
\hline Turbidity, NTU & 8 & 8 & 347 & 2.6 & 2500 & 5 & 6 \\
\hline \multicolumn{8}{|c|}{$K-862-E$} \\
\hline Cadmium, mg/L & 32 & 10 & 0.0069 & $<0.003$ & $<0.019$ & 0.01 & 2 \\
\hline Chromium (total), $\mathrm{mg} / \mathrm{L}$ & 32 & 26 & 0.21 & $<0.01$ & 0.71 & 0.05 & 15 \\
\hline Iron, $\mathrm{mg} / \mathrm{L}$ & 32 & 19 & 7.51 & $<0.004$ & 76 & 0.3 & 9 \\
\hline Lead, mg/L & 64 & 11 & 0.045 & $<0.004$ & $<0.28$ & 0.05 & 3 \\
\hline Manganese, $\mathrm{mg} / \mathrm{L}$ & 32 & 30 & 0.17 & $<0.001$ & 2.7 & 0.05 & 13 \\
\hline Sulfate, $\mathrm{mg} / \mathrm{L}$ & 16 & 16 & 229 & 37 & 559 & 250 & 6 \\
\hline Total coliform bacteria (COI.) & 6 & 1 & 9.0 & $<1.0$ & $<9.0$ & 1 & 1 \\
\hline Trichloroethene, $\mathrm{mg} / \mathrm{L}$ & 16 & 9 & 0.0048 & 0.002 & $<0.01$ & 0.005 & 3 \\
\hline Turbidity, NTU & 16 & 16 & 66 & 0.4 & 550 & 5 & 10 \\
\hline \multicolumn{8}{|c|}{$K-892-G$ and $H$} \\
\hline Chromium (total), mg/L & 23 & 19 & 0.16 & $<.0 .01$ & 0.37 & 0.05 & 16 \\
\hline Iron, $\mathrm{mg} / \mathrm{L}$ & 23 & 8 & 0.48 & $<0.004$ & 2.2 & 0.3 & 3 \\
\hline Lead, mg/L & 46 & 2 & 0.057 & $<0.004$ & $<0.059$ & 0.05 & 2 \\
\hline Manganese, $\mathrm{mg} / \mathrm{L}$ & 23 & 18 & 0.1 & $<0.001$ & 0.76 & 0.05 & 5 \\
\hline Sulfate, $\mathrm{mg} / \mathrm{L}$ & 11 & 11 & 229 & 26 & 435 & 250 & 5 \\
\hline Total coliform bacteria ( $\mathrm{COL})$ & 4 & 1 & 3.0 & $<1.0$ & $<3.0$ & 1 & 1 \\
\hline Turbidity, NTU & 11 & 11 & 27 & 0.54 & 260 & 5 & 3 \\
\hline \multicolumn{8}{|c|}{$K-892-J$} \\
\hline Alpha activity, pCi/L & 9 & 9 & 9.53 & 0.3 & 68 & 15 & 1 \\
\hline Chromium (total), $\mathrm{mg} / \mathrm{L}$ & 20 & 9 & 0.095 & $<0.01$ & 0.31 & 0.05 & 3 \\
\hline Iron, $\mathrm{mg} / \mathrm{L}$ & 20 & 15 & 43 & $<0.004$ & 300 & 0.3 & 9 \\
\hline Lead, mg/L & 39 & 8 & 0.12 & $<0.004$ & 0.22 & 0.05 & 7 \\
\hline Mianganese, $\mathrm{mg} / \mathrm{L}$ & 20 & 19 & 0.71 & $<0.001$ & 5.9 & 0.05 & 8 \\
\hline Trichloroethene, $\mathrm{mg} / \mathrm{L}$ & 9 & 1 & 0.016 & $<0.005$ & $<0.016$ & 0.005 & 1 \\
\hline Turbidity, NTU & 9 & 9 & 1546 & 30 & 6000 & 5 & 9 \\
\hline \multicolumn{8}{|c|}{$K-901-A$} \\
\hline Barium, $\mathrm{mg} / \mathrm{L}$ & 46 & 46 & 0.1 & 0.018 & 1.2 & 1 & 1 \\
\hline Chromium (total), $\mathrm{mg} / \mathrm{L}$ & 46 & 7 & 0.19 & $<0.01$ & $<1.2$ & 0.05 & 1 \\
\hline Iron, $\mathrm{mg} / \mathrm{L}$ & 46 & 30 & 6.9 & $<0.004$ & $<13$ & 0.3 & 10 \\
\hline Lead, $\mathrm{mg} / \mathrm{L}$ & 92 & 16 & 0.032 & $<0.004$ & $<0.19$ & 0.05 & $?$ \\
\hline Manganese, $\mathrm{mg} / \mathrm{L}$ & 46 & 45 & 0.4 & $<0.001$ & 10.0 & 0.05 & 31 \\
\hline Total coliform bacteria (COL) & 7 & 3 & 22 & $<1.0$ & 27 & 1 & 3 \\
\hline Trichloroethene, $\mathrm{mg} / \mathrm{L}$ & 26 & 14 & 0.0094 & $<0.001$ & 0.036 & 0.005 & 8 \\
\hline Turbidity, I'TU & 23 & 23 & 312 & 0.65 & 1800 & 5 & 11 \\
\hline Zinc, $\mathrm{mg} / \mathrm{L}$ & 46 & 31 & 0.27 & $<0.001$ & $<7.1$ & 5 & 1 \\
\hline
\end{tabular}


Table 2.3.15 (Continued)

\begin{tabular}{|c|c|c|c|c|c|c|c|}
\hline \multirow[t]{2}{*}{ Parameter } & \multirow{2}{*}{$\begin{array}{c}\text { Number of } \\
\text { samples }\end{array}$} & \multicolumn{2}{|c|}{$\begin{array}{l}\text { Samples above } \\
\text { detection limit }\end{array}$} & \multirow{2}{*}{$\operatorname{Min}^{b}$} & \multirow{2}{*}{$\operatorname{Max}^{b}$} & \multirow{2}{*}{$\begin{array}{c}\text { Reference } \\
\text { value }\end{array}$} & \multirow{2}{*}{$\begin{array}{c}\text { No. of } \\
\text { samples } \\
>\text { reference }\end{array}$} \\
\hline & & No. & $A v^{a}$ & & & & \\
\hline \multicolumn{8}{|c|}{$K-1004$ (includes $K-1006$ ) } \\
\hline Chromium (total), $\mathrm{mg} / \mathrm{L}$ & 49 & 2 & 0.33 & $<0.01$ & 0.65 & 0.05 & 1 \\
\hline Iron, $\mathrm{mg} / \mathrm{L}$ & 49 & 36 & 0.39 & $<0.004$ & 6.3 & 0.3 & 9 \\
\hline Lead, mg/L & 97 & 7 & 0.024 & $<0.004$ & $<0.081$ & 0.05 & 1 \\
\hline Manganese, $\mathrm{mg} / \mathrm{L}$ & 49 & 43 & 0.5 & $<0.001$ & 2.5 & 0.05 & 29 \\
\hline Total coliform bacteria (COL) & 5 & 2 & 2.5 & $<1.0$ & 4.0 & 1 & 1 \\
\hline Trichloroethene, $\mathrm{mg} / \mathrm{L}$ & 26 & 21 & 0.063 & 0.001 & $<0.24$ & 0.005 & 14 \\
\hline Turbidity, NTU & 23 & 23 & 79 & 0.45 & 1100 & 5 & 11 \\
\hline \multicolumn{8}{|c|}{$K-1007$} \\
\hline Chromium (total), $\mathrm{mg} / \mathrm{L}$ & 14 & 5 & 0.023 & $<0.01$ & 0.061 & 0.05 & 1 \\
\hline Iron, $\mathrm{mg} / \mathrm{L}$ & 14 & 10 & 3.62 & $<0.004$ & 13.0 & 0.3 & 5 \\
\hline Lead, $\mathrm{mg} / \mathrm{L}$ & 28 & 6 & 0.04 & $<0.004$ & $<0.21$ & 0.05 & 1 \\
\hline Manganese, $\mathrm{mg} / \mathrm{L}$ & 14 & 14 & 0.29 & 0.037 & 1.2 & 0.05 & 13 \\
\hline Turbidity, NTU & 7 & 7 & 77 & 1.6 & 26 & 5 & 5 \\
\hline \multicolumn{8}{|c|}{$K-1064-G$} \\
\hline 1,1-Dichloroethene, $\mathrm{mg} / \mathrm{L}$ & 14 & 7 & 0.005 & 0.002 & $<0.009$ & 0.007 & 2 \\
\hline Alpha activity, $\mathrm{pCi} / \mathrm{L}$ & 10 & 10 & 17.4 & -1.0 & 50 & 15 & 4 \\
\hline Arsenic, $\mathrm{mg} / \mathrm{L}$ & 31 & 9 & 0.099 & $<0.005$ & $<0.32$ & 0.05 & 4 \\
\hline Cadmium, mg/L & 33 & 12 & 0.0051 & $<0.003$ & $<0.011$ & 0.01 & 1 \\
\hline Iron, $\mathrm{mg} / \mathrm{L}$ & 31 & 15 & 0.46 & $<0.004$ & 5.0 & 0.3 & 3 \\
\hline Lead, mg/L & 64 & 10 & 0.047 & $<0.004$ & $<0.12$ & 0.05 & 6 \\
\hline Manganese, $\mathrm{mg} / \mathrm{L}$ & 31 & 25 & 0.046 & $<0.001$ & 0.19 & 0.05 & 8 \\
\hline Silver, mg/L & 33 & 9 & 0.026 & $<0.006$ & $<0.15$ & 0.05 & 1 \\
\hline Trichloroethene, $\mathrm{mg} / \mathrm{L}$ & 14 & 3 & 0.011 & $<0.005$ & $<0.013$ & 0.005 & 3 \\
\hline Turbidity, NTU & 10 & 10 & 78 & 0.6 & 750 & 5 & 4 \\
\hline \multicolumn{8}{|c|}{$K-1070-A$} \\
\hline 1,1,1-Trichloroethane, $\mathrm{mg} / \mathrm{L}$ & 16 & 1 & 3.4 & $<0.005$ & $<3.4$ & 0.2 & 1 \\
\hline 1,1-Dichloroethene, $\mathrm{mg} / \mathrm{L}$ & 16 & 1 & 1.5 & $<0.005$ & $<1.5$ & 0.007 & 1 \\
\hline Cadmium, mg/L & 37 & 10 & 0.0054 & $<0.003$ & 0.011 & 0.01 & 1 \\
\hline Iron, $\mathrm{mg} / \mathrm{L}$ & 37 & 15 & 2.15 & $<0.004$ & $<20.0$ & 0.3 & 4 \\
\hline Lead, mg/L & 72 & 10 & 0.062 & $<0.004$ & $<0.12$ & 0.05 & 6 \\
\hline Manganese, $\mathrm{mg} / \mathrm{L}$ & 37 & 2.7 & 0.05 & $<0.001$ & 0.44 & 0.05 & 3 \\
\hline Trichloroethene, $\mathrm{mg} / \mathrm{L}$ & 16 & 8 & 0.36 & $<0.001$ & 2.5 & 0.005 & 6 \\
\hline Turbidity, NTU & 8 & 8 & 140 & 1.0 & 750 & 5 & 6 \\
\hline \multicolumn{8}{|c|}{$K-1070 \cdot C / D$ (includes $K-1414)$} \\
\hline 1,1-Dichloroethene, $\mathrm{mg} / \mathrm{L}$ & 40 & 4 & 0.05 & $<0.002$ & $<0.12$ & 0.007 & 2 \\
\hline Alpha activity, $\mathrm{pCi} / \mathrm{L}$ & 28 & 28 & 2.05 & -1.0 & 17.0 & 15 & 1 \\
\hline Barium, $\mathrm{mg} / \mathrm{L}$ & 59 & 59 & 0.17 & 0.001 & 1.1 & 1 & 1 \\
\hline Cadmium, mg/L & 59 & 15 & 0.0061 & $<0.003$ & $<0.011$ & 0.01 & 2 \\
\hline Chromium (total), $\mathrm{mg} / \mathrm{L}$ & 59 & 9 & 0.063 & $<0.01$ & $<0.17$ & 0.05 & 4 \\
\hline Iron, $\mathrm{mg} / \mathrm{L}$ & 59 & 42 & 9.5 & $<0.004$ & 120 & 0.3 & 25 \\
\hline Lead, mg/L & 116 & 19 & 0.031 & $<0.004$ & $<0.19$ & 0.05 & 3 \\
\hline Manganese, $\mathrm{mg} / \mathrm{L}$ & 59 & 50 & 1.82 & $<0.001$ & $<10$ & 0.05 & 34 \\
\hline Trichloroethene, $\mathrm{mg} / \mathrm{L}$ & 40 & 9 & 0.34 & $<0.002$ & $<1.7$ & 0.005 & 7 \\
\hline Turbidity, NTU & 28 & 28 & 99 & 0.8 & 650 & 5 & 24 \\
\hline Vinyl chloride. $\mathrm{mg} / \mathrm{L}$ & 40 & 1 & 0.003 & $<0.003$ & $<0.1$ & 0.002 & 1 \\
\hline
\end{tabular}


Table 2.3.15 (Continued)

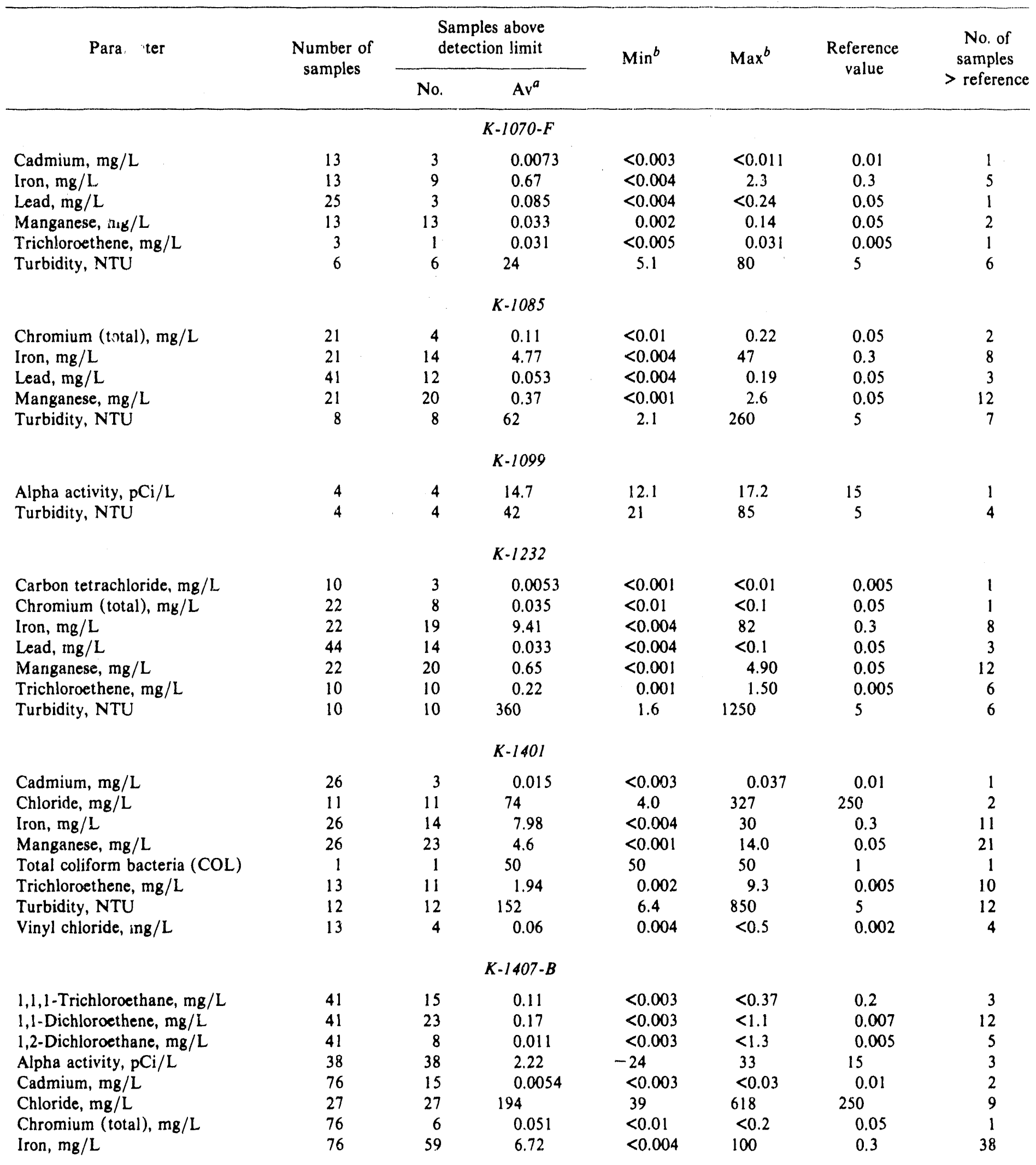


Table 2.3.15 (Cuntinued)

\begin{tabular}{|c|c|c|c|c|c|c|c|}
\hline \multirow[t]{2}{*}{ Parameter } & \multirow{2}{*}{$\begin{array}{l}\text { Number of } \\
\text { samples }\end{array}$} & \multicolumn{2}{|c|}{$\begin{array}{l}\text { Samples above } \\
\text { detection limit }\end{array}$} & \multirow{2}{*}{$\operatorname{Min}^{b}$} & \multirow{2}{*}{$\operatorname{Max}^{b}$} & \multirow{2}{*}{$\begin{array}{l}\text { Reference } \\
\text { value }\end{array}$} & \multirow{2}{*}{$\begin{array}{c}\text { No. of } \\
\text { samples } \\
>\text { reference }\end{array}$} \\
\hline & & No. & $A v^{a}$ & & & & \\
\hline \multicolumn{8}{|c|}{$K-1407-B$ (Continued) } \\
\hline Lead, mg/L & 153 & 27 & 0.041 & $<0.004$ & $<0.5$ & 0.05 & 6 \\
\hline Manganese, $\mathrm{mg} / \mathrm{L}$ & 76 & 76 & 5.94 & 0.0039 & 24 & 0.05 & 71 \\
\hline Sulfate, $\mathrm{mg} / \mathrm{L}$ & 35 & 35 & 140 & 6.0 & 1400 & 250 & 7 \\
\hline Trichloroethene, $\mathrm{mg} / \mathrm{L}$ & 41 & 36 & 1.65 & $<0.003$ & 36 & 0.005 & 35 \\
\hline Turbidity, NTU & 35 & 35 & 116 & 0.43 & 2300 & 5 & 23 \\
\hline Vinyl chloride, $\mathrm{mg} / \mathrm{L}$ & 41 & 30 & 0.12 & $<0.003$ & 0.54 & 0.002 & 30 \\
\hline \multicolumn{8}{|c|}{$K-I 407-C$} \\
\hline Chromium (total), $\mathrm{mg} / \mathrm{L}$ & 37 & 8 & 0.026 & $<0.01$ & $<0.052$ & 0.05 & 1 \\
\hline Iron, $\mathrm{mg} / \mathrm{L}$ & 37 & 32 & 7.96 & $<0.004$ & 88 & 0.3 & 20 \\
\hline Lead, mg/L & 73 & 19 & 0.029 & $<0.004$ & $<0.15$ & 0.05 & 5 \\
\hline Manganese, $\mathrm{mg} / \mathrm{L}$ & 37 & 37 & 4.97 & 0.0071 & 32 & 0.05 & 25 \\
\hline Sulfate, $\mathrm{mg} / \mathrm{L}$ & 18 & 18 & 60 & 4.0 & 298 & 250 & 2 \\
\hline Trichloroethene, $\mathrm{mg} / \mathrm{L}$ & 20 & 7 & 0.0071 & $<0.001$ & $<0.019$ & 0.005 & 3 \\
\hline Turbidity, NTU & 18 & 18 & 46 & 2.0 & 260 & 5 & 15 \\
\hline Vinyl chloride, mg/L & 20 & 4 & 0.0047 & $<0.003$ & $<0.01$ & 0.002 & 4 \\
\hline \multicolumn{8}{|c|}{$K-1407-C$ Upgradient } \\
\hline Alpha activity, $\mathrm{pCi} / \mathrm{L}$ & 6 & 6 & 2.17 & -11 & 17.0 & 15 & 1 \\
\hline Chromium (total), $\mathrm{mg} / \mathrm{L}$ & 12 & 3 & 0.24 & $<0.01$ & $<0.31$ & 0.05 & 3 \\
\hline Iron, mg/L & 12 & 9 & 13.7 & $<0.004$ & 50 & 0.3 & 7 \\
\hline Lead, $\mathrm{mg} / \mathrm{L}$ & 24 & 6 & 0.13 & $<0.004$ & $<0.5$ & 0.05 & 3 \\
\hline Manganese, $\mathrm{mg} / \mathrm{L}$ & 12 & 12 & 0.97 & 0.015 & 2.0 & 0.05 & 9 \\
\hline Turbidity, NTU & 6 & 6 & 174 & 20 & 800 & 5 & 6 \\
\hline \multicolumn{8}{|c|}{$K-1407 \cdot W A G$ (includes $K-1070-B$ and $K-1407-A$ ) } \\
\hline 1,1-Dichloroethene, $\mathrm{mg} / \mathrm{L}$ & 25 & 11 & 0.2 & $<0.002$ & $<0,83$ & 0.007 & 6 \\
\hline Alpha activity, $\mathrm{pCi} / \mathrm{L}$ & 21 & 21 & 10.0 & 0.45 & 47 & 15 & 5 \\
\hline Chromium (total), $\mathrm{mg} / \mathrm{L}$ & 47 & 10 & 0.057 & $<0.01$ & 0.14 & 0.05 & 4 \\
\hline Iron, $\mathrm{mg} / \mathrm{L}$ & 47 & 32 & 10.2 & $<0.004$ & 71 & 0.3 & 21 \\
\hline Lead, $\mathrm{mg} / \mathrm{L}$ & 92 & 13 & 0.025 & $<0.004$ & $<0.058$ & 0.05 & 1 \\
\hline Manganese, $\mathrm{mg} / \mathrm{L}$ & 47 & 46 & 2.43 & $<0.001$ & 14.0 & 0.05 & 36 \\
\hline Trichloroethene, $\mathrm{mg} / \mathrm{L}$ & 25 & 18 & 5.04 & $<0.001$ & 34 & 0.005 & 17 \\
\hline Turbidity, NTU & 21 & 21 & 181 & 0.7 & 1700 & 5 & 17 \\
\hline Vinyl chloride, $\mathrm{mg} / \mathrm{L}$ & 25 & 15 & 0.16 & $<0.005$ & $<2.5$ & 0.002 & 15 \\
\hline \multicolumn{8}{|c|}{$K \cdot 1410$} \\
\hline Iron, $\mathrm{mg} / \mathrm{L}$ & 2 & 1 & 36 & $<0.004$ & 36 & 0.3 & 1 \\
\hline Manganese, mg/L & 2 & 2 & 0.47 & 0.033 & 0.9 & 0.05 & 1 \\
\hline Trichloroethene, $\mathrm{mg} / \mathrm{L}$ & 1 & 1 & 0.064 & 0.064 & 0.064 & 0.005 & 1 \\
\hline Turbidity, NTU & 1 & 1 & 1300 & 1300 & 1300 & 5 & $i$ \\
\hline
\end{tabular}


Table 2.3.15 (Continued)

\begin{tabular}{|c|c|c|c|c|c|c|c|}
\hline \multirow[t]{2}{*}{ Parameter } & \multirow{2}{*}{$\begin{array}{l}\text { Number of } \\
\text { samples }\end{array}$} & \multicolumn{2}{|c|}{$\begin{array}{l}\text { Samples above } \\
\text { detection limit }\end{array}$} & \multirow{2}{*}{$\operatorname{Min}^{b}$} & \multirow{2}{*}{$\operatorname{Max}^{b}$} & \multirow{2}{*}{$\begin{array}{c}\text { Reference } \\
\text { value }\end{array}$} & \multirow{2}{*}{$\begin{array}{c}\text { No. of } \\
\text { samples } \\
>\text { reference }\end{array}$} \\
\hline & & No. & $A v^{a}$ & & & & \\
\hline \multicolumn{8}{|c|}{$K-1413$} \\
\hline Cadmium, mg/L & 49 & 21 & 0.0073 & $<0.003$ & $<0.033$ & 0.01 & 2 \\
\hline Chromium (total), mg/L & 49 & 16 & 0.05 & $<0.01$ & 0.29 & 0.05 & 3 \\
\hline Iron, $\mathrm{mg} / \mathrm{L}$ & 49 & 32 & 10.5 & $<0.004$ & 56 & 0.3 & 19 \\
\hline Lead, mg/L & 96 & 28 & 0.031 & $<0.004$ & $<0.11$ & 0.05 & 6 \\
\hline Manganese, mg/L & 49 & 49 & 0.47 & 0.0023 & 2.3 & 0.05 & 35 \\
\hline Total coliform bacteria $(\mathrm{COL})$ & 2 & 1 & 4.0 & $<1.0$ & 4.0 & 1 & 1 \\
\hline Trichloroethene, $\mathrm{mg} / \mathrm{L}$ & 25 & 23 & 0.7 & 0.001 & 3.8 & 0.005 & 16 \\
\hline Turbidity, NTU & 23 & 23 & 434 & 1.9 & 2200 & 5 & 21 \\
\hline Vinyl chloride, $\mathrm{mg} / \mathrm{L}$ & 25 & 5 & 0.0094 & $<0.003$ & $<0.2$ & 0.002 & 5 \\
\hline \multicolumn{8}{|c|}{$K-1420$} \\
\hline 1,1-Dichloroethene, $\mathrm{mg} / \mathrm{L}$ & 13 & 1 & 0.023 & $<0.005$ & $<0.1$ & 0.007 & 1 \\
\hline Alpha activity, $\mathrm{pCi} / \mathrm{L}$ & 12 & 12 & 9.13 & -1.0 & 49 & 15 & 2 \\
\hline Chromium (total), $\mathrm{mg} / \mathrm{L}$ & 27 & 4 & 0.064 & $<0.01$ & $<0.18$ & 0.05 & 1 \\
\hline Iron, $\mathrm{mg} / \mathrm{L}$ & 27 & 25 & 4.98 & $<0.004$ & 110 & 0.3 & 8 \\
\hline Lead, $\mathrm{mg} / \mathrm{L}$ & 53 & 10 & 0.017 & $<0.004$ & $<0.091$ & 0.05 & 1 \\
\hline Manganese, mg/L & 27 & 27 & 0.81 & 0.0025 & 8.1 & 0.05 & 13 \\
\hline Trichloroethene, $\mathrm{mg} / \mathrm{L}$ & 13 & 6 & 1.17 & $<0.002$ & 3.4 & 0.005 & 5 \\
\hline Turbidity, NTU & 12 & 12 & 34 & 1.8 & 170 & 5 & 8 \\
\hline Vinyl chloride, $\mathrm{mg} / \mathrm{L}$ & 13 & 3 & 0.11 & 0.009 & $<0.25$ & 0.002 & 3 \\
\hline \multicolumn{8}{|c|}{$K-1503$} \\
\hline Chromium (total), $\mathrm{mg} / \mathrm{L}$ & 13 & 4 & 0.048 & $<0.01$ & 0.14 & 0.05 & 1 \\
\hline Iron, $\mathrm{mg} / \mathrm{L}$ & 13 & 13 & 7.5 & 0.01 & 76 & 0.3 & 6 \\
\hline Manganese, $\mathrm{mg} / \mathrm{L}$ & 13 & 12 & 0.82 & $<0.001$ & 3.8 & 0.05 & 9 \\
\hline Trichloroethene, $\mathrm{mg} / \mathrm{L}$ & 7 & 4 & 0.012 & $<0.001$ & $<0.018$ & 0.005 & 3 \\
\hline Turbidity, NTU & 6 & 6 & 202 & 18 & 950 & 5 & 6 \\
\hline
\end{tabular}

${ }^{a}$ Average of samples above detection.

${ }^{b}$ Maximum and minimum of all the samples. 


\subsection{BIOLOGICAL SAMPLING}


Table 2.4.1. 1989 Concentrations of ${ }^{131}$ I in milk"

\begin{tabular}{|c|c|c|c|c|c|c|}
\hline \multirow{2}{*}{ Station $^{b}$} & \multirow{2}{*}{$\begin{array}{l}\text { No. of } \\
\text { samples }\end{array}$} & \multicolumn{4}{|c|}{ Concentration $(\mathrm{pCi} / \mathrm{L})$} & \multirow{2}{*}{$\begin{array}{l}\text { Dose } \\
(\mu \mathrm{S} v)^{c}\end{array}$} \\
\hline & & Max & Min & Av & Std. error & \\
\hline \multicolumn{7}{|c|}{ Immediate environs } \\
\hline 1 & 15 & 2.4 & -1.1 & 0.65 & 0.26 & 3.2 \\
\hline 2 & 17 & 1.4 & -1.1 & 0.27 & 0.17 & 1.4 \\
\hline 3 & 16 & 1.4 & -0.54 & 0.31 & 0.15 & 1.6 \\
\hline 4 & 17 & 1.6 & -2.4 & 0.032 & 0.23 & 0.16 \\
\hline $\begin{array}{l}\text { Network } \\
\text { summary }\end{array}$ & 65 & 2.4 & -2.4 & 0.31 & 0.10 & 1.5 \\
\hline \multicolumn{7}{|c|}{ Remote environs } \\
\hline 51 & 1 & 1.4 & 1.4 & 1.4 & & 6.7 \\
\hline 53 & 1 & 0.27 & 0.27 & 0.27 & & 1.3 \\
\hline $\begin{array}{l}\text { Network } \\
\text { summary }\end{array}$ & 2 & 1.4 & 0.27 & 0.81 & 0.54 & 4.0 \\
\hline
\end{tabular}

${ }^{a}$ Raw milk samples; Station 2 is a dairy.

${ }^{b}$ See Fig. 2.4.1 in Vol. 1.

'Potential 50-year committed effective dose equivalents from drinking $365 \mathrm{~L}$ of milk per year using average radionuclide concentrations at each location.

Table 2.4.2. 1989 Concentrations of total radioactive $\mathrm{Sr}$ in milk"

\begin{tabular}{|c|c|c|c|c|c|c|}
\hline \multirow{2}{*}{ Station $^{b}$} & \multirow{2}{*}{$\begin{array}{c}\text { No. of } \\
\text { samples }\end{array}$} & \multicolumn{4}{|c|}{ Concentration $(\mathrm{pCi} / \mathrm{L})$} & \multirow{2}{*}{$\begin{array}{l}\text { Dose } \\
(\mu \mathrm{Sv})\end{array}$} \\
\hline & & Max & Min & Av & Std. error & \\
\hline \multicolumn{7}{|c|}{ Immediate environs } \\
\hline 1 & 15 & 10 & 0.86 & 3.3 & 0.74 & 42 \\
\hline 2 & 17 & 7.3 & 0.81 & 1.9 & 0.48 & 25 \\
\hline 3 & 16 & 4.9 & 0.32 & 2.4 & 0.30 & 31 \\
\hline 4 & 17 & 12 & -1.6 & 4.0 & 0.82 & 52 \\
\hline
\end{tabular}

Network

$\begin{array}{lllllll}\text { summary } & 65 & 12 & -1.6 & 2.9 & 0.32 & 37\end{array}$

\begin{tabular}{llllll}
\multicolumn{7}{c}{ Remote environs } \\
51 & 1 & 1.8 & 1.8 & 1.8 & 24 \\
53 & 1 & 0.30 & 0.30 & 0.30 & 3.8
\end{tabular}

Network

$\begin{array}{lllllll}\text { summary } & 2 & 1.8 & 0.30 & 1.1 & 0.77 & 14\end{array}$

${ }^{a}$ Raw milk samples; Station 2 is a dairy.

${ }^{b}$ See Fig. 2.4 .1 in Vol. 1.

cPotential 50-year committed effective dose equivalents from drinking $365 \mathrm{~L}$ of milk per year using average radionuclide concentrations at each location. 
Table 2.4.3. 1989 Mercury concentrations in Clinch River bluegill

\begin{tabular}{lccccccc}
\hline Location & \multirow{2}{*}{$\begin{array}{c}\text { No. of } \\
\text { fish } \\
\text { sampled }\end{array}$} & \multicolumn{4}{c}{ Concentration $(\mu \mathrm{g} / \mathrm{g}$ wet wt) } & $\begin{array}{c}\text { Percentage } \\
\text { of action } \\
\text { level }^{b}\end{array}$ \\
\hline CRK 8.0 & 12 & 0.35 & 0.061 & 0.14 & 0.023 & 14 \\
CRK 33.3 & 12 & 0.13 & 0.027 & 0.054 & 0.0090 & 5.4 \\
CRK 40.0 & 12 & 0.10 & 0.016 & 0.041 & 0.0072 & 4.1 \\
\hline
\end{tabular}

${ }^{a}$ See Fig, 2.4.2 in Vol. 1.

${ }^{b}$ Percent of Food and Drug Administration action level of mercury in fish $(1.0 \mu \mathrm{g} / \mathrm{g})$ for the average concentration.

Table 2.4.4. 1989 PCB Concentrations in Clinch River bluegill

\begin{tabular}{|c|c|c|c|c|c|c|c|}
\hline \multirow{2}{*}{ Location ${ }^{a}$} & \multirow{2}{*}{$\begin{array}{l}\text { PCB } \\
\text { type }\end{array}$} & \multirow{2}{*}{$\begin{array}{l}\text { No. of } \\
\text { fish } \\
\text { samipled }\end{array}$} & \multicolumn{4}{|c|}{ Concentration $(\mu g / g$ wet $w t)$} & \multirow{2}{*}{$\begin{array}{c}\text { Percentage } \\
\text { of } \\
\text { tolerance }^{b}\end{array}$} \\
\hline & & & Max & Min & Av & Std. error & \\
\hline CRK 8.0 & 1254 & 12 & 0.03 & $<0.01$ & $<0.018$ & 0.0027 & 0.92 \\
\hline CRK 8.0 & 1260 & 12 & 0.07 & $<0.01$ & $<0.016$ & 0.0050 & 0.79 \\
\hline CRK 33.3 & 1254 & 12 & 0.10 & $<0.01$ & $<0.019$ & 0.0074 & 0.96 \\
\hline CRK 33.3 & 1260 & 12 & 0.10 & $<0.01$ & $<0.025$ & 0.0080 & 1.3 \\
\hline CRK 40.0 & 1254 & 12 & 0.01 & $<0.01$ & $<0.010$ & 0 & 0.50 \\
\hline CRK 40.0 & 1260 & 12 & 0.04 & $<0.01$ & $<0.018$ & 0.0035 & 0.88 \\
\hline
\end{tabular}

${ }^{\circ}$ See Fig. 2.4.2 in Vol. 1.

bercent of Food and Drug Administration action level of PCBs in fish $(2.0 \mu \mathrm{g} / \mathrm{g}$ ) for the average concentration. 
Table 2.4.5. 1989 Radionuclide concentrations in Clinch River bluegill

\begin{tabular}{|c|c|c|c|c|c|c|}
\hline Location $^{a}$ & Radionuclide & $\begin{array}{c}\text { No. of } \\
\text { samples }^{b}\end{array}$ & $\operatorname{Max}$ & Min & Av & $\begin{array}{l}\text { Std. } \\
\text { error }\end{array}$ \\
\hline \multicolumn{7}{|c|}{ Concentration $(p \mathrm{Ci} / \mathrm{g}$ ash $w t)$} \\
\hline CRK 8.0 & ${ }^{60} \mathrm{Co}$ & 6 & 0.51 & -0.057 & 0.22 & 0.087 \\
\hline CRK 8.0 & ${ }^{137} \mathrm{Cs}$ & 6 & 12 & 3.8 & 7.6 & 1.6 \\
\hline CRK 8.0 & Total $\mathrm{Sr}^{c}$ & 6 & 0.68 & 0.027 & 0.32 & 0.086 \\
\hline CRK 33.3 & ${ }^{60} \mathrm{Co}$ & 6 & 0.38 & -0.032 & 0.21 & 0.067 \\
\hline CRK 33.3 & ${ }^{137} \mathrm{Cs}$ & 6 & 16 & 3.5 & 11 & 1.8 \\
\hline CRK 33.3 & Total $\mathrm{Sr}^{c}$ & 6 & 1.9 & 0.51 & 1.5 & 0.22 \\
\hline CRK 40.0 & ${ }^{60} \mathrm{Co}$ & 6 & 0.16 & -0.14 & 0.013 & 0.043 \\
\hline CRK 40.0 & ${ }^{137} \mathrm{Cs}$ & 6 & 1.9 & 0.26 & 0.83 & 0.26 \\
\hline CRK 40.0 & Total $\mathrm{Sr}^{c}$ & 6 & 1.7 & -0.27 & 0.50 & 0.27 \\
\hline \multicolumn{7}{|c|}{ Concentration ( $p \mathrm{Ci} / \mathrm{g}$ wet wt) } \\
\hline CRK 8.0 & ${ }^{60} \mathrm{Co}$ & 6 & 0.0063 & -0.00084 & 0.0027 & 0.0011 \\
\hline CRK 8.0 & ${ }^{137} \mathrm{Cs}$ & 6 & 0.15 & 0.045 & 0.10 & 0.022 \\
\hline CRK 8.0 & Total $\mathrm{Sr}^{c}$ & 6 & 0.0091 & 0.00032 & 0.0042 & 0.0012 \\
\hline CRK 33.3 & ${ }^{60} \mathrm{Co}$ & 6 & 0.0045 & -0.00041 & 0.0025 & 0.00078 \\
\hline CRK 33.3 & ${ }^{137} \mathrm{Cs}$ & 6 & 0.18 & 0.041 & 0.13 & 0.021 \\
\hline CRK 33.3 & Total $\mathrm{Sr}^{c}$ & 6 & 0.022 & 0.0060 & 0.017 & 0.0026 \\
\hline CRK 40.0 & ${ }^{60} \mathrm{Co}$ & 6 & 0.0020 & -0.0018 & 0.00019 & 0.00056 \\
\hline CRK 40.0 & ${ }^{137} \mathrm{Cs}$ & 6 & 0.032 & 0.0032 & 0.012 & 0.0044 \\
\hline CRK 40.0 & Total $\mathrm{Sr}^{c}$ & 6 & 0.020 & -0.0045 & 0.0064 & 0.0035 \\
\hline
\end{tabular}

${ }^{a}$ See Fig. 2.4 .2 in Vol. 1.

${ }^{b} \mathrm{~A}$ sample is a composite of 6 to $10 \mathrm{fish}$.

Total radioactive $\mathrm{Sr}\left({ }^{89} \mathrm{Sr}\right.$ and $\left.{ }^{90} \mathrm{Sr}\right)$. 


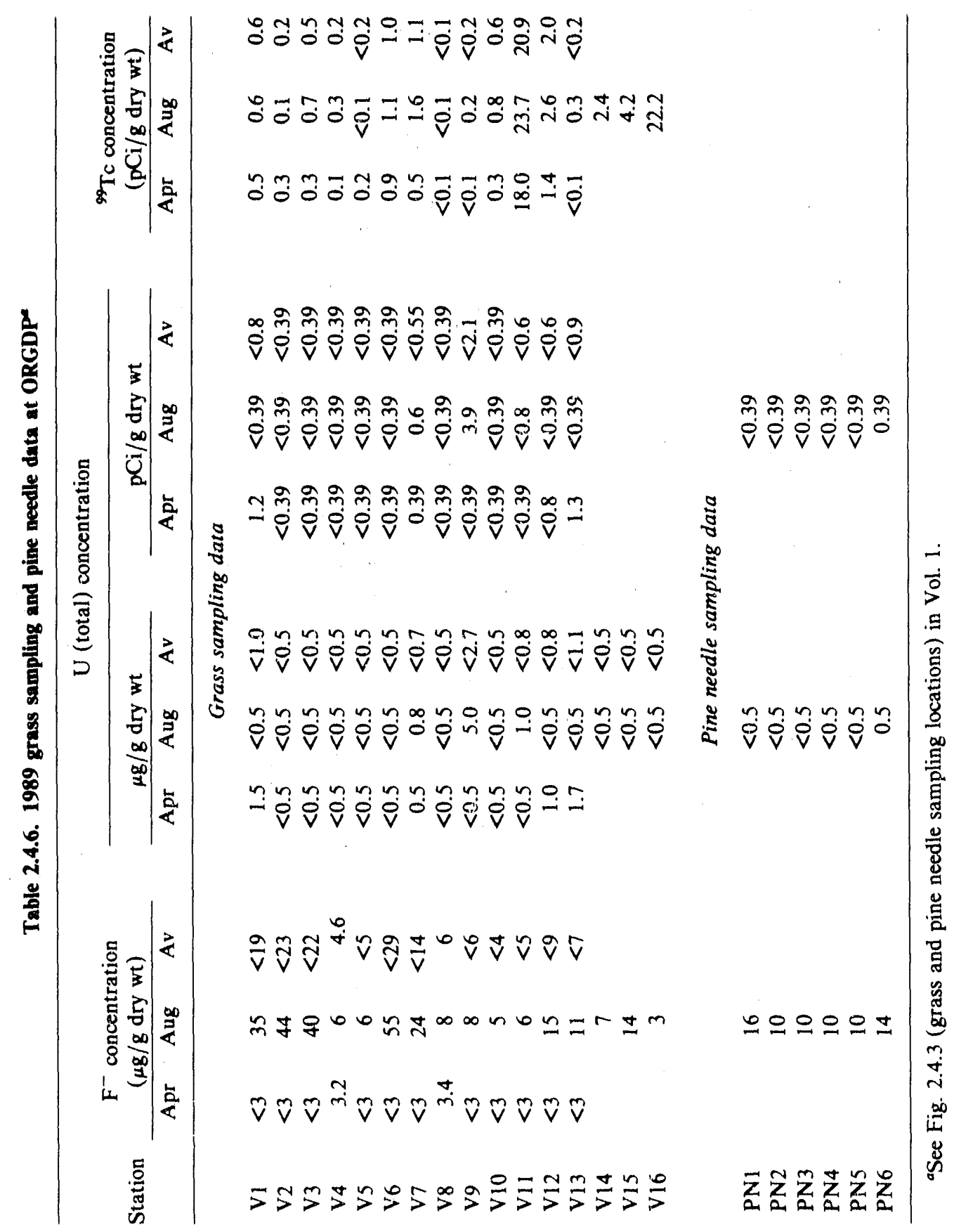




\subsection{SOIL AND SEDIMENT MONITORING}


Table 2.5.1. ${ }^{6} \mathrm{Co}$ concentrations in soil, 1989

\begin{tabular}{|c|c|c|c|c|c|}
\hline \multirow{2}{*}{ Location } & \multirow{2}{*}{$\begin{array}{c}\text { Number of } \\
\text { samples }\end{array}$} & \multicolumn{4}{|c|}{ Concentration ( $\mathrm{pCi} / \mathrm{g}$ dry wt) } \\
\hline & & $\operatorname{Max}$ & $\operatorname{Min}$ & Av & Std. error ${ }^{a}$ \\
\hline \multicolumn{6}{|c|}{ ORNL Perimeter Stations } \\
\hline 03 & 3 & 0.054 & -0.035 & 0.019 & 0.027 \\
\hline 07 & 3 & 0.019 & -0.022 & -0.0054 & 0.012 \\
\hline 09 & 3 & 0.057 & -0.035 & 0.0045 & 0.027 \\
\hline 20 & 3 & -0.0081 & -0.030 & -0.018 & 0.0063 \\
\hline 21 & 3 & 0.057 & -0.0054 & 0.021 & 0.019 \\
\hline \multicolumn{6}{|l|}{ Network } \\
\hline summary & 15 & 0.057 & -0.035 & 0.0041 & 0.0086 \\
\hline \multicolumn{6}{|c|}{ Oak Ridge Reservation Stations } \\
\hline 40 & 3 & 0.032 & -0.0027 & 0.014 & 0.010 \\
\hline 45 & 3 & 0.030 & 0.0081 & 0.022 & 0.0068 \\
\hline 46 & 3 & 0.030 & 0.0054 & 0.015 & 0.0074 \\
\hline \multicolumn{6}{|l|}{ Network } \\
\hline summary & 9 & 0.032 & -0.0027 & 0.017 & 0.0043 \\
\hline
\end{tabular}

${ }^{a}$ Standard error about the average.

Table 2.5.2. ${ }^{137} \mathrm{Cs}$ concentrations in soil, 1989

\begin{tabular}{|c|c|c|c|c|c|}
\hline \multirow{2}{*}{ Location } & \multirow{2}{*}{$\begin{array}{c}\text { Number of } \\
\text { samples }\end{array}$} & \multicolumn{4}{|c|}{ Concentration $(\mathrm{pCi} / \mathrm{g}$ dry $w \mathrm{t})$} \\
\hline & & $\operatorname{Max}$ & Min & Av & Std. error ${ }^{a}$ \\
\hline \multicolumn{6}{|c|}{ ORNL Perimeter Stations } \\
\hline 03 & 3 & 0.92 & 0.70 & 0.80 & 0.063 \\
\hline 07 & 3 & 1.5 & 0.30 & 0.84 & 0.36 \\
\hline 09 & 3 & 1.5 & 0.11 & 0.69 & 0.42 \\
\hline 20 & 3 & 0.27 & 0.19 & 0.23 & 0.024 \\
\hline 21 & 3 & 0.12 & 0.051 & 0.081 & 0.021 \\
\hline \multicolumn{6}{|l|}{ Network } \\
\hline summary & 15 & 1.5 & 0.051 & 0.53 & 0.13 \\
\hline \multicolumn{6}{|c|}{ Oak Ridge Reservation Stations } \\
\hline 40 & 3 & 0.19 & 0.049 & 0.13 & 0.042 \\
\hline 45 & 3 & 0.46 & 0.0081 & 0.19 & 0.14 \\
\hline 46 & 3 & 0.35 & 0.26 & 0.30 & 0.025 \\
\hline \multicolumn{6}{|l|}{ Network } \\
\hline summary & 9 & 0.46 & 0.0081 & 0.21 & 0.049 \\
\hline
\end{tabular}

aStandard error about the average. 
Table 2.5.3. ${ }^{238} \mathrm{Pu}$ concentrations in soil, 1989

\begin{tabular}{|c|c|c|c|c|c|}
\hline \multirow{2}{*}{ Location } & \multirow{2}{*}{$\begin{array}{c}\text { Number of } \\
\text { samples }\end{array}$} & \multicolumn{4}{|c|}{ Concentration ( $\mathrm{pCi} / \mathrm{g}$ dry $w \mathrm{t}$ ) } \\
\hline & & $\operatorname{Max}$ & Min & Av & Std. error ${ }^{a}$ \\
\hline \multicolumn{6}{|c|}{ ORNL Perimeter Stations } \\
\hline 03 & 3 & 0.0012 & -0.00027 & 0.00064 & 0.00046 \\
\hline 07 & 3 & 0.0035 & -0.00027 & 0.0011 & 0.0012 \\
\hline 09 & 3 & 0.0011 & -0.00046 & 0.00023 & 0.00045 \\
\hline 20 & 3 & 0.00027 & -0.00065 & -0.00011 & 0.00028 \\
\hline 21 & 3 & -0.00024 & -0.00070 & -0.00047 & 0.00013 \\
\hline \multicolumn{6}{|l|}{ Network } \\
\hline summary & 15 & 0.0035 & -0.00070 & 0.00028 & 0.00028 \\
\hline \multicolumn{6}{|c|}{ Oak Ridge Reservation Stations } \\
\hline 40 & 3 & 0.00057 & 0.00019 & 0.00042 & 0.00012 \\
\hline 45 & 3 & 0.0012 & -0.0010 & 0.00014 & 0.00063 \\
\hline 46 & 3 & 0.00097 & -0.0014 & 0.000009 & 0.00070 \\
\hline \multicolumn{6}{|l|}{ Network } \\
\hline summary & 9 & 0.0012 & -0.0014 & 0.00019 & $0.000 ? 8$ \\
\hline
\end{tabular}

${ }^{a}$ Standard error about the average.

Table 2.5.4. ${ }^{239} \mathrm{Pu}$ concentrations in soll, 1989

\begin{tabular}{|c|c|c|c|c|c|}
\hline \multirow{2}{*}{ Location } & \multirow{2}{*}{$\begin{array}{c}\text { Number of } \\
\text { samples }\end{array}$} & \multicolumn{4}{|c|}{ Concentration (pCi/g dry wt) } \\
\hline & & $\operatorname{Max}$ & Min & Av & Std. error ${ }^{a}$ \\
\hline \multicolumn{6}{|c|}{ ORNL Perimeter Stations } \\
\hline 03 & 3 & 0.038 & 0.030 & 0.034 & 0.0024 \\
\hline 07 & 3 & 0.022 & 0.0032 & 0.013 & 0.0053 \\
\hline 09 & 3 & 0.049 & 0.0054 & 0.022 & 0.014 \\
\hline 20 & 3 & 0.0032 & 0.0022 & 0.0027 & 0.00031 \\
\hline 21 & 3 & 0.0013 & --0.000027 & 0.00072 & 0.00039 \\
\hline $\begin{array}{l}\text { Network } \\
\text { summary }\end{array}$ & 15 & 0.049 & -0.000027 & 0.014 & 0.0042 \\
\hline \multicolumn{6}{|c|}{ Oak Ridge Reservation Stations } \\
\hline 40 & 3 & 0.0038 & -0.00073 & 0.0020 & 0.0014 \\
\hline 45 & 3 & 0.0049 & -0.0025 & 0.0018 & 0.0022 \\
\hline 46 & 3 & 0.0065 & 0.0035 & 0.0052 & 0.00089 \\
\hline $\begin{array}{l}\text { Network } \\
\text { summary }\end{array}$ & 9 & 0.0065 & -0.0025 & 0.0030 & 0.00097 \\
\hline
\end{tabular}

${ }^{a}$ Standard error about the average. 
Table 2.5.5. Total radioactive $\mathrm{Sr}$ concentrations in soil, 1989

\begin{tabular}{|c|c|c|c|c|c|}
\hline \multirow{2}{*}{ Location } & \multirow{2}{*}{$\begin{array}{l}\text { Number of } \\
\text { samples }\end{array}$} & \multicolumn{4}{|c|}{ Concentration ( $\mathrm{pCi} / \mathrm{g}$ dry wt) } \\
\hline & & $\operatorname{Max}$ & Min & Av & Std. error \\
\hline \multicolumn{6}{|c|}{ ORNL Perimeter Stations } \\
\hline 03 & 3 & 0.78 & 0.24 & 0.43 & 0.18 \\
\hline 07 & 3 & 0.70 & 0.35 & 0.50 & 0.11 \\
\hline 09 & 3 & 0.21 & 0.15 & 0.19 & 0.019 \\
\hline 20 & 3 & 0.21 & 0.014 & 0.085 & 0.062 \\
\hline 21 & 3 & 0.11 & 0.027 & 0.067 & 0.023 \\
\hline $\begin{array}{l}\text { Network } \\
\text { summary }\end{array}$ & 15 & 0.78 & 0.014 & 0.25 & 0.060 \\
\hline \multicolumn{6}{|c|}{ Oak Ridge Reservation Stations } \\
\hline 40 & 3 & 0.19 & 0.011 & 0.076 & 0.058 \\
\hline 45 & 3 & 0.24 & -0.030 & 0.079 & 0.082 \\
\hline 46 & 3 & 0.23 & 0.092 & 0.15 & 0.040 \\
\hline $\begin{array}{l}\text { Network } \\
\text { summary }\end{array}$ & 9 & 0.24 & -0.030 & 0.10 & 0.034 \\
\hline
\end{tabular}

${ }^{a}$ Standard error about the average.

Table 2.5.6. ${ }^{234} \mathrm{U}$ concentrations in soil, 1989

\begin{tabular}{|c|c|c|c|c|c|}
\hline \multirow{2}{*}{ Location } & \multirow{2}{*}{$\begin{array}{c}\text { Number of } \\
\text { samples }\end{array}$} & \multicolumn{4}{|c|}{ Concentration ( $\mathrm{pCi} / \mathrm{g}$ dry wt) } \\
\hline & & Max & Min & Av & Std. error ${ }^{a}$ \\
\hline \multicolumn{6}{|c|}{ ORNL Perimeter Stations } \\
\hline 03 & 3 & 0.30 & 0.20 & 0.24 & 0.029 \\
\hline 07 & 3 & 0.35 & 0.27 & 0.32 & 0.027 \\
\hline 09 & 3 & 0.41 & 0.24 & 0.31 & 0.049 \\
\hline 20 & 3 & 0.30 & 0.25 & 0.27 & 0.014 \\
\hline 21 & 3 & 0.97 & 0.59 & 0.77 & 0.11 \\
\hline $\begin{array}{l}\text { Network } \\
\text { summary }\end{array}$ & 15 & 0.97 & 0.20 & 0.38 & 0.057 \\
\hline \multicolumn{6}{|c|}{ Oak Ridge Reservation Stations } \\
\hline 40 & 3 & 3.2 & 2.4 & 2.8 & 0.25 \\
\hline 45 & 3 & 1.1 & 0.49 & 0.79 & 0.19 \\
\hline 46 & 3 & 0.76 & 0.43 & 0.60 & 0.094 \\
\hline Network & & & & & \\
\hline summary & 9 & 3.2 & 0.43 & 1.4 & 0.36 \\
\hline
\end{tabular}

aStandard error about the average. 
Table 2.5.7. ${ }^{235} \mathrm{U}$ concentrations in soil, 1989

\begin{tabular}{|c|c|c|c|c|c|}
\hline \multirow{2}{*}{ Location } & \multirow{2}{*}{$\begin{array}{c}\text { Number of } \\
\text { samples }\end{array}$} & \multicolumn{4}{|c|}{ Concentration ( $\mathrm{pCi} / \mathrm{g}$ dry $w t)$} \\
\hline & & $\operatorname{Max}$ & Min & Av & Std. error \\
\hline \multicolumn{6}{|c|}{ ORNL Perimeter Stations } \\
\hline 03 & 3 & 0.013 & 0.0065 & 0.0086 & 0.0021 \\
\hline 07 & 3 & 0.014 & 0.011 & 0.012 & 0.00087 \\
\hline 09 & 3 & 0.016 & 0.011 & 0.014 & 0.0014 \\
\hline 20 & 3 & 0.020 & 0.0057 & 0.012 & 0.0042 \\
\hline 21 & 3 & 0.041 & 0.022 & 0.032 & 0.0056 \\
\hline \multicolumn{6}{|l|}{ Network } \\
\hline summary & 15 & 0.041 & 0.0057 & 0.016 & 0.0026 \\
\hline \multicolumn{6}{|c|}{ Oak Ridge Reservation Stations } \\
\hline 40 & 3 & 0.30 & 0.14 & 0.21 & 0.048 \\
\hline 45 & 3 & 0.14 & 0.035 & 0.073 & 0.033 \\
\hline 46 & 3 & 0.030 & 0.012 & 0.022 & 0.0052 \\
\hline \multicolumn{6}{|l|}{ Network } \\
\hline summary & 9 & 0.30 & 0.012 & 0.10 & 0.032 \\
\hline
\end{tabular}

Table 2.5.8. ${ }^{238} \mathrm{U}$ concentrations in soil, 1989

\begin{tabular}{|c|c|c|c|c|c|}
\hline \multirow{2}{*}{ Location } & \multirow{2}{*}{$\begin{array}{c}\text { Number of } \\
\text { samples }\end{array}$} & \multicolumn{4}{|c|}{ Concentration ( $\mathrm{pCi} / \mathrm{g} \mathrm{dry}$ wr) } \\
\hline & & Max & Min & Av & Std. error \\
\hline \multicolumn{6}{|c|}{ ORNL Perimeter Stations } \\
\hline 03 & 3 & 0.21 & 0.14 & 0.17 & 0.021 \\
\hline 07 & 3 & 0.27 & 0.21 & 0.25 & 0.020 \\
\hline 09 & 3 & 0.35 & 0.14 & 0.24 & 0.061 \\
\hline 20 & 3 & 0.23 & 0.17 & 0.19 & 0.019 \\
\hline 21 & 3 & 0.62 & 0.41 & 0.50 & 0.063 \\
\hline \multicolumn{6}{|l|}{ Network } \\
\hline sumniary & 15 & 0.62 & 0.14 & $0.2 i$ & 0.036 \\
\hline \multicolumn{6}{|c|}{ Oak Ridge Reservation Stations } \\
\hline 40 & 3 & 4.3 & 1.4 & 2.5 & 0.93 \\
\hline 45 & 3 & 1.2 & 0.30 & 0.83 & 0.27 \\
\hline 46 & 3 & 0.38 & 0.25 & 0.32 & 0.036 \\
\hline \multicolumn{6}{|l|}{ Network } \\
\hline summary & 9 & 4.3 & 0.2 .5 & 1.2 & 0.43 \\
\hline
\end{tabular}

"Standard error about the average. 
Table 2.5.9. 1989 fluoride and uranium in soil from ORGDP perimeter

\begin{tabular}{|c|c|c|c|c|c|c|c|c|c|c|}
\hline \multirow{3}{*}{$\begin{array}{c}\text { New } \\
\text { station ID }\end{array}$} & \multirow{3}{*}{$\begin{array}{l}\text { No. of } \\
\text { samples }\end{array}$} & \multicolumn{6}{|c|}{$\begin{array}{l}\text { Concentration } \\
(\mu \mathrm{g} / \mathrm{g} \text { dry wt) }\end{array}$} & \multirow{2}{*}{\multicolumn{3}{|c|}{$\begin{array}{c}\mathrm{U} \\
(\mathrm{pCi} / \mathrm{g} \text { dry wt) }\end{array}$}} \\
\hline & & \multicolumn{3}{|c|}{$F$} & \multicolumn{3}{|c|}{$\mathrm{U}$ (total) } & & & \\
\hline & & April & Aug & Av & April & Aug & Av & April & Aug & Av \\
\hline S18 & 2 & 250 & 443 & 347 & 4 & 3 & 4 & 3.1 & 2.4 & 3.1 \\
\hline S19 & 2 & 650 & 436 & 543 & 3 & 2 & 3 & 2.4 & 1.6 & 2.4 \\
\hline $\mathrm{S} .20$ & 2 & 250 & 252 & 251 & 6 & 5 & 6 & 4.7 & 3.9 & 4.7 \\
\hline $\mathrm{S} 21$ & 2 & 300 & 395 & 348 & 5 & 3 & 4 & 3.9 & 2.4 & 3.1 \\
\hline $\mathrm{S} 22$ & 2 & 850 & 693 & 772 & 4 & 2 & 3 & 3.1 & 1.6 & 2.4 \\
\hline $\mathrm{S} 23$ & 2 & 150 & 251 & 201 & 3 & 3 & 3 & 2.4 & 2.4 & 2.4 \\
\hline $\mathbf{S} 24$ & 2 & 50 & 236 & 143 & 2 & 2 & 2 & 1.6 & 1.6 & 1.6 \\
\hline $\mathrm{S} 25$ & 2. & 250 & 466 & 358 & 4 & 5 & 5 & 3.1 & 3.9 & 3.9 \\
\hline S26 & 2 & $<50$ & 246 & $<148$ & 5 & 4 & 5 & 3.9 & 3.1 & 3.9 \\
\hline $\mathbf{S} 27$ & 2 & 450 & 441 & 446 & 2 & 2 & 2 & 1.5 & 1.6 & 1.6 \\
\hline S28 & 2 & 250 & 417 & 334 & 42 & 32 & 37 & 32.9 & 25.1 & 29.0 \\
\hline $\mathbf{S} 29$ & 2 & 550 & 1442 & 996 & 10 & 4 & 7 & 7.8 & 3.1 & 5.5 \\
\hline $\mathrm{S} 30$ & 2 & 50 & 355 & 203 & 7 & 2 & 5 & 5.5 & 1.6 & 3.7 \\
\hline S31 & 2 & - & 444 & 444 & - & 7 & 7 & - & 5.5 & 5.5 \\
\hline
\end{tabular}

${ }^{a}$ See Fig. 2.5 .1 in Vol. 1.

Table 2.5.10. 1989 concentrations of various elements in stream sediment samples near ORGDP

\begin{tabular}{|c|c|c|c|c|c|c|c|c|}
\hline \multirow{2}{*}{ Element } & \multicolumn{8}{|c|}{$\begin{array}{l}\text { Concentration } \\
(\mu \mathrm{g} / \mathrm{g} \text { dry } w t)\end{array}$} \\
\hline & SS 1 & SS2 & SS3 & SS4 & SS5 & SS6 & SS7 & SS8 \\
\hline Aluminum & 9700 & 4300 & 6900 & 9800 & 8800 & 5400 & 6100 & 1800 \\
\hline Cadmium & 0.80 & 0.66 & 0.35 & 0.69 & 0.39 & 0.47 & 0.61 & $<0.26$ \\
\hline Chromium & 15 & 16 & 10 & 18 & 15 & 29 & 9.6 & 4.5 \\
\hline Copper & 11 & 11 & 13 & 24 & 18 & 14 & 9.6 & 2.5 \\
\hline Lead & 24 & 15 & 29 & 31 & 31 & 23 & 28 & 5.1 \\
\hline Mangarese & 460 & 220 & 440 & 200 & 460 & 430 & 630 & 300 \\
\hline Mercury & $<1.0$ & 4.9 & 4.9 & 6.2 & 6.8 & 4.4 & $<1.0$ & $<1.0$ \\
\hline Nickel & 15 & 24 & 18 & 42 & 13 & 19 & 8.6 & 4.7 \\
\hline Thorium & $<16$ & $<13$ & $<17$ & $<16$ & $<16$ & $<17$ & $<19$ & $<18$ \\
\hline Uranium & 2 & 1 & 3 & 5 & 4 & 4 & 2 & $<1$ \\
\hline Zinc & 52 & 43 & 59 & 70 & 62 & 43 & 40 & 13 \\
\hline
\end{tabular}

See Fig. 2.5.2 in Vol. 1. 


\subsection{EXTERNAL GAMMA RADIATION}


Table 2.6.1. 1989 External gamma radiation measurements

\begin{tabular}{|c|c|c|c|c|c|}
\hline \multirow[b]{2}{*}{ Location } & \multirow{2}{*}{$\begin{array}{c}\text { Number of } \\
\text { samples }\end{array}$} & \multicolumn{4}{|c|}{ Exposure rate $(\mu R / h)$} \\
\hline & & Max & Min & Av & $\begin{array}{c}\text { Standard } \\
\text { error }^{a}\end{array}$ \\
\hline \multicolumn{6}{|c|}{ ORNL PAM stations } \\
\hline 3 & 45 & 7.1 & 6.6 & 0.8 & 0.021 \\
\hline 4 & 9 & 110 & 94 & 110 & 2.1 \\
\hline 7 & 22 & 8.9 & 7 & 8.0 & 0.13 \\
\hline 20 & 4 & 9.4 & 8.4 & 8.8 & 0.21 \\
\hline $\begin{array}{l}\text { Network } \\
\text { summary }\end{array}$ & 80 & 110 & 6.6 & 18 & 3.5 \\
\hline \multicolumn{6}{|c|}{ Oak Ridge Reservation stations } \\
\hline 8 & 28 & 7.4 & 6.9 & 7.1 & 0.029 \\
\hline 31 & 39 & 11 & 7.5 & 7.8 & 0.082 \\
\hline 33 & 23 & 8 & 7.1 & 7.5 & 0.052 \\
\hline 34 & 8 & 9.1 & 7.8 & 8.5 & 0.14 \\
\hline 36 & 28 & 7.4 & 6.8 & 7.1 & 0.029 \\
\hline 40 & 16 & 8.2 & 7.6 & 7.9 & 0.039 \\
\hline 41 & 40 & 6.5 & 5.8 & 6.1 & 0.032 \\
\hline 42 & 8 & 7.2 & 6.7 & 6.9 & 0.058 \\
\hline 43 & 19 & 6.9 & 6.4 & 6.7 & 0.033 \\
\hline 44 & 42 & 7.3 & 6.4 & 6.7 & 0.031 \\
\hline 45 & 3 & 7.4 & 7.2 & 7.3 & 0.063 \\
\hline $\begin{array}{l}\text { Network } \\
\text { summary }\end{array}$ & 254 & 11 & 5.8 & 7.1 & 0.043 \\
\hline
\end{tabular}

'Standard deviation of the mean. 
3. POTENTIAL RADIATION AND CHEMICAL DOSE TO THE PUBLIC 
All data for thls section are presented in Vol. 1. 


\section{REMEDIAL ACTION}


All data for this section are presented in Vol. 1. 
5. SOLID WASTE MANAGEMENT PROGRAM 
Table 5.2.1. Y-12 Plant remedial action waste data for 1989

\begin{tabular}{ll}
\hline \multicolumn{1}{c}{ Waste } & $\begin{array}{c}\text { Quantity } \\
\left(\mathrm{yd}^{3}\right)\end{array}$ \\
\hline Nonhazardous & 1000 \\
Harzardous and/or mixed & $3972^{a}$ \\
\hline
\end{tabular}

${ }^{a}$ Does not include treated water discharged from Oil Ponds 1 and 2.

Table 5.3.1. Y-12 Plant on-site waste treatment data for FY 1989

\begin{tabular}{lrcc}
\hline Waste & $\begin{array}{c}\text { Quantity } \\
\text { treated } \\
(\mathrm{kg})^{a}\end{array}$ & $\begin{array}{c}\text { Treatment } \\
\text { method }\end{array}$ & $\begin{array}{c}\text { Residue } \\
\text { type }\end{array}$ \\
\hline Liquids & & \\
Nonhazardous & $4,771,700^{b}$ & $c$ & Sludge \\
Hazardous & $1,967,000$ & $d$ & Sludge \\
Low-level aqueous & 556,000 & $c, e$ & Sludge \\
Mixed & $4,195,000$ & $c$ & Sludge \\
& Sclids & & Solid \\
Low-level solids $\left(\mathrm{ft}^{3}\right)$ & 375,000 & Compaction & S
\end{tabular}

Units are kilograms except as noted.

${ }^{6}$ Does not include wastewater treated at the Steam Plant Wastewater Treatment Facility.

'Batch reactors, settling, filtration, clirome reduction, hydrated lime treatment, dewatering, effluent polishing, biodenitrification, and biological degradation.

${ }^{d}$ Batch reactors, settling, filtration, chrome reduction, hydrated lime treatment, dewatering, effluent polishing, biodenitrification, biological degradation, $\mathrm{pH}$ control, and metal precipitation.

'Batch reactors, settling, filtration, chrome reduction, hydrated lime treatment, dewatering, effluent polishing, and biodenitrification.

Total; cannot be broken down. 
Table 5.3.2. 1989 ORNL waste treatment data

\begin{tabular}{lcll}
\hline Type & $\begin{array}{c}\text { Quantity } \\
(\mathrm{kg})\end{array}$ & Treatment & Residue type \\
\hline Hazardous & 394 & $\begin{array}{l}\text { Detonation } \\
\text { Evaporation }\end{array}$ & $\begin{array}{l}\text { None } \\
\text { This facility is no } \\
\text { longer in operation }\end{array}$ \\
\hline
\end{tabular}

Table 5.3.3. Y-12 Plant on-site waste disposal during 1989

\begin{tabular}{llr}
\multicolumn{1}{c}{ Waste } & Method & $\begin{array}{c}\text { Quantity } \\
(\mathrm{kg})\end{array}$ \\
\hline Sanitary/industrial & Landfill & $6,308,000^{\circ}$ \\
Solid/low level & Landfill & 406,000 \\
Classified & Landfill & 95,000 \\
\hline \multicolumn{2}{c}{${ }^{a}$ This category includes construction/demolition } \\
spoils from Y-12 Plant.
\end{tabular}

Table 5.3.4. 1989 ORNL on-site waste disposal

\begin{tabular}{llc}
\multicolumn{1}{c}{ Waste } & Disposal method & Quantity $(\mathrm{kg})$ \\
\hline $\begin{array}{l}\text { Hazardous } \\
\text { Sanitary } \\
\quad \text { Nonradiological } \\
\text { Radiological } \\
\text { Construction } \\
\text { debris }\end{array}$ & Release to air & 59 \\
$\begin{array}{l}\text { Asbestos } \\
\text { Radiological }\end{array}$ & Landfilling & $1,400 \mathrm{~m}^{36}$ \\
$\begin{array}{c}\text { Scrap metal } \\
\text { Radiological }\end{array}$ & Landfilling & 1,300 \\
\hline
\end{tabular}

${ }^{a}$ This is not RCRA regulated hazardous.

${ }^{b}$ The nonradiological sanitary waste (i.e., garbage) is disposed of in the Y-12 Centralized Sanitary Landfill. 
Table 5.3.5. ORGDP waste disposal at DOE Oak Ridge Facilities during 1989

\begin{tabular}{ccc} 
Waste & Quantity & Disposal method \\
\hline $\begin{array}{c}\text { Asbestos } \\
\text { nonradiological }(\mathrm{kg})\end{array}$ & 33,400 & $\begin{array}{c}\text { Sent to landfill at } \\
\text { Y-12 Plant }\end{array}$ \\
$\begin{array}{c}\text { Miscellaneous } \\
\text { nonradiological }\left(\mathrm{m}^{3}\right)\end{array}$ & 45 & $\begin{array}{c}\text { Buried at ORGDP classified } \\
\text { solids } \\
\text { nonhazardous }\left(\mathrm{m}^{3}\right)\end{array}$ \\
\end{tabular}

Table 5.3.6. Y-12 Plant 1989 off-site waste disposal

\begin{tabular}{llr}
\hline \multicolumn{1}{c}{ Waste } & \multicolumn{1}{c}{ Method } & \multicolumn{1}{c}{$\begin{array}{c}\text { Quantity } \\
(\mathrm{kg})\end{array}$} \\
\hline Oil and solvents & $\begin{array}{c}\text { Fuels program; } \\
\text { incineration; } \\
\text { recycle/recovery }\end{array}$ & 141,000 \\
PCB liquid & $\begin{array}{l}\text { Incineration } \\
\text { PCB solids }\end{array}$ & $\begin{array}{l}\text { Incineration } \\
\text { RCRA }\end{array}$ \\
Scrap metal (clean) & Phipped off-site & 17,900 \\
\hline
\end{tabular}

Table 5.3.7. 1989 ORNL off-site waste disposal

\begin{tabular}{|c|c|c|c|}
\hline Waste & $\begin{array}{l}\text { Quantity } \\
\text { (kg) }\end{array}$ & Disposal method & Location \\
\hline Hazardous & 46,000 & Landfilling & Chem Waste, Emelle, AL \\
\hline Hazardous & 5,400 & Fuel recycle & Chem Waste, Emelle, AL \\
\hline Hazardous & 44,000 & $\begin{array}{l}\text { Incineration (ash } \\
\text { is landfilled) }\end{array}$ & Rollins, Baton Rouge, LA \\
\hline Hazardous & 22,000 & Metal reclamation & Silver Services, Gallatin, TN \\
\hline $\begin{array}{l}\text { Asbestos } \\
\text { nonradiological }\end{array}$ & 7,700 & Landfilling & $\begin{array}{l}\text { Y-12 Plant Central Sanitary Landfill, } \\
\text { Oak Ridge, TN }\end{array}$ \\
\hline $\begin{array}{l}\text { Miscellaneous } \\
\text { nonhazardous }\end{array}$ & 3,500 & Landfilling & $\begin{array}{l}\text { Y-12 Plant Central Sanitary Landfill, } \\
\text { Oak Ridge, TN }\end{array}$ \\
\hline
\end{tabular}


Table 5.3.8. ORGDP off-site disposal activities during 1989

\begin{tabular}{|c|c|c|}
\hline $\begin{array}{c}\text { Waste } \\
\text { description }\end{array}$ & $\begin{array}{l}\text { Quantity } \\
\text { (kg) }\end{array}$ & $\begin{array}{l}\text { Ultimate } \\
\text { disposal }\end{array}$ \\
\hline $\begin{array}{l}\text { Scrap metal- } \\
\text { nonradiological }\end{array}$ & 129,655 & Sold to public \\
\hline $\begin{array}{r}\text { Batteries } \\
\text { (solid) }\end{array}$ & 8,575 & $\begin{array}{l}\text { Sold to public } \\
\text { (for recycle) }\end{array}$ \\
\hline $\begin{array}{l}\text { Film } \\
\text { (solid) }\end{array}$ & 136 & $\begin{array}{l}\text { Sold to public } \\
\text { (for recovery) }\end{array}$ \\
\hline Chemicals & 227 & Sold to public \\
\hline $\begin{array}{l}\text { Office furniture, } \\
\text { tires, etc. }\end{array}$ & 3,308 & Sold to public \\
\hline $\begin{array}{l}\text { Laboratory chemicals, } \\
\text { janitorial supplies, } \\
\text { etc. }\end{array}$ & 11,762 & $\begin{array}{l}\text { Commercial disposal } \\
\text { facility }\end{array}$ \\
\hline Scrap lumber & 908 & Sold to public \\
\hline Lead & 14,240 & Sold to public \\
\hline Brass & 1,430 & Sold to public \\
\hline
\end{tabular}

Table 5.3.9. Waste placed in storage on-site at the Y-12 Plant in 1989

\begin{tabular}{lr}
\hline \multicolumn{1}{c}{ Waste } & $\begin{array}{c}\text { Quantity } \\
(\mathrm{kg})\end{array}$ \\
\hline Low level & $2,274,000^{\circ}$ \\
Mixed & 915,000 \\
PCB & 46,600 \\
PCB/uranium & 1,100 \\
RCRA & 141,000 \\
Scrap metal (contaminated) & 639,000 \\
\hline
\end{tabular}

${ }^{a}$ Includes wastes stored at ORGDP. 
Table 5.3,10. Y-12 Plant total waste in storage at the end of $1989^{a}$

\begin{tabular}{lr}
\hline \multicolumn{1}{c}{ Waste } & $\begin{array}{c}\text { Quantity } \\
(\mathrm{kg})\end{array}$ \\
\hline Low-level & $3,313,400$ \\
Mixed & $5,723,300$ \\
Hazardous & 175,000 \\
PCB & 34,000 \\
PCB/uranium & 263,000 \\
Noncontaminated oils/ & 30,000 \\
$\quad$ solvents & \\
Roofing materials & $3,109,000$ \\
Scrap metal & \\
$\quad$ Clean & \\
$\quad$ Uranium-contaminated & $4,224,500$ \\
Mercury-contaminated soil & $3,436,400$ \\
Other & $1,209,600$ \\
\hline
\end{tabular}

Totals do not include United Nuclear Corporation wastes.

${ }^{b}$ Mercury-contaminated waste materials.
Table 5.3.11. 1989 waste placed in storage at ORNL

\begin{tabular}{lrr}
\hline \multirow{2}{*}{ Waste } & \multicolumn{2}{c}{ Quantity (kg) } \\
\cline { 2 - 3 } & Long-term & Short-term \\
\hline Hazardous & & $70,000^{a}$ \\
Mixed & 2,600 & 3,700 \\
PCB & & \\
$\quad$ Nonradiological & & 33,000 \\
$\quad$ Radiological & 660 & \\
Transuranic & & \\
$\quad$ Contact handled & & \\
$\quad$ Remote handled & $30 \mathrm{~m}^{3}$ & \\
Low-level (m $)^{3}$ & $9 \mathrm{~m}^{3}$ & \\
Asbestos & $610 \mathrm{~m}^{3}$ & \\
$\quad$ Nonradiological & & \\
$\quad$ Radiological & & 7,700 \\
Scrap metal & & 1,800 \\
$\quad$ Nonradiological & & \\
$\quad$ Radiological & & $1,000,000$ \\
Miscellaneous & & 29,000 \\
$\quad$ Radiological (m & & \\
\hline
\end{tabular}

${ }^{a} \mathrm{Almost} 38,000 \mathrm{~kg}$ will be recycled.

${ }^{b}$ Low-level concentrated sludge.

PCB concentration of $\geqslant 2 \mathrm{ppm}$.

Table 5.3.12. Waste remaining in storage at ORNL at year's end for 1989

\begin{tabular}{lr}
\hline \multicolumn{1}{c}{ Waste } & $\begin{array}{c}\text { Quantity } \\
(\mathrm{kg})\end{array}$ \\
\hline Hazardous & 61,000 \\
Mixed & 111,000 \\
PCB & \\
$\quad$ Nonradiological & 27,000 \\
$\quad$ Radiological & 4,100 \\
Transuranic & \\
$\quad$ Contact handled & 110,000 \\
$\quad$ Remote handled & 470,000 \\
Low-level (m $\left.{ }^{3}\right)^{a}$ & 21 \\
Scrap metal radiological & 890 \\
\hline
\end{tabular}

'An additional $275 \mathrm{~m}^{3}$ of ORNL's low-level concentrate sludge is in storage at ORGDP. 
Table 5.3.13. 1989 ORGDP waste placed in on-site storkge

\begin{tabular}{|c|c|c|c|c|}
\hline \multirow{2}{*}{$\begin{array}{c}\text { Waste } \\
\text { description }\end{array}$} & \multirow{2}{*}{ Quantity } & \multicolumn{2}{|c|}{ Ty pe siorage ${ }^{a}$} & \multirow{2}{*}{$\begin{array}{l}\text { Ultimate } \\
\text { disposal }\end{array}$} \\
\hline & & Short-term & Long-term & \\
\hline Scrap metal (kg) & 175,650 & & $\mathrm{X}$ & Under review \\
\hline PCB liquids (L) & 946 & $\mathrm{X}$ & & TSCA incinerator \\
\hline PCB solids $(\mathrm{kg})$ & 22,797 & $X$ & & TSCA incinerator \\
\hline Cenirifuge sludge $(\mathrm{kg})$ & 19,772 & & $\mathbf{X}$ & Under review \\
\hline Plating solutions (kg) & 363 & & $\mathrm{X}$ & $\begin{array}{l}\text { Shipped uff-site } \\
\text { from storage }\end{array}$ \\
\hline Solvents (L) & 9,654 & $X$ & & TSCA incinerator \\
\hline Oils (L) & 52,801 & $\mathrm{X}$ & & TSCA incinerator \\
\hline $\begin{array}{l}\text { Sludge from } \\
\text { K-1232 treatment } \\
\text { of Y-12 Plant } \\
\text { wastewaters }(\mathrm{kg})\end{array}$ & None & & $\mathrm{X}$ & Under review \\
\hline $\begin{array}{l}\text { Laboratory waste }(\mathrm{kg}) \\
\text { BMP }\end{array}$ & 31,679 & $X$ & & $\begin{array}{l}\text { Analyses and } \\
\text { treatment } \\
\text { plan under } \\
\text { review }\end{array}$ \\
\hline $\begin{array}{l}\text { Sludge from close- } \\
\text { out of } \mathrm{K}-1407-\mathrm{B} / \mathrm{C} \\
\text { ponds }\left(\mathrm{L} \times 10^{6}\right)\end{array}$ & 7.0 & & $\mathrm{X}$ & $\begin{array}{l}\text { Delisting } \\
\text { effort under } \\
\text { way }\end{array}$ \\
\hline $\begin{array}{l}\text { Photographic } \\
\text { solutions (L) }\end{array}$ & 619 & $\mathrm{X}$ & & $\begin{array}{l}\text { ORNL for } \\
\text { silver recovery }\end{array}$ \\
\hline $\begin{array}{l}\text { Paint and } \\
\text { aerosoi contents ( } L \text { ) }\end{array}$ & 5,808 & $\mathrm{X}$ & & TSCA incinerator \\
\hline TSCA ash $(\mathrm{kg})^{b}$ & 16,892 & & $\mathrm{X}$ & Under review \\
\hline TSCA sludge (kg) & 23,380 & & $\mathrm{X}$ & Under review \\
\hline Gas cylinders $(\mathrm{kg})$ & 54 & & $\mathrm{X}$ & Under review \\
\hline Metallic mercury $(\mathrm{kg})$ & 7 & $\mathrm{X}$ & & Off-site recycle \\
\hline Solvent-contaminated rags $(\mathrm{kg})$ & 2,000 & $\mathrm{X}$ & & TSCA incinerator \\
\hline Laundry sludge $(\mathrm{kg})$ & 4,738 & $\mathrm{X}$ & & TSCA incinerator \\
\hline
\end{tabular}

${ }^{a}$ Short-term storage $=:<5$ years; long-term storage $=>5$ years.

tTSCA and RCRA test burns; includes caustic sludges from scrubbing system. 
Table 5.3.14. Was 'e placed in storage at ORGDP from other DOE facilities during 1989

\begin{tabular}{|c|c|c|c|c|}
\hline \multirow{2}{*}{$\begin{array}{c}\text { Waste } \\
\text { description }\end{array}$} & \multirow{2}{*}{ Quantity $^{a}$} & \multicolumn{2}{|c|}{ Type storage $^{b}$} & \multirow{2}{*}{$\begin{array}{l}\text { Ultimate } \\
\text { disposal }\end{array}$} \\
\hline & & Short-term & Long-term & \\
\hline Trichloroethane & 3,812 & $\mathrm{X}$ & & $\begin{array}{l}\text { Blended, TSCA } \\
\text { incinerated }\end{array}$ \\
\hline Tetrachloroethylene & 15,925 & $\mathrm{X}$ & & $\begin{array}{l}\text { Blended, TSCA } \\
\text { incinerated }\end{array}$ \\
\hline Metal sludges & $199,285 \mathrm{~kg}$ & & & Under review \\
\hline Trichloroethylene & 6,043 & $\mathbf{X}$ & & TSCA incinerated \\
\hline Waste oils/solvents & 14,754 & $\mathrm{X}$ & & TSCA incinerated \\
\hline Nonhazardous waste & $3,600 \mathrm{~kg}$ & & & Under review \\
\hline Acetonitrile & 8,600 & $\mathrm{X}$ & & $\begin{array}{l}\text { Blended, TSCA } \\
\text { incinerated }\end{array}$ \\
\hline $\begin{array}{l}\text { Hazardous waste liquid contaminated } \\
\text { with lead, mixed }\end{array}$ & 20,414 & & $\mathbf{x}$ & Under review \\
\hline Waste solvent, mixed & 3,180 & $\mathrm{X}$ & & $\begin{array}{l}\text { Blended, TSCA } \\
\text { incinerated }\end{array}$ \\
\hline $\begin{array}{l}\text { Hazardous waste radioactive } \\
\text { materials, mixed }\end{array}$ & $59,052 \mathrm{~kg}$ & & $x$ & Under review \\
\hline $\begin{array}{l}\text { Hazardous waste soidds } \\
\text { contaminated with metals }\end{array}$ & $13,183 \mathrm{~kg}$ & & $X$ & Under review \\
\hline $\begin{array}{l}\text { Hazardous waste contaminated } \\
\text { soils }\end{array}$ & $16,044 \mathrm{~kg}$ & & $x$ & Under review \\
\hline
\end{tabular}

Numbers are liters unless otherwise noted.

${ }^{b}$ Short-term storage $=<5$ years; long-term storage $=>5$ years. 
Table 5.3.15. Total waste in storage at ORGDP-end of 1989

\begin{tabular}{|c|c|c|}
\hline $\begin{array}{c}\text { Waste } \\
\text { description }\end{array}$ & Quantity $^{a}$ & $\begin{array}{l}\text { Ultimate } \\
\text { disposal }\end{array}$ \\
\hline Waste oils (low-level waste) & 12,400 & Incineration \\
\hline $\begin{array}{l}\text { K-1420 nitric } \\
\text { acid (mixed) }\end{array}$ & 17,500 & Under review \\
\hline $\begin{array}{l}\text { K-1420 electro- } \\
\text { less nickel } \\
\text { solution (mixed) }\end{array}$ & 21,163 & Under review \\
\hline $\begin{array}{l}\text { Spent solvents, } \\
\text { oils, \& PCB } \\
\text { liquids (mixed) }\end{array}$ & 649,549 & Incineration \\
\hline Paint waste & 17,241 & Incineration \\
\hline $\begin{array}{l}\text { K-1232 spent carbon } \\
\text { filter agent (mixed) }\end{array}$ & 41,600 & Under review \\
\hline $\begin{array}{l}\text { Sludges from } \\
\text { treatmient of } \\
\text { wastewaters }\end{array}$ & 161,722 & Under review \\
\hline $\begin{array}{l}\text { PCB solids and } \\
\text { liquids ( } \mathrm{PCB} \text { radiological) }\end{array}$ & 965 (drums) & Incineration \\
\hline $\begin{array}{l}\text { Decontamination solutions } \\
\text { (nixed) }\end{array}$ & 229 (drums) & Under review \\
\hline $\begin{array}{l}\text { Waste oils from } \\
\text { WMCO (mixed) }\end{array}$ & 814 (drums) & Incineration \\
\hline Y-12 metal sludges & $280,717(\mathrm{~kg})$ & Under review \\
\hline Waste treatment & 114,000 & Treatment \\
\hline $\begin{array}{l}\text { Portsmouth PCB } \\
\text { contaminated soil }\end{array}$ & $909,000(\mathrm{~kg})$ & Under review \\
\hline Incineration ash/sludge & $137,313(\mathrm{~kg})$ & Under review \\
\hline $\mathrm{K}-1407 \mathrm{~B} / \mathrm{C}$ pond sludge (mixed) & 31,958 (drums) & $\begin{array}{l}\text { To be fixed in } \\
\text { concrete }\end{array}$ \\
\hline $\mathrm{K}-1407 \mathrm{~B} / \mathrm{C}$ pond sludge (mixed) & 45,856 (drums) & Stabilized \\
\hline $\begin{array}{l}\text { RMil lead contaminated liquid } \\
\text { (mixed) }\end{array}$ & $18,585(\mathrm{~kg})$ & Under review \\
\hline $\begin{array}{l}\text { WMCO hazardous waste liquid } \\
\text { (mixed) }\end{array}$ & $25,263(\mathrm{~kg})$ & Incineration \\
\hline $\begin{array}{l}\text { WMCO hazardous waste solid } \\
\text { (mixed) }\end{array}$ & $9,728(\mathrm{~kg})$ & Under review \\
\hline
\end{tabular}

${ }^{a}$ Units are liters except where noted otherwise. 
6. SPECIAL STUDIES 
All data for this section are presented in Vol. 1. 
7. QUALITY ASSURANCE 
Table 7.1.1. Example of inorganic QC results for sampling GW-514-Y-12 Plant, 1989

\begin{tabular}{|c|c|c|c|}
\hline Parameter & GW-514 & $\begin{array}{l}\text { Field } \\
\text { replicate }\end{array}$ & $\begin{array}{l}\text { Field } \\
\text { blank }\end{array}$ \\
\hline \multicolumn{4}{|l|}{ Concentration $(\mathrm{mg} / \mathrm{L})$} \\
\hline As & $<0.005$ & $<0.005$ & $\mathrm{NA}^{a}$ \\
\hline $\mathrm{Ba}$ & 0.008 & 0.008 & NA \\
\hline $\mathrm{Cd}$ & $<0.002$ & $<0.002$ & NA \\
\hline $\mathrm{Cr}$ & $<0.01$ & $<0.01$ & NA \\
\hline $\mathrm{Pb}$ & $<0.004$ & $<0.004$ & NA \\
\hline $\mathrm{Se}$ & $<0.005$ & $<0.005$ & NA \\
\hline $\mathrm{Ag}$ & $<0.01$ & 0.01 & NA \\
\hline $\mathrm{Hg}$ & $<0.0002$ & $<0.0002$ & NA \\
\hline Total organic carbon & $<1.0$ & $<1.0$ & NA \\
\hline $\mathrm{pH}$ (units) & 7.1 & 7.1 & NA \\
\hline \multicolumn{4}{|l|}{ Activity (pCi/L) } \\
\hline Alpha & -1.2 & 3.0 & NA \\
\hline Beta & 3.6 & 7.0 & NA \\
\hline Suspended solids $(\mathrm{mg} / \mathrm{L})$ & 2.0 & 1.0 & NA \\
\hline $\mathrm{U}(\mathrm{mg} / \mathrm{L})$ & $<0.001$ & $<0.001$ & NA \\
\hline Conductivity $(\mu \mathrm{mho} / \mathrm{cm})$ & 345.0 & 342.0 & NA \\
\hline \multicolumn{4}{|l|}{ Alkalinity $(\mathrm{mg} / \mathrm{L})$} \\
\hline $\mathrm{CO}_{3}$ & $<1.0$ & $<1.0$ & NA \\
\hline $\mathrm{HCO}_{3}$ & 176.0 & 176.0 & NA \\
\hline
\end{tabular}

${ }^{a}$ Not applicable. 
Table 7.1.2 Example of organic QC data at GW-514-Y-12 Plant, 1989

\begin{tabular}{llll}
\multicolumn{1}{c}{ Parameter } & GW-514 & $\begin{array}{c}\text { Field } \\
\text { duplicate }\end{array}$ & $\begin{array}{c}\text { Field } \\
\text { blank }\end{array}$ \\
\hline \multicolumn{1}{c}{ Volatile organic compounds } \\
\hline
\end{tabular}

$\mathrm{U}=$ compound analyzed for but not detected.

$\mathrm{J}=$ indicates an estimated value. 
Oak Ridge Environmental Report, Vol. 2-1989

Table 7.2.1. Energy Systems environmental analysis procedures for water

\begin{tabular}{|c|c|c|c|}
\hline Parameter & $\begin{array}{l}\text { Energy } \\
\text { Systems } \\
\text { procedure }\end{array}$ & EPA method & $\begin{array}{l}\text { Lowest } \\
\text { concentration } \\
\text { reported }^{a}\end{array}$ \\
\hline Alkalinity, $\mathrm{CaCO}_{3}(\mathrm{mg} / \mathrm{L})$ & EC-1005 & 310.1 & 5 \\
\hline Gross alpha activity $(\mathrm{pCl} / \mathrm{L})$ & EC-1010 & & 1.0 \\
\hline Gross beta activity (pCi/L) & EC- 1010 & & 4.0 \\
\hline \multicolumn{4}{|l|}{${ }^{24 !} \mathrm{Am}$ and ${ }^{244} \mathrm{Cm}(\mathrm{pCi} / \mathrm{L})$} \\
\hline${ }^{241} \mathrm{Am}$ & EC- 1020 & & $\begin{array}{l}3.0 \\
(\mathrm{ORNL}) 0.05\end{array}$ \\
\hline${ }^{244} \mathrm{Cm}$ & EC- 1020 & & $\begin{array}{l}3.0 \\
\text { (ORNL) } 0.05\end{array}$ \\
\hline \multicolumn{4}{|l|}{ As and Se, gaseous hydride-AA (mg/L) } \\
\hline As & EC-1040 & 206.3 & 0.002 \\
\hline $\mathrm{Se}$ & EC- 1040 & 270.3 & 0.002 \\
\hline Asbestos (fibers/L) & $\mathrm{EC} \cdot 1050$ & & $0.3 \times 10^{6}$ \\
\hline Biochemical oxygen demand, 5-d (mg/L) & EC-1060 & 405.1 & 5 \\
\hline Bromide, spectrophotometric $(\mathrm{mg} / \mathrm{L})$ & EC-1070 & ASTM D $1216-77$ & 0.1 \\
\hline $\begin{array}{l}\text { Chemical oxygen demand (low level) } \\
\text { titration method }(\mathrm{mg} / \mathrm{L})\end{array}$ & EC-1090 & 410.2 & 5 \\
\hline Chloride, titration, $\mathrm{HgNO}_{3}(\mathrm{mg} / \mathrm{L})$ & EC- 1120 & 325.3 & 2 \\
\hline \multicolumn{4}{|l|}{ Anions, ion chromatograph ${ }^{b}(\mathrm{mg} / \mathrm{L})$} \\
\hline Chloride & EC-1130 & 300.0 & 2 \\
\hline Nitrate $(\mathrm{N})$ & EC-1130 & 300.0 & 1 \\
\hline Sulfate & EC. 1130 & 300.0 & 5 \\
\hline Phosphate (P) & EC-1130 & 300.0 & 2 \\
\hline $\mathrm{TRCl}_{2}$, amperometric $(\mathrm{mg} / \mathrm{L})$ & EC- 1150 & 330.1 & $\begin{array}{l}0.05 \\
(\text { PGDP) } 0.01\end{array}$ \\
\hline $\mathrm{Cr}(\mathrm{VI})$, spectrophotometric $(\mathrm{mg} / \mathrm{L})$ & EC-1180 & USGS $^{c}$ & 0.01 \\
\hline Coliform bacteria, fecal (colonies/100 mL) & $\mathrm{EC}-1190$ & $909 \mathrm{C} \mathrm{Std} \mathrm{Mth}$ & 1 \\
\hline Coliform bacteria, total (colonies/100 mL) & EC-1200 & 909A Std Mth & 1 \\
\hline Color (color unit) & EC-1220 & 110.2 & 1 \\
\hline Conductance, specific $(\mu \mathrm{mho} / \mathrm{cm})$ & EC- 1240 & 120.1 & 0.5 \\
\hline Cyanide, total $(5-\mathrm{cm}$ cell $)$ & EC-1270 & 335.2 & 0.004 \\
\hline Dissolved oxygen, membrane electrode method $(\mathrm{mg} / \mathrm{L})$ & $\mathrm{EC}-1300$ & 360.1 & 0.1 \\
\hline Fluoride $(\mathrm{mg} / \mathrm{L})$ & EC-1330 & 340.2 & 0.1 \\
\hline Gamma-ray emitters (pCi/L) & EC-1340 & & 2.5 \\
\hline \multicolumn{4}{|l|}{$\begin{array}{l}\text { Herbicides (chloriilated phenoxy acid), } \\
\text { GC method }(\mu \mathrm{g} / \mathrm{L})\end{array}$} \\
\hline $2,4-\mathrm{D}$ & EC-1370 & 509B Std Mth & 0.1 \\
\hline Silvex & EC. 1370 & 509B Std Mth & 0.02 \\
\hline${ }^{131} \mathrm{I}(\mathrm{pCi} / \mathrm{L})$ & EC-1380 & & 4.3 \\
\hline $\mathrm{Hg}$, total $(\mathrm{mg} / \mathrm{L})$ & EC-1390 & 245.1 & 0.0002 \\
\hline Methylene-blue-a tive substances (mg/L) & $\mathrm{EC}-1450$ & 425.1 & 0.05 \\
\hline${ }^{237} \mathrm{~Np}(\mathrm{pCi} / \mathrm{L})$ & EC- 1460 & & $\begin{array}{l}1.0 \\
(\mathrm{ORNL}) 4 \times 10^{-2}\end{array}$ \\
\hline \multicolumn{4}{|l|}{$N(\mathrm{mg} / \mathrm{L})$} \\
\hline Ammonia, spectrophotometric & EC- 1470 & 350.2 & 0.2 \\
\hline Ammonia, SIE & EC- 1480 & 350.3 & 0.2 \\
\hline Kjeldahl (total), spectrophotometric & $\mathrm{EC}-1500$ & 351.3 & 0.2 \\
\hline Kjeldahl (total), volumetric & EC- 1510 & 351.3 & 0.2 \\
\hline Kjeldahl (total), SYE & $\mathrm{EC} \cdot 1520$ & 351.4 & 0.2 \\
\hline Nitrate, brucine method & EC- 1530 & 352.1 & 0.1 \\
\hline Nitrate-nitrite, Cd-Redn. & EC-1540 & 353.3 & 0.1 \\
\hline $\mathrm{N}$-nitrosomorpholine, spectrophotometric $(\mathrm{mg} / \mathrm{L}$ ) & EC-1550 & & 1.0 \\
\hline$O \& r_{;}$gravimetric $(\mathrm{mg} / \mathrm{L})$ & EC-1560 & 413.1 & 5 \\
\hline$O \& G$, infrared $(\mathrm{mg} / \mathrm{L})$ & EC-1570 & 413.2 & 2.0 \\
\hline Pentachlorophenol, HPLC $(\mu \mathrm{g} / \mathrm{L})$ & EC-1583 & & 50 \\
\hline
\end{tabular}


Table 7.2.1 (continued)

\begin{tabular}{|c|c|c|c|}
\hline Parameter & $\begin{array}{c}\text { Energy } \\
\text { Systems } \\
\text { procedure }\end{array}$ & EPA method & $\begin{array}{l}\text { Lowest } \\
\text { concentration } \\
\text { reported }\end{array}$ \\
\hline \multicolumn{4}{|c|}{ Pesticides (organochlorine), GC method ( $\mu \mathrm{g} / \mathrm{L}$ ) } \\
\hline Lindane & EC. 1586 & $608^{e}$ & 0.01 \\
\hline Endrin & EC-1586 & 608 & 0.05 \\
\hline Toxaphene & EC- 1586 & 608 & 1.0 \\
\hline Methoxychlor & EC. 1586 & 509A Std Mth & 0.2 \\
\hline \multicolumn{4}{|l|}{ Phenols $(\mu \mathrm{g} / \mathrm{L})$} \\
\hline Without conc. & $\mathrm{EC}-1590$ & 420.1 & 50 \\
\hline With conc. & EC- 1590 & 420.1 & 5 \\
\hline $\mathrm{pH}$, electrometric (units) & EC- 1600 & 150.1 & Nearest 0.1 \\
\hline $\mathrm{P}$ (all forms), spectrophotometric $(\mathrm{mg} / \mathrm{L})$ & EC- 1610 & 365.2 & 0.1 \\
\hline $\mathrm{Pu}$ isotopes $(\mathrm{pCi} / \mathrm{L})$ & EC-1615 & & $\begin{array}{l}1.0 \\
(\text { ORNL }) 5 \times 10^{-2}\end{array}$ \\
\hline PCBs, each $(\mu \mathrm{g} / \mathrm{L})$ & EC- 1620 & 608 & 0.5 \\
\hline $\begin{array}{l}\text { Priority pollutants, organic } \\
\text { (base/neutral/acid), each }(\mu \mathrm{g} / \mathrm{L})\end{array}$ & EC-1701 & 625 & Mostly $10.50^{f}$ \\
\hline $\begin{array}{l}\text { Priority pollutants, organic } \\
\text { (volatile, purgeable), each }(\mu \mathrm{g} / \mathrm{L})\end{array}$ & EC-1704 & 624 & Mostly $1030^{8}$ \\
\hline \multicolumn{4}{|l|}{ Solids } \\
\hline Dissolved $(\mathrm{mg} / \mathrm{L})$ & EC- 1760 & 160.1 & 10 \\
\hline Settleable $[\mathrm{mL} /(\mathrm{L} \cdot \mathrm{h})]$ & EC- 1770 & 160.5 & 1.0 \\
\hline Total $(\mathrm{mg} / \mathrm{L})$ & EC- 1790 & 160.3 & 10 \\
\hline Undissolved $(\mathrm{mg} / \mathrm{L})$ & EC- 1800 & 160.2 & 4 \\
\hline Volatile $(\mathrm{mg} / \mathrm{L})$ & EC- 1820 & 160.4 & 5 \\
\hline${ }^{90} \mathrm{Sr}(\mathrm{pCi} / \mathrm{L})$ & EC- 1840 & & $\begin{array}{l}4.0 \\
\text { (ORNL) } 2.0\end{array}$ \\
\hline Sulfate, turbidimetric method $(\mathrm{mg} / \mathrm{L})$ & EC- 1850 & 375.4 & 5 \\
\hline${ }^{99} \mathrm{Tc}(\mathrm{pCi} / \mathrm{L})$ & EC- 1860 & & 300 \\
\hline Th isotopes $(\mathrm{pCi} / \mathrm{L})$ & EC- 1870 & & $\begin{array}{l}0.4 \\
\left(\text { ORNL) } 5 \times 10^{-2}\right.\end{array}$ \\
\hline Th, spectrophotometric $(\mathrm{mg} / \mathrm{L})$ & EC-1871 & & $2 \times 10^{-3}$ \\
\hline $\begin{array}{l}\text { Total organic carbon, combustion or } \\
\text { oxidation }(\mathrm{mg} / \mathrm{L})\end{array}$ & EC-1873 & 415.1 & 1 \\
\hline Tritium $(\mathrm{pCi} / \mathrm{L})$ & EC-1879 & & $\begin{array}{l}5000 \\
(\text { ORNL) } 1500\end{array}$ \\
\hline Turbidity (NTU) & EC- 1880 & 180.1 & 0.05 \\
\hline $\mathrm{U}$ (total), fluorometric $(\mathrm{mg} / \mathrm{L})$ & $\mathrm{EC}-1910$ & & $1 \times 10^{-3}$ \\
\hline $\mathrm{U}$ isotopes $(\mathrm{pCi} / \mathrm{L})$ & EC-1920 & & $\frac{1}{(\text { ORNL }) 5 \times 10^{-2}}$ \\
\hline$U$ isotopic abundances (wt $\%$ ) & EC. 1960 & & 0.001 \\
\hline
\end{tabular}

${ }^{a}$ The lowest concentration reported (LCR) may vary among specific samples, depending on interferences in the sample matrix. However, these LCRs have been assigned to accommodate most minor interferences, $S$, me of the Energy Systems LCRs are higher than the method detection limits (MDLs) listed by EPA. This is consistent with guidance from EPA. However, any data reported below the MDLs must be supported by sound documentation. The LCRs applied by the Energy Systems laboratories meet the needs of the programs they support.

${ }^{b}$ Approved for drinking water only (reagent water).

'Methods for Analysis of Inorganic Substances in Water and Fluvial Sediment, U.S. Department of the Interior, U.S. Geological Survey, Open-File Report 78-679; or "Methods for Determination of Inorganic Substances in Water and Fluvial Sediments, ${ }^{n}$ N. W. Skougstad et al., Techniques of Water-Resources Investigation, Book 5 , Chapter A1, U.S. Geological Survey, 1979.

${ }^{d}$ All references to Standard Methods for the 15th Edition, 1980.

'Federal Register 49 (209), 43, 261, October 26, 1984.

fFor 81 compouinds.

${ }^{8}$ For 31 compounds. 
Table 7.2.2. Energy Systems atomic absorption and ICP environmental analysis procedures for waters

\begin{tabular}{|c|c|c|c|}
\hline \multirow[b]{2}{*}{ Element } & \multicolumn{3}{|c|}{ Lowest concentration reported ${ }^{a}\left(\mathrm{mg}_{\mathrm{g}} / \mathrm{L}\right)$} \\
\hline & $\begin{array}{l}\text { MMES EC- } 1400 \\
\text { EPA } 200 \text { series } \\
\text { flame AA }\end{array}$ & $\begin{array}{c}\text { MMES EC- } 1400 \\
\text { EPA } 200 \text { series } \\
\text { graphite furnace AA }\end{array}$ & $\begin{array}{c}\text { MMES EC- } 1410 \\
\text { EPA } 200.7 \\
\text { ICP }\end{array}$ \\
\hline $\mathrm{Ag}$ & 0.05 & 0.01 & 0.03 \\
\hline $\mathrm{Al}$ & 0.3 & 0.01 & 0.01 \\
\hline As & $b$ & 0.005 & 0.1 \\
\hline $\mathrm{Ba}$ & 0.2 & 0.01 & 0.005 \\
\hline $\mathrm{Ca}$ & 0.05 & $b$ & 0.01 \\
\hline $\mathrm{Cd}$ & 0.02 & 0.002 & 0.01 \\
\hline $\mathrm{Cr}$ & 0.2 & 0.01 & 0.05 \\
\hline $\mathrm{Cu}$ & 0.05 & 0.004 & 0.01 \\
\hline $\mathrm{Fe}$ & 0.05 & $b$ & 0.01 \\
\hline $\mathrm{K}$ & 0.2 & $b$ & 2.0 \\
\hline \multirow[t]{2}{*}{$\mathrm{Li}$} & 0.01 & $b$ & 0.02 \\
\hline & & & $(\mathrm{ORNL}) 0.2$ \\
\hline $\mathrm{Mg}$ & 0.02 & $b$ & 0.002 \\
\hline $\mathrm{Mn}$ & 0.03 & 0.01 & 0.005 \\
\hline Mo & 0.2 & 0.01 & 0.05 \\
\hline \multirow[t]{2}{*}{$\mathrm{Na}$} & 0.05 & $b$ & 0.04 \\
\hline & & & (ORNL) 0.5 \\
\hline $\mathrm{Ni}$ & 0.1 & 0.01 & 0.05 \\
\hline $\mathrm{Pb}$ & 0.2 & 0.004 & 0.2 \\
\hline $\mathrm{Se}$ & $b$ & 0.005 & 0.2 \\
\hline $\mathrm{Zn}$ & 0.02 & $b$ & 0.005 \\
\hline
\end{tabular}

${ }^{a}$ The lowest concentration reported (LCR) may vary among specific samples, depending on interferences in the sample matrix. However, these LCRs have been assigned to accommodate most minor interferences. Some of the Energy Systems LCRs are higher than the method detection limits (MDLs) listed by EPA. This is wnsistent with guidance from EPA. However, any data reported below the MDLs must be supported by sound documentation. The LCRs applied by Energy Systems laboratories meet the needs of the programs they support.

${ }^{b}$ Element not normally determin?d using this technique. 
Table 7.2.3. Energy Systems environmental analysis procedures for air

\begin{tabular}{|c|c|c|c|}
\hline Parameter & $\begin{array}{l}\text { Energy } \\
\text { Systems } \\
\text { procedure }\end{array}$ & $\begin{array}{l}\mathrm{NIOSH}^{a} \\
\text { or EPA } \\
\text { method }\end{array}$ & $\begin{array}{l}\text { Lowest } \\
\text { concentration } \\
\text { reported }\end{array}$ \\
\hline $\begin{array}{l}\text { Gross alpha, beta, air filters, } \\
\text { radiochemistry }\left(\mathrm{pCi} / \mathrm{m}^{3}\right)\end{array}$ & EC-2100 & APHA $601,602^{c}$ & \\
\hline Alpha & & & 0.005 \\
\hline Beta & & & 0.025 \\
\hline Dustfall, gravimetric & $\mathrm{EC}-2270$ & & $d$ \\
\hline Fluoride, air, S:E ( $\mu \mathrm{g} /$ sample) & $\mathrm{EC}-2360$ & & 5 \\
\hline Fluoride, stacks, SIE $\left(\mu \mathrm{g} / \mathrm{m}^{3}\right)$ & $\mathrm{EC}-2370$ & & 30 \\
\hline Gamma-ray spec., air filters & $\mathrm{EC}-2400$ & & $d$ \\
\hline $\begin{array}{l}{ }^{131} \text { I, gamma-ray spec, air filter } \\
\text { (pCi/filter) }\end{array}$ & $\mathrm{E} C-2420$ & & 2.5 \\
\hline $\begin{array}{l}\text { Metals in air particulates, } \\
\text { emission spec. }(\mu \mathrm{g} / \text { sample })\end{array}$ & EC-2440 & & $\begin{array}{l}\text { For } 48 \text { metals, } \\
\text { mostly } 1-10\end{array}$ \\
\hline \multicolumn{4}{|l|}{$\begin{array}{l}\text { Air filters, radiochemistry } \\
\text { (pCi/filter) }\end{array}$} \\
\hline $\mathrm{Pu}$ & $\mathrm{EC}-2500$ & EPA-680/4-75-001 & 0.04 \\
\hline${ }^{90} \mathrm{Sr}$ & EC-2580 & & 2 \\
\hline${ }^{99} \mathrm{Tc}$ & $\mathrm{EC}-2600$ & & 300 \\
\hline $\begin{array}{l}\text { Th alpha isotopes, radiochemistry } \\
(\mathrm{pCi} / \text { filter) }\end{array}$ & EC-2640 & & 0.04 \\
\hline $\begin{array}{l}\mathrm{U}, \text { air filters, fluorometric } \\
(\mu \mathrm{g} / \text { sample })\end{array}$ & $\mathrm{EC}-2850$ & & 0.05 \\
\hline $\begin{array}{l}\mathrm{U} \text { isotopes, air filters, } \\
\text { radiochemistry ( } \mathrm{pCi} / \text { filter })\end{array}$ & EC-2870 & EPA-680/4-75-001 & 0.04 \\
\hline $\begin{array}{l}\text { U, stack gases, spec. /fluoro. } \\
\left(\mu \mathrm{g} / \mathrm{m}^{3}\right)\end{array}$ & EC-2890 & & 1.7 \\
\hline $\begin{array}{l}\text { Dichlorotetrafluoroethane, GC method } \\
\left(\mathrm{mg} / \mathrm{m}^{3}\right)\end{array}$ & IHA-230 & NIOSH S108 & 3500 \\
\hline $\begin{array}{l}\text { Dioctyl phthalate, air, GC method } \\
\left(\mathrm{mg} / \mathrm{m}^{3}\right)\end{array}$ & IHA-235 & NIOSH S40 & 2 \\
\hline Formaldehyde, air $\left(\mathrm{mg} / \mathrm{m}^{3}\right)$ & IHA-237 & NIOSH 125 & 0.1 \\
\hline Isopropanol, air $\left(\mathrm{mg} / \mathrm{m}^{3}\right)$ & IHA-240 & NIOSH S64 & 180 \\
\hline Oil mist, air, infrared $\left(\mathrm{mg} / \mathrm{m}^{3}\right)$ & IHA-247 & & 0.5 \\
\hline $\begin{array}{l}\text { Organic solvents, air, GC method } \\
\text { (mg/sample) }\end{array}$ & IHA-250 & NIOSH 127 & $\begin{array}{l}18 \mathrm{cprls} \\
0.01 \text { to } 1.0\end{array}$ \\
\hline Pentachlorophenol, air, HPLC $\left(\mathrm{mg} / \mathrm{m}^{3}\right)$ & IHA-260 & NIOSH S297 & 0.27 \\
\hline PCBs, air, GC $\left(\mu \mathrm{g} / \mathrm{m}^{3}\right)$ & IHA-270 & NIOSH 244 & 10 \\
\hline Quinoline, air & IHA-273 & & $d$ \\
\hline Toluene disocyanate, air $\left(\mu \mathrm{g} / \mathrm{m}^{3}\right)$ & IHA-239 & NIOSH 141 & 7 \\
\hline $\begin{array}{l}\text { Tributyl phos. air, GC method } \\
\left(\mathrm{mg} / \mathrm{m}^{3}\right)\end{array}$ & IHA-285 & NIOSH S208 & 2.7 \\
\hline $\begin{array}{l}\text { Vinyl chloride, air, GC method } \\
\left(\mu \mathrm{g} / \mathrm{m}^{3}\right)\end{array}$ & IHA-294 & NIOSH 178 & 8 \\
\hline
\end{tabular}

${ }^{a}$ NIOSH Manual of Analytical Mithods, 2nd ed., U.S. Dept. of Health, Education, and Welfare, 1977.

The lowest concentration reported (LCR) may vary among specific samples, depending on interferences in the sample matrix. However, these LCRs have been assigned to accommodate most minor interferences. Some of the Energy Systems LCRs are higher than the method detection limit (MDLs) listed by EPA. This is consistent with guidance from EPA. However, any data reported below the MDLs must be supported by sound documentation. The LCRs applied by Energy Systems laboratories meet the needs of the programs they support.

'APHA Methods, Americar public Health Assoc., !97?.

${ }^{d}$ Procedure in preparation. 
Table 7.2.4. Energy Systems environmental analysis procedures for soll and sediment

\begin{tabular}{|c|c|c|c|}
\hline Parameter & $\begin{array}{l}\text { Energy } \\
\text { Systems } \\
\text { procedure }\end{array}$ & $\begin{array}{l}\text { EPA } \\
\text { method }\end{array}$ & $\begin{array}{l}\text { Lowest } \\
\text { concentration } \\
\text { reported }^{a}\end{array}$ \\
\hline Fluoride & $E C-3050$ & & $b$ \\
\hline Gamma-ray spectrum analysis & EC-3070 & & $b$ \\
\hline $\begin{array}{l}\mathrm{Hg} \text { (total), flameless atomic } \\
\text { absorption }(\mathrm{mg} / \mathrm{kg})\end{array}$ & $\mathrm{EC}-3100$ & 245.5 & 0.2 \\
\hline Metals, atomic absorption & EC-3200 & 200 Series & $c$ \\
\hline $\begin{array}{l}\text { Metals, inductively coupled } \\
\text { plasma-optical emission } \\
\text { spectrometric (ICP-OES) }\end{array}$ & $E C-3250$ & 200.7 & $c$ \\
\hline Np, direct gamma spectrum & $\mathrm{EC}-3300$ & & $b$ \\
\hline${ }^{237} \mathrm{~Np}$, radiochemical ( $\mathrm{pCi} / \mathrm{kg}$ ) & EC-3305 & & 20 \\
\hline $\mathrm{Pu}$, radiochemical $(\mathrm{pCi} / \mathrm{kg})$ & $\mathrm{EC}-3360$ & & 20 \\
\hline $\begin{array}{l}\text { PCBs, gas chromatographic } \\
(\mathrm{mg} / \mathrm{kg})\end{array}$ & $\mathrm{EC}-3400$ & & 0.1 \\
\hline${ }^{90} \mathrm{Sr}$, radiochemical ( $\mathrm{pCi} / \mathrm{kg}$ ) & $\mathrm{EC}-3500$ & $704 \mathrm{Std} \mathrm{Mth}^{d}$ & 200 \\
\hline${ }^{99} \mathrm{Tc}$, radiochemical $(\mathrm{pCi} / \mathrm{kg})$ & $\mathrm{EC}-3550$ & & $2 \times 10^{4}$ \\
\hline Th, spectrophotometric $(\mathrm{mg} / \mathrm{kg})$ & $E C-3600$ & & 3 \\
\hline $\begin{array}{l}\text { Th (alpha-emitting) isotopes, } \\
\text { radiochemical }(\mathrm{pCi} / \mathrm{kg})\end{array}$ & $E^{-}-3650$ & & 4 \\
\hline $\mathrm{U}($ total $)$, fluorometric $(\mathrm{mg} / \mathrm{kg})$ & $\mathrm{EC}-3700$ & & 0.5 \\
\hline $\begin{array}{l}\mathrm{U} \text { (total and isotopic), } \\
\text { isotope dilution mass } \\
\text { spectrometric }(\mathrm{ng})\end{array}$ & $\mathrm{EC}-3740$ & & 10 \\
\hline $\begin{array}{l}U \text { isotopes, radiochemical } \\
(\mathrm{pCi} / \mathrm{kg})\end{array}$ & $\mathrm{EC}-3780$ & & 4 \\
\hline
\end{tabular}

"The lowest concentration reported (LCR) may vary among specific samples, depending on interferences in the sample matrix. However, these LCRs have been assigned to accommodate most minor interferences. Some of the Energy Systems LCRs are higher than the method detection limits (MDLs) listed by EPA. This is consistent with guidance from EPA. However, any data reported below the MDLs must be supported by sound documentaion. The LCRs applied by Energy Systems laboratories meet the needs of the programs they support.

${ }^{b}$ Procedure in preparation.

'See Table 7.2.2.

${ }^{d}$ References to Standard Methods are from the 14th Edition, 1975. 
Table 7.2.5. Energy Systems environmental analysis procedures for biota

\begin{tabular}{|c|c|c|c|}
\hline Parameter & $\begin{array}{l}\text { Energy } \\
\text { Systems } \\
\text { procedure }\end{array}$ & EP.A method & $\begin{array}{l}\text { Lowest } \\
\text { concentration } \\
\text { rep ed } \mathrm{d}^{\mathfrak{d}}\end{array}$ \\
\hline Fluoride in vegetation $(\mathrm{mg} / \mathrm{kg}$ ) & EC. -4100 & & 3 \\
\hline $\begin{array}{l}\text { Gamma-ray spectrometry } \\
\text { of deer muscle }\end{array}$ & EC -4130 & & $b$ \\
\hline Gamma-ray spectrometry of fish & EC-4150 & & $b$ \\
\hline $\begin{array}{l}\text { Gamma-ray spectrometry of } \\
\text { vegetation }\end{array}$ & EC-4170 & & $b$ \\
\hline${ }^{131} \mathrm{I}$ and ${ }^{90} \mathrm{Sr}$ in raw milk $(\mathrm{pCi} / \mathrm{L})$ & $\mathrm{EC}-4180$ & & \\
\hline${ }^{131} \mathrm{I}$ & & & 1 \\
\hline${ }^{90} \mathrm{Sr}$ & & & 1 \\
\hline $\begin{array}{l}\text { Metals in fish, atomic absorption, } \\
\text { furnace } A A(\mathrm{mg} / \mathrm{kg})\end{array}$ & $\mathrm{EC}-4250$ & $600 / 4-81-055^{c}$ & \\
\hline $\mathrm{Cd}$ & & & 0.01 \\
\hline $\mathrm{Cr}$ & & & 0.05 \\
\hline $\mathrm{Cu}$ & & & 0.10 \\
\hline $\mathrm{Ni}$ & & & 0.50 \\
\hline $\mathrm{Pb}$ & & & 0.05 \\
\hline $\begin{array}{l}\text { Metals in vegetation, atomic } \\
\text { absorption, flame } A A(\mathrm{mg} / \mathrm{kg})\end{array}$ & EC. 4300 & & \\
\hline $\mathrm{Cd}$ & & & 0.5 \\
\hline $\mathrm{Cr}$ & & & 3.0 \\
\hline $\mathrm{Cu}$ & & : & 2.0 \\
\hline $\mathrm{Ni}$ & & & 3.5 \\
\hline $\mathrm{Zn}$ & & & 0.5 \\
\hline $\mathrm{Pu}$ isotopes in fish $(\mathrm{pCi} / \mathrm{kg})$ & EC- -4360 & & $\begin{array}{l}20 \\
(\mathrm{ORNL}) 4\end{array}$ \\
\hline $\begin{array}{l}\mathrm{Pu} \text { isotopes in vegetation } \\
(\mathrm{pCi} / \mathrm{kg})\end{array}$ & EC-4380 & & $\begin{array}{l}40 \\
(\text { ORNL) } 4\end{array}$ \\
\hline PCBs in fish and animal tissue & $\mathrm{EC}-4400$ & $600 / 4-81-055^{c}$ & 0.1 \\
\hline${ }^{90} \mathrm{Sr}$ in tïsh $(\mathrm{pCi} / \mathrm{kg})$ & EC -4600 & & $\begin{array}{l}1000 \\
(\text { ORNL) } 200\end{array}$ \\
\hline${ }^{90} \mathrm{Sr}$ in vegetation $(\mathrm{pCi} / \mathrm{kg})$ & $\mathrm{EC}-4620$ & & $\begin{array}{l}100^{n} \\
\text { (ORNL) } 200\end{array}$ \\
\hline${ }^{99} \mathrm{Tc}$ in fish & EC. 4630 & & $b$ \\
\hline${ }^{99} \mathrm{Tc}$ in vegetation & EC-4635 & & $b$ \\
\hline Th isotopes in vegetation $(\mathrm{pCi} / \mathrm{kg})$ & EC-4640 & & $\begin{array}{l}40 \\
(\mathrm{ORNL}) 4\end{array}$ \\
\hline$U($ total) in vegetation $(\mathrm{mg} / \mathrm{kg})$ & $\mathrm{EC}-4700$ & & 0.5 \\
\hline $\begin{array}{l}U \text { (total and isotopic) in } \\
\text { vegetation (ng/sample) }\end{array}$ & EC. 4720 & & 10 \\
\hline$U$ isotopes in animal tissue & EC -4800 & & $b$ \\
\hline $\mathrm{U}$ isotopes in vegetation $(\mathrm{pCi} / \mathrm{kg})$ & EC -4840 & & $\begin{array}{l}40 \\
\text { (ORNL) } 4\end{array}$ \\
\hline
\end{tabular}

${ }^{a}$ The lowest concentration reported (LCR) may vary among specific samples, depending on interferences in the sample matrix. However, these LCRs have been assigned to accommodate most minor interferences. Some of the Energy Syutems LCRs are higher than the method detection limit (MDLs) listed by EPA. This is consistent with guidance from EPA. However, any data reported below the MDLs must be supported by sound documentation. The LCRs applied by Energy Systems laboratories meet the needs of the programs thoy support.

${ }^{b}$ Procedure in preparation.

'Interim Methods for the Sampling and Analysis of $I$ iority Pollutants in Sediments and Fish Tissue, EPA, October 1980. 
Table 7.2.6. EPA EMSL-LV Intercomparison Radionuclide Control Program-Y-12, 1989

\begin{tabular}{|c|c|c|c|c|}
\hline \multirow{2}{*}{$\begin{array}{l}\text { Analysis and } \\
\text { sample date }\end{array}$} & \multicolumn{2}{|c|}{ Values } & \multirow{2}{*}{$\begin{array}{c}\text { Normalized } \\
\text { deviation }\end{array}$} & \multirow{2}{*}{$\begin{array}{c}\text { Performance } \\
\text { evaluation }\end{array}$} \\
\hline & EPA & $Y-12$ & & \\
\hline \multicolumn{5}{|c|}{ Water $(p C i / L)$} \\
\hline \multicolumn{5}{|l|}{${ }^{239} \mathrm{Pu}$} \\
\hline $1 / 89$ & $4.20 \pm 0.40$ & 4.33 & 0.58 & Acceptable \\
\hline $8 / 89$ & $2.80 \pm 0.30$ & 20.7 & 103 & Unacceptable \\
\hline \multicolumn{5}{|l|}{ Gross alpha } \\
\hline $1 / 89$ & $8.00 \pm 5$ & 15.6 & 2.66 & Acceptable \\
\hline $4 / 89$ & $29.00 \pm 7$ & 30.3 & 0.33 & Acceptable \\
\hline $5 / 89$ & $30.00 \pm 8$ & 33.7 & 0.79 & Acceptable \\
\hline $10 / 89$ & $49.00 \pm 12$ & 49.3 & 0.05 & Acceptable \\
\hline \multicolumn{5}{|l|}{ Gross beta } \\
\hline $1 / 89$ & $4.00 \pm 5$ & 8.00 & 1.39 & Acceptable \\
\hline $4 / 89$ & $57.00 \pm 5$ & 55.3 & 0.58 & Acceptable \\
\hline $5 / 89$ & $50.00 \pm 5$ & 46.0 & 1.39 & Acceptable \\
\hline $10 / 89$ & $32.00 \pm 5$ & 29.7 & 0.81 & Acceptable \\
\hline \multicolumn{5}{|l|}{$U$} \\
\hline $3 / 89$ & $5.00 \pm 6$ & 4.33 & 0.19 & Acceptable \\
\hline $4 / 89$ & $3.00 \pm 6$ & 3.33 & 0.10 & Acceptable \\
\hline $10 / 89$ & $12.00 \pm 6$ & 6.33 & 1.64 & Acceptable \\
\hline $11 / \% 9$ & $15.00 \pm 6$ & 16.33 & 0.47 & Acceptable \\
\hline \multicolumn{5}{|l|}{${ }^{134} \mathrm{Cs}$} \\
\hline $4 / 89$ & $20.00 \pm 5$ & 24.7 & 1.62 & Acceptable \\
\hline $6 / 89$ & $39.00 \pm 5$ & 42.0 & 1.04 & Acceptable \\
\hline \multicolumn{5}{|l|}{${ }^{137} \mathrm{Cs}$} \\
\hline $4 / 89$ & $20.00 \pm 5$ & 20.7 & 0.23 & Acceptable \\
\hline $6 / 89$ & $20.00 \pm 5$ & 24.7 & 1.62 & Acceptable \\
\hline \multicolumn{5}{|l|}{${ }^{133} \mathrm{Ba}$} \\
\hline $6 / 89$ & $49.00 \pm 5$ & 59.7 & 3.70 & Unacceptable \\
\hline \multicolumn{5}{|l|}{${ }^{60} \mathrm{Co}$} \\
\hline $6 / 89$ & $31.00 \pm 5$ & 36.0 & 1.73 & Acceptable \\
\hline \multicolumn{5}{|l|}{${ }^{65} \mathrm{Zn}$} \\
\hline $6 / 89$ & $165.00 \pm 17$ & 166.0 & 0.10 & Acceptable \\
\hline \multicolumn{5}{|l|}{${ }^{106} \mathrm{Ru}$} \\
\hline $6 / 89$ & $128.00 \pm 13$ & 196.3 & 9.10 & Unacceptable \\
\hline \multicolumn{5}{|l|}{${ }^{3} \mathrm{H}$} \\
\hline $6 / 89$ & $4503 \pm 450$ & 4123 & 1.46 & Acceptable \\
\hline $10 / 89$ & $3496 \pm 364$ & 1850 & 7.83 & Unacceptable \\
\hline \multicolumn{5}{|l|}{${ }^{226} \mathrm{Ra}$} \\
\hline $7 / 89$ & $17.70 \pm 2.70$ & 24.0 & 4.04 & Unacceptable \\
\hline \multicolumn{5}{|l|}{${ }^{228} \mathrm{Ra}$} \\
\hline $7 / 89$ & $18.30 \pm 2.70$ & 39.7 & 13.7 & Unacceptable \\
\hline
\end{tabular}


212 Oak Ridge Environmental Report, Vol. 2-1989

Table 7.2.6 (continued)

\begin{tabular}{|c|c|c|c|c|}
\hline \multirow{2}{*}{$\begin{array}{l}\text { Analysis and } \\
\text { sample date }\end{array}$} & \multicolumn{2}{|c|}{ Values } & \multirow{2}{*}{$\begin{array}{c}\text { Normalized } \\
\text { deviation }\end{array}$} & \multirow{2}{*}{$\begin{array}{c}\text { Performance } \\
\text { evaluation }\end{array}$} \\
\hline & EPA & $Y-12$ & & \\
\hline \multicolumn{5}{|c|}{ Air filler ( $p$ Ci/filter) } \\
\hline Gross alpha & & & & \\
\hline $3 / 89$ & $21.00 \pm 5$ & 21.7 & 0.23 & Acceprable \\
\hline \multicolumn{5}{|l|}{ Gross beta } \\
\hline $3 / 89$ & $62.00 \pm 5$ & 61.0 & 0.35 & Acceptable \\
\hline \multicolumn{5}{|l|}{${ }^{90} \mathrm{Sr}$} \\
\hline $3 / 89$ & $20.00 \pm 1.50$ & 20.0 & 0 & Acceptable \\
\hline \multicolumn{5}{|l|}{${ }^{137} \mathrm{Cs}$} \\
\hline $3 / 89$ & $20.00 \pm 5$ & 23.3 & 1.15 & Asceptable \\
\hline
\end{tabular}


Table 7.2.7. EPA EMSL-LV Intercomparison Radionuclide Control Program ORNL low-level radiochemical laboratory, 1989

\begin{tabular}{|c|c|c|c|c|}
\hline \multirow{2}{*}{$\begin{array}{l}\text { Analysis atid } \\
\text { sample date }\end{array}$} & \multicolumn{2}{|c|}{ Values } & \multirow{2}{*}{$\begin{array}{c}\text { Ratio }^{b} \\
\text { (ORNL/EPA) }\end{array}$} & \multirow{2}{*}{$\begin{array}{c}\text { Performance } \\
\text { evaluation }\end{array}$} \\
\hline & $\mathrm{EPA}^{a}$ & ORNL & & \\
\hline
\end{tabular}

Water, $p C i / L$

Gross Alpha

$\begin{array}{lrccl}1 / 89 & 8 \pm 5 & 7.33 & 0.92 & \text { Acceptable } \\ 4 / 89 & 29 \pm 7 & 29 & 1.00 & \text { Acceptable } \\ 5 / 89 & 30 \pm 8 & 25 & 0.83 & \text { Acceptable } \\ 9 / 89 & 4 \pm 5 & 3.67 & 0.92 & \text { Acceptable }\end{array}$

Gross Beta

$\begin{array}{lrlll}1 / 89 & 4 \pm 5 & 6 & 1.50 & \text { Acceptable } \\ 4 / 89 & 57 \pm 5 & 62.7 & 1.10 & \text { Acceptable } \\ 5 / 89 & 50 \pm 5 & 55 & 1.10 & \text { Acceptable }\end{array}$

$9 / 89 \quad 6 \pm 5 \quad 7.33 \quad 1.22 \quad$ Acceptable

${ }^{133} \mathrm{Ba}$

$6 / 89 \quad 49 \pm 5 \quad 48 \quad 0.98$

$10 / 89 \quad 59 \pm 6 \quad 54 \quad 0.92$

${ }^{134} \mathrm{Cs}$

$2 / 89$

$4 / 89$

$6 / 89$

$10 / 89$

${ }^{137} \mathrm{Cs}$

$2 / 89$

$4 / 89$

$6 / 89$

$10 / 89$

${ }^{31} \mathrm{Cr}$

$2 / 89$

${ }^{60} \mathrm{Co}$

$2 / 89$

$6 / 89$

$10 / 89$

${ }^{3} \mathrm{H}$

$2 / 89$

$6 / 89$

$10 / 89$

${ }^{239} \mathrm{Pu}$

$1 / 89$

$8 / 89$

${ }^{226} \mathrm{Ra}$

$3 / 89$

$4 / 89$

$7 / 89$

${ }^{228} \mathrm{Ra}$

$4 / 89$

$7 / 89$

$$
\begin{aligned}
& 10 \pm 5 \\
& 20 \pm 5 \\
& 39 \pm 5 \\
& 29 \pm 5
\end{aligned}
$$

10.33

13

36.7

25.33

$10 \pm 5$

$20 \pm 5$

$20 \pm 5$

$59 \pm 5$

10.67

15.3

21

58.33

$235 \pm 24$

213

$10 \pm 5$

$31 \pm 5$

$30 \pm 5$

$$
9.33
$$

31

27.76

$2754 \pm 356$

$4491 \pm 450$

1933

4323

$3496 \pm 364 \quad 2527$

$$
4.2 \pm 0.4
$$

$2.83 \pm 0.3$

3.9

2.83

$4.9 \pm 0.4$

$3.5 \pm 0.5$

$17.7 \pm 2.7$

4.5

3.5

19.1

$3.6 \pm 0.5$

18.3

4.6
17.4
1.03

2.42

0.94

0.87

1.07

0.77

1.05

0.99

0.91

0.93

1.00

0.93

3.99

0.96

0.72

0.93

1.00

0.92

1.00

1.08

1.28

0.95
Acceptable Acceptable

Acceptable Warning Acceptable Acceptable

Acceptable Acceptable Acceptable Acceptable

Acceptable

Acceptable Acceptable Acceptable

Unacceptable Acceptable Unacceptable ${ }^{a}$

Acceptable Acceptable

Acceptable Acceptable Acceptable

Acceptable Acceptable 
Table 7.2 .7 (continued)

\begin{tabular}{|c|c|c|c|c|}
\hline \multirow{2}{*}{$\begin{array}{l}\text { Analysis and } \\
\text { sample date }\end{array}$} & \multicolumn{2}{|c|}{ Values } & \multirow{2}{*}{$\begin{array}{c}\text { Ratio } \\
\text { (ORNL/EPA) }\end{array}$} & \multirow{2}{*}{$\begin{array}{c}\text { Performance } \\
\text { evaluation }\end{array}$} \\
\hline & EPA & ORNL & & \\
\hline \multicolumn{5}{|l|}{${ }^{106} \mathrm{Ru}$} \\
\hline $2 / 89$ & $178 \pm 18$ & 163 & 0.92 & Acceptable \\
\hline $6 / 89$ & 128 & 116 & 0.91 & Acceptable \\
\hline $10 / 89$ & $161 \pm 16$ & 136.33 & 0.85 & Acceptable \\
\hline \multicolumn{5}{|l|}{${ }^{89} \mathrm{Sr}$} \\
\hline $1 / 89$ & $40 \pm 5$ & 42.33 & 1.06 & Acceptable \\
\hline $4 / 89$ & $8 \pm 5$ & 9.7 & 1.21 & Acceptable \\
\hline $6 / 89$ & $6 \pm 5$ & 6.67 & 1.11 & Acceptable \\
\hline $9 / 89$ & $14 \pm 5$ & 14.33 & 1.02 & Acceptable \\
\hline \multicolumn{5}{|l|}{${ }^{\infty} \mathrm{Sr}$} \\
\hline $1 / 89$ & $25 \pm 2$ & 24 & 0.96 & Acceptable \\
\hline $4 / 89$ & $8 \pm 1.5$ & 6.3 & 0.79 & Acceptable \\
\hline $6 / 89$ & $6 \pm 1.5$ & 5.33 & 0.89 & Acceptable \\
\hline $9 / 89$ & $10 \pm 5$ & 8.67 & 0.87 & Acceptable \\
\hline \multicolumn{5}{|l|}{$\mathrm{U}$} \\
\hline $3 / 89$ & $5 \pm 6$ & 5.33 & 1.07 & Acceptable \\
\hline $4 / 89$ & $3 \pm 6$ & 2.0 & 0.67 & Acceptable \\
\hline $7 / 89$ & $41 \pm 6$ & 38.30 & 0.93 & Acceptable \\
\hline \multicolumn{5}{|l|}{$0 \mathrm{Zn}_{\mathrm{n}}$} \\
\hline $2 / 89$ & $159 \pm 16$ & 163 & 1.03 & Acceptable \\
\hline $6 / 89$ & $165 \pm 17$ & 175 & 1.06 & Acceptable \\
\hline $10 / 89$ & $129 \pm 13$ & 121.67 & 0.94 & Acceptable \\
\hline \multicolumn{5}{|c|}{ Air filters, $p C i / f i l t e r$} \\
\hline \multicolumn{5}{|l|}{ Gross alpha } \\
\hline $3 / 89$ & $21 \pm 5$ & 24.3 & 1.16 & Acceptable \\
\hline \multicolumn{5}{|l|}{ Gross Beta } \\
\hline $3 / 89$ & $62 \pm 5$ & 64.3 & 1.04 & Acceptable \\
\hline \multicolumn{5}{|l|}{${ }^{137} \mathrm{Cs}$} \\
\hline $3 / 89$ & $20 \pm 5$ & 18.7 & 0.93 & Acceptable \\
\hline \multicolumn{5}{|l|}{${ }^{90} \mathrm{Sr}$} \\
\hline $3 / 89$ & $20 \pm 2$ & 19.7 & 0.99 & Acceptable \\
\hline
\end{tabular}

${ }^{a}$ Values and uncertainty were provided by the EPA and are published as provided.

${ }^{b}$ Ratio is proved as an indication of performance in comparison to EPA values. Ratic is not used as a measure of acceptability of data and may vary widely in relation to the individual precision associated with a particular radionuclide.

The EPA gives three classes of performance based on the number of standard deviations a result is from the true value; these are acceptable $(<2$ sigma), warning ( 2 to 3 sigma), and unacceptable ( $>3$ sigma). 
Table 7.2.8. EPA EMSL-LV Intercomparison Radionuclide Control Program-ORGDP, 1989

\begin{tabular}{|c|c|c|c|c|}
\hline \multirow{2}{*}{$\begin{array}{l}\text { Analysis and } \\
\text { sample date }\end{array}$} & \multicolumn{2}{|c|}{ Values $\left(\mathrm{pCi} / \mathrm{unit}^{a}\right.$} & \multirow{2}{*}{$\begin{array}{c}\text { Ratio }^{b} \\
\text { (EPA/ORGDP) }\end{array}$} & \multirow{2}{*}{$\begin{array}{l}\text { Performance } \\
\text { evaluation }\end{array}$} \\
\hline & EPA & ORGDP & & \\
\hline \multicolumn{5}{|c|}{ Water } \\
\hline \multicolumn{5}{|l|}{${ }^{239} \mathrm{Pu}$} \\
\hline $1 / 89$ & $4.20 \pm 0.40$ & $3.77 \pm 0.15$ & 1.11 & Acceptable \\
\hline $8 / 89$ & $2.80 \pm 0.30$ & $2.70 \pm 0.10$ & 1.03 & Acceptable \\
\hline \multicolumn{5}{|l|}{$\mathrm{U}$} \\
\hline $3 / 89$ & $5.00 \pm 6.00$ & $5.00 \pm 0.00$ & 1.00 & Acceptable \\
\hline $4 / 89$ & $3.00 \pm 6.00$ & $3.00 \pm 0.00$ & 1.00 & Acceptable \\
\hline $7 / 89$ & $41.00 \pm 6.00$ & $39.33 \pm 0.58$ & 1.04 & Acceptable \\
\hline $10 / 89$ & $12.00 \pm 6.00$ & $6.33 \pm 0.58$ & 1.89 & Acceptable \\
\hline $11 / 89$ & $15.00 \pm 6.00$ & $14.33 \pm 1.15$ & 1.05 & Acceptable \\
\hline \multicolumn{5}{|l|}{ Gross alpha } \\
\hline $1 / 89$ & $8.00 \pm 5.00$ & $5.67 \pm 0.58$ & 1.41 & Acceptable \\
\hline $4 / 89$ & $29.00 \pm 7.00$ & $19.33 \pm 2.08$ & 1.500 & Acceptable \\
\hline $5 / 89$ & $30.00 \pm 8.0$ & $17.67 \pm 0.58$ & 1.70 & Acceptable \\
\hline $9 / 89$ & $4.00 \pm 5.00$ & $3.00 \pm 1.00$ & 1.33 & Acceptable \\
\hline $10 / 89$ & $49.00 \pm 12.00$ & $41.33 \pm 1.53$ & 1.18 & Acceptable \\
\hline \multicolumn{5}{|l|}{ Gross beta } \\
\hline $1 / 89$ & $4.00 \pm 5.00$ & $5.67 \pm 0.58$ & 0.70 & Acceptable \\
\hline $4 / 89$ & $57.00 \pm 5.00$ & $34.33 \pm 2.89$ & 1.66 & Acceptable \\
\hline $5 / 89$ & $50.00 \pm 5.00$ & $57.33 \pm 1.15$ & 0.87 & Acceptable \\
\hline $9 / 89$ & $6.00 \pm 5.00$ & $3.33 \pm 0.58$ & 1.80 & Acceptable \\
\hline $10 / 89$ & $32.00 \pm 5.00$ & $28.33 \pm 0.58$ & 1.13 & Acceptable \\
\hline \multicolumn{5}{|l|}{${ }^{3} \mathrm{H}$} \\
\hline $6 / 89$ & $4503 \pm 450$ & $3491 \pm 367$ & 1.29 & Acceptable \\
\hline $10 / 89$ & $3496 \pm 364$ & $3305 \pm 177$ & 1.06 & Acceptable \\
\hline \multicolumn{5}{|l|}{${ }^{134} \mathrm{Cs}$} \\
\hline $4 / 89$ & $20.00 \pm 5.00$ & $15.00 \pm 1.73$ & 1.33 & Acceptable \\
\hline $6 / 89$ & $39.00 \pm 5.00$ & $31.33 \pm 0.58$ & 1.24 & Acceptable \\
\hline $10 / 89$ & $29.00 \pm 5.00$ & $25.00 \pm 1.00$ & 1.16 & Acceptable \\
\hline \multicolumn{5}{|l|}{${ }^{137} \mathrm{Cs}$} \\
\hline $4 / 89$ & $20.00 \pm 5.00$ & $34.33 \pm 2.89$ & 0.58 & Acceptable \\
\hline $6 / 89$ & $20.00 \pm 5.00$ & $195.33 \pm 4.73$ & 0.10 & Unacceptable \\
\hline $10 / 89$ & $59.00 \pm 5.00$ & $64.33 \pm 3.06$ & 0.92 & Acceptable \\
\hline $10 / 89$ & $5.00 \pm 5.00$ & $5.00 \pm 0.00$ & 1.00 & Acceptable \\
\hline \multicolumn{5}{|l|}{${ }^{133} \mathrm{Ba}$} \\
\hline $6 / 89$ & $49.00 \pm 5.00$ & $41.00 \pm 2.00$ & 1.20 & Acceptable \\
\hline $10 / 89$ & $59.00 \pm 6.00$ & $58.33 \pm 3.21$ & 1.01 & Acceptable \\
\hline \multicolumn{5}{|l|}{${ }^{60} \mathrm{Co}$} \\
\hline $6 / 89$ & $31.00 \pm 5.00$ & $31.33 \pm 0.58$ & 0.99 & Acceptable \\
\hline $10 / 89$ & $30.00 \pm 5.00$ & $31.67 \pm 1.53$ & 0.95 & Acceptable \\
\hline \multicolumn{5}{|l|}{${ }^{65} \mathrm{Zn}$} \\
\hline $6 / 89$ & $165.00 \pm 17.00$ & $185.00 \pm 1.73$ & 0.89 & Acceptable \\
\hline $10 / 89$ & $129.00 \pm 13.00$ & $133.67 \pm 3.06$ & 0.97 & Acceptable \\
\hline \multicolumn{5}{|l|}{${ }^{106} \mathrm{Ru}$} \\
\hline 6/89 & $128.00 \pm 13.00$ & $143.00 \pm 8.66$ & 0.90 & Acceptable \\
\hline $10 / 89$ & $161.00 \pm 16.00$ & $140.33 \pm 3.21$ & 1.15 & Acceptable \\
\hline
\end{tabular}


Table 7.2 .8 (continued)

\begin{tabular}{|c|c|c|c|c|}
\hline \multirow{2}{*}{$\begin{array}{l}\text { Analysis and } \\
\text { sample date }\end{array}$} & \multicolumn{2}{|c|}{ Values $(\mathrm{pCi} / \text { unit })^{0}$} & \multirow{2}{*}{$\begin{array}{c}\text { Ratio }^{b} \\
\text { (EPA/ORGDP) }\end{array}$} & \multirow{2}{*}{$\begin{array}{c}\text { Performance } \\
\text { evaluation }\end{array}$} \\
\hline & EPA & ORGDP & & \\
\hline & & Air filters & & \\
\hline \multicolumn{5}{|l|}{ Gross alpha } \\
\hline $3 / 89$ & $21.00 \pm 5.00$ & $13.00 \pm 1.00$ & 1.62 & Acceptable \\
\hline $8 / 89$ & $6.00 \pm 5.00$ & $4.33 \pm 0.58$ & 1.38 & Acceptable \\
\hline \multicolumn{5}{|l|}{ Gross beta } \\
\hline $3 / 89$ & $62.00 \pm 5.00$ & $63.67 \pm 4.04$ & 0.97 & Acceptable \\
\hline \multicolumn{5}{|l|}{${ }^{90} \mathrm{Sr}$} \\
\hline $3 / 89$ & $20.00 \pm 5.00$ & $22.33 \pm 0.58$ & 0.90 & Acceptable \\
\hline \multicolumn{5}{|l|}{${ }^{137} \mathrm{Cs}$} \\
\hline $3 / 89$ & $20.00 \pm 5.00$ & $20.33 \pm 2.31$ & 0.98 & Acceptable \\
\hline $8 / 89$ & $10.00 \pm 5.00$ & $13.00 \pm 1.00$ & 0.76 & Acceptable \\
\hline
\end{tabular}

"Unit for water is "liter." Unit for air is "filter."

${ }^{b}$ Acceptahle ratio is $0.50-1.50$. 
Table 7.2.9. EML Intercomparison study QAP-30 Y-12 Radiochemical Laboratory May 1989

\begin{tabular}{|c|c|c|c|c|c|}
\hline Parameter & $\begin{array}{l}\text { EML } \\
\text { value }\end{array}$ & $\begin{array}{l}Y-12 \\
\text { value }\end{array}$ & $\begin{array}{c}\text { Y.12 percent } \\
\text { error }^{a}\end{array}$ & $\begin{array}{c}\text { Ratio } \\
Y^{\prime}-12 / E M L\end{array}$ & $\begin{array}{c}\text { Performance } \\
\text { evaluation }\end{array}$ \\
\hline \multicolumn{6}{|c|}{ Soil $(p C i / g)$} \\
\hline${ }^{90} \mathrm{Sr}$ & 1.09 & 1.52 & 10 & $1.39 \pm 0.17$ & Acceptable \\
\hline${ }^{137} \mathrm{Cs}$ & 20.8 & 33.1 & 6 & $1.59 \pm 0.12$ & Unacceptable \\
\hline${ }^{239} \mathrm{Pu}$ & 0.420 & 0.166 & 12 & $0.40 \pm 0.05$ & Unacceptable \\
\hline${ }^{241} \mathrm{Am}$ & 0.210 & 0.223 & 8 & $1.06 \pm 0.11$ & Acceptable \\
\hline \multicolumn{6}{|c|}{ Vegetation (pCi/g) } \\
\hline${ }^{90} \mathrm{Sr}$ & 3.75 & 4.58 & 6 & $1.22 \pm 0.08$ & Acceptable \\
\hline${ }^{137} \mathrm{Cs}$ & 1.60 & 3.73 & 23 & $2.33 \pm 0.55$ & Unacceptable \\
\hline${ }^{239} \mathrm{Pu}$ & 0.022 & 0.016 & 43 & $0.73 \pm 0.33$ & Acceptable \\
\hline${ }^{241} \mathrm{Am}$ & 0.015 & 0.013 & 53 & $0.87 \pm 0.56$ & Acceptable \\
\hline${ }^{238} \mathrm{U}$ & 0.012 & 0.014 & 0 & $1.17 \pm 0.29$ & Acceptable \\
\hline \multicolumn{6}{|c|}{ Water $(p C i / m L)$} \\
\hline${ }^{3} \mathrm{H}$ & 6.31 & 5.43 & 25 & $0.86 \pm 0.23$ & Acceptable \\
\hline${ }^{54} \mathrm{Mn}$ & 0.300 & 0.678 & 16 & $2.26 \pm 0.40$ & Unacceptable \\
\hline${ }^{57} \mathrm{Co}$ & 0.880 & 1.84 & 4 & $2.09 \pm 0.15$ & Unacceptable \\
\hline${ }^{60} \mathrm{Co}$ & 0.940 & 1.93 & 9 & $2.05 \pm 0.23$ & Unacceptable \\
\hline${ }^{90} \mathrm{Sr}$ & 0.550 & 0.58 & 6 & $1.05 \pm 0.08$ & Acceptable \\
\hline${ }^{134} \mathrm{Cs}$ & 2.73 & 5.09 & 2 & $1.86 \pm 0.11$ & Unacceptable \\
\hline${ }^{137} \mathrm{Cs}$ & 2.55 & 5.69 & 3 & $2.23 \pm 0.15$ & Unacceptable \\
\hline${ }^{739} \mathrm{Pu}$ & 0.0059 & 0.0041 & 17 & $0.69 \pm 0.12$ & Acceptable \\
\hline${ }^{241} \mathrm{Am}$ & 0.0045 & 0.0046 & 15 & $1.02 \pm 0.21$ & Acceptable \\
\hline${ }^{238} \mathrm{U}$ & 0.0044 & 0.0112 & 0 & $2.55 \pm 0.17$ & Unacceptable \\
\hline
\end{tabular}

apercent error for the laboratory based on three replicate analyses. 
Table 7.2.10. EML Intercomparison Study QAP-31 Y-12 Radiochemical Laboratory November 1989

\begin{tabular}{lccccc} 
Parameter & $\begin{array}{l}\text { EML } \\
\text { value }\end{array}$ & $\begin{array}{c}\text { Y-12 } \\
\text { value }\end{array}$ & $\begin{array}{c}\text { Y-12 percent } \\
\text { error }^{\circ}\end{array}$ & $\begin{array}{c}\text { Ratio } \\
\text { Y-12/EML }\end{array}$ & $\begin{array}{c}\text { Performance } \\
\text { evaluation }\end{array}$ \\
\hline \multicolumn{6}{c}{ Air, Bq/filter } \\
${ }^{90} \mathrm{Sr}$ & 0.200 & 0.220 & 31 & 1.10 & Acceptable \\
${ }^{239} \mathrm{Pu}$ & 0.018 & 0.012 & 41 & 0.67 & Acceptable \\
${ }^{241} \mathrm{Am}$ & 0.018 & 0.026 & 30 & 1.44 & Acceptable \\
$\mathrm{U}(\mu \mathrm{g})$ & 0.720 & 0.800 & $\ldots$. & 1.11 & Acceptable
\end{tabular}

Soil, $\mathrm{Bq} / \mathrm{kg}$

$\begin{array}{lccccc}{ }^{90} \mathrm{Sr} & 5.73 & 6.29 & 73 & 1.10 & \text { Acceptable } \\ { }^{137} \mathrm{Cs} & 17.1 & 15.9 & 6 & 0.93 & \text { Acceptable } \\ { }^{239} \mathrm{Pu} & 2.22 & 1.82 & 5 & 0.82 & \text { Acceptable } \\ \mathrm{U}(\mu \mathrm{g} / \mathrm{g}) & 1.71 & 1.34 & & 0.78 & \text { Acceptable }\end{array}$

Vegetation, $B q / k g$

$\begin{array}{lcccll}{ }^{90} \mathrm{Sr} & 1830 & 1660 & 5 & 0.91 & \text { Acceptable } \\ { }^{239} \mathrm{Pu} & 0.0745 & 0.103 & 63 & 1.38 & \text { Acceptable } \\ \mathrm{U}(\mu \mathrm{g} / \mathrm{g}) & 0.047 & 0.275 & \ldots . & 5.80 & \text { Unacceptable }\end{array}$

Water, $B q / L$

\begin{tabular}{lccrll}
${ }^{3} \mathrm{H}$ & 395 & 364 & 7 & 0.92 & Acceptable \\
${ }^{90} \mathrm{Sr}$ & 31.7 & 33.9 & 5 & 1.07 & Acceptable \\
${ }^{239} \mathrm{Pu}$ & 0.350 & 0.247 & 10 & 0.71 & Acceptable \\
${ }^{241} \mathrm{Am}$ & 0.333 & 0.340 & 8 & 1.02 & Acceptable \\
$\mathrm{U}(\mu \mathrm{g} / \mathrm{mL})$ & 13.2 & 13.0 & - & 0.98 & Acceptable \\
\hline
\end{tabular}

${ }^{a}$ Percent error for the laboratory based on three replicate analyses.

${ }^{b} \mathrm{~Bq}=$ becquerel. $1 \mathrm{~Bq}=27 \mathrm{pCi}$. 
Table 7.2.11. 1989 EML intercomparison study ORNL low-level radiochemical laboratory April 1989

\begin{tabular}{|c|c|c|c|c|c|}
\hline Parameter & $\begin{array}{l}\text { EML } \\
\text { value }\end{array}$ & $\begin{array}{l}\text { ORNL } \\
\text { value }\end{array}$ & $\begin{array}{l}\text { ORNL } \\
\% \text { error }^{a}\end{array}$ & $\begin{array}{c}\text { Ratio } \\
\text { (ORNL/EML) }\end{array}$ & $\begin{array}{l}\text { Performance } \\
\text { evaluation }\end{array}$ \\
\hline \multicolumn{6}{|c|}{ Air, $p C i / f i l t e r$} \\
\hline${ }^{7} \mathrm{Be}$ & 1950 & 1700 & 5 & 0.87 & Acceptable \\
\hline${ }^{54} \mathrm{Mn}$ & 3.74 & 3.30 & 63 & 0.88 & Acceptable \\
\hline${ }^{60} \mathrm{Co}$ & 126 & 125 & 8 & 0.99 & Acceptable \\
\hline${ }^{90} \mathrm{Sr}$ & 2.39 & 3.64 & 57 & 1.52 & Acceptable \\
\hline${ }^{90} \mathrm{Sr}$ & 2.39 & 2.40 & 41 & 1.00 & Acceptable \\
\hline${ }^{125} \mathrm{Sb}$ & 96.8 & 82.0 & 8 & 0.85 & Acceptable \\
\hline${ }^{137} \mathrm{Cs}$ & 189 & 190 & 5 & 1.01 & Acceptable \\
\hline${ }^{144} \mathrm{Ce}$ & 327 & 305 & 3 & 0.93 & Acceptable \\
\hline${ }^{239} \mathrm{Pu}$ & 0.270 & 0.210 & 47 & 0.78 & Accentable \\
\hline${ }^{239} \mathrm{Pu}$ & 0.270 & 0.150 & 66 & 0.56 & Acceptable \\
\hline${ }^{241} \mathrm{Am}$ & 0.225 & 0.240 & 33 & 1.07 & Acceptable \\
\hline${ }^{241} \mathrm{Am}$ & 0.225 & 0.250 & 40 & 1.11 & Acceptable \\
\hline${ }^{238} \mathrm{U}$ & 0.090 & 0.180 & 27 & 2.00 & Unacceptable \\
\hline${ }^{238} \mathrm{U}$ & 0.090 & 0.170 & 58 & 1.89 & Acceptable \\
\hline \multicolumn{6}{|c|}{ Soil, $p C i / g$} \\
\hline${ }^{40} \mathrm{~K}$ & 24.1 & 25.0 & 4 & 1.04 & Acceptable \\
\hline${ }^{90} \mathrm{Sr}$ & 1.09 & 1.10 & 9 & 1.01 & Acceptable \\
\hline${ }^{90} \mathrm{Sr}$ & 1.09 & 1.60 & 6 & 1.47 & Acceptable \\
\hline${ }^{137} \mathrm{Cs}$ & 20.8 & 20.0 & 5 & 0.96 & Acceptable \\
\hline${ }^{239} \mathrm{Pu}$ & 0.420 & 0.400 & 7 & 0.95 & Acceptable \\
\hline${ }^{241} \mathrm{Am}$ & 0.210 & 0.190 & 15 & 0.90 & Acceptable \\
\hline \multicolumn{6}{|c|}{ Vegetation, $\mathrm{pCi} / \mathrm{g}$} \\
\hline${ }^{40} \mathrm{~K}$ & 26.1 & 28.0 & 3 & 1.07 & Acceptable \\
\hline${ }^{90} \mathrm{Sr}$ & 3.75 & 3.80 & 7 & 1.01 & Acceptable \\
\hline${ }^{137} \mathrm{Cs}$ & 1.60 & 1.65 & 6 & 1.03 & Acceptable \\
\hline${ }^{239} \mathrm{Pu}$ & 0.022 & 0.028 & 35 & 1.27 & Acceptable \\
\hline${ }^{241} \mathrm{Am}$ & 0.015 & 0.018 & 22 & 1.20 & Acceptable \\
\hline${ }^{238} \mathrm{U}$ & 0.012 & 0.013 & 46 & 1.08 & Acceptable \\
\hline \multicolumn{6}{|c|}{ Water, $p C i / m L$} \\
\hline${ }^{3} \mathrm{H}$ & 6.31 & 5.70 & 26 & 0.90 & Acceptable \\
\hline${ }^{54} \mathrm{Mn}$ & 0.300 & 0.310 & 6 & 1.03 & Acceptable \\
\hline${ }^{57} \mathrm{Co}$ & 0.880 & 0.840 & 2 & 0.95 & Acceptable \\
\hline${ }^{60} \mathrm{Co}$ & 0.940 & 0.880 & 2 & 0.93 & Acceptable \\
\hline${ }^{90} \mathrm{Sr}$ & 0.550 & 0.570 & 5 & 1.04 & Acceptable \\
\hline${ }^{137} \mathrm{Cs}$ & 2.55 & 2.40 & 4 & 0.94 & Acceptable \\
\hline${ }^{144} \mathrm{Ce}$ & 1.89 & 1.85 & 5 & 0.98 & Acceptable \\
\hline${ }^{239} \mathrm{Pu}$ & 0.00590 & 0.00620 & 16 & 1.05 & Acceptable \\
\hline${ }^{241} \mathrm{Am}$ & 0.00450 & 0.00420 & 23 & 0.93 & Acceptable \\
\hline${ }^{238} \mathrm{U}$ & 0.00440 & 0.00450 & 22 & 1.02 & Acceptable \\
\hline
\end{tabular}

${ }^{a}$ Counting error, $2 \sigma$. 
Table 7.2.12. 1989 EML intercomparison study ORNL low-level radiochemical laboratory September 1989

\begin{tabular}{lccccc}
\hline Parameter & $\begin{array}{l}\text { EML } \\
\text { value }\end{array}$ & $\begin{array}{c}\text { ORNL } \\
\text { value }\end{array}$ & $\begin{array}{c}\text { ORNL } \\
\text { \% error }\end{array}$ & $\begin{array}{c}\text { Ratio } \\
\text { (ORNL/EML) }\end{array}$ & $\begin{array}{c}\text { Performance } \\
\text { evaluation }\end{array}$ \\
\hline \multicolumn{5}{c}{ Air, pCi/filter } \\
${ }^{7} \mathrm{Be}$ & 123 & 120 & 8 & 0.98 & Acceptable \\
${ }^{54} \mathrm{Mn}$ & 4.17 & 4.40 & 4 & 1.06 & Acceptable \\
${ }^{60} \mathrm{Co}$ & 8.17 & 8.60 & 2 & 1.05 & Acceptable \\
${ }^{90} \mathrm{Sr}$ & 0.200 & 0.230 & 34 & 1.15 & Acceptable \\
${ }^{134} \mathrm{Cs}$ & 9.33 & 8.30 & 2 & 0.89 & Acceptable \\
${ }^{139} \mathrm{Cs}$ & 3.58 & 3.80 & 5 & 1.06 & Acceptable \\
${ }^{144} \mathrm{Ce}$ & 7.08 & 7.40 & 6 & 1.05 & Acceptable \\
${ }^{239} \mathrm{Pu}$ & 0.018 & 0.018 & 222 & 1.00 & Acceptable \\
${ }^{241} \mathrm{Am}$ & 0.018 & 0.021 & 19 & 1.17 & Acceptable \\
${ }^{238} \mathrm{U}$ & 0.090 & 0.015 & 20 & 0.17 & Unacceptable
\end{tabular}

Soil, $p \mathrm{Ci} / \mathrm{g}$

$\begin{array}{lccrll}{ }^{40} \mathrm{~K} & 561 & 600 & 8 & 1.07 & \text { Acceptable } \\ { }^{90} \mathrm{Sr} & 5.73 & 7.70 & 55 & 1.34 & \text { Acceptable } \\ { }^{137} \mathrm{Cs} & 642 & 735 & 6 & 1.14 & \text { Acceptable } \\ { }^{239} \mathrm{Pu} & 17.1 & 13.0 & 7 & 0.76 & \text { Acceptahle } \\ { }^{241} \mathrm{Am} & 2.22 & 2.40 & 16 & 1.08 & \text { Acceptable } \\ { }^{238} \mathrm{U} & 43.4 & 27.0 & 7 & 0.62 & \text { Acceptable }\end{array}$

$\begin{array}{lccccc}{ }^{40} \mathrm{~K} & 1290 & 1550 & 6 & 1.20 & \text { Acceptable } \\ { }^{90} \mathrm{Sr} & 1830 & 1450 & 6 & 0.79 & \text { Acceptable } \\ { }^{137} \mathrm{Cs} & 47.9 & 52.0 & 9 & 1.09 & \text { Acceptable } \\ { }^{241} \mathrm{Am} & 0.060 & 0.087 & 229 & 1.45 & \text { Acceptable } \\ { }^{238} \mathrm{U} & 0.60 & 0.54 & 55 & 0.90 & \text { Acceptable }\end{array}$

Water, $p C i / m L$

$\begin{array}{lccccl}{ }^{3} \mathrm{H} & 395 & 336.00 & 11 & 0.85 & \text { Acceptable } \\ { }^{54} \mathrm{Mn} & 65.0 & 67.0 & 1 & 1.03 & \text { Acceptable } \\ { }^{57} \mathrm{Co} & 135 & 140 & 7 & 1.04 & \text { Acceptable } \\ { }^{60} \mathrm{Co} & 155 & 155 & 6 & 1.00 & \text { Acceptable } \\ { }^{90} \mathrm{Sr} & 31.7 & 35 & 5 & 1.10 & \text { Acceptable } \\ { }^{134} \mathrm{Cs} & 68.3 & 64 & 1 & 0.94 & \text { Acceptable } \\ { }^{137} \mathrm{Cs} & 68.3 & 73 & 1 & 1.07 & \text { Acceptable } \\ { }^{144} \mathrm{Ce} & 132 & 135 & 7 & 1.02 & \text { Acceptable } \\ { }^{239} \mathrm{Pu} & 0.350 & 0.260 & 15 & 0.74 & \text { Acceptable } \\ { }^{241} \mathrm{Am} & 0.333 & 0.340 & 14 & 1.02 & \text { Acceptable } \\ { }^{238} \mathrm{C} & 0.167 & 0.160 & 18 & 096 & \text { Acceptable }\end{array}$

${ }^{a}$ Counting error, $2 \sigma$. 
Table 7.2.13. 1989 EML intercomparison study results for ORGDP in May 1989

\begin{tabular}{|c|c|c|c|c|c|}
\hline Parameter & EML value & ORGDP value & $\begin{array}{c}\text { Error, ORGDP } \\
(\%)\end{array}$ & $\begin{array}{c}\text { Ratio } \\
\text { (ORGDP/EML) }\end{array}$ & $\begin{array}{l}\text { Performance } \\
\text { evaluation }\end{array}$ \\
\hline \multicolumn{6}{|c|}{ Water $(p C i / m L)$} \\
\hline${ }^{3} \mathrm{H}$ & $0.631 \times 10^{1}$ & $0.371 \times 10^{1}$ & 61 & $0.59 \pm 0.37$ & Acceptable \\
\hline${ }^{54} \mathrm{Mn}$ & $0.300 \times 10^{0}$ & $0.316 \times 10^{0}$ & 4 & $1.05 \pm 0.08$ & Acceptable \\
\hline${ }^{57} \mathrm{Co}$ & $0.880 \times 10^{0}$ & $0.893 \times 10^{0}$ & 1 & $1.01 \pm 0.06$ & Acceptable \\
\hline${ }^{60} \mathrm{Co}$ & $0.940 \times 10^{0}$ & $0.921 \times 10^{0}$ & $i$ & $0.98 \pm 0.05$ & Acceptable \\
\hline${ }^{90} \mathrm{Sr}$ & $0.550 \times 10^{0}$ & $0.601 \times 10^{0}$ & 2 & $1.09 \pm 0.05$ & Acceptable \\
\hline${ }^{134} \mathrm{Cs}$ & $0.273 \times 10^{1}$ & $0.251 \times 10^{1}$ & 0 & $0.92 \pm 0.05$ & Acceptable \\
\hline${ }^{137} \mathrm{Cs}$ & $0.255 \times 10^{1}$ & $0.261 \times 10^{1}$ & 1 & $1.02 \pm 0.06$ & Acceptable \\
\hline${ }^{239} \mathrm{Pu}$ & $0.590 \times 10^{-2}$ & $0.652 \times 10^{-2}$ & 12 & $1.11 \pm 0.15$ & Acceptable \\
\hline${ }^{241} \mathrm{Am}$ & $0.450 \times 10^{-2}$ & $0.357 \times 10^{-2}$ & 17 & $0.79 \pm 0.18$ & Acceptable \\
\hline${ }^{238} \mathrm{U} \mu \mathrm{g}$ & $0.440 \times 10^{-2}$ & $0.429 \times 10^{-2}$ & 14 & $0.98 \pm 0.16$ & Acceptable \\
\hline \multicolumn{6}{|c|}{ Air (pCi/filter) } \\
\hline${ }^{60} \mathrm{Co}$ & $0.126 \times 10^{3}$ & $0.129 \times 10^{3}$ & 4 & $1.02 \pm 0.07$ & Acceptable \\
\hline${ }^{134} \mathrm{Cs}$ & $0.158 \times 10^{3}$ & $0.146 \times 10^{3}$ & 3 & $0.92 \pm 0.06$ & Acceptable \\
\hline${ }^{137} \mathrm{Cs}$ & $0.189 \times 10^{3}$ & $0.201 \times 10^{3}$ & 4 & $1.06 \pm 0.07$ & Acceptable \\
\hline${ }^{144} \mathrm{Ce}$ & $0.327 \times 10^{3}$ & $0.348 \times 10^{3}$ & 8 & $1.06 \pm 0.11$ & Acceptable \\
\hline${ }^{239} \mathrm{Pu}$ & $0.270 \times 10^{0}$ & $0.222 \times 10^{0}$ & 60 & $0.82 \pm 0.50$ & Acceptable \\
\hline${ }^{241} \mathrm{Am}$ & $0.225 \times 10^{0}$ & $0.232 \times 10^{0}$ & 46 & $1.03 \pm 0.49$ & Acceptable \\
\hline \multicolumn{6}{|c|}{ Soil $(p C i / g)$} \\
\hline${ }^{90} \mathrm{Sr}$ & $0.109 \times 10^{1}$ & $0.156 \times 10^{1}$ & 24 & $1.43 \pm 0.36$ & Acceptable \\
\hline${ }^{137} \mathrm{Cs}$ & $0.208 \times 10^{2}$ & $0.216 \times 10^{2}$ & 2 & $1.04 \pm 0.04$ & Acceptable \\
\hline${ }^{239} \mathrm{Pu}$ & $0.420 \times 10^{0}$ & $0.431 \times 10^{0}$ & 8 & $1.03 \pm 0.10$ & Acceptable \\
\hline${ }^{241} \mathrm{Am}$ & $0.210 \times 10^{0}$ & $0.158 \times 10^{0}$ & 14 & $0.75 \pm 0.11$ & Acceptable \\
\hline
\end{tabular}

${ }^{a}$ Acceptable ratio is 0.5 to 1.5 . 
Table 7.2.14. 1989 EML intercomparison study results for ORGDP in September 1989

\begin{tabular}{|c|c|c|c|c|c|}
\hline Parameter & EML value & ORGDP value & $\begin{array}{c}\text { Error, ORGDP } \\
(\%)\end{array}$ & $\begin{array}{c}\text { Ratio } \\
\text { (ORGDP/EML) }\end{array}$ & $\begin{array}{l}\text { Performance } \\
\text { evaluation }^{a}\end{array}$ \\
\hline \multicolumn{6}{|c|}{ Water $(p C i / m L)$} \\
\hline${ }^{3} \mathrm{H}$ & $0.395 \times 10^{3}$ & $0.362 \times 10^{1}$ & 12 & $0.92 \pm 0.12$ & Acceptable \\
\hline${ }^{54} \mathrm{Mn}$ & $0.650 \times 10^{2}$ & $0.70 \mathrm{~s} \times 10^{1}$ & 1 & $1.08 \pm 0.09$ & Acceptable \\
\hline${ }^{57} \mathrm{Co}$ & $0.135 \times 10^{3}$ & $0.147 \times 10^{1}$ & 0 & $1.09 \pm 0.09$ & Acceptable \\
\hline${ }^{60} \mathrm{Co}$ & $0.155 \times 10^{3}$ & $0.162 \times 10^{1}$ & 0 & $1.05 \pm 0.09$ & Acceptable \\
\hline${ }^{90} \mathrm{Sr}$ & $0.317 \times 10^{2}$ & $0.360 \times 10^{1}$ & 6 & $1.14 \pm 0.08$ & Acceptable \\
\hline${ }^{134} \mathrm{Cs}$ & $0.683 \times 10^{2}$ & $0.670 \times 10^{1}$ & 0 & $0.98 \pm 0.08$ & Acceptable \\
\hline${ }^{137} \mathrm{Cs}$ & $0.683 \times 10^{2}$ & $0.762 \times 10^{1}$ & 1 & $1.12 \pm 0.08$ & Acceptable \\
\hline${ }^{144} \mathrm{Ce}$ & $0.132 \times 10^{3}$ & $0.150 \times 10^{1}$ & 1 & $1.14 \pm 0.10$ & Acceptable \\
\hline${ }^{239} \mathrm{Pu}$ & $0.350 \times 10^{0}$ & $0.238 \times 10^{1}$ & 10 & $0.68 \pm 0.07$ & Acceptable \\
\hline${ }^{241} \mathrm{Am}$ & $0.333 \times 10^{0}$ & $0.281 \times 10^{1}$ & 11 & $0.84 \pm 0.14$ & Acceptable \\
\hline${ }^{238} \mathrm{U}, \mathrm{pCi}$ & $0.167 \times 10^{0}$ & $0.153 \times 10^{1}$ & 12 & $0.92 \pm 0.12$ & Acceptable \\
\hline \multicolumn{6}{|c|}{ Air ( $p C i / f i l t e r)$} \\
\hline${ }^{54} \mathrm{Mn}$ & $0.417 \times 10^{1}$ & $0.463 \times 10^{1}$ & 8 & $1.11 \pm 0.11$ & Acceptable \\
\hline${ }^{60} \mathrm{Co}$ & $0.817 \times 10^{1}$ & $0.881 \times 10^{1}$ & 5 & $1.08 \pm 0.07$ & Acceptable \\
\hline${ }^{134} \mathrm{Cs}$ & $0.933 \times 10^{1}$ & $0.920 \times 10^{1}$ & 4 & $0.99 \pm 0.05$ & Acceptable \\
\hline${ }^{137} \mathrm{Cs}$ & $0.358 \times 10^{1}$ & $0.395 \times 10^{1}$ & 9 & $1.10 \pm 0.11$ & Acceptable \\
\hline${ }^{144} \mathrm{Ce}$ & $0.708 \times 10^{1}$ & $0.760 \times 10^{1}$ & 18 & $1.07 \pm 0.22$ & Acceptable \\
\hline${ }^{239} \mathrm{Pu}$ & $0.180 \times 10^{-1}$ & $0.102 \times 101$ & 50 & $0.57 \pm 0.29$ & Acceptable \\
\hline${ }^{241} \mathrm{Am}$ & $0.180 \times 10^{-1}$ & $0.163 \times 10^{-1}$ & 31 & $0.91 \pm 0.31$ & Acceptable \\
\hline${ }^{238} \mathrm{U}, \mathrm{pCi}$ & $0.900 \times 10^{-2}$ & $0.121 \times 10^{-1}$ & 41 & $1.34 \pm 0.56$ & Acceptable \\
\hline \multicolumn{6}{|c|}{ Soil $(p C i / g)$} \\
\hline${ }^{137} \mathrm{Cs}$ & $0.642 \times 10^{3}$ & $0.660 \times 10^{1}$ & 3 & $1.03 \pm 0.05$ & Acceptable \\
\hline${ }^{239} \mathrm{Pu}$ & $0.171 \times 10^{2}$ & $0.140 \times 10^{1}$ & 8 & $0.82 \pm 0.21$ & Acceptable \\
\hline${ }^{241} \mathrm{Am}$ & $0.222 \times 10^{1}$ & $0.162 \times 10^{1}$ & 28 & $0.73 \pm 0.23$ & Acceptable \\
\hline $\mathrm{U}, \mathrm{Bq}$ & $0.434 \times 10^{2}$ & $0.281 \times 10^{1}$ & 5 & $0.65 \pm 0.10$ & Acceptable \\
\hline
\end{tabular}

${ }^{a}$ Acceptable ratio is 0.5 to 1.5 . 
Table 7.2.15. Proficiency Environmental Testing Control Program at the Y-12 Plant Environmental Laboratory in 1989-Level 1 concentrations

\begin{tabular}{|c|c|c|c|c|c|}
\hline \multirow{2}{*}{ Paramiter } & \multirow{2}{*}{$\begin{array}{l}\text { Average } \\
\text { recovery" } \\
(\%)\end{array}$} & \multirow{2}{*}{$\begin{array}{l}\text { Average number } \\
\text { of standard } \\
\text { deviat| } n \mathrm{~s}^{b}\end{array}$} & \multicolumn{3}{|c|}{ Performance $^{c}$} \\
\hline & & & Acceptable & Warning & Unacceptable \\
\hline Biochemical oxygen demand & 95.68 & 0.403 & 10 & 0 & 0 \\
\hline Chemical oxygen demand & 88.74 & 0.973 & 11 & 0 & 1 \\
\hline Total organic carbon & 106.34 & 0.806 & 11 & 1 & 0 \\
\hline Ammonia nitroger & 92.71 & 1.389 & 10 & 1 & 1 \\
\hline Nitrate nitrogen & 101.89 & 0.400 & 12 & 0 & 0 \\
\hline Phosphäte-P & $: 02.76$ & 0.198 & 12 & 0 & 0 \\
\hline Kjeldahl nitrogen & 108.45 & 0.609 & 12. & 0 & 0 \\
\hline Total phosphorus & 108.87 & 0.556 & 12 & 0 & 0 \\
\hline Total wspended solids & 87.09 & 0.516 & 12 & 0 & 0 \\
\hline Total dissolved solids & 92.78 & 0.7 .37 & 11 & 1 & 0 \\
\hline Oil and grease & 86.57 & 0.448 & 12 & 0 & 0 \\
\hline Alkalinity & 101.69 & 0.388 & il & 1 & 0 \\
\hline Calciunn & 102.30 & 0.316 & 11 & 0 & 0 \\
\hline Chloride & 100.35 & 0.318 & 12 & 0 & 0 \\
\hline Conductivity & 95.81 & 1.200 & 10 & 0 & 2 \\
\hline Magnesium & 100.01 & 0.303 & 11 & 0 & 0 \\
\hline Potassium & 101.89 & 1.001 & 9 & 2 & 0 \\
\hline Sodium & 103.51 & 0.691 & 11 & 0 & 0 \\
\hline Sulfate & 101.69 & 0.595 & 12 & 0 & 0 \\
\hline Total hardness & 100.98 & 0.390 & 11 & 0 & 0 \\
\hline$\rho \mathrm{H}$ & 104.01 & $0.59 \mathrm{i}$ & 12 & 0 & 0 \\
\hline Aluminurn & 119.53 & 0.600 & 9 & 1 & 1 \\
\hline Arsenic & 89.92 & 0.910 & 8 & 1 & 0 \\
\hline Barium & 105.99 & 0.755 & 11 & 0 & 0 \\
\hline Beryllium & 100.37 & 0.411 & 11 & 0 & 0 \\
\hline Cadmium & 99.52 & 0.545 & 11 & 0 & 0 \\
\hline Chromium & 101.86 & 0.246 & 11 & 0 & 0 \\
\hline Copper & 101.15 & 0.442 & 11 & 0 & 0 \\
\hline Iron & 138.06 & 1.924 & 10 & 0 & 1 \\
\hline Lead & 99.81 & 0.525 & 11 & 0 & 0 \\
\hline Manganese & 103.37 & 0.740 & 11 & 0 & 0 \\
\hline Mercury & 96.26 & 0.508 & 12 & 0 & 0 \\
\hline Nickel & 97.70 & 0.340 & 11 & 0 & 0 \\
\hline Selenium & 95.06 & 0.953 & 12 & 0 & 0 \\
\hline Silver & 93.03 & 0.460 & 11 & 0 & 0 \\
\hline Thallium & 98.49 & 0.654 & 11 & 0 & 0 \\
\hline Zinc & 103.48 & 0.811 & 10 & 1 & 0 \\
\hline Phenol & 103.47 & 1.123 & 11 & 0 & 1 \\
\hline Cyanide & 84.81 & 0.615 & 9 & 1 & 0 \\
\hline Residual chlorine & 92.70 & 0.323 & 12 & 0 & 0 \\
\hline Fluoride & 95.07 & 0.447 & 12 & 0 & 0 \\
\hline Organic halide & 98.24 & 0.815 & 7 & 0 & 0 \\
\hline $\mathrm{Cr}^{6+}$ & 106.48 & 1.000 & 10 & 0 & 0 \\
\hline Uranium & 95.93 & 0.888 & 12 & 0 & 0 \\
\hline Vanadium & 100.76 & 0.270 & 9 & 0 & 0 \\
\hline
\end{tabular}

"Average of all results for the Y.12 laboratory. All parameters were not measured every nonth.

The average number of standard deviations from the mean of all participants. The number of participant laboratories varied depending on the parameter and the month.

The adopted limits place the warning (marginal) level at 1.96 standard deviations and the acceptance level at 2.58 deviations from the mean. 
Table 7.2.16. Proficiency Environmental Testing Control Program at the Y-12 Plant Enrironmental Laboratory in 1989-Level 2 concentrations

\begin{tabular}{|c|c|c|c|c|c|}
\hline \multirow{2}{*}{ Parameter } & \multirow{2}{*}{$\begin{array}{l}\text { Average } \\
\text { recoverye } \\
(\notin)\end{array}$} & \multirow{2}{*}{$\begin{array}{c}\text { Average number } \\
\text { of standard } \\
\text { deviations }\end{array}$} & \multicolumn{3}{|c|}{ Performance ${ }^{r}$} \\
\hline & & & Acceptable & Warning & Unacceptable \\
\hline Biochemical oxygen demand & 94.06 & 0.510 & 10 & 0 & 0 \\
\hline Chemical uxygen demand & 84.75 & 0.520 & 12 & 0 & 0 \\
\hline Total organic carbon & $106 \cap 3$ & 0.674 & 12 & 0 & 0 \\
\hline Ammonia nitrogen & $9<, 44$ & 0.883 & 11 & 0 & 0 \\
\hline Nitrate nitrogen & 103.25 & 0.446 & 12 & 0 & 0 \\
\hline Phosphate-P & 104.22 & 0.328 & 12 & 0 & 0 \\
\hline Kjeldahl nitrogen & 103.09 & 0.403 & 12 & 0 & 0 \\
\hline Total phosphorus & 103.84 & 0.612 & 12 & 0 & 0 \\
\hline Total suspended solids & 93.70 & 0.502 & 12 & 0 & 0 \\
\hline Total dissolved solids & 97.51 & $0.1 \% 9$ & 12 & 0 & 0 \\
\hline Oil and grease & 91.29 & 0.390 & 12 & 0 & 0 \\
\hline Alkalinity & 101.76 & 0.394 & 12 & 0 & 0 \\
\hline Calcium & 102.16 & 0.340 & 11 & 0 & 0 \\
\hline Chloride & 98.75 & 0.264 & 12 & 0 & 0 \\
\hline Conductivity & 91.38 & 1.522 & 10 & 0 & 2 \\
\hline Magnesium & 99.40 & 0.434 & 11 & 0 & 0 \\
\hline Potassium & 105.50 & 1.402 & 8 & 1 & 2 \\
\hline Sodium & 102.23 & 0.647 & 11 & 0 & 0 \\
\hline Sulfate & 103.52 & 0.635 & 10 & 2 & 0 \\
\hline Total hardness & 100.24 & 0.312 & 11 & 0 & 0 \\
\hline $\mathrm{pH}$ & 104.43 & 0.731 & 12 & 0 & 0 \\
\hline Aluminum & 100.07 & 0.474 & 11 & 0 & 0 \\
\hline Arsenic & 89.12 & 0.654 & 11 & 1 & 0 \\
\hline Barium & 105.41 & 0.633 & 11 & 0 & 0 \\
\hline Beryllium & $1010^{\circ}$ & 0.293 & 11 & 0 & 0 \\
\hline Cadmium & 98.69 & 0.685 & 11 & 0 & 0 \\
\hline Chromium & 100.89 & 0.222 & 11 & 0 & 0 \\
\hline Copper & 99.88 & 0.710 & 10 & 0 & 1 \\
\hline Iron & 103.79 & 0.624 & 11 & 0 & 0 \\
\hline Lead & 98.74 & 0.907 & 11 & 0 & 0 \\
\hline Manganese & 102.88 & 0.896 & 11 & 0 & 0 \\
\hline Mercurv & 95.54 & 0.374 & 12 & 0 & 0 \\
\hline Nickel & 97.71 & 0.638 & 11 & 0 & 0 \\
\hline Selenium & 95.48 & 1.525 & 11 & 0 & 1 \\
\hline Silver & 90.76 & 0.944 & 10 & 0 & 1 \\
\hline Thallium & 101.64 & 0.532 & 11 & 0 & 0 \\
\hline Zinc & 95.84 & 0.643 & 11 & 0 & 0 \\
\hline Phenol & 99.37 & 0.629 & 12 & 0 & 0 \\
\hline Cyanide & 94.88 & 0.335 & 10 & 0 & 0 \\
\hline Residual chlorine & 94.68 & 0.277 & 12 & 0 & 0 \\
\hline Fluoride & 97.02 & 0.559 & 12 & 0 & 0 \\
\hline Organic halides & 93.98 & 0.615 & 7 & 0 & 0 \\
\hline $\mathrm{Cr}^{+}+$ & 106.66 & 0.892 & $\dot{y}$ & 0 & 1 \\
\hline Uranium & 96.46 & 0.815 & 12 & 0 & 0 \\
\hline Vanadiurn & 100.73 & 0.404 & 8 & 1 & 0 \\
\hline
\end{tabular}

-Average of all reaulis for the Y-12 laboratory. All parameters were not measured every month.

The average number of siandard deviations from the mei.: of all participants. The number of participant laboratorie varied depending on the parameter and the month.

The adopted limits place the warning (marginal) level at 1.96 standard deviations and the acceptance level at 2.58 deviations from the mean. 
Table 7.2.17. Proficiency Environmental Testing Control Program ar ORNL in 1989-Level 1 concentration

\begin{tabular}{|c|c|c|c|c|c|}
\hline \multirow{2}{*}{ Parameter } & \multirow{2}{*}{$\begin{array}{l}\text { Average } \\
\text { recovery } \\
(\%)\end{array}$} & \multirow{2}{*}{$\begin{array}{c}\text { Average number } \\
\text { of standard } \\
\text { deviations }\end{array}$} & \multicolumn{3}{|c|}{ Performance } \\
\hline & & & Acceptable ${ }^{c}$ & Marginal ${ }^{c}$ & Unacceptable ${ }^{c}$ \\
\hline Biochemical $O$ demand & 97 & 0.45 & 12 & 0 & 0 \\
\hline TOC & 99 & 0.27 & 12 & 0 & 0 \\
\hline Ammonia $\mathrm{N}$ & 111 & 0.34 & 19 & 0 & 1 \\
\hline Nitrate $\mathbf{N}$ & 101 & 0.10 & 10 & 0 & 0 \\
\hline Orthophosphate as $\mathrm{P}$ & 104 & 0.12 & 3 & 0 & 0 \\
\hline Total P & 90 & 0.59 & 2 & 0 & 0 \\
\hline Suspended solids & 88 & 0.79 & 11 & 1 & 0 \\
\hline Dissolved solin. & 103 & 0.57 & 12 & 0 & 0 \\
\hline$O \& G$ & 83 & 0.45 & 11 & 0 & 0 \\
\hline Alkalinity & 101 & 0.42 & 11 & 0 & 0 \\
\hline $\mathrm{Ca}$ & 99 & 0.20 & 9 & 0 & 0 \\
\hline Chloride & 99 & 0.32 & 12 & 0 & 0 \\
\hline Conductivity & 95 & 0.54 & 12 & 0 & 0 \\
\hline $\mathrm{Mg}$ & 96 & 0.33 & 8 & 0 & 0 \\
\hline $\mathrm{K}$ & 100 & 0.47 & 8 & 0 & 0 \\
\hline $\mathrm{Na}$ & 99 & 0.36 & 10 & 0 & 0 \\
\hline Sulfate & 101 & 0.45 & 12 & 0 & 0 \\
\hline $\begin{array}{l}\text { Total hardness } \\
\left(\text { as } \mathrm{CaCO}_{3}\right)\end{array}$ & 100 & 0.38 & 12 & 0 & 0 \\
\hline $\mathrm{pH}$ & 102 & 0.55 & 12 & 0 & 0 \\
\hline As & 98 & 0.42 & 6 & 0 & 1 \\
\hline $\mathrm{Ba}$ & 99 & 0.33 & 6 & 0 & 0 \\
\hline $\mathrm{Cd}$ & 79 & 1.54 & 4 & 1 & 1 \\
\hline $\mathrm{Cr}_{\mathrm{r}}$ & 90 & 0.81 & 4 & 1 & 0 \\
\hline $\mathrm{Cu}$ & 100 & 0.32 & 6 & 0 & 0 \\
\hline $\mathrm{Fe}$ & 107 & 0.14 & 3 & 0 & 0 \\
\hline $\mathrm{Pb}$ & 87 & 0.96 & 3 & 0 & 0 \\
\hline $\mathrm{Mn}$ & 105 & 2.23 & 4 & 0 & 1 \\
\hline $\mathrm{Hg}$ & 97 & 0.33 & 12 & 0 & 0 \\
\hline $\mathrm{Ni}$ & 97 & 0.31 & 4 & 0 & 0 \\
\hline $\mathrm{Se}$ & 120 & 0.99 & 2 & 1 & 0 \\
\hline $\mathrm{Ag}$ & 88 & 0.86 & 5 & 0 & 0 \\
\hline $\mathrm{Zn}$ & 100 & 0.31 & 5 & 0 & 0 \\
\hline Phenol & 113 & 0.58 & 11 & 1 & 0 \\
\hline Cyanide & 105 & 0.42 & 12 & 0 & 0 \\
\hline Total residual $\mathrm{Cl}$ & 101 & 0.53 & 12 & 0 & 0 \\
\hline Al & $170^{d}$ & 0.76 & 3 & 0 & 0 \\
\hline V & 98 & 0.36 & 6 & 0 & 0 \\
\hline $\mathrm{Be}$ & 97 & 0.35 & 5 & 0 & 0 \\
\hline Total organic halides & 142 & 0.27 & 3 & 0 & 0 \\
\hline $\mathrm{Cr}^{6+}$ & 106 & 0.40 & 1 & 0 & 0 \\
\hline
\end{tabular}

average of 12 months results at ORNL. All parameters were not analyzed each month.

oThe average number of standard deviations from the mean of all participants. The number of participant laboratories varied depending on the parameters and the month.

'For EPA, the warning level is 1.96 standard deviations, and the acceptance level is 2.58 standard deviations from the mean.

Would be $99 \%$ and 0.44 if outlier is removed. 
Table 7.2.18. Proficiency Environmental Testing Control Program at ORNL in 1989-Level 2 concentrations.

\begin{tabular}{|c|c|c|c|c|c|}
\hline \multirow{2}{*}{ Parameter } & \multirow{2}{*}{$\begin{array}{c}\text { Average } \\
\text { recovery } \\
(\%)\end{array}$} & \multirow{2}{*}{$\begin{array}{c}\text { Average number } \\
\text { of standard } \\
\text { deviations }^{b}\end{array}$} & \multirow[b]{2}{*}{ Acceptable ${ }^{r}$} & \multicolumn{2}{|c|}{ Performance } \\
\hline & & & & Marginal ${ }^{c}$ & Unacceptable ${ }^{r}$ \\
\hline Biochemical O demand & 95 & 0.32 & 12 & 0 & 0 \\
\hline TOC & 101 & 0.24 & 12 & 0 & 0 \\
\hline Amrnonia $\mathrm{N}$ & 104 & 0.58 & 18 & 2 & 0 \\
\hline Nitrate $\mathbf{N}$ & 101 & 0.18 & 10 & 0 & 0 \\
\hline Orthophosphate as P & 102 & 0.13 & 3 & 0 & 0 \\
\hline Total $\mathbf{P}$ & 96 & 0.37 & 4 & 0 & 0 \\
\hline Suspended solids & 94 & 0.58 & 12 & 0 & 0 \\
\hline Dissolved solids & 99 & 0.64 & 12 & 0 & 0 \\
\hline$O \& G$ & 87 & 0.30 & 10 & 0 & 0 \\
\hline Alkalinity & 95 & 0.53 & 11 & 0 & 0 \\
\hline $\mathrm{Ca}$ & 99 & 0.28 & 9 & 0 & 0 \\
\hline Chloride & 99 & 0.28 & 12 & 0 & 0 \\
\hline Conductivity & 93 & 0.49 & 12 & 0 & 0 \\
\hline $\mathrm{Mg}$ & 95 & 0.30 & 8 & 0 & $u$ \\
\hline $\mathrm{K}$ & 101 & 0.13 & 8 & 0 & 0 \\
\hline $\mathrm{Na}$ & 99 & 0.27 & 10 & 0 & 0 \\
\hline Sulfate & 102 & 0.41 & 12 & 0 & 0 \\
\hline $\begin{array}{l}\text { Total hardness } \\
\text { (as } \mathrm{CaCO}_{3} \text { ) }\end{array}$ & 99 & 0.33 & 12 & 0 & 0 \\
\hline $\mathrm{pH}$ & 102 & 0.44 & 12 & 0 & 0 \\
\hline As & 98 & 0.35 & 11 & 0 & 0 \\
\hline $\mathrm{Ba}$ & 98 & 0.48 & 6 & 0 & 0 \\
\hline $\mathrm{Cd}$ & 96 & 0.60 & 6 & 0 & 0 \\
\hline $\mathrm{Cr}$ & 98 & 0.45 & 6 & 0 & 0 \\
\hline $\mathrm{Cu}$ & 100 & 0.45 & 6 & 0 & 0 \\
\hline$F_{g}$ & 97 & 0.62 & 5 & 0 & 0 \\
\hline $\mathrm{Pb}$ & 92 & 0.94 & 5 & 0 & 0 \\
\hline $\mathrm{Mn}$ & 96 & 0.59 & 6 & 0 & 0 \\
\hline $\mathrm{Hg}$ & 97 & 0.25 & 12 & 0 & 0 \\
\hline $\mathrm{Ni}$ & 98 & 0.46 & 6 & 0 & 0 \\
\hline $\mathrm{Se}$ & 96 & 0.99 & 4 & 1 & 0 \\
\hline $\mathrm{Ag}$ & 101 & 0.42 & 6 & 0 & 0 \\
\hline $\mathrm{Zn}$ & 101 & 0.39 & 6 & 0 & 0 \\
\hline Phenol & 105 & 1.04 & 11 & 0 & 1 \\
\hline Cyanide & 108 & 0.40 & 12 & 0 & 0 \\
\hline Total residual $\mathrm{Cl}$ & 100 & 0.44 & 12 & 0 & 0 \\
\hline Al & 96 & 1.07 & 5 & 1 & 0 \\
\hline V & 98 & 0.39 & 6 & 0 & 0 \\
\hline $\mathrm{Be}$ & 97 & 0.45 & 6 & 0 & 0 \\
\hline Tctal organic halides & 94 & 0.71 & 6 & 0 & 0 \\
\hline $\mathrm{Cr}^{6+}$ & 104 & 0.12 & 1 & 0 & 0 \\
\hline
\end{tabular}

${ }^{a}$ Average of 10 months results at ORNL. All parameters were not analyzed each month.

"The average number of standard deviations from the mean of all participants. The number of participant laboratories varied depending on the parameters and the $m$ onth.

${ }^{c}$ For EPA, the warning level is 1.96 . tandard deviations, and the acceptance level is 2.58 standard deviations from the mean. 
Table 7.2.19. Proficiency Environmental Testing Control Program at ORGDP in 1989-Level 1 concentrations

\begin{tabular}{|c|c|c|c|c|c|}
\hline \multirow{2}{*}{ Parameter } & \multirow{2}{*}{$\begin{array}{l}\text { Average } \\
\text { recovery } \\
(\%)\end{array}$} & \multirow{2}{*}{$\begin{array}{l}\text { Average number } \\
\text { of standard } \\
\text { deviations }\end{array}$} & \multicolumn{3}{|c|}{ Performance ${ }^{c}$} \\
\hline & & & Acceptable & Marginal & Unacceptable \\
\hline Alkalinity & 93.693 & -0.67 & 10 & 0 & 1 \\
\hline $\mathrm{Al}$ & 142.546 & 1.27 & 9 & 0 & 3 \\
\hline Ammonia nitrogen & 119.254 & 0.28 & 10 & 1 & 0 \\
\hline As & 92.741 & -0.35 & 12 & 0 & 0 \\
\hline $\mathrm{Ba}$ & 96.313 & -0.49 & 12 & 0 & 0 \\
\hline $\mathrm{Be}$ & 98.019 & -0.03 & 12 & 0 & 0 \\
\hline Biochemical oxygen demand & 94.634 & 0.02 & 10 & 0 & 0 \\
\hline $\mathrm{Cd}$ & 95.811 & -0.49 & 12 & 0 & 0 \\
\hline $\mathrm{Ca}$ & 96.546 & -0.52 & 11 & 0 & 0 \\
\hline Chemical oxygen demand & 98.382 & 0.23 & 9 & 1 & 0 \\
\hline Chloride & 106.984 & 0.75 & 9 & 0 & 2 \\
\hline $\mathrm{Cr}_{\mathrm{r}}$ & 99.191 & -0.27 & 12 & 0 & 0 \\
\hline Conductivity & 90.444 & -0.22 & 10 & 1 & 0 \\
\hline $\mathrm{Cu}$ & 93.405 & -0.80 & 10 & 1 & 1 \\
\hline Cyanide & 80.701 & -0.68 & 10 & 0 & 1 \\
\hline $\mathrm{Fl}$ & 99.795 & C.14 & 11 & 0 & 0 \\
\hline $\mathrm{Cr}^{6+}$ & 84.525 & -0.35 & 11 & 0 & 0 \\
\hline $\mathrm{Fe}$ & 90.541 & -0.53 & 12 & 0 & 0 \\
\hline $\mathrm{Pb}$ & 97.522 & -0.16 & 12 & 0 & 0 \\
\hline $\mathrm{Mg}$ & 96.649 & -0.16 & 11 & 0 & 0 \\
\hline $\mathrm{Mn}$ & 97.524 & -0.16 & 12 & 0 & 0 \\
\hline $\mathrm{Hg}$ & 100.042 & -0.01 & 12 & 0 & 0 \\
\hline $\mathrm{Ni}$ & 93.357 & -0.24 & 12 & 0 & 0 \\
\hline Nitrate-N & 107.355 & 0.35 & 11 & 0 & 0 \\
\hline Oil and grease & 89.399 & 0.16 & 11 & 0 & 0 \\
\hline Orthophosphate as $\mathbf{P}$ & 96.159 & -0.39 & 11 & 0 & 0 \\
\hline $\mathrm{pH}$ & 99.338 & -0.29 & 11 & 0 & 0 \\
\hline Phenol & 94.561 & -0.18 & 11 & 0 & 0 \\
\hline $\mathrm{K}$ & 104.293 & 0.16 & 10 & 0 & 1 \\
\hline $\mathrm{Se}$ & 104.796 & -0.13 & 12 & 0 & 0 \\
\hline $\mathrm{Ag}$ & 89.889 & -0.59 & 10 & 2 & 0 \\
\hline $\mathrm{Na}$ & 99.674 & -0.30 & 11 & 0 & 0 \\
\hline Sulfate & 98.631 & 0.06 & 11 & 0 & 0 \\
\hline $\mathrm{Tl}$ & 100.461 & 0.11 & 12 & 0 & 0 \\
\hline Total dissolved solids & 111.681 & 0.22 & 11 & 0 & 0 \\
\hline Total hardness, as $\mathrm{CaCO}_{3}$ & 101.579 & 0.10 & 11 & 0 & 0 \\
\hline Total Kjeldahl $\mathbf{N}$ & 171.708 & 0.88 & 8 & 0 & 2 \\
\hline Total organic carbon & 105.735 & 0.50 & 9 & 1 & 0 \\
\hline Total organic halides, $\mathrm{TO}_{\mathrm{x}}$ & 101.870 & -0.32 & 10 & 1 & 0 \\
\hline Total phosphorus as $\mathbf{P}$ & 110.551 & 0.20 & 11 & 0 & 0 \\
\hline Total residual chlorine & 112.753 & 0.70 & 9 & 1 & 1 \\
\hline Total suspended solids & 90.965 & -0.16 & 11 & 0 & 0 \\
\hline $\mathrm{U}$ & 100.653 & -0.02 & 11 & 0 & 0 \\
\hline $\mathrm{V}$ & 97.291 & -0.36 & 9 & 0 & 0 \\
\hline $\mathrm{Zn}$ & 137.821 & 2.42 & 10 & 0 & 2 \\
\hline
\end{tabular}

${ }^{a}$ Average of all results for ORGDP. All parameters were not measured every month.

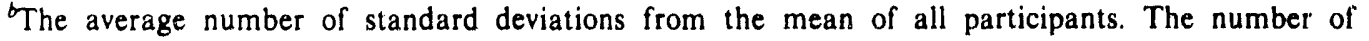
participant laboratories varied depending on the parameters and the month.

'For the EPA, the warning (marginal) level is 1.96 standard deviations and the acceptance level is 2.58 standard deviations from the mean. 
Table 7.2.20. Proficiency Environmental Testing Control Program at ORGDP in 1989-Level 2 concentrations

\begin{tabular}{|c|c|c|c|c|c|}
\hline \multirow{2}{*}{ Parameter ${ }^{a}$} & \multirow{2}{*}{$\begin{array}{l}\text { Average } \\
\text { recovery } \\
(\%)\end{array}$} & \multirow{2}{*}{$\begin{array}{c}\text { Average number } \\
\text { of standard } \\
\text { deviations }\end{array}$} & \multicolumn{3}{|c|}{ Performance ${ }^{c}$} \\
\hline & & & Acceptable & Marginal & Unacceptable \\
\hline Alkalinity & 92.949 & -0.54 & 9 & 2 & 0 \\
\hline $\mathrm{Al}$ & 98.054 & -0.32 & 11 & 1 & 0 \\
\hline Ammonia nitrogen & 113.765 & 1.02 & 8 & 3 & 0 \\
\hline As & 95.702 & -0.12 & 12 & 0 & 0 \\
\hline $\mathbf{B a}$ & 96.242 & -0.66 & 12 & 0 & 0 \\
\hline $\mathrm{Be}$ & 98.268 & -0.25 & 12 & 0 & 0 \\
\hline Biochemical oxygen demand & 98.395 & 0.04 & 9 & 1 & 0 \\
\hline $\mathrm{Cd}$ & 96.293 & -0.64 & 12 & 0 & 0 \\
\hline $\mathrm{Ca}$ & 97.658 & -0.59 & 11 & 0 & 0 \\
\hline Chemical oxygen demand & 108.447 & 0.68 & 9 & 1 & 0 \\
\hline Chloride & 98.520 & -0.22 & 11 & 0 & 0 \\
\hline $\mathrm{Cr}$ & 99.345 & -0.24 & 12 & 0 & 0 \\
\hline Conductivity & 89.486 & 0.11 & 10 & 1 & 0 \\
\hline $\mathrm{Cu}$ & 97.860 & -0.46 & 12 & 0 & 0 \\
\hline Cyanide & 89.719 & -0.45 & 10 & 0 & 1 \\
\hline $\mathrm{Cr}^{6+}$ & 92.090 & -0.37 & 11 & 0 & 0 \\
\hline Fluoride & 100.765 & 0.17 & 11 & 0 & 0 \\
\hline $\mathrm{Fe}$ & 97.670 & -0.56 & 12 & 0 & 0 \\
\hline $\mathrm{Pb}$ & 99.601 & 0.01 & 11 & 1 & 0 \\
\hline $\mathrm{Mg}$ & 96.676 & -0.14 & 11 & 0 & 0 \\
\hline $\mathrm{Mn}$ & 97.680 & -0.02 & 12 & 0 & 0 \\
\hline $\mathrm{Hg}$ & 97.774 & -0.06 & 12 & 0 & 0 \\
\hline $\mathrm{Ni}$ & 96.697 & -0.57 & 12 & 0 & 0 \\
\hline Nitrate-N & $10 i .385$ & 0.09 & 11 & 0 & 0 \\
\hline Oil and grease & 90.477 & 0.04 & 11 & 0 & 0 \\
\hline Orthophosphate as $\mathrm{P}$ & 98.425 & -0.08 & 11 & 0 & 0 \\
\hline $\mathrm{pH}$ & 100.250 & -0.17 & 11 & 0 & 0 \\
\hline Phenol & 93.615 & -0.40 & 11 & 0 & 0 \\
\hline K & 101.866 & -0.12 & 11 & 0 & 0 \\
\hline $\mathrm{Se}$ & 97.542 & -0.01 & 12 & 0 & 0 \\
\hline $\mathbf{A g}$ & 88.330 & -1.38 & 10 & 1 & 1 \\
\hline $\mathrm{Na}$ & 99.897 & -0.17 & 11 & 0 & 0 \\
\hline Sulfate & 98.758 & 0.16 & 11 & 0 & 0 \\
\hline $\mathrm{Tl}$ & 101.145 & 0.30 & 12 & 0 & 0 \\
\hline Total dissolved solids & 106.324 & 0.35 & 11 & 0 & 0 \\
\hline Total hardness, as $\mathrm{CaCO}_{3}$ & 98.276 & -0.01 & 11 & 0 & 0 \\
\hline Total Kjeldahl-N & 107.090 & 0.37 & 9 & 1 & 0 \\
\hline Total organic carbon & 114.115 & 1.04 & 9 & 0 & 1 \\
\hline Total organic halides, $\mathrm{TO}_{\mathrm{x}}$ & 87.426 & -0.42 & 10 & 0 & 0 \\
\hline Total phosphorus as $\mathbf{P}$ & 98.400 & -0.06 & 11 & 0 & 0 \\
\hline Total residual chlorine & 103.451 & 0.38 & 10 & 0 & 1 \\
\hline Total suspended solids & 92.541 & 0.00 & 11 & 0 & 0 \\
\hline $\mathrm{U}$ & 101.776 & 0.19 & 11 & 0 & 0 \\
\hline V & 97.168 & -0.54 & 9 & 0 & 0 \\
\hline $\mathrm{Zn}$ & 99.775 & -0.46 & 12 & 0 & 0 \\
\hline
\end{tabular}

${ }^{a}$ Average of all results for ORGDP. All pararneters were not measured every month.

The average number of standard deviations from the mean of all participants. The number of participant laboratories varied depending on the parameters and the month.

'For the EPA, the warning (marginal) level is 1.96 standard deviations and the acceptance level is 2.58 standard deviations from the mean. 
Table 7.2.21. EPA performance evaluation DMR-QA study number 009-Y-12 Plant, 1989

\begin{tabular}{|c|c|c|c|c|c|}
\hline \multirow{2}{*}{ Analytes } & \multicolumn{2}{|c|}{ Values } & \multicolumn{2}{|c|}{ Limits } & \multirow{2}{*}{$\begin{array}{l}\text { Performance } \\
\text { evaluation }\end{array}$} \\
\hline & Reported & True & Acceptance & Warning & \\
\hline \multicolumn{6}{|c|}{ Trace metals $(\mu \mathrm{g} / L)$} \\
\hline Al & 1230 & 1150 & $931-1340$ & $982-1290$ & Acceptable \\
\hline As & 148 & 226 & $171-272$ & $184-259$ & Unacceptable \\
\hline $\mathrm{Be}$ & 78.9 & 80.1 & $68.4-91.2$ & $71.3-88.3$ & Acceptable \\
\hline $\mathrm{Cd}$ & 327 & 320 & $277-375$ & $289-363$ & Acceptable \\
\hline $\mathrm{Cr}$ & 161 & 150 & $118-179$ & $125-171$ & Acceptable \\
\hline Co & 953 & 900 & $787-1010$ & $816-983$ & Acceptable \\
\hline $\mathrm{Cu}$ & 77 & 76.2 & $65.4-86.0$ & $67.9-83.4$ & Acceptable \\
\hline $\mathrm{Fe}$ & 744 & 749 & $652-859$ & $678-833$ & Acceptable \\
\hline $\mathrm{Pb}$ & 100 & 91.7 & $71.5-115$ & $77.0-110$ & Acceptable \\
\hline $\mathrm{Mn}$ & 1010 & 970 & $886-1050$ & $906-1030$ & Acceptable \\
\hline $\mathrm{Hg}$ & 0.9 & 0.853 & $0.457-1.23$ & $0.554-1.13$ & Acceptable \\
\hline $\mathrm{Ni}$ & 635 & 622 & $547-691$ & $565-673$ & Acceptable \\
\hline $\mathrm{Se}$ & 12.5 & 12.0 & $6.24-16.7$ & $7.56-15.4$ & Acceptable \\
\hline V & 498 & 479 & $420-553$ & $437-536$ & Acceptable \\
\hline $\mathrm{Zn}$ & 205 & 210 & $181-237$ & $188-230$ & Acceptable \\
\hline \multicolumn{6}{|c|}{ Miscellaneous analytes $(\mathrm{mg} / L)$} \\
\hline pH (units) & 7.78 & 7.80 & $7.55-7.97$ & $7.60-7.92$ & Acceptable \\
\hline $\begin{array}{l}\text { Total sus- } \\
\text { pended solids }\end{array}$ & 41.8 & 41.9 & $33.3-46.6$ & $34.9-45.0$ & Acceptable \\
\hline O\&G & 19.6 & 19.8 & $10.7-24.8$ & $12.5-23.0$ & Acceptable \\
\hline \multicolumn{6}{|c|}{ Nutrients $(m g / L)$} \\
\hline Ammonia-nitrogen & 12.4 & 13.0 & $10.2-15.5$ & $10.9-14.9$ & Acceptable \\
\hline Nitrate-nitrogen & 8.7 & 8.50 & $6.94-10.1$ & $7.32-9.72$ & Acceptable \\
\hline Kjeldahl-nitrogen & 15.0 & 15.0 & $11.4-18.2$ & $12.2-17.4$ & Acceptable \\
\hline Total P & 7.08 & 7.01 & $5.60-8.38$ & $5.93-8.05$ & Acceptable \\
\hline Orthophosphate & 3.85 & 4.80 & $3.25-4.36$ & $3.39-4.23$ & Acceptable \\
\hline \multicolumn{6}{|c|}{ Dernands $(m g / L)$} \\
\hline COD & 92 & 91.7 & $74.4-109$ & $78.8-105$ & Acceptable \\
\hline TOC & 37.7 & 37.0 & $30.6-43.2$ & $32.3-41.6$ & Accentable \\
\hline 5-day BOD & 60 & 59.7 & $41.7-85.7$ & $47.2-80.3$ & Acceptable \\
\hline \multicolumn{6}{|c|}{ Additional miscellaneous analytes $(\mathrm{mg} / \mathrm{L})$} \\
\hline Total cyanide & 0.054 & 0.070 & $0.0300-7.0998$ & $0.0388-0.0910$ & Acceptable \\
\hline Total phenolics & 0.54 & 0.645 & $0.307-1.01$ & $0.395-0.920$ & Acceptable \\
\hline $\begin{array}{l}\text { Total residual } \\
\text { chlorine }\end{array}$ & 1.91 & 2.00 & $1.35-2.53$ & $1.51-2.38$ & Acceptable \\
\hline
\end{tabular}


Table 7.2.22. EPA performance evaluation DMR-QA study number 009_-ORNL, 1989

\begin{tabular}{|c|c|c|c|c|c|}
\hline \multirow{2}{*}{ Analytes } & \multicolumn{2}{|c|}{ Values } & \multicolumn{2}{|c|}{ Limits } & \multirow{2}{*}{$\begin{array}{l}\text { Performance } \\
\text { evaluation }\end{array}$} \\
\hline & Reported & True $^{a}$ & Acceptable & Warning & \\
\hline \multicolumn{6}{|c|}{ Trace metals; $\mu \mathrm{g} / L$} \\
\hline $\mathrm{Al}$ & 393 & 350 & $269-439$ & $290-418$ & Acceptable \\
\hline As & 60 & 45.1 & $35.6-55: 5$ & $38.0-53.0$ & Unusable data \\
\hline $\mathrm{Be}$ & 429 & 400 & $347-449$ & $360-436$ & Acceptabie \\
\hline $\mathrm{Cd}$ & 24.1 & 85.1 & $74.1-100$ & $77.4-97.0$ & Not acceptable \\
\hline $\mathrm{Cr}$ & 627 & 500 & $485-703$ & $512-676$ & Acceptable \\
\hline $\mathrm{Co}$ & 222 & 200 & $171-229$ & $179-222$ & Acceptable \\
\hline $\mathrm{Cu}$ & 845 & 820 & $753-893$ & $770-876$ & Acceptable \\
\hline $\mathrm{Fe}$ & 1920 & 1827 & $: 650-2030$ & $1690-1980$ & Acceptable \\
\hline $\mathrm{Pb}$ & 404 & 415 & $361-479$ & $376-464$ & Acceptable \\
\hline $\mathrm{Mn}$ & 287 & 292 & $263-318$ & $270-311$ & Acceptable \\
\hline $\mathrm{Hig}$ & 8.86 & 8.69 & $6.40-11.1$ & $6.99-10.5$ & Acceptable \\
\hline $\mathrm{Ni}$ & 403 & 370 & $319-419$ & $331-407$ & Acceptable \\
\hline $\mathrm{Se}$ & 48.2 & 48.0 & $33.0-58.5$ & $36.2-55.3$ & Acceptable \\
\hline $\mathrm{V}$ & 1610 & 1497 & $1270-1710$ & $1330-1650$ & Acceptable \\
\hline $\mathrm{Zn}$ & 398 & 401 & $347-446$ & $359-434$ & Acceptable \\
\hline \multicolumn{6}{|c|}{ Miscellaneous analytes, $\mathrm{mg} / \mathrm{L}^{b}$} \\
\hline $\begin{array}{l}\mathrm{pH} \text {, units } \\
\text { Total }\end{array}$ & 5.81 & 5.80 & $5.66-5.91$ & $5.69-5.88$ & Acceptable \\
\hline suspended solids & 26.0 & 29.7 & $24.2-33.3$ & $25.3-32.2$ & Acceptable \\
\hline Oil and grease & 12.0 & 12.0 & $6.32-16.4$ & $7.57-15.1$ & Acceptable \\
\hline \multicolumn{6}{|c|}{ Nutrients, $m g / L$} \\
\hline Ammonia as $\mathrm{N}$ & 2.97 & 3.00 & $2.31-3.66$ & $2.47-3.50$ & Acceptable \\
\hline Nitrate as $\mathrm{N}$ & 1.04 & 0.950 & $0.745-1.16$ & $0.794-1.11$ & Acceptable \\
\hline \multicolumn{6}{|l|}{ Total } \\
\hline phosphorous & 2.30 & 2.50 & $2.03--3.01$ & $2.15-2.89$ & Acceptable \\
\hline \multicolumn{6}{|c|}{ Demands, $m g / L$} \\
\hline TOC & 11.6 & 11.5 & $9.45-16.0$ & $10.3-15.2$ & Acceptable \\
\hline 5-d BOD & 22.0 & 18.6 & $13.1-30.9$ & $15.3-28.7$ & Acceptable \\
\hline \multicolumn{6}{|c|}{ Additional miscellaneous analytes, $\mathrm{mg} / \mathrm{L}$} \\
\hline Total cyanide & 0.976 & 0.890 & $0.562 \cdots 1.14$ & $0.635-1.07$ & Acceptable \\
\hline Total phenolics & 0.268 & 0.268 & $0.127-0.409$ & $0.162-0.374$ & Acceptable \\
\hline Total residual & & & & & \\
\hline chlorine & 0.710 & 0.700 & $0.440-0.925$ & $0.504-0.861$ & Acceptable \\
\hline
\end{tabular}

${ }^{a}$ Based on theoretical calculations or a reference value when necessary.

${ }^{b}$ Units are $\mathrm{mg} / \mathrm{L}$ except for $\mathrm{pH}$, which is reported in $\mathrm{pH}$ units. 
Table 7.2.23. Water supply performance evaluation study number WS-024-Y-12 Plant, 1989

\begin{tabular}{|c|c|c|c|c|c|}
\hline \multirow{2}{*}{ Analytes } & \multirow{2}{*}{$\begin{array}{l}\text { Sample } \\
\text { number }\end{array}$} & \multicolumn{2}{|c|}{ Values } & \multirow{2}{*}{$\begin{array}{c}\text { Acceptance } \\
\text { limits }\end{array}$} & \multirow{2}{*}{$\begin{array}{c}\text { Performance } \\
\text { evaluation }\end{array}$} \\
\hline & & Reported & True & & \\
\hline \multicolumn{6}{|c|}{ Trace nietals $(\mu \mathrm{g} / L)$} \\
\hline $\mathrm{Sb}$ & $\begin{array}{l}3 \\
4\end{array}$ & $\begin{array}{l}5.25 \\
36\end{array}$ & $\begin{array}{l}9.00 \\
42.0\end{array}$ & $\begin{array}{l}6.27-12.3 \\
34.3-53.1\end{array}$ & $\begin{array}{l}\text { Unacceptable } \\
\text { Acceptable }\end{array}$ \\
\hline As & $\begin{array}{l}1 \\
2\end{array}$ & $\begin{array}{l}11 \\
82\end{array}$ & $\begin{array}{l}10.2 \\
80.7\end{array}$ & $\begin{array}{l}7.88-11.6 \\
67.1-92.4\end{array}$ & $\begin{array}{l}\text { Acceptable } \\
\text { Acceptable }\end{array}$ \\
\hline $\mathrm{Ba}$ & $\begin{array}{l}1 \\
2\end{array}$ & $\begin{array}{l}896 \\
43.2\end{array}$ & $\begin{array}{l}853 \\
41.0\end{array}$ & $\begin{array}{c}729-944 \\
31.7-49.7\end{array}$ & $\begin{array}{l}\text { Acceptable } \\
\text { Acceptable }\end{array}$ \\
\hline Be & $\begin{array}{l}3 \\
4\end{array}$ & $\begin{array}{l}0.622 \\
0.956\end{array}$ & $\begin{array}{l}0.600 \\
1.00\end{array}$ & $\begin{array}{l}0.467-0.771 \\
0.832-1.19\end{array}$ & $\begin{array}{l}\text { Acceptable } \\
\text { Acceptable }\end{array}$ \\
\hline $\mathrm{Cd}$ & $\begin{array}{l}1 \\
2\end{array}$ & $\begin{array}{l}15.9 \\
11.0\end{array}$ & $\begin{array}{l}15.4 \\
10.4\end{array}$ & $\begin{array}{l}13.5-17.5 \\
8.40-11.8\end{array}$ & $\begin{array}{l}\text { Acceptable } \\
\text { Acceptable }\end{array}$ \\
\hline $\mathrm{Cr}$ & $\begin{array}{l}1 \\
2\end{array}$ & $\begin{array}{l}130 \\
28.1\end{array}$ & $\begin{array}{l}127 \\
25.5\end{array}$ & $\begin{array}{c}111-144 \\
21.4-29.8\end{array}$ & $\begin{array}{l}\text { Acceptable } \\
\text { Acceptable }\end{array}$ \\
\hline $\mathrm{Pb}$ & $\begin{array}{l}1 \\
2\end{array}$ & $\begin{array}{l}12 \\
6.25\end{array}$ & $\begin{array}{l}15.0 \\
3.20\end{array}$ & $\begin{array}{l}11.7-18.6 \\
1.45-5.37\end{array}$ & $\begin{array}{l}\text { Acceptable } \\
\text { Unacceptable }\end{array}$ \\
\hline $\mathrm{Hg}$ & $\begin{array}{l}1 \\
2\end{array}$ & $\begin{array}{l}5.59 \\
2.06\end{array}$ & $\begin{array}{l}5.76 \\
2.16\end{array}$ & $\begin{array}{l}4.65-6.75 \\
1.42-2.69\end{array}$ & $\begin{array}{l}\text { Acceptable } \\
\text { Acceptable }\end{array}$ \\
\hline $\mathrm{Ni}$ & $\begin{array}{l}3 \\
4\end{array}$ & $\begin{array}{l}3.00 \\
12.7\end{array}$ & $\begin{array}{l}2.00 \\
14.0\end{array}$ & $\begin{array}{r}0.977-3.03 \\
11.6-16.3\end{array}$ & $\begin{array}{l}\text { Acceptable } \\
\text { Acceptable }\end{array}$ \\
\hline $\mathrm{Se}$ & $\begin{array}{l}1 \\
2\end{array}$ & $\begin{array}{l}48.5 \\
11\end{array}$ & $\begin{array}{l}48.0 \\
12.0\end{array}$ & $\begin{array}{l}37.2-57.1 \\
8.79-14.4\end{array}$ & $\begin{array}{l}\text { Acceptable } \\
\text { Acceptable }\end{array}$ \\
\hline $\mathrm{Ag}$ & $\begin{array}{l}1 \\
2\end{array}$ & $\begin{array}{l}108 \\
5.68\end{array}$ & $\begin{array}{r}103 \\
6.45\end{array}$ & $\begin{array}{l}88.4-117 \\
5.22-8.04\end{array}$ & $\begin{array}{l}\text { Acceptable } \\
\text { Acceptable }\end{array}$ \\
\hline $\mathrm{Tl}$ & $\begin{array}{l}3 \\
4\end{array}$ & $\begin{array}{l}1.33 \\
14.6\end{array}$ & $\begin{array}{l}2.00 \\
18.0\end{array}$ & $\begin{array}{l}1.20-2.85 \\
14.3-21.6\end{array}$ & $\begin{array}{l}\text { Acceptable } \\
\text { Acceptable }\end{array}$ \\
\hline \multicolumn{6}{|c|}{ Nitrate/nitrite/fluoride (mg/L) } \\
\hline Nitrate as $\mathrm{N}$ & $\begin{array}{l}1 \\
2\end{array}$ & $\begin{array}{l}0.81 \\
9.33\end{array}$ & $\begin{array}{l}0.600 \\
8.50\end{array}$ & $\begin{array}{c}0.433-0.820 \\
7.21-10.0\end{array}$ & $\begin{array}{l}\text { Acceptable } \\
\text { Acceptable }\end{array}$ \\
\hline Nitrite as $\mathrm{N}$ & $\begin{array}{l}1 \\
2\end{array}$ & $\begin{array}{l}0.16 \\
0.91\end{array}$ & $\begin{array}{l}0.150 \\
0.900\end{array}$ & $\begin{array}{l}0.123-0.177 \\
0.779-1.02\end{array}$ & $\begin{array}{l}\text { Acceptable } \\
\text { Acceptable }\end{array}$ \\
\hline Fluoride & $\begin{array}{l}1 \\
2\end{array}$ & $\begin{array}{l}1.26 \\
1.69\end{array}$ & $\begin{array}{l}1.30 \\
1.72\end{array}$ & $\begin{array}{l}1.17-1.43 \\
1.55-1.89\end{array}$ & $\begin{array}{l}\text { Acceptable } \\
\text { Acceptable }\end{array}$ \\
\hline \multicolumn{6}{|c|}{ Trihalomethanes $(\mu \mathrm{g} / L)$} \\
\hline Bromodichloromethane & $\begin{array}{l}1 \\
2\end{array}$ & $\begin{array}{l}24.5 \\
57.9\end{array}$ & $\begin{array}{l}22.5 \\
57.8\end{array}$ & $\begin{array}{l}18.0-27.0 \\
46.2-69.4\end{array}$ & $\begin{array}{l}\text { Acceptable } \\
\text { Asceptable }\end{array}$ \\
\hline Bromoform & $\begin{array}{l}1 \\
2\end{array}$ & $\begin{array}{l}13.3 \\
77.6\end{array}$ & $\begin{array}{l}12.3 \\
66.9\end{array}$ & $\begin{array}{l}9.84-14.8 \\
53.5-80.3\end{array}$ & $\begin{array}{l}\text { Acceptable } \\
\text { Acceptable }\end{array}$ \\
\hline Chlorodibromomethane & $\begin{array}{l}1 \\
2\end{array}$ & $\begin{array}{l}7.78 \\
79.4\end{array}$ & $\begin{array}{l}7.66 \\
80.5\end{array}$ & $\begin{array}{l}6.13-9.19 \\
64.4-96.6\end{array}$ & $\begin{array}{l}\text { Acceptable } \\
\text { Acceptable }\end{array}$ \\
\hline Chloroform & $\begin{array}{l}1 \\
2\end{array}$ & $\begin{array}{l}12.6 \\
59.1\end{array}$ & $\begin{array}{l}10.6 \\
63.8\end{array}$ & $\begin{array}{l}8.48-12.7 \\
51.0-76.6\end{array}$ & $\begin{array}{l}\text { Acceptable } \\
\text { Acceptable }\end{array}$ \\
\hline $\begin{array}{l}\text { Total } \\
\text { Trihalomethanes }\end{array}$ & $\begin{array}{l}1 \\
2\end{array}$ & $\begin{array}{l}58.18 \\
274.0\end{array}$ & $\begin{array}{l}53.1 \\
269\end{array}$ & $\begin{array}{c}42.5 \cdots 63.7 \\
215 \cdots 323\end{array}$ & $\begin{array}{l}\text { Acceptable } \\
\text { Acceptable }\end{array}$ \\
\hline
\end{tabular}


Table 7.2.23 (continued)

\begin{tabular}{|c|c|c|c|c|c|}
\hline \multirow{2}{*}{ Analytes } & \multirow{2}{*}{$\begin{array}{l}\text { Sainple } \\
\text { number }\end{array}$} & \multicolumn{2}{|c|}{ Values } & \multirow{2}{*}{$\begin{array}{l}\text { Acceptance } \\
\text { limits }\end{array}$} & \multirow{2}{*}{$\begin{array}{c}\text { Performance } \\
\text { evaluation }\end{array}$} \\
\hline & & Reported & True & & \\
\hline \multicolumn{6}{|c|}{ Insecticides $(\mu \mathrm{g} / L)$} \\
\hline Endrin & $\begin{array}{l}1 \\
2\end{array}$ & $\begin{array}{l}5.05 \\
0.351\end{array}$ & $\begin{array}{l}4.29 \\
0.293\end{array}$ & $\begin{array}{c}2.91-5.38 \\
0.182-0.414\end{array}$ & $\begin{array}{l}\text { Acceptable } \\
\text { Acceptable }\end{array}$ \\
\hline Lindane & $\begin{array}{l}1 \\
2\end{array}$ & $\begin{array}{l}4.46 \\
0.520\end{array}$ & $\begin{array}{l}3.16 \\
0.474\end{array}$ & $\begin{array}{c}2.15-4.15 \\
0.267-0.623\end{array}$ & $\begin{array}{l}\text { Unacceptable } \\
\text { Acceptable }\end{array}$ \\
\hline Methoxychlor & $\begin{array}{l}1 \\
2\end{array}$ & $\begin{array}{l}61.5 \\
5.06\end{array}$ & $\begin{array}{l}73.2 \\
5.37\end{array}$ & $\begin{array}{l}38.5-87.2 \\
3.28-6.22\end{array}$ & $\begin{array}{l}\text { Acceptable } \\
\text { Acceptable }\end{array}$ \\
\hline Toxaphene & $\begin{array}{l}3 \\
4\end{array}$ & $\begin{array}{l}7.90 \\
2.25\end{array}$ & $\begin{array}{l}7.58 \\
2.33\end{array}$ & $\begin{array}{l}4.57-9.85 \\
1.50-3.33\end{array}$ & $\begin{array}{l}\text { Acceptable } \\
\text { Acceptable }\end{array}$ \\
\hline \multicolumn{6}{|c|}{ Herbicides $(\mu \mathrm{g} / L)$} \\
\hline $2,4-5$ & $\begin{array}{l}1 \\
2\end{array}$ & $\begin{array}{l}3.24 \\
43.1\end{array}$ & $\begin{array}{l}5.70 \\
68.3\end{array}$ & $\begin{array}{l}2.11-8.09 \\
26.0-93.0\end{array}$ & $\begin{array}{l}\text { Acceptable } \\
\text { Acceptable }\end{array}$ \\
\hline $2,4,5-T P($ silvex) & $\begin{array}{l}1 \\
2\end{array}$ & $\begin{array}{c}1.76 \\
30.7\end{array}$ & $\begin{array}{l}4.30 \\
73.1\end{array}$ & $\begin{array}{l}1.56-5.76 \\
23.7-100\end{array}$ & $\begin{array}{l}\text { Acceptable } \\
\text { Acceptable }\end{array}$ \\
\hline \multicolumn{6}{|c|}{ Volatile organic compounds $(\mu \mathrm{g} / L)$} \\
\hline Benzene & 1 & 4.17 & 4.32 & $2.59-6.05$ & Acceptable \\
\hline Carbon tetrachloride & 1 & 8.20 & 4.56 & $2.74-6.38$ & Unacceptable \\
\hline 1,4-Dichlorobenzene & 1 & 2.88 & 2.50 & $1.50-3.50$ & Acceptable \\
\hline 1,2-Dichloroethane & 1 & 13.6 & 13.2 & $10.6 \cdots 15.8$ & Acceptable \\
\hline 1,1-Dichloroethylene & 1 & 5.18 & 5.36 & $3.22-7.50$ & Acceptable \\
\hline 1,1,1-Trichloroethane & 1 & 5.65 & 3.21 & $1.93-4.49$ & Unacceptable \\
\hline Trichloroethylene & 1 & 7.88 & 7.36 & $4.42-10.3$ & Acceptable \\
\hline Vinyl chloride & 1 & 5.29 & 4.35 & $2.61-6.09$ & Acceptable \\
\hline Bromobenzene & 2 & 5.54 & 5.78 & $3.47-8.09$ & Acceptable \\
\hline 4-Chlorotoluene & 2 & 7.06 & 6.59 & $3.95-9.23$ & Acceptable \\
\hline 1,3-Dichlorobenzene & 2 & 8.53 & 8.09 & $4.85-11.3$ & Acceptable \\
\hline C 1,2 Dichloroethylene & 2 & 7.16 & 7.24 & $4.34-10.1$ & Acceptable \\
\hline 1,3-Dichloropropane & 2 & 10.5 & 10.8 & $8.64-13.0$ & Acceptable \\
\hline 1,1-Dichloropropene & 2 & 9.04 & 8.88 & $5.33-12.4$ & Acceptable \\
\hline 1,1,1,2-Tetrachloroethane & 2 & 5.44 & 5.90 & $3.54-8.26$ & Acceptable \\
\hline Total xylenes & 2 & 8.76 & 14.0 & $11.2-16.8$ & Unacceptable \\
\hline
\end{tabular}


Table 7.2.23 (continued)

\begin{tabular}{|c|c|c|c|c|c|}
\hline \multirow{2}{*}{ Analytes } & \multirow{2}{*}{$\begin{array}{l}\text { Sample } \\
\text { number }\end{array}$} & \multicolumn{2}{|c|}{ Values } & \multirow{2}{*}{$\begin{array}{l}\text { Acceptance } \\
\text { limits }\end{array}$} & \multirow{2}{*}{$\begin{array}{l}\text { Performance } \\
\text { evaluation }\end{array}$} \\
\hline & & Reported & True & & \\
\hline \multicolumn{6}{|c|}{ Miscellaneous analytes } \\
\hline $\begin{array}{l}\text { Residual free } \\
\mathrm{Cl}(\mathrm{mg} / \mathrm{L})\end{array}$ & $\begin{array}{l}1 \\
2\end{array}$ & $\begin{array}{l}0.63 \\
1.14\end{array}$ & $\begin{array}{l}0.616 \\
1.16\end{array}$ & $\begin{array}{l}0.363-0.861 \\
0.840-1.44\end{array}$ & $\begin{array}{l}\text { Acceptable } \\
\text { Acceptable }\end{array}$ \\
\hline $\begin{array}{r}\text { Turbidity } \\
\text { (NTUs) }\end{array}$ & $\begin{array}{l}1 \\
2\end{array}$ & $\begin{array}{l}3.70 \\
1.15\end{array}$ & $\begin{array}{l}3.40 \\
1.00\end{array}$ & $\begin{array}{r}2.85-3.8 .3 \\
0.735-1.13\end{array}$ & $\begin{array}{l}\text { Acceptable } \\
\text { Unacceptable }\end{array}$ \\
\hline $\begin{array}{l}\left.\text { Calcium (as } \mathrm{CaCO}_{3}\right) \\
(\mathrm{mg} / \mathrm{L})\end{array}$ & 1 & 155 & 120 & $109-128$ & Unacceptable \\
\hline $\mathrm{pH}$ (units) & 1 & 9.15 & 9.12 & $8.84-9.34$ & Acceptable \\
\hline $\begin{array}{l}\text { Alkalinity }\left(\text { as } \mathrm{CaCO}_{3}\right) \\
(\mathrm{mg} / \mathrm{L})\end{array}$ & 1 & 37 & 34.8 & $32.0-40.5$ & Acceptable \\
\hline Sodium $(\mathrm{mg} / \mathrm{L})$ & 1 & 17.6 & 16.0 & $14.6-18.0$ & Acceptable \\
\hline Sulfate $(\mathrm{mg} / \mathrm{L})$ & $\begin{array}{l}1 \\
2\end{array}$ & $\begin{array}{c}6.25 \\
59.6\end{array}$ & $\begin{array}{r}5.30 \\
51.0\end{array}$ & $\begin{array}{l}3.01-7.48 \\
43.5-56.2\end{array}$ & $\begin{array}{l}\text { Acceptable } \\
\text { Unacceptable }\end{array}$ \\
\hline Total cyanide $(\mathrm{mg} / \mathrm{L})$ & $\begin{array}{l}1 \\
2\end{array}$ & $\begin{array}{l}0.272 \\
0.412\end{array}$ & $\begin{array}{l}0.310 \\
0.430\end{array}$ & $\begin{array}{l}0.222-0.364 \\
0.311-0.533\end{array}$ & $\begin{array}{l}\text { Acceptable } \\
\text { Acceptable }\end{array}$ \\
\hline
\end{tabular}


Table 7.2.24. Water supply performance evaluation study WP-023-ORNL, 1989

\begin{tabular}{|c|c|c|c|c|c|}
\hline \multirow{2}{*}{ Parameter } & \multirow{2}{*}{$\begin{array}{l}\text { Sumple } \\
\text { number }\end{array}$} & \multicolumn{2}{|c|}{ Values } & \multirow{2}{*}{$\begin{array}{c}\text { Acceptance } \\
\text { limits }\end{array}$} & \multirow{2}{*}{$\begin{array}{c}\text { Performance } \\
\text { evaluation }\end{array}$} \\
\hline & & Reported & True $^{a}$ & & \\
\hline
\end{tabular}

Trace metals, $\mu \mathrm{g} / \mathrm{L}$

Al

As

$\mathrm{Be}$

$\mathrm{Cd}$

Co

Cr

$\mathrm{Cu}$

$\mathrm{Fe}$

$\mathrm{Hg}$

$\mathrm{Mn}$

$\mathrm{Ni}$

$\mathrm{Pb}$

Se

V

$\mathrm{Zn}$

$\mathrm{Sb}$

Ag

$\mathrm{Tl}$

Mo

$\mathrm{Sr}$

$\mathrm{Ti}$

$\mathrm{pH}$, units

Specific conductivity $\mu \mathrm{mhos} / \mathrm{cm}$ at $25^{\circ} \mathrm{C}$

TDS at $180^{\circ} \mathrm{C}$

Total hardness, as $\mathrm{CaCO}_{3}$

Calcium, as $\mathrm{CaCO}_{3}$
1580

$51.5 \quad 51.9$

134

6.74

504

13.5

125

5.42

7.93

142

814

5.55

545

13.7

1680

10.2

26.7

3.3

702

13.5

593

10.2

1110

15.9

144

12.3

21.8

1390

1260

13.1

123

13.2

0.470

152

7.20

500

13.9

133

3.55

$1260-1820$

20.2-107

$\cdot 118-181$

$4.06-10.1$

434-553

8.15-20.0

$112-149$

150

$2.73-14.9$

150

834

$121-175$

6.65

578

696-974

$2.17-10.5$

497-633

16.0

$11.6-20.5$

1704

14.0

1500-1890

$2.76 \cdots 25.8$

30.0

$21.5-39.8$

3.59

700

$2.56-4.65$

16.3

630-752

606

12.4

1108

$9.00-22.2$

532-675

$3.83-21.5$

$942-1270$

16.3

$10.6-23.7$

140

99.4160

11.1

$5.81-15.2$

22.4

1459

1267

$13.6 \cdots 31.0$

$1270-1650$

$1110-1420$

12.6

135

$7.71-16.8$

$83.5-169$

$15.0 \quad 7.57-20.4$

$0.560 \quad 0.250-0.827$

7.20

8.12

$6.16-10.0$

13.8

$9.25 \cdots 19.5$

40.0
28.2

$30.1-51.7$

39.9

15.5-38.4

5.10

5.14

$2.40-7.83$

29.1

30.4

$22.7-37.8$

175

45.7

$136-21.5$

$30.7-60.5$
Acceptable

Acceptable

Acceptable

Acceptable

Acceptable

Acceptable

Acceptable

Check for error

Acceptable

Acceptable

Acceptable

Acceptable

Acceptable

Acceptable

Acceptable

Acceptable

Acceptable

Acceptable

Acceptable

Acceptable

Acceptable

Acceptable

Acceptable

Acceptable

Acceptable

Acceptable

Acceptable

Acceptable

Acceptable

Acceptable

Acceptable

Acceptable

Acceptable

Acceptable

Acceptable

Acceptable

Acceptable

Acceptable

Acceptable

Acceptable

Acceptable

Minerals, $m g / L$ (except as noted)

$\begin{array}{ccccl}3 & 7.94 & 7.9 & 7.62 \cdots 8.12 & \text { Acceptable } \\ 4 & 4.20 & 4.2 & 4.12 \cdots 4.28 & \text { Acceptable } \\ 1 & 249 & 234 & 214-257 & \text { Acceptable } \\ 2 & 1030 & 1030 & 922-1140 & \text { Acceptable } \\ 1 & 134 & 133 & 90.2-179 & \text { Acceptable } \\ 2 & 656 & 647 & 380-967 & \text { Acceptable } \\ 1 & 51.4 & 50.6 & 43.458 .0 & \text { Acceptable } \\ 2 & 350 & 342 & 312 \cdots 368 & \text { Acceptable } \\ 1 & 20.8 & 19.0 & 16.5 \cdots 22.2 & \text { Acceptable } \\ 2 & 101 & 93.3 & 80.9 \cdots 108 & \text { Acceptable }\end{array}$


Tab' 7.2 .24 (continued)

\begin{tabular}{|c|c|c|c|c|c|}
\hline \multirow{2}{*}{ Parameter } & \multirow{2}{*}{$\begin{array}{l}\text { Sample } \\
\text { number }\end{array}$} & \multicolumn{2}{|c|}{ Values } & \multirow{2}{*}{$\begin{array}{c}\text { Acceptance } \\
\text { limits }\end{array}$} & \multirow{2}{*}{$\begin{array}{c}\text { Performance } \\
\text { evaluation }\end{array}$} \\
\hline & & Reported & True $^{a}$ & & \\
\hline \multirow[t]{2}{*}{ Magnesium } & 1 & 0.775 & 0.771 & $0.599-0.934$ & Acceptable \\
\hline & 2 & 27.6 & 26.5 & $22.5-30.5$ & Acceptable \\
\hline \multirow[t]{2}{*}{ Sodium } & 1 & 15.3 & 14.9 & $12.9-16.9$ & Acceptable \\
\hline & 2 & 36.0 & 35.2 & $31.3-39.2$ & Acceptable \\
\hline \multirow[t]{2}{*}{ Potassium } & 1 & 14.0 & 14.0 & $11.7-15.8$ & Acceptable \\
\hline & 2 & 36.0 & 36.6 & $30.9-41.7$ & Acceptable \\
\hline Total alkalinity, & 1 & 24.0 & 23.4 & $20.4-27.9$ & Acceptable \\
\hline \multirow[t]{2}{*}{ as $\mathrm{CaCO}_{3}$} & 2 & 71.0 & 69.0 & $63.4-77.1$ & Acceptable \\
\hline & \multicolumn{4}{|c|}{ Minerals, $m_{\phi} / L$ (except as noted) } & \\
\hline \multirow[t]{2}{*}{ Chloride } & 1 & 40.1 & 38.6 & $33.5-44.6$ & Acceptable \\
\hline & 2 & 250 & 244 & $220-267$ & Acceptable \\
\hline \multirow[t]{2}{*}{ Fluoride } & 1 & 3.32 & 3.40 & $2.92-3.91$ & Acceptable \\
\hline & 2 & 0.406 & 0.219 & $0.149-0.304$ & Not acceptable \\
\hline \multirow[t]{2}{*}{ Sulfate } & 1 & 13.8 & 13.6 & $9.94-16.5$ & Acceptable \\
\hline & 2 & 50.5 & 50.0 & $40.7-57.7$ & Acceptable \\
\hline \multicolumn{6}{|c|}{ Nutrients, $m g / L$} \\
\hline \multirow{2}{*}{ Ammonia as $\mathrm{N}$} & 1 & 0.700 & 0.692 & $0.446-0.975$ & Acceptable \\
\hline & 2 & 3.49 & 3.50 & $2.65-4.33$ & Acceptable \\
\hline \multirow[t]{2}{*}{ Nitrate as $\mathrm{N}$} & 1 & 0.45 & 0.451 & $0.320-0.573$ & Acceptable \\
\hline & 2 & 2.30 & 2.41 & $1.83-2.92$ & Acceptable \\
\hline \multirow[t]{2}{*}{ Orthophosphate } & 1 & 0.30 & 0.299 & $0.244-0.356$ & Acceptable \\
\hline & 2 & 1.10 & 1.11 & $0.932-1.28$ & Acceptable \\
\hline \multirow[t]{2}{*}{ Kjeldahl-nitrogen } & 3 & 3.40 & 0.451 & D.L. ${ }^{b}-1.05$ & Not acceptable \\
\hline & 4 & 0.460 & 3.50 & $2.35-4.60$ & Not acceptable \\
\hline \multirow[t]{2}{*}{ Total phosphorous } & 3 & 2.90 & 0.351 & $0.273-0.455$ & Not acceptable \\
\hline & 4 & 0.350 & 2.75 & $2.23-3.41$ & Not acceptable \\
\hline \multicolumn{6}{|c|}{ Demands, $m g / L$} \\
\hline \multirow[t]{2}{*}{ TOC } & 1 & 38.2 & 79.6 & $65.7-91.0$ & Not acceptable \\
\hline & 2 & 5.34 & 10.4 & $8.24-13.3$ & Not acceptable \\
\hline \multicolumn{6}{|c|}{ Miscellaneous parameters, $m g / L$} \\
\hline \multirow[t]{2}{*}{ Total cyanide } & 1 & 0.291 & 0.270 & $0.164-0.347$ & Acceptable \\
\hline & 2 & 0.892 & 0.800 & $0.561-1.01$ & Acceptable \\
\hline \multirow{2}{*}{$\begin{array}{l}\text { Nonfilterable } \\
\text { residue }\end{array}$} & 1 & 91.6 & 90.7 & $83.1-95.6$ & Acceptable \\
\hline & 2 & 27.1 & 29.2 & $24.0-32.4$ & Acceptable \\
\hline \multirow[t]{2}{*}{ Oil and grease } & 1 & 44.1 & 43.9 & $20.9-54.5$ & Acceptable \\
\hline & 2 & 15.7 & 16.0 & $6.97-21.5$ & Acceptable \\
\hline & Mis & ellaneous $p$ & imeters, $n$ & & \\
\hline Total phenolics & 1 & 0.404 & 0.406 & $0.206-0.607$ & Acceptable \\
\hline & 2 & 1.91 & 1.99 & $1.07-2.91$ & Acceptable \\
\hline Total residue $\mathrm{Cl}$ & 1 & 0.650 & 0.602 & $0.374-0.788$ & Acceptable \\
\hline & 2 & 1.38 & 1.41 & $0.832-1.82$ & Acceptable \\
\hline
\end{tabular}

${ }^{a}$ Based upon theoretical calculations, or a reference value when necessary.

${ }^{b}$ D.L. $=$ Detection Limit. 
Table 7.2.25. Water supply performance evaluation study number WP-022-ORGDP, 1989

\begin{tabular}{|c|c|c|c|c|c|c|}
\hline \multirow{2}{*}{ Parameter } & \multirow{2}{*}{$\begin{array}{l}\text { Sample } \\
\text { No. }\end{array}$} & \multicolumn{2}{|c|}{ Values } & \multicolumn{2}{|c|}{ Limits } & \multirow{2}{*}{$\begin{array}{l}\text { Performance } \\
\text { evaluation }\end{array}$} \\
\hline & & Reported $^{a}$ & True $^{b}$ & Acceptance & Warning & \\
\hline \multicolumn{7}{|c|}{ Minerals $(m g / L)$} \\
\hline $\mathrm{pH}$ & $\begin{array}{l}3 \\
4\end{array}$ & $\begin{array}{l}5.83 \\
7.80\end{array}$ & $\begin{array}{l}5.80 \\
7.80\end{array}$ & $\begin{array}{l}5.66-5.91 \\
7.55-7.97\end{array}$ & $\begin{array}{l}5.69-5.88 \\
7.60-7.92\end{array}$ & $\begin{array}{l}\text { Acceptable } \\
\text { Acceptable }\end{array}$ \\
\hline $\begin{array}{l}\text { Special conditions, } \\
\mu \mathrm{mhos} / \mathrm{cm} \text { at } 25^{\circ} \mathrm{C}\end{array}$ & $\begin{array}{l}1 \\
2\end{array}$ & $\begin{array}{l}239 \\
757\end{array}$ & $\begin{array}{l}191 \\
755\end{array}$ & $\begin{array}{l}164-208 \\
665-824\end{array}$ & $\begin{array}{l}169-203 \\
684-804\end{array}$ & $\begin{array}{l}\text { Unacceptable } \\
\text { Acceptable }\end{array}$ \\
\hline TDS at $180^{\circ} \mathrm{C}$ & $\begin{array}{l}1 \\
2\end{array}$ & $\begin{array}{l}99.0 \\
424\end{array}$ & $\begin{array}{l}100 \\
401\end{array}$ & $\begin{array}{r}73.1-136 \\
298-.522\end{array}$ & $\begin{array}{r}80.9-128 \\
326-494\end{array}$ & $\begin{array}{l}\text { Acceptable } \\
\text { Acceptable }\end{array}$ \\
\hline $\begin{array}{l}\text { Total hardness, } \\
\text { as } \mathrm{CaCO}_{3}\end{array}$ & $\begin{array}{l}1 \\
2\end{array}$ & $\begin{array}{c}19.0 \\
167\end{array}$ & $\begin{array}{l}18.1 \\
170\end{array}$ & $\begin{array}{c}15.3-22.0 \\
154-183\end{array}$ & $\begin{array}{l}16.1-21.2 \\
158-179\end{array}$ & $\begin{array}{l}\text { Acceptable } \\
\text { Acceptable }\end{array}$ \\
\hline $\begin{array}{l}\text { Total alkalinity, } \\
\text { as } \mathrm{CaCO}_{3}\end{array}$ & $\begin{array}{l}1 \\
2\end{array}$ & $\begin{array}{r}8.00 \\
45.0\end{array}$ & $\begin{array}{l}8.59 \\
52.9\end{array}$ & $\begin{array}{l}5.73-12.7 \\
47.3-57.4\end{array}$ & $\begin{array}{l}6.59-11.8 \\
48.6-56.1\end{array}$ & $\begin{array}{l}\text { Acceptable } \\
\text { Unacceptable }\end{array}$ \\
\hline Chloride & $\begin{array}{l}1 \\
2\end{array}$ & $\begin{array}{l}14.0 \\
202\end{array}$ & $\begin{array}{l}14.9 \\
191\end{array}$ & $\begin{array}{c}12.4-17.3 \\
177-207\end{array}$ & $\begin{array}{c}13.0-16.7 \\
180-203\end{array}$ & $\begin{array}{l}\text { Acceptable } \\
\text { Acceptable }\end{array}$ \\
\hline Fluoride & $\begin{array}{l}1 \\
2\end{array}$ & $\begin{array}{l}1.62 \\
0.160\end{array}$ & $\begin{array}{l}1.60 \\
0.160\end{array}$ & $\begin{array}{c}1.39-1.78 \\
0.0837-0.235\end{array}$ & $\begin{array}{c}1.44-1.73 \\
0.103-0.216\end{array}$ & $\begin{array}{l}\text { Acceptable } \\
\text { Acceptable }\end{array}$ \\
\hline Sulfate & $\begin{array}{l}1 \\
2\end{array}$ & $\begin{array}{l}37.0 \\
6.00\end{array}$ & $\begin{array}{c}38.0 \\
6.10\end{array}$ & $\begin{array}{l}30.6-44.3 \\
2.92-8.80\end{array}$ & $\begin{array}{l}32.3-42.6 \\
3.65-8.07\end{array}$ & $\begin{array}{l}\text { Acceptable } \\
\text { Acceptable }\end{array}$ \\
\hline \multicolumn{7}{|c|}{ Nutrients $(\mathrm{mg} / L)$} \\
\hline Ammonia-N & $\begin{array}{l}1 \\
2\end{array}$ & $\begin{array}{l}3.22 \\
14.6\end{array}$ & $\begin{array}{c}3.00 \\
13.0\end{array}$ & $\begin{array}{l}2.31-3.66 \\
10.2-15.5\end{array}$ & $\begin{array}{l}2.47-3.50 \\
10.9-14.9\end{array}$ & $\begin{array}{l}\text { Acceptable } \\
\text { Acceptable }\end{array}$ \\
\hline Nitrate-N & $\begin{array}{l}1 \\
2\end{array}$ & $\begin{array}{l}0.980 \\
8.78\end{array}$ & $\begin{array}{l}0.950 \\
8.50\end{array}$ & $\begin{array}{r}0.745-1.16 \\
6.94-10.1\end{array}$ & $\begin{array}{r}0.794-1.11 \\
7.32-9.72\end{array}$ & $\begin{array}{l}\text { Acceptable } \\
\text { Acceptable }\end{array}$ \\
\hline Orthophosphate & $\begin{array}{l}1 \\
2\end{array}$ & $\begin{array}{l}0.910 \\
2.84\end{array}$ & $\begin{array}{l}0.940 \\
3.80\end{array}$ & $\begin{array}{r}0.789-1.09 \\
3.25-4.36\end{array}$ & $\begin{array}{r}0.825-1.05 \\
3.39-4.23\end{array}$ & $\begin{array}{l}\text { Acceptable } \\
\text { Unacceptable }\end{array}$ \\
\hline Kjeldahl-N & $\begin{array}{l}3 \\
4\end{array}$ & $\begin{array}{l}7.78 \\
15.7\end{array}$ & $\begin{array}{l}8.00 \\
15.0\end{array}$ & $\begin{array}{l}5.88-9.90 \\
11.4-18.2\end{array}$ & $\begin{array}{l}6.36-9.42 \\
12.2-17.4\end{array}$ & $\begin{array}{l}\text { Acceptable } \\
\text { Acceptable }\end{array}$ \\
\hline Total phosphorus & $\begin{array}{l}3 \\
4\end{array}$ & $\begin{array}{l}1.57 \\
6.85\end{array}$ & $\begin{array}{l}2.50 \\
7.01\end{array}$ & $\begin{array}{l}2.03-3.01 \\
5.60-8.38\end{array}$ & $\begin{array}{l}2.15-2.89 \\
5.93-8.05\end{array}$ & $\begin{array}{l}\text { Unacceptable } \\
\text { Acceptable }\end{array}$ \\
\hline \multicolumn{7}{|c|}{ Demands $(m g / L)$} \\
\hline COD & $\begin{array}{l}1 \\
2\end{array}$ & $\begin{array}{l}37.0 \\
93.0\end{array}$ & $\begin{array}{l}28.5 \\
91.7\end{array}$ & $\begin{array}{l}19.7-44.0 \\
74.4-109\end{array}$ & $\begin{array}{l}22.7-40.9 \\
78.8-105\end{array}$ & $\begin{array}{l}\text { Acceptable } \\
\text { Acceptable }\end{array}$ \\
\hline TOC & $\begin{array}{l}1 \\
4\end{array}$ & $\begin{array}{l}13.0 \\
42.0\end{array}$ & $\begin{array}{l}11.5 \\
37.0\end{array}$ & $\begin{array}{l}9.45-16.0 \\
30.6-4 j .2\end{array}$ & $\begin{array}{l}10.3-15.2 \\
32.3-41.6\end{array}$ & $\begin{array}{l}\text { Acceptable } \\
\text { Check for error }\end{array}$ \\
\hline 5-d BOD & $\begin{array}{l}1 \\
2\end{array}$ & $\begin{array}{l}22.0 \\
69\end{array}$ & $\begin{array}{l}18.6 \\
59.7\end{array}$ & $\begin{array}{l}13.1-30.9 \\
41.7-85.7\end{array}$ & $\begin{array}{l}15.3-2.8 .7 \\
47.2-80.3\end{array}$ & $\begin{array}{l}\text { Acceptable } \\
\text { Acceptable }\end{array}$ \\
\hline \multicolumn{7}{|c|}{ Miscellaneous parameters $(m g / L)$} \\
\hline Total cyanide & $\begin{array}{l}1 \\
2\end{array}$ & $\begin{array}{l}0.960 \\
0.070\end{array}$ & $\begin{array}{l}0.890 \\
0.070\end{array}$ & $\begin{array}{c}0.562-1.14 \\
0.0300-0.0998\end{array}$ & $\begin{array}{c}0.635-1.07 \\
0.0388-0.0910\end{array}$ & $\begin{array}{l}\text { Acceptable } \\
\text { Acceptable }\end{array}$ \\
\hline Nonfilterable residue & $\begin{array}{l}1 \\
2\end{array}$ & $\begin{array}{l}28.0 \\
41.0\end{array}$ & $\begin{array}{l}29.7 \\
41.9\end{array}$ & $\begin{array}{l}24.2 \cdots 33.3 \\
33.3 \div 46.6\end{array}$ & $\begin{array}{l}25.3-\cdots 2.2 \\
34.9-45.0\end{array}$ & $\begin{array}{l}\text { Acceptable } \\
\text { Acceptable }\end{array}$ \\
\hline
\end{tabular}


Table 7.2.25 (continued)

\begin{tabular}{|c|c|c|c|c|c|c|}
\hline \multirow{2}{*}{ Parameter } & \multirow{2}{*}{$\begin{array}{c}\text { Sample } \\
\text { No. }\end{array}$} & \multicolumn{2}{|c|}{ Values } & \multicolumn{2}{|c|}{ Limits } & \multirow{2}{*}{$\begin{array}{l}\text { Performance } \\
\text { evaluation }\end{array}$} \\
\hline & & Reported $^{a}$ & True $^{b}$ & Acceptance & Warning & \\
\hline Oil and grease & $\begin{array}{l}1 \\
2\end{array}$ & $\begin{array}{l}10.6 \\
18.1\end{array}$ & $\begin{array}{l}12.0 \\
19.8\end{array}$ & $\begin{array}{l}6.32-16.4 \\
10.7-24.8\end{array}$ & $\begin{array}{l}7.57-15.1 \\
12.5-23.0\end{array}$ & $\begin{array}{l}\text { Acceptable } \\
\text { Acceptable }\end{array}$ \\
\hline Total phenolics & $\begin{array}{l}1 \\
2\end{array}$ & $\begin{array}{l}20.5 \\
0.500\end{array}$ & $\begin{array}{l}0.268 \\
0.646\end{array}$ & $\begin{array}{l}0.127-0.409 \\
0.307-1.01\end{array}$ & $\begin{array}{l}0.162-0.374 \\
0.395-0.920\end{array}$ & $\begin{array}{l}\text { Unacceptable } \\
\text { Acceptable }\end{array}$ \\
\hline Total residual chlorine & $\begin{array}{l}1 \\
2\end{array}$ & $\begin{array}{l}2.06 \\
4.12\end{array}$ & $\begin{array}{l}1.40 \\
4.00\end{array}$ & $\begin{array}{r}0.906-1.72 \\
2.76-5.01\end{array}$ & $\begin{array}{l}1.01-1.61 \\
3.05-4.71\end{array}$ & $\begin{array}{l}\text { Unacceptable } \\
\text { Acceptable }\end{array}$ \\
\hline
\end{tabular}

${ }^{a}$ Based on theoretical calculations or a reference value when necessary. 
Table 7.2.26. Water supply performance evaluation study number WP-023_ORGDP, 1989

\begin{tabular}{|c|c|c|c|c|c|c|}
\hline \multirow{2}{*}{ Parameter } & \multirow{2}{*}{$\begin{array}{l}\text { Sample } \\
\text { No. }\end{array}$} & \multicolumn{2}{|c|}{ Values } & \multicolumn{2}{|c|}{ Limits } & \multirow{2}{*}{$\begin{array}{l}\text { Performance } \\
\text { evaluation }\end{array}$} \\
\hline & & Reported $^{a}$ & True $^{b}$ & Acceptance & Warning & \\
\hline \multicolumn{7}{|c|}{ Minerals $(m g / L)$} \\
\hline $\mathrm{pH}$ & $\begin{array}{l}3 \\
4\end{array}$ & $\begin{array}{l}7.91 \\
4.22\end{array}$ & $\begin{array}{l}7.9 \\
4.2\end{array}$ & $\begin{array}{l}7.62-8.12 \\
4.12-4.28\end{array}$ & $\begin{array}{l}7.68-8.06 \\
4.14-4.26\end{array}$ & $\begin{array}{l}\text { Acceptable } \\
\text { Acceptable }\end{array}$ \\
\hline $\begin{array}{l}\text { Special conditions, } \\
\mu \text { mhos } / \mathrm{cm} \text { at } 25^{\circ} \mathrm{C}\end{array}$ & $\begin{array}{l}1 \\
2\end{array}$ & $\begin{array}{r}238 \\
1040\end{array}$ & $\begin{array}{r}234 \\
1030\end{array}$ & $\begin{array}{l}214-257 \\
922-1140\end{array}$ & $\begin{array}{l}220-252 \\
949-1110\end{array}$ & $\begin{array}{l}\text { Acceptable } \\
\text { Acceptable }\end{array}$ \\
\hline TDS at $180^{\circ} \mathrm{C}$ & $\begin{array}{l}1 \\
2\end{array}$ & $\begin{array}{l}109 \\
659\end{array}$ & $\begin{array}{l}133 \\
647\end{array}$ & $\begin{array}{r}90.2-179 \\
380-967\end{array}$ & $\begin{array}{l}101-16^{2} \\
453-394\end{array}$ & $\begin{array}{l}\text { Acceptable } \\
\text { Acceptable }\end{array}$ \\
\hline $\begin{array}{l}\text { Total hardness, } \\
\text { as } \mathrm{CaCO}_{3}\end{array}$ & $\begin{array}{l}1 \\
2\end{array}$ & $\begin{array}{l}50.8 \\
337\end{array}$ & $\begin{array}{l}50.6 \\
342\end{array}$ & $\begin{array}{c}43.4-58.8 \\
312-368\end{array}$ & $\begin{array}{c}45.2-56.2 \\
319 \cdots 361\end{array}$ & $\begin{array}{l}\text { Acceptable } \\
\text { Acceptable }\end{array}$ \\
\hline $\begin{array}{l}\text { Total alkalinity, } \\
\text { as } \mathrm{CaCO}_{3}\end{array}$ & $\begin{array}{l}1 \\
2\end{array}$ & $\begin{array}{l}24 \\
70.0\end{array}$ & $\begin{array}{l}23.4 \\
69.0\end{array}$ & $\begin{array}{l}20.4-27.9 \\
63.4-77.1\end{array}$ & $\begin{array}{l}21.3-27.0 \\
65.1-75.4\end{array}$ & $\begin{array}{l}\text { Acceptable } \\
\text { Acceptable }\end{array}$ \\
\hline Chloride & $\begin{array}{l}1 \\
2\end{array}$ & $\begin{array}{l}39.3 \\
238\end{array}$ & $\begin{array}{l}38.6 \\
244\end{array}$ & $\begin{array}{c}33.5-44.5 \\
220-267\end{array}$ & $\begin{array}{c}34.9-43.3 \\
226261\end{array}$ & $\begin{array}{l}\text { Acceptable } \\
\text { Acceptable }\end{array}$ \\
\hline Fluoride & $\begin{array}{l}1 \\
2\end{array}$ & $\begin{array}{l}3.60 \\
0.24\end{array}$ & $\begin{array}{l}3.40 \\
0.219\end{array}$ & $\begin{array}{c}2.92-3.91 \\
0.149-0.304\end{array}$ & $\begin{array}{c}3.04 \cdots 3.79 \\
0.168-0.285\end{array}$ & $\begin{array}{l}\text { Acceptable } \\
\text { Acceptable }\end{array}$ \\
\hline Sulfate & $\begin{array}{l}1 \\
2\end{array}$ & $\begin{array}{l}13.0 \\
50.5\end{array}$ & $\begin{array}{l}13.6 \\
50.0\end{array}$ & $\begin{array}{l}9.94-16.5 \\
40.7-57.7\end{array}$ & $\begin{array}{l}10.8-15.7 \\
42.8-55.6\end{array}$ & $\begin{array}{l}\text { Acceptable } \\
\text { Acceptable }\end{array}$ \\
\hline \multicolumn{7}{|c|}{ Nutrients $(m g / L)$} \\
\hline Ammonia- $N$ & $\begin{array}{l}1 \\
2\end{array}$ & $\begin{array}{l}0.76 \\
3.64\end{array}$ & $\begin{array}{l}0.692 \\
3.50\end{array}$ & $\begin{array}{c}0.446-0.975 \\
2.65-4.33\end{array}$ & $\begin{array}{c}0.510-0.9: 1 \\
2.85-4.13\end{array}$ & $\begin{array}{l}\text { Acceptable } \\
\text { Acceptable }\end{array}$ \\
\hline Nitrate $\mathbf{N}$ & $\begin{array}{l}1 \\
2\end{array}$ & $\begin{array}{l}0.473 \\
2.7 !\end{array}$ & $\begin{array}{l}0.451 \\
2.41\end{array}$ & $\begin{array}{c}0.320-0.573 \\
1.83-2.92\end{array}$ & $\begin{array}{c}0.350-0.542 \\
1.96-2.79\end{array}$ & $\begin{array}{l}\text { Acceptable } \\
\text { Acceptable }\end{array}$ \\
\hline Orthophosphate & $\begin{array}{l}1 \\
2\end{array}$ & $\begin{array}{l}0.28 \\
1.04\end{array}$ & $\begin{array}{l}0.299 \\
1.11\end{array}$ & $\begin{array}{l}0.2: 4-0.356 \\
0.932-1.28\end{array}$ & $\begin{array}{l}0.257-0.342 \\
0.973-1.24\end{array}$ & $\begin{array}{l}\text { Acceptable } \\
\text { Acceptable }\end{array}$ \\
\hline Kjeldahl-N & $\begin{array}{l}3 \\
4\end{array}$ & $\begin{array}{l}0.336 \\
3.76\end{array}$ & $\begin{array}{l}0.451 \\
3.50\end{array}$ & $\begin{array}{c}\mathrm{DL}^{b}-1.05 \\
2.35-4.60\end{array}$ & $\begin{array}{c}0.121-0.927 \\
2.62-4.33\end{array}$ & $\begin{array}{l}\text { Acceptable } \\
\text { Acceptable }\end{array}$ \\
\hline Total phosphorus & $\begin{array}{l}3 \\
4\end{array}$ & $\begin{array}{l}0.388 \\
3.00\end{array}$ & $\begin{array}{l}0.351 \\
2.75\end{array}$ & $\begin{array}{c}0.273-0.455 \\
2.23-3.41\end{array}$ & $\begin{array}{c}0.294-0.433 \\
2.37-3.27\end{array}$ & $\begin{array}{l}\text { Acceptable } \\
\text { Acceptable }\end{array}$ \\
\hline \multicolumn{7}{|c|}{ Demands $(m g / L)$} \\
\hline COD & $\begin{array}{l}1 \\
2\end{array}$ & $\begin{array}{r}206 \\
28\end{array}$ & $\begin{array}{l}201 \\
26.3\end{array}$ & $\begin{array}{c}161-221 \\
15.3-33.5\end{array}$ & $\begin{array}{c}168-213 \\
17.5-31.5\end{array}$ & $\begin{array}{l}\text { Acceptable } \\
\text { Acceptable }\end{array}$ \\
\hline TOC & $\begin{array}{l}1 \\
2\end{array}$ & $\begin{array}{l}76.7 \\
10.6\end{array}$ & $\begin{array}{l}79.6 \\
10.4\end{array}$ & $\begin{array}{l}65.7-91.0 \\
8.24-13.3\end{array}$ & $\begin{array}{l}69.0-87.6 \\
8.90 \cdots 12.6\end{array}$ & $\begin{array}{l}\text { Acceptable } \\
\text { Acceptable }\end{array}$ \\
\hline$j-d$ BOD & $\begin{array}{l}1 \\
2\end{array}$ & $\begin{array}{l}96.2 \\
12.9\end{array}$ & $\begin{array}{l}127 \\
18.0\end{array}$ & $\begin{array}{l}78.9-176 \\
9.42-26.5\end{array}$ & $\begin{array}{l}91.0-164 \\
11.5-24.4\end{array}$ & $\begin{array}{l}\text { Acceptable } \\
\text { Acceptable }\end{array}$ \\
\hline \multicolumn{7}{|c|}{ Miscellaneous parameters $(\mathrm{mg} / \mathrm{L})$} \\
\hline Total cyanide & $\begin{array}{l}1 \\
2\end{array}$ & $\begin{array}{l}0.30 \\
0.78\end{array}$ & $\begin{array}{l}0.270 \\
0.800\end{array}$ & $\begin{array}{l}0.164-0.347 \\
0.561 \cdots 1.01\end{array}$ & $\begin{array}{l}0.187-0.324 \\
0.618-0.955\end{array}$ & $\begin{array}{l}\text { Acceptable } \\
\text { Acceptable }\end{array}$ \\
\hline Nonfilterable residue & $\begin{array}{l}1 \\
2\end{array}$ & $\begin{array}{l}89.5 \\
27.0\end{array}$ & $\begin{array}{l}90.7 \\
29.2\end{array}$ & $\begin{array}{l}83.1-95.6 \\
24.0-32.4\end{array}$ & $\begin{array}{l}84.7 \ldots 94.1 \\
25.1 \cdots 31.4\end{array}$ & $\begin{array}{l}\text { Acceptable } \\
\text { Acceptable }\end{array}$ \\
\hline
\end{tabular}


Table 7.2 .26 (continued)

\begin{tabular}{|c|c|c|c|c|c|c|}
\hline \multirow{2}{*}{ Parameter } & \multirow{2}{*}{$\begin{array}{c}\text { Sample } \\
\text { No. }\end{array}$} & \multicolumn{2}{|c|}{ Values } & \multicolumn{2}{|c|}{ Limits } & \multirow{2}{*}{$\begin{array}{l}\text { Performance } \\
\text { evaluation }\end{array}$} \\
\hline & & Reported $^{a}$ & True $^{b}$ & Acceptance & Warning & \\
\hline Oil and grease & $\begin{array}{l}1 \\
2\end{array}$ & $\begin{array}{l}41.9 \\
14.2\end{array}$ & $\begin{array}{l}43.9 \\
16.0\end{array}$ & $\begin{array}{l}20.9-54.5 \\
6.97-21.5\end{array}$ & $\begin{array}{l}25.1-50.3 \\
8.79 \cdots 19.7\end{array}$ & $\begin{array}{l}\text { Acceptable } \\
\text { Acceptable }\end{array}$ \\
\hline Total phenolics & $\begin{array}{l}1 \\
2\end{array}$ & $\begin{array}{l}0.39 \\
2.00\end{array}$ & $\begin{array}{l}0.406 \\
1.99\end{array}$ & $\begin{array}{c}0.206-0.607 \\
1.07-2.91\end{array}$ & $\begin{array}{c}0.257-0.556 \\
1.30-2.68\end{array}$ & $\begin{array}{l}\text { Acceptable } \\
\text { Acceptable }\end{array}$ \\
\hline Total residual chlorine & $\begin{array}{l}1 \\
2\end{array}$ & $\begin{array}{l}0.5 ! \\
1.26\end{array}$ & $\begin{array}{l}0.602 \\
1.41\end{array}$ & $\begin{array}{l}0.374-0.788 \\
0.832-1.82\end{array}$ & $\begin{array}{l}0.429-0.733 \\
0.961-1.69\end{array}$ & $\begin{array}{l}\text { Acceptable } \\
\text { Acceptable }\end{array}$ \\
\hline
\end{tabular}

${ }^{a}$ Based on theoretical calculations or a reference value when necessary.

${ }^{b}$ Detection limit $(D L)=$ acceptance limit. 
Table 7.2.27. Water supply performance evaluation study number WS-023-ORGDP, 1989

\begin{tabular}{|c|c|c|c|c|c|}
\hline \multirow{2}{*}{ Parameter } & \multirow{2}{*}{$\begin{array}{c}\text { Sample } \\
\text { No. }\end{array}$} & \multicolumn{2}{|c|}{ Values } & \multirow{2}{*}{$\begin{array}{l}\text { Acceptance } \\
\text { limits }\end{array}$} & \multirow{2}{*}{$\begin{array}{l}\text { Performance } \\
\text { evaluation }\end{array}$} \\
\hline & & Reported & True $^{a}$ & & \\
\hline \multicolumn{6}{|c|}{ Trace metals $(\mu g / L)$} \\
\hline As & $\begin{array}{l}1 \\
2\end{array}$ & $\begin{array}{l}59.8 \\
3.95\end{array}$ & $\begin{array}{l}60.5 \\
3.56\end{array}$ & $\begin{array}{l}49.7-68.7 \\
2.29-4.41\end{array}$ & $\begin{array}{l}\text { Acceptable } \\
\text { Acceptable }\end{array}$ \\
\hline $\mathrm{Ba}$ & $\begin{array}{l}1 \\
2\end{array}$ & $\begin{array}{l}117 \\
53.8\end{array}$ & $\begin{array}{l}120 \\
53.3\end{array}$ & $\begin{array}{l}98.4-137 \\
39.9-64.2\end{array}$ & $\begin{array}{l}\text { Acceptable } \\
\text { Acceptable }\end{array}$ \\
\hline $\mathrm{Cd}$ & $\begin{array}{l}1 \\
2\end{array}$ & $\begin{array}{l}5.82 \\
35.2\end{array}$ & $\begin{array}{l}6.05 \\
33.6\end{array}$ & $\begin{array}{l}4.78-6.98 \\
26.0-39.4\end{array}$ & $\begin{array}{l}\text { Acceptable } \\
\text { Acceptable }\end{array}$ \\
\hline $\mathrm{Cr}$ & $\begin{array}{l}1 \\
2\end{array}$ & $\begin{array}{l}20.9 \\
54.4\end{array}$ & $\begin{array}{l}19.7 \\
53.2\end{array}$ & $\begin{array}{l}15.9-24.6 \\
45.9-60.5\end{array}$ & $\begin{array}{l}\text { Acceptable } \\
\text { Acceptable }\end{array}$ \\
\hline $\mathrm{Cu}$ & $\begin{array}{l}1 \\
2\end{array}$ & $\begin{array}{l}42.5 \\
674^{b}\end{array}$ & $\begin{array}{l}44.0 \\
660\end{array}$ & $\begin{array}{c}37.5-48.8 \\
598-696\end{array}$ & $\begin{array}{l}\text { Acceptable } \\
\text { Acceptable }\end{array}$ \\
\hline $\mathrm{Pb}$ & $\begin{array}{l}1 \\
2\end{array}$ & $\begin{array}{l}8.30 \\
60.8\end{array}$ & $\begin{array}{c}8.80 \\
51.0\end{array}$ & $\begin{array}{l}6.25-11.9 \\
45.3-59.4\end{array}$ & $\begin{array}{l}\text { Acceptable } \\
\text { Not acceptable }\end{array}$ \\
\hline $\mathrm{Hg}$ & $\begin{array}{l}1 \\
2\end{array}$ & $\begin{array}{l}3.32^{b} \\
4.62\end{array}$ & $\begin{array}{l}2.80 \\
4.00\end{array}$ & $\begin{array}{l}1.98-3.28 \\
2.98-4.84\end{array}$ & $\begin{array}{l}\text { Not acceptable } \\
\text { Acceptable }\end{array}$ \\
\hline Se & $\begin{array}{l}1 \\
2\end{array}$ & $\begin{array}{l}18.8 \\
60.7\end{array}$ & $\begin{array}{l}19.0 \\
57.0\end{array}$ & $\begin{array}{r}14.0-22.9 \\
44.6-67.3\end{array}$ & $\begin{array}{l}\text { Acceptable } \\
\text { Acceptable }\end{array}$ \\
\hline$A g$ & $\begin{array}{l}1 \\
2\end{array}$ & $\begin{array}{l}30.1 \\
4.00\end{array}$ & $\begin{array}{l}26.0 \\
3.42\end{array}$ & $\begin{array}{l}22.7-30.5 \\
2.48-4.88\end{array}$ & $\begin{array}{l}\text { Acceptable } \\
\text { Acceptable }\end{array}$ \\
\hline \multicolumn{6}{|c|}{ Nitrate/nitrite/fluoride $(\mathrm{mg} / \mathrm{L})$} \\
\hline Nitrate, as $\mathbf{N}$ & $\begin{array}{l}1 \\
2\end{array}$ & $\begin{array}{l}0.49 \\
4.30\end{array}$ & $\begin{array}{l}0.492 \\
4.00\end{array}$ & $\begin{array}{c}0.296-0.766 \\
3.00-5.27\end{array}$ & $\begin{array}{l}\text { Acceptable } \\
\text { Acceptable }\end{array}$ \\
\hline Nitrite, as $\mathrm{N}$ & $\begin{array}{l}1 \\
2\end{array}$ & $\begin{array}{l}0.30 \\
0.76\end{array}$ & $\begin{array}{l}0.197 \\
0.800\end{array}$ & $\begin{array}{l}0.165-0.230 \\
0.691-0.910\end{array}$ & $\begin{array}{l}\text { Not acceptable } \\
\text { Acceptable }\end{array}$ \\
\hline Fluoride & $\begin{array}{l}1 \\
2\end{array}$ & $\begin{array}{l}0.22 \\
1.24\end{array}$ & $\begin{array}{l}0.196 \\
1.30\end{array}$ & $\begin{array}{c}0.163-0.235 \\
1.17-1.43\end{array}$ & $\begin{array}{l}\text { Acceptable } \\
\text { Acceptable }\end{array}$ \\
\hline \multicolumn{6}{|c|}{ Insecticides $(\mu g / L)$} \\
\hline Chlordane & $\begin{array}{l}5 \\
6\end{array}$ & $\begin{array}{l}5.96^{b} \\
1.64^{b}\end{array}$ & $\begin{array}{l}8.83 \\
2.21\end{array}$ & $\begin{array}{r}2.22-12.1 \\
0.796-3.01\end{array}$ & $\begin{array}{l}\text { Acceptable } \\
\text { Acceptable }\end{array}$ \\
\hline Endrin & $\begin{array}{l}1 \\
2\end{array}$ & $\begin{array}{l}0.24 \\
2.19\end{array}$ & $\begin{array}{l}0.195 \\
2.34\end{array}$ & $\begin{array}{c}0.101-0.281 \\
1.55-2.99\end{array}$ & $\begin{array}{l}\text { Acceptable } \\
\text { Acceptable }\end{array}$ \\
\hline Heptacnlor & $\begin{array}{l}7 \\
8\end{array}$ & $\begin{array}{l}0.50^{b} \\
0.14^{b}\end{array}$ & $\begin{array}{l}0.836 \\
0.183\end{array}$ & $\begin{array}{r}0.334-0.977 \\
0.0728-0.245\end{array}$ & $\begin{array}{l}\text { Acceptable } \\
\text { Acceptable }\end{array}$ \\
\hline Heptachlor epoxide & $\begin{array}{l}7 \\
8\end{array}$ & $\begin{array}{l}0.89 \\
0.27\end{array}$ & $\begin{array}{l}1.03 \\
0.275\end{array}$ & $\begin{array}{l}0.611-1.32 \\
0.144-0.365\end{array}$ & $\begin{array}{l}\text { Acceptable } \\
\text { Acceptable }\end{array}$ \\
\hline Lindane & $\begin{array}{l}1 \\
2\end{array}$ & $\begin{array}{l}0.25 \\
1.64\end{array}$ & $\begin{array}{l}0.237 \\
1.74\end{array}$ & $\begin{array}{c}0.0909-0.388 \\
0.936-2.64\end{array}$ & $\begin{array}{l}\text { Acceptable } \\
\text { Acceptable }\end{array}$ \\
\hline Methoxychlor & $\begin{array}{l}1 \\
2\end{array}$ & $\begin{array}{c}3.29^{b} \\
19.8^{b}\end{array}$ & $\begin{array}{l}4.39 \\
26.9\end{array}$ & $\begin{array}{l}2.61-5.40 \\
15.9-31.1\end{array}$ & $\begin{array}{l}\text { Acceptable } \\
\text { Acceptable }\end{array}$ \\
\hline Toxaphene & $\begin{array}{l}3 \\
4\end{array}$ & $\begin{array}{l}4.53 \\
8.83\end{array}$ & $\begin{array}{l}3.20 \\
10.2\end{array}$ & $\begin{array}{l}1.67-4.30 \\
6.35-13.7\end{array}$ & $\begin{array}{l}\text { Not acceptabie } \\
\text { Acceptable }\end{array}$ \\
\hline
\end{tabular}


Table 7.2.27 (continued)

\begin{tabular}{|c|c|c|c|c|c|}
\hline \multirow{2}{*}{ Parameter } & \multirow{2}{*}{$\begin{array}{c}\text { Sample } \\
\text { No. }\end{array}$} & \multicolumn{2}{|c|}{ Values } & \multirow{2}{*}{$\begin{array}{l}\text { Acceptance } \\
\text { limits }\end{array}$} & \multirow{2}{*}{$\begin{array}{l}\text { Performance } \\
\text { evaluation }\end{array}$} \\
\hline & & Reported & True $^{a}$ & & \\
\hline \multicolumn{6}{|c|}{ Herbicides $(\mu \mathrm{g} / \mathrm{L})$} \\
\hline $2,4-\mathrm{D}$ & $\begin{array}{l}1 \\
2\end{array}$ & $\begin{array}{l}83.4 \\
1.85\end{array}$ & $\begin{array}{c}84.2 \\
1.79\end{array}$ & $\begin{array}{c}30.3-121 \\
0.320-3.14\end{array}$ & $\begin{array}{l}\text { Acceptable } \\
\text { Acceptable }\end{array}$ \\
\hline 2,4,5-TP, Silvex & $\begin{array}{l}1 \\
2\end{array}$ & $\begin{array}{l}249 \\
11.0^{b}\end{array}$ & $\begin{array}{c}57.1 \\
2.30\end{array}$ & $\begin{array}{r}7.16-91.8 \\
0.881-3.02\end{array}$ & $\begin{array}{l}\text { Not acceptaiole } \\
\text { Not acceptable }\end{array}$ \\
\hline
\end{tabular}

${ }^{a}$ Based on theoretical calculations or a reference value when necessary.

${ }^{b}$ Significant general method bias is anticipated for this result. 
Table 7.2.28. Water suppiy performance evaluation study number WS-024-ORGDP, 1989

\begin{tabular}{|c|c|c|c|c|c|}
\hline \multirow{2}{*}{ Parameter } & \multirow{2}{*}{$\begin{array}{c}\text { Sample } \\
\text { No. }\end{array}$} & \multicolumn{2}{|c|}{ Values } & \multirow{2}{*}{$\begin{array}{l}\text { Acceptance } \\
\text { limits }\end{array}$} & \multirow{2}{*}{$\begin{array}{c}\text { Performance } \\
\text { evaluation }\end{array}$} \\
\hline & & Reported & True & & \\
\hline \multicolumn{6}{|c|}{ Trace metals $(\mu g / L)$} \\
\hline $\mathrm{Sb}$ & $\begin{array}{l}3 \\
4\end{array}$ & $\begin{array}{c}6.10 \\
34.9\end{array}$ & $\begin{array}{c}9.00 \\
42.0\end{array}$ & $\begin{array}{l}6.27-12.3 \\
34.3-53.1\end{array}$ & $\begin{array}{l}\text { Unacceptable } \\
\text { Acceptable }\end{array}$ \\
\hline As & $\begin{array}{l}1 \\
2\end{array}$ & $\begin{array}{l}9.40 \\
75.5\end{array}$ & $\begin{array}{l}10.2 \\
80.7\end{array}$ & $\begin{array}{l}7.88-11.6 \\
67.1-92.4\end{array}$ & $\begin{array}{l}\text { Acceptable } \\
\text { Acceptable }\end{array}$ \\
\hline $\mathrm{Ba}$ & $\begin{array}{l}1 \\
2\end{array}$ & $\begin{array}{l}798 \\
33.2\end{array}$ & $\begin{array}{l}853 \\
41.0\end{array}$ & $\begin{array}{c}729-944 \\
31.7-49.7\end{array}$ & $\begin{array}{l}\text { Acceptable } \\
\text { Acceptable }\end{array}$ \\
\hline $\mathrm{Be}$ & $\begin{array}{l}3 \\
4\end{array}$ & $\begin{array}{l}0.580 \\
0.970\end{array}$ & $\begin{array}{l}0.600 \\
1.00\end{array}$ & $\begin{array}{l}0.467-0.771 \\
0.832 \cdots 1.19\end{array}$ & $\begin{array}{l}\text { Acceptable } \\
\text { Acceptable }\end{array}$ \\
\hline $\mathrm{Cd}$ & $\begin{array}{l}1 \\
2\end{array}$ & $\begin{array}{l}14.4 \\
10.9\end{array}$ & $\begin{array}{l}15.4 \\
10.4\end{array}$ & $\begin{array}{l}13.5-17.5 \\
8.40-11.8\end{array}$ & $\begin{array}{l}\text { Acceptable } \\
\text { Acceptable }\end{array}$ \\
\hline $\mathrm{Cr}$ & $\begin{array}{l}1 \\
2\end{array}$ & $\begin{array}{l}121 \\
30.5\end{array}$ & $\begin{array}{l}127 \\
25.5\end{array}$ & $\begin{array}{c}111-144 \\
21.4-29.8\end{array}$ & $\begin{array}{l}\text { Acceptable } \\
\text { Acceptable }\end{array}$ \\
\hline $\mathrm{Cu}$ & $\begin{array}{l}1 \\
2\end{array}$ & $\begin{array}{l}309 \\
23.8\end{array}$ & $\begin{array}{l}330 \\
33.0\end{array}$ & $\begin{array}{c}292 \cdots 364 \\
27.7-37.6\end{array}$ & $\begin{array}{l}\text { Acceptable } \\
\text { Unacceptable }\end{array}$ \\
\hline $\mathrm{Pb}$ & $\begin{array}{l}1 \\
2\end{array}$ & $\begin{array}{l}16.5 \\
4.10\end{array}$ & $\begin{array}{l}15.0 \\
3.20\end{array}$ & $\begin{array}{l}11.7-18.6 \\
1.45-5.37\end{array}$ & $\begin{array}{l}\text { Acceptable } \\
\text { Acceptable }\end{array}$ \\
\hline $\mathrm{Hg}$ & $\begin{array}{l}1 \\
2\end{array}$ & $\begin{array}{l}5.47 \\
1.98\end{array}$ & $\begin{array}{l}5.76 \\
2.16\end{array}$ & $\begin{array}{l}4.65-6.75 \\
1.42-2.69\end{array}$ & $\begin{array}{l}\text { Acceptable } \\
\text { Acceptable }\end{array}$ \\
\hline $\mathrm{Ni}$ & $\begin{array}{l}3 \\
4\end{array}$ & $\begin{array}{l}4.67 \\
18.3\end{array}$ & $\begin{array}{l}2.00 \\
14.0\end{array}$ & $\begin{array}{r}0.977-3.03 \\
11.6-16.3\end{array}$ & $\begin{array}{l}\text { Unacceptable } \\
\text { Unacceptable }\end{array}$ \\
\hline $\mathrm{Se}$ & $\begin{array}{l}1 \\
2\end{array}$ & $\begin{array}{l}40.7 \\
10.4\end{array}$ & $\begin{array}{l}48.0 \\
12.0\end{array}$ & $\begin{array}{l}37.2-57.1 \\
8.79-14.4\end{array}$ & $\begin{array}{l}\text { Acceptable } \\
\text { Acceptable }\end{array}$ \\
\hline $\mathrm{Ag}$ & $\begin{array}{l}1 \\
2\end{array}$ & $\begin{array}{l}118 \\
6.40\end{array}$ & $\begin{array}{l}103 \\
6.45\end{array}$ & $\begin{array}{l}88.4-117 \\
5.22-8.04\end{array}$ & $\begin{array}{l}\text { Unacceptable } \\
\text { Acceptable }\end{array}$ \\
\hline $\mathrm{Tl}$ & $\begin{array}{l}3 \\
4\end{array}$ & $\begin{array}{r}<10.0 \\
13.5\end{array}$ & $\begin{array}{l}2.00 \\
18.0\end{array}$ & $\begin{array}{l}1.20-2.85 \\
14.3-21.6\end{array}$ & $\begin{array}{l}\text { Unusable data } \\
\text { Unacceptable }\end{array}$ \\
\hline \multicolumn{6}{|c|}{ Nitrate/nitrite/fluoride $(\mathrm{mg} / L)$} \\
\hline Nitrate, as $\mathbf{N}$ & $\begin{array}{l}1 \\
2\end{array}$ & $\begin{array}{l}0.620 \\
8.48\end{array}$ & $\begin{array}{l}0.600 \\
8.50\end{array}$ & $\begin{array}{c}0.433-0.820 \\
7.21-10.0\end{array}$ & $\begin{array}{l}\text { Acceptable } \\
\text { Acceptable }\end{array}$ \\
\hline Nitrite, as $\mathrm{N}$ & $\begin{array}{l}1 \\
2\end{array}$ & $\begin{array}{l}0.155 \\
0.907\end{array}$ & $\begin{array}{l}0.150 \\
0.900\end{array}$ & $\begin{array}{l}0.123-0.177 \\
0.779-1.02\end{array}$ & $\begin{array}{l}\text { Acceptable } \\
\text { Acceptable }\end{array}$ \\
\hline Fluoride & $\begin{array}{l}1 \\
2\end{array}$ & $\begin{array}{l}1.27 \\
1.68\end{array}$ & $\begin{array}{l}1.30 \\
1.72\end{array}$ & $\begin{array}{l}1.17-1.43 \\
1.55-1.89\end{array}$ & $\begin{array}{l}\text { Acceptable } \\
\text { Acceptable }\end{array}$ \\
\hline \multicolumn{6}{|c|}{ Insecticides $(\mu g / L)$} \\
\hline Chlordane & $\begin{array}{l}5 \\
6\end{array}$ & $\begin{array}{l}1.38^{b} \\
4.84^{b}\end{array}$ & $\begin{array}{l}1.32 \\
4.86\end{array}$ & $\begin{array}{r}0.611-1.75 \\
2.06-6.31\end{array}$ & $\begin{array}{l}\text { Acceptable } \\
\text { Acceptable }\end{array}$ \\
\hline Endrin & $\begin{array}{l}1 \\
2\end{array}$ & $\begin{array}{l}4.20 \\
0.25\end{array}$ & $\begin{array}{l}4.29 \\
0.293\end{array}$ & $\begin{array}{c}2.91-5.38 \\
0.182-0.414\end{array}$ & $\begin{array}{l}\text { Acceptable } \\
\text { Acceptable }\end{array}$ \\
\hline Heptachlor & $\begin{array}{l}7 \\
8\end{array}$ & $\begin{array}{l}0.23^{b} \\
2.25^{b}\end{array}$ & $\begin{array}{l}0.263 \\
3.15\end{array}$ & $\begin{array}{c}0.107-0.350 \\
1.32-4.14\end{array}$ & $\begin{array}{l}\text { Acceptable } \\
\text { Acceptable }\end{array}$ \\
\hline Heptachlor epoxide & $\begin{array}{l}7 \\
8\end{array}$ & $\begin{array}{l}0.16 \\
1.40\end{array}$ & $\begin{array}{l}0.161 \\
1.61\end{array}$ & $\begin{array}{c}0.0913-0.206 \\
0.992-2.09\end{array}$ & $\begin{array}{l}\text { Acceptable } \\
\text { Acceptable }\end{array}$ \\
\hline
\end{tabular}


Table 7.2.28 (continued)

\begin{tabular}{|c|c|c|c|c|c|}
\hline \multirow{2}{*}{ Parameter } & \multirow{2}{*}{$\begin{array}{l}\text { Sample } \\
\text { number }\end{array}$} & \multicolumn{2}{|c|}{ Values } & \multirow{2}{*}{$\begin{array}{l}\text { Acceptance } \\
\text { limits }\end{array}$} & \multirow{2}{*}{$\begin{array}{c}\text { Performance } \\
\text { evaluation }\end{array}$} \\
\hline & & Reported & True & & \\
\hline Lindane & $\begin{array}{l}1 \\
2\end{array}$ & $\begin{array}{l}3.74 \\
0.56\end{array}$ & $\begin{array}{l}3.16 \\
0.474\end{array}$ & $\begin{array}{c}2.15 \cdots 4.15 \\
0.267-0.623\end{array}$ & $\begin{array}{l}\text { Acceptable } \\
\text { Acceptable }\end{array}$ \\
\hline Methoxychlor & $\begin{array}{l}1 \\
2\end{array}$ & $\begin{array}{l}51.8^{b} \\
4.60^{b}\end{array}$ & $\begin{array}{c}73.2 \\
5.37\end{array}$ & $\begin{array}{l}38.5-87.2 \\
3.28-6.22\end{array}$ & $\begin{array}{l}\text { Acceptable } \\
\text { Acceptable }\end{array}$ \\
\hline Toxaphene & $\begin{array}{l}3 \\
4\end{array}$ & $\begin{array}{l}7.36 \\
2.26\end{array}$ & $\begin{array}{l}7.58 \\
2.33\end{array}$ & $\begin{array}{l}4.57-9.85 \\
1.50-3.33\end{array}$ & $\begin{array}{l}\text { Acceptable } \\
\text { Acceptable }\end{array}$ \\
\hline \multicolumn{6}{|c|}{ Herbicides $(\mu g / L)$} \\
\hline 2,4-D & $\begin{array}{l}1 \\
2\end{array}$ & $\begin{array}{l}4.88 \\
6.2^{b}\end{array}$ & $\begin{array}{l}5.70 \\
68.3\end{array}$ & $\begin{array}{l}2.11--8.09 \\
26.0-93.0\end{array}$ & $\begin{array}{l}\text { Acceptable } \\
\text { Acceptable }\end{array}$ \\
\hline 2,4,5-TP, silvex)1 & $\begin{array}{l}3.62^{b} \\
2\end{array}$ & $\begin{array}{r}4.30 \\
58.7^{b}\end{array}$ & $\begin{array}{c}1.56-5.76 \\
73.1\end{array}$ & $\begin{array}{c}\text { Acceptable } \\
23.7-100\end{array}$ & Acceptable \\
\hline \multicolumn{6}{|c|}{ Polychlorinated biphenyls $(\mu \mathrm{g} / L)$} \\
\hline PCB-Aroclor 1016/1242 & 1 & $<0.50$ & 0.100 & $0.290-0.209$ & Unacceptable \\
\hline РСB-Aroclor 1254 & 1 & $<1.00$ & 0.113 & $0.0518-0.172$ & Unusable data \\
\hline \multicolumn{6}{|c|}{ Trihalomethanes $(\mu \mathrm{g} / \mathrm{L})$} \\
\hline Bromodichloromethane & $\begin{array}{l}1 \\
2\end{array}$ & $\begin{array}{l}21.5 \\
57.6\end{array}$ & $\begin{array}{l}22.5 \\
57.8\end{array}$ & $\begin{array}{l}18.0-27.0 \\
46.2-69.4\end{array}$ & $\begin{array}{l}\text { Acceptable } \\
\text { Acceptable }\end{array}$ \\
\hline Bromoform & $\begin{array}{l}1 \\
2\end{array}$ & $\begin{array}{l}11.7 \\
73.3\end{array}$ & $\begin{array}{l}12.3 \\
66.9\end{array}$ & $\begin{array}{l}9.84-14.8 \\
53.5-80.3\end{array}$ & $\begin{array}{l}\text { Acceptable } \\
\text { Acceptable }\end{array}$ \\
\hline Chlorodibromomethane & $\begin{array}{l}1 \\
2\end{array}$ & $\begin{array}{l}6.98 \\
81.3\end{array}$ & $\begin{array}{l}7.66 \\
80.5\end{array}$ & $\begin{array}{l}6.13-9.19 \\
64.4-96.6\end{array}$ & $\begin{array}{l}\text { Acceptable } \\
\text { Acceptable }\end{array}$ \\
\hline Chloroform & $\begin{array}{l}1 \\
2\end{array}$ & $\begin{array}{l}11.1 \\
66.8\end{array}$ & $\begin{array}{l}10.6 \\
63.8\end{array}$ & $\begin{array}{l}8.48-12.7 \\
51.0-76.6\end{array}$ & $\begin{array}{l}\text { Acceptable } \\
\text { Acceptable }\end{array}$ \\
\hline Total trihalomethane & $\begin{array}{l}1 \\
2\end{array}$ & $\begin{array}{l}51.28 \\
279.0\end{array}$ & $\begin{array}{l}53.1 \\
269\end{array}$ & $\begin{array}{c}42.5-63.7 \\
215-323\end{array}$ & $\begin{array}{l}\text { Acceptable } \\
\text { Acceptable }\end{array}$ \\
\hline \multicolumn{6}{|c|}{ Volatile organic compounds $(\mu g / L)$} \\
\hline Benzene & 1 & 3.90 & 4.32 & $2.59 \cdots 6.05$ & Acceptable \\
\hline Carbon tetrachloride & 1 & 4.19 & 4.56 & $2.74-6.38$ & Acceptable \\
\hline 1,4-Dichlorobenzene & 1 & 2.37 & 2.50 & $1.50-3.50$ & Acceptable \\
\hline 1,2-Dichloroethane & 1 & 13.5 & 13.2 & $10.6-15.8$ & Acceptable \\
\hline 1,1-Dichloroethylene & 1 & 4.78 & 5.36 & $3.22-7.50$ & Acceptable \\
\hline 1,1,1-Trichloroethane & 1 & 2.82 & 3.21 & $1.93-4.49$ & Unacceptable \\
\hline Trichloroethylene & 1 & 6.22 & 7.36 & $4.42-10.3$ & Acceptable \\
\hline Vinyl chloride & 1 & 5.29 & 4.35 & $2.61-6.09$ & Acceptable \\
\hline Bromobenzene & 2 & 5.25 & 5.78 & $3.47-8.09$ & Acceptable \\
\hline 4-Chlorotoluene & 2 & 5.53 & 6.59 & $3.95 \cdots 9.23$ & Acceptable \\
\hline 1,2-Dibromochloropropanes & 3 & $<5.00$ & 0.804 & $0.482 \cdots 1.13$ & Unusabie data \\
\hline
\end{tabular}


Table 7.2 .28 (continued)

\begin{tabular}{|c|c|c|c|c|c|}
\hline \multirow{2}{*}{ Parameter } & \multirow{2}{*}{$\begin{array}{l}\text { Sample } \\
\text { number }\end{array}$} & \multicolumn{2}{|c|}{ Values } & \multirow{2}{*}{$\begin{array}{l}\text { Acceptance } \\
\text { limits }\end{array}$} & \multirow{2}{*}{$\begin{array}{c}\text { Performance } \\
\text { evaluation }\end{array}$} \\
\hline & & Reported & True & & \\
\hline 1,3-Dichlorobenzene & 2 & 6.80 & 8.09 & $4.85 \cdots 11.3$ & Acceptable \\
\hline C 1,2-Dichloroethylene & 2 & 6.35 & 7.24 & $4.34-10.1$ & Acceptable \\
\hline 1,3-Dichloropropane & 2 & 10.3 & 10.8 & $8.64-13.0$ & Acceptable \\
\hline 1,1-Dichloropropene & 2 & $<5.00$ & 8.88 & $5.33 \cdots 12.4$ & Acceptable \\
\hline 1,3-Dichloropropene & 2 & 6.88 & & $\mathrm{DL}^{c} \ldots \mathrm{DL}^{c}$ & Unacceptable \\
\hline Ethylene dibromide, EDB & 3 & $<5.00$ & 0.480 & $0.288-0.672$ & Unusable data \\
\hline 1,1,1,2-Tetrachloroethane & 2 & 5.00 & 5.90 & $3.54-8.26$ & Acceptable \\
\hline Total xylenes & 2 & 12.2 & 14.0 & $11.2-16.8$ & Unacceptable \\
\hline \multicolumn{6}{|c|}{ Miscellaneous parameters } \\
\hline Residual free $\mathrm{Cl}, \mathrm{mg} / \mathrm{L}$ & $\begin{array}{l}1 \\
2\end{array}$ & $\begin{array}{l}0.560 \\
0.950\end{array}$ & $\begin{array}{l}0.616 \\
1.16\end{array}$ & $\begin{array}{l}0.363-0.861 \\
0.840-1.44\end{array}$ & $\begin{array}{l}\text { Acceptable } \\
\text { Acceptablc }\end{array}$ \\
\hline Turbidity, NTUs & $\begin{array}{l}1 \\
2\end{array}$ & $\begin{array}{l}3.35 \\
1.40^{b}\end{array}$ & $\begin{array}{l}3.40 \\
1.00\end{array}$ & $\begin{array}{r}2.85-3.83 \\
0.735-1.13\end{array}$ & $\begin{array}{l}\text { Acceptable } \\
\text { Unacceptable }\end{array}$ \\
\hline Sulfate, $\mathrm{mg} / \mathrm{L}$ & $\begin{array}{l}1 \\
2\end{array}$ & $\begin{array}{l}4.75 \\
47.2\end{array}$ & $\begin{array}{l}5.30 \\
51.0\end{array}$ & $\begin{array}{l}3.01-7.48 \\
43.5-56.2\end{array}$ & $\begin{array}{l}\text { Acceptable } \\
\text { Unacceptable }\end{array}$ \\
\hline Total cyanide, $\mathrm{mg} / \mathrm{L}$ & $\begin{array}{l}1 \\
2\end{array}$ & $\begin{array}{l}0.180 \\
0.260\end{array}$ & $\begin{array}{l}0.310 \\
0.430\end{array}$ & $\begin{array}{l}0.222-0.364 \\
0.311-0.533\end{array}$ & $\begin{array}{l}\text { Unacceptable } \\
\text { Unacceptable }\end{array}$ \\
\hline
\end{tabular}

${ }^{a}$ Based on theoretical calculations or a reference value when necessary.

${ }^{b}$ Significant general method bias is anticipated for this result.

'Detection limit $(\mathrm{DL})=$ acceptance limit. 
Table 7.2.29. Water supply performance evaluation study number WS-025-ORGDP, 1989

\begin{tabular}{|c|c|c|c|c|c|}
\hline \multirow{2}{*}{ Parameter } & \multirow{2}{*}{$\begin{array}{c}\text { Sampie } \\
\text { No. }\end{array}$} & \multicolumn{2}{|c|}{ Values } & \multirow{2}{*}{$\begin{array}{l}\text { Acceptance } \\
\text { limits }\end{array}$} & \multirow{2}{*}{$\begin{array}{l}\text { Performance } \\
\text { evaluation }\end{array}$} \\
\hline & & Reported & True $^{a}$ & & \\
\hline \multicolumn{6}{|c|}{ Trace metals $(\mu g / L)$} \\
\hline $\mathrm{Sb}$ & $\begin{array}{l}3 \\
4\end{array}$ & $\begin{array}{c}20.3 \\
5.80\end{array}$ & $\begin{array}{c}21.0 \\
6.00\end{array}$ & $\begin{array}{l}14.8-29.6 \\
3.16-9.09\end{array}$ & $\begin{array}{l}\text { Acceptable } \\
\text { Acceptable }\end{array}$ \\
\hline As & $\begin{array}{l}1 \\
2\end{array}$ & $\begin{array}{r}52.3^{b} \\
8.00\end{array}$ & $\begin{array}{l}51.0 \\
8.50\end{array}$ & $\begin{array}{l}41.1-56.5 \\
6.16-10.1\end{array}$ & $\begin{array}{l}\text { Acceptable } \\
\text { Acceptable }\end{array}$ \\
\hline $\mathrm{Ba}$ & $\begin{array}{l}1 \\
2\end{array}$ & $\begin{array}{l}105 \\
365\end{array}$ & $\begin{array}{l}115 \\
369\end{array}$ & $\begin{array}{r}96.9-128 \\
318-402\end{array}$ & $\begin{array}{l}\text { Acceptable } \\
\text { Acceptable }\end{array}$ \\
\hline $\mathrm{Be}$ & $\begin{array}{l}3 \\
4\end{array}$ & $\begin{array}{l}2.01 \\
0.412\end{array}$ & $\begin{array}{l}2.00 \\
0.400\end{array}$ & $\begin{array}{c}1.81-2.23 \\
0.252-0.529\end{array}$ & $\begin{array}{l}\text { Acceptable } \\
\text { Acceptable }\end{array}$ \\
\hline $\mathrm{Cd}$ & $\begin{array}{l}1 \\
2\end{array}$ & $\begin{array}{c}2.69 \\
31.0\end{array}$ & $\begin{array}{l}2.30 \\
27.6\end{array}$ & $\begin{array}{l}1.79-2.92 \\
21.8-33.0\end{array}$ & $\begin{array}{l}\text { Acceptable } \\
\text { Acceptable }\end{array}$ \\
\hline $\mathrm{Cr}$ & $\begin{array}{l}1 \\
2\end{array}$ & $\begin{array}{l}15.4 \\
61.2\end{array}$ & $\begin{array}{l}15.0 \\
60.0\end{array}$ & $\begin{array}{l}12.0-18.1 \\
52.2-68.7\end{array}$ & $\begin{array}{l}\text { Acceptable } \\
\text { Acceptable }\end{array}$ \\
\hline $\mathrm{Cu}$ & $\begin{array}{l}1 \\
2\end{array}$ & $\begin{array}{l}55.2 \\
996\end{array}$ & $\begin{array}{l}59.4 \\
990\end{array}$ & $\begin{array}{c}51.2-67.2 \\
895-1060\end{array}$ & $\begin{array}{l}\text { Acceptable } \\
\text { Acceptable }\end{array}$ \\
\hline $\mathrm{Pb}$ & $\begin{array}{l}1 \\
2\end{array}$ & $\begin{array}{l}7.10 \\
22.1\end{array}$ & $\begin{array}{c}6.26 \\
20.0\end{array}$ & $\begin{array}{l}4.47-8.51 \\
16.2-23.0\end{array}$ & $\begin{array}{l}\text { Acceptable } \\
\text { Acceptable }\end{array}$ \\
\hline $\mathrm{Hg}$ & $\begin{array}{l}1 \\
2\end{array}$ & $\begin{array}{l}0.713 \\
4.27\end{array}$ & $\begin{array}{l}0.720 \\
4.32\end{array}$ & $\begin{array}{r}0.287-1.06 \\
3.12-5.50\end{array}$ & $\begin{array}{l}\text { Acceptable } \\
\text { Acceptable }\end{array}$ \\
\hline $\mathrm{Ni}$ & $\begin{array}{l}3 \\
4\end{array}$ & $\begin{array}{l}6.77 \\
3.56\end{array}$ & $\begin{array}{l}7.00 \\
7.00\end{array}$ & $\begin{array}{l}5.33-8.19 \\
1.20-4.68\end{array}$ & $\begin{array}{l}\text { Acceptable } \\
\text { Acceptable }\end{array}$ \\
\hline $\mathrm{Se}$ & $\begin{array}{l}1 \\
2\end{array}$ & $\begin{array}{l}13.4^{b} \\
64.5\end{array}$ & $\begin{array}{l}14.4 \\
72.0\end{array}$ & $\begin{array}{l}10.4-16.7 \\
56.6-86.1\end{array}$ & $\begin{array}{l}\text { Acceptable } \\
\text { Acceptable }\end{array}$ \\
\hline Ag & $\begin{array}{l}1 \\
2\end{array}$ & $\begin{array}{c}4.72 \\
43.3\end{array}$ & $\begin{array}{l}4.30 \\
43.0\end{array}$ & $\begin{array}{l}3.37--5.57 \\
35.8-51.0\end{array}$ & $\begin{array}{l}\text { Acceptable } \\
\text { Acceptable }\end{array}$ \\
\hline \multicolumn{6}{|c|}{ Trace metals } \\
\hline $\mathrm{Tl}$ & $\begin{array}{l}3 \\
4\end{array}$ & $\begin{array}{c}34.5 \\
3.40\end{array}$ & $\begin{array}{c}36.0 \\
3.00\end{array}$ & $\begin{array}{l}29.0-42.7 \\
1.75-4.25\end{array}$ & $\begin{array}{l}\text { Acceptable } \\
\text { Acceptable }\end{array}$ \\
\hline \multicolumn{6}{|c|}{ Miscellaneous analytes } \\
\hline Residual free chlorine, $\mathrm{mg} / \mathrm{L}$ & $\begin{array}{l}1 \\
2\end{array}$ & $\begin{array}{l}0.49 \\
1.76\end{array}$ & $\begin{array}{l}0.505 \\
1.40\end{array}$ & $\begin{array}{c}0.292-0.727 \\
1.11-1.66\end{array}$ & $\begin{array}{l}\text { Acceptable } \\
\text { Not acceptable }\end{array}$ \\
\hline Turbidity, NTU & $\begin{array}{l}1 \\
2\end{array}$ & $\begin{array}{l}0.95 \\
0.95\end{array}$ & $\begin{array}{l}0.450 \\
0.600\end{array}$ & $\begin{array}{l}0.265-0.660 \\
0.415-0.755\end{array}$ & $\begin{array}{l}\text { Not acceptable } \\
\text { Not acceptable }\end{array}$ \\
\hline Sulfate, $\mathrm{mg} / \mathrm{L}$ & $\begin{array}{l}1 \\
2\end{array}$ & $\begin{array}{l}9.64 \\
34.1\end{array}$ & $\begin{array}{l}9.70 \\
34.0\end{array}$ & $\begin{array}{l}6.92-12.2 \\
28.5-38.1\end{array}$ & $\begin{array}{l}\text { Acceptable } \\
\text { Acceptable }\end{array}$ \\
\hline Total cyanide, $\mathrm{mg} / \mathrm{L}$ & $\begin{array}{l}1 \\
2\end{array}$ & $\begin{array}{l}0.46 \\
0.19\end{array}$ & $\begin{array}{l}0.500 \\
0.220\end{array}$ & $\begin{array}{l}0.348-0.608 \\
0.150-0.269\end{array}$ & $\begin{array}{l}\text { Acceptable } \\
\text { Acceptable }\end{array}$ \\
\hline
\end{tabular}

${ }^{a}$ Based on theoretical calculations or a reference value when necessary.

${ }^{b}$ Significant general method bias is anticipated for this result. 
Table 7.2.30. CLP performance evaluation results-inorganics (ORNL, 1989)

\begin{tabular}{|c|c|c|c|c|}
\hline \multirow{2}{*}{$\begin{array}{l}\text { Scoring } \\
\text { classification }\end{array}$} & \multicolumn{4}{|c|}{ Points deducted } \\
\hline & $\begin{array}{c}\text { Ist } \\
\text { quarter }\end{array}$ & $\begin{array}{c}\text { 2nd } \\
\text { quarter }\end{array}$ & $\begin{array}{c}\text { 3rd } \\
\text { quarter }\end{array}$ & $\begin{array}{l}4 \text { th } \\
\text { quarter }\end{array}$ \\
\hline Duplicate precision $^{a}$ & 0 & 0 & 0 & 1.0 \\
\hline Matrix spikes ${ }^{b}$ & 0.5 & 0 & 0 & 2.0 \\
\hline \multicolumn{5}{|c|}{ Water sample } \\
\hline Identification & 0 & 0 & 0 & 0 \\
\hline Quantitation & 4.3 & 0 & 0 & 9.9 \\
\hline False positives and unmet CRDLs ${ }^{c}$ & 0 & 0 & 0 & 0 \\
\hline \multicolumn{5}{|c|}{ Soil sample } \\
\hline Identification & 0 & 0 & 0 & 0 \\
\hline Quantitation & 8.5 & 0 & 0 & 3.3 \\
\hline False positives and unmet CRDLs ${ }^{c}$ & 0 & 0 & 0 & 0 \\
\hline Total points deducted & 13.3 & 0 & 0 & 16.2 \\
\hline Laboratory score ${ }^{d}$ & $86.7^{\circ}$ & 0 & 0 & 83.8 \\
\hline
\end{tabular}

${ }^{\circ}$ Maximum of 10 points deducted based on number of duplicate results that are outside of the control limits.

${ }^{b}$ Maximum of 10 points deducted based on number of matrix spike results that are outside of the control limits.

'Points deducted for false positive values and for not meeting the contractrequired detection limits (CRDLs).

${ }^{d}$ The maximum number of possible points is 100 .

Table 7.2.31. CLP performance evaluation results-organics (ORNL, 1989)

\begin{tabular}{lcccc}
\hline \multicolumn{1}{c}{$\begin{array}{c}\text { Scoring } \\
\text { classification }\end{array}$} & \multicolumn{4}{c}{ Points deducted } \\
\cline { 2 - 5 } & $\begin{array}{c}\text { 1st } \\
\text { quarter }\end{array}$ & $\begin{array}{c}\text { 2nd } \\
\text { quarter }\end{array}$ & $\begin{array}{c}\text { 3rd } \\
\text { quarter }\end{array}$ & $\begin{array}{c}\text { 4th } \\
\text { quarter }\end{array}$ \\
\hline No. of TCL con pounds not identified & 8.1 & 0 & 0 & 7.9 \\
No. of TCL compounds misquantified & 20.3 & 6.0 & 0 & 7.9 \\
No. of TCL contaminants & 0 & 0 & 0 & 0 \\
No. of non-TCL compounds not identified & 5.5 & 0 & 0 & 0 \\
No. of non-TCL contaminants & 5.5 & 0 & 0 & 0 \\
Total points deducted & 39.4 & 6.0 & 0 & 15.8 \\
Laboratory score & 60.6 & 94.0 & 0 & 84.2 \\
\hline
\end{tabular}

${ }^{a}$ The maximum number of possible points is 100 . 
Table 7.2.32. CLP performance evaluation results-inorganics (ORGDP, FY 1989)

\begin{tabular}{|c|c|c|c|c|}
\hline \multirow{2}{*}{$\begin{array}{c}\text { Scoring } \\
\text { classification }\end{array}$} & \multicolumn{4}{|c|}{ Points deducted } \\
\hline & $\begin{array}{c}\text { 1st } \\
\text { quarter }\end{array}$ & $\begin{array}{l}\text { 2nd } \\
\text { quarter }\end{array}$ & $\begin{array}{c}\text { 3rd } \\
\text { quarter }\end{array}$ & $\begin{array}{c}4 \text { th } \\
\text { quarter }\end{array}$ \\
\hline Duplicate precision $^{a}$ & 0 & 0 & 0 & 0 \\
\hline Matrix spikes ${ }^{b}$ & 2 & 2 & 3 & 1 \\
\hline \multicolumn{5}{|c|}{ Water sample } \\
\hline Identification & 0 & 0 & 0 & 0 \\
\hline Quantitation & 0 & 3 & 0 & 0 \\
\hline False positives and unmet CRDLs ${ }^{c}$ & 0 & 0 & 0 & 0 \\
\hline \multicolumn{5}{|c|}{ Soil sample } \\
\hline Identification & 0 & 0 & 0 & 0 \\
\hline Quantitation & 0 & 1 & 0 & 0 \\
\hline False positives and unmet CRDLs ${ }^{c}$ & 0 & 0 & 0 & 0 \\
\hline Total points deducted & 5.7 & 16.1 & 1.5 & 1.5 \\
\hline Laboratory score $^{d}$ & 94.3 & 83.9 & 98.5 & 98.5 \\
\hline
\end{tabular}

${ }^{a}$ Maximum of 10 points deducted based on number of duplicate results that are outside of the control limits.

${ }^{b}$ Maximum of 10 points deducted based on number of matrix spike results that are outside of the control limits.

'Points deducted for false positive values and for not meeting the contractrequired detection limits (CRDLs).

${ }^{d}$ The maximum number of possible points is 100 .

Table 7.2.33. CLP performance evaluation results-organics (ORGDP, FY 1989)

\begin{tabular}{lcccc}
\multicolumn{1}{c}{$\begin{array}{c}\text { Scoring } \\
\text { classification }\end{array}$} & \multicolumn{3}{c}{ Points deducted } \\
\cline { 2 - 5 } & $\begin{array}{c}1 \mathrm{st} \\
\text { quarter }\end{array}$ & $\begin{array}{c}2 \mathrm{~d} \\
\text { quarter }\end{array}$ & $\begin{array}{c}\text { 3rd } \\
\text { quarter }^{b}\end{array}$ & $\begin{array}{c}\text { 4th } \\
\text { quarter }\end{array}$ \\
\hline No. of TCL compounds not identified & 3 & 0 & 0 \\
No. of TCL compounds misquantified & 7 & 5 & 3 \\
No. of TCL contaminants & 0 & 0 & 0 \\
No. of non-TCL compounds not identified & 0 & 0 & 1 \\
No. of non-TCL contaminants & 0 & 0 & 2 \\
Total points deducted & 52.7 & 15.0 & 18.4 \\
Laboratory score $^{a}$ & 47.3 & 85.0 & 81.6 \\
\hline
\end{tabular}

a The maximum number of possible points is 100 .

${ }^{b}$ EPA did not evaluate any data. 


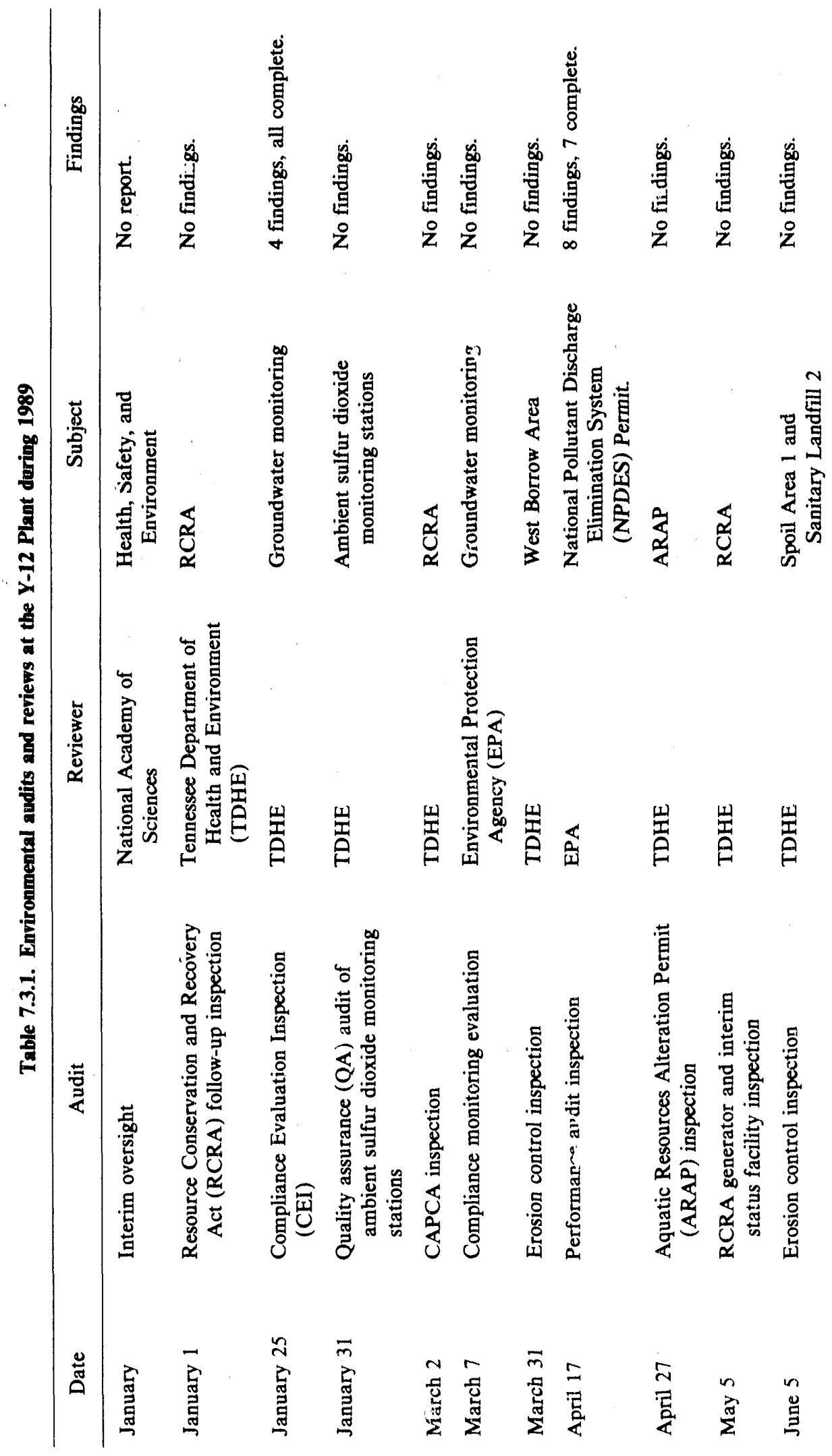




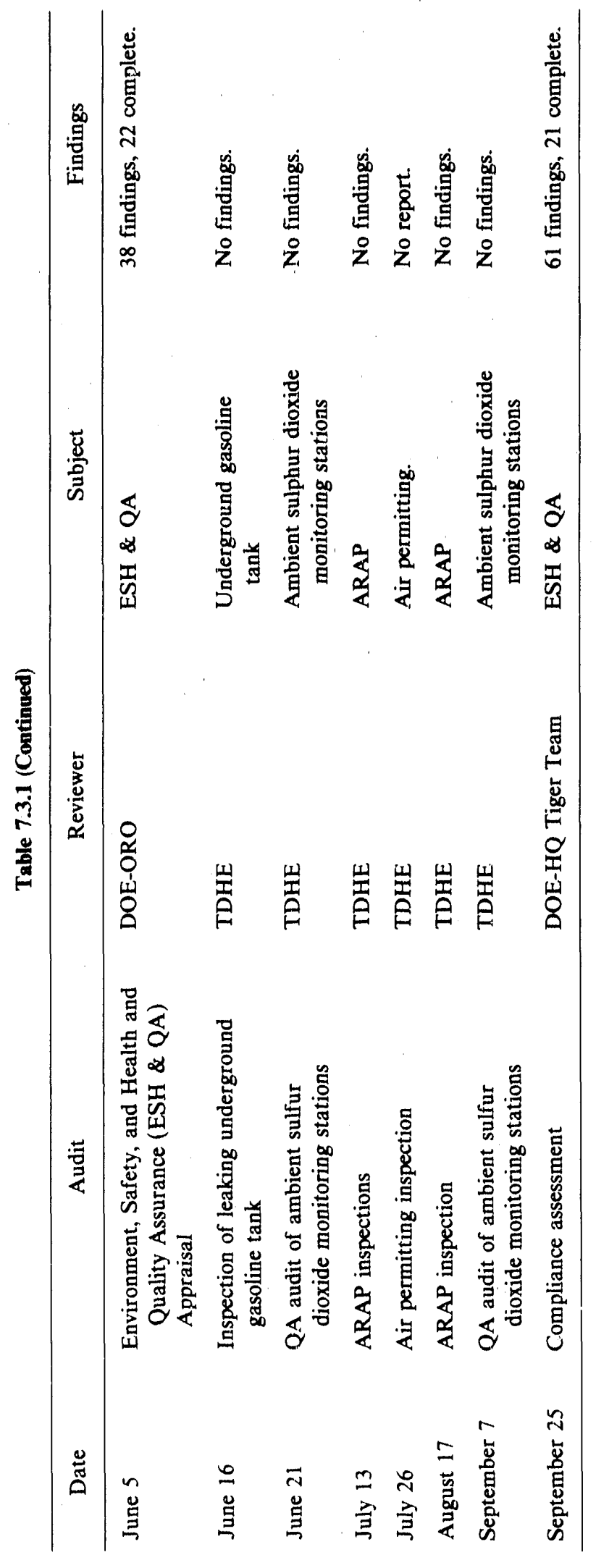




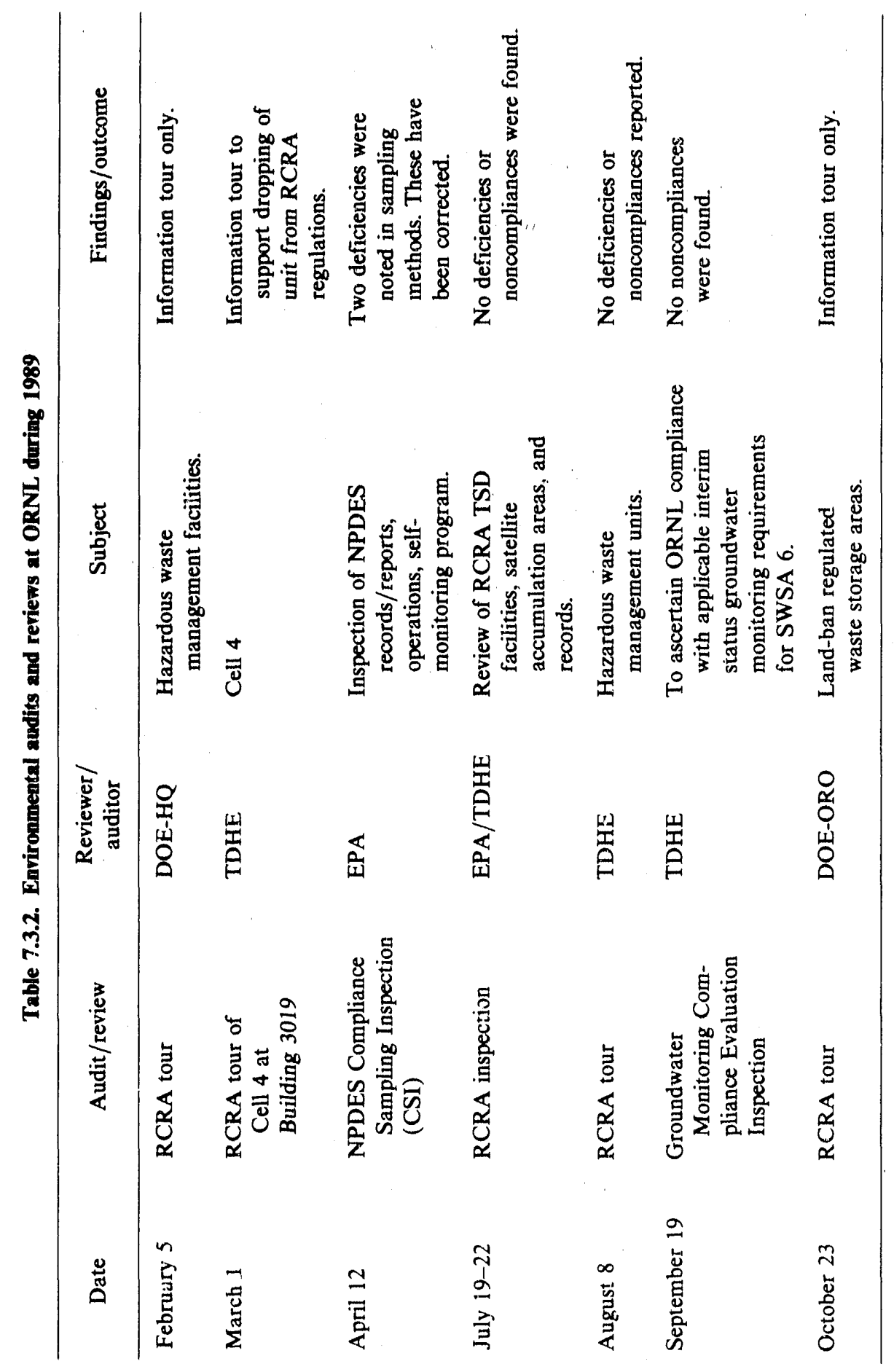




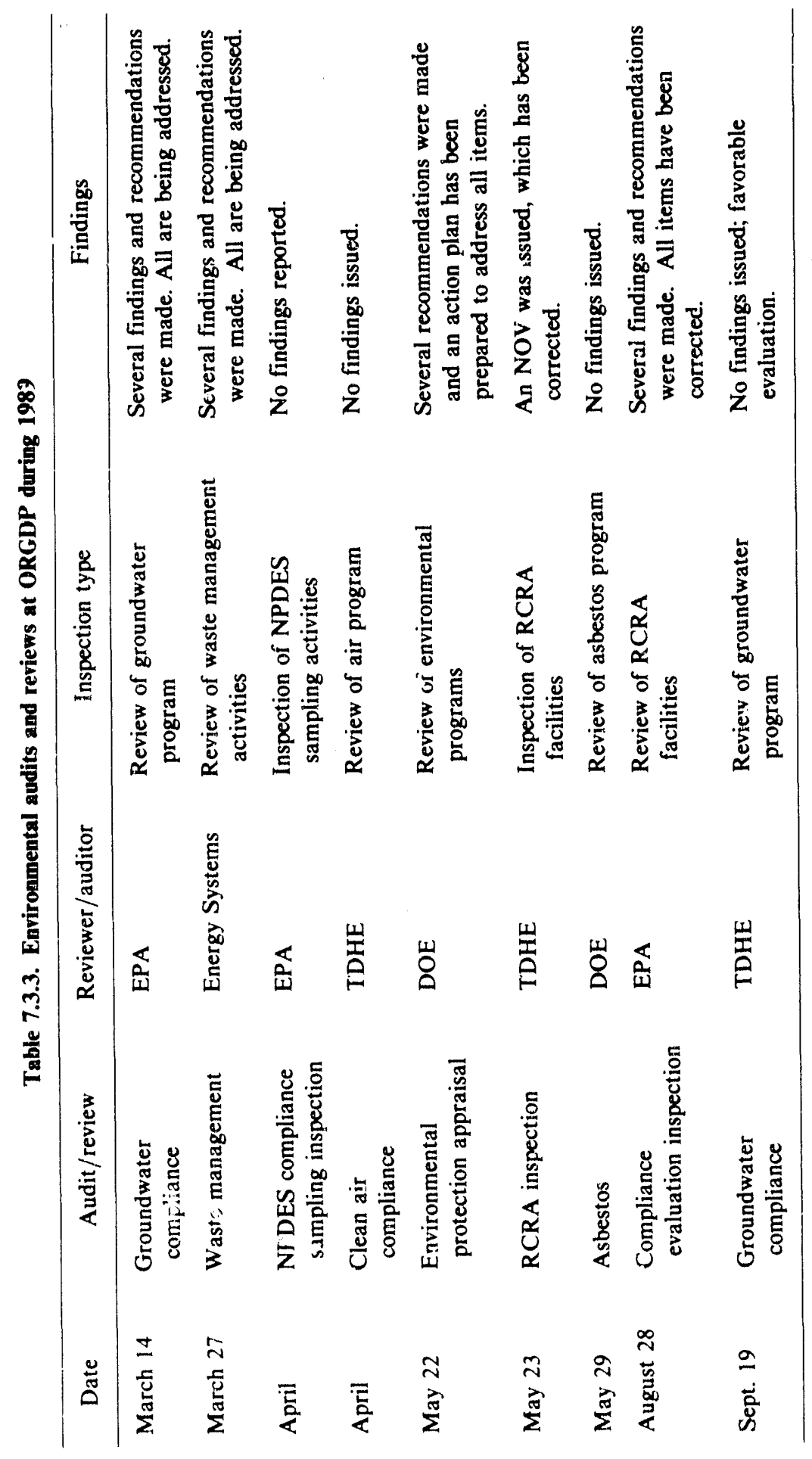



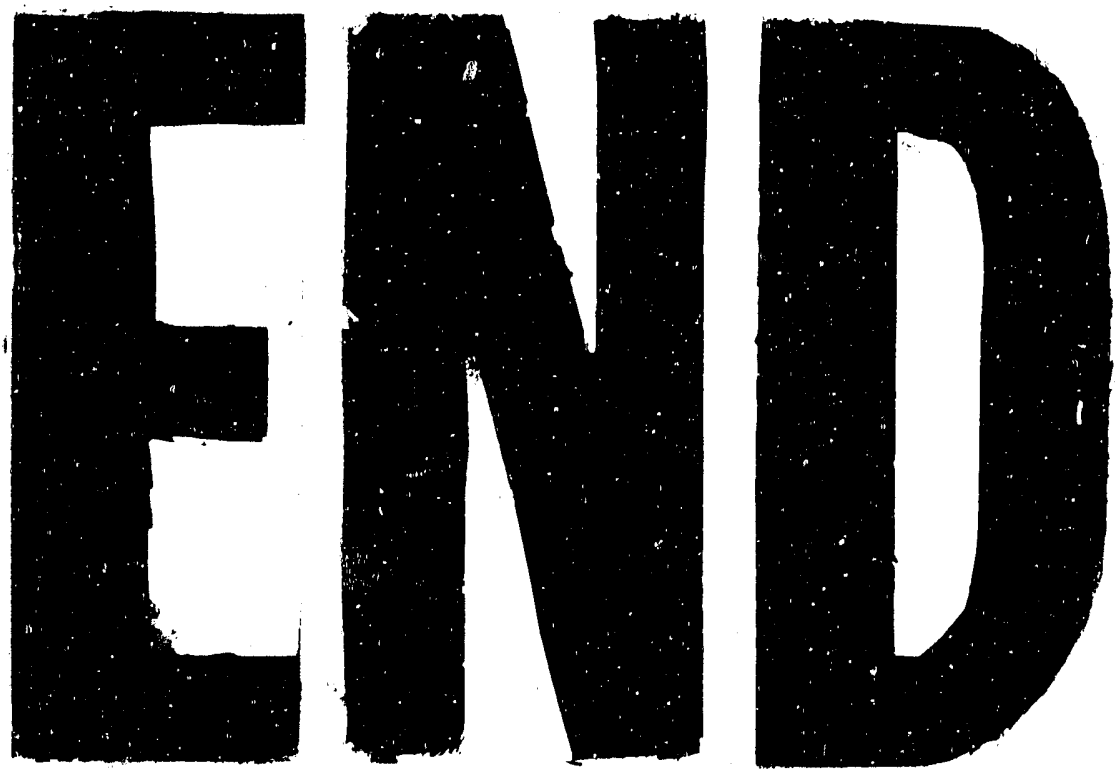

4
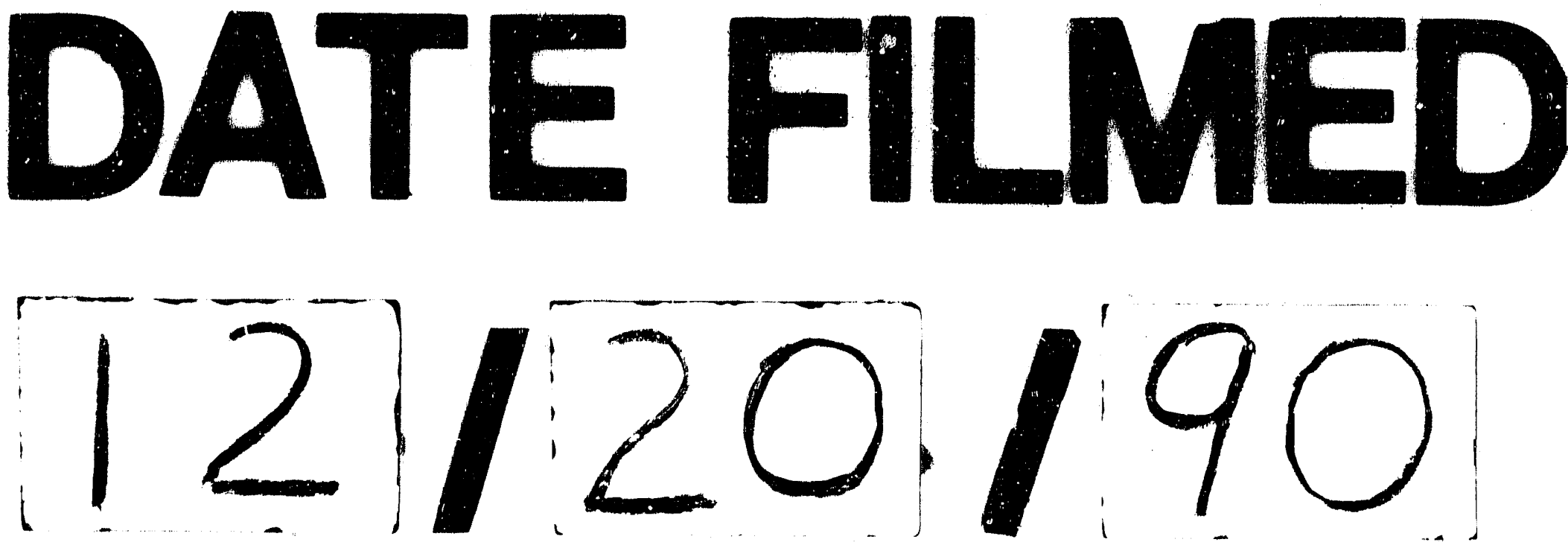
